\title{
Proceedings
}

\section{Future Directions of Music Cognition}

February 22 - May 31, 2021

Ohio State University, Virtual

Edited by Joshua Albrecht, Lindsay Warrenburg,

Lindsey Reymore, Daniel Shanahan 


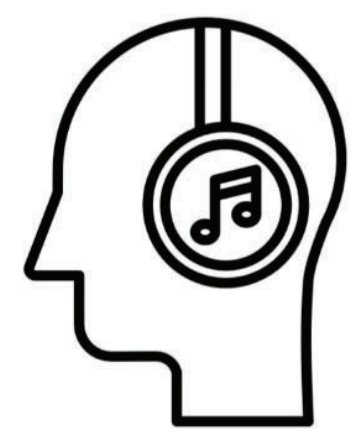

\section{Future Directions of Music Cognition February - May 2021}

\section{Proceedings}

Edited by Joshua Albrecht, Lindsay Warrenburg,

Lindsey Reymore, Daniel Shanahan

Ohio State University

Virtual 


\section{Table of contents}

\section{Editorial introduction}

Albrecht, J., Warrenburg, L., Reymore, L., \& Shanahan, D.

Introduction to Future Directions of Music Cognition proceedings

\section{Section 1: Speaker series}

Huron, D.

On the future of music research

Loui, P.

Use-inspired music cognition: Designing cognitively informed musical interventions for the brain

\section{Section 2: Corpus Studies}

Blake, A.

Tempering the clavier: Examining the intervallic content of Bach's Well-Tempered Clavier through the lens of historical temperaments

Cumming, J.E. \& McKay, C.

Using corpus studies to find the origins of the madrigal

de Clercq, $T$.

How should corpus studies of harmony in popular music handle the minor tonic?

$\mathrm{Hu}, \mathrm{T}$. \& Arthur, C.

A statistical model for melody reduction

Light, L. \& Arthur, C.

Voice-leading in Palestrina's masses: A comparison of interval-succession definitions

Lyon, J. \& Yorgason, B.

Tagging film music: A corpus study of Max Steiner's film scores

Miyake, J.

Implications of thematic reuse in Haydn's sonata forms

O’ Connor, R. \& Wu Fu, P.

Musical instrument, personality and interpretation: Music cognition at a college-conservatory

O'Hara, W.

Upham, F. \& Cumming, J.

Streaming complexity in the Renaissance Mass Ordinary cycle

White, C.W.

Deployments of change and novelty in a corpus of popular music

White, C.W., Fulmer, J., Cordova, B., Black, A., Danitz, C., Evans, W., Fischer, A., Greene, R., He, J., Kenyon, E., Miller, J., Moylan, M., Ring, A., Schwitzgebel, E., \& Wang, Y.

\section{Section 3: Emotion}

Al Alam, T. \& Dibben, N.

A comparison of presence and emotion between immersive virtual reality and desktop displays for musical multimedia 
Chen, E., Yohannes, E., Burgess, S., \& Shanahan, D.

Dropping the bass: The relationship between heart rate and expectation in electronic dance music

Lopez, G. \& VanHandel, L.

Hearing, seeing, liking: The effects of audio-visual listening conditions on perceptual ratings

Madden, G.B. \& Jabusch, H.

Regulating functional and hedonic emotions in the pursuit of musical practice goals

Miskinis, A., Lin, X., \& Kanaan, S.

Virtual Harmony: Music interaction with virtual reality to reduce stress

Riggle, $\mathrm{M}$.

Pleasurable music selects for enhanced music memory, hence music emotions: The evolutionary forces laid bare

Sankhe, P. \& Madan, R.

Cortical representations of auditory perception using graph independent component analysis on EEG

Tate, M., Greenberg, D.M., \& O’Neill, S.

Sound and feeling: Musical training moderates the association between adult ADHD and emotion regulation

Wang, A.Y.

Stimulus accessibility and music theory/therapy

Warrenburg, L., Centa, N., Li, X., Park, H., Sari, D., \& Xie, F.

Sonic intimacy in the music of Billie Eilish and recordings that induce ASMR

Emotion Abstracts

\section{Section 4: Pedagogy}

Chenette, T., Phillips, A., \& Wood, E.

Does Gestalt hearing exist?

Okada, B.M. \& Slevc, L.R.

What is "musical ability" and how do we measure it?

Pomerleau-Turcotte, J., Sala, M.T.M, Dubé, F., \& Vachon, F.

Does cognitive load differ among sight-singers? An exploratory study using pupillometry and interviews

Summa-Chadwick, M.

Reaching across the aisle: The essential need for collaboration among researchers, medical personnel, therapists, musicians, and educators

Pedagogy Abstracts

\section{Section 5: Perception}

Gardner, S., Berezina-Blackburn, V., \& Shanahan, D.

The effect of gesture on the perception of linearity in instrumental music

Ho, $\mathrm{H}$.

Rethinking gesture theory via embodiment and acousmatic music

Kuusi, T., Jimenez, I., \& Schulkind, M.D.

Identifying Beatles songs from their chord progressions: New evidence of the effect of specialized harmonic familiarity, melodic cues, and transposition on the identification of songs from chord progressions 
Schmuckler, M.

Music surface and musical structure: The role of abstraction in musical processing

Teo, Y.

Post-tonal harmonic tension and theoretical hybridity

\section{Section 6: Rhythm \& Meter}

Breen, M. \& Fitzroy, A.

Pitch cues to hierarchical metric structure in children's poetry

Delasanta, L.

Information specification during singing: A theoretical approach to music performance

Ferguson, B.

Form and intertextuality in movie music videos

Fink, L.K.

Computational models of temporal expectations

Kowalewski, D., Kratzer, T., \& Friedman, R.

Social music: Investigating the link between personal liking and perceived groove

Lookenbill, Z. \& VanHandel, L.

Effect of metrical primes on perceived complexity of 2:3 and 3:4 polyrhythms

Percival, $\mathrm{H}$.

Find your groove: A pilot study on the influences of beat salience and social connectedness on groove perception

Schmuckler, M. \& Moranis, R.

Rhythm contour drives musical memory

Rhythm Abstracts

\section{Section 7: Timbre}

Bulla, W.A. \& Chon, S.H.

An investigation of loudness effect on pitch and timbre discrimination

Fakhrtabatabaie, A. \& Jennings, S.G.

Evaluating Tenney's critical band using a computational model of the human cochlea

Timbre's function in the perception of affective intentions: Contextual information and effects of learning

Kahrs, N. \& Chiu, M.

Chord spacing and quality: Lessons from timbre research

Maxwell, B.N., Fritzinger, J.B., \& Carney, L.H.

A new auditory theory and its implications for the study of timbre

Winikoff, J.R.

Kuvunga: Timbre, interlocking, and composite melodies in Zambian Luvale ngoma

\section{$\underline{\text { Editorial Coda }}$}

Albrecht, J.

Coda: Charting Future Directions of Music Cognition in turbulent times 


\title{
Introduction to Future Directions of Music Cognition proceedings
}

\author{
Joshua Albrecht ${ }^{1 \dagger}$ \\ Lindsay Warrenburg ${ }^{2}$ \\ Lindsey Reymore ${ }^{2}$ \\ Daniel Shanahan ${ }^{2}$ \\ ${ }^{1}$ Hugh A. Glauser School of Music, Kent State University, Kent, OH, USA \\ ${ }^{2}$ School of Music, Ohio State University, Columbus, OH, USA \\ Published 16 December 2021; https://doi.org/10.18061/FDMC.2021.0001 \\ † Corresponding author: jalbrec6@kent.edu
}

\begin{abstract}
The Future Directions of Music Cognition conference developed parallel to many of the societal changes brought about by the COVID-19 pandemic. The result was an innovative approach to online, remote conferencing. This proceedings journal reflects the research presented at the conference and speaker series, but the virtual nature of the conference also provides opportunities for this journal to be multimedia in new and exciting ways. Most of the articles in this journal are accompanied by an online multimedia presence, with articles linked to videos of the authors presenting, slides, and access to original data and stimuli. This article reviews the story of how the conference and speaker series developed during the pandemic, some of the achievements of the conference, and how the journal is structured.
\end{abstract}

\section{Retrospective: How we got here}

It takes a certain degree of boldness to invoke the idea of Future Directions when starting a new project. Research develops quickly, especially in the sciences. Rapidly developing technology consistently drives methodological innovations, and new knowledge is constantly being generated through the collection of new experimental data. The field of music cognition is a case in point, with increasing expansion in recent years, reflected both in new research questions and ever deepening subfield specializations.

Nevertheless, in the summer of 2019, Lindsay Warrenburg, Lindsey Reymore, Daniel Shanahan, and I sat down to begin planning the Future Directions of Music Cognition conference, originally scheduled for the summer of 2020 at The Ohio State University in Columbus, Ohio. The development of the conference was motivated precisely by these recent rapid developments in the field. Given the increasing size and scope of the field, one goal for the conference was to provide a resource for researchers wanting to evaluate methods and technologies outside of their primary area of specialization. We also wanted to create new vehicles for participants to engage in dialogue about the field and its future directions while having a centralized forum to review current methodological innovations and trends.

To accomplish these goals, the conference was designed in part as a deep dive into five popular subspecialities to review the current state of the field and possibilities for future directions of research. These five topics - corpus studies, emotion, rhythm and meter, timbre, and pedagogy - would each have been featured for one of the conference's five days. Each day would have featured a blend of activities related to the sub-discipline, including a keynote presentation and a methodology workshop led by an expert on the topic, presentations and posters on the topic by conference attendees, and concentrated working groups led by the expert to design and potentially run studies on that topic to be presented at the end of the conference.

Of course, it's always challenging to accurately predict the future. But 2019 might have been one of the single worst times in recent history to make long term plans. After we had secured our five topic experts, received 114 submissions for presentations and posters, and recruited a panel of 25 expert reviewers to recommend submissions for acceptance, the global COVID-19 pandemic broke out, followed by an unprecedented shutdown of the worldwide economy and the near elimination of face-to-face academic discourse. Consequently, our original plans for an inperson conference were first postponed and then redirected entirely.

\section{The new shape of the conference}

In grappling with the realities of a worldwide pandemic, we quickly decided that it would be better to 
reimagine the conference rather than to simply substitute subpar virtual imitations for our original inperson plans. Given the realities of screen fatigue, we were also wary of a week-long online conference schedule. The challenges of moving online, however, also brought opportunities for us to more fully incorporate some of our values into the structure of the conference. For example, our organizing principles included free and open access to conference resources, an emphasis on advancing opportunities for young scholars, and increasing the inclusivity of our community.

With these values and constraints in mind, Future Directions of Music Cognition was reimagined as two complementary events. The first of these events was an online conference, held March 6-7, 2021. This conference featured the originally accepted talk and poster presentations in sessions in topical groupings, roughly corresponding to the original conference. The two-day long conference featured 49 long talks of 12 minutes and 21 lightning talks ("posters") of 5 minutes. A full schedule can be found at the MASCATS website (MASCATS, March 6-7 Schedule, 2021).

To increase accessibility, conference registration was free and open to the public. With travel costs eliminated, over 100 attendees from around the world were able to participate in the two-day conference. In our efforts to support young scholars, we were able to honor six exemplary student presentations with awards totaling \$1,800 (MASCATS, Student Awards, 2021). The conference also featured several opportunities for participants to socialize. These included video conferencing sites available during coffee and lunch breaks, and three sessions in which participants were randomly paired up for five-minute social sessions. A Slack channel, dedicated to conference chat about sessions and presentations, happened in real time and retrospectively (Slack, 2021). Over 500 people from 16 different time zones participated on this Slack channel, consistent with our goal of expanding access to the conference. Finally, through hosting with the Open Science Framework, we have uploaded all conference presentations for permanent and free online access, including both pre-recorded presentations and live recordings at the conference (Open Science Framework, Future Directions of Music Cognition March Conference, 2021). Readers of this proceedings journal are encouraged to supplement their reading with videos of the authors presenting, in which sound examples and other multimedia enrichment are often included.
The second component of Future Directions was a speaker series. The shift to a virtual conference allowed us to greatly expand the number of experts involved. Fifteen presentations were delivered weekly in a synchronous online format to our worldwide audience between February and May 2021. Consistent with our goal of opening up resources to a wide audience, over 1,200 people signed up for some aspect of the conference, representing 427 universities and 32 industries in 46 countries on 6 continents. Topics aligned with the original five sub-specialties within music cognition along with several other topic areas, such as neuroscience, music theory, music industry and technology, genetics, and sociology. The speaker series included thirteen hour-long keynote talks and two additional initiatives. The first initiative, an "alt-ac" panel discussion, featured four researchers pursuing careers outside of academia and provided a variety of resources for those interested in following alternative career paths beyond the traditional academic route (Open Science Framework, Alt-ac Resources, 2021). The second initiative was an interactive town hall discussion centered on the future role of anti-racism efforts in the Society for Music Perception and Cognition (Gather Town, 2021). Speaker series presentations were recorded and uploaded to the Open Science Framework, and will be stored in perpetuity as a freely accessible resource available to researchers and the public (Open Science Framework, Future Directions of Music Cognition - Speaker Series, 2021).

Together, the online virtual conference and the speaker series represent a significant effort of both established and new scholars to grapple with the current and future state of our scientific communities and research in light of the evolving nature of our field and new methods and theories.

\section{How to read this journal}

The structure of the proceedings journal reflects the conception of the conference. There are seven main sections, starting with articles by David Huron (pp. 524) and Psyche Loui (pp. 25-31), two of the speaker series presenters. Huron's paper, "On the future of music research," is a wide ranging article on the nature of music research, examining some first principles, reviewing some of the most significant achievements, and looking forward to new fruitful directions music research may take, followed by a reproduction of the question and answer period after his talk. Loui's paper, 
"Use-inspired music cognition: Designing cognitively informed musical interventions for the brain," casts light on the future of music cognition research by reviewing and drawing lessons from the early days of the field, arguing for a deeper integration of pure music cognition research with practical applications of musicbased interventions that can genuinely help people and make the world a better place.

Following this first section, the remaining sections reflect the conference's contributions in six of the music cognition sub-disciples represented by the conference, including corpus studies, music and emotion, pedagogy, music perception, rhythm and meter, and timbre. Each section consists of full-length articles organized by first author last name. Sections end with the published abstracts for that sub-discipline. Finally, the journal ends with an editorial epilogue that reflects on how the Future Directions of Music Cognition conference, speaker series, and proceedings interact with some of the societal changes that have happened between the original conception of the conference and its conclusion as a way of looking forward to what future directions the field may take.

As mentioned above, all conference presentations were recorded and posted in perpetuity on the Open Science Framework's (OSF) Future Directions of Music Cognition - March Conference web page (2021). Speaker series talks are also freely available at OSF's Future Directions of Music Cognition Speaker Series website (2021). Most of the articles in this journal were also presented at the conference, and so readers can watch video presentations on OSF. Additionally, several OSF pages also include slides of presentations, original data from studies, stimuli used, or other background materials. DOI links for each presentation are included in the header of each article below author information. Interested readers are encouraged to further examine online content.

Finally, as of this publication date the conference Slack channel (2021) remains available, with separate channels for each session. Visitors to the Slack channel can review the history of questions, answers, and comments associated with each paper. By joining the Slack channel, readers may even post their own questions to authors and continue public dialogue about the research in this journal.

\section{References}

Gather Town. (2021). Town Hall on Anti-Racism in Music Cognition.

https://gather.town/app/BYatfy3d9rD3cwA0/FD antirac ismTH

MASCATS. (2021). March 6-7 schedule. http://org.osu.edu/mascats/march-6-7-schedule/

MASCATS. (2021). Student Awards. http://org.osu.edu/mascats/student-awards

Open Science Framework. (2021). Alt-ac Resources Future Directions. https://osf.io/gbcp6

Open Science Framework. (2021). Future Directions of Music Cognition - March Conference. https://osf.io/meetings/FD2021Conf/

Open Science Framework. (2021). Future Directions of Music Cognition - Speaker Series. https://osf.io/meetings/FD2021Sp

Slack. (2021). Future Directions of Music Cognition. futuredirecti-nvq6273.slack.com 


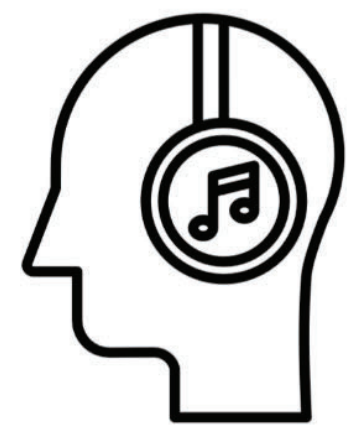

Speaker Series

Future Directions of Music Cognition February - May 2021

Ohio State University

Virtual 


\title{
On the future of music research
}

\author{
David Huron ${ }^{1 \dagger}$ \\ ${ }^{1}$ Ohio State University, Columbus, Ohio, USA \\ † Corresponding author: huron.1@osu.edu
}

Published 16 December 2021; https://doi.org/10.18061/FDMC.2021.0002

Author video presentation and/or other conference material: https://doi.org/10.17605/OSF.IO/BGD8Q

\begin{abstract}
Several observations are made regarding the state of music research. An extended list of research questions is presented and descriptive, hermeneutic, explanatory, and poetic approaches to arts scholarship distinguished. It is proposed that a valuable area for future research is policy-related scholarship, especially research oriented toward advancing the public interest in cultural matters. While the goals of public/applied musicology are endorsed, attention is drawn to the risks of relying on practices that are not evidence based. The critical role played by explanatory research as an agent of change is highlighted. At the same time, doubts are expressed regarding the potential for music to precipitate major political transformation. It is suggested that corpus studies offer a promising future research avenue. Multimeasure research methods are endorsed and an integrated biopsycho-socio-cultural approach to musical understanding is advocated. Public questions and answers follow. ${ }^{1}$
\end{abstract}

KEYWORDS: music research, motivating questions, applied musicology, music and politics, music science, cultural policy

\section{Introduction}

Over the centuries, music has become an increasingly prominent part of people's lives. I don't need to show you data in order to convince you that people today listen to much more music than was the case, say, two or three hundred years ago. But it's not just the case that people engage music more often than in the past. It's also the case that people engage in talking and writing about music much more than in the past.

Figure 1 traces the frequencies of the words "music" and "musique" as they appear in English and French language books since 1700. What's important to understand here is that these graphs don't simply indicate that there has been more published material concerning music over time. That's pretty much true of every topic. The graphs show the relative appearance of the words "music" and "musique" in published books over a three-hundred year period. That is, music has become proportionally much more important as a topic for writers over the past three centuries.

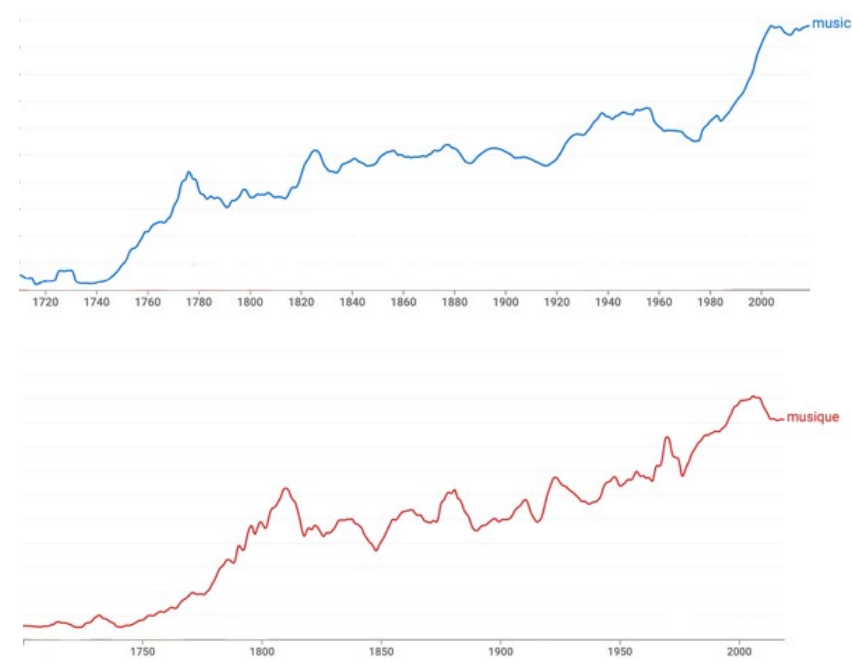

Figure 1: Relative frequencies of appearance for the words "music" and "musique" in English and French texts (Google ngram).

Figure 2 shows the notable prominence of this musical enthusiasm compared with other human passions like sports, movies, television, dance, theater, and the internet:

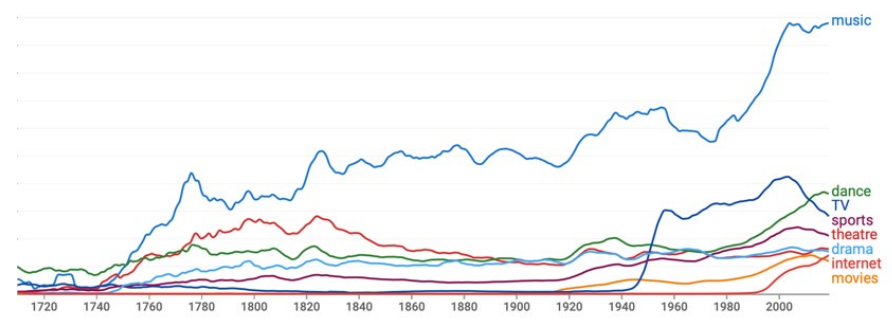

Figure 2: Comparison of relative frequencies for various entertainment-related words in English texts from 1700 to present.

I think what this testifies to is that, it's not simply the case that music-making and listening has become an increasingly prominent activity in people's lives, but that people are interested in learning and talking about 
music; that is, people are much more engaged in ideas about music. Like the music itself, there are pleasures to be had from simply reading about, thinking about, and discussing music.

There are lots of different ways we can talk about music: we talk about our musical likes and dislikes; we gossip about our favorite musicians; we read biographies and pedagogical works on music. And there are plenty of philosophical discussions and speculations. It is this propensity to talk about music where music research fits in. Research contributes an important part to the thoughts and conversations we have about music.

Of course, research doesn't merely provide compelling stories about music. It also can have practical utility. At its best, research can help us solve problems, can offer new insights into how music works its wonders, suggest alternative approaches to music and music-making, and potentially point to possible unexplored musical realms - that is, novel regions of musical expression that have yet to be investigated by creative artists.

As musicians and music scholars, we surely want music to thrive - to be all that it can be. We want to promote and extend music to serve human well-being in as many ways as music is capable of doing that.

What we can predict about the future of music research is that researchers will surely explore all the ways in which sounds can be compelling to human minds. And we might further expect that, except insofar as people have only limited leisure time, the disposition to write and talk about music will continue well into the future.

\section{Types of Arts Research}

Of course, there are different types of research. Research is inherently an open-ended enterprise, so any effort to create a taxonomy of research is necessarily suspect. Nevertheless, broadly speaking, we might distinguish at least four approaches to arts scholarship, which I will refer to as descriptive, hermeneutic, explanatory, and poetic.

Descriptive Scholarship aims to describe some practice, work, or culture without offering any interpretation. Examples of descriptive scholarship are readily found in music history, music theory, and ethnomusicology. For example, much historical research is descriptive - as when a biography simply chronicles the events in a musician's life. Many music analyses are also descriptive, as when a roman numeral analysis identifies a sequence of harmonic events. And the simplest ethnography might, for example, provide a detailed description of a ritual or performance without necessarily offering any interpretation other than to report or relay the interpretations given by the people involved. Descriptive scholarship also includes firstperson or phenomenological reports of the writer's subjective experiences.

Hermeneutic Scholarship, by contrast, aims to interpret some practice, work, or culture. Traditionally, hermeneutic scholarship aimed to decode or decipher the meaning of some text, such as attempting to clarify what an author might have meant by a particular passage (Zimmermann, 2015). An ethnography moves from descriptive to hermeneutic when the researcher proposes some novel interpretation that differs or augments what the people involved might say. It's common in musicology to offer hermeneutic interpretations of musical works, such as Susan McClary's famously controversial interpretation of a passage from Beethoven's ninth symphony as a representation or manifestation of sexual violence (McClary, 1987). Hermeneutic scholarship might also involve practical suggestions regarding how to perform or interpret a given work or passage.

Explanatory Research might be considered a special type of hermeneutic scholarship. Like hermeneutic scholarship, explanatory research has the aim of interpreting some phenomenon. However, explanatory research focuses exclusively on causal interpretations. The goal is to provide an account of how and why something occurs. Explanatory scholarship might propose why a particular work is organized the way it is, why listeners respond the way they do, or why certain practices, tendencies, or dispositions might be observed. The narratives are restricted to plausible causal scenarios or accounts.

Finally, there's what might be called Poetic Scholarship. This form of scholarship is unique to the arts and is not found in the sciences. It's imaginative scholarship that offers a creative response to some phenomenon.

An example of poetic scholarship would be Robert Schumann's programmatic stories describing Chopin's music through the imaginary characters of Florestan and Eusebius. But there's plenty of formal music analysis that fits into this category as in what David Temperley has referred to as "suggestive" analysis (Temperley, 1999). The scholarship may suggest how a work, performance, or experience might be approached or interpreted, without implying that this is the only way, 
the right way, a better way, a useful way, or even a beautiful way. (By contrast, hermeneutic suggestions for performance assume that some interpretations are better than others.)

Poetic scholarship is itself best viewed as art: it's art in response to art. The artist-scholar responds to some phenomenon by creating another work of imagination.

Poetic scholarship differs from descriptive, hermeneutic and explanatory research in that it makes no knowledge claims. For example, poetic scholarship differs from introspective description in that there is no implied claim that the description is accurate or representative of one's experience. We appreciate poetic scholarship, not according to its truth claims, but by such criteria as enjoyment, novelty, inventiveness, interest, beauty, and humor. Poetic scholarship is assessed largely exempt from criticism. One might claim, for example, that a work of poetic scholarship is not in the spirit of the original phenomenon; however, such criticism implies evaluating the scholarship from a hermeneutic perspective.

In all four approaches to arts scholarship, researchers commonly hope that the resulting scholarship will enhance our appreciation of a practice, work, performance, or culture.

\section{Motivating Questions}

For me, the best research starts with questions. I often set my students the following task: If you were granted an audience with God, and could ask anything at all, what would you want to know about music? It's worth spending a minute or two to consider some music related questions.

1. Why do people make music?

2. Does music-making contribute to human survival in some way?

3. Do non-human animals make or enjoy "music"?

4. Why doesn't every culture in the world have similar music?

5. Why are some people more musical than others?

6. What are the elements of musical ability?

7. Is musical "intelligence" independent of general intelligence?

8. Can we predict which children are likely to be most musically gifted?

9. Why are some people "tone-deaf?"

10. Can something be done to alleviate tonedeafness?
11. Is there something wrong with people who don't like music?

12. How does music give pleasure?

13. Why does the sound of fingernails scratching a blackboard sound so bad?

14. What makes certain sonorities or chords sound more pleasant than others?

15. Why does "rubato" exist-why isn't music played strictly according to the notated timing?

16. What makes some interpretations of a work sound better than others?

17. Does everyone "hear" music the same way?

18. How do children experience music differently from adults?

19. How does our musical "hearing" change as we grow-up and grow old?

20. Are there different ways of "listening?"

21. With training or effort, how differently might we be able to hear music?

22. Why do people disagree about musical likes and dislikes?

23. What makes us actively hate some songs?

24. Why do our musical preferences sometimes change over time?

25. Are musical preferences related to personality?

26. What does a person's musical tastes say about them?

27. How and why do musical styles change?

28. To what extent can we hear or understand the music of another culture in the same way as people from that culture do?

29. How do musical canons form?

30. Do we need to have a musical canon?

31. Why do we need so much music and so much musical variety-why don't we limit our listening to just a dozen of our favorite works?

32. How does repeated listening to a work change our experience of it?

33. What is the origin of various compositional rules, such as the rules of "voice-leading?"

34. What is the relationship between music and speech or language?

35. What is the relationship between music and movement or dance?

36 . Why do some melodies get stuck in your head?

37 . Why don't all melodies get stuck in your head?

38. What goes on when we imagine music?

39. What makes something sound sad or happy? Angry, cute, disgusting, tender, devotional, aggressive, exciting, powerful?

40. How does music evoke emotions in listeners? 
41. Are there some emotions that can't be evoked by music - such as shame or guilt?

42. Why does some music make us nostalgic?

43. Why do people willingly listen to music that makes them report feeling sad?

44. Why do musicians have to practice so much?

45. Is there a better way to practice?

46. What is the best way to teach music?

47. Is there anything that can be done to lessen or avoid stage fright?

48. How are groups of performers able to coordinate their activities?

49. How is it that some musicians are able to improvise?

50. Why does some music make people want to dance? Why doesn't music make people want to cook, work in the garden, or go swimming?

51. Why do most people prefer tonal music to atonal music?

52. What is tonality anyway?

53. Why is it easy to drive a car and listen to loud music at the same time, yet it is often difficult to solve math problems and listen to loud music?

54. Can music "heal" people?

55. Can listening to music make you smarter?

56. Can music somehow corrupt or enhance moral behavior?

57. Can the music you listen to influence your political views? If so, how?

58. Is background music bad for you?

59. Can a person listen to too much music?

60. Does the complete absence of music have a detrimental effect on people?

61. Why are some people more enthusiastic about music than others?

62. What makes a good musical culture?

63. How can economies be organized so as to facilitate musical employment?

64. How do we engage the music of other cultures without being inadvertently exploitive?

65. Does our personal physiology affect our experience of music?

66. How does illness or physiological abnormality influence musical experience?

67. How can certain drugs enhance or detract from musical pleasure?

68. Are there brain structures specialized for just music?

69. What makes something "musical?"

70. Are there limits to what music could be?
Of course, there are many more questions that could be added to this list, and I'm sure readers will have their own pet questions that should be added. Although we have provisional answers for some of these questions, these are early days, and the questions themselves remind us of just how little we know at this point.

\section{The Importance of Causal Research}

What I think is important to understand is that there are real and pressing questions related to music. Questions like music's capacity for well-being, and questions of how to approach the music of another culture - these are questions that warrant careful consideration and sustained efforts to find good answers.

Ideally, research helps us change the world for the better. Of course, much change occurs by happenstance - without us having any say or influence. It would certainly be preferable if we had some choice in the matter. If we want to achieve some preferred state, it would be helpful to know what actions will make that happen. It would also be helpful to know what other actions might impede our goal, and what actions look useful, but aren't. In short, if we want to change the world, then we need to understand how change happens. We need to know that doing $X$ will likely result in $Y$, or that doing $X$ will have no effect on achieving $Y$. Of the four types of scholarship I've identified, the one that gives us the best tools for change is explanatory research - the scholarly approach that delves into causes.

Let me say that again: If we want to change the world for the better, the most useful research is research that identifies causes, and that's explanatory research. Sadly, explanatory research is the hardest to do. In the case of historical scholarship, we have no good causal methods. Identifying the causes of past historical events is necessarily correlational and so always speculative. Even in the sciences, a large proportion of the research is correlational rather than experimental. Moreover, when beginning an investigation, it's generally wise to cast a wide net, so descriptive and hermeneutic approaches offer good exploratory approaches for getting started.

\section{Multi-Measure Research}

In conducting explanatory research, we need to have good intuitions about the source of the causation. Is the phenomenon caused principally by a person's cultural background? Or is the main cause personality? Might 
there be some sort of genetic component (Harden, 2021)?

We have no hope of identifying possible causal factors unless we're measuring the right variables. However, what people measure is commonly determined by their disciplinary backgrounds. If you work in an EEG lab, then you're going to measure EEG. If you're an ethnomusicologist working in the field, then what you collect will surely be ethnographic descriptions. The cliché here is that for the person who has a hammer, all the world looks like a nail. As researchers, we tend to rely on the same descriptive methods or the same dependent measures over and over again.

In 2012, I happened to attend a meeting of ethologists at Hunter College in New York City. Ethologists study animal behavior, and I watched presentation after presentation in which researchers discussed their work investigating the behavior of some particular animal. What struck me was the multiple methods used. Here's a presentation on some chinchilla behavior: here's the social behavioral data, and here's the brain-imagining data, and here's the genetic data. And now here's a presentation on gold finches: Here's the parenting behavioral data, and here's the hormonal assay data, and here's the brain-imagining data, and here's the genetic data. And so it went, for presentation after presentation.

Ultimately, I expect the future of empirical music research will follow this multi-measure approach. As the costs of various measurement methods become more affordable, music researchers will find it easier to embrace multi-measure methods. Imagine some future music conference where the presentations are of the following form: Here's this aspect of musical behavior. Here's the behavioral data, here's the socio-cultural, economic, and historical context, here's the personality data, here's the physiological data, here's the neuroimaging data, here's the genetic data, here's the proteomic data, and here's the introspective/ phenomenological report data from the participants. Once again, if we're not measuring the right things, we have no hope of identifying the locus of influence. In order to cast a wide net, we need to embrace multimeasure methods as the opportunities arise.

In conducting research, it's important not to view your disciplinary background as the source of some sort of allegiance. Think of your discipline as your limitation. If you think culture has little effect on how people experience music, I recommend reading Steven Feld's book on the Kaluli (Feld, 1990/2012) — or my book on expectation (Huron, 2006). If you think biology has little effect on how people experience music, try reading the literature on the effects of lysergic acid diethylamide (LSD) - or consider reading my book on voice leading (Huron, 2016). We now have excellent empirical evidence that musical experience is influenced by physiology, by psychology, by personality, by social context, and by enculturation. This means that there is only one proper approach to the study of music and that's an integrated bio-psychosocio-cultural approach.

I think there are two immediate take-home messages from the promise of future multi-measure methods. First, always be prepared to learn and apply new measurement methods. And secondly, aim to collaborate with people who collect data that is contrasting or complementary to what you tend to do.

\section{Corpus Studies}

Of course, not all empirical research need focus on identifying causes. Nor should empirical research focus exclusively on how humans experience music. As already mentioned, the study of history is necessarily correlational. Moreover, most historical questions center on phenomena other than subjective human experience. From a traditional musicological perspective, I think a ripe area for research is the application of empirical methods to the study of music history. In particular, I think the future looks especially rosy for digital corpus studies. Let me offer three examples of how empirical methods have led to new insights regarding music history.

One of my favorite studies is a corpus study conducted by Matthias Mauch and colleagues from 2015 (Mauch, et al., 2015). They used a technique devised by Jonathan Foote called "Foote Similarity" to examine stylistic changes in Western popular music (Foote, 1999, 2000).

Foote Similarity is simply a method for measuring similarity over time. In commercial applications it's used, for example, to identify discontinuities as a way of navigating through movies or audio recordings. Suppose you have an audio file containing a conversation between a man and a woman. You're looking for a particular point in the recording where the woman says something of special interest. Unfortunately, the recording is long, and its mostly dominated by the man talking. Instead of scanning forward through the file, playing bits and pieces of the conversation, it would be more convenient if you could use a "skip" button that would take you to the next 
moment when the speaker changes-from man to woman, or from woman to man.

A simple point-by-point measure of the audio spectrum will pin-point places in the recording where there is a large change, and those points would typically be associated with moments when the speaker changes. You could apply the same method to have a skip button that would move you in a song between verse and chorus passages. In effect, what Foote Similariity provides is an automatic method for audio segmentation using a measure of sonic novelty.

Now instead of characterizing the changes in an audio track over several minutes or hours of music, suppose that you decide to characterize changes in music over a 50-year period. That's what Mauch and his colleagues did. They applied Foote Similarity measures to what-you-might-think-of as one very long music file consisting of (30-second excerpts from) 17,000 pop songs on the US charts, from 1960 to 2010 . What this technique allows you to do is to identify moments in time where the music becomes significantly different from what existed before.

When calculating an audio spectrum, one consideration is the size of the analysis window used. In audio applications, we often employ windows whose sizes amount to a few milliseconds. Especially when the audio consists of many individual recordings, there's merit to using window sizes that are much longer than the songs themselves. In this case, we might consider window sizes as large as an entire year or more.

Figure 3 shows the results of their study. The horizontal axis represents time (from 1960 to 2010) whereas the vertical axis represents the window size (measured in years). Dark blue here means high similarity. Light blue, yellow, and red represent periods where the music becomes increasingly different from what came before. As the window size increases you can see that there emerge particular years in which notable discontinuities appear - that is, where the music changes noticeably. The three most prominent discontinuities coincide with the years 1964, 1983, and 1991.

If you're a popular American music scholar, you would surely be able to label these particular changes: 1964 is associated with the so-called British Invasion, with the popularity of groups like the Beatles and the Rolling Stones. 1983 is associated with the introduction of New Wave and Hard Rock, and 1991 is associated with the introduction of Rap and Hip Hop.

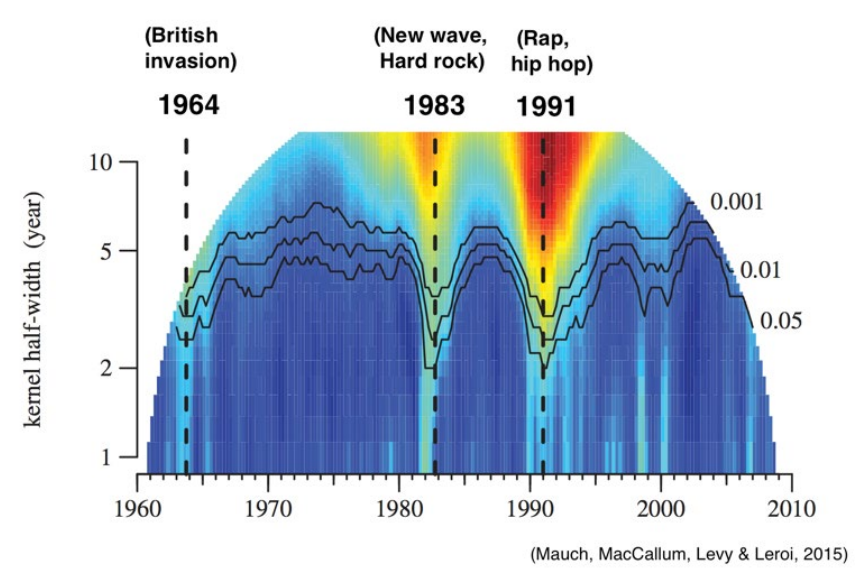

Figure 3: Sonic discontinuities by date of release for 17,000 pop songs appearing on US charts from 1960 to 2010, measured using Foote Similarity. Reproduced from Mauch, et al., 2015.

\section{Stylistic Change}

Now apart from identifying moments of historical change, corpus studies can also tell us something about the nature of the stylistic changes. An example is a study I conducted with Katlyn Horn where we assembled a large random sample of Western art music spanning a one-and-a-half century period from 1750 to 1900 (Horn \& Huron, 2015).

For each sample passage we coded just four pieces of information: the tempo, the dynamic level, the mode (major or minor), and the predominant articulationfrom predominantly staccato to predominantly legato. We then conducted cluster analyses for each of three successive 50-year periods. For each cluster type, we provided a descriptive label. For example, the combination of loud/major/fast/staccato we labeled 'joyful' passages. The combination of quiet/major/slow/legato, we labeled 'tender lyrical.' What we called 'sad-relaxed' passages were quiet/minor/slow/legato, and what we called 'lighteffervescent' passages were quiet/major/fast/staccato. Our aim was to determine what combinations of musical features tend to predominate, and whether the different types of passages tend to change over time.

Figure 4 shows the resulting cluster analysis for the entire 150-year period. The vast majority of passages can be classified as falling into just eight categories: joyful, regal, tender-lyrical, light-effervescent, serious, passionate, sneaky, and sad-relaxed. (Incidentally, what we mean by 'sneaky' music are passages that are quiet, fast, staccato, and in the minor mode - think Grieg's In the Hall of the Mountain King.) 


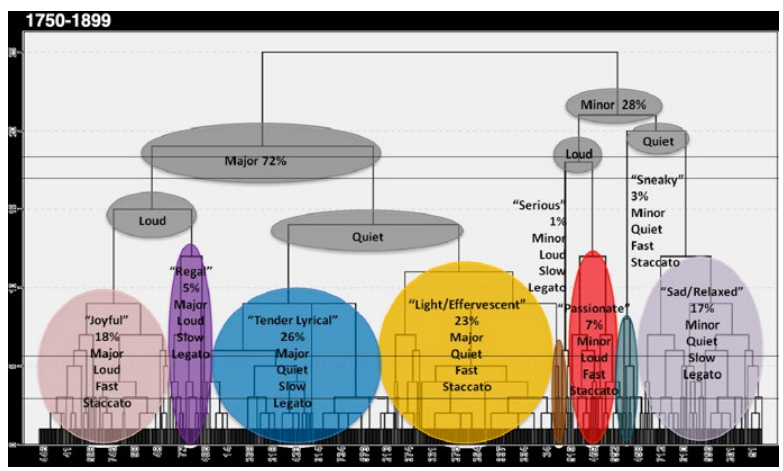

Figure 4: Cluster analysis for a random sample of musical passages of Western art music spanning the period 1750 to 1900. Reproduced from Horn \& Huron, 2015.

What's interesting is how the proportions of these different types of passages change over time. For example, in the late 18th century (Figure 5), you can see that the most prominent category is 'light-effervescent' music (shown in yellow) - and representing nearly 40 percent of all sampled passages.

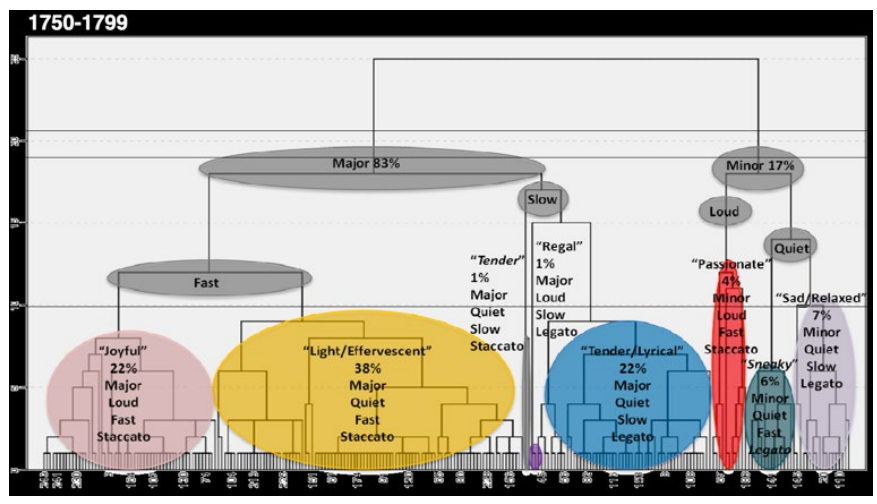

Figure 5: Cluster analysis for a random sample of musical passages of Western art music spanning the period 1750 to 1800. (Horn \& Huron, 2015)

By the early 19th century (Figure 6), light-effervescent music is less than 30 percent.

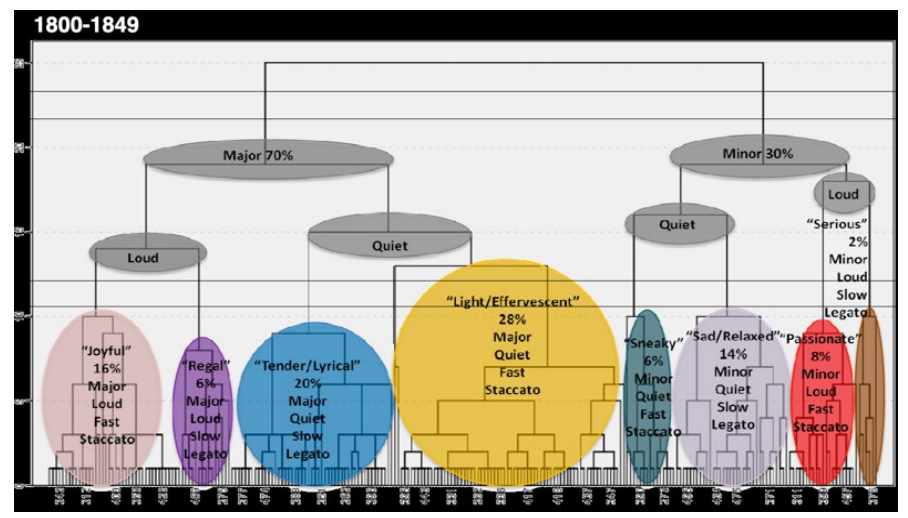

Figure 6: Cluster analysis for a random sample of musical passages of Western art music spanning the period 1800 to 1850. (Horn \& Huron, 2015)

And by the last half of the 19th century (Figure 7), there simply weren't enough passages to result in an independent cluster. That is to say, light-effervescent passages nearly completely disappeared in our sample of Western art music.

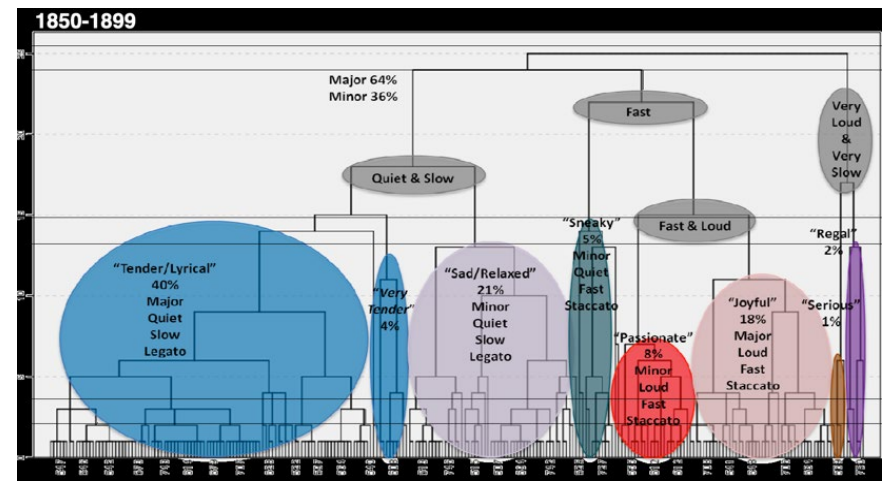

Figure 7: Cluster analysis for a random sample of musical passages of Western art music spanning the period 1850 to 1900. (Horn \& Huron, 2015)

Figure 8 summarizes the changes over the 150 -year period. Musical passages we might call 'tender-lyrical' expanded dramatically, especially in the last half of the 19 th century, and represent almost half of the music we sampled. At the same time, 'sad-relaxed' music triples in size over the 150-year period. And finally, 'lighteffervescent' passages, which predominate in the 18th century, virtually disappear by the end of the 19th century. 


\begin{tabular}{|l|l|l|l|}
\hline \multicolumn{5}{|c|}{ Proportion of Each Cluster in Each Epoch } \\
\hline & $\mathbf{1 7 5 0 - 1 7 9 0}$ & $\mathbf{1 8 0 0 - 1 8 4 0}$ & $\mathbf{1 8 5 0 - 1 8 9 0}$ \\
\hline Tender/Lyrical & $22 \%$ & $20 \%$ & $44 \%$ \\
\hline Light/Effervescent & $38 \%$ & $28 \%$ & $0 \%$ \\
\hline Joyful & $22 \%$ & $16 \%$ & $18 \%$ \\
\hline Regal & $1 \%$ & $6 \%$ & $2 \%$ \\
\hline Sad/Relaxed & $7 \%$ & $14 \%$ & $21 \%$ \\
\hline Passionate & $4 \%$ & $8 \%$ & $8 \%$ \\
\hline Sneaky & $6 \%$ & $6 \%$ & $5 \%$ \\
\hline Serious & $0 \%$ & $2 \%$ & $1 \%$ \\
\hline
\end{tabular}

Figure 8: Comparison of relative proportions of different types of passages for three 50-year epochs $\begin{array}{llll}\text { from } & 1750 & \text { to } & \end{array}$

In general, we might say that music became less lighthearted or joyful and more solemn, serious, or passionate. The results are certainly consistent with how we generally think of late Romanticism.

\section{Geography}

Apart from how music changes over time, music also changes over space. There's a geography to music history, and especially interesting are those changes where musical ideas or patterns disperse over time.

Some years ago, Bret Aarden and I published a study where we mapped some 6,000 European folksongs. Bret spent the summer using an electronic gazetteer to resolve village place names into latitude and longitude values. That allowed us to use mapping software to create musical maps (Aarden \& Huron, 2001).

Figure 9 shows an example of one of the maps we generated. This is a map showing the distribution of songs in the major and minor modes in western Europe. White regions represent areas where major-mode songs predominate whereas dark regions represent areas where the minor mode predominates.

Historical musicologists have long observed the tendency for musical innovations originating in Italy to slowly make their way north into northern Europe. One of these innovations was the major/minor system which developed in Italy around 1600. Prior to the major/minor system, the most common mode in European music was the Dorian mode - a mode that most resembles the modern minor rather than major mode. So the main evidence for the spread of the major/minor system is the increasing use of the major mode.

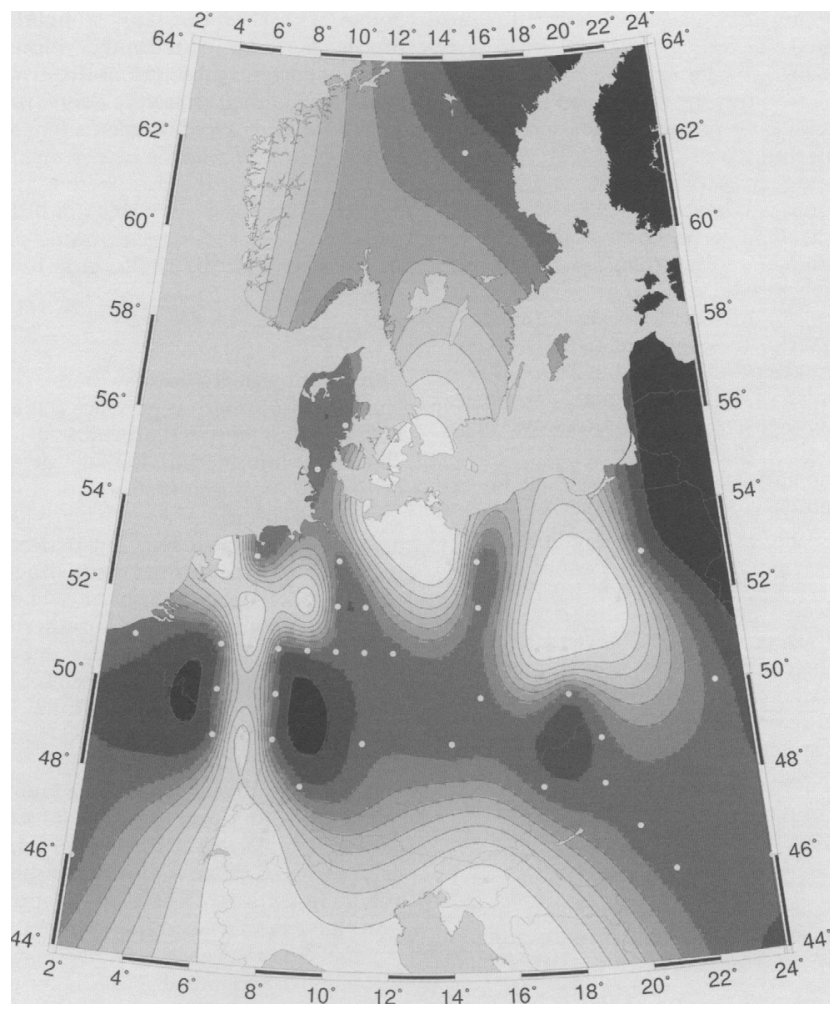

Figure 9: Geographical distribution showing the predominance of major mode (white) and minor mode (black) folksongs. (from Aarden \& Huron, 2001)

These cultural dispersions happened long before the invention of railroads. They occurred during times when rivers were the main conveyors of people, goods, ideas - and of music. Mountains were especially formidable cultural barriers.

In particular, let me draw your attention to a whitecolored north-south strip between Switzerland and the Netherlands. What's striking about this corridor is that it coincides almost perfectly with the Rhine River valley. It's consistent with the conjecture that the major mode dispersed north from Italy through the Rhine River corridor.

The right-most white region on our map similarly coincides nearly perfectly with the Vistula river valley in modern Poland. The Vistula flows through the major cities of Kraków and Warsaw, entering the Baltic Sea at Gdansk (German: Danzig), Poland's foremost port. In general, notice that white (major mode) regions tend to coincide with either river valleys or coastal areas. Evidently, the geographical spread of the major mode can still be observed in folksong repertoires hundreds of years later. 
Certainly, more research is needed. This map represents merely a smoking gun. But it is suggestive, and the results imply that there may be much to learn from considering how musical features are distributed over geographical space.

\section{The Promise of Corpus Research}

As a group, I think these three studies illustrate the enormous potential and future promise for corpus studies. Big data offers opportunities to pinpoint musical changes in time and place, trace possible patterns of musical dispersion and influence, and identify the specific musical and stylistic features that are the main targets of musical change.

At some point in the future, someone is going to apply a technique like Foote Similarity measures to the entire history of documented music - not just Western music, but all music around the world for which we have historical records.

These techniques are going to give us much more refined insights into the kinds of changes music undergoes in different places and times. Moreover, bottom-up measures might even be tailored so that a measure of similarity itself (for example) might reflect what we know or conjecture about how people in different times or cultures might have perceived musical similarity. Techniques such as these offer a much more comprehensive approach to understanding the history of musical change.

\section{Realism}

As I mentioned earlier, one of the overarching aims of music research is to ultimately identify and explore all of the ways that music can contribute to human wellbeing. Of course, our dreams about what music might be able to achieve are not always realistic.

\section{Public/Applied Musicology}

Most recently, we might consider the exciting and inspiring initiatives known as public or applied musicology. Although initiated some decades ago, the applied musicology movement has gained considerable momentum in the past few years. Several specialty conferences have explicitly focused on applied musicology and special sessions on applied musicology are now routine at general music conferences.

The basic idea is for musicologists, ethnomusicologists, music theorists, and music scholars of all stripes to better serve the public, and improve the societies in which we live. The idea is to tear-down the town-and-gown barriers and better connect ivory-tower institutions to their local and regional communities.

In the case of musicology, proposals for public outreach include music journalism, organizing concerts and festivals, writing program notes, curating exhibits and public events, involvement with broadcast media, web design, repatriating indigenous musical knowledge, sponsoring or nurturing various performance troupes (especially from minority communities), and aiding in disaster recovery and other social and physical emergencies.

An example of public musicology is starting up a community choir among (say) Somali immigrants in order to enhance a sense of identity among the participants.

I think the aims of applied musicology are laudable. I think "Make the world a better place" is part of everyone's job description. Once again, our hope is surely to support every effort to use music to improve human well-being in as many ways as that's possible. But I do have concerns related to the more social service elements you find in the public musicology movement.

If we're considering Applied Musicology as a form of social service, then there is something to be said for looking at the experiences of those already involved in social services - most notably social workers. Social workers occupy the front lines. One would be hardpressed to find a cadre of people more eager to improve the human condition, especially improving the lives of those among us who are less fortunate. Social workers are typically idealistic and eager to be the agents of positive change.

The history of social work, however, is spotty. Ideas, initiatives, and programs come and go. If there is one overarching lesson to be learned from the history of social services, it's that what we think might be a good idea, doesn't always pan-out. The history of social intervention is littered with ineffective programs.

Two well-known programs in the U.S. were the D.A.R.E. program whose aim was to reduce drug abuse, and the Scared Straight program which exposed juvenile delinquents to prisons as a way of scaring them away from future criminal activity. They were both popular programs that spread beyond the U.S. to other countries. The only problem was that long-term studies showed they didn't work.

The D.A.R.E. program had no effect on illegal drug use and actually increased alcohol and tobacco use among participants compared with matched at-risk kids who didn't participate in the program. And in long-term studies, participants in the Scared Straight program were 
more likely to engage in future criminal activity compared with those juvenile delinquents who had been random assigned not to participate in the program.

What social workers understand is that although we can hold the very best of intentions, our intuitions are very often wrong. Of course, intuition is essential. When we're dealing with some problem, there often isn't pertinent research to draw on, and so we have no other option but to rely on our intuitions. Musicians certainly understand that, perhaps more than others.

But social workers have learned over and over again just how fallible our intuitions can be. What social workers have learned is that it's essential to maintain a healthy skepticism about the effectiveness of various programs and that you shouldn't introduce a program without a formal process in place for evaluating its effectiveness. In recruiting students to social work programs, it's not enough just to seek committed social or community activists. You need people who are skeptical of their own intuitions and who understand that good intentions can backfire. You need people who are careful thinkers and understand the importance of evidence-based practice.

Returning again to the example of starting a community choir among Somali immigrants, it's indeed very likely that the activity will contribute to a sense of identity among the participants. But research on group identity suggests that this will weaken their sense of belonging to larger groups, such as the feeling of being a resident of their host town or city, a feeling of kindship with other African immigrants, or feelings of being citizens of the world. So just what precisely is being accomplished?

I don't want to suggest that public musicology is misguided, or that we should leave social services to the professionals. When it comes to building better and more just lives, we need all the resources we can muster. What I'm saying is that we're headed for trouble if we don't take research seriously and just assume our good intentions and intuitions are sufficient.

It all hinges on understanding the effectiveness of what we're doing and that hinges on program assessment.

There is, of course, a lot of expertise that already exists for measuring program effectiveness. But let's not deceive ourselves: you won't find that expertise in music departments or conservatories. Social workers and other professionals won't be impressed by our amateur efforts and they will be exasperated that we'll be competing against them for scarce government and philanthropic support.
It looks like we're creating a generation of new arts graduates who regard themselves as social activists, but who are badly trained, and who won't initially realize the extent of their poor training.

Despite the good intentions, my reading of the applied or public musicology (and ethnomusicology) movements is that we're headed for failure if practitioners aren't properly trained in how to conduct best-practices assessment research.

We need to stop thinking in terms of good and bad people and think instead in terms of effective and ineffective practices. And that requires that we focus on research rather than focusing on moral judgments of others. My fear for the applied musicology movement is that we're headed for a repeat performance of the Mozart Effect blunder, only on a much larger scale.

\section{Political Value of Music}

Music has long been regarded as playing a major role in political transformation. Music has indeed figured prominently in many historical and current protest movements. But its capacity for political change is often over-exaggerated as in Lord Fletcher of Saltoun's famous claimed that "Were I able to make the ballads of a nation, I need not write its laws."

Lord Fletcher's statement is certainly poetic. But it is more optimistic than realistic. Music is important in people's lives, but it is unlikely that music holds the sort of dramatic political power that some music scholars often suppose.

Theodor Adorno believed in the politically transformative power of twelve-tone music: that living in the false age, serial music would hold up the false to the false (as he said) and so help to bring about revolutionary change. So just how much political impact can we attribute to Schoenberg, Webern, Berg, and their colleagues? Adorno proposed, for example, that if people had listened more carefully to music, the holocaust would have been avoided. Quoting the pertinent passage from Adorno, New Musicologist, Susan McClary noted approvingly, "So the stakes are enormous" (McClary, 2021).

Even in the case of popular music, there are grounds to be skeptical of common claims regarding its political influence. There is first of all the question of causality: does music lead or follow, and if it leads, does it compel in some way?

There's also the issue of relying on lyrics. There is more to music's influence than lyrics, but in political movements lyrics ostensibly play a critical role. However, the extant research shows that only about half 
of music listeners even pay attention to sung lyrics (Condit-Schultz \& Huron, 2015). For many people around the world, much of the popular music they hear is in a language they don't even understand. And, of course, that's long been the case for classical music.

If I were a multi-billionaire intent on using my wealth to bring about political change, I'm not really convinced that funneling money to musicians would be as effective or more effective than supporting individual politicians, writers, or media commentors.

Madeleine Albright was right that music is a useful "soft power" political tool. But so are banquets and gifts. Music offers a useful way to build bridges, but that's likely attributable to the pleasure it affords. Of course, music does have some political-ideological influence. But it's doubtful that it can effectively write a nation's laws or instigate a revolution.

In understanding the political value of music, once again, it's incumbent upon researchers to neither overestimate nor underestimate its effectiveness.

\section{Cultural Policy}

Let me offer an alternative suggestion regarding the goal of political change.

Universities house lots of different kinds of research. A notable class of research that we rarely hear about in the arts is policy research. If you visit other university departments, such as engineering, medicine, social work, education, environment, etc., you'll find many scholars who work explicitly on public policy issues. For example, in medicine you'll find researchers with expertise on public health policy. In economics, there are scholars who work on taxation policy and banking regulation. In computer science, there are faculty who worry about data security and privacy. In chemistry, there're people who are concerned about handling of toxic products and occupational safety. In agriculture, you'll find researchers intent on reconciling good environmental regulation with productive farming practices. In veterinary medicine, there are people who study policies related to animal welfare. In psychology, you'll find faculty who work on issues of clinical certification and therapeutic practice.

In education, law, geography, economics, urban planning, business, biology, and dozens of other departments, you'll find a proportion of scholars who spend their lives preparing for the moment when they can offer advice to legislators. If a government is contemplating revising laws or regulations related to welfare, or fisheries, or transportation, you'll find academics and academic organizations ready to travel to the seats of government and ready to submit carefully reasoned policy position papers. These policy scholars understand the legislative history; they are knowledgeable about the policies in other jurisdictions and countries, and they have followed the good, bad, and indifferent outcomes from various initiatives. They can point to unexpected repercussions of different regulatory approaches and they can provide useful advice intended to serve the public good.

Not so in the arts. In the arts, we have some scholars who engage in educational policy research. But I know of no music scholar engaged in cultural policy research.

At the moment, the Web is utterly transforming the way in which culture is created and disseminated, how cultural industries bring in income, how nonprofit organizations do fundraising, who holds power, how distribution is organized, how musicians make money, the role of amateurs, whether music distribution will become entirely subservient to advertising, and myriad other issues. Not since the invention of sound recording in the late 19th century has music been impacted the way it is now.

All over the world, countries are faced with legislative and regulatory challenges concerning intellectual property, cultural organizations, maintaining cultural identities in the face of globalization, repatriating intangible cultural artifacts, and many other challenges.

I'd like to suggest that the most important task for music scholars (and arts scholars in general) is to nurture and advance culture. In the same way that an agronomist can ask "What is good agricultural practice?" We can ask, "what makes a good musical culture?" And like the agronomist, we can then take practical steps to try to change the world for the better. There are surely many facets that define a vibrant and valuable musical culture. And part of what shapes culture is the legal and regulatory environment in which musical culture operates.

In October of 2018, the U.S. Music Modernization Act was signed into law. That law emerged from a battle between the two main commercial stakeholders, namely representatives of copyright owners and producers, and media conglomerates involved in music distribution. This year, 2021, saw the creation of the new music licensing collective which will act as a toll booth for companies like Spotify, Pandora Soundcloud, and other digital music providers. Once again, in formulating this legislation, the people around the table who thrashed out the agreements were the commercial groups with vested interests. According to public records and minutes of the 
various meetings leading to the legislation, there apparently wasn't a single individual present representing the public interest.

The United States isn't alone in this. All over the world, we see this same scenario repeated. What policies will best serve the public? Alas, music scholars have virtually nothing to offer. I don't know of any policy expertise in music departments or conservatories.

I'd like to propose that an important area for future music-related research is in the domain of cultural policy. If we don't engage in public policy research related to arts and culture, then cultural policy will be shaped almost exclusively by commercial and industrial interests.

Cultural policy research would include both basic research as well as specific policy recommendations. Basic research on cultural values and cultural policy might focus on questions like the following:

1. What is the purpose of cultural preservation?

2. How do we maintain cultural traditions while ensuring creative freedom?

3. At what point does promoting a sense of cultural identity lead to cultural intolerance?

4. Is cultural policy something we should create $a$ priori? Or should policy arise through a laissez faire process of benign neglect?

5. How do people express cultural preferences?

6. How can we infer the cultural values of some group of people?

7. In comparison to unpaid amateur musicmaking, what is the role of professional musicians in a vibrant musical culture?

8. How do we align the interests of commercial cultural industries with the public good?

This is only a sample of what is surely a long list of questions regarding musical and cultural values.

We need, I believe, to develop a cadre of music scholars with cultural policy expertise, who will act in the public interest, paying attention to the nurturing and development of musical culture in all its various manifestations. Once again, the aim is to serve human well-being in as many ways as music is capable of doing that.

Historically, social and political critique in the arts has tended to remain at an abstract and rhetorical level. What is needed, I propose, is concrete suggestions and evidence-based legislative recommendations.

Most music programs are funded through public taxes. We receive public support because our activities are thought to serve the public good. The ultimate stakeholders in these decisions, are not merely copyright holders and media conglomerates; the ultimate stakeholders are - all of us.

At the very moment when decisions are being made that will affect musical culture - possibly for the next several centuries - arts scholars appear to have nothing to contribute. Scholars in the law schools, in engineering, and business have thought more about the future of music than have musicians and music scholars.

\section{Music Science}

Empirical research in music has grown considerably in recent decades. Figure 10 shows a Google ngram graph for the relative frequency of occurrence for the phrases "music psychology" and "music cognition" since the end of the second world war.

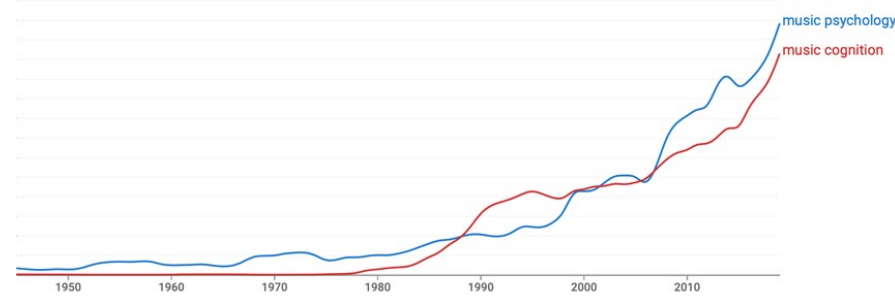

Figure 10: Relative frequencies for the phrases "music psychology" and "music cognition" in English texts from 1940 to present.

It's exciting to see such growth. However, it's appropriate to put this growth in perspective (Figure 11). When we compare music cognition and music psychology to musicology, music theory, and ethnomusicology, we can see that the field is what the British would call "small beer."

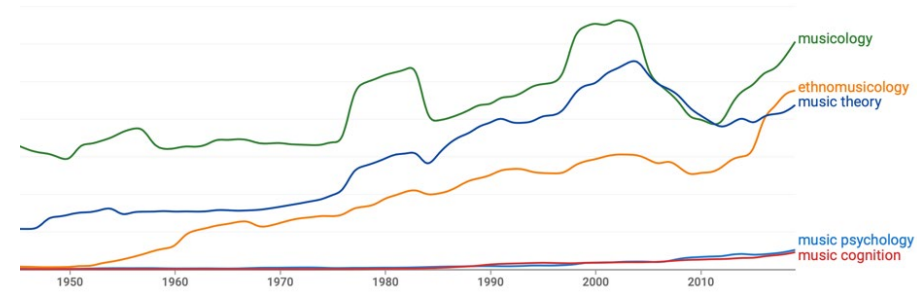

Figure 11: Relative frequencies of "music psychology" and "music cognition" compared with musicology, ethnomusicology, and music theory.

Within the broader realm of music scholarship, music psychology is a bit player. Moreover, that's also true in the world of psychological research: the psychology of music is a very small part of the modern field of psychology. 
Apart from music psychology, a marginal status is also evident for the musical 'sciences' in general. In an article from 1885, the Austrian musicologist Guido Adler famously distinguished two main branches of music studies: historically-oriented music scholarship and a music science which he dubbed systematische Musikwissenschaft or "systematic musicology". Initially, systematic musicology included "comparative musicology" which later morphed into ethnomusicology and abandoned any affiliation with a science of music.

Today, systematic musicology is the one homegrowth science within the world of music scholarship. But unlike the other subdisciplines of music scholarship, systematic musicology has been in long-term decline. When I was a student, there were three systematic musicology graduate programs in North America. All three are now closed.

In Europe-especially in German-speaking countries - we see that systematic musicology programs continue to exist, but the field as a whole is not thriving. Focusing on literature in German, Figure 12 suggests the field has been in decline after peaking at the turn of the century.

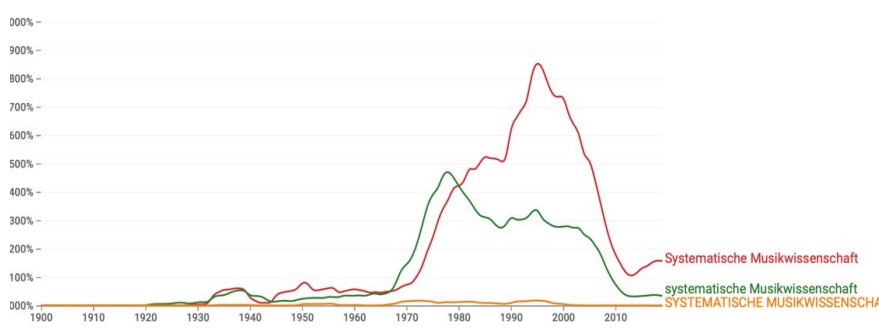

Figure 12: Relative frequency for the phrase "systematische Musikwissenschaft" in German texts from 1900 to present.

The sciences of music mostly thrive outside of music scholarship. It is psychologists, engineers, and computer scientists who are the principal torchbearers for a science of music.

I happen to love both science and the arts, so for me the culture wars have been simply dispiriting. But I am hopeful that, in the future, music scholarship will again warmly welcome empirical and scientific scholarship into the fold. But I'm aware that that will only happen if the research offers compelling insights that attract mainstream music scholars.

\section{Reprise}

So let me bring my presentation here to a close by offering some observations and some advice.

Over at least the past three centuries, music has become increasingly present in people's lives. Moreover, as we saw, even talking or writing about music has also become increasingly popular. Apparently, people hunger for information and ideas about music.

As musicians and music scholars, we surely want music to serve human well-being in as many ways as music is capable of doing that. However, from time-totime, our zeal and enthusiasm for music can outstrip the evidence. Our task is neither to overestimate or underestimate music's capacities.

Even when we harbor the best of intentions, our intuitions can fail us. In fact, sometimes our intuitions can be counterproductive. Intuition is essential in life, but in matters of importance we are remiss if we don't aim for evidence-based practice.

If we want to change the world, we need to know that the changes we propose or make will have the intended effect. The only type of research that allows us to understand change is explanatory research whose aim is to identify causality.

There are many possible causal sources, so in conducting explanatory research we should cast a wide net by collecting multiple measures-spanning the biological to the cultural. In order to pursue a multimeasure approach, we need to be open to learning new methods and aim to collaborate with researchers who employ contrasting or complementary approaches.

There are real questions to be answered about music. The best music research starts with good questions in which the researcher then assembles the methods and tools that will help address that question. Questions should dictate methods rather than the other way around.

In this presentation, I've suggested that a particularly good question is: What makes for a good musical culture? It's certainly a thorny challenging question. But it's also an essential question that's worthy of our attention. It's a question that leads to a follow-up practical question: How do we go about improving musical culture? I've suggested in this presentation that this question is deserving of a central place motivating music scholarship. To the extent that musical culture is shaped by technology, commerce, legislation, and regulation, the public interest in culture requires engagement with the formation of pertinent policy. 
I've suggested that if we care about the state of musical culture, then we need to invest in policy-related research. That is, we need to engage in the sort of policy research one finds in nearly every other department in the university academy. Specifically, we need to go beyond abstract political rhetoric, and focus on concrete suggestions for evidence-based legislative recommendations. In shaping future musical cultures, I propose that we need to develop an informed cadre of scholars who can act in the public interest.

Empirical music scholarship has shown considerable growth in recent decades. However, scientific and systematic approaches to music-research still represent only a small fraction of music-related scholarship. As we've seen, musicology's home-grown science of music - the field of systematic musicology-has struggled. Efforts by some arts and humanities scholars have done much to undermine confidence in science. As a result, it has been psychologists rather than music scholars, who have been at the forefront and leaders in music science.

Much of the misunderstanding can be traced to a problem of language. Many arts and humanities critics have rightly challenged scientific claims to truth. While scientific research is motivated by the aim or hope of attaining truth, truth cannot be established through empirical observation - something we've known since David Hume and philosophers of even earlier centuries. Practical science is not about truth, it's about evidence. It's about collecting and interpreting evidence. If we want to regain the respect of philosophers, we need to stop defending science by talking about truth. The proper way to defend science and scientific method is by talking about evidence and how evidence can make one narrative more plausible than another.

More important than the philosophical arguments, the most effective way to build interest in music science is by demonstrating its value. If we want empirical music research to be impactful, we need to address and solve musical problems. We need to produce stories that musicians and music scholars find compelling and interesting. Among other auspicious approaches, corpus studies appear to be especially promising.

If we want music research to be impactful: we need to keep focused on musical problems. We need to resist chasing after funding that supports tangential studies, in which musical concerns are sidelined. We need to embrace an integrated bio-psycho-socio-cultural approach. A full accounting of music is not possible by focusing solely on biology or psychology. Nor will we have a full account of music by limiting our work to social and cultural accounts.

Don't think of your discipline as your allegiance, think of it as your limitation.

There is room for poetic music scholarship in which scholars respond to art with imaginative interpretations. However, when the scholarship moves into the territory of knowledge claims, artistic inventiveness needs to step to-the-side and allow careful empirical testing to be front-and-center. We need to place more emphasis on hermeneutic and explanatory research over descriptive and poetic musicological scholarship.

One of the first methodological no-no's taught to both arts and sciences scholars is the illegitimacy of the ad hominem argument, where the attack focuses on people rather than ideas. As someone who has taught research methodology for more than two decades it has personally been disconcerting to see the recent explosion of personal attacks in the academic world. Let's stop talking about good and bad people, and instead focus on good and bad ideas.

There is much to learn about research methods; and it's important for every scholar not to be satisfied with what they were taught. Everyone needs to read widely beyond their discipline and look for methodological insights wherever you can find them. If you don't know what reification is, or the correlation/causality conflation, or if you don't recognize acquiescence bias, or don't know what counterfactual control is; if you can't recognize an ad hominem argument when you see one- - then you're not as effective a scholar as you could be. Everyone needs to develop a healthy understanding of the innumerable ways by which research can go awry.

Methodology is not some sort of rationalist obsession. It is the way we pay tribute to our scholarly predecessors whose hard-won lessons include the discovery of yet additional ways where research can go wrong.

Having said all of that, BE HOPEFUL! I'm thoroughly convinced that music scholarship's best moments lie ahead. I think the future of music research is actually very rosy. I only wish I could be around for all those future discoveries. And with that, I thank you for your attention.

\section{Questions and Answers}

Bob Sturm: 1) What did the work of Mauch et al. show that wasn't already known from popular music studies? (Their paper makes some very strange claims.) 2) Do you think the kinds of features they extract (e.g. 12 MFCCs from $43 \mathrm{~ms}$ frames) from 30-second extracts 
(which might not even be "representative") may mean they are not analyzing music at all?

David Huron: In my talk I highlighted those dates that people might recognize as interpretable: 1964, 1983, and 1991. First, notice that the magnitude of the three corresponding Foote similarity measures differ: the data suggests that the musical changes associated with the advent of Rap and Hip Hop are larger than for New Wave and Hard Rock, and are much greater than for the British Invasion. Also notice that there are lesser but noticeable moments of change around 1998 and 2000.

What's useful about a quantitative approach is that it makes it clear that there is a hierarchy to different musical changes: some are more important than others. A quantitative approach also alerts us to more subtle musical changes.

I agree that there are technical issues that mar this particular paper by Mauch et al. However, I think the general approach is inspiring. Future researchers will surely refine the methodology, and I expect historians will find it stimulating to interpret the various quantitative results.

Eric Elder: I'm curious to know more about how you define corpus content, or define your terms, as in your study with Horn. For example, it seems to me that highly successful American (at least) "light-Classical" composers - who accounted for the lion's share of sheet music-didn't make the cut in fitting into your definition of "art music." I'm thinking here of composers like M. L. Gottschalk or Basile Bares. How do we make decisions of definition and corpus content responsibly?

DH: I'm glad you phrased your question in terms of "responsibility." There's no such thing as an unbiased musical sample. At this point in our history, the main problem is not building unbiased corpora; the main problem is building any corpora at all. Everybody wants data, but in my experience very few researchers want to tackle the thankless job of assembling a corpus. Especially if assembling a corpus leads to charges of bias, there's even less incentive for scholars to do the necessary work.

Ironically, criticism itself can lead to even worse bias. Suppose I'm interested in Czech music and want to build an appropriate musical database. No matter what I include, of course, I'll be criticized for excluding composers $\mathrm{X}, \mathrm{Y}$, and $\mathrm{Z}$. I can avoid such criticism, for example, by focusing just on the music of Smetana. No one can criticize me now because I can say my database is music by Smetana.
The danger here is we end up with corpora for individual musicians and avoid corpora representing national, stylistic, cultural, or period musics. That is, because of the fear of criticism, we're less likely to have corpora for Indonesian music, Hip Hop, Romani (gypsy) music, LGBTQ+ music, or 12th century Gregorian chant, and more likely to have corpora for Bach, Mozart, and Palestrina - which is indeed the case now.

Of course, we need to be vigilant about the biases attending any database. When we publish, we need to identify as best we can the limitations of any corpus we use.

In the case of the Horn and Huron study, the sampled composers were randomly drawn from two Wikipedia articles entitled "List of Classical-Era Composers" and "List of Romantic-Era Composers." Together, these articles list 860 composers. Gottschalk was included in the list from which we randomly selected composers, but not Bares.

If I were to offer advice, I would say: Do the best you can to have a clear definition of the population of interest; be as inclusive and representative as you can. But above all, please build a corpus!

As we gain experience building more musical databases, researchers will inevitably become more sophisticated in identifying and controlling various forms of sampling bias.

David Sherry: Has neuroscience research produced in the explanatory category of music research?

DH: When I was an undergraduate, I read quite a bit of basic hearing science with the hope that it might contribute to my understanding of music. It didn't help at all.

At least, that was the case for the first couple of decades of my career. But over time, connections emerged, and now we can see that there are important musical phenomena (like the high voice superiority effect) whose origins can be traced directly to the physiology of hearing.

In the case of neuroscience, I think we need to be similarly patient. I'm not a neuroscientist, but my understanding is that the cliche criticism is that neuroscience-more specifically brain imaging research-tends to point to anatomical correlates, sometimes using weak data, sometimes using suspect statistical procedures, while failing to inform us about the causal mechanisms - that is, offering explanations.

Neuroscience these days is mostly correlational rather than explanatory. But neuroscientists are smart people facing especially difficult problems. These are early days in our understanding of the brain. I expect it 
will take a while before neuroscientists can remove the training wheels and really start moving ahead. My experience with studying hearing sciences I think offers a hopeful tale: we simply need to be patient.

I think it would be a grave mistake for a music scholar to assume that music can be understood without recourse to understanding the brain. That's a bad idea in the same way that ignoring culture is a bad idea.

Michael Schutz: Can you give examples of individuals in other fields who have been particularly successful in translating basic research into public policy? This seems like a very important challenge for us as music scholars.

DH: No. I don't know of anyone I could point to. It's more of a wish on my part for what I hope happens in the future.

Song Hui Chon: There must be some noise-related public policy and regulations based on acoustics and psychoacoustics (and medicine). Not exactly musicbased research, but a closely related field.

DH: Good point.

Assaf Suberry: Isn't a good music policy advice will be to invest in music education? Not for its cognitive outcomes but for its cultural/moral or just because of the inner value of music making and listening.

DH: I agree. We don't need to promote music simply for its instrumental value. Music gives pleasure to billions of people, and unlike the pleasure of smoking, eating, alcohol, gambling, and other enjoyable activities, music is remarkably benign. Music has intrinsic value in people's lives apart from how it might be used for other purposes.

Christ Billy Aryanto: I want to ask regarding music training and cognitive abilities. Most past research measured musical training by years of music training only or self-report questionnaire. Do you think there is a problem of measurement objectivity so there is no strong relationship found between those variables?

DH: That's an interesting proposal. Your suggestion is that perhaps we haven't seen much cognitive transfer from musical skills because our measures of musical skill are poor. I suppose that's quite possible. That sounds like a good research project.

Laurie Heller: Any advice for psychology in particular?

DH: I have a great admiration for psychologists. I've interacted with a lot of scientists over my career, and the very best scientists I've encountered are not acousticians, biologists, or neuroscientists - they're experimental psychologists. They're generally the most knowledgeable about experimental design and statistics, and more careful in applying best-practices methodology. The only advice I can offer to psychologists is: keep doing what you're doing!

Courtney Hilton: You identified demonstrating 'value' of more scientific research as being key to better integrating it within the broader field of musicology. I think I agree. But, to play devil's advocate, what if the different fields have fundamentally incommensurate values (i.e., like what someone like Kuhn, in the philosophy of science, would describe as arising from fundamentally different research paradigms)? How optimistic should we be about achieving better integration.

DH: People do indeed disagree about values, but those disagreements usually aren't as irreconcilable as is commonly supposed. Philosophers have noted that Kuhn's concept of "incommensurability" is overstated. In the real world, people don't have trouble trading apples for oranges. The key is conversation.

I think we'd be surprised at how readily folks from different backgrounds would come to some basic agreements about shared values, even if some disagreements persist. I think much of the problem arises because there's no conversation going on. When it comes to values, people tend to want to be affirmed rather than informed. It helps a lot to talk directly with people you think of as the enemy.

Byan Bell: What other research methods do you think are promising besides corpus studies?

DH: The gold standard for empirical research is still the double-blind controlled experiment. As I've argued elsewhere, what we really need is a lot more sensitive and mindful cross-cultural experimental research.

Sarah Sauve: Perhaps I'm misunderstanding the context, but as a predominantly Western and white researcher field, how can we fairly define what a "good musical culture" looks like?

DH: Yes, it would be a grave mistake not to cast a wide net when considering what makes a good musical culture. Once again, I think conversation is the key. We will learn to identify good musical cultures only through experience, debate, and interaction.

Incidentally, there's precedent for this with various research efforts to measure general human well-being. You know, for decades, governments have tended to rely on GDP - gross domestic product - as an index of a nation's well-being. GDP has a certain utility, but its deficiencies have also been long recognized, not least by economists themselves. When GDP is used to shape social policy, there is plenty of opportunity for mischief. 
Can we come up with a better index of human wellbeing?

You probably know the story about what happened in Bhutan when the King there voiced his dissatisfaction with GDP and instead said that his policies would be oriented to maximizing what he called Gross National Happiness. Over the past three decades or so, dozens of new measurements have been devised to better estimate human well-being-like the Med Jones Gross National Well-being index, the Oxford Poverty and Human Development Initiative, the Multidimensional Poverty Index, the Gallup-Healthways Well-Being Index, and the OECD Better Life Index. And probably the best known of these measures is the United Nations Human Development Index.

Of course, none of these measures is perfect. But as we gain experience and input from lots of different people, these sorts of measures do, I think, help us better understand various implicit or explicit human values. When decisions are made on the basis of these more refined indices, we have the potential to better tailor policies and regulations so as to increase their benefits.

My aim here isn't to define "good musical culture." My aim is simply to raise the concern and to encourage research in this area. In my own career I've done no policy-related research at all. It's only since I retired that I've really thought about the state of music research in general and what we haven't been doing that we ought to be doing. And my conclusion is that we haven't been attending to an important part of our research mission: nurturing the sort of musical culture that best contributes to human well-being.

Niels Christian Hansen: If corpus studies hold the promise for future scholarship, it becomes key for us to identify and/or create the best corpora. I'm curious what your favorite corpus is? And how do we go about identifying and creating optimal corpora in the future?

DH: I'm not sure I have a favorite corpus because they're all flawed in various ways. I suppose I'm partial at the moment to a cross-cultural sample of melodic transcriptions we created recently based on the French Ocora and the Smithsonian Folkways collections. It's a symbolic rather than an audio corpus. It's far from ideal, but it's a real effort to create a musical database that draws on a range of musics from around the world. Unfortunately, it needs to be bigger.

How do we go about creating optimal corpora for future research? That's a great question, but I'm afraid that would require a very lengthy response. There are just so many considerations, so I'll pass on answering that for now.
Juan Pablo Vigneaux: Could you elaborate a bit on the role of psychology and cognitive science in future music research and to what extent this line of work might be relevant to general musicologists? What are the future perspectives of those approaches? (I'm trying to distinguish them from the sort of data analysis methods applied to corpus studies and to the public policy recommendations.)

DH: In retrospect, I guess my presentation has unduly emphasized my enthusiasm for corpus studies and policy research. Many of the questions I posed in my talk are essentially psychological in character. For example, can a person listen to too much music? How does music evoke emotions in listeners? Can music somehow corrupt or enhance moral behavior? Is background music bad for you? These are questions that can't be answered through corpus studies, but they are questions that can be addressed through experiments. Surely, much of the most informative music research in the future will be psychological.

Pauline Larrouy-Maestri: You say "what is a good music culture?" and "how to improve musical culture?" are good questions. Can you tell us more about what you mean by "good" in this context?

DH: As I mentioned earlier, my aim is really to start a conversation rather than to propose specific solutions. But I understand that it would be helpful to have some idea of the sorts of values one might imagine characterizing a good musical culture. So in general, what I mean by "good" is something that contributes to human well-being. That can be cached out in various ways, including better prospects for employment opportunities for musicians, better opportunities for amateur music-making, greater freedom of musical access for listeners, musical experiences that people find meaningful and rewarding, music that contributes to mental and physical health, and a culture that encourages people to be respectful and understanding of folks different from themselves. How's that for a start?

William O'Hara: How might music departments embrace the public policy agenda you describe? Researchers pivoting to new areas? Interdisciplinary collaborations? New grad/undergrad degree programs, etc.?

DH: It would require leadership at the level of department heads or directors. It's going to be challenging because currently I don't know of any scholar who does cultural policy research in music. So even if a department head or director wanted to hire such a person they're going to find it difficult to find someone. I suppose one might begin by arranging one 
or more conferences related to public policy in musical culture and see what happens. Lots of people have musical backgrounds, so perhaps there's an existing scholar in economics, law, or government programs who could be inspired to focus on this topic. In the end, I suspect that an existing music industry program might be encouraged to expand its offerings and hire someone in the area.

Eric Clarke: My question relates to what you characterize as poetic investigation and in particular to the relationship between practice-as-research and explanatory research. Arguably, David Sudnow's 1978 book 'Ways of the Hand' is close to the former, but perhaps help to for instance Jonathan De Souza's 2017 'Music at Hand'. So is there really such a gulf between them as your presentation might have seemed to suggest?

DH: I recall loving Sudnow's 'Ways of the Hand' and his later book 'Talk's Body.' Although Sudnow's writing is wonderfully poetic, in my taxonomy, I'd characterize it primarily as phenomenological and so descriptive. He's describing in intimate detail his personal subjective experience. And that's grist for the mill for trying to interpret and understand it. So I think it feeds pretty directly into the later, more analytic work, such as De Souza's work on idiomaticity.

Aditya Chandler: Given how little consensus there is on the best approach to music studies, how do you approach communicating your findings in an engaging way with those who may not have the expertise or trust in empirical, explanatory work that you have?

DH: Many people (myself included) are interested in making the world a better place. That is, we'd like to bring about certain changes. Causal ("how" and "why") accounts should be inherently more interesting to us because explanatory accounts offer opportunities to effect change. (If you don't believe there are knowable causes, then you can't change the world except by accident.) So for people who want to change the world, explanatory work should already be intrinsically more engaging.

Secondly, there is something captivating about evidence. Like a good detective story where the missing fireplace poker means the butler couldn't have perpetrated the crime, explanatory research often assembles evidence that people will find absorbing, forceful, and compelling. There is something satisfying about a persuasive argument.

Yes, there are times when we are offended by explanatory accounts because they feel too deterministic. All you can do is talk about it.
Logan Rutledge: I feel as though some poetic music theory papers deal more strongly with Gender Studies, Critical Race Theory, LBTGQIA+ Studies, etc. (For example, Marianne Kielian Gilbert's work on Nina Simone and Miriam Gideon). Is it possible that while poetic papers might be more based on personal observation creating a paper embedded in subjectivity, the interdisciplinary and cultural depth and meaning is more significant and thus is important in moving legislation through affect?

DH: There's a lot here in your question to talk about. Let me pick up on just one thread.

There's a long history of debate about whether change is best achieved through logical argument or by emotional appeals. I suppose the division between Art and Science reflects these two different aspects of who we are. The cliché is that Art appeals predominantly to feeling whereas Science appeals predominantly to our sense of rationality.

I think it's important to recognize the limits of both approaches. You can't debate what people feel, but sometimes the feelings people experience arise from misunderstanding a situation. On the other hand, logical arguments can be devoid of moral or aesthetic value. I'm not ready to give up, either on the value of human emotions, nor the value of rationality. Or said another way, I love both art and science.

Lydia Snyder: I found the study on changes in musical elements across time fascinating. Has there been any studies like this done in other cultures?

DH: None that I know of. I'd encourage young scholars to consider pursuing more such studies and, as you suggest, to broaden the mandate to study all of the world's music-making.

Anonymous Attendee: What advice would you give to a university music student who aspires to conduct research in music cognition, but lives in an area in which music cognition is not offered in many universities? How does one pave the way for music cognition in a university where there is currently no program?

DH: First, I sympathize with people who don't have access to the same resources as others do. It's an unfortunate if perennial problem.

Of course when I was a student (many years ago) there were no music cognition programs in existence. In my case I thought the study of music needed to be more than simply the study of music history. For both my undergraduate and masters' degrees I enrolled in interdisciplinary programs that allowed me greater freedom to pursue different things. I took courses in music. But I also took courses in acoustics, psychology, 
computer science, philosophy, aesthetics, history, physiology, and sociology. Apart from the coursework, I did a lot of reading, predominantly in music, but later in cognitive sciences.

If you're hoping to do an advanced degree related to music cognition, most programs are looking for students who have strong backgrounds in both music and science.

Bryan Bell: Of the "big questions" you listed at the beginning of your talk, which questions do you think are most pertinent to music research now?

DH: I think there are a number of interesting questions related to the flexibility or plasticity of human musical hearing. For example, music theorists have long argued that good musical analyses change the way people experience the music. These claims need empirical support. That is, I think a worthwhile area of future research would address questions like: Are there different ways of "listening?" And with training or effort, how differently might we be able to hear music?

Similarly, questions arise from our engagement with world music and cultural difference. For example, to what extent can we hear or understand the music of another culture in the same way as people from that culture do?

It would be interesting to know whether different musical preferences relate to different ways of hearing. For example, I'm not a fan of Heavy Metal. So why not? Is there something about the way I listen-my listening habits, my personality, my cultural background - that prevent me from appreciating this genre? And, of course, I favor the question: What makes a good musical culture?

Eleonora Beier: Given the importance of focusing future research on applied questions, do you think there is still value to basic research about the cognitive/neural mechanisms of music even if it does not provide obvious applications to policy and society?

DH: Absolutely. For two reasons. First, there's a long history of tangential research bearing unexpected fruit. I think of basic research as second-order pragmatic research.

The second reason is more important. The most important resource any researcher has is her or his passion. Without passion, there's little motivation to work on problems. You're going to be your most productive as a scholar if you are doing things you're passionate about. If you're passionate about doing applied research, then do applied research. If you're passionate about doing basic research, then do basic research. Also, don't be afraid to change your research focus. Your interests are apt to change over the course of your career. Following your passion is key to maintaining your enthusiasm and productivity.

Michael Schutz: The findings of Mauch et al. (2015) regarding changes over music history are fascinating. At the same time, seeing that I can't help but be reminded of your example of tendencies to construct stories after the fact. There is a great example in the "American Soldier" survey looking at how we can easily generate explanations for nearly any outcome of complex data. Nearly any outcome of "what predicts success as a soldier" sort of seems right when you think about it (I think I learned this from your workshop!).

How do we guard against this kind of post-hoc explanation for what could (in theory) be artifacts of complex statistical analyses. In other words, if the dips had come in different years, could we not have come up with musical/cultural events happening around then that might (might) explain them? Music is so complex, this seems like a real danger.

DH: As you rightly note, the best research posits $a$ priori hypotheses and then tests them. Post hoc accounts aren't nearly so convincing. I liken a priori hypothesis testing to guessing what's inside a box and then opening the box to see if you're right. In post hoc research, you open the box, and then tell everyone what's inside. Clearly, a priori research is more compelling because the researcher better invites failure.

But post hoc or exploratory research also has an important role. There are plenty of cases where you don't have a clue what's inside the box, and so you have little choice but to open it up and have a look. Especially in novel fields where we don't yet have any good theories or intuitions, post hoc or exploratory research is the only viable approach.

As you note, in corpus studies there's a real danger in conducting exploratory research when the corpus represents a complete population of interest. In this case, a post hoc approach amounts to opening all of the boxes. That's not good because it preempts the possibility of doing later a priori hypothesis testing. If you're interested, I've written about this in a 2013 paper entitled "On the virtuous and the vexatious in an age of big data."

\section{References}

Aarden, B. \& Huron, D. (2001). Mapping European folksong: Geographical localization of musical features. Computing in Musicology, 12, 169-183.

Bigand, E., \& Tillmann, B. (2021, February 12). Near and 
far transfer: Is music special? PsyArXiv. https://doi.org/10.31234/osf.io/gtnza

Condit-Schultz, N. \& Huron, D. (2015). Catching the lyrics: Intelligibility in twelve song genres. Music Perception, 32(5), 470-483. https://doi.org/10.1525/mp.2015.32.5.470

Costa-Giomi, E. (2004). Effects of three years of piano instruction on children's academic achievement, school performance and self-esteem. Psychology of Music, 32(2), 139-152. https://doi.org/10.1177/0305735604041491

Feld. S. (1990/2012). Sound and Sentiment: Birds, Weeping, Poetics, and Song in Kaluli Expression. Duke University Press. https://doi.org/10.1515/9780822395898

Foote, J. (1999). Visualizing music and audio using selfsimilarity. In Proceedings of the seventh ACM international conference on Multimedia (Part 1) (pp. 7780). https://doi.org/10.1145/319463.319472

Foote, J. (2000). Automatic audio segmentation using a measure of audio novelty. In 2000 IEEE International Conference on Multimedia and Expo. ICME2000. Proceedings. Latest Advances in the Fast Changing World of Multimedia (Cat. No. 00TH8532) (Vol. 1, pp. 452-455). IEEE. https://doi.org/10.1109/ICME.2000.869637

Harden, K. P. (2021). The science of terrible men. Aeon. https://aeon.co/essays/what-do-we-do-with-the-scienceof-terrible-men Accessed March 31, 2021.

Huron, D. (2006). Sweet Anticipation: Music and the Psychology of Expectation. MIT Press. https://doi.org/10.7551/mitpress/6575.001.0001

Huron, D. (2013). On the virtuous and the vexatious in an age of big data. Music Perception, 31(1), 4-9.

\footnotetext{
1 The text for this article is based on the inaugural keynote address for the Future Directions of Music Cognition International Conference, delivered online on February 22, 2021.
}

https://doi.org/10.1525/mp.2013.31.1.4

Huron, D. (2016). Voice Leading: The Science Behind a Musical Art. MIT Press. https://doi.org/10.7551/mitpress/ $\underline{9780262034852.001 .0001}$

Horn, K. \& Huron, D. (2015). On the changing use of the major and minor modes 1750-1900. Music Theory Online, 20(1), March 2015. https://doi.org/10.30535/mto.21.1.4

McClary, S. (1987). Minnesota Composers Forum Newsletter.

Sala, G., \& Gobet, F. (2020). Cognitive and academic benefits of music training with children: A multilevel meta-analysis. Memory \& Cognition, 48, 1429-1441. https://doi.org/10.3758/s13421-020-01060-2

Schellenberg, E. G., \& Weiss, M. W. (2013). Music and cognitive abilities. In D. Deutsch (Ed.), The psychology of music (3rd ed., pp. 499-550). Amsterdam, the Netherlands: Elsevier. https://doi.org/10.1016/B978-012-381460-9.00012-2

Schellenberg, E. G. (2020). Music training, individual differences, and plasticity. In M.S.C. Thomas, D. Marescha \& I. Dumontheil (Eds.), Educational Neuroscience, Frontiers of Developmental Science, (pp. 415-442). Routledge Taylor \& Francis Group: New York and London. https://doi.org/10.4324/9781003016830-21

Temperley, D. (1999). The question of purpose in music theory: Description, suggestion, and explanation. Current Musicology, 66, 66-85.

Zimmermann, Jens (2015). Hermeneutics: A Very Short Introduction. Oxford University Press. https://doi.org/ 10.1093/actrade/9780199685356.003.0006 


\author{
Use-inspired music cognition: \\ Designing cognitively informed musical interventions for the brain \\ Psyche Loui ${ }^{\dagger}$ \\ ${ }^{1}$ Department of Music, College of Arts Media and Design, Northeastern University, Boston, MA, USA \\ ${ }^{\dagger}$ Corresponding author: p.loui@northeastern.edu \\ Published 16 December 2021; https://doi.org/10.18061/FDMC.2021.0003 \\ Author video presentation and/or other conference material: https://doi.org/10.17605/OSF.IO/6TNES
}

\begin{abstract}
As the field of music cognition is rapidly burgeoning, researchers are beginning to consider how the unique amalgam of scientific and humanistic study of music may translate towards large-scale interventions that may improve cognition for many, including but not limited to people from neurodiverse populations. Here I examine novel ways in which music cognition research may help improve cognition, in ways that move away from overused tropes (e.g. the Mozart Effect) towards future directions of use-inspired music cognition research. As use cases, I will describe some recent studies in my lab that capitalize on new musical technology, developed from first principles from music cognition research, to help those with attention deficits, dementia and memory disorders, and Parkinson's Disease. Our results show how music cognition can help refine and target music-based interventions for multiple special populations, by pinpointing ways in which music capitalizes on fundamental operating characteristics of the brain.
\end{abstract}

KEYWORDS: interventions, cognitive, neuroscience, entrainment, Alzheimer's Disease

\section{Introduction}

In considering future directions in the field of music cognition, perhaps it is helpful to start with the early days of our field. In doing so I begin by acknowledging that technology has always informed music cognition. Figures such as Max Matthews and John Chowning, who were some of the founders of electronic music and sound synthesis, changed how we think about how the mind can perceive and produce music. Innovations in sound design gave rise to sounds like the Shepard tone, which helped shed light on the difference between pitch direction and pitch height. The work by Bregman on scene analysis (Bregman, 1990) was informed by sound design technology, and has since then informed sound design technology as well.

On the other hand, music cognition has always been inspired by subjective experience. The work by Diana Deutsch, for example, on the speech to song illusion and the tritone paradox, informed our study of individual differences. The work by Carol Krumhansl and colleagues on probe tone profiles (Krumhansl, 1990) was really a subjective rating of fit within a musical context that gave us some understanding into our mental representation of music. And that was coupled with the use of multidimensional scaling to define musical spaces, such as the work of David Wessel on timbre spaces (Wessel, 1979) and by Carol Krumhansl and colleagues on pitch spaces and tonal spaces (Krumhansl $\&$ Kessler, 1982). These examples were relatively objective ways to look at something that felt subjective at first. I was initially drawn to this field because I felt that it was a way to use somewhat objective methods to look at subjective experiences. The more I do research, however, the more I learn that these seemingly objective tools are certainly still implemented by humans. And of course, humans can be quite subjective at times. Thus, we have to be careful and responsible in how we think about data and why we acquire data, as well as how we use and reuse data.

\section{Lessons from History of Music Cognition}

It bears mention that there were some relatively dark pages in the history of music perception and cognition. One of these dark pages, perhaps, is the Mozart effect in 1993, when it was discovered that if you listen to Mozart Two-Piano Sonata K. 448 for 10 minutes or so, afterwards there was a boost in IQ scores, more specifically in mental rotation scores, that was related to IQ (Rauscher, Shaw, \& Ky, 1993). It turns out there were lots of problems with that claim. First of all, we learned a lot about the importance of control conditions, or the ruling out of possible confounds and covariates that might contribute to this effect. Secondly, most of that effect is attributable to affective arousal. So the controversial question remains, and that is: Can music listening affect cognition? There is ample evidence for long-term musical training affecting brain and cognition (Habibi, Ilari, Heine, \& Damasio, 2020), but can short 
term music listening also affect cognition? I think that is still an open question. As extraordinary claims require extraordinary evidence (Sagan, 1980), we need some extraordinary evidence to be able to make these extraordinary claims about music improving cognition. So what are some ways in which we can go about finding these extraordinary pieces of evidence? And so to that, I turn to Pasteur's quadrants for scientific research (Stokes, 2011), a way to think systematically about how different kinds of research directions or research programs can really inform different goals.

One such goal is the quest for fundamental understanding. Basic research is focused on the quest for advancing fundamental understanding. Discoveries such as the electron or the structure of the atom are research that changed how we know how the world works. On the other hand, it's hard to think immediately about how that's going to apply to your life, even though it obviously has fundamental and important implications for many fields afterwards.

On the other hand, another goal is societal need. To meet this goal we should think about something that's useful: applied research. For example, the invention of the light bulb, a patentable invention that improved many lives, is built upon fundamental understanding, but it did not necessarily change fundamental understanding. I hope that music cognition research could turn towards something that's use-inspired, in that it can both be improvement in consideration of use, but also a quest for fundamental understanding.

\begin{tabular}{|c|c|c|c|}
\cline { 3 - 4 } \multicolumn{2}{c|}{} & \multicolumn{2}{c}{ Considerations of use? } \\
\cline { 3 - 4 } \multicolumn{2}{c|}{} & No & Yes \\
\hline $\begin{array}{c}\text { Quest for } \\
\text { fundamental } \\
\text { understanding? }\end{array}$ & Yes & Bosic research & Use-inspired research \\
\cline { 2 - 4 } & No & - & Applied research \\
\hline
\end{tabular}

Figure 1: Pasteur's quadrants for classification of scientific research.

My claim is that music cognition can help refine and target music-based interventions for multiple populations. Trained as a cognitive neuroscientist, I think about ways in which music cognition can help reveal the fundamental operating characteristics of the brain. In this regard, there could be a use-inspired cycle between purely thinking about music and the mind, and applications for how this can be used to help people.

Here I will review a few different use cases from work in my lab. Our work on music and the reward system (Sachs, Ellis, Schlaug, \& Loui, 2016) has inspired some ongoing projects in my lab on musicbased intervention for older adults (Loui et al., 2017; Quinci et al., 2021). We are also interested in music and the auditory-motor system, and for that, we have some work that's ongoing on dance for Parkinson's disease (Krotinger \& Loui, 2021). Thirdly, I will discuss music and neural entrainment, as more and more research is showing that music is a very effective way of entraining or tuning the neural activity or populations of neurons in the brain (Tichko, Kim, Large, \& Loui, 2020). Can we capitalize on this knowledge towards making some music that might be useful for cognition?

\section{Music and the Reward System}

Let us begin by talking about music and the reward system. As we know, different individuals have different aesthetic responses to art (Harrison \& Loui, 2014). When I listen to Rachmaninoff's second piano concerto, I often get the chills. I often feel like my hair is standing on end. But maybe that is not your experience at all. Maybe you find that when you listen to punk rock, you suddenly feel like your heart is racing and you get very excited and you feel very strong emotions.

Several years ago, Matt Sachs and I surveyed two hundred undergraduate students on how frequently they experienced these strong aesthetic responses (Sachs et al., 2016). We then used multidimensional scaling to give a two-dimensional space of how closely related these different, strong aesthetic responses were. In this two-dimensional space, the points that are closer together mean that they're more frequently co-reported by multiple individuals. From this you can see heart racing, heart skip a beat, hair stand on end, pit in stomach, lump in throat - items that actually pertain to part of the body show up on one side of the plot, whereas items that are more abstract and cognitive and maybe even transcendental (feeling like you're somewhere else, or losing sense of time, or feeling in awe, or feeling touched) show up on the other side of the plot. Chills shows up in the middle, which means that it is a fairly commonly reported strong emotional or aesthetic response to music that people get when they experience music. We can also do the same multidimensional scaling on people's ratings for how much they like certain musical genres. And then you can make certain predictions with this space. For example, if you enjoy listening to blues, you probably also enjoy listening to folk and reggae and bluegrass. If you enjoy listening to pop you probably also like rock and oldies and classical. 
Again, no one size fits all, but there's a variety of musical genres that are all equally good parts of the experience.

We then looked into brain connectivity differences between groups of people who either got chills all the time and people who did not get chills, controlling for many different variables, including the amount of musical training people had and personality differences, and also gender and age. What we found was that controlling for all these differences, people who get chills when they listen to music, tend to have a larger volume of white matter connectivity that was identified using diffusion tensor imaging, between the superior temporal gyrus, which is important for auditory perception, and the insula and the medial prefrontal cortex, which are important for emotion and for social reward processing. In other words, there are structural differences between the brains of people who get chills all the time and people who do not particularly feel emotional or feel chills when they listen to music.

After we published these results, we were approached by BW who is a musical anhedonic. He scored more than five standard deviations below the mean on all the variables within the Barcelona music reward questionnaire (Mas-Herrero, Marco-Pallares, Lorenzo-Seva, Zatorre, \& Rodriguez-Fornells, 2013), and he reported a socially-debilitating lack of emotional responses to music. When we looked at his brain, we saw that there was a difference in structural connectivity in the white matter between areas that are important for auditory perception, specifically the superior temporal gyrus, and the areas that are important for reward, specifically the nucleus accumbens (Loui et al., 2017).

Amy Belfi and I defined, based on a review of the literature, a neuroanatomical model that's important for music and reward (Belfi \& Loui, 2020). On one hand the auditory system is useful for making predictions about specific musical features as they come in. On the other hand, the reward system is important for learning and motivating the shaping and testing of predictions. Musical emotions and music reward probably hinge upon the successful interaction between these two networks within the brain.

\section{From Model to Music-Based Intervention}

This neuroanatomical model was implemented by Diana Wang, who was an undergraduate in my lab (Wang, Belden, Hanser, Geddes, \& Loui, 2020). She looked at the regions of interest that were in the auditory network and in the reward and valuation network, and she quantified the functional connectivity of these reward and auditory regions in a large group of individuals. This is using an open source dataset called ADNI (Alzheimer's Disease Neuroimaging Initiative) (Jack et al., 2008). ADNI enabled us to look at 108 brains without having scanned any of those brains ourselves. Among healthy older adults, there was significant functional connectivity from both the auditory and the reward systems. There was also an overlap between these two systems, centering around the anterior insula, but really spanning many different areas of the brain. This functional connectivity was generally decreased in people with Alzheimer's disease, but there was still some preserved functional connectivity in the anterior insula. Thirdly, when looking at functional connectivity in those with mild cognitive impairment, we saw functional connectivity patterns that was closer to the healthy controls, again with the overlap between auditory and reward connectivity centering around the anterior insula.

This correspondence between the functional connectivity data and our anatomical model provides an actual therapeutic target for how the auditory system and the reward system might be talking to each other, as we design music-based interventions for healthy aging. Working with music therapists in the Berkelee college of music, especially Dr. Suzanne Hanser, we designed an eight-week-long intervention where a therapist comes in and works with older adults. We are starting with healthy, older adults, but we are moving towards people with mild cognitive impairment and hopefully eventually people with Alzheimer's disease as well. For our intervention, we are testing how much mindful listening every day, for an hour a day, can change your brain's activity, connectivity, and performance on a variety of neuropsychological tests. While we are doing this music-based intervention right now, I will specifically present some preliminary results from a functional magnetic resonance imaging task. In this task, participants listened to 24 different twenty-second long clips of musical stimuli, and made ratings of how much they liked the music and how familiar that music was to them. The musical stimuli included some songs that we used from previous studies, but also some songs importantly that were self-selected by the listeners. Listeners brought in what they thought they most enjoyed listening, and then we cropped them to the length that fits into the time they spent in the scanner.

When listening to self-selected music, we saw activation unsurprisingly in the auditory areas. There was also significant activation in the medial prefrontal cortex, which is part of the reward system. Co-activated 
with the medial prefrontal cortex we also saw the posterior cingulate cortex, and these two regions together are part of the default mode network. Interestingly, there was also deactivation in some other areas. This deactivation was stronger when listening to other-selected music. A contrast between self-selected music listening and other-selected music listening revealed a strong effect where there was massively higher activity throughout the brain of self-selected music compared to other-selected music. Music that you choose to listen to is much better at engaging your brain than music that someone else chooses for you.

While these results are still new and data collection is still ongoing, what's maybe not so new is the idea that music is important for social bonding, across many cultures. People across many cultures play music together, they sing together, they dance together. People in different musical cultures have love songs for example, and also lullabies, and people across many different cultures move together in response to music. And this is not something that we are taught to do, but it is just something that we do. There is something about music that compels movement together. Pat Savage and other colleagues and I mapped out how we thought this might be implemented in the brain (Savage et al., 2020). Musical features engage perception, action systems of multiple people, and that might be linked to, on one hand, the dopaminergic reward system, and on the other hand, the oxytocin and endogenous opioid systems. These systems together might be important for social bonding. Underlying areas in the brain that might be important for perception and action include superior temporal areas and motor areas which work together to enable perception-action coupling, but also in conjunction with the basal ganglia and the ventromedial prefrontal cortex. There are many other areas that are important that are involved in music listening, but this was one way to simplify it at this point.

\section{Perception-Action Coupling and Parkinson's Disease}

Perception and action coupling is what I want to turn to next. We know that music is quite effective at driving sensorimotor coupling, especially auditory-motor coupling, and rhythm is an important driver of that. And among different rhythmic patterns there are some that are more effective at driving sensorimotor coupling. We might call them groovy music. Groovy music is what makes you want to dance, the kind of music that when you listen to it, there's something about the auditory features that seem to compel your motor urge to move.
One group of people that suffer from a motor disorder are people with Parkinson's disease. Parkinson's disease is characterized by movement problems, including difficulty with initiating movement and difficulty in maintaining rhythmic movement. There is good anecdotal evidence that dancing can really help the impaired movements in Parkinson's disease.

We started to ask the question, what is it that dance experience might do? Can we look at the effects of an intervention that uses dance, on people with Parkinson's disease, and can we actually assess how rhythm might mediate the way dance might help improve the motor symptoms of people with Parkinson's disease?

We used various gold standards to assess rhythm, motor symptoms, and sensorimotor coupling in Parkinson's disease. To assess the motor problems in Parkinson's disease, we used the UPDRS, which stands for the Unified Parkinson's Disease Rating Scale (Goetz et al., 2008), which assesses a variety of different motor as well as non-motor symptoms, that are part of Parkinson's disease. To look at rhythmic ability we looked at the Beat Assessment Test (BAT) (Iversen \& Patel, 2010). To look at sensorimotor coupling, we looked at tapping entropy: participants had to listen to music that was either highly groovy or more chill, and they had to tap their fingers in time to the music. And we looked at how much variability there was in the tapping. Participants who are better at synchronizing to music tend to have lower variability, or lower entropy in their tapping data.

Does a dance intervention for Parkinson's disease actually reduce your Parkinsonian symptoms? Anna Krotinger and I saw a reduction in Parkinson's symptoms, that is an improvement in behavior, following four months of a dance intervention as assessed using the gold standard UPDRS score (Krotinger \& Loui, 2021). Does sensory-motor coupling have anything to do with it? To look at that, we looked at finger tapping for high-groove songs and for lowgroove songs. Some high-groove songs are Superstition, Sing, sing, sing, In the mood, Cheek to cheek. Some low-groove songs are Carolina on my mind, Till there was you, and Comfortably numb.

Parkinson's patients showed a reduction in tapping entropy after the intervention, suggesting that sensory motor coupling ability might be tuned or be improved.

Furthermore, dance experience predicted how successful dancing intervention was at reducing symptoms of Parkinson's disease. People who had more dance experience coming in to the intervention tended to show more of an improvement in UPRS. In other 
words, both dance intervention and dance experience are important for the reduction of symptoms in Parkinson's disease. And we think that the underlying mechanism involves perception and action, or the ability for rhythm to engage sensorimotor coupling.

\section{Music as Brain Stimulation}

Music listening engages multiple areas of the brain. From recent work in auditory neuroscience, we know that neural oscillations, rhythmic bands of brain activity, are linked to cognitive behavior by tuning different networks of the brain. These different rhythmic oscillatory networks, observable using EEG, are important for aging, and show differences between healthy aging and Alzheimer's disease and related disorders. We did a review recently on the multiple scales of dementia and related pathologies (Tichko et al., 2020). At the top, the behavioral impairments from dementia include learning and memory impairments, as well as deficits of motivation. These are subserved by neural systems-level differences, including lower gamma-band activity and lower theta-band activity in healthy aging, but especially in Alzheimer's disease. The neural systems differences in turn are subserved by neuronal degradation and the loss of synaptic activity at the cellular level and then amyloid plaques and tau tangles, unwanted protein deposits that are at the molecular level.

In recent years, exciting work had shown that gamma-band light stimulation, first optogenetically delivered directly to the brain and then visually delivered by flickering lights to the eyes, can reduce the levels of plaques and tangles that are in a mouse model of Alzheimer's disease, essentially reversing the biomarkers of Alzheimer's disease (Iaccarino et al., 2016). In humans, new research is emerging looking specifically at theta-gamma coupling, or the coupling between theta and gamma frequency bands, of rhythmic oscillatory brain activity in young adulthood and in older adults. Normally a young adult has theta-band and gamma-band activity that are well coupled together, and that enables good working memory performance. Now in older adults, there is a decrease in working memory performance; this is coupled with a decrease in the coupling between the theta band and the gamma band. Now, new research has shown that by restoring this theta and gamma coupling, by using transcranial alternating current stimulation, it was possible to reverse the working memory deficits so that the working memory performance of a 70 year old could match the level of a 20 year old. In other words, brain stimulation can revive working memory in older adults, specifically by synchronizing rhythmic brain circuits (Reinhart \& Nguyen, 2019).

Now we know some ways to synchronize rhythmic brain circuits. We know that music is, of course, a very rich rhythmic stimulus. We can even simulate the patterns of brain activity under musical stimulation. Work by Ed Large and Ji Chul Kim, and their company Oscilloscape, has been able to make a neural network model that can find the beat from musical sounds. Starting from the acoustic stimulus, they can figure out the frequency of the beat and then deliver that beat frequency by flashing lights. And so you could be looking at lights that are smart, like Christmas lights, but that can detect the downbeat. This is possible even when there's no energy at the downbeat, such as in a syncopated piece of music. Then, by adding a level of gamma stimulation to lights that are already dancing to the music, we can use this system as a new form of brain stimulation. And in doing so we could take advantage of the natural phase-amplitude coupling that's in the brain, and also take advantage of the fact that people are already very motivated to listen to music, to use musicmodulated lights as a form of brain stimulation and to test the ability of this system to affect cognitive behavior. We are currently testing this on young adults as well as on older adults. And then we hope to move on to people with mild cognitive impairment and with Alzheimer's disease.

\section{Summary and Conclusions}

I have reviewed a few examples of use-inspired cases for music cognition. The different projects that I have covered are all very new; they are all in different stages of completion.

To summarize, the music listening intervention project takes advantage of work from music and the reward system, and informs us about the role of agency in selecting stimuli. Again, we see evidence that not one size fits all, but the need to respect individuals' interest and desires and tastes in selecting music for an intervention that works. The work on dance for Parkinson's highlights the role of groove and sensorimotor coupling, and the possibility of harnessing that towards an intervention that helps reduce symptoms in a needy population.

The work on functional music for cognition, points at the role of oscillations and entrainment at both behavioral and neural levels for cognition. While these studies are just getting started, they highlight the need for designing control studies and asking tough questions 
that get at the active ingredients in an intervention, and the ways in which a research program may adequately control for extraneous factors that covary with the intervention in affecting outcomes.

To get back to the future directions of music cognition, I believe that music cognition research can be useful as well as informative. While we pursue research for our own interests, it is also useful to be guided by other people's needs in identifying possible research questions. This understanding, when applied to one's preferred level of analysis, may yield important information for how we design interventions to help those who need it the most. Finally, working across disciplines and across different lines of inquiry will be useful for informing future musical experiences. In some ways, perhaps the best music might not have been heard yet, and perhaps the best musical experiences might not have happened yet. There is need for working with people from different backgrounds and training, such as with music therapy and with sound design, that can contribute towards useful research.

\section{Acknowledgements}

I would like to thank my lab and collaborators for their wonderful work over the years, and their support and their inspiration. Supported by NSF-CAREER \#1945436, NSF-STTR \#2014870, Grammy Foundation, Kim \& Glen Campbell Foundation, and the Provost's Tier 1 award from Northeastern University.

\section{References}

Belfi, A. M., \& Loui, P. (2020). Musical anhedonia and rewards of music listening: current advances and a proposed model. Annals of the New York Academy of Sciences, 1464, 99-114. https://doi.org/10.1111/nyas.14241

Bregman, A. S. (1990). Auditory Scene Analysis: MIT Press.

https://doi.org/10.7551/mitpress/1486.001.0001

Goetz, C. G., Tilley, B. C., Shaftman, S. R., Stebbins, G. T., Fahn, S., Martinez - Martin, P., . . . Dodel, R. (2008). Movement Disorder Society - sponsored revision of the Unified Parkinson's Disease Rating Scale (MDS - UPDRS): scale presentation and clinimetric testing results. Movement disorders: official journal of the Movement Disorder Society, 23(15), 2129-2170.

https://doi.org/10.1002/mds.22340

Habibi, A., Ilari, B., Heine, K., \& Damasio, H. (2020). Changes in auditory cortical thickness following music training in children: converging longitudinal and cross-sectional results. Brain Struct Funct. https://doi.org/10.1007/s00429-020-02135-1

Harrison, L. D., \& Loui, P. (2014). Thrills, Chills, Frissons, and Skin Orgasms: Toward an Integrative Model of Transcendent Psychophysiological Moments in Music. Frontiers in Psychology, 5. https://doi.org/10.3389/fpsyg.2014.00790

Iaccarino, H. F., Singer, A. C., Martorell, A. J., Rudenko, A., Gao, F., Gillingham, T. Z., . . . Tsai, L.-H. (2016). Gamma frequency entrainment attenuates amyloid load and modifies microglia. Nature, $\quad$ 540(7632), 230-235. https://doi.org/10.1038/nature20587

Iversen, J. R., \& Patel, A. D. (2010). The Beat Alignment Test (BAT). Paper presented at the International Conference for Music Perception and Cognition, Sapporo, Japan.

Jack, C. R., Bernstein, M. A., Fox, N. C., Thompson, P., Alexander, G., Harvey, D., . . . Weiner, M. W. (2008). The Alzheimer's disease neuroimaging initiative (ADNI): MRI methods. Journal of Magnetic Resonance Imaging, 27(4), 685-691. https://doi.org/10.1002/jmri.21049

Krotinger, A., \& Loui, P. (2021). Rhythm and Groove as Cognitive Mechanisms of Dance Intervention in Parkinson's Disease. PLoS ONE, 2020.2009.2014.297325.

https://doi.org/10.1371/journal.pone.0249933

Krumhansl, C. (1990). Cognitive Foundations of Musical Pitch: Oxford University Press.

Krumhansl, C. L., \& Kessler, E. J. (1982). Tracing the dynamic changes in perceived tonal organization in a spatial representation of musical keys. Psychol Rev, 89(4), 334-368. https://doi.org/10.1037/0033295X.89.4.334

Loui, P., Patterson, S., Sachs, M. E., Leung, Y., Zeng, T., \& Przysinda, E. (2017). White Matter Correlates of Musical Anhedonia: Implications for Evolution of Music. Frontiers in Psychology, 8(1664). https://doi.org/10.3389/fpsyg.2017.01664

Mas-Herrero, E., Marco-Pallares, J., Lorenzo-Seva, U., Zatorre, R. J., \& Rodriguez-Fornells, A. (2013). Individual differences in Music Reward experiences. Music Perception: An Interdisciplinary Journal, 31(2), 118-138. https://doi.org/10.1525/mp.2013.31.2.118

Quinci, M. A., Goutama, V., Gong, D., Hanser, S., Donovan, N. J., Geddes, M., \& Loui, P. (2021). Effects of Music-Based Intervention on Loneliness 
and Reward Processing in Aging. Paper presented at the Cognitive Neuroscience Society.

Rauscher, F. H., Shaw, G. L., \& Ky, K. N. (1993). Music and spatial task performance. Nature, 365(6447), 611. https://doi.org/10.1038/365611a0

Reinhart, R. M. G., \& Nguyen, J. A. (2019). Working memory revived in older adults by synchronizing rhythmic brain circuits. Nat Neurosci, 22(5), 820827. https://doi.org/10.1038/s41593-019-0371-x

Sachs, M. E., Ellis, R. J., Schlaug, G., \& Loui, P. (2016). Brain connectivity reflects human aesthetic responses to music. Soc. Cogn. Affect. Neurosci., $\quad 11(6), \quad 884-891$. https://doi.org/10.1093/scan/nsw009

Savage, P. E., Loui, P., Tarr, B., Schachner, A., Glowacki, L., Mithen, S. J., \& Fitch, W. T. (2020). Music as a coevolved system for social bonding. Behavioral \& Brain Sciences, In press. https://doi.org/10.1017/S0140525X20000333

Stokes, D. E. (2011). Pasteur's quadrant: Basic science and technological innovation: Brookings Institution Press.

Tichko, P., Kim, J. C., Large, E., \& Loui, P. (2020). Integrating music-based interventions with Gamma-frequency stimulation: Implications for healthy ageing. European Journal of Neuroscience, $n / a(n / a)$. https://doi.org/10.1111/ejn.15059

Wang, D., Belden, A., Hanser, S., Geddes, M. R., \& Loui, P. (2020). Resting-State Connectivity of Auditory and Reward Systems in Alzheimer's Disease and Mild Cognitive Impairment. bioRxiv, 2020.2003.2011.986125. https://doi.org/10.1101/2020.03.11.986125

Wessel, D. L. (1979). Timbre Space as a Musical Control Structure. Computer Music Journal, 3(2), 45-52. https://doi.org/10.2307/3680283 


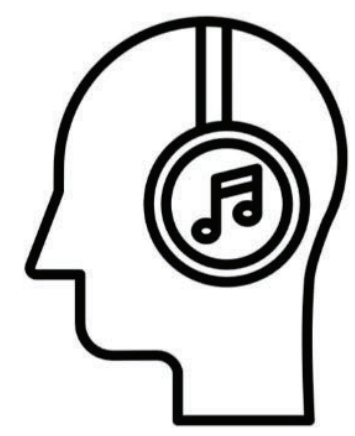

\section{Corpus Studies}

Future Directions of Music Cognition February - May 2021

Ohio State University

Virtual 


\title{
Tempering the clavier: Examining the intervallic content of Bach's Well-Tempered Clavier through the lens of historical temperaments
}

\author{
Andrew Blake ${ }^{1 \dagger}$ \\ ${ }^{1}$ Eastman School of Music, University of Rochester, Rochester, NY, USA \\ † Corresponding author: ablake10@u.rochester.edu \\ Published 16 December 2021; https://doi.org/10.18061/FDMC.2021.0004 \\ Author video presentation and/or other conference material: https://doi.org/10.17605/OSF.IO/UGW86
}

\begin{abstract}
While it is understood today that J.S. Bach's Well-Tempered Clavier was intended for a system of "well temperament," which (if any) specific temperament remains unknown. Excerpts from the work have been analyzed in the context of Werkmeister-III (Gann, 2019), but also within other tuning frameworks (Lehman, 1995). This study addressed the question of temperament from a computational standpoint in other words, the aim was to determine whether a certain temperament would be a better "fit" for Bach's WTC when frequency of harmonic intervals and deviation from pure (just) intonation are considered. This study examined accented (simultaneous onset) imperfect consonances' frequencies as a proportion of all accented harmonic intervals in the WTC fugues, weighting each according to its notated duration in the score. The average expected frequency for each interval was calculated on a basis of key (e.g. major third between tonic and mediant) to control for differences in usage arising from tonal function. Intervallic "emphasis," then, was used as a metric. This study found no significant correlation between intervallic emphasis for Werkmeister-III, and a small yet significant positive correlation for Bach-Lehman. This indicates that Bach was not avoiding less purely-tuned intervals in these fugues, and emphasizing the "color" of each key.
\end{abstract}

KEYWORDS: Bach, Temperament, WellTempered Clavier, Werkmeister, Bach-Lehman

\section{Introduction}

Not all harmonic intervals are handled equally in Western music. In the contrapuntal practices of J.S. Bach, for example, consonant intervals are a much larger part of the harmonic language than dissonant intervals (Huron, 1991). For example, the perfect fifth is used more frequently and freely than the minor second. Furthermore, certain combinations of scale degrees are more frequent than others as a consequence of tonal function, to such an extent that difference in the handling of pitch classes has been used to predict the key of an excerpt or work (Temperley, 1999, Albrecht \& Shanahan, 2013). As a result of this, one might expect the perfect fifth between the tonic and dominant in the major scale would likely be emphasized (used more frequently) over that between the mediant and leadingtone.

When considering tuning systems such as quartercomma meantone, however, some fifths will be more "in-tune" than others. In this system, 11 of the perfect fifths are tuned slightly flat (696.6c), while one is significantly sharp (737.7c). This is a consequence of the quarter-comma meantone system privileging the major third as a consonance: 8 of the 12 major thirds are justly tuned, but four are similarly sharp (427.4c). As a result of these stark differences between keys, it might be expected that composers would be more likely to write in keys which are not affected by the detuned intervals.

Regarding J.S. Bach's Well-Tempered Clavier, the question of which (if any) tuning system intended is not conclusively answered. It is clear that "well temperament" was intended, but this represents a class of temperaments, rather than one specific solution. In well temperament, all keys are considered to be equally viable for composition. Whereas it was previously thought that this work was intended for equal temperament, it is understood today that this was not necessarily the case (Gann, 2019, 90). Rather than relying solely on historical writings, it is possible that a systematic study of Bach's Well Tempered Clavier might provide insight into the "well temperament" intended for the work. This study examines Bach's WTC in the contexts of Werkmeister-III (Gann, 2019, 87-101) and the Bach/Lehman (Lehman, 1995) well temperaments, and additionally considers 12 -tone equal temperament and quarter-comma meantone (Gann, $2019,71-74)$ as controls.

The expectation of this study is that one or more nonequal well temperaments (Bach-Lehman, WerkmeisterIII) will better explain the harmonic interval content in the WTC fugues than other tunings (12-equal temperament, quarter-comma meantone). In short, this study addresses the question of whether Bach is using 
certain intervals more or less frequently than expected (based on their tonal function) in a manner consistent with their intonation in each key.

\section{Method}

\section{Scope of Corpus}

The corpus for this study consists of 48 fugues, from Bach's Well-Tempered Clavier (WTC). These fugues were already encoded in the CCARH corpus (Sapp, 1984) by David Huron in 1995, and the sic editions were used in lieu of the ossia versions. While Gann (2019, $97-$ 98) analyzes selected preludes from the WTC, the fugues were chosen in this study for their consistency in texture and number of voices. The preludes of the WTC include vastly differing textures, even between the preludes in $\mathrm{C}$ major in books 1 and 2. As this study was concerned with the harmonic interval content of Bach's WTC, certain preludes (e.g. Prelude No. 21 in B-flat Major, WTC Book 2) would create significant outliers in cases containing very few harmonic intervals. More specifically, the data of interest in the WTC fugues is the emphasis and de-emphasis of tonal intervals.

\section{Tonal Interval ("Scale-Degree Class Pair")}

For the purposes of this study, a "tonal interval" consists of a pair of chromatic scale degree classes - a pitch class space rotated such that the tonic pitch is always 0 , the dominant pitch 7 , etc. The notation of these tonal intervals always places the lower pitch first, thus $(0,4)$ and $(4,0)$ are different intervals (despite membership in interval class 4). This method is used to account for differences in intervallic usage with respect to key: for example, the perfect fifth $(0,7)$ would be expected much more frequently than $(6,11)$. Inversional equivalence is not assumed; $(0,7)$ represents a perfect fifth whereas $(7$, 0 ) is a perfect fourth. Additionally, octave equivalence (e.g. M3 = M10) is assumed for this study. Rather than considering all harmonic intervals, this study only examines those which are "accented" - intervals where two pitches are articulated simultaneously.

\section{Measurement of Interval Frequency}

Intervallic frequency was first measured as an interval's frequency of use, weighted using the durations of intervals in which two pitches have simultaneous onsets, as a proportion of all intervals used in a piece. For example, the tonal interval $(0,4)$ has a frequency of $4.716 \%$ in the C Major Fugue from WTC Book 1, whereas $(4,8)$ only has frequency $0.736 \%$. This discrepancy is consistent with the structure of the fugue - while the tonic key is $\mathrm{C}$ major, there are a few brief tonicizations of A minor using the major third E - G\#. The spelling of these intervals was considered, thus the interval E - G\# would be registered as a major third whereas $\mathrm{E}-\mathrm{Ab}$ would be analyzed as a diminished fourth. While the frequencies of intervals can be measured in this way, it could be argued that $(0,4)$ is not more common in this fugue "because it is more in tune," but rather due to the significance of this tonal interval over $(4,8)$. For this reason, all tonal intervals were measured using "deviation from expected frequency," and were compared to an average frequency across all fugues of the same mode (major/minor). The major and minor mode frequencies were calculated separately to avoid skewing of data, particularly around scale degree 3 (scale degree classes 3 and 4). Using "deviation from expected frequency" as a measure, $(0,4)$ in the C Major Fugue is actually $3.297 \%$ below the average for a major key fugue, while $(4,8)$ is $0.049 \%$ above average. Similarly, the minor sixth $(4,0)$ is $0.054 \%$ less frequent than expected, which might be indicative of this fugue spending more time in other tonal regions than others.

\section{Rationale for Focus on Imperfect Consonances}

While any interval in the chosen temperaments could be explained as an approximation of just intonation (e.g. the $700 \mathrm{c}$ fifth to the $3: 2$ harmonic ratio of $702 \mathrm{c}$ ), this study is only concerned with two intervals - minor thirds and major thirds - and their respective inversions. The more pure harmonic ratios of these intervals draw greater attention to their tuning when they are significantly mistuned, as might be most evident with an out-of-tune octave. Thirds are also prioritized over fifths in this study due to the lack of voice-leading constraints on their use. Fifths or octaves, on the other hand, are subject to constraints (e.g. avoidance of parallel or similar fifths). Regarding dissonances, some intervals such as the major second have multiple valid tunings in just intonation. The first three degrees of a justly tuned major scale, for example, consist of two major seconds tuned at 204c (9:8) and 182c (10:9) - a combination which yields a just major third (5:4) of 386c. Additionally, the tuning systems in question all entail varying degrees of preference for the major third or the perfect fifth, with quarter-comma meantone favoring thirds the most. Pythagorean tuning, which is not in the scope of this study, is the system which prioritizes the fifth. All tuning systems of interest here compromise the fifth slightly to prioritize the tuning of most (well temperaments) or all (equal temperament) thirds. For these reasons, only the four imperfect consonances are 
in the scope of this study, although they are still measured as a proportion of all intervals (including perfect fifths, minor seconds, etc.).

Hypothesis

The hypothesis is that for some temperament Bach's use of tonal intervals in the WTC fugues will emphasize those which are more in-tune and de-emphasize those which are farther out of tune. The statistical test used for this study was Pearson's R correlation, specifically between "deviation from expected frequency of use" and "absolute deviation from just intonation." There are 12 combinations of experimental conditions, as there are 4 tuning systems (12-equal temperament, quartercomma meantone, Werkmeister-III, and BachLehman), which can be examined in the "major" and "minor" key fugues, as well as both major and minor together. The null hypothesis was that there is no negative correlation between Bach's patterns of interval emphasis and some temperament $(\mathrm{p}<0.05)$. If such a negative correlation were found, rejection of this null hypothesis would provide evidence consistent with the hypothesis, as well as a possible clue regarding the temperament Bach might have had in mind.

\section{Results}

Of the four temperaments examined in this study, two were treated as potential candidates for the tuning of Bach's WTC - Werkmeister-III and Bach-Lehman. 12equal temperament and quarter-comma meantone were included as control cases, and it was expected that there would be no meaningful correlation with either. Table 1 provides Pearson correlation values for each of the 12 configurations (3 modes, each considered in 4 temperaments). Any interval which does not occur in any fugue of the corresponding mode was removed to prevent these from skewing the results (hence the discrepancy in population size for the "Major" and "Minor" cases).

\section{2-Equal Temperament}

As expected, there was no correlation between intervallic intonation and Bach's pattern of intervallic emphasis and de-emphasis. No matter which key is used, every interval is tuned equally.

\section{Quarter-Comma Meantone}

As with 12-equal temperament, there was no significant correlation between interval tuning and patterns of emphasis. Even though the effect size was larger than that of 12-equal temperament, the p-value indicates that these correlations are not significant.
Table 1. Correlation coefficients and p-values for 12 combinations of experimental conditions.

\begin{tabular}{|l|l|l|l|l|}
\hline Temperament & Mode & $\mathbf{n}$ & $\mathbf{r}$ & $\mathbf{p}$ \\
\hline 12-ET & Major & 1128 & $1.946 \mathrm{e}-6$ & .9999 \\
\hline 12-ET & Minor & 1080 & $2.755 \mathrm{e}-6$ & .9999 \\
\hline 12-ET & Both & 2208 & $2.361 \mathrm{e}-6$ & .9999 \\
\hline Meantone & Major & 1128 & .0102 & .7316 \\
\hline Meantone & Minor & 1080 & -.0047 & .8776 \\
\hline Meantone & Both & 2208 & .0026 & .9042 \\
\hline Werkmeister & Major & 1128 & .0460 & .1224 \\
\hline Werkmeister & Minor & 1080 & .0486 & .1035 \\
\hline Werkmeister & Both & 2208 & .0478 & .0247 \\
\hline Bach-Lehman & Major & 1128 & .0847 & .0044 \\
\hline Bach-Lehman & Minor & 1080 & .0673 & .0270 \\
\hline Bach-Lehman & Both & 2208 & .0757 & .0004 \\
\hline
\end{tabular}

\section{Werkmeister-III}

Under the "major" $(p=.12)$ and "minor" $(p=.10)$ key conditions, there is not a significant correlation between interval emphasis and intonation. When both major and minor are considered together, however, the result is a slightly positive correlation $(\mathrm{p}=.004)$. Considering the lack of a significant correlation in the previous tests (and similarity in effect size to the two insignificant correlations), this is likely a result of the larger population size $(\mathrm{n}=2208)$.

\section{Bach-Lehman}

Under all three experimental conditions, the BachLehman tuning indicates a slight positive correlation, with all three conditions significant at $p<.05$. While significant (if small in terms of effect size), this result is striking in the valence of correlation - a negative correlation would be consistent with the hypothesis, whereas a positive correlation would imply that Bach is, if anything, emphasizing de-tuned intervals in the WTC fugues.

\section{Discussion}

As the results of this study were not sufficient to reject the null hypothesis, the evidence is inconsistent with the initial hypothesis regarding Bach's patterns of intervallic emphasis and de-emphasis. While there are some potential confounding factors, the implications of these results - that perhaps Bach was not targeting a specific tuning system for the WTC - should also be addressed. 


\section{Fugal Constraints}

Many of the possible confounding effects on this study stem from the repertoire selected for the corpus - the 48 fugues from Bach's WTC. The fugues were selected to reduce any confounding effect of textural difference as can be found in the preludes. While the fugues are much more uniform in texture (contrapuntal with generally 3 to 4 voices), the art of writing fugues introduces many constraining factors to the compositional process. In other words, even if Bach might have emphasized or deemphasized certain intervals according to their tuning, factors such as voice-leading, imitative counterpoint, and playability (one performer with two hands, as opposed to a choral and instrumental ensemble where each part can be more independent) are all factors which might have outweighed any preferred handling of intervallic intonation.

\section{Tonal Considerations}

While the results of the study are not consistent with the initial hypothesis, the data are consistent with the earlier assumption that a tonal interval's function affects its emphasis or de-emphasis when compared to other intervals. Table 1 displays the expected frequency (as a proportion of total intervallic content) for the top 15 imperfect consonances in major key fugues. All tonal intervals except for the lowest column in Table 1 (the minor third between scale degree classes 6 and 9) are diatonic to the major scale and make up a total of $59.51 \%$ of all accented harmonic intervals in the majorkey fugues. All perfect consonances, intervals with chromatic pitches, and dissonances are encompassed by the remaining $40.49 \%$. Even among these 14 intervals, the tonic-mediant scale degree class pair has a total frequency of $13.33 \%$, whereas the second most frequent is mediant-dominant $(4,7)$ at $8.70 \%$. In other words, over one third of these imperfect consonances are part of the tonic triad, indicating that the major-key fugues emphasize these consonances over others.

While Table 2 includes the top 15 imperfect consonances from the major-key fugues, inclusion of the perfect consonances would introduce only one interval - the perfect fifth $(0,7)$ at expected frequency $2.40 \%$. This emphasis of almost all diatonic thirds and sixths over even the most common perfect consonance is likely a result of contrapuntal constraints which apply only to perfect intervals. Whereas successions of thirds are permitted in $18^{\text {th }}$-century contrapuntal practice, parallel fifths are not permitted. Leaps to perfect intervals are similarly constrained when compared to imperfect consonances. Furthermore, counterpoint which is invertible at the octave transforms fifths into fourths - an interval which is considered dissonant when formed against the bass voice.

Table 2. Top 15 imperfect consonances in the major-key fugues of Bach's WTC.

\begin{tabular}{|l|l|l|l|}
\hline SD 1 & SD 2 & Interval & Expected Frequency \\
\hline 0 & 4 & M3 & $8.01 \%$ \\
\hline 7 & 11 & M3 & $5.62 \%$ \\
\hline 4 & 7 & $\mathrm{~m} 3$ & $5.56 \%$ \\
\hline 9 & 0 & $\mathrm{~m} 3$ & $5.32 \%$ \\
\hline 4 & 0 & $\mathrm{~m} 6$ & $5.21 \%$ \\
\hline 2 & 5 & $\mathrm{~m} 3$ & $4.83 \%$ \\
\hline 5 & 9 & $\mathrm{M} 3$ & $3.81 \%$ \\
\hline 11 & 2 & $\mathrm{~m} 3$ & $3.80 \%$ \\
\hline 7 & 4 & $\mathrm{M} 6$ & $3.14 \%$ \\
\hline 5 & 2 & $\mathrm{M} 6$ & $3.11 \%$ \\
\hline 0 & 9 & $\mathrm{M} 6$ & $3.03 \%$ \\
\hline 2 & 11 & $\mathrm{M} 6$ & $2.92 \%$ \\
\hline 11 & 7 & $\mathrm{~m} 6$ & $2.92 \%$ \\
\hline 9 & 5 & $\mathrm{~m} 6$ & $2.23 \%$ \\
\hline 6 & 9 & $\mathrm{~m} 3$ & $1.35 \%$ \\
\hline
\end{tabular}

"Well Temperament" as a Class of Tuning Systems As Bach does not seem to de-emphasize tonal intervals according to intonation in the Well-Tempered Clavier, it may be the case that the work was intended not for a specific well temperament, but rather for any which would be classified as a "well temperament." Some tuning systems prioritize just intervals at the cost of a handful of significantly out-of-tune ones. For example, Pythagorean tuning prioritizes the just perfect fifth but includes a "wolf" fifth which is 24c flat. Quarter-comma meantone includes 8 just major thirds, but the remaining 4 are $41 \mathrm{c}$ sharp (more than 20c sharper than equal temperament). While these tunings can be rotated to start on a different pitch in order to move the out-of-tune intervals, keyboard instruments lack this flexibility.

A "well temperament", on the other hand, eliminates these starker contrasts. Whereas the major third in meantone is either in tune or 41c sharp, for example, major thirds in Werkmeister-III vary between $4 \mathrm{c}$ and $22 \mathrm{c}$ sharp, or $6 \mathrm{c}$ and $22 \mathrm{c}$ in Bach-Lehman. Consequentially, these intervals remain usable across all keys. If Bach's WTC were intended for something within this "class" of temperaments, the handling of interval tuning would have been a "solved problem": all keys are equally usable, meaning there would be no 
need to treat certain keys significantly different from the others.

\section{Acceptance of Slight Mistuning (Key Color)}

Bach's decision to not emphasize or de-emphasize intervals based on tuning (either through indifference to the tuning of specific keys or through emphasis of greater tuning deviations) might also have served to emphasize the unique profile of each key. Composers of the late Baroque and Classical style periods would eventually take an interest in this phenomenon of "key color." Christian Schubart, an early Classical composer, described the emotional affect which he considered to be expressed through each of the 24 keys (Dubois, 1983). For example, the key of C Major is described as "pure innocence," whereas D-flat Major is a "leering key, degenerating into grief and rapture" (Dubois 1983, 433). Perhaps an apparent indifference toward or emphasis of out-of-tune intervals, then, is meant to showcase the uniqueness of the 24 keys rather than to homogenize them as the original hypothesis would have expected.

\section{Conclusion}

It is obvious from the title that J.S. Bach's WellTempered Clavier is intended for "well temperament," but this refers to a class of temperaments rather than one specific system which is today referred to as "well temperament." Preludes from the WTC have been analyzed in the context of Werkmeister-III by some, and others have considered other temperaments to be Bach's own system of tuning (Lehman, 1995). While it was not in use at the time for keyboard tuning, equal temperament is a type of "well temperament," as all keys would be considered viable for composition.

In testing for a negative correlation between emphasis or de-emphasis of tonal intervals (pitch class pairs transposed such that the tonic is 0) and deviation from just intonation across Werkmeister-III and BachLehman tuning, the evidence was not sufficient to reject the null hypothesis. The results indicate that Bach either emphasized out-of-tune intervals slightly (BachLehman temperament) or was seemingly indifferent to the tuning of intervals in the fugues. In either of these cases, it is apparent that the "colors" of the 24 keys are emphasized, rather than homogenized. A suitable follow-up to this study might consist of an experiment in which participants listen to the expositions of these fugues in various temperaments, to measure for an effect of tuning on perceived emotional affect of the music itself.

\section{References}

Albrecht, J., \& Shanahan, D. (2013). The Use of Large Corpora to Train a New Type of Key-Finding Algorithm: An Improved Treatment of the Minor Mode. Music Perception: An Interdisciplinary Journal, 31(1), 59-67. https://doi.org/10.1525/mp.2013.31.1.59

DuBois, Ted A. "Christian Friedrich Daniel Schubart's "Ideen zu einer Aesthetik der Tonkunst": An annotated translation." PhD diss., University of Southern California, 1983. USC Digital Library.

Gann, Kyle. The Arithmetic of Listening: Tuning Theory and History for the Impractical Musician. Urbana, IL: University of Illinois Press, 2019. https://doi.org/10.5406/j.ctvpj7j1r

Huron, David. "Tonal Consonance versus Tonal Fusion in Polyphonic Sonorities." Music Perception: An Interdisciplinary Journal 9, no. 2 (1991): 135-54. https://doi.org/10.2307/40285526

Lehman, Bradley. "Bach's Extraordinary Temperament: Our Rosetta Stone: 1." Early Music 33, no. 1 (2005): 3-23. https://doi.org/10.1093/em/cah037

Sapp, C. (1984). Center for Computer Assisted Research in the Humanities. Retrieved from http://www.ccarh.org/.

Temperley, D. (1999). What's Key for Key? The KrumhanslSchmuckler Key-Finding Algorithm Reconsidered. Music Perception: An Interdisciplinary Journal, 17(1), 65-100. https://doi.org/10.2307/40285812 


\title{
Using corpus studies to find the origins of the madrigal
}

\author{
Julie E. Cumming ${ }^{\dagger}$ \\ Cory $\mathrm{McKay}^{2}$ \\ ${ }^{1}$ Schulich School of Music, McGill University, Montréal, QC, Canada \\ ${ }^{2}$ Marianopolis College, Montréal, QC, Canada, \\ †julie.cumming@mcgill.ca \\ Published 16 December 2021; https://doi.org/10.18061/FDMC.2021.0005 \\ Author video presentation and/or other conference material: https://doi.org/10.17605/OSF.IO/MQEZT
}

\begin{abstract}
A recurring topic in musicology is the origin of the madrigal. Did it come from the frottola, the motet and chanson, or other Italian traditions? MS Florence, BNC, 164-167 (c. 1520) has four sections, each devoted to a different genre: madrigals, other Italian-texted genres, chansons, and motets. These sections provide evidence of genre classification from the period. We encoded the 82 pieces in the manuscript and used jSymbolic to extract 801 features from each file. We then used Weka to train classifiers to identify the pieces in the different sections. This allowed us to test the claims of earlier scholars as to similarity or difference between the madrigals and the other genres. The classifiers could distinguish the other Italian-texted genres from the madrigals only $72 \%$ of the time, compared to $100 \%$ of the time for the motets and chansons, suggesting that the madrigals are more similar to other Italian-texted pieces than to the other genres. Features based on rhythm were particularly effective in separating the genres, especially in discriminating madrigals from motets.
\end{abstract}

KEYWORDS: madrigal, corpus studies, machine learning, genre, feature extraction, musical style

\section{Introduction}

Einstein's view that "the genesis of the madrigal...is the transformation of the frottola from an accompanied song...into a motet-like polyphonic construction with four parts of equal importance" (1949, p. 21; Rubsamen, 1964, p. 35) has been rejected. Iain Fenlon and James Haar's study of the sources (1988) suggests "less an evolution from one genre to another than an existence of two distinct traditions" (Carter, 1992, p. 87): the frottola is associated with North Italian courts and print sources, while the first madrigals are found in Florentine manuscript sources (Fenlon and Haar, pp. 6$8,14-17)$. Haar proposed that the new style derived from the motet and chanson (1986, 53, pp. 64-66), while Fenlon, Haar, and Carter emphasized the importance of the chanson (Fenlon and Haar, 1988, p. 7; Carter, 1992, p. 89). Cummings (2004, 12, pp. 5362) stresses Florentine traditions of carnival song and improvised solo song, as well as the role of the villotta (a popular genre from Northern Italy often found in Florentine sources).

In any case, if the madrigal did not derive from the frottola, what were its stylistic roots? What are its distinguishing features? Unlike the chanson and motet, genres that emerged in the middle ages, the madrigal appeared quite suddenly. Zoey Cochran and Cumming connect the emergence of the madrigal with the claim that modern Florentine was the best candidate for a standardized literary Italian, as part of the debate on the "questione della lingua" (Cumming \& Cochran, 2018). We suggest that members of the Orti oricellari group (Florentine intellectuals who met at the Rucellai Gardens; Cummings, 2004) commissioned local composers to create a new genre, the madrigal, that set poems in modern Florentine Italian by Petrarch and by local poets in a high style quite different from that of the northern Italian frottola. The earliest surviving madrigals are by Bernardo Pisano and Sebastiano Festa. If they invented a new genre, did they draw some of the musical features from contemporary genres? If so, which genres, and which features?

To investigate this question we chose to focus on the contents of a key manuscript source of the earliest madrigals, copied c. 1520, Florence, Biblioteca Nazionale Centrale, MSS Magl. XIX. 164-167 (Florence 164), identified by Cummings (2004, p. 62) as "a kind of musical manifesto of the 'program' of the Rucellai group." There are no composer attributions in the manuscript, but most of the pieces in the manuscript have concordant sources with attributions, and can be connected to known composers. The manuscript serves as a snapshot of Florentine musical culture of the period: it has four sections that correspond to the gathering structure of the manuscript (Cummings, 2006, pp. 6-7), and each one is devoted to a different musical genre, or group of associated genres (Table 1). Section 2, while varied, is dominated by the villotta and closely related genres such as the zibaldone, the protovillotta, and the canzone di Maggio. 
Table 1: Contents of Florence 164.

\begin{tabular}{|l|l|}
\hline Piece nos. & \multicolumn{1}{|c|}{ Section, contents, composers } \\
\hline $1-26$, & 1. Madrigals \\
45.5 & Pisano (12: 2-12, 16) \\
$(27)$ & Anon. Pisano? (7: 1, 13-15, 17-19) \\
& S. Festa (5: 20, 22-25) \\
& Anon. S. Festa? (3: 21, 26, 45.5) \\
\hline $27-45$ & 2. Other Italian-texted pieces (OIT) \\
$(19)$ & a. Northern proto-villotte by Compere, \\
& Obrecht, Josquin (3: 35, 37-38) \\
& b. Canzone di Maggio by Isaac (1: 34) \\
& c. Italian villotte by Pesenti, F.P., S. Festa, \\
& Anon. (6: 31-3, 42-45) \\
& d.Zibaldoni (quodlibets), Anon. (3: 39-41) \\
& e. Frottole by Tromboncino (2: 27, 36) \\
f. Unclear by Anon. (3: 28, 29, 30)
\end{tabular}

\section{Method}

Comparing multiple pieces in one genre to multiple pieces in another is a complex task, and difficult to do by hand. We therefore chose to utilize an automated approach involving feature extraction, machine learning and information gain analysis.

The first step was to digitize Florence 164 using a consistent editorial and encoding workflow; as noted by Cumming et al. (2018), inconsistent digitization practices can produce biased results when employing automated analysis techniques. The music was manually transcribed with Sibelius, using original note values, and including only accidentals found in the MS. A MIDI file was exported for each of the 82 pieces.

Next, we used the open source jSymbolic 2.2 (McKay et al., 2018) software to extract features from each of these MIDI files. The term "features" has different meanings in different disciplines; here, we define a feature as a numerical measurement of a single, precisely defined musical characteristic that can be extracted from a digital score. In this case, features were extracted globally for each piece. jSymbolic 2.2 can extract 1497 feature values measuring a diverse range of characteristics, including: pitch statistics, melody / horizontal intervals, chords / vertical intervals, texture, rhythm, instrumentation, and dynamics. Only 801 of these features were used in this study, however, as features associated with instrumentation, dynamics, tempo, etc. are not relevant to this repertoire.

Once the features were extracted, they were used as the input to machine learning and statistical analysis processing. Supervised learning involves building a classification model that can map novel inputs into classes of interest by first training on dedicated training data. We trained such models on all four sections of Florence 164, in order to see how effectively the features could differentiate the genres. As discussed in more detail below, we used classification accuracy as an imperfect but useful proxy for similarity; the more difficult it is to distinguish between pieces belonging to two given genres, the more similar those genres may be said to be, at least with respect to the particular features considered. We used the broad jSymbolic feature catalogue specifically because we wanted to be able to consider as diverse a range of characteristics as possible.

We used a ten-fold cross-validation methodology to carry out classification experiments. This meant segmenting the data into ten pairs of training / testing folds, such that each piece served as a training instance nine times and as a test instance once. This permits one to evaluate the effectiveness of classifiers in a way that minimizes risks of overfitting. The well-known support vector machine (SVM) algorithm was used to train the classifiers, as it performs well on relatively small datasets like Florence 164. More specifically, we used the SMO SVM implementation from the open source Weka (Witten et al., 2016) data mining package, with a linear kernel and default hyperparameters.

We were also interested in seeing which features separated madrigals from each of the other genres. This can be a difficult thing to do perfectly: groups of features can vary together in subtle ways that may be modelled successfully by classifiers, but which are difficult to express clearly in human interpretable ways. For the sake of simplicity, we used information gain as a simple entropy-based metric for measuring how well features considered individually separate the genres. In particular, we used Weka's InfoGainAttributeEval metric, which outputs a value between zero and one for each feature, with a higher value indicating that the feature is more useful in distinguishing the genres in question. 
Table 2. Classification accuracies found in a series of tenfold cross-validation experiments. The first row of results shows the accuracy for an experiment involving all four classes. The following rows show the results for each possible pairwise experiment.

\begin{tabular}{|l|r|}
\hline \multicolumn{1}{|c|}{ Comparison } & \multicolumn{1}{c|}{$\begin{array}{c}\text { Classification } \\
\text { Accuracy }\end{array}$} \\
\hline $\begin{array}{l}\text { Madrigals vs. Motets } \\
\text { vs. OITs vs. Chansons }\end{array}$ & $65.9 \%$ \\
\hline Madrigals vs. Motets & $100.0 \%$ \\
\hline Madrigals vs. OITs & $71.7 \%$ \\
\hline Madrigals vs. Chansons & $100.0 \%$ \\
\hline Motets vs. OITs & $93.5 \%$ \\
\hline Motets vs. Chansons & $80.6 \%$ \\
\hline OITs vs. Chansons & $74.4 \%$ \\
\hline
\end{tabular}

Table 3. Confusion matrix for the cross-validation experiment involving all four genres (Row 1 of Table 2). Each row indicates the true, ground truth genre, and the columns other than the first indicate how many pieces were assigned to each class by the classifier. The diagonal corresponds to correct classifications.

\begin{tabular}{|l|r|r|r|r|}
\hline & Madrigal & \multicolumn{1}{|c|}{ Motet } & OIT & \multicolumn{1}{c|}{ Chanson } \\
\hline Madrigal & 26 & 0 & 1 & 0 \\
\hline Motet & 0 & 8 & 0 & 4 \\
\hline OIT & 10 & 0 & 4 & 5 \\
\hline Chanson & 1 & 3 & 4 & 16 \\
\hline
\end{tabular}

\section{Results and Discussion}

Table 2 shows the classification accuracies resulting from the cross-validation experiments. The first row of results indicates that $65.9 \%$ classification accuracy can be attained when considering all four genres as candidates. However, not all genres are equally represented in the dataset, which can potentially bias results; additionally, the classifier may not necessarily be equally effective with respect to all classes.

It is therefore useful to examine the confusion matrix for this experiment (Table 3), which provides more detail on how the individual genres performed in this experiment. Table 3 reveals that madrigals could be distinguished from the other genres almost perfectly in this four-genre experiment (only one of the twentyseven madrigals was misclassified), but that chansons were sometimes confused with other Italian-texted pieces (OITs) and motets. It is also interesting to note that, although only one madrigal was misidentified as an OIT, ten of the nineteen OITs were misclassified as madrigals.
Further insights can be gleaned from pairwise crossvalidation experiments, where the classifier only needs to choose between two candidate genres, rather than four, an easier and more specialized kind of classification problem. The additional rows of Table 2 outline the results of such pairwise experiments, which are mostly consistent with the findings from the fourgenre experiment. Chansons prove once again to be relatively difficult to distinguish from motets and OITs, but not madrigals. Motets were once again relatively easy to distinguish from OITs, and madrigals could be easily distinguished from motets and chansons, but not OITs.

Since difficulty in accurately discriminating between classes can be considered an imperfect but useful proxy for similarity, we can interpret these results as suggesting that madrigals are closer in terms of musical content to OITs than to motets or chansons, since they are harder to distinguish from one another. It is worth reemphasizing, however, that this classification difficulty is associated more with confusing OITs with madrigals than the reverse.

There is a caveat that should be mentioned here: the OIT group includes music belonging to several genres, so it is possible that the difficulty in distinguishing madrigals from OITs could be at least partially due to difficulty in training a model that fully encompasses the range of music in this diverse group. However, Table 2 shows that chansons (to a small extent) and motets (to a large extent) could be better separated from OITs than madrigals, so although the possibility of an occluding influence resulting from the diversity of OIT's sub-genres should not be discounted, this influence, even if present, probably does not account for all the difficulty in separating OITs from madrigals. This would ideally be investigated using more music from each of the OIT sub-genres, but given the limited data available, it is still reasonable to suspect that the cross-validation results do ultimately indicate stronger similarity between madrigals and OITs than between madrigals and either motets or chansons.

As noted above, we used information gain as a rough metric for providing insight into which features were individually statistically most effective in separating the genres. Tables 4 to 6 show the ranked top ten individual features in each of the pairwise comparisons involving madrigals. The jSymbolic manual (http://jmir.sourceforge.net/manuals/jSymboli c_manual/home.html) can be consulted for detailed explanations of the features appearing in these tables; 
some of the features measure qualities familiar to music theorists, but others are novel.

It is notable that there are a good number of features with high information gains in the madrigals vs. motets and madrigals vs. chansons comparisons. There are important rhythmic differences between the madrigals and motets, as indicated by the fact that the top nine features in Table 4 are all associated with rhythm. Motets tend to use many more long note values and have more varied rhythmic values, while madrigals tend to have long strings of minims (half notes).

Table 6 shows that vertical and rest-associated features are most effective individually in separating madrigals from chansons. These features are associated with imitative texture: the chansons have fewer simultaneous pitches, and more partial rests (rests in one to three voices) than do the madrigals.

The features distinguishing madrigals from OITs on Table 5 also include strong rhythmic representation, but are supplemented by features measuring texture, verticality and melody. The features are more varied, and it is harder to associate them with musical features that are easy to describe. There are no individually strong features in the madrigals vs. OITs comparison; indeed, the highest information gain of 0.388 in Table 5 would not be anywhere near the top ten in either of the other two comparisons. This further supports the similarity between madrigals and OITs suggested by the cross-validation experiments, since no individual features exhibit patterns that easily distinguish madrigals from OITs in a broad sense.

Overall, these results suggest many intriguing areas of further investigation, including detailed analyses of how the individual features vary from piece to piece and genre to genre, and a more sophisticated statistical investigation of the discriminatory power of feature groups (as opposed to just individual features).

It is also important to emphasize that statistical feature analyses of these types ultimately require expert musicological confirmation of salience, in order to verify that the statistically discriminative power of any given feature is not just due to statistical coincidences without musical significance. However, the initial exploratory approach employed here is quite useful for highlighting areas for further inquiry.
Table 4. Ranked ten features with the highest information gain in separating madrigals from motets.

\begin{tabular}{|l|l|}
\hline $\begin{array}{c}\text { Informa- } \\
\text { tion Gain }\end{array}$ & \multicolumn{1}{c|}{ Feature Name } \\
\hline 0.890 & Variability in rhythmic value run lengths \\
\hline 0.890 & Prevalence of very long rhythmic values \\
\hline 0.890 & Mean rhythmic value run length \\
\hline 0.890 & Rhythmic value variability \\
\hline 0.890 & Partial rests fraction \\
\hline 0.890 & Rhythmic value histogram (half notes) \\
\hline 0.731 & Mean rhythmic value \\
\hline 0.731 & $\begin{array}{l}\text { Rhythmic value median run lengths } \\
\text { histogram (half notes) }\end{array}$ \\
\hline 0.731 & Prevalence of medium rhythmic values \\
\hline 0.678 & $\begin{array}{l}\text { Average number of simultaneous pitch } \\
\text { classes }\end{array}$ \\
\hline
\end{tabular}

Table 5. Ranked ten features with the highest information gain in separating madrigals from OITs.

\begin{tabular}{|l|l|}
\hline $\begin{array}{c}\text { Informa- } \\
\text { tion Gain }\end{array}$ & \multicolumn{1}{c|}{ Feature Name } \\
\hline 0.388 & Relative note durations of lowest line \\
\hline 0.351 & Rhythmic value histogram 2 \\
\hline 0.351 & Prevalence of short rhythmic values \\
\hline 0.343 & Total number of notes \\
\hline 0.316 & Relative note density of highest line \\
\hline 0.290 & $\begin{array}{l}\text { Beat histogram tempo standardized } \\
\text { (eighth bin) }\end{array}$ \\
\hline 0.271 & Average length of melodic arcs \\
\hline 0.265 & $\begin{array}{l}\text { Prevalence of most common vertical } \\
\text { interval }\end{array}$ \\
\hline 0.261 & $\begin{array}{l}\text { Rhythmic value median run lengths } \\
\text { histogram (dotted breves or longer) }\end{array}$ \\
\hline 0.261 & Voice equality - number of notes \\
\hline
\end{tabular}

Table 6. Ranked ten features with the highest information gain in separating madrigals from chansons.

\begin{tabular}{|l|l|}
\hline $\begin{array}{c}\text { Informa- } \\
\text { tion Gain }\end{array}$ & \multicolumn{1}{|c|}{ Feature Name } \\
\hline 0.798 & Partial chords \\
\hline 0.798 & Average number of simultaneous pitches \\
\hline 0.798 & $\begin{array}{l}\text { Average number of simultaneous pitch } \\
\text { classes }\end{array}$ \\
\hline 0.798 & $\begin{array}{l}\text { Chord type histogram (just two pitch } \\
\text { classes) }\end{array}$ \\
\hline 0.721 & Relative note durations of lowest line \\
\hline 0.680 & Average rest fraction across voices \\
\hline 0.680 & Average number of independent voices \\
\hline 0.616 & Median partial rest duration \\
\hline 0.601 & Standard triads \\
\hline 0.573 & Mean partial rest duration \\
\hline
\end{tabular}




\section{Conclusion}

Our results indicate that the madrigals are closer in musical style to the OITs than they are to either the chansons or the motets, and cast doubt on the claims of Fenlon, Haar, and Carter that the madrigal derived its style from the chanson and motet. The similarities between the madrigals and the OITs support Cummings's emphasis on Italian song traditions, and especially to the villotta (although our particular experiments here did not separate out the villotte from other OITs).

Correlation is not equivalent to historical causation - so musical similarities between madrigals and villotte do not necessarily mean that the madrigal "came from" or "evolved out of" the villotta. However, causation normally does involve correlation. So if we observe similarities (correlations) between the madrigal and the OITs, for example, it is possible that causation is involved, that composers of the first madrigals may have taken the villotta as a model or a source for their new genre. Practices of Italian text setting (such as long strings of syllabic minims) could also have had an impact, although jSymbolic does not provide direct insight here, as it does not extract features from the texts, only from the music.

Connections between the villotta and the madrigal are supported by other extra-musical factors as well. Sebastiano Festa wrote both madrigals and villotte; Pesenti, known for his villotte in Florence 164, also wrote one surviving madrigal. The fact that many villotte are mixed in with madrigals in the manuscripts of the later 1520 s also suggests that the two were seen as related genres.

In future work we will break down the various genres included in the OITs, add more villotte to our corpus, expand our research to include the madrigals of Verdelot copied in the $1520 \mathrm{~s}$, and analyze feature values more deeply. The ability to extract musical features and analyze them statistically has already shed new light on one of the enduring problems of musicology.

\section{Corpus}

All the pieces in Florence 164 used in this study are available as MIDI and PDF files at https://zenodo.org/record/4451464\#.YAdwE-hKj-g.

Pre-extracted feature values are also posted there.

\section{Acknowledgements}

Thank-you to Ian Lorenz, Jonathan Stuchbery, Linda Pearse, Sara Sabol, Vi-An Tran, Zoey Cochran, Tristan Tenaglio, Rían Adamían, Ichiro Fujinaga, and SIMSSA for their contributions and expertise. Financial support was generously provided by the granting agencies FRQSC (Fonds de recherche du Québec - Société et culture) and SSHRC (Social Sciences and Humanities Research Council of Canada).

\section{References}

Carter, T. 1954. (1992). Music in late Renaissance \& early baroque Italy. Amadeus Press. http://www.gbv.de/dms/bowker/toc/9780931340536.pd f

Cumming, J. E., \& Cochran, Z. M. (2018, November 1). The Questione della musica: Revisiting the origins of the Italian madrigal [Paper presentation]. American Musicological Society Annual Meeting, San Antonio, Texas.

Cumming, J. E., McKay, C., Stuchbery, J., \& Fujinaga, I. (2018). Methodologies for creating symbolic corpora of Western music before 1600. Proceedings of the 19th International Society for Music Information Retrieval Conference, 491-498.

Cummings, A. M. (2004). The Maecenas and the madrigalist: Patrons, patronage, and the origins of the Italian madrigal. American Philosophical Society.

Cummings, A. M. (2006). Ms Florence, Biblioteca nazionale centrale, Magl. XIX, 164-167. Ashgate.

Einstein, A. (1949). The Italian madrigal (W. O. Strunk Trans.). Princeton University Press.

Fenlon, I., \& Haar, J. (1988). The Italian madrigal in the early sixteenth century: Sources and interpretation. Cambridge University Press.

Haar, J. (1986). The early madrigal: Humanistic theory in practical guise. In Essays on Italian poetry and music in the Renaissance, 1350-1600 (49-75, 171-184). University of California Press. https://doi.org/10.1525/9780520329966-006

McKay, C., Cumming, J., \& Fujinaga, I. (2018). jSymbolic 2.2: Extracting features from symbolic music for use in musicological and MIR research. Proceedings of the 19th International Society for Music Information Retrieval Conference, 348-354.

Witten, I., Frank, E., Hall, M. A., Pal, C. J. (2016). Data mining: Practical machine learning tools and techniques. Morgan Kaufmann. https://doi.org/10.1016/C2015-0-02071-8 


\title{
How should corpus studies of harmony in popular music handle the minor tonic?
}

\author{
Trevor de Clercq ${ }^{1 \dagger}$ \\ ${ }^{1}$ Department of Recording Industry, Middle Tennessee State University, Murfreesboro, TN, USA \\ $\dagger$ Corresponding author: tdeclerca@mtsu.edu \\ Published 16 December 2021; https://doi.org/10.18061/FDMC.2021.0006 \\ Author video presentation and/or other conference material: https://doi.org/10.17605/OSF.IO/WXCTV
}

\begin{abstract}
Corpus studies of harmony in popular music normally assume a singular tonic pitch assigned to scale-degree 1, which highlights similarities in chord organization between parallel keys. Recently, Nobile (2020) posits a "double-tonic complex" in rock, where two tonics-a major chord and its relative minor-are active simultaneously, such that similarities in chord organization manifest between relative keys. Using Kullback-Leibler divergence as a metric, I assess in a corpus of classical music and a corpus of popular music how well chord organization given a minor tonic is modeled by chord organization in the parallel and relative major. I show that chord organization in the classical corpus is modeled well by the parallel key encoding, but chord organization in the popular music corpus shows mixed results. I thus suggest that corpus studies of harmony in popular music should account for the two different ways (parallel and relative) that chord organization given a minor tonic corresponds to a major key. Possible strategies include separate analyses, dual encodings, and six-based minor.
\end{abstract}

KEYWORDS: corpus study, harmony, tonality, popular music, minor keys

\section{Introduction}

A decade ago, David Temperley and I published a corpus study of harmony in rock music (de Clercq \& Temperley, 2011). One motivation for this work was that musicologists had proposed different theories about the harmonic organization of rock (Everett, 2004; Moore, 1992; Stephenson, 2002), and we hoped our research could offer some empirical evidence in that regard. Our original corpus, called the "RS 5x20," included Roman numeral encodings for the top twenty songs from five consecutive decades (the 1950s through the 1990s) on the 2004 Rolling Stone magazine list of the "500 greatest songs of all time."

The results from our study showed various distinguishing aspects of harmony in rock as compared to common-practice music. In the overall distribution of chord roots (Table 1), for example, scale-degree b7 was the most common root after 1,4 , and 5-a stark contrast with common-practice norms (Temperley, 2009). We also provided data on how many times one chord root changed to another (Table 2). Chords with a root of scale-degree $b 7$, for example, most often moved to chords with a root of 1,4 , and $b 6$ and not often to chords with a root of 2,5 , or 6 . That said, the question of whether these findings supported one theory of harmonic organization or another was, we admitted, a matter of interpretation.

Table 1: Distribution of the eight most common chromatic roots in the RS $5 \times 20$ corpus.

\begin{tabular}{r|rr} 
Root & $\mathbf{n}$ & $\mathbf{n} / \mathbf{N}$ \\
\hline $\mathbf{1}$ & 3,058 & .328 \\
$\mathbf{4}$ & 2,104 & .226 \\
$\mathbf{5}$ & 1,516 & .163 \\
$\mathbf{b 7}$ & 748 & .081 \\
$\mathbf{6}$ & 674 & .072 \\
$\mathbf{b 6}$ & 372 & .040 \\
$\mathbf{2}$ & 336 & .036 \\
$\mathbf{b 3}$ & 240 & .026
\end{tabular}

Table 2: Chord transitions in the RS 5x20 for the eight most common chromatic roots, with each cell indicating the number of occurrences from an antecedent chord root ("Ant") to its consequent ("Cons").

\begin{tabular}{r|rrrrrrrr}
\hline Ant & $\begin{array}{r}\text { Cons } \\
\mathbf{1}\end{array}$ & $\mathbf{2}$ & $\mathbf{b 3}$ & $\mathbf{4}$ & $\mathbf{5}$ & $\mathbf{b 6}$ & $\mathbf{6}$ & b7 \\
& & 132 & 94 & 1052 & 710 & 104 & 302 & 470 \\
$\mathbf{2}$ & 120 & & 2 & 58 & 97 & 0 & 24 & 0 \\
$\mathbf{b 3}$ & 50 & 6 & & 64 & 2 & 67 & 0 & 41 \\
$\mathbf{4}$ & 1162 & 30 & 98 & & 514 & 57 & 72 & 90 \\
$\mathbf{5}$ & 788 & 36 & 6 & 392 & & 6 & 191 & 48 \\
b6 & 208 & 1 & 20 & 22 & 22 & & 10 & 78 \\
$\mathbf{6}$ & 144 & 87 & 0 & 260 & 124 & 21 & & 3 \\
b7 & 386 & 0 & 11 & 188 & 26 & 114 & 6 &
\end{tabular}

In the years since our 2011 publication, Temperley and I expanded the corpus to include harmonic and melodic encodings for 200 songs, called the "RS 200" (2013). Subsequent published work, both by us and others, has used this corpus to study various topics, including rhythm and meter (Biamonte, 2014), the interaction of harmony and form (de Clercq, 2017), and 
probabilistic models of harmony (Korzeniowski, Sears, \& Widmer, 2018). In addition, other corpora of chord annotations for popular music have become available (Burgoyne et al., 2011; Koops et al., 2019).

Alongside this corpus work, musicologists continued to propose and refine theories about the harmonic organization of popular music (Biamonte, 2017; Doll, 2017). Of particular relevance here, Nobile recently posited that some rock songs may have not one but two active tonics, a situation he calls the "double-tonic complex" (2020). In short, Nobile asserts that the tonic chords of two relative keys - such as $\mathrm{C}$ major and $\mathrm{A}$ minor-can coexist, without one necessarily taking precedence over the other. A common example of this is the Axis progression (Richards, 2017), which consists of the four-chord sequence Am-F-C-G and its various rotations and transpositions, such as the Bm-G-D-A loop found in the song "Building a Mystery" by Sarah McLachlan (1997). Prior to Nobile's work, music theorists would assign Roman numerals to this progression in one of two ways: either as (1) vi-IV-I$\mathrm{V}$, with the third chord as tonic; or as (2) i-bVI-bIIIbVII, with the first chord as tonic. See Doll (2017) for examples of both methods. What does it mean for corpus studies of harmony that both Roman numeral analyses may be correct, given that current corpora of chord annotations indicate only a single tonic or key?

\section{Parallel or Relative Key Relationships?}

Consider the implications of the double-tonic complex on a corpus study of harmony. The corpus might, for example, include many instances of the Axis progression, which has been found in upwards of $30 \%$ of hit songs in the past fifteen years (Richards, 2017). Assuming for the sake of argument that roughly half of the Axis progressions were analyzed in a major key and the other half in a minor key, we would find a variety of chord roots in the overall distribution. In addition to scale-degree 1 , we would find many instances of scaledegrees $b 3,4,5, b 6,6$, and $b 7$, making popular music appear to contain a rich palette of chord roots. Yet underlying these results is a rather simple set of merely four diatonic chords.

The problem is that Roman numerals, which traditionally take the tonic chord whether major or minor to be "one," excel at highlighting the similarity of chord progressions between parallel keys. For classical music, this is a useful feature, since theorists posit similar chord function between parallel keys. Compare, for example, the normative harmonic functions of chords in a major key (Figure 1) and chords in a minor key (Figure 2), as diagrammed in a prominent music theory textbook (Kostka, Payne, \& Almén, 2013, p. 105). Notice that, aside from modifications in chord quality to account for the mode of the key, the diagrams are otherwise identical. We would thus expect scaledegree behavior to be the same between major and minor tonalities. In the key of $\mathrm{C}$ major, for example, we expect an A minor chord (vi) to move to an F major (IV) or $\mathrm{D}$ minor chord (ii), similar to our expectation in the key of $\mathrm{C}$ minor for an $\mathrm{A} b$ major chord (bVI) to move to an $\mathrm{F}$ minor (iv) or D diminished chord (ii ${ }^{\circ}$ ).

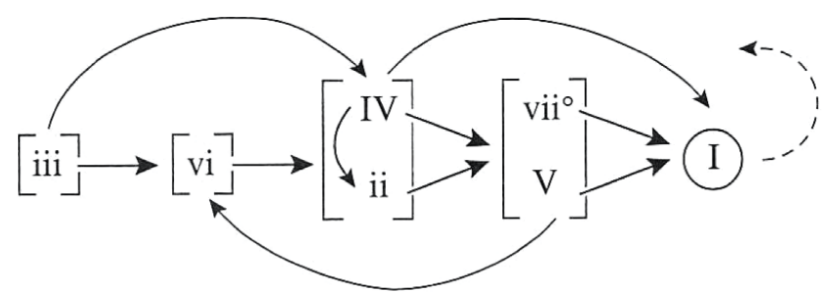

Figure 1: Normative harmonic functions in major keys, as diagrammed by Kostka, Payne, and Almén.

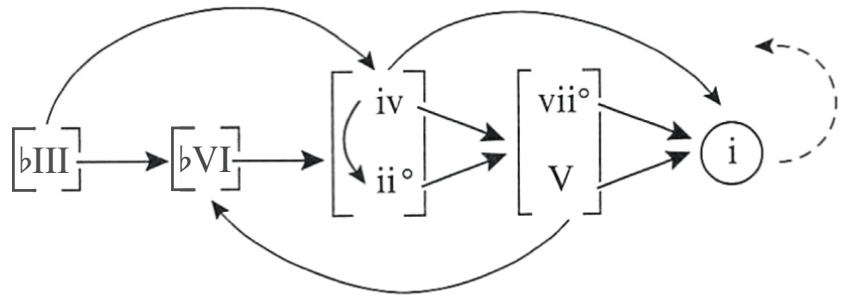

Figure 2: Normative harmonic functions in minor keys, adapted from the diagram by Kostka, Payne, and Almén.

The Axis progression, however, does not display similar chord behavior between parallel keys. In an Axis progression in $\mathrm{C}$ major, for example, an A minor chord (vi) goes to an $\mathrm{F}$ major chord (IV), whereas an Axis progression in $\mathrm{C}$ minor, an $\mathrm{A} b$ major chord (bVI) goes to an $\mathrm{Eb}$ major chord (bIII). The Axis progression instead displays similar chord behavior between relative keys.

This type of relative-key similarity between chord progressions is not limited to the Axis progression. Consider, for example, the song "Rockin' in the Free World" by Neil Young (1989). The opening chords for the verse and chorus sections are shown in Figure 3. My encoding in the RS 200 (TdC) takes G major to be the tonic of the chorus and $\mathrm{E}$ minor to be the tonic of the verse. As a result, the chorus includes the chords I, IV, $\mathrm{V}$, and vi, whereas the verse includes the chords i, bVI, and bVII. Yet most of the chord symbols in both sections are identical: C, D, and Em. Moreover, the behavior of 
these chords is the same between sections, the D chord moving to $\mathrm{C}$ and $\mathrm{C}$ to $\mathrm{Em}$. My Roman numeral analysis, therefore, obfuscates the very clear similarity in chord palette and chord behavior between the two sections.

\begin{tabular}{r|c|cc|c|cc|} 
Verse & Em & D & C & Em & D & C \\
\hline$(T d C) ~ E=1$ & i & bVII & bVI & i & bVII & bVI \\
G =1 & vi & V & IV & vi & V & IV
\end{tabular}

Figure 3: The first four bars of harmonic content for the verse and chorus of "Rockin' in the Free World" by Neil Young (1989), showing chord symbols and Roman numerals with different pitches as scale-degree 1.

How much, then, is this a problem for corpus studies of harmony? The central question, I would argue, is whether chord syntax in popular music given a minor tonic is better modeled by the parallel major, the relative major, or neither. In what follows, I present a statistical analysis of the RS 200 as an initial attempt towards an answer. I then close by suggesting some workarounds and alternative encoding schemes.

\section{Some Statistical Scrutiny}

To assess whether harmonic patterns in popular music given a minor tonic correspond more with harmonic patterns in the parallel or relative major, I will use a metric known as Kullback-Leibler divergence (or "KL divergence"), also known as relative entropy. In essence, KL divergence shows how surprised we will be by events given a certain set of expectations (McElreath 2020). If we have certain expectations for chord progressions given a major tonic, for example, are we more surprised by the behavior of chords given a minor tonic if we encode that minor tonic as parallel or as relative to the major tonic? Formally, KL divergence $\left(D_{\mathrm{KL}}\right)$ is determined by calculating the weighted average quotient in $\log$ probabilities (here, the natural $\log$ ) between a target distribution $(p)$ and a model $(q)$ :

$$
\text { (1) } \quad D_{\mathrm{KL}}=\sum_{i} p_{i} * \ln \left(\frac{p_{i}}{q_{i}}\right)
$$

Note that KL divergence is not symmetrical. A situation where we expect some events to be rare but in reality all events are fairly common (some low $q_{i}$ values, moderate $p_{i}$ values) generates a greater $\mathrm{KL}$ divergence-i.e., is more surprising - than a situation where we expect all events to be fairly common but in reality some events are fairly rare (moderate $q_{i}$ values, some low $p_{i}$ values). In other words, we are more surprised by something rare happening than something common not happening.

Table 3 illustrates the application of KL divergence using data from a corpus study by Temperley (2009) that tallied the distribution of chord roots in the musical examples from the third edition of the Kostka-Payne workbook (the "KP corpus"). Presuming chord behavior in these examples follows the diagrams in Figures 1 and 2 , we would expect the distribution of chord roots in a minor key to be less surprising when compared to the distribution of chord roots in the parallel major than the relative major. Notice that the greatest proportions $(\mathrm{n} / \mathrm{N})$ of chord roots for examples in a major key are scaledegrees 1 (.36) and 5 (.23). Similarly, the greatest proportions of chord roots for examples in a minor key encoded with tonic equal to 1 (a parallel method) are also scale-degrees 1 (.35) and 5 (.27).

Table 3: Diatonic root distributions in the KP corpus, showing KL divergence for excerpts with a minor tonic encoded as parallel or relative to the major tonic.*

\begin{tabular}{|c|c|c|c|c|c|c|c|}
\hline \multicolumn{2}{|c|}{ Major } & \multicolumn{3}{|c|}{ Minor as Parallel } & \multicolumn{3}{|c|}{ Minor as Relative } \\
\hline Root & $\mathrm{n} / \mathbf{N}$ & Root & $n / N$ & $D_{\mathrm{KL}}$ & Root & $n / \mathbf{N}$ & $D_{\mathrm{KL}}$ \\
\hline 1 & .36 & 1 & .35 & -0.004 & 1 & .03 & -0.077 \\
\hline 2 & .14 & 2 & .07 & -0.048 & 2 & .08 & -0.041 \\
\hline 3 & .03 & b3 & .03 & 0.004 & 3 & 27 & 0.607 \\
\hline 4 & .07 & 4 & .08 & 0.009 & 4 & .08 & 0.009 \\
\hline 5 & .23 & 5 & .27 & 0.044 & 5 & .03 & -0.054 \\
\hline 6 & .07 & b6 & .08 & 0.009 & 6 & .35 & 0.540 \\
\hline 7 & .04 & b7 & .03 & -0.014 & 7 & .07 & 0.025 \\
\hline chr & .06 & chr & .09 & 0.044 & chr & .09 & 0.044 \\
\hline & & & $\Sigma$ & 0.043 & & $\Sigma$ & 1.052 \\
\hline
\end{tabular}

The three rightmost columns in Table 3 rotate the data from the "Minor as Parallel" columns so that the minor tonic is taken to be scale-degree 6 in the relative major. Doing so, we can assess how well our expectations from the major key model chord behavior in the relative minor. As the divergence totals in the bottom row show, encoding these excerpts with a minor tonic as parallel to the major key generates less total divergence (0.043) than encoding minor tonics as relative to the major key (1.052). The key of A minor, 
for example, thus contains many chords with a root of $\mathrm{A}$ and $\mathrm{E}$, which is more like $\mathrm{A}$ major than $\mathrm{C}$ major.

Table 4 shows the same methodology applied to the RS 200 corpus. This table divides the corpus into songs with a major tonic (I) only (154 songs) and songs with a minor tonic (i) only (32 songs). Songs with both a major and minor tonic were ignored (14 songs). Note that chord roots of scale-degree 1,4 , and 5 are the most common in songs with a major tonic, as in Table 1. But also note that in songs with a major tonic, scale-degree b7 - which was the next most common root in Table 1is now subsumed in the chromatic category ("chr"), which in total accounts for a smaller proportion of chord roots than scale-degree 6 .

Table 4: Diatonic root distributions in the RS 200, showing KL divergence for excerpts with a minor tonic encoded as parallel or relative to the major tonic.

Major $\quad$ Minor as Parallel $\quad$ Minor as Relative

\begin{tabular}{rr|rrr|rrr}
\hline Root & $\mathbf{n} / \mathbf{N}$ & Root & $\mathbf{n} / \mathbf{N}$ & $\mathbf{D}_{\mathbf{K L}}$ & Root & $\mathbf{n} / \mathbf{N}$ & $\mathbf{D}_{\text {KL }}$ \\
\hline $\mathbf{1}$ & .34 & $\mathbf{1}$ & .33 & -0.009 & $\mathbf{1}$ & .08 & -0.116 \\
$\mathbf{2}$ & .04 & $\mathbf{2}$ & .02 & -0.015 & $\mathbf{2}$ & .13 & 0.139 \\
$\mathbf{3}$ & .03 & $\mathbf{b 3}$ & .08 & 0.088 & $\mathbf{3}$ & .13 & 0.209 \\
$\mathbf{4}$ & .26 & $\mathbf{4}$ & .13 & -0.090 & $\mathbf{4}$ & .13 & -0.090 \\
$\mathbf{5}$ & .19 & $\mathbf{5}$ & .13 & -0.047 & $\mathbf{5}$ & .16 & -0.029 \\
$\mathbf{6}$ & .08 & $\mathbf{b 6}$ & .13 & 0.072 & $\mathbf{6}$ & .33 & 0.488 \\
$\mathbf{7}$ & .00 & $\mathbf{b 7}$ & .16 & 0.610 & $\mathbf{7}$ & .02 & 0.049 \\
$\mathbf{c h r}$ & .06 & $\mathbf{c h r}$ & .02 & -0.020 & $\mathbf{c h r}$ & .02 & -0.020 \\
\hline & & & $\mathbf{\Sigma}$ & $\mathbf{0 . 5 8 8}$ & & $\mathbf{\Sigma}$ & $\mathbf{0 . 6 3 0}$
\end{tabular}

As the KL divergence totals in the bottom row of Table 4 show, encoding the minor key in a parallel way to the major tonic has a roughly similar divergence as encoding it in a relative way $(0.588$ versus 0.630$)$. To understand why, consider the chord roots in the "Minor as Parallel" column in Table 4 in the key of A minor. The proportions of chord roots on D (4), E (5), F (b6), and $G(b 7)$ are roughly equivalent. This means that the A minor key can be modeled by A major (the parallel major), in which chords with a root of D (4) and E (5) are common, about as well as it can be modeled by $\mathrm{C}$ major (the relative major), in which chords with a root of F (4) and G (5) are common. In other words, the chord distribution for a minor key in popular music exhibits similarities with both the parallel and relative major, unlike the chord distribution for a minor key in classical music (at least as presented in a prominent textbook).

Admittedly, the divergence of chord distributions in a minor key compared to the parallel major (0.588) is less than the divergence as compared to the relative major (0.630). We might thus consider that thinking about minor keys in a parallel way is still more appropriate, albeit it perhaps less so than for classical music. This outcome reverses, however, if we consider chord transitions. KL divergence values for root transition probabilities (using Laplace smoothing in cases of zero probability) are shown in Table 5, which compares chord behavior in a minor key with its parallel major, and Table 6, which compares chord behavior in a minor key with its relative major. (For display purposes, the values represent "milli-divergences," e.g., 371 is actually a divergence of 0.371 .) As the overall KL divergences in the bottom righthand corners show, chord transition probabilities in a minor key are closer to those of the relative major (1.353) than the parallel major (1.575). The difference is small, though, and neither shows a close modeling of expectation.

Table 5: KL divergence table (in milli-values) for root transition probabilities in the RS 200, comparing songs with a minor tonic encoded as parallel keys to songs with a major tonic.

\begin{tabular}{|c|c|c|c|c|c|c|c|c|}
\hline \multicolumn{9}{|c|}{ Cons } \\
\hline Ant & 1 & 2 & $3 / b 3$ & 4 & 5 & $6 / b 6$ & $7 / b 7$ & chr \\
\hline 1 & & -6 & 60 & -45 & -22 & -8 & 396 & -11 \\
\hline 2 & -4 & & 0 & 0 & -4 & 0 & 3 & -1 \\
\hline $3 / b 3$ & 25 & -2 & & 14 & 4 & 33 & 58 & 1 \\
\hline 4 & -56 & -2 & 45 & & -26 & 13 & 31 & -3 \\
\hline 5 & -6 & 18 & 0 & -13 & & -8 & 8 & -2 \\
\hline $6 / b 6$ & 104 & 0 & 1 & -6 & 5 & & 204 & 0 \\
\hline $7 / b 7$ & 371 & 0 & 3 & 94 & 31 & 290 & & 1 \\
\hline chr & -8 & 0 & 0 & -8 & 0 & 0 & 0 & \\
\hline
\end{tabular}

Table 6: KL divergence table (in milli-values) for root transition probabilities in the RS 200, comparing songs with a minor tonic encoded as relative keys to songs with a major tonic.

\begin{tabular}{|c|c|c|c|c|c|c|c|c|}
\hline & \multicolumn{8}{|l|}{ Cons } \\
\hline Ant & 1 & 2 & 3 & 4 & 5 & 6 & 7 & chr \\
\hline 1 & & 4 & -4 & -46 & -26 & -14 & 0 & -5 \\
\hline 2 & 24 & & 93 & 11 & -6 & 110 & 10 & 0 \\
\hline 3 & 0 & 1 & & -2 & 2 & 257 & 43 & 1 \\
\hline 4 & -22 & -2 & 21 & & -26 & 162 & 0 & 0 \\
\hline 5 & -12 & 11 & 11 & 4 & & 115 & 0 & -2 \\
\hline 6 & 39 & 17 & 124 & -1 & 188 & & 24 & 18 \\
\hline 7 & 2 & 0 & 37 & 0 & 2 & 16 & & 0 \\
\hline chr & 0 & 8 & 0 & -2 & 0 & 14 & 1 & \\
\hline
\end{tabular}




\section{Conclusion}

How then should we handle the minor tonic in corpus studies of harmony in popular music?

One recommendation is simply to not merge data from major and minor keys into a single statistical analysis. That is easier said than done, since it is often unclear whether the tonic chord of a song is major or minor. But to the extent that it is possible to separate out tonalities that are clearly major or clearly minor, we should try to do so.

A second recommendation is that when we hope to analyze a corpus that contains encodings of songs in a minor tonality, we must reconcile the possibility that chord behavior in that minor tonality may exhibit similarities to chord behavior in the parallel major, the relative major, or neither. Accommodating for these possibilities requires at least two encodings for a single song. If a song contains the progression Am-F-Dm-F, our encoding (or our analysis of the encoding) should allow for the fact that this could be represented as viIV-ii-IV or i-bVI-iv-bVI, even if we clearly hear A minor to be the tonic. These two approaches need to be strictly distinguished from one another in the final analysis, though, so as not to overstate the complexity of harmonic syntax in popular music.

In this regard, it is worth mentioning that encoding the minor tonic as "one," which is the default method for classically trained musicians, is not the standard practice among many commercial musicians. Users of the Nashville number system, for example, normally encode the root of minor tonic as scale-degree 6 (de Clercq, 2019), which highlights similar chord patterns between the minor key and its relative major. I am not advocating here that we always encode the root of the minor tonic as scale-degree 6 or as scale-degree 1 . Until we have a better understanding of harmonic behavior in popular music, we probably need both methods.

In closing, I will admit that I cannot offer a tidy answer to the central question of this paper. Instead, what I have hoped to accomplish is to show how in a corpus of songs drawn from the last century, harmonic behavior in songs with a clear minor tonic has no consistent parallel-key correspondence to harmonic behavior in songs with a clear major tonic, even though it is these parallel-key correspondences that a traditional "one-based minor" Roman numeral analysis highlights. So when I think about the future direction of corpus work on harmony in popular music, I think we need to follow more than the current path we have been on.

\section{References}

Biamonte, N. (2014). Formal functions of metric dissonance in rock music. Music Theory Online, 20(2). https://doi.org/10.30535/mto.20.2.1

Biamonte, N. (2017). Pop/rock tonalities. In F. Wörner, U. Scheideler, and P. Rupprecht (Eds.), Tonality Since 1950 (pp. 89-101). Franz Steiner.

Burgoyne, J. A., Wild, J., \& Fujinaga, I. (2011). An expert ground truth set for audio chord recognition and music analysis. In A. Klapuri \& C. Leider (Eds.), Proceedings of the 12th international society for music information retrieval conference (pp. 633-638).

de Clercq, T. (2017). Interactions between harmony and form in a corpus of rock music. Journal of Music Theory, 61(2), 143-170. https://doi.org/10.1215/002229094149525

de Clercq, T. (2019). The Nashville number system: A framework for teaching harmony in popular music. Journal of Music Theory Pedagogy, 33, 3-28.

de Clercq, T., \& Temperley, D. (2011). A corpus analysis of rock harmony. Popular Music, 30(1), 143-170. https://doi.org/10.1017/S026114301000067X

Doll. C. (2017). Hearing harmony: Toward a tonal theory for the rock era. University of Michigan. https://doi.org/10.3998/mpub.3079295

Everett, W. (2004). Making sense of rock's tonal systems. Music Theory Online, 10(4).

Koops, H. V., de Haas, W. B., Burgoyne, J. A., Bransen, J., Kent-Muller, A., \& Volk, A. (2019). Annotator subjectivity in harmony annotations of popular music. Journal of New Music Research, 48(3), 232-252. https://doi.org/10.1080/09298215.2019.1613436

Korzeniowski, F., Sears, D., \& Widmer, G. (2018). A largescale study of language models for chord prediction. In Proceedings of the 2018 IEEE international conference on acoustics, speech, and signal processing (pp. 91-95). https://doi.org/10.1109/ICASSP.2018.8462285

Kostka, S., Payne, D., \& Almén, B. (2013). Tonal harmony: With an introduction to twentieth-century music (7th ed.). McGraw-Hill.

McElreath, R. (2020). Statistical rethinking: A Bayesian course with examples in $R$ and STAN (2nd ed.). CRC. https://doi.org/10.1201/9780429029608

Moore, A. (1992). Patterns of harmony. Popular Music, 11(1), 73-106.

https://doi.org/10.1017/S0261143000004852

Nobile, D. (2020). Double-tonic complexes in rock music. Music Theory Spectrum, 42(2), 207-226. https://doi.org/10.1093/mts/mtaa003

Richards, M. (2017). Tonal ambiguity in popular music's Axis progressions. Music Theory Online, 23(3). https://doi.org/10.30535/mto.23.3.6 
Stephenson. K. (2002). What to listen for in rock: A stylistic analysis. Yale University. https://doi.org/10.12987/yale/9780300092394.001.0001

Temperley. D. (2009). A statistical analysis of tonal harmony. http://davidtemperley.com/kp-stats/

Temperley, D., \& de Clercq, T. (2013). Statistical analysis of harmony and melody in rock music. Journal of $\mathrm{New}$ Music Research, 42(3), 187-204. https://doi.org/10.1080/09298215.2013.788039 


\title{
A statistical model for melody reduction
}

\author{
Tianxue $\mathrm{Hu}^{1 \dagger}$ \\ Claire Arthur ${ }^{2}$ \\ ${ }^{1,2}$ Computational \& Cognitive Musicology Lab, Georgia Tech Center for Music Technology, \\ Georgia Institute of Technology, Atlanta, Georgia, United States \\ ${ }^{\dagger}$ Corresponding author: thu82@gatech.edu \\ Published 16 December 2021; https://doi.org/10.18061/FDMC.2021.0007
}

Author video presentation and/or other conference material: https://doi.org/10.17605/OSF.IO/326CH

\begin{abstract}
A commonly-cited reason for the poor performance of automatic chord estimation (ACE) systems within music information retrieval (MIR) is that non-chord tones (i.e., notes outside the supporting harmony) contribute to error during the labeling process (e.g., Chen \& Su, 2018; Masada \& Bunescu, 2017). Despite the prevalence of machine-learning approaches in MIR, there are cases where alternative approaches provide a simpler alternative while allowing for insights into musicological practices.

In this project, we present a statistical model for predicting chord tones based on music theory rules. Our model is currently focused on predicting chord tones in classical music, since composition in this style is highly constrained, theoretically making the placement of chord tones highly predictable. Indeed, music theorists have labeling systems for every variety of non-chord tone, primarily classified by the note's metric position and intervals of approach and departure. Using metric position, duration, and melodic intervals as predictors, we build a statistical model for predicting chord tones using the TAVERN dataset (Devaney et al. 2015). While our probabilistic approach is similar to other efforts in the domain of automatic harmonic analysis (e.g., Temperley 1997; Temperley \& Sleator 1999), our focus is on melodic reduction rather than predicting harmony. However, we hope to pursue applications for ACE in the future. Finally, we implement our melody-reduction model using an existing symbolic visualization tool, to assist with melody reduction and non-chord tone identification for computational musicology researchers and music theorists.
\end{abstract}

KEYWORDS: melody reduction, melodic analysis, statistical modeling, non-chord tone identification, automatic chord estimation

\section{Introduction}

Automatic chord estimation (ACE) of symbolic music (i.e., the automatic labeling of polyphonic symbolic data) has become a popular task over the past decades in the field of computational musicology. Many chord recognition models have been built based on music theory rules, statistical approaches, or machine learning algorithms (Chen \& Su, 2018; Masada \& Bunescu, 2017; Temperley, 1997). However, a common obstacle across all three approaches is the appearance of nonchord tones in the score (Chen \& Su, 2018; Masada \& Bunescu, 2017; Radicioni \& Esposito, 2010). Therefore, this paper focuses on building an automatic non-chord tone recognition model for a given melody in the common practice classical style.

In Western classical music theory, non-chord tones (NCTs) are categorized in many ways based on a melody note's metric position, and intervallic distance and direction from a prior and subsequent note (i.e., the intervals of arrival and departure) (Kostka \& Payne, 2013, p.184). Each category of NCT is given a label based on their function (i.e., passing tone, neighbor tone, suspension, etc). To predict, then, whether a note is a chord tone (CT) in a melody and given no harmonic information, the important features are a note's arriving and departure intervals, metric position, and duration.

In this project, we propose a logistic regression model to distinguish NCTs and CTs using the Theme And Variation Encodings with Roman Numerals (TAVERN) corpus (Devaney et al., 2015). This model is then used to perform melodic reduction via non-chord tone identification for computational musicology researchers and music theorists.

\section{Background}

With the prevalence of machine-learning approaches in the field of music information retrieval (MIR), several studies on ACE in symbolic music have indicated the presence of NCTs in a score add a significant amount of "noise" to a model, thus reducing accuracy. Prior studies have demonstrated relatively high prediction accuracy in datasets in strict chorale style (e.g., Bach) (Masada \& Bunescu, 2017) which are known to contain mostly CTs. However, on non-homorhythmic and especially virtuosic styles, such as the TAVERN (Devaney et al., 2015) and Beethoven Piano Sonata (Chen \& Su, 2018) corpora, model performances worsen. We believe NCT 
identification could be an essential task in the data processing stage for $\mathrm{ACE}$ in order to reduce the noisiness in a dataset.

A few researchers have attempted to either isolate NCTs directly--such as the dissonant filter tool in Verovio Humdrum Viewer (VHV) for identifying ornamental tones of different types (Sapp, 2017) - or else included NCTs as a feature in modeling harmony. For example, Temperley and Sleator used a rule-based system to identify ornamental dissonances in a model for predicting harmony labels (1999). However, both of these studies consider elements from a full polyphonic score in order to identify NCTs.

Recent research involving $\mathrm{CT}$ and $\mathrm{NCT}$ recognition has been more centered around statistical and machine learning models relying on parameters such as melodic features, segmentation, and vertical sonority (ConditSchultz et al., 2018; Arthur 2016). Arthur (2016) created a backward stepwise regression model using duration, metric levels, approach and departure intervals only from melody to predict NCTs with the themes portion of the TAVERN corpus. The current project follows Arthur's research by exploring model performance using the whole TAVERN dataset. We additionally provide a visual implementation of the model to recognize NCTs in a melody more intuitively.

\section{Methods}

The project methodology can be broken into two stages: data collection and pre-processing, and model fitting.

\section{Data Collection and Pre-processing}

The data collection and pre-processing part contains two steps. The first is to extract musical features from the original score, to construct a data frame from the selected dataset, and the second step is to generate ground truth for each note based on its underlying labeled harmony in the corpus. The ground truth is simply whether or not a given melody note is a member of the underlying harmony or not (i.e., CT/NCT).

For this project we relied exclusively on the TAVERN corpus (Devaney et al., 2015),[2] which contains 27 sets of themes and variations $(\mathrm{T} \& \mathrm{~V})$ for piano by Mozart and Beethoven with 281 themes and variations in total. Each musical phrase is encoded as a separate.$k r n$ file containing the full score alongside Roman numeral and Functional interpretations.

We 'extracted' the melody using the Humdrum toolkit (Huron, 1995), which is a command-line tool that performs various tasks for symbolic music such as converting musical representations (e.g., pitch class to scale degree), extracting and calculating musical features (e.g., metric position, neighboring intervals, etc.), et cetera. Due to the complexity in tracing "the melody" for a given theme or variation, we extracted the upper-most voice and operationalized that as "melody" for the purposes of our study. With each melody note as a data point, a data frame was then constructed by extracting each note's scale degree under the current key, metric position, duration, arriving and departure intervals, along with the corresponding Roman numeral (RN) and the current key. The ground truth of each note-whether it is a chord tone or not-was then labeled via a NCT identification algorithm given its scale degree, associated $\mathrm{RN}$, and the key. All information was then concatenated into one data frame in $\mathrm{R}$ for statistical evaluation.

The total number of eligible data points in the TAVERN dataset is 45,299 , with approximately $72 \%$ CTs and 28\% NCTs. The variations subset of TAVERN has a higher percentage of NCTs compared with only 19\% NCTs in the themes (Arthur, 2016). This is likely due to the stylistic tendency for variations to exhibit extreme ornamentation and elaboration techniques; these decorations are most commonly passing tones, neighboring tones, or appoggiaturas (Giraud et al., 2013).

\section{Model Fitting}

We proposed a logistic regression model because the dependent variable - CT or NCT - is binary. Since Arthur's NCT model was only trained on the themes from the TAVERN dataset (Arthur, 2016), we wished to explore a model's performance trained on the whole dataset. As the distribution of CTs / NCTs in the dataset is skewed, the initial goal of the model was to demonstrate improvement over a baseline model that simply identifies every single note as a CT (i.e., accuracy is just the percentage of the CTs in the test set, or $\sim 72 \%$ ).

The independent variables in our model are: duration (linear), on-beat/off-beat (binary), arriving interval (coded as a step or a leap), and departure interval (step/leap). After splitting the TAVERN dataset into an $80 / 20 \%$ train and test set, we built a logistic regression model. Considering only the main effects, the accuracy on the test set is $75.33 \%$ with AUC $=0.78$, which is higher than the baseline model's $70.30 \%$ accuracy (percentage of CTs in the test set). The AUC score in this case reflects the model's ability to distinguish between $\mathrm{CT}$ and $\mathrm{NCT}$, where $\mathrm{AUC}=1$ represents perfect classification accuracy, while $\mathrm{AUC}=0.5$ 
implies zero discrimination ability. Since the testing accuracy will presumably increase when considering interactions between independent variables, we then applied a forward stepwise logistic regression until achieving the best AIC score, to find the variables and interactions that significantly impact the model's performance. The result of the model (referred as Model 1) with the best AIC (AIC $=34871.22)$ is shown in Table 1:

Table 1: Significant Factors in Model 1.

$\begin{array}{ll}\text { Main Effects } & \text { Interactions } \\ \text { Departure Interval(DI) } & \text { AI:Dur } \\ \text { Arriving Interval(AI) } & \text { DI:AI } \\ \text { Duration(Dur) } & \text { AI:Beat } \\ \text { On/off-beat(Beat) } & \text { DI:Beat } \\ & \text { DI:Dur } \\ & \text { DI:Beat } \\ & \text { DI:AI:Beat }\end{array}$

The AIC of Model 1 is 34871.22, with an accuracy of $75.34 \%$ and an AUC of 0.79 . Our next goal was to attempt to achieve an equal or higher testing accuracy using a simpler model. However, testing accuracy failed to improve as expected. A likely reason is the substantial noisiness in the variations as described earlier. Accordingly, we attempted to replicate Arthur's (2016) approach of training with only the themes (but testing on the full dataset). The logic here was that "rules" are more likely to be followed in simple themes but difficult to learn from the full variations. By applying the forward stepwise logistic regression, the best model (referred as Model 2) parameters are shown in Table 2:

Table 2: Significant Factors in Model 2.

$\begin{array}{ll}\text { Main Effects } & \text { Interactions } \\ \text { Departure Interval(DI) } & \text { AI:Beat } \\ \text { Arriving Interval(AI) } & \text { DI:Beat } \\ \text { Duration(Dur) } & \text { DI:Dur } \\ \text { On/off-beat(Beat) } & \end{array}$

The AIC of Model 2 is 1202.98, and its accuracy on the test set is $75.39 \%$ with an AUC of 0.78 . Model 2 successfully replicates Arthur's findings (Arthur, 2016). With themes as the training data, though the accuracy and AUC did not significantly improve, the model is much simpler with a substantially lower AIC. In other words, we achieved the same accuracy with a simpler model. As a result, we further investigated Model 2 in the following subsections.

\section{Exploring Other Factors}

Besides duration, on-beat/off-beat, arriving and departure intervals, Arthur's model also included whether a note is a boundary note, referring to whether a note is a starting or an ending note of a piece, as another factor. Accordingly, we not only introduced a new variable, boundary note (binary), but also included whether a note was arriving by a rest (binary) or departing to a rest (binary) along with the previous factors. After repeating training on the themes dataset using forward stepwise logistic regression, the best model was exactly the same as Model 2, allowing us to conclude that these new factors do not contribute to the performance of the model.

\section{Results}

This subsection evaluates the performance and generalizability of Model 2 by performing crossvalidation and testing on other classical music datasets. Cross-validation is applied to test the model's ability to classify CTs/NCTs in new (unseen) data, and addresses problems like overfitting or selection bias, and gives insights on the model's overall generalizability. As a first litmus test, we wanted to test Model 2 on random subsets of the themes portion of the dataset itself. The themes portion of the dataset has 2,039 data points with $81 \%$ CTs and $19 \%$ NCTs. Our logic was that given the simplicity and uniformity in the themes compared with the variations, the model ought to perform better. Using an $80 \% / 20 \%$ split training and testing, we performed a 10 -fold cross-validation on the themes data, the results of this test of Model 2 (using parameters shown in Table 2 ) is shown in Table 3.

Table 3: Cross-validation of Model 2 on Themes Only.

\begin{tabular}{|l|}
\hline Percentage of chord tones in the training set: $83.19 \%$ \\
\hline Cross-validation: \\
Accuracy: $84.87 \%$ \\
Precision: 0.8756 \\
Recall: 0.9508 \\
f1: 0.9116 \\
AUC: 0.8747 \\
\hline
\end{tabular}

The results indicate that the model makes sufficient predictions on the themes dataset itself. Of course, it is still biased due to the extreme proportion of CTs. To examine Model 2's generalizability, we applied the model on other datasets as well. Previously we tested the model on only a part of the TAVERN dataset (i.e., a fixed $20 \%$ test set). Since the melodic complexity can 
vary significantly across different variations, we use cross-validation to re-test the model on the complete TAVERN dataset by randomly selecting 2,400 data points over 50 separate trials and take the average over all trials (Table 4). In addition to TAVERN, we test Model 2 on another dataset with harmonic annotations of Joseph Haydn's "Sun Quartets" dataset in kern format (López, 2017), which contains 6 string quartets (12,616 data points). The Model 2 results tested on the two datasets (full TAVERN and Sun Quartets) are displayed in Table 4. The prediction accuracy is higher than the baseline model in both cases, and Model 2 is capable of distinguishing CTs and NCTs in the melodies from the two datasets.

Table 4: The Baseline accuracy, predicting accuracy, and AUC on different datasets.

\begin{tabular}{|c|c|c|}
\hline Dataset & TAVERN & Haydn \\
\hline Baseline & $71.2 \%$ & $66.0 \%$ \\
\hline Model 2 & $76.4 \%$ & $70.6 \%$ \\
\hline AUC & 0.7766 & 0.6852 \\
\hline
\end{tabular}

So far, we investigated and evaluated the best model trained with the themes dataset (Model 2), using the full set of parameters as shown in Table 2. In the next subsection, we introduce a visualization tool for melodic reduction using our model.

\section{Visualization in Verovio Humdrum Viewer}

This section presents an application of the model to a score visualization program, using color to allow easy identification of notes predicted as NCTs in the score. We use the Verovio Humdrum Viewer (VHV) as our visualization platform, which is an online symbolic music editor and interactive notation rendering interface for .krn and .mei files (Sapp, 2017).

The data processing of a new score is similar to the procedure in Section Data Collection and Preprocessing. After feeding the data from the new score into our model, users can modify a probability threshold after which the model generates prediction probabilities for each note. The default threshold is 0.5 , which takes probability larger than 0.5 as CTs and smaller than 0.5 as NCTs. Next, the program creates another spine **color in the original score to distinguish CTs and NCTs: CTs are labeled as black where NCTs are labeled as pink by matching index. An example is shown in Figure 1. Note that RNs are not used in the model and are only shown as a visualization aid.
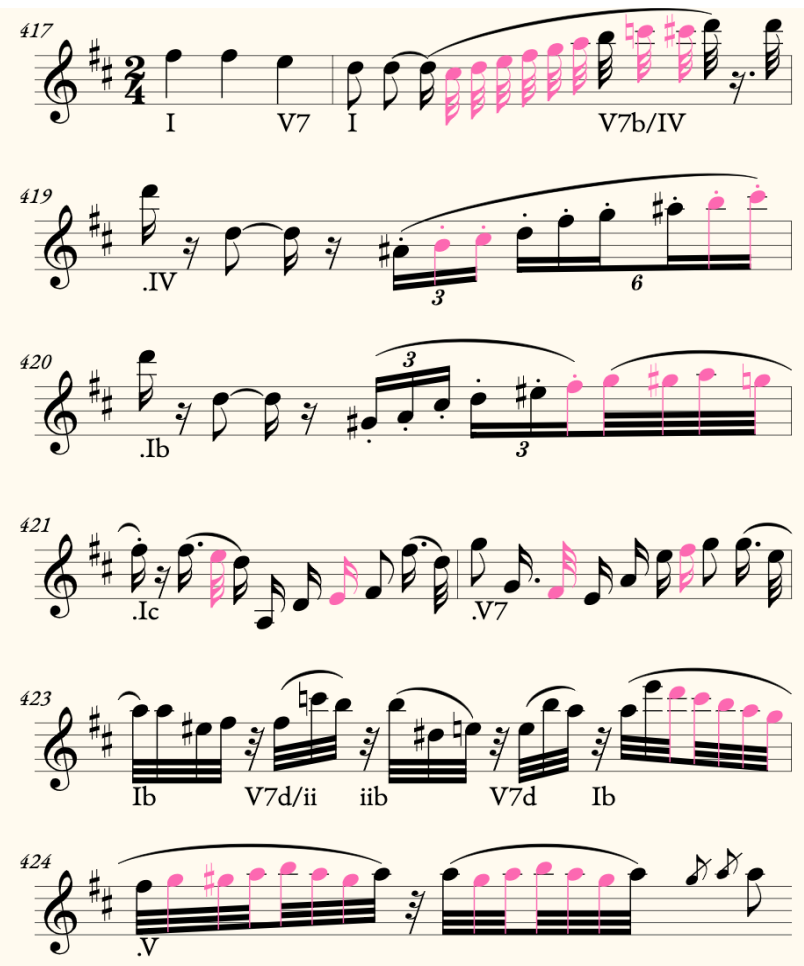

Figure 1: Visualization of a phrase in the TAVERN dataset (with harmony labeled), where black notes are chord tones and pink notes are non-chord tones.

The visual implementation of the model in VHV provides an intuitive way to recognize NCTs in a melody or to evaluate our model's performance. The visualization would assist computational musicologists and music theorists with music analysis.

\section{Conclusion}

This paper proposed a logistic regression model to predict non-chord tones (NCTs) from a single melodic line from a classical score. The best model, trained on the themes of the TAVERN dataset, used several melodic features as NCT predictors (i.e., duration, beat, arriving and departure intervals). The model can successfully distinguish CTs and NCTs across multiple datasets with an average accuracy of about $73.5 \%$. Finally, we introduced a visualization tool using the model, which performs melodic reduction on a monophonic symbolic score for computational musicology researchers and music theorists.

\section{Discussion}

Future improvements will include simplified user experience of the visualization program, building a web 
page or a pipeline tool, and/or implementing the function as a permanent feature in Verovio Humdrum Viewer. Our model does not guarantee the best performance for several reasons. First, since the ratio of NCT to CT are usually unbalanced in classical music this leads to a higher possibility for a model to predict a note as CT. Second, our ground truth is based on humanannotated Roman numerals, which, despite being 'expert' is far from an objective process. For instance, Koops et al. (2020) indicated some annotators may prefer to annotate fewer chord changes to emphasize the longer-range structure of the piece, while others may analyze harmonies in more detail. Thus, the ground truth of a dataset - whether a note is an CT/NCT - impacts the information a model is trained on, which therefore will further affect the performance of a model. Finally, our operationalization of "melody" was oversimplified, and longer-range contextual features (beyond $n=2$ ) were not considered.

Other applications of the model would include expanding to apply it to other genres. Our eventual goal is to use the model as a melody reduction process to potentially improve ACE accuracy in symbolic music. We are currently working on implementing the model as a preprocessing stage for ACE in polyphonic symbolic music using a deep learning method. The study focuses on classical string quartets, applying the model to each independent voice in order to perform a full score reduction. Our preliminary results indicate a modest improvement in recognition accuracy over a baseline model (feedforward neural network) after applying score reduction. We look forward to presenting the work in the near future.

\section{End Notes}

[1] Project code at

https://github.com/TianxueHu/Auto NCT

[2] The TAVERN Corpus:

https://github.com/musicnerd/TAVERN

\section{References}

Arthur, C. A Corpus Approach to the Classification of Nonchord Tones Across Genres. In 14th International Conference on Music Perception and Cognition, San Francisco, CA, United States.

Charyton, C. (Ed.). (2015). Creativity and innovation among science and art: A discussion of the two cultures. Springer, London. https://doi.org/10.1007/978-1-4471-6624-5

Chen, T. P., \& Su, L. (2018). Functional Harmony Recognition of Symbolic Music Data with Multi-task Recurrent Neural
Networks. In International Society for Music Information Retrieval Conference (ISMIR), Paris, France (pp. 90-97).

Condit-Schultz, N., Ju, Y., \& Fujinaga, I. (2018). A Flexible Approach to Automated Harmonic Analysis: Multiple Annotations of Chorales by Bach and Prætorius. In International Society for Music Information Retrieval Conference (ISMIR), Paris, France (pp. 66-73).

Devaney, J., Arthur, C., Condit-Schultz, N., \& Nisula, K. (2015, October). Theme and variation encodings with roman numerals (TAVERN): A new data set for symbolic music analysis. In International Society for Music Information Retrieval Conference (ISMIR), Malaga, Spain.

Giraud, M., Déguernel, K., \& Cambouropoulos, E. (2013, October). Fragmentations with pitch, rhythm and parallelism constraints for variation matching. In International Symposium on Computer Music Multidisciplinary Research (pp. 298-312). Springer, Cham. https://doi.org/10.1007/978-3-319-12976-1 19

Huron, D. (1995). The Humdrum Toolkit: Reference manual. Menlo Park, California: Center for Computer Assisted Research in the Humanities.

Klapuri, A. P. (2004). Automatic music transcription as we know it today. Journal of New Music Research, 33(3), 269282. https://doi.org/10.1080/0929821042000317840

Koops, H. V., de Haas, W. B., Bransen, J., \& Volk, A. (2020). Automatic chord label personalization through deep learning of shared harmonic interval profiles. Neural Computing and Applications, 32(4), 929-939. https://doi.org/10.1007/s00521-018-3703-y

Kostka, S., \& Payne, D. (2013). Tonal harmony. McGraw-Hill Higher Education.

Masada, K., \& Bunescu, R. C. (2017). Chord Recognition in Symbolic Music Using Semi-Markov Conditional Random Fields. In International Society for Music Information Retrieval Conference (ISMIR), Suzhou, China.

Salzer, F. (1949). The Technique of Variation; A Study of the Instrumental Variation from Antonio de Cabezón to Max Reger. University of California. https://doi.org/10.2307/829725

Sapp, C.S. (2017, Mar 4) Verovio Humdrum Viewer Documentation. https://doc.verovio.humdrum.org/

López, N. N. (2017). Automatic harmonic analysis of classical string quartets from symbolic score (Doctoral dissertation, Master's thesis, Universitat Pompeu Fabra). https://doi.org/10.5281/zenodo.1095617

Radicioni, D. P., \& Esposito, R. (2010). BREVE: an HMPerceptron-based chord recognition system. In Advances in Music Information Retrieval (pp. 143-164). Springer, Berlin, Heidelberg. https://doi.org/10.1007/978-3-642-11674-2 7

Temperley, D. (1997). An algorithm for harmonic analysis. Music Perception, 15(1), 31-68. https://doi.org/10.2307/40285738

Temperley, D., \& Sleator, D. (1999). Modeling meter and harmony: A preference-rule approach. Computer Music Journal, 23(1), 10-27. https://doi.org/10.1162/014892699559616 


\title{
Voice-leading in Palestrina's masses: A comparison of interval-succession definitions
}

\author{
Laney Light ${ }^{1 \dagger}$ \\ Claire Arthur ${ }^{2}$ \\ ${ }^{1}$ School of Music, Georgia Institute of Technology, Atlanta, GA, USA \\ ${ }^{2}$ School of Music, Georgia Institute of Technology, Atlanta, GA, USA \\ ${ }^{\dagger}$ Corresponding author: 1light7@gatech.edu \\ Published 16 December 2021; https://doi.org/10.18061/FDMC.2021.0008 \\ Author video presentation and/or other conference material: https://doi.org/10.17605/OSF.IO/G8ZX6
}

\begin{abstract}
Computational musicology methods allow us to perform a systematic analysis of all intervals in a corpus, but are all note successions equally important? In traditional music theory, the pulse beats tend to be given greater "weight" or relevance. In Renaissance music, it is unclear whether voice-leading guidelines should apply to any consecutive intervals (note level), or merely those that traverse from one pulse beat to the next (pulse level, defined by the whole note or semibreve). Since this question bears important implications for computational musicology, we set out to empirically evaluate it via a corpus study of Palestrina's masses.

We investigated the voice-leading patterns in Palestrina's corpus of 104 masses using music21. For each pair of voices, we systematically investigated whether Palestrina's voiceleading patterns differed at the note level compared to the pulse level. Our results showed that the distribution of voiceleading patterns was significantly different at the pulse level than the note level. Violations of traditional "rules" prohibiting parallel or similar motion between perfect intervals were more common at the pulse level than the note level $(p<.05)$, while factors associated with breaking the rules (e.g., having more voices in the texture) were similar between the two levels.
\end{abstract}

KEYWORDS: Voice leading, Renaissance,

Palestrina, corpus study

\section{Introduction}

Voice-leading, an integral aspect of Western music, refers to the way individual voices, or musical parts, move together in a multi-part texture. This movement must consider each voice's melodic motion (horizontal intervals) as well as the harmony between voices active at the same time (vertical intervals). Voice leading in Western music originated in early forms of polyphonic singing in the Middle Ages and evolved into complex counterpoint in the late Renaissance (Fuller, 2002). Treatises by Renaissance music theorists included guidelines for what was generally considered "good" and "bad" polyphonic writing. However, there were seldom explicit "rules" as one might find in a modern theory textbook (e.g., Aldwell et al, 2019) or a $20^{\text {th }}$ century text on writing in the Renaissance style (e.g., Gauldin, 1985). Guidelines in these modern textbooks are developed from the authors' internalization of "inherent rules" abstracted from years of study of early musical treatises and familiarization with a vast body of early contrapuntal composition. However, given the magnitude of musical material from this period, it is all but impossible to examine voice-leading practices in a systematic manner using traditional humanistic methods alone. In this paper we comprehensively examine the voice-leading practices in a large body of Renaissance polyphony to clarify certain ambiguities (or inconsistencies) in ancient and modern texts.

Examining voice-leading patterns in Renaissance music using computational methods has implications for Renaissance voice-leading pedagogy because it can highlight subtle variations between Renaissance theory and practice, clarify the context under which certain rules operate, and potentially identify a set of more general principles guiding certain compositional behaviors. Since Renaissance polyphony formed the basis for voice-leading practices through the Baroque, Classical, and into the modern era, any insights gained from this research are likely to have implications for voice leading in later musical styles as well.

Although modern computational methods provide powerful tools for analyzing scores, defining compositional rules algorithmically requires careful consideration. For example, it is often unclear how the "rules" of voice leading should be applied regarding meter. Isolated musical examples-especially in Renaissance treatises - are often presented in a prototypical notation that appears agnostic to the "beat" (or position), duration, or voice position, or else are demonstrated with a very specific selection of musical material. One question that arises, then, when trying to interpret these examples is whether they represent a rule to be followed at any "hierarchical level?" Or, for example, whether there are separate rules that would apply at the "note level" (any consecutive intervals) 
versus the "pulse level" (from one strong beat to the next). That is, are voice-leading rules to be applied differently at different hierarchical levels?

Andrews (1958) describes a voice-leading "violation" where direct consecutive octaves at the pulse level are (ineffectively) interrupted by a passing tone. However, we do find evidence of this pattern (and other "violations") used occasionally in Palestrina's masses, as illustrated in Figure 1. Using computational techniques, we can analyze millions of individual intervals to quantify how often these types of patterns occur in compositional practice, and under what circumstances.

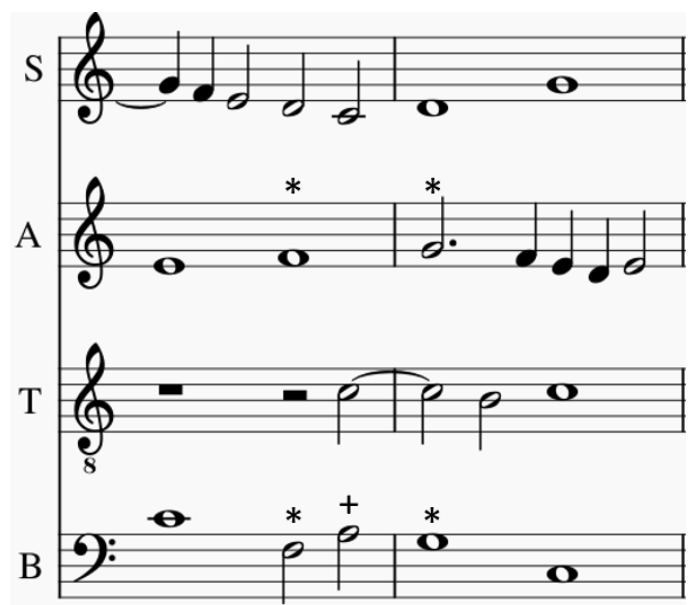

Figure 1. Excerpt from Palestrina's Missa Sine nominee (Mantuan), Agnus. The prohibition on direct consecutive perfect intervals is violated at the pulse level (*) but not at the note level because of the passing tone (+).

In this study we take a computational approach to investigate whether Renaissance compositions differ in their voice-leading treatment at the note level versus the pulse level. To answer this question, we used a digitally encoded corpus of Palestrina's masses. This corpus is ideal for this analysis since Palestrina is well known as an exemplar of Renaissance vocal polyphony (Benjamin, 2005), and therefore his work should be representative of the voice-leading practices of the time. Moreover, given Palestrina's huge influence on the development of contrapuntal practice (Marvin, 2002), we would expect to find evidence of similar voiceleading practices in later polyphonic music.

\section{Related Works}

There have been many studies on Renaissance counterpoint and Palestrina in particular (e.g., Jeppesen,
1927; Marshall, 1963; Hanson, 1983). These have covered a wide range of historical and music theory topics, but most have used traditional musicology approaches. Using computational methods, we can build on these theoretical works to examine scores systematically and identify patterns from large datasets in ways that are not possible with traditional approaches.

Palestrina's compositions provide a rich data source for computational analysis because of the large number of works that have been encoded as symbolic score data. Several studies have leveraged this data source for computational analyses (e.g., Arthur, 2021; Sigler, 2015; Knopke et al, 2009; Farbood \& Schoner, 2001), but to date only Arthur (2021) has examined Palestrina's voice-leading specifically.

There have been a few computational studies focusing on voice leading. Wall et al (2020) performed an empirical study of the effects of voice leading and harmony on musical expectancy, but the perceptual study did not rely on data from symbolic scores. Huron and Collins (1999), whose work most resembles our own, investigated the degree to which voice leading guidelines set by Zarlino and Berardi agreed with compositional practice. As it was unclear whether melodic rules should apply to all intervals or only those on the strong beats, the authors created two separate "inventories" of melodic intervals, one containing noteto-note intervals and the other containing intervals between notes on the strong beats. However, their work only examined voice-leading in a stricter and highly imitative style (canon for 3 voices). In addition, while their sample was formidable in size and scope (79 canons and 13 didactic examples by 13 composers over a period of 250 years), the overall sample of intervals is comparatively small due to the short length of the canon form itself. Most recently, Arthur (2021) performed a comprehensive examination of Palestrina's voice leading to examine the role of vocal texture on voiceleading rules and preferences.

How intervals are represented (or tallied) affects one's results. There are many ways in which voiceleading patterns can be represented symbolically. For example, Conklin (2002) fully expanded each piece, creating a new vertical "slice" at each onset to produce a "viewpoint representation" that can be sampled at regular intervals (quarter notes, for example). Sears et al (2002) presented a "skip-gram" representation. Based on the n-gram representation (e.g., a trigram is a sequence of $n=3$ events), the skip-gram is defined as non-adjacent sequences that skip over $n$ number of events. This method, which was extended by Finkensiep 
eta al (2018) to allow for nested skip-grams, can uncover higher-level structural patterns that may be "hidden" by other passing tones. However, the skips are not dependent on the beat positions of the notes. Arthur (2021) only examines voice-leading as a function of simple 2-gram (i.e., note level) patterns. In our analysis, we build on Arthur's work, but examine several of these representation models (vertical slices from a full expansion of the score, sampled at the note (bigram) level and at the pulse beats to obtain two separate inventories of intervals).

\section{Method}

\section{Voice-Leading Patterns}

We used a systematic computational approach to analyze voice-leading patterns in a corpus of 104 masses by Palestrina using music21 (Cuthbert, 2010) [1]. We performed a full expansion of the scores, "slicing" the scores vertically at any new onset in any voice. Next, we calculated the vertical bigrams (harmonic intervals) between every pair of voices in each slice, as well as the horizontal bigrams (melodic intervals) for each voice. Then we categorized the types of contrapuntal motion leading to each vertical interval, as shown in Figure 2: parallel, contrary, similar, oblique, and stasis (one or both voices resting on the previous vertical interval, or both voices repeating the same notes).

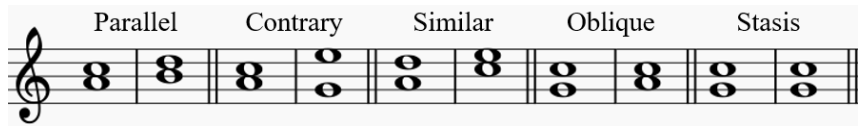

Figure 2. Examples of voice-leading motion types.

We created two non-mutually exclusive inventories of intervals, "note level" and "pulse level" [2]. Within each inventory, we identified a set of features associated with each interval, including the position of each voice within the texture (inner vs. outer voice), total number of voices in the texture at that slice, and whether each interval was approached by a step or a leap. Next, building on the work of Arthur (2021), we searched each inventory for specific patterns that violate two voiceleading "rules" governing harmonic intervals.

- R1: perfect harmonic interval (P5, P8, or P1) should not be approached by parallel motion

- R2: perfect harmonic interval (P5, P8, or P1) should not be approached by similar motion

\section{Statistical Analysis}

We ran statistical tests to assess the associations between metric position, hierarchical levels, and voiceleading patterns. All statistical tests were conducted using JMP® software [3], with an alpha level of .05.

First, we tested whether the usage of certain types of harmonic intervals (consonances and dissonances) differed for pulse-beat onsets compared to other metric positions. We categorized the harmonic intervals into perfect consonances (P1, P5, and P8), imperfect consonances (m3, M3, m6, and M6), and dissonances (all other intervals). Using a Pearson chi-square test, we compared the distributions of the interval categories for harmonic intervals landing on a pulse beat, compared to all other metric positions.

Next, we evaluated whether Palestrina used different voice-leading patterns at different hierarchical levels. To do this, we compared the distribution of motion types (parallel, contrary, similar, oblique, and stasis) in the note-level inventory vs. the pulse-level inventory, using a chi-square test. We also calculated the percentage of intervals that violate each of the above rules within each inventory, using a chi-square test to determine whether the prevalence of 'rule violations' differed at the pulse level and note level.

Finally, we investigated the specific conditions under which the rules were violated using logistic regression models, with separate models for each voiceleading rule and for the note-level and pulse-level inventories. The dependent variable for each model was whether the rule was violated (yes vs. no). The independent variables were: relative position of the consequent interval (inner/inner, outer/outer, or inner/outer); total number of voices in the texture at the consequent interval (categorized as 2 to 3,4 to 5 , or $6+$ voices); whether the upper voice moved by a leap; and whether the lower voice moved by a leap.

\section{Results}

The note-level inventory contained 1,705,371 harmonic intervals with their associated features, which was approximately 2.8 times the size of the pulse-level inventory (615,730 intervals).

The distribution of perfect, imperfect, and dissonant intervals on the pulse beats differed significantly from the distribution at other metric positions, $X^{2}(2, N=$ $1,705,371)=109,993, p<.05$. As shown in Figure 3, consonances were more common on the pulse beats compared to other metric positions, consistent with trends described by Andrews (1958, p. 63). 
Dissonances

Imperfect Consonances

Perfect Consonances

Pulse beats

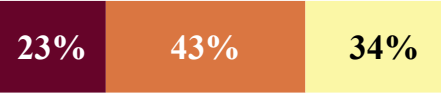

Other

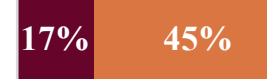

$39 \%$

Figure 3. The distribution of harmonic interval types varies by metric position, with consonant intervals more prevalent on the pulse beats.

Voice-leading patterns also differed at the note level compared to the pulse level (see Figure 4). Similar, parallel, and contrary motion were more prevalent at the pulse level, while stasis and oblique motion were more prevalent at the note level, $X^{2}(4, N=2,321,101)=$ $116,356, p<.05$.

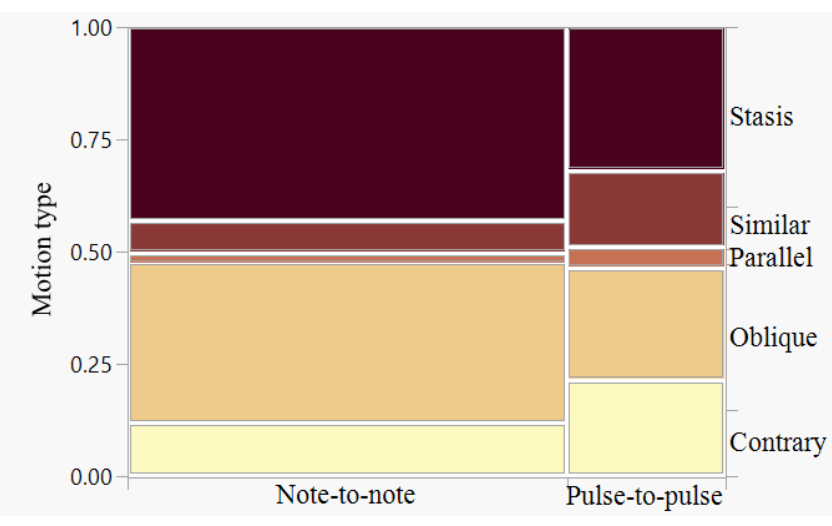

Figure 4. Similar, parallel, and contrary motion are more prevalent at the pulse level than the note level. The column width is proportional to the total number of intervals in the inventory.

Both voice-leading rules ( $\mathrm{R} 1$ and $\mathrm{R} 2$ ) were broken significantly more often at the pulse level than the note level. We observed that R1 (parallel motion to perfect interval) was almost never broken at the note level $(0.01 \%)$, but this voice-leading pattern was used $2.2 \%$ of the time at the pulse level, $X^{2}(1, N=2,321,101)=$ $35,976, p<.05$. R2 was also broken more often at the pulse level than the note level $(3.6 \%$ vs. $1.2 \%), X^{2}(1, N$ $=2,321,101)=13,387, p<.05$.
The factors associated with breaking both voiceleading rules were similar at the note and pulse levels, although the effect sizes tended to be smaller at the pulse level ( $p<.05$ for all effects). Outer/outer voice pairings were more likely to break both rules than inner/inner pairings. At the pulse level, outer/inner pairings were more likely to break R1 at the pulse level but less likely at the note level, compared to inner/inner voice pairings. Having larger numbers of voices in the texture and moving by leaps (as opposed to steps) were associated with greater likelihood of breaking both voice-leading rules.

Table 1. Parameter estimates from logistic regression models. The dependent variable for each model is rule violation (yes vs. no).

\begin{tabular}{|l|c|c|c|c|}
\hline \multirow{2}{*}{ Effect } & \multicolumn{2}{|c|}{ Rule 1 } & \multicolumn{2}{c|}{ Rule 2 } \\
\cline { 2 - 5 } & $\begin{array}{l}\text { Note } \\
\text { level }\end{array}$ & $\begin{array}{c}\text { Pulse } \\
\text { level }\end{array}$ & $\begin{array}{l}\text { Note } \\
\text { level }\end{array}$ & $\begin{array}{l}\text { Pulse } \\
\text { level }\end{array}$ \\
\hline $\begin{array}{l}\text { Position } \\
\text { outer/outer } \\
\text { (vs. inner/inner) }\end{array}$ & 1.00 & 0.47 & 0.63 & 0.42 \\
$\begin{array}{l}\text { outer/inner } \\
\text { (vs. inner/inner) }\end{array}$ & -0.54 & 0.39 & 0.36 & 0.25 \\
\hline $\begin{array}{l}\text { Number of voices } \\
\text { 6+ (vs. 2 to 3) } \\
4 \text { to 5 (vs. 2 to 3) }\end{array}$ & 0.20 & 0.06 & 0.30 & 0.25 \\
\hline $\begin{array}{l}\text { Lower voice movement } \\
\text { leap (vs. step) }\end{array}$ & 0.48 & 0.08 & 1.70 & 0.16 \\
\hline $\begin{array}{l}\text { Upper voice movement } \\
\text { leap (vs. step) }\end{array}$ & 0.79 & 0.28 & 0.58 & 0.16 \\
\hline
\end{tabular}

\section{Discussion}

We found that consonances were more prevalent on the pulse beats, consistent with Andrews' (1958) observation that intervals on pulse beats are usually consonant (p. 62). Our analysis of voice-leading patterns showed that contrary, similar, and parallel motion types made up a larger proportion of the total intervals at the pulse level than at the note level.

Specific voice-leading "rules" (approaching perfect intervals by parallel or similar motion) were broken more often at the pulse level. This is seemingly contrary to what we could expect based on theoretical texts. Assuming any rule violations would be more obvious at perceptually salient levels, we would have expected fewer violations at the pulse level, "hiding" them instead at other beat positions. 
We also identified features that were associated with a greater likelihood of breaking certain voice-leading rules. Both rules were more likely to be broken in textures with larger numbers of voices, supporting Arthur (2016)'s observation that it becomes more difficult to follow the rules as the number of voices increases.

\section{Conclusion}

We found that voice-leading patterns in Palestrina's masses differ at the pulse level compared to the note level. Our findings are relevant for voice-leading pedagogy because they suggest that Palestrina used certain types of "forbidden" voice-leading patterns more often than previously assumed, albeit at a higher metric level. This also has implications for other types of computational analyses using note-to-note successions, because using higher-level hierarchical structures could uncover different patterns or relationships. However, selecting the best unit of analysis for voice leading is still unclear. The most perceptually salient beats should be used for the pulse level analysis, but determining which beats are the most salient depends on many factors, including the tempo at which a piece would have been performed, which is difficult to determine from the symbolic music alone.

\section{End Notes}

[1] The pieces are encoded in kern format and publicly available via music21's built-in corpus.

[2] We defined the pulse as the semibreve (or whole note). While the pulse level can change (Morgan, 2016) DeFord notes that the semibreve is the most common case (DeFord, 2015, p.375).

[3] JMP ${ }^{\circledR}$, Version 15. SAS Institute Inc., Cary, NC, 1989-2020.

\section{References}

Aldwell, E., Schachter, C., \& Cadwallader A. (2019). Harmony \& Voice Leading (5th ed.). Cengage.

Andrews, H.K. (1958). An Introduction to the Technique of Palestrina. Novello and Company.

Arthur, C. (2021). Vicentino versus Palestrina: A computational investigation of voice leading across changing vocal densities. Journal of New Music Research. Advance online publication. https://doi.org/10.1080/09298215.2021.1877729

Benjamin, T. (2005). The Craft of Modal Counterpoint (2nd ed.). Routledge. https://doi.org/10.4324/9780203997208

Conklin, D. (2002). Representation and Discovery of Vertical Patterns in Music. In C. Anagnostopoulou, M.
Ferrand, \& A. Smaill (Eds.), Music and Artificial Intelligence (Vol. 2445, pp. 32-42). Springer Berlin Heidelberg. https://doi.org/10.1007/3-540-45722-4 5

Cuthbert, M. S., \& Ariza, C. (2010). Music21: A toolkit for computer-aided musicology and symbolic music data. 11th International Society for Music Information Retrieval Conference (ISMIR), Utrecht, Netherlands, 637-642.

DeFord, R. (2015). Tactus, Mensuration, and Rhythm in Renaissance Music. Cambridge University Press. https://doi.org/10.1017/CBO9781107587717

Finkensiep, C., Neuwirth, M., \& Rohrmeier, M. (2018). Generalized skipgrams for pattern discovery in polyphonic streams. 19th International Society for Music Information Retrieval Conference (ISMIR), 547-553.

Fuller, S. (2002). Organum - Discantus - Contrapunctus in the Middle Ages. In T. Christensen (Ed.), The Cambridge History of Western Music Theory (pp. 477-502). Cambridge University Press. https://doi.org/10.1017/CHOL9780521623711.017

Huron, D., \& Collins, D. (1999). Voice leading in cantus firmus-based composition: A comparison between theory and practice in Renaissance and Baroque music using computer-assisted inferential measures. Computers in Music Research, 6 (Spring), 53-95.

Gauldin, R. (1985). A Practical Approach to SixteenthCentury Counterpoint. Prentice Hall, Inc.

Hanson, J. R. (1983). Enumeration of Dissonance in the Masses of Palestrina. College Music Symposium, 23 (1): 50-64.

Hanson, John. (1983). Enumeration of dissonance in the masses of Palestrina. College Music Symposium, 23 (1): 50-64. College Music Symposium, 23(1), 50-64.

Jeppesen, K. (1927). The Style of Palestrina and the Dissonance. Translated by Nargaret N. Hamerik. Oxford University Press; revised English edition, 1946; reprint of the 1946 edition: Dover Publications, 1970.

Marshall, R. (1963). The paraphrase technique of Palestrina in his masses based on hymns. Journal of the American Musicological Society, 16(3). https://doi.org/10.2307/829827

Marvin, C. (2002). Giovanni Pierluigi Da Palestrina: A Research Guide. Routledge Music Bibliographies Ser. (1st ed., Vol. 56). Routledge Publishing, Inc.

Morgan, A. (2016). Renaissance interval-succession theory: Treatises and analysis. [Doctoral dissertation, McGill University]. Proquest.

Sears, D. R. W., Arzt, A., Frostel, H., Sonnleitner, R., \& Widmer, G. (2017, July 18). Modeling harmony with skip-grams. 18th International Society for Music Information Retrieval Conference (ISMIR), Suzhou, China, 332-338. 


\title{
Tagging film music: A corpus study of Max Steiner's film scores
}

\author{
Jeff Lyon ${ }^{1 \dagger}$ \\ Brent Yorgason ${ }^{2}$ \\ ${ }^{1}$ School of Music, Brigham Young University, Provo, UT, USA \\ ${ }^{2}$ Harold B. Lee Library, Brigham Young University, Provo, UT, USA \\ $\dagger$ Corresponding author: jeff lyon@byu.edu \\ Published 16 December 2021; https://doi.org/10.18061/FDMC.2021.0009 \\ Author video presentation and/or other conference material: https://doi.org/10.17605/OSF.IO/ZNVJQ
}

\begin{abstract}
The Max Steiner Digital Thematic Catalog is an ongoing corpus study project which will incorporate thematic data from all of Steiner's existing film scores (from 1930-1965). This film music corpus is significant because Steiner's work is representative of over three decades of Hollywood film scoring practices. Here we describe our methods and procedures in tagging film music data for an online database. Consistent metadata and tagging will help researchers to more effectively use the data in this corpus.
\end{abstract}

KEYWORDS: Max Steiner, film music, analysis, corpus studies, tagging

\section{Introduction}

The Max Steiner Digital Thematic Catalog is an ongoing corpus study project which will incorporate thematic data from all of Steiner's existing film scores. Max Steiner's film score collection at Brigham Young University comprises over 31,000 pages of film sketches spanning over 300 films from 1930 to 1965.[1] This corpus is significant because Steiner's work was quite influential and is representative of over three decades of Hollywood film scoring practices.

A central goal of the corpus study is to identify and analyze themes, theme variants, gestures, and other types of underscoring in each film. Our online database (accessible at https://maxsteinerinstitute.org) describes each musical cue in detail, providing a transcription of the score (either from existing sketches or by ear), a transcription of Steiner's handwritten annotations, precise timings, film stills, music-analytical data, and tags that relate each cue to the characters, events, places, and narrative structure of the film.

A detailed tagging system has been developed that will allow scholars to make comparisons between film cues and to find patterns across Steiner's sizable corpus. The average film has over 500 tags and the process of tagging them will likely take us over a decade. Thus, it is important to have a consistent tagging process with established procedures and categorization methods.
In order to consistently tag the cues in a film, we have established specific tagging categories. The core tagging categories are mood, action, character, setting, and scene (MACSS). Additional categories include object tags, music tags, film tags, and analytical tags. To further ensure tagging consistency, we have developed a set of automated tools to help us find conflicts and redundancies.

Most film tagging systems (such as the user-supplied tags found at IMDb) focus on the visual elements and plot points of a film. Our tagging system incorporates a specific focus on film music analysis, which introduces numerous terms not found in typical tagging schemas or established thesauri. We believe this will be very useful to scholars in the growing field of film music research.

\section{Method}

Here we describe further each of the tagging categories and the tools that we use to ensure consistency and to enable discovery.

\section{Tagging Categories}

The most significant step in the development of our tagging system was the creation of specific tagging categories. Every musical cue in the database is given a tag in each of the five core categories of MACSS (mood, action, character, setting, and scene).

\section{Table 1: The five core categories of MACSS}

\begin{tabular}{|l|l|l|}
\hline Category & Description & Examples \\
\hline Mood & $\begin{array}{l}\text { mood or emotion } \\
\text { of a musical cue }\end{array}$ & $\begin{array}{l}\text { tense, mysterious, dark, } \\
\text { comical, romantic }\end{array}$ \\
\hline Action & $\begin{array}{l}\text { action associated } \\
\text { with the cue }\end{array}$ & $\begin{array}{l}\text { walking, running, } \\
\text { dancing, falling, dying }\end{array}$ \\
\hline Character & $\begin{array}{l}\text { character types } \\
\text { involved in the cue }\end{array}$ & $\begin{array}{l}\text { hero, gangster, leading } \\
\text { lady, doctor, lawyer }\end{array}$ \\
\hline Setting & $\begin{array}{l}\text { location of the } \\
\text { musical cue }\end{array}$ & $\begin{array}{l}\text { prison, street, office, } \\
\text { barn, courtroom, boat }\end{array}$ \\
\hline Scene & $\begin{array}{l}\text { the scene type for } \\
\text { the current cue }\end{array}$ & $\begin{array}{l}\text { establish character, } \\
\text { conflict, flashback, } \\
\text { climax, love scene }\end{array}$ \\
\hline
\end{tabular}


There are also several optional tagging categories to be used whenever possible and applicable: film (or movie) tags, music tags, analytical tags, physical object tags, and concept tags.[2]

\section{Table 2: Additional optional tagging categories}

\begin{tabular}{|l|l|l|}
\hline Category & Description & Examples \\
\hline $\begin{array}{l}\text { Film (or } \\
\text { Movie) }\end{array}$ & $\begin{array}{l}\text { film techniques } \\
\text { used in the cue }\end{array}$ & $\begin{array}{l}\text { montage, voice-over, } \\
\text { double exposure }\end{array}$ \\
\hline Music & $\begin{array}{l}\text { significant aspects } \\
\text { relating to music }\end{array}$ & $\begin{array}{l}\text { source music, quotation, } \\
\text { stinger, mickey-mousing }\end{array}$ \\
\hline Analytical & $\begin{array}{l}\text { music-analytical } \\
\text { terminology }\end{array}$ & $\begin{array}{l}\text { ostinato, sequence, tone- } \\
\text { cluster, AABA structure, } \\
\text { octatonic, parallelism }\end{array}$ \\
\hline $\begin{array}{l}\text { Thing } \\
\text { (physical) }\end{array}$ & $\begin{array}{l}\text { important physical } \\
\text { objects in the cue } \\
\text { phonograph, newspaper }\end{array}$ & $\begin{array}{l}\text { death, murder, fear, } \\
\text { deception, love, war }\end{array}$ \\
\hline $\begin{array}{l}\text { Thing } \\
\text { (conceptual) }\end{array}$ & $\begin{array}{l}\text { central concepts } \\
\text { involved in the cue }\end{array}$ & $\begin{array}{l}\text { deater, } \\
\text { dection, }\end{array}$ \\
\hline
\end{tabular}

Overall, our philosophy is to tag each film cue with as much data as possible, with the categories providing a crucial guide for potential tags.

\section{Tools for Consistency}

Since the tagging process will take place over a period of several years, we needed to develop tools to ensure consistency over time. The Tag Data tool in the database lists and enumerates all tags across all categories (see Figure 1). It provides a quick reference for the most commonly-used tags in the database and a reminder of the proper formatting and spelling of tags.[3]

\begin{tabular}{|l|l|l|l|l|l|}
\hline \multicolumn{2}{|l|}{ Mood/Imotion } & \multicolumn{2}{l|}{ Action } & Character \\
\hline tense & 455 & conversing & 278 & leading man & 514 \\
dramatic & 263 & walking & 157 & leading lady & 473 \\
mysterious & 219 & running & 97 & hero & 422 \\
dark & 167 & singing & 65 & heroine & 419 \\
somber & 145 & arguing & 63 & gangster & 265 \\
cheerful & 101 & driving & 55 & antagonist & 221 \\
calm & 82 & dancing & 47 & lawyer & 107 \\
gentle & 77 & falling & 46 & teenager & 107 \\
romantic & 59 & escaping & 45 & FBI & 81 \\
\end{tabular}

\section{Figure 1: Tag Data tool}

Before adding a new tag to the database, we often check the Tag Data to determine if there is an existing tag that could be a better fit. We are also developing an integrated thesaurus that creates a link between related terms in the database (such as turbulent, agitated, and distressed) even if we decide to use them separately.
After we have finished tagging a film, we use the Unique Tags tool to identify tags that were used only in that film. If we have formatted a tag differently than usual (such as "onscreen" vs. "on-screen"), it will appear as a unique tag and we will standardize it. For instance, the unique tags shown in Figure 2 for the 1939 film Dust Be My Destiny include the tag "end credits," which we would standardize as "end title sequence."'[4]

\section{dark room bank sincere barn inversion theater expectant milkman jailer buzzy thankful road end credits kind COW resting pensive photography hitch= hiking bribe settling down Rimsky-Korsakov oppressive denial devotion hiccuping hook injustice mooing car horn millk intervallic expansion}

\section{Figure 2: Unique Tags tool}

More recently we have started to use OpenRefine, an open source tool for data cleanup (https://openrefine.org). This tool has helped us to more efficiently identify inconsistently formatted tags as well as tags that have been assigned to multiple categories (as described further in the Discussion section below).

\section{Tools for Discovery}

We have also developed tools to enable discovery. All of the tags displayed in the online database are hyperlinked to the Tag Search feature, which allows users to search for tags across all films (see the "murder" search in Figure 3). The Thesaurus provides users with alternative search terms ("execution," "murdering," and "death"). And Tag Clouds (similar to the one shown in Figure 2) allow users to easily see the most-used tags within a film.

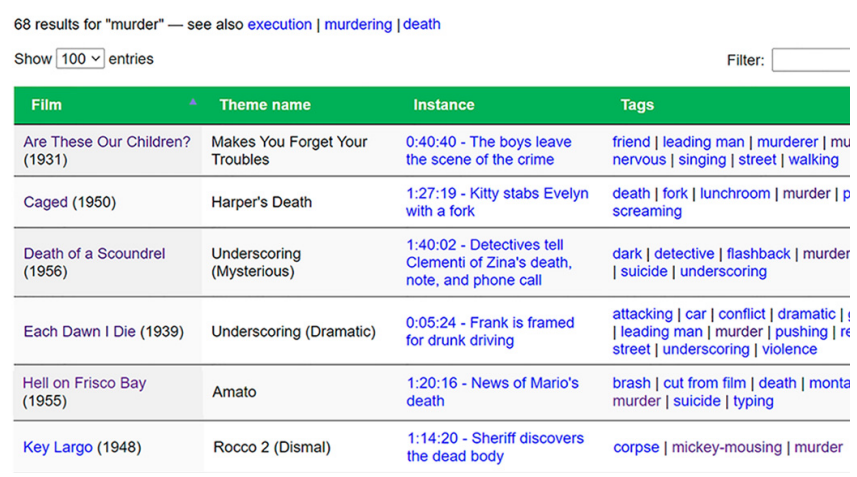

Figure 3: Tag Search tool 


\section{Results}

Although the tagging process for the Steiner corpus is still underway, the data collected so far has already provided ample material for film music research (Lyon 2021; Yorgason 2020, 2021). Here, we provide the results of a hypothetical research question using information currently in the online database. Steiner is known for his use of the technique of "mickey-mousing," which means that he frequently "catches the action" on the screen with musical gestures in his score. A film music scholar might pose the following questions: (1) How frequently does Steiner use mickey-mousing? (2) Does mickeymousing increase or decrease over time? (3) Which actions are most frequently mickey-moused? (4) Which film genres use mickey-mousing the most?

\section{Frequency}

Using the Tag Data tool, we find that the tag "mickeymousing" is used 272 times out of 1,674 total cues. Thus, Steiner uses the technique of mickey-mousing in $16.2 \%$ of all musical cues in the current database.

\section{Increase or Decrease over Time}

Consulting the data for individual films in the database, we find "mickey-mousing" tagged more than twice as many times in Steiner's later Warner Bros. years (211 out of 1,245 cues $=16.9 \%$ ) than in his earlier career at RKO ( 31 out of 429 cues $=7.2 \%$ ).

\section{Actions Most Often Mickey-moused}

157 distinct action tags in the database were associated with a "mickey-mousing" tag. The actions most often mickey-moused were "running" (44 times), "falling" (26 times), and "walking" (25 times). 16.2\% of all mickey-mousing cues involved running, and $66.7 \%$ of all cues tagged "running" were mickey-moused.

\section{Film Genres}

All of the films currently in the database are in the crime genre, which makes it difficult to answer this question satisfactorily. However, the data does show that two of the films with a strong comedic element have the highest ratio of cues tagged "mickey-mousing": Crime School (36.7\%) and Angels with Dirty Faces (32.6\%). By contrast, two of the most serious and dark films in the database have the lowest ratio of cues tagged "mickeymousing": The Letter (2\%) and The Unfaithful (3.1\%).

These results are likely to change as more films from a wider variety of genres are added to the database.

\section{Discussion}

In order to more thoroughly evaluate our tagging process, we now consider some numerical data regarding the work that has been completed so far. Of the 32 films that have been fully tagged (about $10 \%$ of Steiner's film scores overall), there have been 15,983 total tags assigned. Here it is worth noting that the earlier RKO films (1930-1936) are generally more sparsely scored (with 4,057 total tags) than the later, more lavish scores for Warner Bros. (11,926 tags). [5] The average number of tags per film is 500 , with a much lower average number of tags for RKO films (194) than for Warner Bros. films (543). Of the films remaining to be tagged, about $60 \%$ will be of the more lavish type, which means that our tagging progress will slow a bit.

Table 3 outlines the distribution of the tags overall. The top 10 tags (such as "leading man," "main theme," and "advance plot") account for $22 \%$ of tags overall. It is possible that some of these tags may be too general or too liberally used, so we might consider replacing them with more specific tags. On the other end of the spectrum, 392 tags were used only once ( $2 \%$ of tags overall). Some of these are specific objects ("shark," "see-saw") or character types ("violinist," "milkman") that we may encounter in later films. Other tags might be too specific (e.g., "punching" = 1 usage while "fighting" = 31) and may need to be generalized in a second pass.

\section{Table 3: Tag distribution}

\begin{tabular}{|l|l|}
\hline Most common tags & \% of all tags \\
\hline Top 10 tags & $22 \%$ \\
\hline Top 20 tags & $34 \%$ \\
\hline Top 50 tags & $50 \%$ \\
\hline Top 100 tags & $63 \%$ \\
\hline Top 200 tags & $77 \%$ \\
\hline Least common tags & $18 \%$ \\
\hline Bottom 996 tags (used 10 times or less) & $18 \%$ \\
\hline Bottom 392 tags (used only once) & $2 \%$ \\
\hline
\end{tabular}

Table 4 further breaks down the distribution of tags by category. The data shows that the five core MACSS categories tend to be more heavily tagged than the others (representing $72 \%$ of all tags assigned). The "film" category is the least heavily tagged (with only $2.3 \%$ ), which might suggest that we need to focus more on this category of tags.[6] The uniformity of scene tags (only $1.5 \%$ unique) is deliberate, since we want to have a limited number of scene types. The abundance of unique "thing" tags is also not surprising, since the objects onscreen tend to be more variable than anything else. 
Table 4: Tag distribution by category

\begin{tabular}{|l|l|l|l|l|}
\hline Category & $\begin{array}{l}\text { Total } \\
\text { tags }\end{array}$ & $\begin{array}{l}\text { \% of all } \\
\text { tags }\end{array}$ & $\begin{array}{l}\text { Tags used } \\
\text { once }\end{array}$ & $\begin{array}{l}\text { \% of tags } \\
\text { used once }\end{array}$ \\
\hline Mood & 2303 & $14.4 \%$ & 35 & $8.9 \%$ \\
\hline Action & 2298 & $14.4 \%$ & 109 & $27.8 \%$ \\
\hline Character & 3553 & $22.2 \%$ & 25 & $6.4 \%$ \\
\hline Setting & 1670 & $10.4 \%$ & 12 & $3.1 \%$ \\
\hline Scene & 1673 & $10.5 \%$ & 6 & $1.5 \%$ \\
\hline Music & 2208 & $13.8 \%$ & 41 & $10.5 \%$ \\
\hline Thing & 1220 & $7.6 \%$ & 138 & $35.2 \%$ \\
\hline Analytical & 689 & $4.3 \%$ & 19 & $4.8 \%$ \\
\hline Film & 369 & $2.3 \%$ & 7 & $1.8 \%$ \\
\hline
\end{tabular}

OpenRefine has helped us to identify additional issues in our current tagging process. Using the "Cluster \& Edit" tool,[7] we found 37 clusters of inconsistently formatted tags, seven of which needed attention. These clusters are made up of tags that should be narrowed down to a single tag, resolving issues such as verb tense, plurality, spelling, and hyphen usage. One example of such a cluster is "photograph/photographs/photography." The cluster tool also found 98 clusters of tags appearing in multiple categories, 46 of which needed attention. Although the same tag in different categories sometimes has a different meaning (such as the tag "Chinese" in the music category vs. the character category), other tags were indeed categorized incorrectly. These inconsistencies are largely found in the earlier films that we tagged, indicating how our procedures have improved over time.

\section{Conclusion}

Consistent tagging of metadata is crucial to finding results in a corpus. We will continue to refine our tagging process as our analysis of Steiner's corpus progresses. We expect that as data from films in other genres (beyond crime) is added to the database, new sets of tags will need to be created in each of the categories. For example, Westerns will have different settings than films noir. Romances will have different characters than thrillers. As the tagging data in the Max Steiner Digital Thematic Catalog grows, film scholars will be able to answer many more questions about practices in Classic Hollywood film scoring.

\section{Acknowledgements}

We would like to acknowledge the Film Music Archives in the L. Tom Perry Special Collections department of the Harold B. Lee Library at Brigham Young University for their role in preserving and providing access to the materials in the Max Steiner Collection.

\section{End Notes}

[1] Currently, our corpus includes 315 films by Steiner, with an additional 34 films categorized as lost, hard to find, or difficult to determine actual authorship based on current information.

[2] Combined with the core categories of MACSS, these optional categories form the acronym MMMAACTTSS (both being a tribute to Max).

[3] For example, we have decided that mood tags should be adjectives (anxious, playful, sentimental), action tags should be gerunds (jumping, spinning, climbing), and character tags should be nouns (guard, sheriff, victim).

[4] The Unique Tags and Tag Data tools also help us to identify underused and newly-established tags that we will want to consider in future tagging sessions.

[5] If we omit one notable outlier, Steiner's 1935 Oscarwinning score for The Informer, the average number of music cues for each RKO film is only 14 , as compared to an average of 104 cues for Warner Bros. films.

[6] Although the "analytical" category also appears to be low in this table $(4.3 \%)$, the tagging process for this category actually lags behind the others due to the time needed to complete the separate step of score analysis (from the transcriptions).

[7] We used the "nearest neighbor - Levenshtein" function using a radius of 1.0 and 6 block characters.

\section{References}

Lyon, J. \& Yorgason, B. (2021). Cataloguing Max Steiner: A Corpus Study of Film Scores. Journal of Film Music, 9(1-2).

Yorgason, B. \& Lyon, J. (2020). Fanfare as Fulcrum: A Pivotal Event in Max Steiner's Theme for Warner Brothers. Music Theory Online, 26(2). https://doi.org/10.30535/mto.26.2.12

(2021). Fanning out from the Fanfare: Max Steiner's Theme for Warner Brothers. Journal of Film Music, 9(1-2). 


\title{
Implications of thematic reuse in Haydn's sonata forms
}

\author{
Jan Miyake \\ Oberlin College \& Conservatory, Oberlin, OH, USA \\ jan.miyake@oberlin.edu \\ Published 16 December 2021; https://doi.org/10.18061/FDMC.2021.0010 \\ Author video presentation and/or other conference material: https://doi.org/10.17605/OSF.IO/Z7BXU
}

\begin{abstract}
Haydn's approach to form is underserved by current theories as discussed by Burstein (2016), Duncan (2011), Fillion (2012), Korstvedt (2013), Ludwig (2012), Neuwirth (2011, 2013), and Riley (2015). Comparing Haydn to composers a generation younger (Mozart and Beethoven) instead of with his contemporaries (such as Dittersdorf and Vanhal) distorts what is, and what is not, idiosyncratic about his compositional form. His inclination to reuse the opening theme later in the movement can impact the melody of his $\mathrm{S}$ theme, the path to and through his recapitulations, and the construction of his phrase's middles (Miyake, 2011). This compositional feature, however, often leads to forms that do not fit neatly into theories of Classical Era form forwarded by Caplin (2001) and Hepokoski and Darcy (2006). The concept of thematic saturation provides a window into investigating how Haydn reuses themes. The quantity and density of thematic saturation measure different aspects of thematic reuse and further our understanding of Haydn's approach to form. This project is part of a larger project that investigates whether patterns of thematic returns are independent of traditional formal designations (sonata form, sonata rondo, ABACA).
\end{abstract}

KEYWORDS: sonata form, thematic saturation, monothematicism, Haydn

\section{Introduction}

Monothematicism is connected with Haydn by almost every writer on the topic (Tilmouth, 2001). Similarly, overviews of Haydn's instrumental works invariably discuss Haydn's use of monothematicism. Monothematic sonata forms, however, account for only a fraction of the many ways Haydn recycles his opening theme. Investigating thematic reuse across a movement allows an analyst to move beyond the conception of form as a static container to be filled, to the conception of form as a process that unfolds in time. Thematic reuse often impacts the paths that Haydn's sonata forms travel, and it can require Procrustean efforts to fit these journeys into a mold made for Beethoven or Mozart. Burstein (2016), Duncan (2011), Fillion (2012), Korstvedt (2013), Ludwig (2012), Neuwirth (2011,
2013), and Riley (2015) all document how Haydn's music is underserved by current theories of form.

The term monothematic specifically refers to a sonata form exposition in which the secondary theme (S) parallels the primary theme (P), but in the new key. Rosen (1998) and Miyake (2011) problematize the term "monothematic" because (1) the P-based S typically diverges from $\mathrm{P}$ to create a contrasting theme and (2) monothematic expositions usually include a non Pbased C-theme. While Haydn frequently uses P-based S themes, a related trademark of his style is his proclivity for reusing his $\mathrm{P}$ theme to initiate a wide range of formal areas. These formal areas include sections of a sonata form (e.g., the development or coda), subsections of a sonata-form section (e.g., sequences in the development or the transition of an exposition), and beginnings within phrases (e.g., the latter phrase of a period, a or module of a multi-modular theme).

Scholars have noted Haydn's thematic reuse, but few engage deeply with it. Hepokoski and Darcy's Sonata Theory (2006) develops language for describing techniques of thematic return. Two examples of this language are "The P-based C" (p. 184) and "The Onset of the Development; P-Material as the Norm" (p. 207). Caplin (2001) also accounts for thematic reuse in his Formenlehre. Two examples, chosen to parallel the Hepokoski and Darcy examples, are subsections on "Main-theme material" when discussing beginning the transition (p. 117) and on "Main theme's basic idea" in a description of ways to begin the pre-core of a development (p. 151). Neither of these theories are designed to look at the impact that thematic reuse has across an entire form. Unlike Caplin, Hepokoski \& Darcy, Haimo (1995) wrestles with the impact that thematic reuse can have on a movement. His unity, redundancy, and variation principles work together to address the impact of thematic saturation on Haydn's compositions. This current work has more in common with Haimo than Caplin, Hepokoski and Darcy because it forwards the concept of thematic saturation to deepen our understanding of how thematic reuse unfolds across a sonata form. 


\section{Method}

\section{Measuring thematic saturation}

Investigating thematic reuse starts with building a corpus of thematic returns. I analyzed nearly 200 movements to list every instance of a thematic return. All movements are last movements, and their attribution to Haydn has not been disproven. Compared with first movements, final movements are less bound by convention and can be organized in a variety of ways, not just as a sonata form. Thematic returns have a formal function of beginning and reuse at least the basic idea of the original theme. Returns can initiate passages at any level of form (e.g., development, consequent phrase, sequence, or S-theme). Thematic returns are a subset of motivic returns. The former must have a formal function of a beginning while the latter can have any formal function. The analyses of the final movements of Haydn's symphonies, keyboard trios, and keyboard sonatas generated 1684 thematic returns from 189 last movements, of which 781 thematic returns come from 99 last movements organized as a sonata form. [1]

The data measure thematic saturation in two ways. Quantity is the percentage of the bars, including those constituting the first theme, that participate in thematic saturation. Density is a count of the number of instances of thematic saturation normalized by dividing the count by the total number of heard measures. Quantity accounts for the length of the return while density does not.

\section{Thematic Plans of Expositions \& Monothematicism}

The thematic plan of an exposition details how thematic reuse impacts the zones of an exposition. By definition, all P-themes are launched by the first theme. Later zones-TR, S, and $\mathrm{C}$ - can also be initiated by the $\mathrm{P}$ theme. There are eight possible plans (Table 1), of which half would be labeled monothematic.

Table 1: Possible thematic plans of a sonata form exposition. $y / n$ indicates whether or not the main theme initiates that zone of the exposition.

\begin{tabular}{|c|c|c|c|c|c|c|c|c|}
\hline \multicolumn{4}{|c|}{ Not monothematic } & \multicolumn{5}{|c|}{ Monothematic } \\
\hline $\mathrm{P}$ & $\mathrm{TR}$ & $\mathrm{S}$ & $\mathrm{C}$ & $\mathrm{P}$ & $\mathrm{TR}$ & $\mathrm{S}$ & $\mathrm{C}$ \\
\hline $\mathrm{y}$ & $\mathrm{n}$ & $\mathrm{n}$ & $\mathrm{n}$ \\
$\mathrm{y}$ & $\mathrm{y}$ & $\mathrm{n}$ & $\mathrm{n}$ \\
$\mathrm{y}$ & $\mathrm{n}$ & $\mathrm{n}$ & $\mathrm{y}$ \\
$\mathrm{y}$ & $\mathrm{y}$ & $\mathrm{n}$ & $\mathrm{y}$ & $\mathrm{n}$ & $\mathrm{y}$ & $\mathrm{n}$ \\
\hline $\mathrm{y}$ & $\mathrm{y}$ & $\mathrm{y}$ & $\mathrm{y}$ & $\mathrm{y}$ & $\mathrm{n}$ \\
\hline & $\mathrm{y}$ & $\mathrm{n}$ & $\mathrm{y}$ & $\mathrm{y}$ \\
\hline & $\mathrm{y}$ & $\mathrm{y}$ & $\mathrm{y}$ & $\mathrm{y}$ \\
\hline
\end{tabular}

Table 2 organizes the possible thematic plans by how many times the main theme initiates a thematic area, highlighting the monothematic plans with a blue font, and providing a count of Haydn's use of each plan in the corpus. While monothematic expositions represent one way an exposition can be thematically saturated, the term omits other equally saturated thematic plans.

Table 2: Possible thematic plans presented from least saturated by the opening theme to most saturated. Monothematic expositions are in blue.

\begin{tabular}{|c|c|c|c|c|c|}
\hline $\mathbf{P}$ & TR & $\mathbf{S}$ & $\mathbf{C}$ & $\begin{array}{l}\text { \# of thematic } \\
\text { areas initiated by } \\
\text { the main theme }\end{array}$ & $\begin{array}{c}\% \text { of } \\
\text { corpus } \\
(\text { size }=99) \\
\end{array}$ \\
\hline $\mathrm{y}$ & $\mathrm{n}$ & $\mathrm{n}$ & $\mathrm{n}$ & 1 & 41 \\
\hline $\mathrm{y}$ & $\mathrm{y}$ & $\mathrm{n}$ & $\mathrm{n}$ & \multirow{3}{*}{2} & 25 \\
\hline$y$ & $n$ & $y$ & $n$ & & 13 \\
\hline $\mathrm{y}$ & $\mathrm{n}$ & $\mathrm{n}$ & $\mathrm{y}$ & & 2 \\
\hline$y$ & $y$ & $y$ & $n$ & \multirow{3}{*}{3} & 10 \\
\hline $\mathrm{y}$ & $\mathrm{y}$ & $\mathrm{n}$ & $\mathrm{y}$ & & 3 \\
\hline$y$ & $n$ & $y$ & $y$ & & 4 \\
\hline$y$ & $y$ & $y$ & $y$ & 4 & 1 \\
\hline
\end{tabular}

Impacts of thematic reuse are investigated by calculating the average quantity and density for each possible exposition plan as well as by the presence of each P-based zone of the exposition.

\section{Results}

Table 3 reports the corpus's average quantity and density of thematic saturation for each type of P-based theme. As the exposition unfolds, the presence of a Pbased theme generally increases the quantity and density of thematic saturation.

Table 3: Average quantity and density of thematic saturation given the existence of a P-based theme

\begin{tabular}{|c|c|c|c|}
\hline $\begin{array}{c}\text { P-based } \\
\text { thematic zone }\end{array}$ & Quantity & Density & $\begin{array}{c}\text { Count in corpus } \\
\text { (size = 99) }\end{array}$ \\
\hline only P & $22 \%$ & .034 & 41 \\
\hline Includes TR & $27 \%$ & .061 & 39 \\
\hline Includes S & $26 \%$ & .069 & 28 \\
\hline Includes C & $29 \%$ & .079 & 10 \\
\hline All sonata forms & $24 \%$ & .051 & 99 \\
\hline
\end{tabular}

Table 3 double or triple counts several expositions because $19 \%$ of the corpus have three or four expositional zones initiated by P. Tables 4 and 5 break the quantity and density of thematic saturation down by 
thematic plan while figures 1 and 2 visually represent the data.

Table 4: Possible thematic plans presented from least saturated by the opening theme to most saturated. Monothematic expositions are in blue.

\begin{tabular}{|c|c|c|c|c|c|}
\hline $\mathbf{P}$ & TR & $\mathbf{S}$ & $\mathbf{C}$ & \multicolumn{2}{|c|}{$\begin{array}{r}\text { Average Quantity of Thematic } \\
\text { Saturation }\end{array}$} \\
\hline $\mathrm{y}$ & $\mathrm{n}$ & $\mathrm{n}$ & $\mathrm{n}$ & $22 \%$ & $22 \%$ \\
\hline $\mathrm{y}$ & $\mathrm{y}$ & $\mathrm{n}$ & $\mathrm{n}$ & $25 \%$ & \multirow{2}{*}{$24 \%$} \\
\hline$y$ & $n$ & $y$ & $n$ & $22 \%$ & \\
\hline $\mathrm{y}$ & $\mathrm{n}$ & $\mathrm{n}$ & $\mathrm{y}$ & $30 \%$ & \multirow{2}{*}{$30 \%$} \\
\hline$y$ & $y$ & $y$ & $n$ & $29 \%$ & \multirow{2}{*}{$50 \%$} \\
\hline $\mathrm{y}$ & $\mathrm{y}$ & $\mathrm{n}$ & $\mathrm{y}$ & $36 \%$ & \\
\hline$y$ & $n$ & $y$ & $y$ & $27 \%$ & $50 \%$ \\
\hline$y$ & $y$ & $y$ & $y$ & $50 \%$ & \\
\hline
\end{tabular}

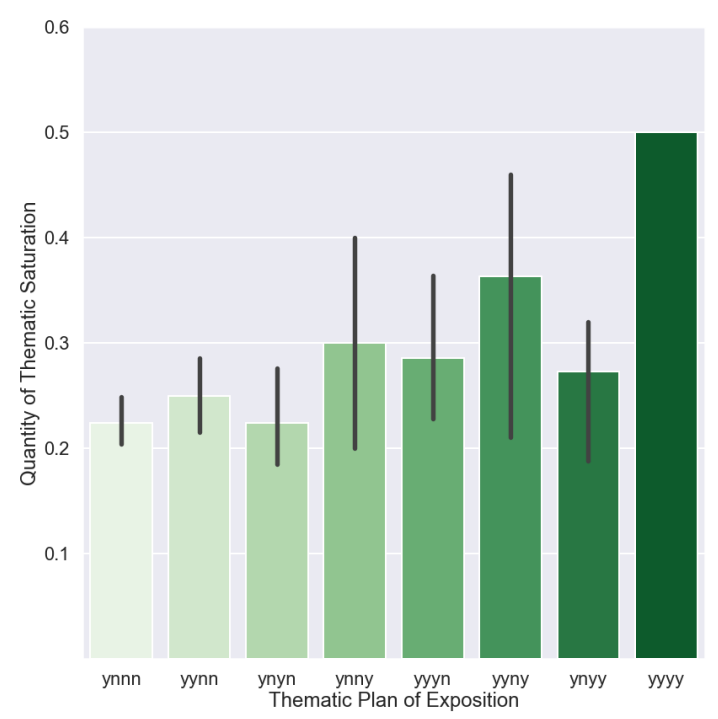

Figure 1: Quantity of Thematic Saturation means for each exposition plan

Table 5: Possible thematic plans presented from least saturated by the opening theme to most saturated. Monothematic expositions are in blue.

\begin{tabular}{|c|c|c|c|c|c|}
\hline $\mathbf{P}$ & $\mathbf{T R}$ & $\mathbf{S}$ & $\mathbf{C}$ & \multicolumn{2}{|c|}{$\begin{array}{c}\text { Average Density of Thematic } \\
\text { Saturation }\end{array}$} \\
\hline $\mathrm{y}$ & $\mathrm{n}$ & $\mathrm{n}$ & $\mathrm{n}$ & .034 & .034 \\
\hline $\mathrm{y}$ & $\mathrm{y}$ & $\mathrm{n}$ & $\mathrm{n}$ & .051 & \multirow{2}{*}{.056} \\
\hline$y$ & $n$ & $y$ & $n$ & .058 & \\
\hline $\mathrm{y}$ & $\mathrm{n}$ & $\mathrm{n}$ & $\mathrm{y}$ & .087 & \\
\hline$y$ & $y$ & $y$ & $n$ & .068 & \multirow{2}{*}{.075} \\
\hline $\mathrm{y}$ & $\mathrm{y}$ & $\mathrm{n}$ & $\mathrm{y}$ & .091 & \multirow{2}{*}{.075} \\
\hline$y$ & $n$ & $y$ & $y$ & .082 & .144 \\
\hline$y$ & $y$ & $y$ & $y$ & .144 & \\
\hline
\end{tabular}

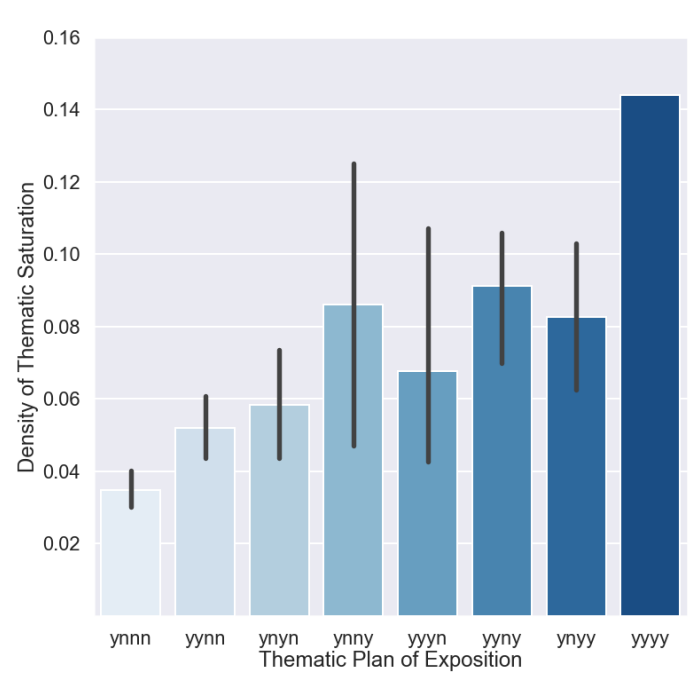

Figure 2: Density of Thematic Saturation means for each exposition plan

\section{Discussion}

In this corpus, Haydn's penchant for thematic reuse within the exposition is clear: Table 2 shows that approximately $60 \%$ of his expositions launch at least one future zone with the P-theme. While Haydn was equally likely to initiate one future exposition section with $\mathrm{P}(40 \%$ of corpus) as he was to initiate no future exposition sections ( $41 \%$ of corpus), the 19\% of expositions with higher degrees of thematic saturation skew the balance towards reusing the P-theme later in the exposition. Even though we believe Haydn writes more monothematic expositions than Beethoven and Mozart, we do not yet have the data to compare these composers' overall thematic reuse in the exposition. Clearly P-based TRs and P-based Cs are common enough to be defaults in Hepokoski and Darcy's typology of exposition possibilities (Hepokoski and Darcy 2006, pp. 101 and 184).

Table 3 shows that the presence of future exposition zones initiated by the P-theme impacts the movement's quantity and density of thematic saturation. The impact on quantity is less pronounced than the impact on density. Since density measures the number of returns and quantity the number of measures in the return, it is clear that Haydn does not recycle the complete theme. Additionally, the later the thematic recycling occurs in the exposition, the larger the impact on the number of future returns; P-based $\mathrm{C}$ sections lead to more 
thematically saturated movements than P-based TR sections.

When considering how intensely a piece is saturated by its main theme, it is tempting to connect thematic saturation with monothematicism. The two concepts, however, refer to distinctly different phenomenon. Monothematicism is defined solely by the contents of an exposition, focusing on comparing the opening bars of $\mathrm{S}$ with the opening bars of $\mathrm{P}$. It ignores other thematic events in the exposition and in the rest of the movement (development, recapitulation, and coda). Thematic saturation, on the other hand, examines the reuse of P's opening throughout the entire movement. It has the potential to capture thematic reuse in other exposition sections, the development, and in Haydn's varied approaches to the recapitulation.

Tables 4 and 5 demonstrate that monothematic expositions from this corpus lead to less saturated movements than non-monothematic exposition with thematic reuse (P-based TR and/or P-based C). In thematic plans with two or three exposition zones initiated by the P-theme, those including a P-based S theme, in a blue font, were the least saturated of the group, with one exception: the density of thematic satuation of the "ynyn" exposition plan. In fact, the existence of a P-based $\mathrm{C}$ theme leads to the highest degrees of density and quantity of thematic saturation. This observation about P-based $\mathrm{C}$ themes is also supported by Table 3 .

The data additionally capture Haydn's penchant for launching a section with $\mathrm{P}$ material and then proceeding to develop it in new ways (Figure 3 ). In Table 4, the average quantity of thematic saturation was the same for the least saturated expositional plan ("ynnn," no future P-based themes) and the least saturated monothematic plan ("ynyn," only a S-based P theme): $22 \%$. But, when comparing these two plans' density of thematic saturation in Table 5, drastically different results emerge: .034 compared with .058 . This divergence in similarity shows that, for these two plans, Haydn created equal amounts of content (quantity) based on reusing $\mathrm{P}$ 's beginning as a future beginning but did so far more frequently (density) in the monothematic plan. In other words, in the more thematically saturated work, Haydn does not bring back the entire P-theme. Rather, he both uses that opening more frequently to launch future areas and then recycles less of the theme before developing it in new ways. This approach to thematic reuse is noted in any significant discussion of Haydn's monothematicism, but the pairing of these two expositional plans quantifies and captures the impact that an exposition's degree of thematic saturation has on the entire movement.
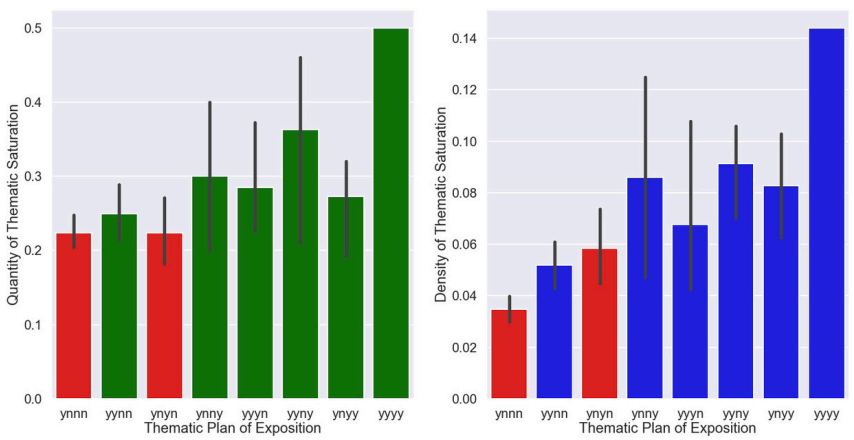

Figure 3: Highlighting how two plans with equal quantity of thematic saturation have unequal density of thematic saturation.

\section{Conclusion}

Thematic reuse is an aspect of Haydn's style that is important and undertheorized. In a sonata form, the thematic saturation of the expositional plan impacts how the rest of the movement unfolds. Monothematicism, a trademark of Haydn's style, and thematic saturation connect in counterintuitive ways. The presence of a Pbased $\mathrm{S}$ theme has a dampening effect on the overall level of a movement's thematic saturation. Investigating the connection between thematic saturation and expositional plan also provides a window into understanding how Haydn balances thematic returns (density) with the amount of material recycled (quantity).

\section{Acknowledgements}

I would like to thank my research assistants Sabine Gross and Adam Willson for their early contributions to collecting and checking data in the symphonies and for contributing to the analytical style sheet. Funding for their assistance was graciously granted by Oberlin College.

\section{End Notes}

[1] Continuous expositions are included in the corpus. Their thematic plan will always have an " $n$ " in the S column. Sonata rondos are not included in the corpus.

\section{References}

Burstein, P. (2016). Expositional Journeys and Resting Points. Composition as a Problem, 7, 5-16.

Caplin, W. E. (2001). Classical Form: A Theory of Formal Functions for the Instrumental Music of Haydn, Mozart, 
and Beethoven. Oxford University Press.

Duncan, S. P. (2011). Blurring the Boundaries: Toward a Multivalent Reading of Three First-Movement Sonata Forms in Haydn's Op. 50 String Quartets. Musical Explorations, 12, 5-40.

Fillion, M. (2012). Form, rhetoric, and the reception of Haydn's rondo finales. In Engaging Haydn: Culture, Context, and Criticism (pp. 187-210). Cambridge University Press. https://doi.org/10.1017/CBO9781139057714.011

Haimo, E. (1995). Haydn's Symphonic Forms: Essays in Compositional Logic. Oxford University Press.

Hepokoski, J., \& Darcy, W. (2006). Elements of Sonata Theory: Norms, Types, and Deformations in the LateEighteenth-Century Sonata. Oxford University Press. https://doi.org/10.1093/acprof:oso/9780195146400.001. $\underline{0001}$

Korstvedt, B. (2013). On Not Inviting Difficulties in Haydn's Symphonies. Haydn: Online Journal of the Haydn Society of North America, 3(2), 35 pages.

Ludwig, A. (2012). Hepokoski and Darcy's Haydn. HAYDN: Online Journal of the Haydn Society of North America, 2(2), 31 pages.

Miyake, J. (2011). Middles and Muddles: Haydn's Compositional Style and Sonata Forms. In S. E. Murray (Ed.), Haydn and His Contemporaries.

Neuwirth, M. (2011). Joseph Haydn's “witty" play on Hepokoski and Darcy's Elements of Sonata Theory. Zeitschrift Der Gesellschaft Für Musiktheorie [Journal of the German-Speaking Society of Music Theory], 8(1), 199-220. https://doi.org/10.31751/586

Neuwirth, M. (2013). Recomposed Recapitulations in the Sonata-Form Movements of Joseph Haydn and His Contemporaries [Dissertation].

Riley, M. (2015). The Sonata Principle Reformulated for Haydn Post-1770 and a Typology of his Recapitulatory Strategies. Journal of the Royal Musical Association, 140(1), 1-39. https://doi.org/10.1080/02690403.2015.1008862

Rosen, C. (1998). The Classical Style: Haydn, Mozart, Beethoven (Expanded edition). W. W. Norton \& Company.

Tilmouth, M. (2001). Monothematic. Grove Music Online. https://doi.org/10.1093/gmo/9781561592630.article.189 $\underline{83}$ 


\title{
Musical instrument, personality and interpretation:
} Music cognition at a college-conservatory

\author{
Rachel O' Connor ${ }^{\dagger 1}$
}

Puo (Roger) $\mathrm{Wu} \mathrm{Fu}^{\dagger 2}$

\author{
${ }^{1}$ Peabody Institute, Johns Hopkins University, Baltimore, MD, USA \\ ${ }^{2}$ Peabody Institute, Johns Hopkins University, Baltimore, MD, USA \\ tunded in part by award from Peabody Conservatory of the Johns Hopkins University \\ ${ }^{\dagger}$ Corresponding author: Susan Weiss, PhD sweiss@jhu.edu \\ Published 16 December 2021; https://doi.org/10.18061/FDMC.2021.0011
}

Author video presentation and/or other conference material: https://doi.org/10.17605/OSF.IO/DNUYR

\begin{abstract}
Orchestral musicians have a tendency to stereotype one another based on their instruments. While research shows that musicians frequently hold these views of other players (Lipton, 1987), there is less research that links personality traits to instrument played. In large ensembles instruments often play 'roles:' "basses determine rhythmic pulse," or "oboes' solos necessitate high artistic interpretation." Much of this is determined by training, reception history and instrumental sound. Our research sought to explore the feasibility of examining both personality traits and interpretation among a small sample of musicians focusing on a comparison of instrumental groups (strings, brass, woodwinds) as the independent variable. Our pilot study explored two primary questions: first, do musicians who play strings, woodwinds or brass exhibit different personality traits? Second, do musicians who play these instruments interpret music differently? Our study looked at differences in the ways instrumentalists interpreted three musical examples without markings other than time and key signatures. The $40+$ students also took the "Big-Five" personality test. Preliminary data revealed that the Big-Five scores aligned with stereotypes (e.g., brass scoring lowest on neuroticism and woodwinds low on extraversion but high on neuroticism). Groups also displayed consistent differences in their interpretive approach to the musical examples.
\end{abstract}

KEYWORDS: Orchestra, instrumental groups, personality differences, stereotypes, music, training, musical interpretation

\section{Introduction}

Stereotypes surrounding the personality traits of instrumentalists are commonly held among classical musicians. For example, oboe players are sometimes thought of as being neurotic individuals, violin players as being "high-maintenance", and trumpet players for having inflated egos. A number of studies conducted in the 1970s-1980s examined stereotypes and personalities of musicians. Davies (1976) determined that orchestral musicians held strong stereotypic convictions about the personality traits of their colleagues, grouped by strings, brass and woodwinds. Lipton (1987) found similar results with a much larger sample size (227 musicians vs Davies' 20). While both of these studies established that instrumentalists had these beliefs of each other, there is less evidence of the actual existence of these personality types. Individual studies have found some evidence to corroborate the commonly-held stereotypes of instrumentalists, although there has been little success with replicating their findings. Kemp's (1981) study of music students found that woodwind players showed greater shyness, self-sufficiency and radicalism than other instrumentals. Bell and Cresswell (1984) had similar findings that alluded to introversion in wind players. Kemp (1981) also described brass players as possessing "the most clearly defined pattern of traits", primarily insensitivity and aggression. Kaplan (1961) talked about self-confidence being integral to high achievement on brass instruments, while sensitivity was counter-productive.

More recently, Langendörfer (2008) studied differences in personality traits amongst 122 professional orchestral musicians in Germany, using the German version of the NEO-Five-Factors-Inventory. This commonly used and well-validated measure assesses five broad dimensions (Openness to experience; Conscientiousness; Extraversion; Agreeableness; Neuroticism) of personality. The Big Five uses factor analysis methods on personality data to ascribe the basic building blocks of personality; it has been considered the apex of personality research for decades. Unlike the results of previous studies on stereotyped views of musicians' personality traits, Langendörfer only found one significant personality difference among instrumentalists; strings had significantly higher scores on conscientiousness compared to woodwind and brass players, with woodwind players scoring lowest. However, stereotypes can go beyond personality traits and into the realm of musicianship - as much can be said about the way that one plays music being inextricably shaped by 
the instrument that one plays. Perhaps one of the most commonly held assumptions about musicians (both by musicians and others) is that one's interpretations, their "musicianship" is a measure of who they are as a person.

While there are countless pedagogical instrumentspecific books that address musicality and interpretation, to our knowledge there are no systematic studies that investigate interpretative tendencies across instruments trained in the style of Western Art Music. Existing research on focuses on identifying the psychological and cognitive processes underlying musical interpretation. Silverman (2008) created a pedagogic model of the creative processes that shape musical interpretation, concluding that it is created through the interaction of a performer's self-efficacy with various high-level musical processes. Losseff (2011) identifies the psychoanalytic concept of "projective identification" as central to musical interpretation, particularly in the process of a performer inserting their sense of self into musical performance. In their pursuit of creating an "epistemology of performance" Shaffer (1995) identifies the invention of "musical characters" through structurally significant metric and dynamic choices as central to interpretation. Lampl (1996) describes the process as a simultaneous interaction of musical context and directives and the tastes and temperaments of a performer.

Thus, this pilot study explored two primary questions of orchestrally trained musicians: Do musicians who play strings, woodwinds or brass exhibit different personality traits? Do musicians who play strings, woodwinds or brass interpret music differently? A common thought about musicians is that "musicality 1 is what is inside of you". Our research sought to explore this question through measures of personality and interpretation.

\section{Method}

\section{Sample}

A total of 42 participants: strings $=18$ (7 violins, 6 celli, 2 violas, 3 bass); woodwinds $=9$ ( 3 flutes, 2 clarinets, 1 bassoon, 3 alto saxophones) and brass $=15$ (6 trumpets, 3 horns, , 3 tuba, 3 trombones). Eligibility was limited to those holding at least a bachelor's degree in performance from an American institution or Conservatory. Participants were recruited online, with a majority drawn from the student body of the Peabody Conservatory. Participants completed the entirety of the three-part survey online which took about 20 minutes. Upon completion, participants received a $\$ 10$ award. This project grew out of a final project from a Music
Cognition class at the Peabody Conservatory of the Johns Hopkins University, with the first phase taking place in Fall 2018, and the second between July 2020January 2021 . The data in this paper is exclusively from the 2020-2021 edition of the project.

\section{Outcome 1: Big-Five Personality Test}

The short version of the Big Five personality test, IPIPNEO-PI-R consists of 120 items measuring the five major domains of personality: openness to experience, conscientiousness, extraversion, agreeableness, and neuroticism (Johnson, 2014). A unique barcode was generated for each participant that allowed us to view and compare their results. Participants completed the IPIP-NEO-PI-R through www.bigfive-test.com.

\section{Outcome 2: Interpretation}

Participants recorded themselves playing three given musical examples (shown in Figure 1) that had no expressive markings to indicate tempo, dynamics, articulation, phrasing, or style. They were instructed to "interpret" the music, and to add tempi, dynamics, articulation and phrasing as they saw fit. The examples provided were the same for each instrument, with appropriate transposition for the key center of specific instruments to avoid unnecessary and additional technical difficulty.

The recordings were evaluated by researchers on the factors of phrasing, dynamics, tempo/rubato/rhythm, and articulation. The tempos of each example were also recorded in beats per minute (BPM), and an average tempo was calculated for each participant. Each participant was given an "interpretation score" from 110 to indicate the amount and variety of deviations from the written music that they employed. A low interpretation score would indicate a performance with little use of and variety of the expressive devices listed above, such as a performance of all three excerpts at the same tempo and dynamic, with no added articulations. A high score would indicate frequent use of several expressive devices with variety within each category, such as a performance that uses contrasting tempi, dynamics and phrasing throughout the three examples. Interpretation was rated by the first two authors of this paper. Inter-rater reliability was assessed by the first two authors independently rating the first five musicians' tapes and meeting to assess concordance of ratings, which established consistency based off of similar measures. Authors also met for four hour-long sessions to discuss interpretation results and the grading scale in greater detail to ensure consistency. 
Research Study - Trumpet, French horn, Clarinet As written (no transposition)

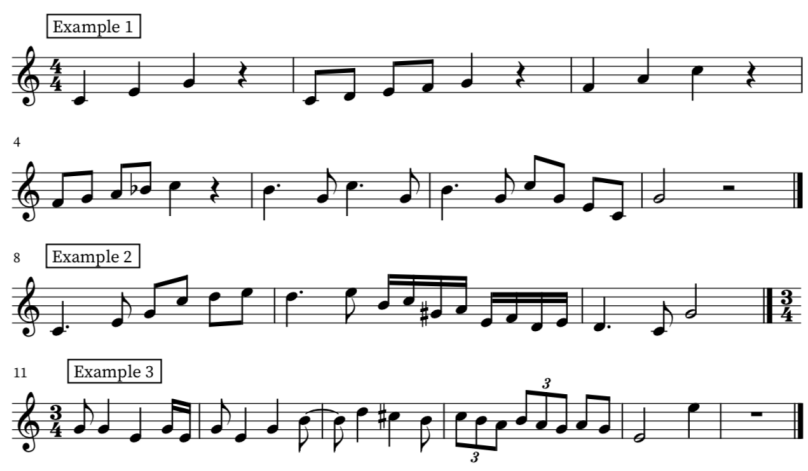

Figure 1: Musical examples given to participants

\section{Results}

The results of the personality test and interpretation showed some preliminary success in creating measurable outcomes when analyzed by instrument group. Some preliminary possibilities of patterns between instrument groups and different interpretive choices also were observed.

\section{Personality traits by instrument family}

Table 1 presents our preliminary pilot data. The data does not show large ranges of scores for the big five personality traits. Since the sample size of 42 is too small to conduct statistical analyses, statistical differences between instrument families for the five personality traits cannot be determined. Numerically, for neuroticism, extraversion and agreeableness, some diversion of scores is observed. For example, for neuroticism brass scores were numerically lower than strings and winds. While for extraversion strings showed a higher numerical score than brass and winds, and for agreeableness, brass scores were higher than strings and winds.

Table 1: presents preliminary pilot data on Big-Five scores organized by instrument family

\begin{tabular}{|c|c|c|c|c|c|}
\hline $\begin{array}{l}\text { Instrument } \\
\text { Family }\end{array}$ & Neuroticism: & Extraversion: & $\begin{array}{l}\text { Openess to } \\
\text { experience: }\end{array}$ & Agreeableness: & Conscientousness: \\
\hline Strings & \begin{tabular}{|r|}
75.3 \\
\end{tabular} & \begin{tabular}{|r|}
84.8 \\
\end{tabular} & 88.9 & 91.7 & $\begin{array}{r}89.1 \\
\end{array}$ \\
\hline Winds & 84.2 & 74 & 90 & 84.2 & 87.6 \\
\hline Brass & 68.7 & 78.5 & 91.9 & 93.8 & 90.3 \\
\hline Group Average: & 73.6 & 80.5 & 90.3 & 90.1 & 88.9 \\
\hline
\end{tabular}

\section{Interpretation and instrument family}

The average interpretation score for the group was 6.1 on a 10-point scale. When grouped by families, string instruments had the highest average interpretation scores of 6.87 , followed by woodwinds at 6.5 , and the brass instruments at 5.205. Within the woodwind family, there was the biggest range of interpretation scores, with the clarinets at 3.5 and the alto saxophones at 9. Brass instruments generally all played at mediumloud dynamic or louder, and all played a clear, forward tenuto start to notes on the border of heavy starts of notes. String instruments showed the most variety as a family in each category.

\section{Table 2: String Instruments interpretation data}

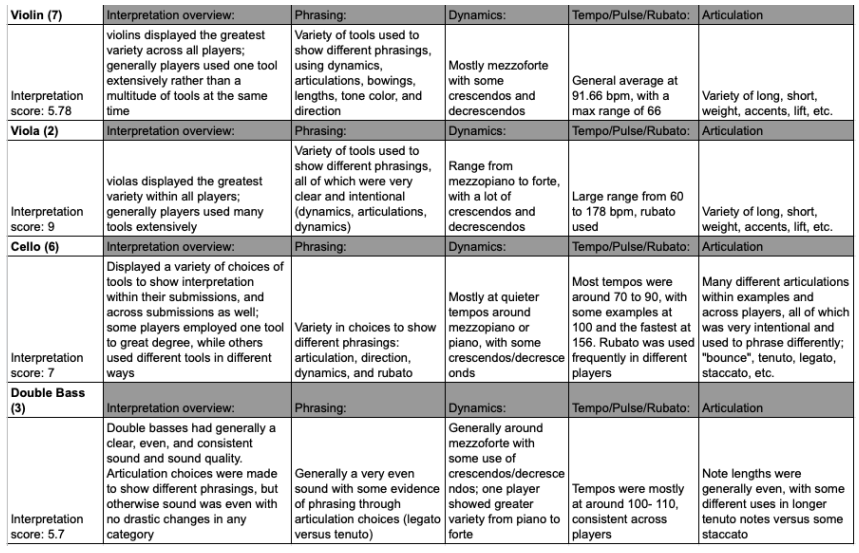

Table 2 shows brief summarizations of individual instruments' use of each expressive device, listed together with the rest of the instruments in the family they belong to. Interpretation scores are given as well as general overviews. All participating instruments were grouped in this way according to their families and compared to one another.

\section{Discussion}

Analyzing our respondents' scores by instrument family produced some interesting data, some of which aligns with existing research and others which contrast it. As our interpretation task is previously unstudied, there is no research to contrast it with, but findings can be related to practical and pedagogical factors.

Our research revealed preliminary evidence that instrumental groups of winds, brass and strings might share personality and interpretative characteristics. With personality, scores from the big-five test indicate some numerical diversion of scores that might indicate a basis for existing stereotypes of players. Other studies of musicians' personalities have found wind players to have unique personalities (Kemp, 1981; Bell and Cresswell, 1984). Our study also found that wind players deviated from our test averages on several 
accounts, with above-average neuroticism (84.2), below average extraversion (74) and below-average agreeableness (84.2). This aligns with the stereotype of woodwind players as being withdrawn, serious and anxious. Our brass players also exhibited some personality traits consistent with the stereotype of brass players being more "laid-back" and "social", having below-average neuroticism and high agreeableness.

We also found trends in interpretation by instrument family: strings were above our group average on Interpretation score while brass were below the average. The higher interpretation scores displayed by string players in general may correspond to both the high amount of melodic content in orchestra repertoire, as well as the relative ease of producing sound through a bow on a string, as compared to air-based instruments. This may make it easier for string players to develop the necessary muscles to produce different kinds of sounds, allowing for earlier access to these skills to try to employ them in different interpretive ways. Additionally, further research into the variety of solo repertoire may show that string players could be more exposed to soloistic music, embedding it into standard string pedagogy. The relative ease of sound production compared to the higher amount of tension in brass playing (and to some degree, woodwind playing) may give string players more time to play and practice on a physical level as well.

The primary limitation of our study is the participant pool size, which is too small to conduct statistical analysis or to draw concrete conclusions on any outcome. Many instruments had 2 or 3 participants, with our biggest sample of participants being the violins at 7 . Musician age, training, and experience is also another factor to consider, in that our participant pool stretched from first year undergraduates to doctoral students in their last semester. As musical interpretation across instrumental groups is previously unstudied, our outcome task and our "interpretation score" represent starting points for future research rather than a definitive model. Despite our best attempt to make the "interpretation score" objective, it still carries a great potential for subjectivity.

As our research lies at the intersection of several sub disciplines: personality, pedagogy and musical interpretation, it could have wide-ranging practical applicability. Our finding that instrumental groups of aspiring professional musicians may hold some observable differentiated personality traits may also reflect personality traits that promote success on those instruments. If we can develop a greater awareness of the environmental and psychosocial factors that create the conditions for certain personalities to thrive on a given instrument but not others, perhaps this awareness could be woven into pedagogy and performance practice so that there is greater opportunity for all to succeed. This knowledge could also be integrated into instrument selection/assignment process for beginners of all ages, at individual, institutional and collegial levels. Similarly, our finding that instrumental groups held some consistencies of musical interpretation which reflect their role in the orchestra could enhance and expand upon existing instrument-specific pedagogies and performance training at all levels.

Despite the improvements that need to be made to our study's design and execution, we think the model holds promise for future research. Beyond the findings discussed in this paper, the study also allows for the examination of personality and interpretation as well as aspects of musical literacy. While our main focus in this paper was the aspects of interpretation that differed across musical groups, there could be great interest in the elements of interpretation that were the same. With a larger sample, information could also be analyzed by instrument, which could hold great implications for comparison to and integration with the wealth of instrument-specific pedagogy that currently exists.

Implications of this research are important in understanding the effects our specific instrumental training have on our general approaches to thinking about and interpreting music. If patterns persist given larger sample sizes, an individual's training may affect the different aspects of music they may focus on or listen for, and potentially limit other aspects of music because of their instrumental "bias". This may imply that a more varied education on different instruments may provide for a more varied capacity to listen and interpret music in different ways.

\section{Conclusion}

Participant sample sizes are too small to draw incontrovertible conclusions, though the data indicate potential patterns in interpretation: string instruments generally employed the greatest variety in dynamics, articulations, phrasing, and tempo/rhythm, woodwind instruments saw the least consistency in interpretation within the family, and brass players generally played louder and focused on a clear and heavy articulation. Big-Five personality scores reflect existing stereotypes and some research by instrument group. Brass neuroticism scores were numerically lower than strings and winds. Woodwinds had lower agreeableness and 
extraversion scores. Strings showed a higher numerical score in extraversion than brass and winds, and for agreeableness, brass scores were higher than strings and winds. Therefore, there are measurable outcomes for personality traits and musical interpretation when grouped by instrument family. These findings could have wide-ranging practical applicability for musicians and music educators and warrant future study. With improvements, our self-created interpretation task and assessment could also hold promise as a method for future studies.

\section{Acknowledgements}

This research was generously supported by the Mollie $G$ and Joseph L. Forscher Award for Research in Music and Cognition. Our deepest thanks go to Dr. Susan Weiss for her unwavering support on this project. We would also like to thank Dr. Monica Lopez-Gonzalez for her support of the initial states of this work in our Music and Cognition Seminar: PY.610.637 (01) FA18.

\section{End Notes}

${ }^{1}$ Note on interchangeability of terms "musicality" and "interpretation". Musicality is a poorly defined word. We are considering "interpretation" a measure of "musicality". Many of our respondent's described musicality as "what is inside of you" and "a culmination of everything you have heard."

\section{References}

Bell CR, Cresswell A. Personality Differences among Musical Instrumentalists. Psychology of Music. 1984;12(2):83-93. https://doi.org/10.1177/0305735684122002

Davies, J. (1976). Orchestral dischord. New Society, 35, 4647.

Kaplan, L. (1961) The relationship between certain personality characteristics and achievement in instrumental music. Doctoral dissertation, New York University.

Kemp, A. (1981). Personality Differences between the Players of String, Woodwind, Brass and Keyboard Instruments, and Singers. Bulletin of the Council for Research in Music Education, (66/67), 33-38. Retrieved February 2, 2021, from http://www.jstor.org/stable/40317663

Lampl, H. (1996). Turning notes into music: An introduction to musical interpretation. The Scarecrow Press, Inc.
Langendörfer, Franziska. Personality differences among orchestra instrumental groups: Just a stereotype? Personality and Individual Differences, Volume 44, Issue 3, 2008, Pages 610-620, ISSN 0191-8869. https://doi.org/10.1016/j.paid.2007.09.027

Lipton, J. P. (1987). Stereotypes concerning musicians within symphony orchestras. Journal of Psychology, 121, 85-93. https://doi.org/10.1080/00223980.1987.9712647

Losseff, N. (2011). Projective identification, musical interpretation and the self. Music Performance Research. 2011; 4:49-59.

Shaffer, L. H. (1995). Musical Performance as Interpretation. Psychology of Music, 23(1), 17-38. https://doi.org/10.1177/0305735695231002

Silverman, M. (2008). A performer's creative processes: implications for teaching and learning musical interpretation. Music Education Research. 2008; 10(2):249-269. https://doi.org/10.1080/14613800802079114 


\title{
Phrase extension in Haydn's string quartet minuets: A preliminary corpus study
}

\author{
William O'Hara ${ }^{1 \dagger}$ \\ ${ }^{1}$ Gettysburg College, Gettysburg, PA, USA \\ ${ }^{\dagger}$ Corresponding author: williamevanohara@gmail.com \\ Published 16 December 2021; https://doi.org/10.18061/FDMC.2021.0012 \\ Author video presentation and/or other conference material: https://doi.org/10.17605/OSF.IO/XZ4E9
}

\begin{abstract}
This study examines a small but well-defined corpus: Franz Joseph Haydn's string quartet minuets and trios $(\mathrm{n}=76$ paired dances, or 152 individual minuets), composed between 1764 and 1803. Seeking to identify the metrical differences between a minuet intended for dancing and one intended for the salon or the concert stage, this study parses Haydn's 8and 10-measure minuet and trio sections (using the models of "tight-knit" theme types proposed by Caplin 1998), identifies patterns in Haydn's phrase extensions, and discusses challenges and opportunities for further corpus-informed studies of phrase rhythm and hypermeter.
\end{abstract}

KEYWORDS: Haydn, phrase rhythm, form, corpus analysis

\section{Introduction}

A triple-meter dance generally written in rounded binary form, the Minuet was a popular eighteenth-century social dance, and a common inner movement in large instrumental works. In symphonies and quartets, the second or third movement is usually a "minuet and trio": a higher-level ternary form that pairs two minuets, the second of which is often simpler, and written in a complementary key [1]. Gretchen Wheelock (1993) has emphasized the importance of the minuet form for Haydn and his contemporaries: The genre was a pedagogical prototype for larger forms, and its formulaic layout made it an ideal vehicle for unexpected and often humorous compositional procedures.

As a dance, the minuet demanded consistent meter and phrase structure. The minuet step (pas de minuet) required six beats (or two measures) to execute, and these steps were chained together into groups of four to eight units-requiring musical phrases of eight to sixteen measures. Eighteenth-century composers and theorists accordingly emphasized the need for symmetry and balance in the minuet. However, these theorists were well aware of the different options available, based on whether the minuet in question was meant to be danced, or only listened to. As H.C. Koch wrote, "If [the minuet] is arranged for dancing, then its melodic sections must have a rhythmical relation of an even number of measures; and it must consist of two sections or reprises, each containing no more than eight measures. If, however, it is not designed for a dance, then not only can its reprises be of quite arbitrary length, but also its melodic sections can be of an uneven number of measures" (Koch 1983, 79). It is this formularity of phrase structure and harmonic scheme - two phrases of eight measures each, with varied figuration over substantially similar harmonies - that made the minuet a popular subject for combinatorial methods of composition such as musical dice games (see Zbikowski 2002).

While several excellent studies of the minuet exist, and individual minuets have often been analyzed, there is still more to learn. This study contributes to the understanding of phrase structure in Classical dance forms by assembling and analyzing a small corpus based on a single repertoire: the minuets and trios found in Franz Joseph Haydn's string quartets [2]. In my analysis, I am concerned primarily with movements that break the minuet form's prevailing four-bar hypermeter, in an attempt to understand precisely how "arbitrary"or not-Haydn's concert minuets are in their meter, phrase structure, and formal design.

\section{Background}

Among Haydn's sixty-eight string quartets, there are 76 minuet \& trio movements, for a total of 152 discrete dances. Generally, each quartet features a minuet and trio, but Haydn's earliest quartets feature two minuet/trio pairs each, as part of a five-movement layout that places minuets $\&$ trios as the second and the fourth movements.

Every minuet and all but two of the trios are divided into two sections by repeat signs, leaving 300 distinct sections for study [3]. These range from 8 measures (the most common length, and the one called for by Koch and his contemporaries) to an upper limit of 58 measures. Table 1 lists the works studied.

\section{Phrase Extension and Expansion}

Music theorists have discussed phrase expansion and contraction for more than 200 years, and have invented various schemas with which to classify these deviations. 
Table 1: Quartets included in this study [4]

$\begin{array}{lll}\text { Work } & \begin{array}{l}\text { \# of quartets } \\ \text { Yp. } 1\end{array} & \begin{array}{l}\text { Year } \\ 5^{* \dagger}\end{array} \\ \text { Op. } 2 & 4^{* \dagger} & 1764 \\ \text { Op. } 9 & 6 & 1765 \\ \text { Op. } 17 & 6 & 1769 \\ \text { Op. } 20 & 6 & 1771 \\ \text { Op. } 33 & 6 & 1772 \\ \text { Op. } 42 & 1 & 1781 \\ \text { Op. } 50 & 6 & 1784 \\ \text { Op. } 54 & 3 & 1787 \\ \text { Op. } 55 & 3 & 1788 \\ \text { Op. } 64 & 6 & 1788 \\ \text { Op. } 71 & 3 & 1790 \\ \text { Op. } 74 & 3 & 1793 \\ \text { Op. } 76 & 6 & 1793 \\ \text { Op. } 77 & 2 & 1797 \\ \text { Op. } 103 & 1 & 1799 \\ & & 1803\end{array}$

* Each of these quartets features two minuets/trios + Some Opp. 1 and 2 quartets have been proven inauthentic

Koch (1983, pp. 41-54), for instance, identified phrase extensions that use internal repetition, appendices that extend the phrase beyond a logical ending (such as a cadence), and parentheses, which interpolate "unessential melodic ideas" (p. 53) within the phrase.

William Caplin's (1998) theory of "form functionality" analyzes the construction of large forms from small syntactic units. Caplin's model holds that "tight-knit" themes such as the period and the sentence are constructed in predictable and consistently sized units - most often two measures. The two-measure building blocks that normatively make up such theme types are analyzed according to the formal functions that they express: standard musical processes such as presentation or continuation.

While tight-knit themes are defined according to eight-measure exemplars, exceptions are common, and their effects on a phrase's grouping structure and formfunctional content can be analyzed according to their harmonic and melodic content. Phrase extension, Caplin writes, "results from 'adding on' material to stretch out a particular formal function in time" (Caplin 1998, p. 20). Expansion, on the other hand, results from "the internal lengthening of component members of the [formal] function over their normative size in tight-knit themes" [5]. In other words, extension describes an extra subphrase unit comprised of repeated or interpolated material, while expansion describes a lengthening departure from a phrase's established harmonic or melodic rhythm, akin to the durational augmentations associated with contrapuntal technique.

Though separated by two centuries, Koch and Caplin (the latter synthesizing a great deal of intervening music and thought) approach issues of phrase rhythm in a remarkably similar way. Both rely on informal accounts of perception, defining phrase modification and repetition through the response of an idealized listener. Koch is interested primarily in four-measure phrases, and his analyses of slightly longer units - five and six measure phrases - hinge upon whether the listener is thought to hear an obvious repetition of the phrase's material, or if the longer phrase is perceived as an integrated whole. In the former case, Koch considers the phrase to be essentially a four-measure unit, for the purposes of composition and analysis; only in the latter case, when no repetition or segmentation can be detected, would the phrase actually be considered to be five or six measures long (see Koch 1983, pp. 41-44).

Similarly, Caplin's clearest explications of formal functionality appeal to a listener's perception of pseudogrammatical functions of initiation, continuation, and conclusion. And his distinction between "real" and "notated" meter hinges upon a subjective interpretation of a piece's metric or grouping structure- "the only valid measure for an analysis of form based on our musical experience" (Caplin 1998, p. 35).

\section{Method}

This study surveys formal designs and phrase structures in Haydn's String Quartet minuets, with the initial goal of establishing some central facts for a larger study of compositional exemplars and the use of musical "templates" in 18th- and 19th-century compositional theory and pedagogy [6]. For each minuet, I have tabulated the number of measures in each section. My analysis then focused on sections that deviate from the minuet's usual template of four-bar hypermeter and balanced construction. Informed by Caplin's theory of formal functions, I determine the generic theme type of each of Haydn's eight- and ten-measure phrases. After surveying the theme types present in normative eightmeasure phrases, I explore the ways that these phrases are extended into ten-measure units, with an eye towards laying the foundation for a more extensive study of hypermeter and phrase rhythm in Haydn's minuets. Such further study will involve larger syntactical units, and phrase contractions from larger phrases. 


\section{Results}

Short, Regular "A Sections" are the Norm

Table 2 lists the number of sections of each type (Minuet A section, Minuet B, Trio A and B) with each given length. As noted above, eighteenth-century music theorists like Koch described eight measures as the ideal length for a minuet section. Even when removed from the constraints of writing for the dance floor, this guideline remains very strong in Haydn's quartet minuets. The effect is especially clear in the A sections of either minuets or trios. $46 \%$ of A sections are 8 measures long. The next most common lengths are 12 measures $(16 \%)$ and 10 measures (14\%). Taken together, these lengths account for $76 \%$ of minuet and trio A sections; the remainder are between 16 and 20 measures, with only two outliers (24 and 36, occurring once each). B sections tend to be notably longer, most likely because they are compositionally more "loosely knit" and because they often contain a reprise of the A section ("rounded binary form"). While $77.3 \%$ of all A sections are 12 bars or less, only $17.3 \%$ of B sections are that short.

\section{Sections with an Odd Number of Measures}

As we might expect from a form that originates in dance, the vast majority of the minuet and trio sections studied feature an even number of measures. Only 10 out of 300 sections $(0.033 \%)$ include an odd number of measures. These are equally distributed throughout Haydn's compositional career, and there is no generic distinction to be found (six occur in minuets, while four occur in trios). All but two are found in B sections, however, providing evidence that $\mathrm{B}$ sections are less structured than A sections. These odd-grouping sections can be as short as 9 measures, or as long as 45, indicating that the length or brevity does not predict an odd number of measures.

\section{Characteristics of Eight-Measure Phrase Structures}

As noted above, eight-measure phrase templates are both common in music theory-they are the form taken by the period and the sentence, for example-and ideally suited to the minuet style, for which the twomeasure minuet step serves as an ideal unit of compositional invention and analytical measurement.

My study of Haydn's phrase structures employs a taxonomy of theme types developed by Caplin (1998), which uses two-measure units as the basic level of analysis. Caplin's model is centered on the period and sentence, two conventional theme types that are strongly
Table 2: Number of Measures, by Each Formal Section

\begin{tabular}{|l|l|l|l|l|l|}
\hline $\begin{array}{c}\text { \# of } \\
\text { measures }\end{array}$ & $\begin{array}{c}\text { Minuet } \\
\text { A }\end{array}$ & $\begin{array}{c}\text { Minuet } \\
\text { B }\end{array}$ & $\begin{array}{c}\text { Trio } \\
\text { A }\end{array}$ & $\begin{array}{c}\text { Trio } \\
\text { B }\end{array}$ & Total \\
\hline $\mathbf{8}$ & 33 & & 35 & 8 & 76 \\
\hline $\mathbf{9}$ & 1 & & 1 & & 2 \\
\hline $\mathbf{1 0}$ & 11 & 2 & 10 & 2 & 25 \\
\hline $\mathbf{1 1}$ & 1 & & & & 1 \\
\hline $\mathbf{1 2}$ & 12 & 4 & 12 & 9 & 37 \\
\hline $\mathbf{1 3}$ & & & & 1 & 1 \\
\hline $\mathbf{1 4}$ & 5 & 1 & 9 & 6 & 21 \\
\hline $\mathbf{1 6}$ & 5 & 4 & 4 & 9 & 22 \\
\hline $\mathbf{1 8}$ & 2 & 7 & 1 & 6 & 16 \\
\hline $\mathbf{2 0}$ & 3 & 8 & 1 & 10 & 22 \\
\hline $\mathbf{2 1}$ & & & & 1 & 1 \\
\hline $\mathbf{2 2}$ & & 4 & & 6 & 10 \\
\hline $\mathbf{2 3}$ & & 2 & & & 2 \\
\hline $\mathbf{2 4}$ & 3 & 10 & & 2 & 15 \\
\hline $\mathbf{2 5}$ & & & & 2 & 2 \\
\hline $\mathbf{2 6}$ & & 4 & & 3 & 7 \\
\hline $\mathbf{2 8}$ & & 2 & & 3 & 5 \\
\hline $\mathbf{3 0}$ & & 5 & & 3 & 8 \\
\hline $\mathbf{3 2}$ & & 3 & & & 3 \\
\hline $\mathbf{3 3}$ & & 1 & & & 1 \\
\hline $\mathbf{3 4}$ & & 2 & & & 2 \\
\hline $\mathbf{3 5}$ & & 1 & & & 1 \\
\hline $\mathbf{3 6}$ & & 5 & 1 & 1 & 7 \\
\hline $\mathbf{3 8}$ & & 2 & & 1 & 3 \\
\hline $\mathbf{4 2}$ & & 3 & & 1 & 4 \\
\hline $\mathbf{4 4}$ & & 2 & & & 2 \\
\hline $\mathbf{4 5}$ & & 1 & & & 1 \\
\hline $\mathbf{4 6}$ & & 1 & & & 1 \\
\hline $\mathbf{5 4}$ & & 1 & & & 1 \\
\hline $\mathbf{5 8}$ & & 1 & & & 1 \\
\hline Total & 76 & 76 & 74 & 74 & 300 \\
\hline $\mathbf{A v e r a g e}$ & 11.025 & 26.0125 & 10.7 & 17.9 & \\
\hline & & & & & \\
\hline & & & & & \\
\hline & & & & & \\
\hline
\end{tabular}

identified with the Viennese Classical repertoire. Broadly, the period is characterized by an antecedent/consequent structure with a medial cadence in the fourth measure and a repetition of the phrase's basic idea in mm. 5-6. The sentence is characterized by its use of two-measure subphrases, consisting of a "presentation" phrase that consists of a basic idea (mm. 1-2) and its immediate, varied repetition (mm. 3-4); and a continuation phrase that often presents shorter, "fragmented" versions of the basic idea $(\mathrm{mm} .5-6)$ on the way to a weak cadence in $\mathrm{m}$. 8. [7]

Along with these two basic theme types, Caplin presents four hybrids that mix some characteristics of each, roughly summarized as follows: 
Hybrid 1: antecedent + continuation (begins like a period, ends like a sentence)

Hybrid 2: antecedent + cadential (similar to Hybrid 1 , but with a second half that presents a simplified cadential figure rather than motivic variation)

Hybrid 3: compound basic idea + continuation (a period's antecedent without the medial cadence, followed by sentential continuation (such as a fragmentation)

Hybrid 4: compound basic idea + consequent (essentially a period without the medial cadence).

Caplin (1998, p. 63) arranges these theme types by similarity, beginning with the sentence, through the four hybrids (arranged from most to least sentential), to the period; I have presented this spectrum in my tables.

The first stage in my analysis was to group each phrase by its theme type, using Caplin's harmonic, motivic, and metric criteria. Table 3 tabulates the theme types in Haydn's 66 eight-measure phrases, taken from the A sections of minuets and trios in the corpus (B sections have generally been omitted from this analysis, because their harmonic instability can lead to unexpected formal procedures). As Table 4 shows, conventional sentences and periods are well-represented within Haydn's opening phrases, while all four of Caplin's hybrids appear.

Table 3: Tight-knit Theme Types in Haydn's Minuet and Trio A Sections

\begin{tabular}{|l|l|l|}
\hline Theme type & \# of works & Percentage \\
\hline Sentence & 22 & $33.3 \%$ \\
\hline Hybrid 3 & 6 & $9.1 \%$ \\
\hline Hybrid 1 & 10 & $15.2 \%$ \\
\hline Hybrid 2 & 4 & $6 \%$ \\
\hline Hybrid 4 & 7 & $10.6 \%$ \\
\hline Period & 17 & $25.7 \%$ \\
\hline
\end{tabular}

\section{A Taxonomy of Ten-Measure Phrases}

Caplin's taxonomy of tight-knit phrases makes it possible to pinpoint how their extensions work. Haydn's ten-measure phrases are almost always extended by the inclusion of additional material-Koch's "interpolations"-while a few phrases use internal repetitions instead. As shown in Table 4, most of these interpolations and repetitions act at the level of the twomeasure subphrase, expanding the sentential/periodic grouping structure that prevails throughout the minuets.

As Table 5 shows, Haydn's phrase extensions almost always happen in the second half of the phrase. Only
Table 4: Types of Extension by Theme Type in TenMeasure Phrases

\begin{tabular}{|c|c|c|c|c|}
\hline \multirow{2}{*}{$\begin{array}{c}\text { Theme } \\
\text { type }\end{array}$} & Total & \multicolumn{3}{|c|}{ Type of extension } \\
\cline { 3 - 5 } & & $\begin{array}{c}\text { Extra } \\
\text { subphrase }\end{array}$ & $\begin{array}{c}\text { Multiple } \\
\text { extra } \\
\text { measures }\end{array}$ & Repetition \\
\hline Sentence & 12 & 9 & 2 & 1 \\
\hline Hybrid 3 & 2 & 2 & - & - \\
\hline Hybrid 1 & 1 & 1 & - & - \\
\hline Hybrid 2 & 3 & 3 & - & - \\
\hline Hybrid 4 & - & - & - & - \\
\hline Period & 9 & 6 & 3 & - \\
\hline Total & 27 & 21 & $5(18.5 \%)$ & $1(3.7 \%)$ \\
\hline
\end{tabular}

Table 5: Location of Phrase Extensions in Ten-Measure Phrases

\begin{tabular}{|c|c|c|c|c|c|c|c|}
\hline $\begin{array}{c}\text { Theme } \\
\text { type }\end{array}$ & $1 / 2$ & $3 / 4$ & $5 / 6$ & $7 / 8$ & $\begin{array}{c}9 / \\
10\end{array}$ & Other & Separate \\
\hline Sent. & - & 1 & 5 & 4 & - & - & 2 \\
\hline Hyb. 3 & - & - & 1 & - & 1 & - & - \\
\hline Hyb. 1 & - & - & - & 1 & - & - & - \\
\hline Hyb. 2 & - & - & - & 1 & - & 2 & - \\
\hline Per. & 1 & - & 2 & 3 & - & - & 3 \\
\hline Total & 1 & 1 & 8 & 9 & 1 & 2 & 5 \\
\hline
\end{tabular}

three extensions occur in antecedent or presentation phrases. The minuet from Op. $71 / 2$ begins with arpeggios in the cello and first violin, before continuing as a conventional period; and B section of the Op. 64/5 trio features an immediate repetition of the basic idea, before its more conventional complementary repetition. The density of mid-phrase interpolations is unsurprising; when determining formal classifications, the beginning of the second half of the phrase is of central importance. Fragmentation, for instance, is a crucial component of a sentence's continuation phrase, as is the return of the basic idea in a period's consequent phrase. Seventeen out of Haydn's 27 expansions (63\%) occur in either mm. 5-6, or mm. 7-8: either delaying the expected subphrase, or immediately following it.

Only one two-measure interpolation positions itself against this grouping structure: Op. 33/5 features a full measure of silence and a metrically unaccented cadence in mm. 8-9. A few examples include two separate onemeasure interpolations. When found in period themes (Op. 20/3, Op. 54/1, and Op. 64/4), interpolate new material into mm. 4 and 9 , delaying each cadence by a 
measure. Perhaps the most interesting case is the second minuet from Op. 2/2, a rare modification to the first half of a phrase in which the basic idea is extended by a measure, and then repeated. This produces the only example of Caplinian expansion, as opposed to extension: rather than adding an additional subphrase, the size of the basic idea itself is expanded.

\section{Discussion}

The analyses presented here are concerned with extremely small numbers of pieces-subcorpora, as it were-and thus serve as a pilot study for a more comprehensive analysis of phrase rhythm in Haydn's quartets. Future research will include longer phrases, and will deal with metric contraction alongside the need to more finely parse phrases and formal elements within the large formal sections studied in this paper. While identifying theme types and locating phrase extensions is a subjective analytical activity, the method shows promise for the ability to detect adaptations of phrase structure. An analysis of harmonic rhythm based on theme type may also combine fruitfully with recompositional analysis (see Rothstein, 1988; O'Hara, forthcoming). Results from this and future studies may contribute to computational studies of phrase rhythm, which will need to identify motivic patterns and cadences alongside harmonic and metric elements.

\section{Conclusion}

Phrase rhythm in Haydn's string quartets is varied: many examples stick to 8- and 16-measure models derived from social dance, while others incorporate odd numbers of measures and unbalanced phrase structures. His phrase expansions fall into few categories, and his frequently operate by interpolating additional material into tight-knit phrase models. Caplin's tight-knit themes are a promising method for future studies, and are able to help analysts identify and understand phrase extensions in works of larger scale.

\section{End Notes}

[1] On minuet and trio style, see Lowe (2002) and McKee $(2005,2011)$.

[2] Corpus studies on rhythm, meter, and style include Cortens (2014), Ito (2014), and Ohriner (2016).

[3] The undivided trios are found in Op. 76/6 and Op. 77/1. At 96 and 100 measures, respectively, these trios are by far Haydn's longest. They are excluded from the analyses of form and phrase rhythm.
[4] Supplemental materials for this paper can be found at http://www.williamohara.net/blog/haydnminuets.

[5] For instance, the minuet from Mozart's G Minor Symphony includes a 14-measure sentence constructed from a 3-measure basic idea and expanded continuation phrase (analyzed in Caplin, 1998, p. 40).

[6] See O'Hara (forthcoming).

[7] See Caplin (1998, pp. 35-58) for a fuller treatment of periods and sentences.

\section{References}

Caplin, W. (1998). Classical Form: A Theory of Formal Functions for the Instrumental Music of Haydn, Mozart, and Beethoven. Oxford University Press.

Cortens, E. (2014). The Expositions of Haydn's String Quartets: A Corpus Study. HAYDN: Online Journal of the Haydn Society of North America 4(1), 1-42.

Fort, J. (2015). Incorporating Haydn's Minuets: Towards a Somatic Theory of Music [Doctoral dissertation, Harvard University]. Digital Access to Scholarship at Harvard.

Ito, J. (2014). Koch's Metrical Theory and Mozart's Music: A Corpus Study. Music Perception 31(3), 205-222. https://doi.org/10.1525/mp.2014.31.3.205

Koch, H. (1983). Introductory Essay on Composition. (Nancy Kovaleff Baker, trans.). Yale University Press. (Original work published 1782-93).

Lowe, M. (2002). Falling From Grace: Irony and Expressive Enrichment in Haydn's Symphonic Minuets. Journal of Musicology 19(1), 171-221. https://doi.org/10.1525/jm.2002.19.1.171

Mirka, D. (2009). Metric Manipulations in Haydn and Mozart: Chamber Music for Strings, 1787-1791. Oxford University Press.

McKee, E. (2005). Mozart in the Ballroom: Minuet-Trio Contrast and the Aristocracy in Self-Portrait. Music Analysis 24(3), 383434. https://doi.org/10.1111/j.1468-2249.2006.00226.x

McKee, E. (2011). Decorum of the Minuet, Delirium of the Waltz: A Study of Dance-Music Relations in 3/4 Time. Indiana University Press. https://doi.org/10.2307/j.ctt2005r6k

O'Hara, W. (forthcoming). Recomposing Music Theory. Unpublished manuscript.

Ohriner, M. (2016). Metric Ambiguity and Flow in Rap Music: A Corpus-Assisted Study of Outkast's "Mainstream" (1996). Empirical Musicology Review 11(2): 153-179. https://doi.org/10.18061/emr.v11i2.4896

Rothstein, William. (1988). Phrase Rhythm in Tonal Music. Schirmer Books.

Wheelock, G. (1993). Haydn's Ingenious Jesting with Art. Schirmer Books.

Zbikowski, L. (2002) Conceptualizing Music: Cognitive Structure, Theory, and Analysis. Oxford University Press. https://doi.org/10.1093/acprof:oso/9780195140231.001.0001 


\author{
Streaming complexity in the Renaissance Mass Ordinary cycle \\ Finn Upham ${ }^{1 \dagger}$ \\ Julie Cumming ${ }^{2}$ \\ ${ }^{1}$ RITMO, Department of Musicology, University of Oslo, Oslo, Norway \\ ${ }^{2}$ Schulich School of Music, McGill University, Montreal, QC, Canada \\ $\dagger$ Corresponding author: finn.upham@gmail.com \\ Published 16 December 2021; https://doi.org/10.18061/FDMC.2021.0013 \\ Author video presentation and/or other conference material: https://doi.org/10.17605/OSF.IO/8D45J
}

\begin{abstract}
Complexity of a piece should also be related to its intended use and context. The Mass Ordinary cycles of 15 th- and 16thc. Europe were works of exceptional formal consistency, and if complexity is relevant to the role of a Kyrie, Gloria, or Agnus Dei, this should be measurable from the scores of surviving examples. We evaluate an aspect of complexity generalizable to past church goers from the consequences of low-level auditory streaming principles. The streaming complexity estimate presumes that this music tends to be heard as an integrated stream within the rich scene of the church service unless the voices diverge through separation cues such as independent onsets and contrary motion. In a corpus of more than 200 mass cycles composed over 150 years, we find significant differences in average streaming complexity over time and between movements. The Agnus Dei tends to be more complex, the Credo, with a long text, is usually low, depending on the composer. Results suggest streaming complexity may indeed be tuned for the intended use of a work. By bringing musicological evidence into corpus analysis, we can consider this feature in relation to the role of music beyond present day patterns of consumption.
\end{abstract}

KEYWORDS: auditory streaming, Renaissance mass ordinary cycles, complexity, corpus studies

\section{Introduction}

Complex polyphony bloomed in Renaissance church music as the mass ordinary cycle emerged in the early $15^{\text {th }}$ century. Was variation in this complexity a formdefining feature that could have been followed by those attending services in the $15^{\text {th }}$ and $16^{\text {th }}$ centuries? Without sound recordings or detailed reports by contemporary listeners, we can only approximate what could have been perceived from symbolic representations of these scores and records of listening conditions. The auditory streaming complexity estimate is calculated as a continuous feature reporting a moment-by-moment tally of how many independent streams or objects might be heard by a casual listener, without explicitly trying to count. This estimate evaluates how individual notes contribute to the blending or separation of each part from the rest. While some cues for sound stream merging and segregation have very robust effects on the attentive listener (Bregman, 1994), attention also plays an important role in how they interpret the auditory scene (Cusack et al., 2004). Perception of sound sources comes from interactions between bottom-up sensory processing and top-down expectations tuned to the signals detected and to the orientation of the listener's auditory focus (Shinn-Cunningham et al., 2017). The exact perceptions of a $15^{\text {th }}$-century European church goer cannot be fully modelled today, but we expect that much of their low-level sensory processing to be similar to those of participants in $20^{\text {th }}$ - and $21^{\text {st }}$-century psychoacoustic experiments with moderate attentiveness.

The principles of auditory scene analysis have been applied to counterpoint and polyphony before. David Huron's work on voice leading identified many connections between the cues influencing sound stream integrity and the rules of species counterpoint (Huron, 2001). The relationship between streaming cues and the merging of parts in polyphonic works has also been looked at experimentally with perceptual judgments. Ben Duane asked participants to judge the audible merging of parts in string quartet excerpts (played in midi) and compared these perceptual accounts to quantifications of stream separation cues discerned from the scores (2013). Duane's results suggested asynchronous note onsets and harmonic dissonance were important factors decreasing the merging of two string parts into a blended stream. Emilios Cambouropoulos combined similar features to identify voices within polyphonic piano music, using onset synchrony, pitch comodulation, and stream continuity to calculate which notes would be integrated vertically and horizontally (2008). This project applies these principles to a corpus of Renaissance mass ordinary cycles to evaluate whether this complexity varies meaningfully with form.

The polyphonic mass ordinary cycle in the Renaissance is an ideal genre for a computational study of streaming complexity. The mass ordinary cycle is a 
polyphonic setting of five texts that were said or sung at every Catholic mass, and each of the mass ordinary texts came into the mass at a different time and served a different liturgical function. It is the largest and most complex musical genre in the Renaissance, and there are many surviving mass cycles. To a 15th-century churchgoer, this polyphonic music sounded elaborate, festive, and special. The alternation between the complex music of the mass ordinary cycle (where each movement begins with similar music) and the chant and spoken text of the rest of the mass could have provided an overall unity to the ceremony. The standardized sequence of the five movements, each with a different approach to text-setting and structure, might have allowed listeners to locate themselves in the ceremonial time of the mass, even if they could not follow what was being said. The melismatic Kyrie and Agnus Dei, both of which have three sections, serve as a kind of internal frame for the mass as a whole. The long syllabic Gloria and Credo movements articulate the liturgy of the word. The melismatic Sanctus and Agnus frame the mystery of transubstantiation. The music of the polyphonic mass ordinary would have provided an aural focus for parishioners, for being "present with the body and intent with the mind" when these services were not expected to be fully heard and understood by all attending.

This project brings these concepts of separation and merging cues to calculate streaming complexity on a large symbolic corpus of polyphonic vocal music and evaluates whether this feature of polyphonic writing changes substantively over time and in ways that might have been relevant to the composers and audiences of the day.

\section{Method}

\section{Dataset of symbolic music files}

The symbolic music files for this corpus analysis came from two public collections of polyphonic Renaissance music: The Josquin Research Project (JRP), an open access database of Renaissance scores in multiple symbolic formats (Rodin \& Sapp, 2019) and RenComp7, a repository of MIDI scores with additional polyphonic vocal works of the Renaissance (McKay et al., 2017). The RenComp7 dataset provided later works: 18 mass ordinary cycles by Tomás Luis de Victoria and the 101 by Giovanni Pierluigi da Palestrina. Another 84 mass ordinary cycles were downloaded from JRP in kern format, works by 13 identified composers and a few without clear attribution. In total, the corpus includes 216 masses by at least 15 different composers.
Using available musicological evidence, these works were additionally grouped by estimated time of composition to the quarter century.

Nearly all of these masses included the typical five movements of the mass ordinary: Kyrie, Gloria, Credo, Sanctus, and Agnus Dei. A few were accompanied by mass proper movements such as the Introit, and these individual movements were discarded prior to analysis. Mass cycles from later periods often contained six or seven or eight movements if the ordinary movements were broken into first and second parts (Agnus Dei), or the Benedictus separated from the first part of the Sanctus. For this analysis, these components were concatenated to match the standard five movements, all sharing the same text and liturgical role. A few mass cycles were incomplete because of missing movements or files that did not render properly; the remainder of these cycles were retained for analysis. Across all 216 masses, 1042 movements were studied, each containing 3 to 12 voice parts.

\section{Calculating Auditory Streaming Complexity}

Unison chant was a common part of church music at this time, in which coordination would favour hearing a group of singers as a single sound source. Polyphonic music emerged in contrast to this aural unity, with an emphasis on counterpoint resulting from the independence of voices in pitch, rhythm, and text. With a combination of Music21, SciPy, Numpy, and Pandas (Oliphant, 2007; Cuthbert, 2019; McKinney, 2010), we evaluated in every score three types of sound events that can contribute to the separation or merging of voices:

1. Voice Entries: when a part enters for the first time or after a rest.

2. Pitch Changes: when a part changes pitch and in which direction.

3. Note Onsets: the beginning of each articulated note in the score.

These are then reduced by their alignment with the same events in other (higher) parts, and scaled by importance. We treat voice entries as stronger separation cues than pitch changes, which are stronger than note onsets in the sustained singing style of this genre.

Auditory streaming is cumulative and a bit sticky: once sounds in the auditory field have been attributed to separate sources, we try to hold on to that organisation until contradictory cues overwhelm it (Bregman, 1994). Because the ear rapidly reassess soundscapes (ShinnCunningham et al., 2017), the effect of separation cues per part were integrated with a short linear decay, 
reducing at a rate of 0.2 per semi-minim or quarter note. These calculations translate to the separation effect of a solo voice entry (against other sound parts) dissipating within 1-1.5 seconds depending on performance tempo.

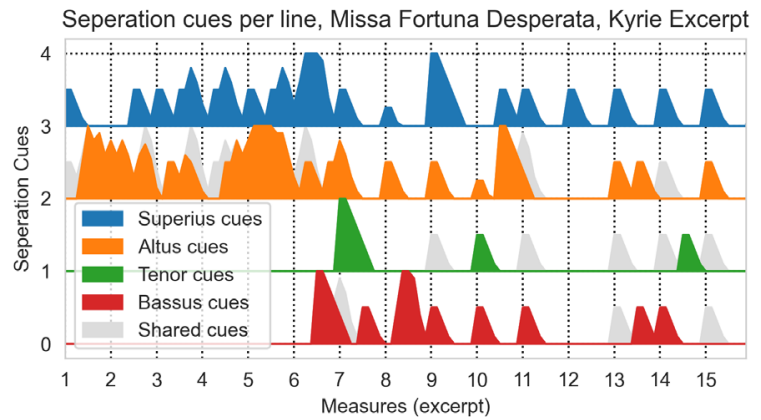

Figure 1: Integrated stream separation cues per part for a 16-m excerpt of a mass by Josquin de Prez. In grey are cues shared with higher voices, contributing to merging of parts, in colour are the integrated separation cues.

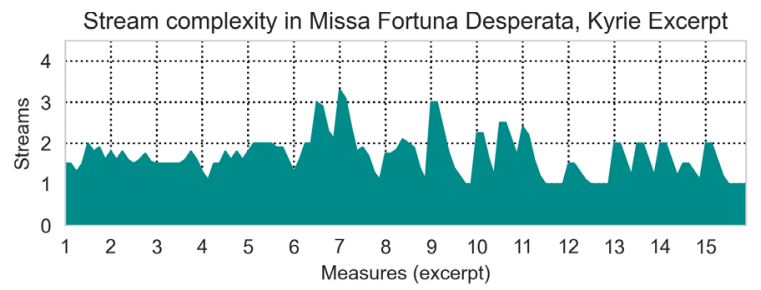

Figure 2: Auditory streaming complexity estimate for the 16-m excerpt reported in Figure 1. The opening duo $(1-5 \mathrm{~mm})$ maintains strongly separated voices. In $\mathrm{mm}$ 12-15, four parts are highly homorhythmic and reduced.

For the corpus analysis, the feature of streaming complexity was averaged per movement and the relative mean streaming complexity calculated by subtracting the average mean per mass cycle. These summary statistics are better interpreted as the center of mass for streaming complexity than representative of the most common values.

\section{Results}

Streaming complexity over time

The first result demonstrates that streaming complexity was not a static quality in renaissance polyphony. Linear regression on random 50 movement subsets from each quarter century era showed a significant increase in estimate values over time (Figure 3 ). Era significantly predicted mean streaming complexity, $\mathrm{b}=.12, t(258)=$ $17.3, p<.001$. Era explained a significant portion of variance between mass cycles, $R^{2}=.55, F(4,245)=$ $79.9, p<.001$.

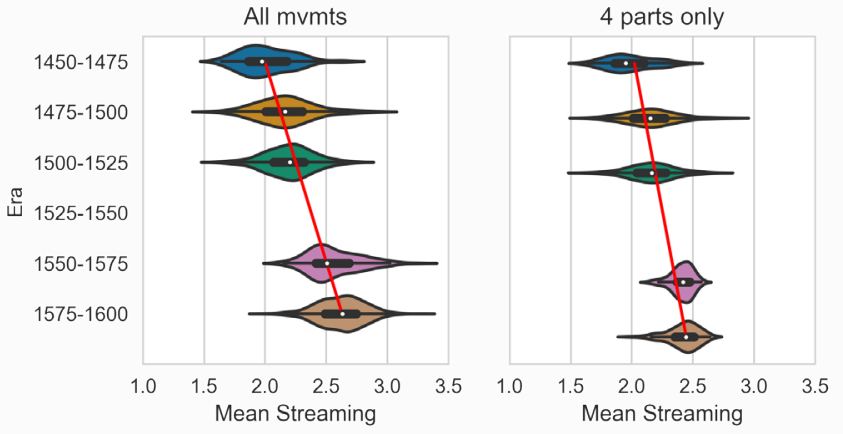

Figure 3: Increasing streaming complexity over time. The distribution of mean streaming complexity per movement increases substantially over successive eras. The effect is substantial when all movements are considered and when considering only movements with 4 voice parts. Regression line in red.

It is known that later works tended to include more parts, which makes higher streaming complexity values possible. However, the increase cannot be attributed only to additional voices, as the effect is noticeable across works with the same number of parts. The 4-part movements shown in the second plot of Figure 3 shows a comparably significant trend: $\mathrm{b}=.088, t(258)=$ $15.2, p<.001, R^{2}=.48, F(4,245)=63.2, p<.001$.

\section{Differences between movements}

While masses changed in streaming complexity over time, the hierarchy between movements appears to be fairly robust. A two-factor ANOVA (movement and composer) on relative mean streaming values showed a significant effect of movement and a significant interaction between movement and composer for both statistics (Vallat, 2018). The main effect of movement yielded an $\mathrm{F}$ ratio of $\mathrm{F}(4,788)=88.1, \mathrm{p}<.001$, indicating

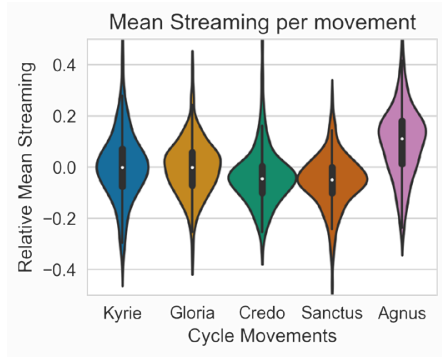

Figure 4: Distributions of relative complexity values per Mass Ordinary Cycle movement. Violin plots report the median, quartiles, and smoothed distribution. 
a significant difference in relative mean streaming complexity between movements. Pairwise differences between most movements were strong $(\mathrm{p}<.001)$, except for Credo-Gloria and Gloria-Sanctus $(\mathrm{p}<.01)$ and no significant difference between Credo-Sanctus and Gloria-Kyrie (Games-Howell post hoc test, allowing for unequal variance). The significant interaction between composer and movement, $\mathrm{F}(64,788)=3.4, \mathrm{p}<.001$, suggests analysis of individual composers' patterns is warranted.

Most composers followed this softened V shape, shown most clearly in Palestrina's works (Figure 5). The one well-represented composer to differ substantially was Josquin des Prez. Most mass cycles confidently attributed to Josquin reaching relatively high streaming complexity in the Credo, a distinctive strategy.

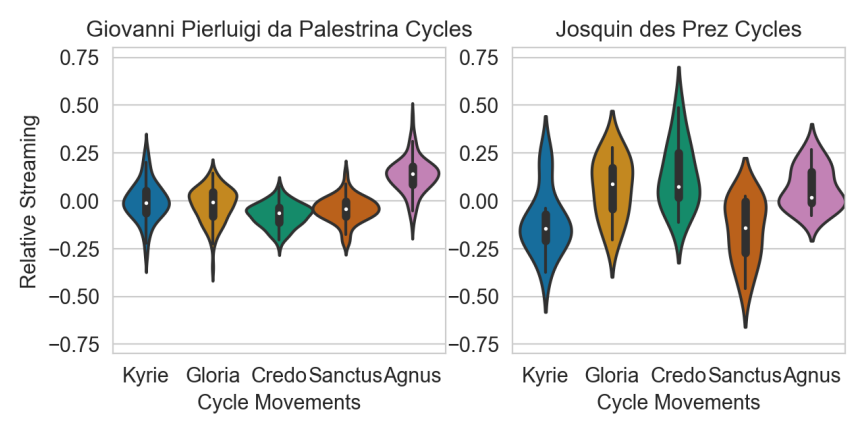

Figure 5: Movement trends for streaming complexity in masses by Palestrina and Josquin in violin plots.

\section{Discussion}

Masses composed between 1450 and 1600 show a trend of increasing complexity. Streaming Complexity Estimate averages fit a strong regression to the five quarter-century sets in the corpus. And the change was not only a consequence of more parts: a parallel analysis of movements with exactly 4 parts shows a similarly strong increase in streaming complexity. These findings correspond to what musicologists and performers already know about style change in the Renaissance, and in the mass in particular. Four-voice writing was typical before 1540, followed by more five-voice writing and experimentation with large numbers of voices in the late $16^{\text {th }}$ century. Not only do we have more voices in the score, we now know that this increase in streaming complexity means masses were composed in a way that individuated voices from each other more of the time: less merging, more separation.

The relative streaming complexity of the different movements also corresponds to musicologists' understanding of the conventions associated with each movement (Fallows, 2012). The subtle "V" shape indicates that streaming complexity decreases from the Kyrie to the Credo, and then ascends through the Sanctus to the highest complexity in the Agnus Dei. The Credo is normally the lowest in streaming complexity; it has the longest text (163 words), and tends to use syllabic text setting and homorhythm. The Sanctus, in contrast, is very melismatic; it has five sections (Sanctus-Pleni-Hosanna-Benedictus-Hosanna), and the Pleni and the Benedictus are normally written for a smaller number (2-3) of voices. It is sung during the liturgical high point of the mass, transubstantiation. The Agnus normally adds voices in the last of its three sections and often features special effects: canonic structures, a cantus firmus in very long notes, or wider ranges (Fallows, 2012). The Agnus is designed to go out with a bang. Overall, the consistency of the "V" shape in our corpus of mass cycles reinforces our initial claim that the sung mass ordinary cycle served as a way to mark experiential time in the context of the larger mass liturgy, perceptible without needing to understand the words.

As in any musical genre, composers could make different choices about which movements should be most complex. Within this corpus only a few composers were represented by enough works to test quantitatively, but the one whose masses stood out most clearly was Josquin des Prez. Unlike the general average hierarchy in the masses of Palestrina, Victoria, and de la Rue, the seventeen masses that are reliably attributed to Josquin have streaming complexity averages that put the Credo movement high in complexity and the Sanctus relatively low. This inversion of the normal hierarchy raises questions about whether those influenced by his work would have followed suit, but more scores are needed for such a study.

\section{Conclusion}

The Auditory Streaming Complexity Estimate was constructed out of principles of auditory perception without optimisation of parameters to fit reported perception. But even without the opportunity to sharpen the model, this measure appears to demonstrate meaningful patterns in this corpus of Renaissance masses.

Over 150 years, this liturgical genre increased in the average streaming complexity, as calculated from the interactions among the voices, and beyond the effect of the number of voices. The five movements of the mass, with their own liturgical functions and formal 
contributions to the mass service, tended to differ in their streaming complexity, creating a hierarchy where some movements tended to be more complex than others across the repertory, according to either feature. These differences were not absolute, but they were still common enough for multiple composers to show the same tendency, a soft $\mathrm{V}$ shape with the central textheavy Credo maintaining lower streaming complexity and the closing glorifying Agnus Dei reaching the greatest heights. This persistent form allows us to imagine how the cycle could have functioned to mark time for the Renaissance listener. The exception, Josquin des Prez, appears to have had his own rules for which movements were most complex in terms of auditory streams.

This estimate of auditory streaming complexity could be adapted to include more information-related stream formation. Motivic repetition, for example, sets up expectations. This corpus covers the period in which imitative texture became dominant for sacred music, and it may well play a role in the growth in complexity already shown. Another research question opened by this analysis is whether composer-specific trends carried forward in ways consistent with records of teaching and region.

It would be immensely useful to tune the Streaming Complexity Estimate directly to reported or measured experiences of listeners. But even without this stage of validation, the strength of the results in this corpus analysis suggest that it establishes the importance of texture as a musical feature that changes within a multi -movement work, and over time. Our calculations were tailored to this genre of music, to specific types of listeners (non-expert), and to a particular listening context. Similar evaluations could be performed on other genres of polyphonic music, such as motets and madrigals, and with some additional changes, to classical genres such as the string quartet.

\section{Acknowledgements}

Thanks to Ichiro Fujinaga and the SIMSSA team for their contributions and expertise. Financial support was generously provided by the granting agencies FRQSC (Fonds de recherche du Québec - Société et culture) and SSHRC (Social Sciences and Humanities Research Council of Canada).

\section{References}

Bregman, A. S. (1990). Auditory Scene Analysis: The Perceptual Organisation of Sound. MIT Press.

\section{https://doi.org/10.7551/mitpress/1486.001.0001}

Cambouropoulos, E. (2008). Voice and stream: Perceptual and computational modeling of voice separation. Music Perception, 26(1), 75-94. https://doi.org/10.1525/mp.2008.26.1.75

Cusack, R., Decks, J., Aikman, G., \& Carlyon, R. P. (2004). Effects of location, frequency region, and time course of selective attention on auditory scene analysis. Journal of Experimental Psychology: Human Perception and Performance, 30(4), 643. https://doi.org/10.1037/0096$\underline{1523.30 .4 .643}$

Cuthbert, M. S. (2019). Music21 Toolkit (Version 5) [Python]. cuthbertLab. https://web.mit.edu/music21/

Duane, B. (2013). "Auditory Streaming Cues in Eighteenthand Early Nineteenth-Century String Quartets: A CorpusBased Study." Music Perception: An Interdisciplinary Journal https://doi.org/10.1525/mp.2013.31.1.46 31(1),46-58.

Fallows, D. (2012). The last Agnus Dei: Or: The cyclic Mass, 1450-1600, as forme fixe. In A. Ammendola, D. Glowotz, \& J. Heidrich (eds.), Polyphone Messen im 15. Und 16. Jahrhundert: Funktion, Kontext, Symbol (pp. 5364). V \& R unipress.

Huron, D. (2001). Tone and Voice: A Derivation of the Rules of Voice-Leading from Perceptual Principles. Music Perception: An Interdisciplinary Journal 19(1), 164. https://doi.org/10.1525/mp.2001.19.1.1

McKay, C., Cumming, J., and Fujinaga, I. (2017). Characterizing Composers Using JSymbolic2 Features. Extended Abstracts for the Late-Breaking Demo Session of the 18th International Society for Music Information Retrieval Conference. Suzhou, China.

McKinney, W. (2010). Data structures for statistical computing in Python. In S. van der Walt \& J. Millman (Eds.), Proceedings of the 9th Python in Science Conference (pp. 51-56). https://doi.org/10.25080/Majora-92bf1922-00a

Oliphant, T. E. (2007). Python for scientific computing. Computing in Science \& Engineering, 9(3), 10-20. https://doi.org/10.1109/MCSE.2007.58

Rodin, J., Sapp, C. The Josquin Research Project. Accessed December 12, 2019. http://josquin.stanford.edu/.

Vallat, R. (2018). Pingouin: Statistics in Python. The Journal of Open Source Software, 3(31), 1026. https://doi.org/10.21105/joss.01026 


\title{
Deployments of change and novelty in a corpus of popular music
}

\author{
Christopher Wm. White ${ }^{1 \dagger}$ \\ ${ }^{1}$ University of Massachusetts Amherst, Amherst, MA, USA \\ ${ }^{\dagger}$ Corresponding author: cwwhite@umass.edu \\ Published 16 December 2021; https://doi.org/10.18061/FDMC.2021.0014 \\ Author video presentation and/or other conference material: $\underline{\text { https://doi.org/10.17605/OSF.IO/MPBT5 }}$
}

\begin{abstract}
This paper considers aspects of form and texture in 20thcentury American popular music using a corpus of textural/lyrical/vocal annotations added to the McGillBillboard corpus (Burgoyne, 2012). The analysis tracks two categories of events: 1) changes differ from immediately prior events; while 2) novelties have not happened previously within a song. I additionally divide these events into their specific species of change (i.e., whether the change involves the introduction of new instrumentation, new harmonizations, etc.), and correlate these events with their position in each track in terms of both clock time and formal zone. These interconnections allow me to demonstrate the types of events that appear at particular places within a song, and suggest ways that listeners may use musical cues to form expectations about a song's form and length.
\end{abstract}

KEYWORDS: corpus analysis, popular music, lyrics, timbre, texture, expectation

\section{Introduction}

One utility of large-scale musical forms is their ability to orient a listener within the timeline of a musical event (Hepokoski and Darcy, 2006). For instance, a listener familiar with the norms of $18^{\text {th }}$ century Viennese sonata forms will know that, upon hearing the beginning of the piece's development section, that the movement is roughly at its halfway point.

$20^{\text {th }}$-century American popular music, especially that based on the rotation of the verse/chorus pair (Margulis, 2014; Temperley 2018), poses a problem for such formal wayfinding: forms that repeat the same musical materials in rotation pose a potential difficulty for a listener to orient themselves, given that the events of the beginning, middle, and end share much of the same musical material (Brody, 2020; Puri, 2020).

This project uses corpus analyses to demonstrate ways that certain musical invariants - specifically, textural, timbral, lyrical, rhytmic, and harmonic changes - are consistently associated with certain points in the timelines of American popular songs. This work combines the notion that studies in form should be grounded in the norms of some corpus (Brody, 2017; Greenberg, 2017; Hepokoski \& Darcy, 2006) with the idea that texture, timbre, and repetition play fundamental roles in popular music's construction (Barna, 2019; deClercq \& Temperley 2011; Lavengood, 2017; Temperley, 2018). To this end, this study analyzes the McGill-Billboard corpus (a dataset of chord and formal annotations of American popular music, 19581991; Burgoyne, 2012) supplemented with the textural annotations of White et al. (2021) to show that certain events can be associated with particular points within a popular song's form. In the end, I suggest that these formal associations may provide a way for listeners to orient themselves within the rotations and repetition that characterize American popular music.

\section{General Method}

\section{The Corpus}

For this analysis, I use the McGill Billboard Corpus (Burgoyne, 2012), a corpus that spans 1958 to 1991, and uses songs selected from the Billboard Top 100 for each year. The corpus itself consists of text files indicating the song's title, key, likely time signature, and finally lines of text corresponding to each musical phrase. These lines indicate the phrases' chords in an adapted lead-sheet-style format, along with measure delineations, designations for the beginning of formal zones, and micro-timing stamps for the phrase beginnings. Further annotations have been added to 665 of these files (White, et al. 2021). These annotations correspond to moments of change within the texture, timbre, text, or other sonic parameters of the song. The annotations each have three values, with the first value representing the category of change (indicating the musical domain or instrument group corresponding to the change, like "percussion" or "voice"), the second adding specificity to the change (e.g., specifying "backup singer" within the broader "voice" category), and the third indicating the type of event (either "change," "entry," or "exit"). These annotations use 50 unique three-character annotations, with 14,376 total annotations.

\section{Methodologies}

Given that this project's motivation is to identify general trends within a dataset, an exploratory (rather than 
experimental) stance was adopted. Three main properties of this dataset were examined. First, information concerning the clock-time length of each track was compiled, along with the annotations' relative placement within this clock-time. The latter of these values is calculated as the proportion between an event's clock time and the overall clock-time duration of the track (i.e., the relative placement of an annotation that appears one minute into a four-minute song would be .25). Second, changes indicated by the corpus's annotations were also associated with formal zones as indicated by the original corpus. Third, "novel" events - event types that were yet to occur within the track were tracked both in connection to their position in proportional timelines and in regard to formal zones.

\section{Observations}

\section{Clock Time}

Figure 1 shows the distribution of the clock-time duration of songs within this corpus. The distribution shows the influence of the "three minute rule" in popular music, a norm that arose from the limitations of early recording technologies in which each side of a record could encode between roughly 3 minutes (for a 10" 78 RPM recording) and 5 minutes (for a 12" 78 RPM recording; Mudge \& Hoek, 2001) of material. Indeed, $90 \%$ of songs within this dataset clock between 2.2 and 4.8 minutes. Such a constraint upon the length of a song is well documented within popular music literature (McKinney, 2015; Temperley 2018). However, it is an important baseline consideration when building a notion of form within this repertoire.

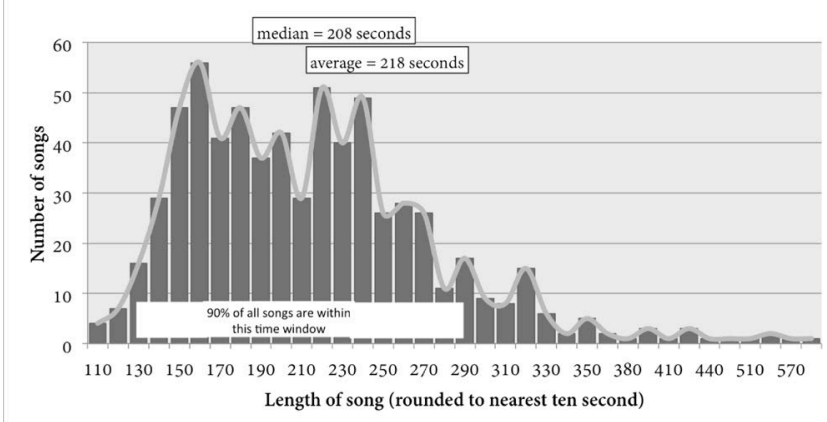

Figure 1: Clock-time duration of songs within the corpus

\section{Changes within a Timeline}

Table 1 shows twelve of the corpus's most salient annotations and associates them with the average position at which they tend to appear within a track.
Positioning is shown in two ways. The mean value simply averages the relative placements of each annotation; the standard deviation (SD) and number of observations $(n)$ associated with this mean are provided as well. Given that these distributions often do not all approximate a normal - let alone a unimodal distribution, "spikes" within the distributions are also identified. Events' relative placements were rounded to the closest single decimal, and distributions of those rounded values are created for each event type. "Spikes" were identified as points in these distributions that peaked $10 \%$ or more from surrounding values; if two consecutive values remained high, they were considered part of the same peak. Table 1's leftmost column shows the three approaches used to identify salient annotations: 1) identifying the three most-frequent annotations, 2) identifying the three most-consistent annotations (i.e., those with the lowest SD values), and finally identifying those six further annotations that maximize frequency and consistency (approximated by the lowest standard error values, excluding event types that appear in the prior two categories).

Table 1: Associations between event-types and position within a track

\begin{tabular}{|c|c|c|c|c|c|}
\hline & Type & $\begin{array}{l}\text { Spike-points } \\
\text { ( } \% \text { of track) }\end{array}$ & $\begin{array}{l}\text { Mean } \\
\text { point in } \\
\text { timeline: }\end{array}$ & SD: & $n$ \\
\hline \multirow{3}{*}{$\begin{array}{l}\text { Most } \\
\text { Frequent }\end{array}$} & New Lyrics & $0-10 \%$ & .34 & .27 & 4493 \\
\hline & $\begin{array}{l}\text { New Singer } \\
\text { Enters }\end{array}$ & $\begin{array}{l}0-20 \% \\
60-80 \%\end{array}$ & .33 & .28 & 1296 \\
\hline & $\begin{array}{c}\text { Accompaniment } \\
\text { instrument } \\
\text { enters } \\
\end{array}$ & $0-10 \%$ & .28 & .27 & 990 \\
\hline \multirow{3}{*}{$\begin{array}{c}\text { Most } \\
\text { Consistent } \\
\text { (lowest SD) }\end{array}$} & Fadeout & $80-100 \%$ & .91 & .06 & 283 \\
\hline & $\begin{array}{l}\text { Repeated lyrics } \\
\text { set in new way }\end{array}$ & $90-100 \%$ & .8 & .14 & 70 \\
\hline & Key change & $50-80 \%$ & .56 & .19 & 119 \\
\hline \multirow{6}{*}{$\begin{array}{c}\text { Most } \\
\text { Frequent and } \\
\text { Consistent } \\
\text { (lowest SE) }\end{array}$} & $\begin{array}{l}\text { Entry of backup } \\
\text { singers }\end{array}$ & $20-40 \%$ & .42 & .25 & 958 \\
\hline & $\begin{array}{c}\text { Exit of lead } \\
\text { singer }\end{array}$ & $\begin{array}{l}40-60 \%, \\
90-100 \% \\
\end{array}$ & .54 & .25 & 778 \\
\hline & $\begin{array}{c}\text { Exit of backup } \\
\text { singers }\end{array}$ & $30-60 \%$ & .47 & .22 & 582 \\
\hline & $\begin{array}{c}\text { Enter solo } \\
\text { instrument } \\
\end{array}$ & $\begin{array}{c}0-10 \%, \\
50-70 \%\end{array}$ & .34 & .26 & 521 \\
\hline & $\begin{array}{c}\text { Full percussion } \\
\text { section enters }\end{array}$ & $0-10 \%$ & .2 & .27 & 458 \\
\hline & $\begin{array}{c}\text { Full } \\
\text { instrumental } \\
\text { ensemble enters }\end{array}$ & $0-10 \%$ & .2 & .29 & 488 \\
\hline
\end{tabular}

Two main observations arise from this table. First, there are a handful of events that most often appear at either the very beginning or end of tracks, with the entry of new lyrics, singers, percussion, and accompaniment textures instantiating the former, and fadeouts and exits of singers instantiating the latter. On the one hand, this observation may seem trivial: of course these types of 
events begin these tracks. After all, in order for a song to begin, singers and instrumentalists need to begin playing and singing. However, within this repertoire, such observations are not trivial. With the exception of live concerts, these songs are presented within seamless sonic streams often with little or no silence in between (consider a progression of songs played on the radio, the sequence of a playlist, the background music of an exercise class, or a wedding DJ's setlist). These cues, therefore, are important to unambiguously indicate that a new form has begun, and that the prior song is complete - they define what it means to begin a popular song's form. Second, several of these events occur at particular intermediate points within a song's timeline. For instance, the entry of a new singer or solo instrument most often occurs $60-80 \%$ or $50-70 \%$ through a track, respectively. Such events potentially serve as markers within these songs' overall forms.

Table 2 shows the annotations most often associated with each formal zone used in the McGill-Billboard corpus. To compensate for the different frequencies of each formal zone, I recorded annotations whose percentage of entries met or exceeded 1.5 times the percentage with which a formal zone occurred. (For instance: $22.8 \%$ of all formal zones are choruses: for an annotation to be associated with this zone, more than $34.2 \%$ of the annotation's occurrences had to occur within that zone.) Only events that occurred more than 50 times in the dataset were considered. Events that are shared by zones are italicized and color-coded.

Table 2: Associations between event-types and formal zones

\begin{tabular}{|c|c|c|c|}
\hline Verse & Interlude & Outro & Intro \\
\hline $\begin{array}{l}\text { Entry of lead } \\
\text { inger } \\
\text { Call/response or } \\
\text { cho format begins } \\
\text { New Lyrics }\end{array}$ & $\begin{array}{l}\text { Rhythmic } \\
\text { density } \\
\text { decreases } \\
\text { Percussion } \\
\text { solo/break } \\
\text { begins/ends }\end{array}$ & $\begin{array}{l}\text { Repeated lyrics set } \\
\text { in new way } \\
\text { - Fadeout }\end{array}$ & $\begin{array}{l}\text { - Enter full ensemble } \\
\text { - Enter full percussion } \\
\text { - Enter accompaniment } \\
\text { instrument } \\
\text { - Enter solo instrument }\end{array}$ \\
\hline \multicolumn{4}{|c|}{ Chorus } \\
\hline \multicolumn{2}{|c|}{$\begin{array}{l}\text { - Entry of backup singers } \\
\text { - Choir entry/exit } \\
\text { - Fadeout } \\
\text { - Full percussion enters } \\
\text { - Key change } \\
\text { - Enter/exit full ensemble } \\
\text { - Overall range expands }\end{array}$} & \multicolumn{2}{|c|}{$\begin{array}{l}\text { - Rhythmic density increases } \\
\text { - Call/response format begins and } \\
\text { ends } \\
\text { - Begin new vocal register } \\
\text { - New vocal or harmonization } \\
\text { technique used } \\
\text { - Repeated lyrics set in new way }\end{array}$} \\
\hline Bridge & Solo & \begin{tabular}{|l|} 
Pre-chorus \\
\end{tabular} & Transition \\
\hline $\begin{array}{l}\text { - Key change } \\
\text { - Accompaniment } \\
\text { ensemble changes } \\
\text { - Spoken lyrics } \\
\text { begin }\end{array}$ & $\begin{array}{l}\text { Enter/exit } \\
\text { solo } \\
\text { instrument }\end{array}$ & $\begin{array}{l}\text { - Accompaniment } \\
\text { ensemble changes } \\
\text { - Entry/exit of backup } \\
\text { singers } \\
\text { - Add digital sounds } \\
\text { to acoustic texture } \\
\text { Begin new vocal } \\
\text { egister }\end{array}$ & \begin{tabular}{|l} 
Overall range \\
contracting \\
Rhythmic density \\
decreases \\
- Key change \\
- New Lyrics \\
- Exit lead singer
\end{tabular} \\
\hline
\end{tabular}

The table illustrates several notable features of this corpus. First, only a handful of event types are not shared between formal zones (i.e., the black, nonitalicized events). The presence of these events would seem to uniquely point to a particular formal zone. For instance, a listener hearing the overall range of the song's texture expand could reasonably conclude that they are hearing a chorus. Second, each of these zones has a unique slate of associated events. Such groups of events may contribute to these zone's definitions and identity. For instance, part of the definition of "chorus" may be the inclusion of the event types included in Table 2. Similarly, these zones can also be defined by what they do not feature: part of the identity of a chorus may be that it does not feature the events associated with other zones.

However, as indicated by the colored italics of Table 2 , there exists a reasonable amount of overlap between zones. Table 3 undertakes a $k$-means cluster of zones using normalized frequencies of all event types that occur greater than 50 times in the corpus. (Using an analysis of silhouette widths for $k=[1 \ldots 8], k=4$ was shown as the optimal clustering; White and Quinn, 2018.) The table shows the families of zones that are associated with similar event types with similar frequencies, along with a brief description of the sorts of events types uses in that cluster (i.e., the "character"). Finally, the "secondary cluster" shows the cluster to which the zone is next closest, affinities which illustrate secondary characteristics of each zone.

Table 3: k-means clustering of each zone's event-type associations $(k=4)$

\begin{tabular}{|c|c|c|c|}
\hline \multirow{3}{*}{$\begin{array}{c}\text { bridge } \\
\text { trans }\end{array}$} & cluster & "Character" & $\begin{array}{l}\text { secondary } \\
\text { cluster }\end{array}$ \\
\hline & 1 & \multirow{3}{*}{$\begin{array}{c}\text { Many } \\
\text { changes, } \\
\text { decreases } \\
\text { in energy, } \\
\text { exits }\end{array}$} & 3 \\
\hline & 1 & & 3 \\
\hline \multirow[b]{2}{*}{ prechorus } & 1 & & 2 \\
\hline & 2 & \multirow{2}{*}{$\begin{array}{l}\text { Increased } \\
\text { thickness of } \\
\text { textures } \\
\text { range } \\
\text { increases, } \\
\text { new entries }\end{array}$} & 1 \\
\hline chorus & 2 & & 3 \\
\hline \multirow{3}{*}{$\begin{array}{l}\text { instrumental } \\
\text { solo } \\
\text { interlude }\end{array}$} & 3 & \multirow{5}{*}{$\begin{array}{l}\text { Decreases } \\
\text { in rhythm } \\
\text { and texture, } \\
\text { entries and } \\
\text { exits of } \\
\text { non-vocal } \\
\text { instruments }\end{array}$} & 1 \\
\hline & 3 & & 1 \\
\hline & 3 & & 1 \\
\hline \multirow{3}{*}{$\begin{array}{c}\text { pre-verse } \\
\text { outro } \\
\text { intro }\end{array}$} & 3 & & 4 \\
\hline & 3 & & 1 \\
\hline & 4 & Entries & 1 \\
\hline
\end{tabular}




\section{Disposition of Novelty}

Figure 2 shows the percentage of annotated events that have not occurred within the song before (i.e., "novelties") versus events that have occurred before, again using relative placement values rounded to the nearest $10 \%$. Naturally, the initial events of a song are mostly novel - there has not yet been an opportunity to repeat these events. As the song continues, fewer and fewer of the annotations are novel: unsurprisingly, as verses and choruses repeat, they also repeat the same types of events. However, the last moments of these songs feature an uptick in novelty - the last several moments of a song tend to issue musical changes unlike those that have occurred previously. A listener who hears an increase in novel events after a period of repetition might be cued to a song's impending ending (at least if they are attuned to this particular norm of this repertoire).

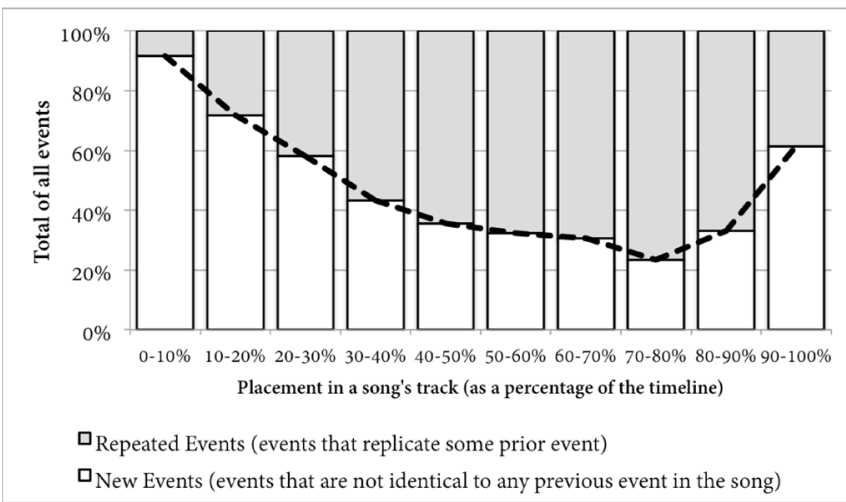

Figure 2: Use of "Novelty" within songs' timelines

Disposition of Formal Zones and the Interaction of Parameters

Figure 3 shows the overall distribution of formal zones within this corpus, with choruses and verses dominating the graph with nearly $60 \%$ of the mass. As has been observed by many observers (and argued in Temperley, 2018), these two zones act as a formal backbone in this repertoire. Indeed, every song in this corpus has at least one of these zones. Due to their frequency and ubiquity, we can imagine these zones acting as core modules within this repertoire: each song features one or both of the chorus/verse zones, and the repetition and rotation of these modules is a central feature of the song's form.

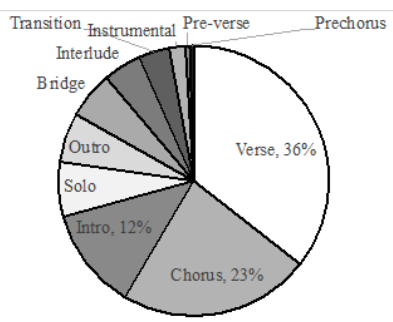

Figure 3: Distribution of formal zones

Figure 4 shows how these core modules are deployed, how they interact with other formal zones, and how other musical parameters are used alongside these zones. The top of the figure presents a flowchart of probabilities derived from the corpus, showing a series of decisions concerning the construction of core modules, how often to rotate the core, and what other formal zones might be included within the song. Clocktime duration is shown above the flowchart, and a proportional timeline is shown below. Finally, a selection of musical changes are shown below the proportional timeline, with their position indicating approximate positions within the track, and percentages again indicating how often such events appear. Overall, this figure provides a birds-eye view of how formal zones are ordered and rotated within this repertoire, and how these orderings interact with certain musical and lyrical changes.

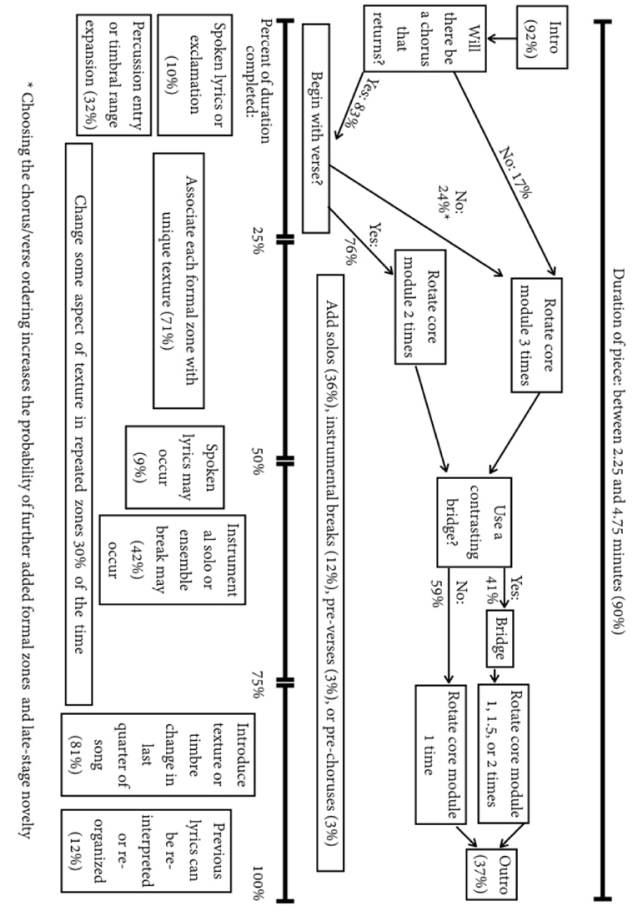

Figure 4: Disposition of formal zones and selected changes within an overall timeline 


\section{Discussion and Conclusion}

Overall, this study suggests four strategies for articulating the progression of a form within a repertoire of American popular songs. First, clock time presents an important overall constraint, with most songs lasting somewhere between 2.2 and 4.8 minutes. Second, certain types of events are often associated either with particular locations within a track, or are associated with particular formal zones. Third, novel events (types of events that have not yet been heard within a track) are clustered at the beginning of a track, and again at the end, with intervening points repeating prior event types. Finally, there exist certain norms concerning the deployment and rotation of formal zones within a song.

This paper began by suggesting that the repetition inherent in American popular music poses a potential problem for listeners: it is difficult to know one's position in a timeline if the song consists primarily of repetitions of the same musical material. However, this study has shown several musical events that have a normative placement within songs' overall durations, and that these events can even be uniquely associated with particular formal zones within this repertoire. These norms may be available to listeners when they are creating expectations about a song's form, and if they are sufficiently internalized can help orient listeners within a song's overall timeline. Furthermore, such norms are based not solely on melodic and harmonic parameters, but on textural, timbral, lyrical, and rhythmic variations. Indeed, this suggests that these domains - and the variation contained therein - may play crucial roles in listener's understanding of form in $20^{\text {th }}$-century American popular music.

\section{References}

Barna, A. 2019. Examining contrast in rock and popular music. Ph.D. Dissertation, Eastman University.

Brody, C. (2016). Parametric interaction in tonal repertoires. Journal of Music Theory, 60 (2), 97-148. https://doi.org/10.1215/00222909-3651814

Brody, C. (2020). Two Langerian sonata-Form problems, with solutions by Beach and Medtner. Paper delivered at Society for Music Theory Annual Meeting.

Burgoyne, J.A. (2012). Stochastic processes and datasetdriven musicology. Ph.D. dissertation, McGill University.

Byros, V. (2009). Foundations of Tonality as Situated Cognition, 1730-1830. Ph.D. Dissertation, Yale University.

deClercq, T, \& Temperley, D. (2011). A corpus analysis of rock harmony. Popular Music, 30(1), 47-70.
https://doi.org/10.1017/S026114301000067X

Greenberg, Y. (2017). Of beginnings and ends: A corpusbased inquiry into the rise of the recapitulation. Journal $\begin{array}{llll}\text { of Music Theory, } 61 & \text { (2), 171-200. }\end{array}$ https://doi.org/10.1215/00222909-4149546

Hepokoski, J \& Darcy, W. (2006). Elements of Sonata Theory: Norms, Types, and Deformations in the LateEighteenth-Century Sonata. New York: Oxford University Press. https://doi.org/10.1093/acprof:oso/ 9780195146400.001 .0001

Lavengood, M. (2017). A new approach to the analysis of musical timbre. Ph.D. diss, CUNY Graduate Center.

Margulis, L. (2014). On repeat: how music plays the mind. New York: Oxford University Press. https://doi.org/10.1093/acprof:oso/9780199990825.001. 0001

McKinney, K. (2015). A hit song is usually 3 to 5 minutes. here's why. Vox. vox.com/2014/8/18/6003271/why-aresongs-3-minutes-long

Mudge, S., D.J. Hoek. (2001). Describing jazz, blues, and popular 78 RPM sound recordings: suggestions and guidelines. Cataloging \& Classification Quarterly, 29(3). 21-48. https://doi.org/10.1300/J104v29n03 03

Puri, M. (2020). Rituals of circularity: on the conceptual underpinnings of rotational form." Paper delivered at the Society for Music Theory Annual Meeting.

Riley, M. (2010). Hermeneutics and the new Formenlehre: an interpretation of Haydn's 'Oxford' symphony, first movement. Eighteenth-Century Music, 7(2): 199 - 219. https://doi.org/10.1017/S1478570610000047

Temperley, D. (2018). The musical language of rock. New York: Oxford University Press. https://doi.org/10.1093/oso/9780190653774.001.0001

White, C. and Quinn, I. (2018). Chord content and harmonic function in tonal music. Music theory spectrum, 40(2), 314-350. https://doi.org/10.1093/mts/mty021

White, CW., Fulmer, J., Cordova, B., Black, A., Danitz, C., Evans, W., Fischer, A., Greene, R., He, J., Kenyon, E., Miller, J., Moylan, M., Ring, A., Schwitzgebel, E., \& Wang, Y. (2021). A New Corpus of Texture, Timbre, and Change in 20th-Century American Popular Music. in Proceedings of the Future Directions of Music Cognition International Conference, 6-7 March 2021. https://doi.org/10.18061/FDMC.2021.0015 


\title{
A new corpus of texture, timbre, and change in 20th-century American popular music
}

\author{
Christopher Wm. White ${ }^{1 \dagger}$, Jeffrey Fulmer ${ }^{2}$, Brian Cordova ${ }^{1}$, Alexandria Black ${ }^{1}$, Chloe Danitz ${ }^{1}$, \\ William Evans ${ }^{1}$, Aidan Fischer ${ }^{1}$, Rashaad Greene, Jinhan He, Emily Kenyon, Joan Miller, \\ Madeline Moylan $^{1}$, Abigail Ring ${ }^{1}$, Emily Schwitzgebel ${ }^{3}$, Yatong Wang ${ }^{1}$ \\ ${ }^{, 1}$ University of Massachusetts Amherst, Amherst, MA, USA \\ ${ }^{2}$ Hampshire College, Amherst, MA, USA \\ ${ }^{3}$ Northwestern University, Evansville, IN, USA \\ $\dagger$ Corresponding author: cwmwhite@umass.edu \\ Published 16 December 2021; https://doi.org/10.18061/FDMC.2021.0015 \\ Author video presentation and/or other conference material: https://doi.org/10.17605/OSF.IO/W32K6
}

\begin{abstract}
This paper describes a new corpus of texture, timbre, and lyrical information within 20th century American popular music, parameters which recent research has suggested play crucial roles in how this music is consumed and perceived. Our corpus applies 3-dimensional annotations to the McGillBillboard corpus (a corpus of harmonic and formal annotations of songs within the Billboard Top-100 between 1958 and 1991; Burgoyne, 2012). These annotations indicate moments of sonic change within each song. The first dimension indicates the broader musical domain of the change (instrumental, percussion, voice, extramusical sounds, lyrics, or rhythm); the second specifies the domain's particular category (e.g.: "backup singers" as a subcategory of the "voice" domain); and, the third indicates whether the texture begins, ends, or alters the previous texture. This paper outlines the methods used to assign these annotations, and some characteristics and notable aspects of the resulting corpus.
\end{abstract}

KEYWORDS: corpus analysis, popular music, lyrics, timbre, texture

\section{Introduction}

To date, corpus analyses of American popular music have primarily focused on the repertoire's use of melody (Tan, Lustig, \& Temperley 2019), harmony (deClercq \& Temperley, 2011; White \& Quinn, 2018), and form (Temperley, 2018). Recent scholarship, however, has suggested that texture and timbre play crucial roles in the production and consumption of American popular music (Barna, 2019; Lavengood, 2017), as well as the repetition of these elements (Acevedo, 2020; Margulis, 2014). This paper outlines annotations added to the McGill-Billboard corpus of $20^{\text {th }}$ century American popular music (Burgoyne, 2012) that indicate moments of instrumental, textural, timbral or other types of sonic changes not explicitly present in the corpus as it currently stands. Our goal is for these annotations to assist research into the interactions between texture, timbre, lyrics and form within this repertoire.

\section{Method}

The McGill-Billboard corpus includes individual text files for each song that specify metadata associated with the song (title, artist, meter, and tonic), and represent each of the song's phrases separate lines, with chords annotated for each measure within the phrases. Microtiming information is associated with each phrase onset, and formal zones (e.g., verse, chorus, intro) are additionally included.

\section{Procedures}

Each of this paper's authors served as annotators. To begin the annotation process, each studied five files along with recordings of the songs, and notated all "moments of change" within the track. As a group, we then compiled a list of all such changes, and both normalized this list using consistent labels, and also decided upon categories with which to organize these changes. Pairs of annotators then independently applied these preliminary annotations to five new songs in the corpus, then compared their results. From our discussions of these comparisons, we developed a style guide, along with a more expansive and rigorous list of possible annotations and categorizations. After repeating this pairwise process a further time, a final list and style guide were decided upon. Using these guides, annotators then progressed through the entire corpus. If a new event type was encountered during this process, a new annotation could be suggested to the collaborators for potential inclusion within the annotation list.

\section{The annotations}

The annotations each have three values, with the first value representing the category of change, the second the genus, and the third the type. Categories involve the broadest band of similarity, and indicate the musical 
domain or instrument group expressing the change. They are: percussion $(\mathrm{P})$, voice $(\mathrm{V})$, extramusical sounds $(\mathrm{E})$, harmony $(\mathrm{H})$, words/lyrics $(\mathrm{W})$, rhythm $(\mathrm{R})$, and instrumental/texture (T). The second value indicates the genera specific to each category. These involve the kind of change being affected (e.g., there is a "solo" genus under the "texture" category, as a solo is a kind of texture). Categories have different numbers of genera, with texture containing the most (12) and rhythm having the fewest (1). The final value then indicates the three event types: Change, Entry, Exit. These types were held in common throughout the categories and genera. The process results in 50 unique three-character annotations, with 14,376 annotations added to 665 McGill-Billboard text files. Figure 1 shows the disposition of annotations between categories.

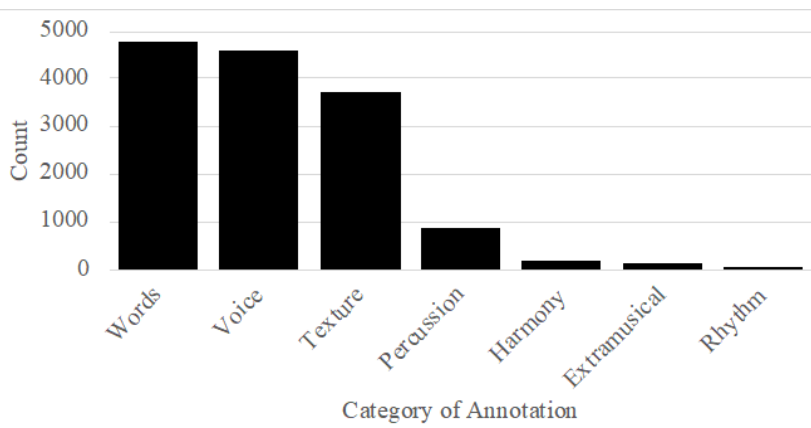

Figure 1: Distribution of categories in the annotation dataset

Each three-member annotation is preceded by a " $\$$ " for querying purposes. Figure 2 shows an example of these annotations from James Brown's "I Don't Mind." Red circles indicate our added annotations, first showing the entry of an accompanying instrument (\$TYI) followed by the entry of backup singers (\$VBI) and a further new singer (\$VSI). A complete dictionary of annotations and the style guide can be found along with the full corpus of annotations at chriswmwhite.com/popannotations.

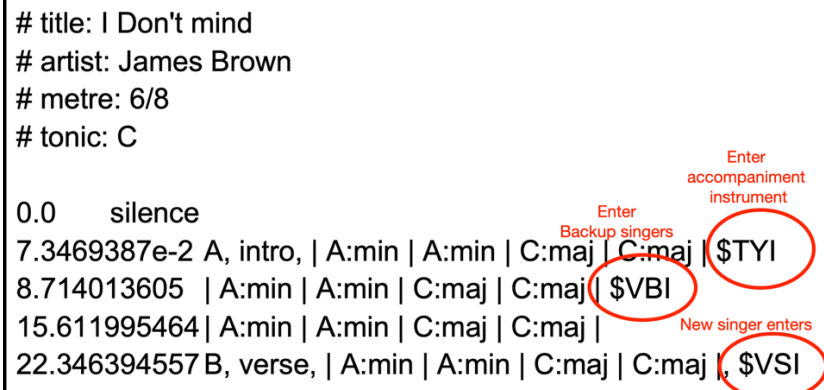

Figure 2: Example annotations

\section{Some Corpus Properties}

Figure 3 displays a series of box-and-whisker plots for each category. The horizontal line within each box shows the median; the upper and lower bounds of the boxes outline the quartiles of data points higher and lower than the median; x's show means; and the range is shown by the "whiskers" emanating from the boxes. This graph illustrates that each category of change can occur throughout a piece, but different categories appear to favor different points within a track's duration. For instance, changes involving percussion seem to occur closer to the beginning of the track, while rhythmic variations occur closer to the end. Figure 4 investigates the disposition of percussion-categorized events, showing each of this category's individual events types: PAI (additional percussion enters, e.g., shaker or wood block); PAO (additional percussion exits); PBI (drums/percussion solo/break starts); $\quad$ PBO (drums/percussion solo/break exits); PTI (full percussion/drum texture enters), PTO (full percussion/drum texture exits). This graph shows that the overall early placement of the percussion category is primarily due to early entries of the full percussion texture and/or additional percussion sounds.

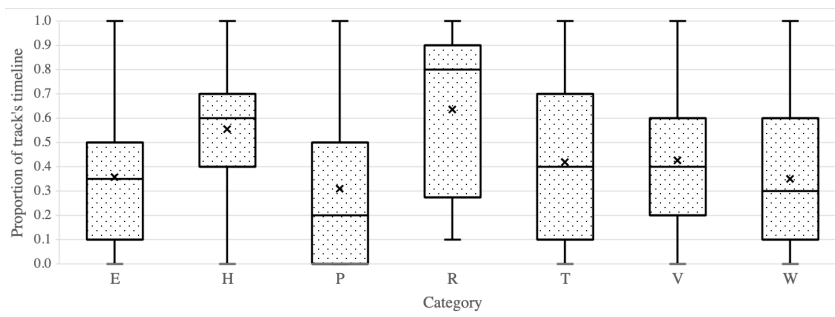

Figure 3: Distribution of categories within pieces' timelines

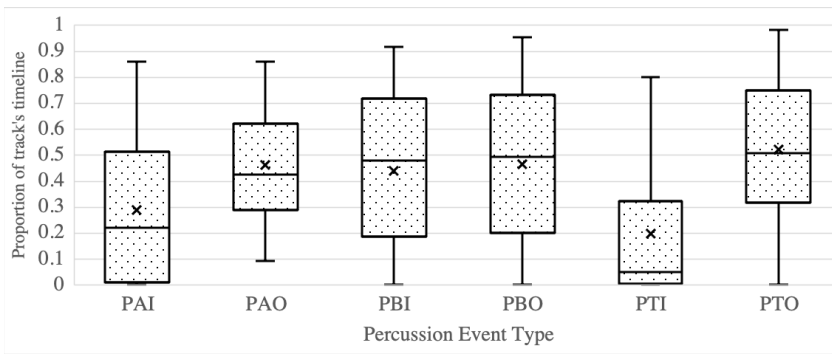

Figure 4: Distribution of events within the Percussion category

Figures 5 and 6 demonstrate some connections between popular music's formal zones and moments of change. Figure 5 shows the average annotations applied to each formal zone in this corpus, with incidental modules (e.g., solos, outros, instrumental interludes) receiving 
the most on average, and choruses and pre-choruses hosting the least. Figure 6 investigates the distribution of these annotations specifically within different rotations of songs' verses. This distribution illustrates that first verses receive the most annotations, while the remainder of verses retain a somewhat steady number of annotations.

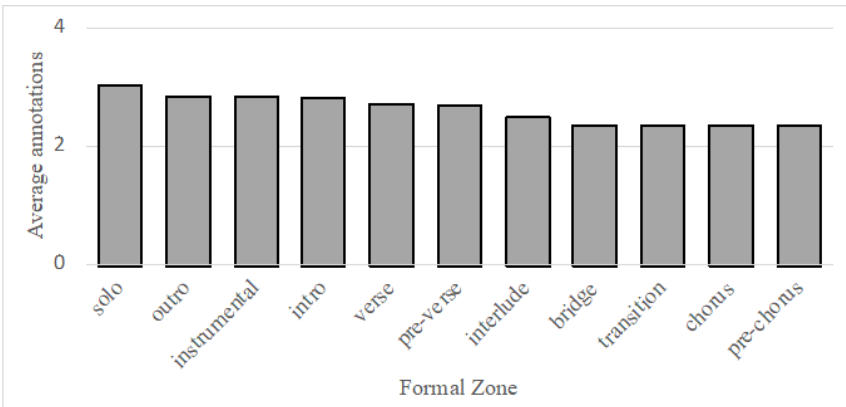

Figure 5: Average amounts of annotations for each formal zone

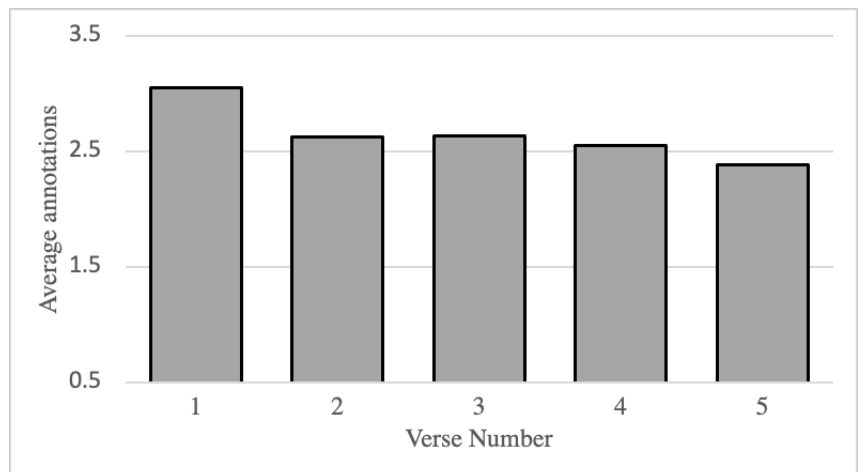

Figure 6: Average amounts of annotations for the first five rotations of verse material

\section{Future Directions}

In an effort to provide maximum coverage of the McGill Billboard Corpus, the final annotations were made by a single collaborator. Individual decisions are likely to vary slightly between annotators, and mistakes and inconsistencies can be expected within the corpus as it currently stands. While we believe the size of the corpus and number of collaborators somewhat mitigate these issues, a second wave of verification and editing would be useful to check for consistency and completeness.

\section{Acknowledgements}

Many thanks to the graduate students of "Analysis of Pop and Rock" at UMass Amherst for participating in this annotation project.

\section{References}

Acevedo, S. (2020). Harmonic schemata of popular music: an empirical representation of analytical patterns and their representation. Ph.D. Dissertation, Yale University.

Barna, A. (2019). Examining contrast in rock and popular music. Ph.D. Dissertation, Eastman University.

Burgoyne, J.A. (2012). Stochastic processes and datasetdriven musicology. Ph.D. dissertation, McGill University.

deClercq, T, \& Temperley, D. (2011). A corpus analysis of rock harmony." Popular Music 30(1), 47-70. https://doi.org/10.1017/S026114301000067X

Lavengood, M. (2017). A new approach to the analysis of musical timbre. Ph.D. diss, CUNY Graduate Center.

Margulis, L. (2014). On repeat: how music plays the mind. New York: Oxford University Press. https://doi.org/10.1093/acprof:oso/9780199990825.001. 0001

Tan, I., Lustig, E., Temperley, D. (2019). Anticipatory syncopation in rock: a corpus study. Music Perception, 36(4), 353-370. https://doi.org/10.1525/mp.2019.36.4.353

Temperley, D. (2018). The musical language of rock. New York: Oxford University Press. https://doi.org/10.1093/oso/9780190653774.001.0001

White, CW. and Quinn, I. (2018). Chord content and harmonic function in tonal music. Music theory spectrum, 40(2), 314-350. https://doi.org/10.1093/mts/mty021 


\title{
Analyzing expressive differences in historic prelude sets using cluster analysis
}

\author{
Cameron J. Anderson ${ }^{1 \dagger}$ \\ Michael Schutz ${ }^{1,2}$ \\ ${ }^{1}$ Department of Psychology, Neuroscience \& Behaviour, McMaster University, Hamilton, Ontario, Canada \\ ${ }^{2}$ School of the Arts, McMaster University, Hamilton, Ontario, Canada \\ ${ }^{\dagger}$ Corresponding author: andersoc@mcmaster.ca \\ Author video presentation and/or other conference material: https://doi.org/10.17605/OSF.IO/QBZ7G
}

Diverging patterns in mode's associations with other musical cues carry distinctive expressive connotations. The major and minor modes' relationship with loudness and timing are often understood in absolute terms, with major pieces described as being faster and louder than their minor counterparts. However, recent findings suggest mode's relationship to other cues shifted markedly in the Romantic era (Horn \& Huron, 2015). Here we expand on previous work using cluster analysis to track expressive changes in music history, applying this technique to Bach's The Well-Tempered Clavier (1722) and Chopin's Preludes (1839). Analyzing clusters of each composer reveals empirical support for mode's changing expressive associations. Specifically, Chopin's minor pieces are distinguished by fast attack rates and louder dynamics than Bach's, consistent with research highlighting mode's changing musical meaning. In tandem with our team's work performing perceptual experiments with these pieces, this analysis provides a valuable complement to the small but growing body of research exploring changes in the use of emotive acoustic cues over musical history.

KEYWORDS: empirical musicology, cluster analysis, cue analysis, historic changes, preludes

\section{Biases in language production are reflected in musical improvisation: Evidence from large-scale corpus analysis}

\author{
Hannah M. Merseal ${ }^{1 \dagger}$ \\ Roger E. Beaty ${ }^{1}$ \\ Klaus Frieler ${ }^{2}$ \\ Martin Norgaard ${ }^{3}$ \\ Maryellen C. MacDonald ${ }^{4}$ \\ Daniel J. Weiss ${ }^{1}$ \\ ${ }^{1}$ Pennsylvania State University, University Park, PA, United States \\ ${ }^{2}$ University of Music Franz Liszt, Weimar, Germany \\ ${ }^{3}$ Georgia State University, Atlanta, GA, United States \\ ${ }^{4}$ University of Wisconsin - Madison, Madison, WI, United States \\ ${ }^{\dagger}$ Corresponding author: hmerseal@psu.edu \\ Author video presentation and/or other conference material: https://doi.org/10.17605/OSF.IO/B7KFV
}

Producing language involves the real-time sequencing of words into phrases, leading to considerable demands on working memory that can be relieved by ordering biases in spoken utterances. One such bias is called easy-first: the tendency for more easily-accessible phrases to occur earlier in an utterance, allowing for incremental planning of more complex phrases (MacDonald, 2013). Recent evidence suggests that this bias may extend beyond language to effect other domains involving real-time action sequencing. In the current study, we sought to test for the presence of the easy-first bias in a creative domain that similarly requires real-time action sequencing: musical improvisation (e.g. Pressing, 1987). Using a corpus of 456 transcribed improvisations from eminent jazz musicians (e.g., Charlie Parker, John Coltrane), we tested for easy-first on multiple definitions of easiness over the phrase and over the corpus: interval frequency, 
interval size, interval variety, pitch variety, and direction changes. Similar to language production, our findings suggest that expert improvisers consistently retrieve "easier" melodic sequences before generating more complex and novel sequences, indicating a similarity in the domain-general sequencing biases that facilitate the spontaneous production of music and language.

KEYWORDS: improvisation, corpus studies, language, action sequencing, hierarchical planning

\section{References}

MacDonald, M. C. (2013). How language production shapes language form and comprehension. Frontiers in Psychology, 4, 226, 1-16. https://doi.org/10.3389/fpsyg.2013.00226

Pressing, J. (1988). Improvisation: Methods and models. In J. A. Sloboda (Ed.), Generative processes in music, (129-178). Oxford. https://doi.org/10.1093/acprof:oso/9780198508465. 003.0007

\title{
Discovering the line of fifths in a large historical corpus
}

\author{
Fabian C. Moss ${ }^{1 \dagger}$ \\ Markus Neuwirth ${ }^{2}$ \\ Martin Rohrmeier ${ }^{1}$ \\ ${ }^{1}$ École Polytechnique Fédérale de Lausanne, Lausanne, Switzerland \\ ${ }^{2}$ Anton Bruckner University, Linz, Austria \\ $\dagger$ Corresponding author: fabian.moss@epfl.ch
}

Author video presentation and/or other conference material: https://doi.org/10.17605/OSF.IO/J5W6T

An increasing number of corpus studies relies on pitchclass distributions in order to infer characteristics of musical pieces under a historical perspective (Albrecht \& Shanahan, 2013; Albrecht \& Huron, 2014; Quinn \& White, 2017; Weiß, Mauch, \& Dixon, 2018; Yust, 2019; Harasim, Moss, Ramirez, \& Rohrmeier, 2021).

This contribution shows that the line of fifths (LOF; Temperley, 2000) is the fundamental underlying tonal space in a large historical corpus (ca. 1360-1940) of Western classical pieces in MusicXML format. Modeling the pieces' pitch-class distributions as vectors in a high-dimensional simplicial space and visualizing them via Principal Component Analysis reveals that the distance to the center of the LOF as well as the distinction between the natural (F, C, G, D, A, E, B) and the altered tonal pitch-classes (e.g., Abb, Db, F\#, C\#\#) are the most important factors for the dispersion of the data. These findings are robust with respect to different dimensionality reduction methods. Moreover, we introduce the concept of pitch-class coevolution and demonstrate that the LOF also underlies striking changes in the usage of pitch-classes between different historical periods.

Any empirical study is based on certain implicit or explicit modeling assumptions, some of which are given by the encoding of a corpus, e.g. whether enharmonic equivalence is assumed (e.g. MIDI-encoding) or not (e.g. MusicXML encoding). Relying on pitch-class distributions without assuming enharmonic equivalence, our findings emphasize the structural importance of the LOF for the organization of the pitchclass content of tonal music across a large historical timespan.

KEYWORDS: line of fifths, tonal pitchclasses, dimensionality reduction, pitch-class coevolution, corpus study 


\section{Does order matter? \\ Examining syntactic priming for tonal harmony using corpus-driven methods

\author{
David R. W. Sears ${ }^{1 \dagger}$ \\ Jonathan E. Verbeten ${ }^{2}$ \\ ${ }^{1}$ Performing Arts Research Laboratory (PeARL), Texas Tech University, Lubbock, TX, USA \\ ${ }^{2}$ School of Music, Texas Tech University, Lubbock, TX, USA \\ †Corresponding author: david.sears@ttu.edu \\ Author video presentation and/or other conference material: https://doi.org/10.17605/OSF.IO/QZFYA
}

Harmonic priming studies have repeatedly demonstrated that a tonal context primes listeners to expect a tonally related target chord (e.g., Bigand \& Pineau, 1997). Tillmann and Bigand (2001) have shown, however, that scrambling the order of chords in the context fails to inhibit processing, suggesting temporal order only weakly contributes to harmonic priming effects. Given the recent claims emerging out of corpus studies of tonal harmony that temporal order is a fundamental organizing principle in many musical styles (e.g., Moss et al., 2019), this study replicates Tillmann and Bigand's experimental design, but trains a probabilistic model on a corpus of chord annotations to select the scrambled conditions.

Sixteen 9-chord sequences were selected from Bach's chorales that either remain unchanged (thereby reflecting low temporal incoherence), or were scrambled to produce increasingly incoherent sequences (i.e., medium or high). To produce the scrambled conditions, a variable-order $n$-gram model (Pearce, 2005) was trained on a corpus of Roman numeral annotations from 100 Bach chorales. It then identified the scrambled versions that produced either medium or high estimates of model uncertainty. Sixty participants (30 musicians) indicated as quickly as possible whether the target chord was in or out of tune. In contrast to previous findings, correct response times from both groups replicated the ascending low-to-high staircase found in the model estimates. Nonmusicians also demonstrated an ascending low-to-high in-tune bias. To our knowledge, these data provide the first behavioral evidence that harmonic priming effects reflect the order of chords in a sequence.

KEYWORDS: tonal harmony, harmonic priming, n-gram model

\section{References}

Bigand, E., \& Pineau, M. (1997). Global context effects on musical expectancy. Perception and Psychophysics, 59(7), 1098-1107. https://doi.org/10.3758/BF03205524

Moss, F. C., Neuwirth, M., Harasim, D., \& Rohrmeier, M. (2019). Statistical characteristics of tonal harmony: A corpus study of Beethoven's string quartets. PLoS ONE, 14(6), e0217242. https://doi.org/10.1371/journal.pone.0217242

Pearce, M. T. (2005). The Construction and Evaluation of Statistical Models of Melodic Structure in Music Perception and Composition. (Unpublished doctoral dissertation), City University, London.

Tillmann, B., \& Bigand, E. (2001). Global context effect in normal and scrambled musical sequences. Journal of Experimental Psychology: Human Perception and Performance, 27(5), 1185-1196. https://doi.org/10.1037/0096-1523.27.5.1185 


\author{
Phrase-rhythmic norms in classical expositions: \\ A corpus study of Haydn's and Mozart's piano sonatas \\ Joseph Chi-Sing Siu ${ }^{1 \dagger}$ \\ ${ }^{1}$ Department of Music, University of Maryland Baltimore County, Baltimore, Maryland, USA \\ †Corresponding author: jsiu@umbc.edu \\ Author video presentation and/or other conference material: https://doi.org/10.17605/OSF.IO/ANGX4
}

Recent research in phrase rhythm and hypermeter have found that some phrase rhythmic patterns, such as the end-accented "closing-theme schema," appear regularly in certain parts of the Classical sonata exposition. These phrase rhythmic norms can, therefore, be regarded as the first-level defaults according to the compositional preference hierarchy in Hepokoski \& Darcy's Sonata Theory. However, besides the closing-theme schema, there has been no systematic study to examine the phrase rhythmic norms in the other locations of the sonata exposition. Therefore, this study aims to fill that research gap by conducting a corpus analysis of phrase rhythmic usage in all the first-movement piano sonata expositions composed by Haydn and Mozart (52 by Haydn and 19 by Mozart). This corpus study can then inform our understanding of phrase rhythmic default levels in Classical sonata form as well as any individual differences in the compositional styles of Haydn and Mozart.
In Haydn's and Mozart's piano sonatas, phrase rhythm in the primary themes are generally regular, while the secondary themes are mostly irregular. However, in the transitions, Haydn and Mozart have different first-level defaults, with regular phrase rhythm occurring more often in Haydn while irregular phrase rhythm is the norm in Mozart. When irregular phrase rhythms occur, Haydn's sonatas demonstrate a strong preference to focus on a single loosening device, nonquadruple hypermeasures, while Mozart's sonatas tend to also include the use of metrical reinterpretations and end-accented phrases. This study also reports on the phrase-rhythmic norms at the boundaries of the sonata formal sections and the hypermetric placements for the MCs, the dominant-locks, and the EECs.

KEYWORDS: phrase rhythm, hypermeter, Classical form, Sonata Theory, corpus study

\title{
Modeling melodic expectations with expectation networks
}

\author{
Niels Verosky ${ }^{1 \dagger}$ \\ Emily Morgan ${ }^{2}$ \\ ${ }^{1}$ San Francisco, CA, United States \\ ${ }^{2}$ University of California-Davis, Davis, CA, United States \\ ${ }^{\dagger}$ Corresponding author: niels.verosky@gmail.com \\ Author video presentation and/or other conference material: https://doi.org/10.17605/OSF.IO/U4CED
}

Expectation networks have been proposed as a computationally simple method for learning tonal expectations associated with individual scale degrees (Verosky, 2019). Using principles of activation and decay, expectation networks infer the expectation of encountering a given event type followed in the near (but not necessarily immediate) future by any other event type. The current work outlines how these learned expectations can be used to predict melody continuations and tests the predictions against listener responses to a melodic cloze task previously used to compare two other models of melodic expectation,
IDyOM and Temperley's Gaussian model (Morgan, Fogel, Nair, \& Patel, 2019; Pearce, 2005; Temperley, 2008). Results of multinomial logistic regression indicate that all three models account for unique variance in listener predictions, with coefficient estimates highest for expectation networks. Despite expectation networks' computational simplicity relative to IDyOM, direct comparisons between IDyOM and expectation networks similarly yielded higher coefficient estimates for the latter. Although all three models are limited in their ability to incorporate global, hierarchical information about pitch structure, 
expectation networks seem to benefit from a tendency to predict all three notes of the tonic triad at cadence points while ranking the tonic as the most probable continuation. Our findings suggest that generalized scale degree expectations as captured by expectation networks, stereotypical pitch sequences as captured by IDyOM, and immediate intervallic expectations as captured by Temperley's model all factor into real-time listener predictions to varying extents, highlighting several possible areas for future work.

KEYWORDS: statistical learning, melody, expectation, prediction, expectation networks 


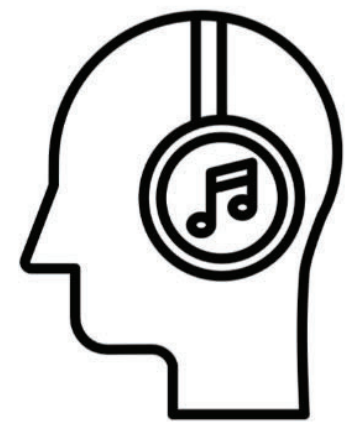

\section{Emotion}

Future Directions of Music Cognition

February - May 2021

Ohio State University

Virtual 


\title{
A comparison of presence and emotion between immersive virtual reality and desktop displays for musical multimedia
}

\author{
Thery Al Alam ${ }^{\dagger}$ \\ Nicola Dibben \\ Department of Music, University of Sheffield, Sheffield, UK \\ † Corresponding author: thery.alam@live.com \\ Published 16 December 2021; https://doi.org/10.18061/FDMC.2021.0017 \\ Author video presentation and/or other conference material: https://doi.org/10.17605/OSF.IO/86K93
}

\begin{abstract}
Visual and auditory immersion influence sense of presence, and in some cases emotional response. However, these have previously been tested separately and it is unknown whether and how immersion influences experiences combining both, as found in musical multimedia. A within-participants experiment compared participants' sense of presence (feeling of 'being there'), and induced and perceived emotions, when experiencing a classical concert performance presented over headphones on a desktop PC (2D video with headlocked sound reproduction) or in virtual reality (VR) (head-mounted display presenting $360^{\circ}$ video and reactive spatial sound reproduction). Emotion was measured by self-report questionnaires based on a three-dimensional model (pleasantness, tense arousal and energetic arousal) and presence was measured using four items (Sense of physical space, Engagement, Ecological Validity and Negative Effects). Participants experienced a significant increase in pleasantness and all dimensions of presence in VR compared to the desktop condition. Ratings of felt arousal and perceived emotions did not significantly differ between conditions. The "engagement" component of presence was positively correlated with emotion felt and perceived, whereas "ecological validity" and "sense of physical space" were related to emotions experienced in the desktop condition only. The role of presence should therefore be considered when investigating emotional responses to musical multimedia.
\end{abstract}

KEYWORDS: virtual reality, music, emotion, immersion, presence

\section{Introduction}

'Immersive' technologies for three-dimensional sound and visual reproduction present new means to engage and emotionally affect audiences. Understanding the experience of immersive musical multimedia can extend existing theories of emotional responses to multimedia and potentially inform their design.

Immersive VR is a market-leading technology that enables a high sense of interaction with multimodal experiences. A VR environment often consists of a 3D audiovisual space with visual information presented in $360^{\circ}$ and spatialised audio that responds to users' movement. As we use the term, immersion describes objective properties of the technology (Slater \& Wilbur, 1997). Virtual environments (VEs) mediated by VR technologies ('Immersive VR') may be considered 'high immersion' because they usually involve more than two spatially oriented sensorial modalities, a highfidelity representation of sensorial information and the ability to modify the VE in response to body movement. This contrasts with the most commonly encountered technologies for audio-visual consumption, namely desktop and mobile video. Environments mediated by a desktop PC ('Desktop Video') may be considered 'low immersion' because they offer limited field of vision and sound reproduction and do not respond to body motion (Miller \& Bugnariu, 2016).

Immersive VR increases users' sense of presence, or feeling of 'being there', in a VE (Slater \& Wilbur, 1997). Presence is associated with allocation of attention to the VE and reduction in resources available for processing other stimuli (Kober \& Neuper, 2012), although the definition of presence remains controversial (Coelho et al., 2006). In this paper, 'presence' describes the feeling evoked in a user of being physically located in a display environment mediated by technology (e.g. in a video, game or film), and comprises different experiential domains (Lessiter et al., 2001).

There is evidence that presence is heightened by increased immersion: in the visual domain, presence increases with headset relative to desktop display (Nichols et al., 2000), and with stereoscopic information, head tracking and field of view (Hendrix \& Barfield, 1996); in the auditory domain, presence increases with spatialized sound relative to no sound or non-spatialized sound (Kobayashi et al., 2015). However, it is difficult to predict the sense of presence in a VE solely by manipulation of technological factors due to other variables, such as previous experience with VR (Coelho et al., 2006).

Whether emotion is influenced by immersion is less clear as is the relationship between presence and 
emotions. Some authors suggest that presence and emotions are related only when the media content is emotionally arousing (Freeman et al., 2005) but further evidence is required.

This study investigated whether levels of visual and auditory immersion together influence emotional experiences of music. We compared induced and perceived emotions, sense of presence, and the relationship between presence and perceived and induced emotions with a Desktop Video (low immersion) and Immersive VR (high immersion) musical multimedia product.

We expected that: (H1): Participants' sense of presence will be higher in the high than the low immersion condition. (H2): Ratings of induced and perceived emotions will differ between the high and low immersion conditions. (H3): sense of presence will correlate with induced and perceived emotions.

\section{Method}

\section{Participants}

The twenty-eight participants ( 13 female; $M$ age $=26.4$ years, $\mathrm{SD}=6.98$ years, range $19-52$ years) comprised students $(82.1 \%)$ and staff $(17.8 \%)$ at the University of Sheffield, of varied nationality, most of whom had prior experience with VR (82.2\%). Participants had no history of seizures, blackouts or psychiatric problems nor implanted medical devices. Participants gave informed written consent in keeping with approval granted by the Ethics Committee of the University of Sheffield.

\section{Stimuli}

The experimental manipulation of immersion comprised a 'high immersion' head-mounted display (HMD) $360^{\circ}$ spherical video (hereafter 'Immersive VR') and 'low immersion' desktop PC (2D) video (hereafter 'Desktop Video') of the same performance of Mozart's 'Agnus Dei', from Mozart's Coronation Mass (mass No.15 in C Major, K.317), scored for soloists, choir and orchestra, and lasting 4 minutes. This duration was sufficient to induce emotions ( $>30 \mathrm{~s}$ ) (Garrido, $2014)$ and avoid adverse physiological reactions $(<20$ mins) (Regan, 1995). This performance is in slow (54 bpm) three-four time, with a relatively simple texture and structure featuring solo soprano and orchestra in a cathedral without an audience.

The VE "Mozart 360: Agnus Dei" (Within Unlimited, 2016) places the viewer at a fixed position that follows the movement of a camera amongst the orchestra. The video is 360 degree spherical and the sound, described as ' $3 \mathrm{D}$ spatial audio' is reactive to head rotation. Participants can interact with the VE via head rotation. The video includes neutrally valenced text overlays that directs the experiencer's attention to particular musicians and compositional processes. An additional VE of 'Google earth VR' (Google, 2016) was used as an introductory activity to reduce novelty effects (Hopp \& Gangadharbatla, 2016), and was chosen because it was familiar to participants from Google Street View technology and was unrelated in content to the experimental stimuli.

The 2D music video was created by recording a playback of 'Mozart 360: Agnus Dei' (Within Unlimited, 2016) from the WITHIN mobile application on an iPhone 8 (Software version 12.2). The movement of the camera, determined by the authors, tracked the appearance of the text on the image. The resulting properties of the sound were $108 \mathrm{kbps}$ in mono transmitted on two channels with $44.100 \mathrm{kHz}$ sample rate. The total bit rate for the image was $3206 \mathrm{kbps}$ at 59.84 frames/second. Unlike the VE version, the sound was headlocked.

\section{Equipment}

Stimuli were presented via i) desktop PC (including 17inch monitor with a $2560 \times 1440$ pixels resolution, a $60 \mathrm{~Hz}$ refresh rate and Intel ${ }^{\circledR}$ Xeon ${ }^{\circledR}$ CPU E5-2630 v3 (a) $2.40 \mathrm{GHz}$ as processor) and, ii) VR HMD with two base stations tracking head movement (HTC vive GPU NVIDIA Quadro K5200, GPU driver 416.78, the custom resolution was $1524 \times 1696$ for each eye with $90 \mathrm{~Hz}$ refresh rate). Music was presented over circumaural headphones (Sony MDR-V150).

\section{Measures}

The Goldsmiths Musical Sophistication Index (GoldMSI) indicates the population sample is representative of the level of musical sophistication in the UK population $(M d n=71.5, S D=19.25$, range $=40-105)$ (Müllensiefen et al., 2014). Familiarity with the piece and the performance, and attention to the music were each measured on 5-point rating scales $(1=$ not at all and $5=$ very). Perceived and felt emotions in the music were measured on a 9-point bipolar scale for each emotion and emotion dimension (felt pleasantness, felt tense arousal, felt energetic arousal, perceived pleasantness, perceived tense arousal and perceived energetic arousal) (Schimmack \& Grob, 2000). The ITC-sense of presence inventory was chosen due to its use in cross-media comparisons (Lessiter et al., 2001). Background information collected included participants' experience with audio media. 


\section{Procedure}

Participants were tested individually. They were screened for general well-being, completed the background information questionnaire and Gold-MSI. Differences between emotions induced and perceived in music were explained. Participants then spent 3 to 5 minutes in 'Google Earth VR' VE presented through a HMD. They walked in a limited room scale and explored the environment using controllers and head motion. Next, they were instructed to focus on the music in the two conditions, to isolate the implications for presentation of the musical performance. Participants experienced the Desktop Video while seated at a desktop screen. Then completed questionnaires on familiarity, attention, emotions and presence. In the Immersive VR condition, participants stood, and explored the VE (presented through a HMD) via head rotation. Afterwards they completed the same questionnaires as for the Desktop Video condition. The order of presentation of the conditions was reversed for half the participants. The same headphones were used in both conditions and the sound level was kept constant. The experiment duration was 45 to 60 minutes.

\section{Results}

Group means were calculated for presence's subscales, emotion felt and perceived, familiarity, and levels of attention. Participants' familiarity with the composition $(M=1.96, S D=1.23)$ and the performance $(M=1.6, S D$ $=1.07)$ were low. Levels of attention to the music were very similar in the Desktop Video $(M=3.43, S D=0.83)$ and Immersive VR $(M=3.43, S D=1.1)$ conditions. There were no significant carry over effects. These variables were excluded from further analysis.

Scores were compared to check for differences in the experience of the Desktop Video and Immersive VR versions. Variables were normally distributed except for felt tense arousal, spatial presence (i.e. sense of physical space) and negative effects in the Desktop Video condition. A paired t-test was run to compare the ratings of perceived pleasantness, perceived tense and energetic arousal, felt pleasantness and energetic arousal, ecological validity and engagement in the two conditions (Table1). Wilcoxon signed-rank test compared the ratings of felt tense arousal, spatial presence and negative effects in the two conditions (Table 2).

Ratings were significantly higher in Immersive VR for felt pleasantness $t(27)=2.09, p=.047, d=.45$, and all measures of presence: engagement $t(27)=8.15, p$
$<.001, d=2.01$, spatial presence $(z=-4.47, p<.001, r$ $=-.844)$, ecological validity $t(27)=6.08, p<.001, d=$ 1.17 , and negative effects $(z=-3.04, p<.001, r=-.57)$ (calculated as one-tailed comparisons due to the directional hypothesis). However, there was no significant difference in the ratings of perceived pleasantness $t(27)=1.78, p=.086, d=.29$, perceived energetic arousal $t(27)=1.87, p=.073, d=.37$, perceived tense arousal $t(27)=.72, p=.477, d=.15$, felt energetic arousal $t(27)=1.31, p=.202, d=.35$ nor felt tense arousal $(z=-1.19, p=.234, r=-.22)$ between the two conditions.

Table 1: Means and standard deviations of Engagement, Ecological validity, Pleasantness, Energetic arousal and Tense arousal.

\begin{tabular}{|c|c|c|c|c|}
\hline \multirow[t]{2}{*}{ Variables } & \multicolumn{2}{|c|}{ Desktop } & \multicolumn{2}{|c|}{ VR } \\
\hline & $M$ & $S D$ & $M$ & $S D$ \\
\hline Engagement & 2.79 & 0.70 & 4.02 & 0.53 \\
\hline Ecological validity & 2.95 & 0.85 & 3.82 & 0.64 \\
\hline $\begin{array}{c}\text { Perceived } \\
\text { pleasantness }\end{array}$ & 6.36 & 1.45 & 6.75 & 1.24 \\
\hline $\begin{array}{c}\text { Perceived energetic } \\
\text { arousal }\end{array}$ & 4.39 & 1.23 & 4.96 & 1.82 \\
\hline $\begin{array}{l}\text { Perceived tense } \\
\text { arousal }\end{array}$ & 4.21 & 2.04 & 3.89 & 2.36 \\
\hline Felt pleasantness & 6.54 & 1.57 & 7.21 & 1.42 \\
\hline $\begin{array}{l}\text { Felt energetic } \\
\text { arousal }\end{array}$ & 4.29 & 1.44 & 4.82 & 1.63 \\
\hline
\end{tabular}

Table 2: Medians and interquartile ranges of Spatial presence, Negative effects, Tense arousal.

\begin{tabular}{|c|l|l|l|l|}
\hline \multirow{2}{*}{ Variables } & \multicolumn{2}{|c|}{ Desktop } & \multicolumn{2}{c|}{ VR } \\
\cline { 2 - 5 } & \multicolumn{1}{|c|}{$\boldsymbol{M d n}$} & \multicolumn{1}{|c|}{$\boldsymbol{I Q R}$} & \multicolumn{1}{c|}{$\boldsymbol{M d n}$} & \multicolumn{1}{c|}{ IQR } \\
\hline Spatial presence & 1.74 & 1.08 & 3.44 & 1.04 \\
\hline Negative effects & 1.57 & 0.83 & 2.17 & 1.12 \\
\hline Felt tense arousal & 3 & 2 & 3 & 3 \\
\hline
\end{tabular}

Spearman's correlation test revealed significant associations between factors of presence and induced and perceived emotions in both conditions (Table 3 and 4). 
Table 3: Spearman's correlations between the sense of presence and felt and perceived emotions in the Desktop Video condition.

\begin{tabular}{|c|l|l|l|l|}
\hline & $\begin{array}{c}\text { Spatial } \\
\text { presence }\end{array}$ & $\begin{array}{c}\text { Engage- } \\
\text { ment }\end{array}$ & $\begin{array}{c}\text { Ecologic- } \\
\text { al validity }\end{array}$ & $\begin{array}{c}\text { Negative } \\
\text { effects }\end{array}$ \\
\hline $\begin{array}{c}\text { Perceived } \\
\text { pleasant- } \\
\text { ness }\end{array}$ & $\begin{array}{l}\mathrm{r}=+.45 \\
(p=.016)\end{array}$ & $\begin{array}{l}\mathrm{r}=+.63 \\
(p<.001)\end{array}$ & $\begin{array}{l}\mathrm{r}=+.55 \\
(p=.002)\end{array}$ & $\begin{array}{r}\mathrm{r}=-.16 \\
(p=.425)\end{array}$ \\
\hline $\begin{array}{c}\text { Perceived } \\
\text { energetic } \\
\text { arousal }\end{array}$ & $\mathrm{r}=+.05$ \\
$(p=.786)$ & $\mathrm{r}=+.21$ & $\mathrm{r}=+.28$ \\
$(p=.273)$ & $(p=.145)$ & $\begin{array}{l}\mathrm{r}=-.28 \\
(p=.149)\end{array}$ \\
\hline $\begin{array}{c}\text { Perceived } \\
\text { tense } \\
\text { arousal }\end{array}$ & $\begin{array}{l}\mathrm{r}=-.19 \\
(p=.323)\end{array}$ & $\begin{array}{r}\mathrm{r}=-.13 \\
(p=.494)\end{array}$ & $\begin{array}{l}\mathrm{r}=-.11 \\
(p=.581)\end{array}$ & $\begin{array}{l}\mathrm{r}=+.01 \\
(p=.945)\end{array}$ \\
\hline $\begin{array}{c}\text { Felt } \\
\text { pleasant- } \\
\text { ness }\end{array}$ & $\begin{array}{l}\mathrm{r}=+.39 \\
(p=.04)\end{array}$ & $\begin{array}{l}\mathrm{r}=+.66 \\
(p<.001)\end{array}$ & $\begin{array}{l}\mathrm{r}=+.60 \\
(p<.001)\end{array}$ & $\begin{array}{r}\mathrm{r}=-.38 \\
(p=.047)\end{array}$ \\
\hline $\begin{array}{c}\text { Felt } \\
\text { energetic } \\
\text { arousal }\end{array}$ & $\begin{array}{l}\mathrm{r}=+.24 \\
(p=.222)\end{array}$ & $\begin{array}{l}\mathrm{r}=+.38 \\
(p=.047)\end{array}$ & $\begin{array}{l}\mathrm{r}=+.34 \\
(p=.077)\end{array}$ & $\begin{array}{l}\mathrm{r}=-.28 \\
(p=.145)\end{array}$ \\
\hline $\begin{array}{c}\text { Felt tense } \\
\text { arousal }\end{array}$ & $\begin{array}{l}\mathrm{r}=-.19 \\
(p=.34)\end{array}$ & $\begin{array}{l}\mathrm{r}=-.34 \\
(p=.075)\end{array}$ & $\begin{array}{l}\mathrm{r}=-.27 \\
(p=.162)\end{array}$ & $\begin{array}{l}\mathrm{r}=+.31 \\
(p=.111)\end{array}$ \\
\hline
\end{tabular}

Table 4: Spearman's correlations between the sense of presence and felt and perceived emotions in the Immersive VR condition.

\begin{tabular}{|c|c|c|c|c|}
\hline & $\begin{array}{c}\text { Spatial } \\
\text { presence }\end{array}$ & $\begin{array}{c}\text { Engage- } \\
\text { ment }\end{array}$ & $\begin{array}{l}\text { Ecologic- } \\
\text { al validity }\end{array}$ & $\begin{array}{c}\text { Negative } \\
\text { effects }\end{array}$ \\
\hline $\begin{array}{c}\text { Perceived } \\
\text { pleasant- } \\
\text { ness }\end{array}$ & $\begin{array}{l}\mathrm{r}=+.21 \\
(p=.275)\end{array}$ & $\begin{array}{l}\mathrm{r}=+.49 \\
(p=.008)\end{array}$ & $\begin{array}{l}\mathrm{r}=+.24 \\
(p=.229)\end{array}$ & $\begin{array}{l}\mathrm{r}=-.28 \\
(p=.150)\end{array}$ \\
\hline $\begin{array}{c}\text { Perceived } \\
\text { energetic } \\
\text { arousal }\end{array}$ & $\begin{array}{l}\mathrm{r}=+.12 \\
(p=.555)\end{array}$ & $\begin{array}{l}\mathrm{r}=+.16 \\
(p=.410)\end{array}$ & $\begin{array}{l}\mathrm{r}=+.09 \\
(p=.637)\end{array}$ & $\begin{array}{l}\mathrm{r}=+.05 \\
(p=.792)\end{array}$ \\
\hline $\begin{array}{c}\text { Perceived } \\
\text { tense } \\
\text { arousal }\end{array}$ & $\begin{array}{l}\mathrm{r}=-.07 \\
(p=.740)\end{array}$ & $\begin{array}{l}\mathrm{r}=-.05 \\
(p=.809)\end{array}$ & $\begin{array}{l}\mathrm{r}=-.16 \\
(p=.424)\end{array}$ & $\begin{array}{l}\mathrm{r}=+.39 \\
(p=.042)\end{array}$ \\
\hline $\begin{array}{c}\text { Felt } \\
\text { pleasant- } \\
\text { ness }\end{array}$ & $\begin{array}{l}\mathrm{r}=+.33 \\
(p=.087)\end{array}$ & $\begin{array}{l}\mathrm{r}=+.76 \\
(p<.001)\end{array}$ & $\begin{array}{l}\mathrm{r}=+.41 \\
(p=.030)\end{array}$ & $\begin{array}{l}\mathrm{r}=-.44 \\
(p=.021)\end{array}$ \\
\hline $\begin{array}{c}\text { Felt } \\
\text { energetic } \\
\text { arousal }\end{array}$ & $\begin{array}{l}\mathrm{r}=0 \\
(p=.999)\end{array}$ & $\begin{array}{l}\mathrm{r}=+.22 \\
(p=.267)\end{array}$ & $\begin{array}{l}\mathrm{r}=+.02 \\
(p=.932)\end{array}$ & $\begin{array}{l}\mathrm{r}=-.2 \\
(p=.307)\end{array}$ \\
\hline $\begin{array}{c}\text { Felt tense } \\
\text { arousal }\end{array}$ & $\begin{array}{l}\mathrm{r}=-.16 \\
(p=.406)\end{array}$ & $\begin{array}{l}\mathrm{r}=-.26 \\
(p=.177)\end{array}$ & $\begin{array}{l}\mathrm{r}=-.16 \\
(p=.407)\end{array}$ & $\begin{array}{l}\mathrm{r}=+.48 \\
(p=.011)\end{array}$ \\
\hline
\end{tabular}

\section{Discussion}

The study investigated whether people's experience of musical multimedia differs with audiovisual formats and their associated levels of immersion. It revealed a significantly higher sense of presence, specifically of engagement and ecological validity, in an Immersive VR musical experience as opposed to the Desktop Video version, suggesting that presence increases with the level of immersion afforded by the technology (H1). This is the first time to our knowledge that this relationship has been empirically confirmed with commercially available musical multimedia.

Level of immersion had no significant effect on the perception nor induction of emotion, with the exception of increased pleasantness reported with the Immersive VR condition (H2). The lack of influence of immersion on other aspects of emotional experience may be due to the low-arousal character of the stimulus (Freeman et al., 2005); it scored a maximum mean rating of 3.89 and 4.96 on a 9-point scale respectively for perceived tense and energetic arousal.

The results confirmed associations between presence, and emotion felt and perceived (H3) for both levels of immersion in this low arousal performance, despite previous suggestions that presence and emotions are only related in the case of arousing stimuli (Freeman et al., 2005). The 'engagement' factor of presence was positively correlated with felt and perceived pleasantness in both conditions, indicating its importance regardless of the immersive properties of the technology. 'Ecological validity' and 'physical presence' seem to play a more important role in the Desktop Video condition: both factors correlated positively with perceived and felt pleasantness, whereas in Immersive VR, 'ecological validity' was positively associated with felt pleasantness only. It could be that in media experiences that lack ecological validity and do not elicit strong feelings of presence, participants' affective experience is related to the resolution of the presentation and how much interaction and control is offered, since these variables influence presence (Lessiter et al., 2001). Finally, the relationship between 'negative effects' and emotion seems to be more comprehensive in Immersive VR: as negative effects of Immersive VR increased, so too did perceived and felt tense arousal, and felt pleasantness decreased, whereas negative effects of the Desktop Video condition were associated with a reduction in felt pleasantness only. Further empirical studies are needed. First, both highand low-arousal stimuli should be included to determine 
whether immersion influences other dimensions of emotional experience. Second, features which coincide in the multimedia products (i.e. visual immersion (2D versus $360^{\circ}$ video), auditory immersion (mono versus 3D spatialised sound), interactivity (none versus head rotation) and body position (sitting versus standing)) should be investigated together and separately to understand how they interact to influence presence and emotions.

\section{Conclusion}

This study provides empirical confirmation that Immersive VR can afford an enhanced sense of 'being present' at virtual music performances relative to Desktop Video and suggests that presence is related to users' emotional experience of musical multimedia. Finally, the study suggests that presence should be considered in theories of musical emotion applied to multimedia contexts.

\section{References}

Coelho, C., Tichon, J. G., Hine, T. J., Wallis, G. M., \& Riva, G. (2006). Media presence and inner presence: the sense of presence in virtual reality technologies. From communication to presence: Cognition, emotions and culture towards the ultimate communicative experience (pp. 25-45). IOS Press, Amsterdam.

Freeman, J., Lessiter, J., Pugh, K., \& Keogh, E. (2005). When presence and emotion are related, and when they are not. Proceedings of the 8th annual international workshop on presence (PRESENCE 2005) (pp. 213-219). International Society for Presence Research.

Garrido, S. (2014). A systematic review of the studies measuring mood and emotion in response to music. Psychomusicology: Music, Mind, and Brain, 24(4), 316. https://doi.org/10.1037/pmu0000072

Google (2016). Google Earth VR [Computer software] Retrieved from https://store.steampowered.com/app/348250/Google Ea rth VR/

Hendrix, C., \& Barfield, W. (1996). Presence within virtual environments as a function of visual display parameters. Presence: Teleoperators \& Virtual Environments, 5(3), 274-289. https://doi.org/10.1162/pres.1996.5.3.274

Hopp, T., \& Gangadharbatla, H. (2016). Novelty effects in augmented reality advertising environments: The influence of exposure time and self-efficacy. Journal of Current Issues \& Research in Advertising, 37(2), 113130. https://doi.org/10.1080/10641734.2016.1171179

Kobayashi, M., Ueno, K., \& Ise, S. (2015). The effects of spatialized sounds on the sense of presence in auditory virtual environments: a psychological and physiological study. Presence: Teleoperators and Virtual Environments, 24(2), 163-174. https://doi.org/10.1162/PRES a 00226

Kober, S. E., \& Neuper, C. (2012). Using auditory eventrelated EEG potentials to assess presence in virtual reality. International Journal of Human-Computer Studies, $\quad 70(9), \quad 577-587$. https://doi.org/10.1016/j.ijhcs.2012.03.004

Lessiter, J., Freeman, J., Keogh, E., \& Davidoff, J. (2001). A cross-media presence questionnaire: The ITC-Sense of Presence Inventory. Presence: Teleoperators \& Virtual Environments, 10(3), 282-297. https://doi.org/10.1162/105474601300343612

Miller, H. L., \& Bugnariu, N. L. (2016). Level of immersion in virtual environments impacts the ability to assess and teach social skills in autism spectrum disorder. Cyberpsychology, Behavior, and Social Networking, 19(4), 246-256. https://doi.org/10.1089/cyber.2014.0682

Müllensiefen, D., Gingras, B., Musil, J., \& Stewart, L. (2014). The musicality of non-musicians: an index for assessing musical sophistication in the general population. PloS one, 9(2), e89642. https://doi.org/10.1371/journal.pone.0089642

Nichols, S., Haldane, C., \& Wilson, J. R. (2000). Measurement of presence and its consequences in virtual environments. International Journal of HumanComputer Studies, 52(3), 471-491. https://doi.org/10.1006/ijhc.1999.0343

Regan, C. (1995). An investigation into nausea and other side-effects of head-coupled immersive virtual reality. Virtual Reality, $1(1), \quad 17-31$. https://doi.org/10.1007/BF02009710

Schimmack, U., \& Grob, A. (2000). Dimensional models of core affect: A quantitative comparison by means of structural equation modeling. European Journal of Personality, 14(4), 325-345. https://doi.org/10.1002/10990984(200007/08)14:4<325::AID-PER380>3.0.CO;2-I

Slater, M., \& Wilbur, S. (1997). A framework for immersive virtual environments (FIVE): Speculations on the role of presence in virtual environments. Presence: Teleoperators \& Virtual Environments, 6(6), 603-616. https://doi.org/10.1162/pres.1997.6.6.603

Within Unlimited, Inc. (2016). Mozart 360: Agnus Dei. WITHIN [Computer software]. Retrieved from : https://store.steampowered.com/app/458890/WITHIN 


\author{
Dropping the bass: The relationship between heart rate and \\ expectation in electronic dance music (A preliminary report) \\ Emily $\mathrm{Chen}^{1 \dagger}$ \\ Ellena Yohannes ${ }^{1}$ \\ Samantha Burgess ${ }^{1}$ \\ Daniel Shanahan ${ }^{1}$ \\ ${ }^{1}$ The Ohio State University, Columbus, Ohio, USA \\ † Corresponding author: chen.8217@osu.edu
}

Published 16 December 2021; https://doi.org/10.18061/FDMC.2021.0018

Author video presentation and/or other conference material: https://doi.org/10.17605/OSF.IO/XDU2J

\begin{abstract}
The relationship between musical expectation and physiological stress has been examined with increasing frequency in recent years (Huron, 2006; Tarr, et al. 2016). Stress induced from either an unexpected musical event can be studied through the measurement of heart rate (Steinbeis et al., 2006). Previous studies have focused on the interaction between global features (e.g., beat subdivision and pitch height) and stress (Liu, et al. 2018), while others have found no effect of local harmonic violations on heart rate (Steinbeis et al., 2006) and little effect of rhythmic entrainment (Mütze, Kopiez, \& Wolf, 2020). More research is needed that focuses on the relationship between predictors of stress through local textural and rhythmic features inherent in musical genres. This study examines the role of specific musical and stylistic features on heart rate. Specifically, we examined elements of electronic dance music (EDM), including fulfilled and avoided "bass drops", defined as a climax of beat subdivision and pitch height which acts as a resolution for the musical phrase. Due to COVID-19, the data collection for this study is still ongoing, although we hypothesize that musical markers of stress, such as the avoidance of bass drops, are positively correlated with a heart rate.
\end{abstract}

KEYWORDS: Heart rate, stress, electronic dance music, EDM, musical expectation

\section{Introduction}

Listening to music has been attributed to a number of health benefits. In a meta-analysis of 400 studies that examine the neurochemical effects of music, researchers found that listening to music can broadly translate into physiological benefits (Chanda and Levitin, 2013). For example, music can help slow a person's heart rate, lower blood pressure and ultimately reduce stress hormones such as adrenaline and cortisol. In a study testing the effect of the human stress response to music, 60 females were categorized into one of three groups (classical music, sound of water, and no acoustic stimulation), and were given a standardized psychosocial stress test. Salivary cortisol levels, heart rate (HR), respiratory sinus arrhythmia (RSA), subjective stress perception and anxiety were analyzed to show whether or not music impacted the human stress response, and as a result, the study concluded that music does impact the psychobiological stress system (Thoma, 2013). Other studies have similarly shown that specifically, certain aspects of music can affect the degree of stress or relaxation a person experiences (de Witte et. al, 2020; Umbrello et. al, 2019). Van Dyck conducted research on auditory stimuli tempi and its effects on listeners' heart rate using photoplethysmography (PPG), and found that the tempo of music has an effect on human physiological responses. He concluded that faster music can trigger arousal, and thus increases heart rate (Van Dyck, 2017). However, there is a lack of research in the field that manipulates the specific musical features to see if an expectation is or is not fulfilled. Furthermore, if the expectation is not fulfilled, we would like to examine if it leads to an increase in stress.

Musical expectation can lead to feelings of relaxation or tension in listeners with tension followed by relaxation in Western music. Oftentimes, there is no dissonance in the end of a piece because of the tendency what Lerdahl and Jackendoff (1983) refer to as a "happy-end". Previous studies have demonstrated that musical expectations can contribute to our physiological and emotional responses through measuring heart rate, electrodermal activity, and electroencephalogram (EEG) activity (Steinbeis et. al, 2006). This study further investigates whether or not the reason for a feeling of fulfillment or unfulfillment of expectation is due to activating the human stress response (Tarr, et al. 2016).

We are currently examining whether or not an unfulfilled expectation increases cortisol levels and 
elicits a demonstrably different reaction in comparison to musical stimuli that contains a fulfilled expectation. While previous research has examined the relationship between music and stress levels (Schaefer, et al, 2015), more research is needed that centers on specific stylistic predictors of stress. More specifically, we will be observing stylistic predictors of stress present in electronic dance music since there is little research that examines musical expectations in contemporary music. More research is needed that examines the relationship between one's musical expectations and their musical preferences. For this study, we aim to use excerpts of electronic dance music (EDM) which is a genre of music that has not been used as often as genres such as classical music or pop/rock.

This study aims to look closely into how the fulfillment of stylistic musical expectations or lack thereof influences one's stress response. Additionally, this study aims to observe how natural exposure to different musical genres may impact stress responses to musical stimuli. The study will also aim to examine if differences in one's overall self-perceived emotional state will affect their HR in response to the musical stimuli. With this study, we hope to focus on the question regarding how musical features that increase expectations, such as those in bass drops in EDM can be measured in terms of HR.

\section{Method}

Prior to the experiment, participants would be asked to download the app, Cardiio, which is the instrument used to measure heart rate. Each participant will schedule a Zoom meeting that will last approximately 10 minutes long. The Research Assistants will administer the survey containing questions from the Goldsmiths Musical Sophistication Index as well as demographic questions. Before any excerpts are played, the participants heart rate will be recorded using the PPG app. The participant will be instructed to show the researchers the results on their phone. Research Assistants will present one musical stimulus (either the EDM with a bass drop, or the EDM without a bass drop). After the presentation of the stimulus, the participant will record their heart rate again using the app.

Each "odd" numbered participant will listen to the excerpt with bass drop and each "even" numbered participant will listen to the excerpt without bass drop. Participants will be either listening to EDM with a bass drop or EDM without a bass drop.

\section{Results and Discussion}

As a result of COVID-19, we have not been able to collect data at this point, but are hoping to be able to in the near future with this new online paradigm. We have instead chosen to include our method and hypotheses here as a form of preregistration. An accompanying preregistration can be found on aspredicted.org.[1]

\section{Conclusion}

We are hoping that results will demonstrate the effects of specific musical features of EDM on heart rate and other possible physiological markers of stress.

\section{Endnotes}

[1] For a copy of the preregistered report, see: https://aspredicted.org/an9tb.pdf.

\section{References}

Chanda, M. L., \& Levitin, D. J. (2013). The neurochemistry of music. Trends in Cognitive Sciences, 17(4), 179-193. https://doi.org/10.1016/j.tics.2013.02.007

de Witte, M., Spruit A., van Hooren, S., Moonen X., Stams, G.-J. (2020). Effects of music interventions on stressrelated outcomes: a systematic review and two metaanalyses, Health Psychology Review, 14:2, 294-324. https://doi.org/10.1080/17437199.2019.1627897

Huron, D. (2008). Sweet Anticipation: Music and the Psychology of Expectation. MIT Press. https://doi.org/10.7551/mitpress/6575.001.0001

Lerdahl F., Jackendoff, R (1983). A Generative Theory of Tonal Music. Cambridge, MA: MIT Press.

Liu Y, Liu G, Wei D, Li Q, Yuan G, Wu S, Wang G and Zhao X (2018). Effects of Musical Tempo on Musicians' and Non-musicians' Emotional Experience When Listening to Music. Front. Psychol. 9:2118. https://doi.org/10.3389/fpsyg.2018.02118

Müllensiefen, D., Gingras, B., Musil, J., \& Stewart, L. (2014). The musicality of non-musicians: An index for assessing musical sophistication in the general population. PLOS ONE, 9(2), Article e89642. https://doi.org/10.1371/journal.pone.0089642

Mütze, H., Kopiez, R., \& Wolf, A. (2020). The effect of a rhythmic pulse on the heart rate: Little evidence for rhythmical 'entrainment' and 'synchronization'. Musicae Scientiae. 24. https://doi.org/10.1177/1029864918817805

Schäfer, T., Huron, D., Shanahan, D., \& Sedlmeier, P. (2015). The sounds of safety: stress and danger in music perception. Frontiers in Psychology, 6. https://doi.org/10.3389/fpsyg.2015.01140

Steinbeis N, Koelsch S, Sloboda JA (2006). The role of harmonic expectancy violations in musical emotions: 
evidence from subjective, physiological, and neural responses. Journal of Cognitive Neuroscience, 18:1380 1393. https://doi.org/10.1162/jocn.2006.18.8.1380

Tarr, B., Launay, J., \& Dunbar, R. I. M. (2016). Silent disco: Dancing in synchrony leads to elevated pain thresholds and social closeness. Evolution and Human Behavior: Official Journal of the Human Behavior and Evolution Society, $37(5)$,

343-349. https://doi.org/10.1016/j.evolhumbehav.2016.02.004

Thoma, M. V., La Marca, R., Brönnimann, R., Finkel, L., Ehlert, U., \& Nater, U. M. (2013). The Effect of Music on the Human Stress Response. PLOS ONE, 8(8), e70156. https://doi.org/10.1371/journal.pone.0070156

Umbrello, M., Sorrenti, T., Mistraletti, G., Formenti, P., Chiumello, D. \& Terzoni, S. (2019). Music therapy reduces stress and anxiety in critically ill patients: A systematic review of randomized clinical trials. Minerva Anestesiologica. 85. https://doi.org/10.23736/S03759393.19.13526-2

Van Dyck, E., Six, J., Soyer, E., Denys, M., Bardijn, I., \& Leman, M. (2017). Adopting a music-to-heart rate alignment strategy to measure the impact of music and its tempo on human heart rate. Musicae Scientiae: The Journal of the European Society for the Cognitive Sciences of Music, 21(4). 390-404. https://doi.org/10.1177/1029864917700706 


\title{
Hearing, seeing, liking: The effects of audio-visual listening conditions on perceptual ratings
}

\author{
Gerardo Lopez ${ }^{\dagger}$ \\ Leigh VanHandel ${ }^{2}$ \\ ${ }^{1}$ Michigan State University, East Lansing, MI, USA \\ ${ }^{2}$ University of British Columbia, Vancouver, British Columbia, CA \\ $\uparrow$ Corresponding author: spotgerry@gmail.com \\ Published 16 December 2021; https://doi.org/10.18061/FDMC.2021.0019 \\ Author video presentation and/or other conference material: https://doi.org/10.17605/OSF.IO/BY2HW
}

\begin{abstract}
This study investigates whether listening to an excerpt of music in audio-only or audio-video contexts affects perceptual ratings, and what the relationship of the context presentation is to perceptual ratings. Participants listened to music in audio-only and audio-video contexts and completed a survey with self-reported ratings for familiarity, emotional and mental engagement, and likability. Results suggest there was no effect of audio-only or audio-visual presentation on perceptual ratings. This conclusion is similar to work done by Huang and Krumhansl (2011). Both emotional engagement and mental engagement had strong correlations with likability ratings. Emotional ratings had a slightly larger effect than mental ratings in the audio-only condition, but results are mixed in the video condition. This suggests a need for closer examination of the emotional and mental rating categories and their relationship to likability.
\end{abstract}

KEYWORDS: perception, audio-visual modalities, emotional engagement, mental engagement, atonal/post-tonal

\section{Introduction}

This study investigated whether listening to an excerpt of atonal/post-tonal music through audio-only or audiovisual contexts affect participants perceptual ratings, and if so, how the perceptual ratings relate to the presentation context.

Prior research in this area includes studies by Behen (1990) and Davidson (1993), both looking at effects of performance on expressivity or other evaluatory measures. Both found that auditory perception could be influenced by visual stimuli, specifically with musical performers. Huang and Krumhansl (2011) focused on potential modality effects (audio-only vs. audiovisual) on performance evaluation with a limited stimuli sample of three excerpts, of which only one was atonal/posttonal; they found no significant differences attributable to modality of presentation.

One critical distinction between this study and previous work (including Behne, 1990; Davidson, 1993;
Huang and Krumhansl, 2011; Vuoskoski, Thompson, Spence and Clarke, 2016; Griffiths and Reay, 2018) is the prior studies' focus on performance ratings. In Huang and Krumhansl (2011), for example, they asked questions like "How appropriate is the performer's expressed emotion?" and "How well does the performance maintain your interest?", whereas the current study asked "How emotionally engaging did you find the musical excerpt?" and "How likeable did you find the musical excerpt?". In addition, this study is focusing on the effects of modality in both tonal and atonal/post-tonal repertoire.

\section{Method}

\section{Participants}

There were 31 participants $($ female $=17$, male $=14$ ). The age range was $18-46$, with the average being 26 years old $(\mathrm{SD}=7.986)$. The majority of participants were undergraduate or graduate students with 6-9 years of musical instruction and 2-3 years of music theory instruction. Participants were recruited via convenience sampling from online forums and were not compensated for their participation.

\section{Design}

The experiment was administered through a Qualtrics survey. The presentation of stimuli was randomized, each with the same set of four ratings questions regarding familiarity, emotional engagement, mental engagement, and likability (Figure 1). No specific definition for these terms was provided other than the familiarity rating requesting their level of familiarity with the excerpt before the study, in an effort to prevent higher ratings later in the repeated measures design.

\section{Procedures}

Participants heard and rated all of the stimuli, which consisted of 6 musical excerpts presented under two conditions, audio-only or audio-visual, resulting in 12 
individual stimuli total (Table 1). The audio for the audio-only condition was sourced from the audio-visual stimuli and was not altered in any way. Participants heard between 30-40 seconds of the musical excerpt, from the beginning of the piece until an appropriate moment in the music (end of a phrase, rest, etc.). Afterwards, they were asked to self-report responses to the survey questions on a 1-6 Likert scale.

\begin{tabular}{|c|c|c|c|c|c|}
\hline \multicolumn{6}{|c|}{$\begin{array}{l}\text { On a scale from } 1-6,1 \text { being not at all and } 6 \text { being extrem } \\
\text { you with the musical excerpt before starting this study? }\end{array}$} \\
\hline 1 & 2 & 3 & 4 & 5 & 6 \\
\hline 0 & 0 & 0 & 0 & 0 & 0 \\
\hline \multicolumn{6}{|c|}{$\begin{array}{l}\text { On a scale from } 1-6,1 \text { being not at all and } 6 \text { being extremely, how emotionally } \\
\text { engaging did you find the musical excerpt? }\end{array}$} \\
\hline 1 & 2 & 3 & 4 & 5 & 6 \\
\hline 0 & 0 & 0 & 0 & 0 & 0 \\
\hline \multicolumn{6}{|c|}{$\begin{array}{l}\text { On a scale from } 1-6,1 \text { being not at all and } 6 \text { being extremely, how mentally } \\
\text { engaging did you find the musical excerpt? }\end{array}$} \\
\hline 1 & 2 & 3 & 4 & 5 & 6 \\
\hline 0 & 0 & 0 & 0 & 0 & 0 \\
\hline \multicolumn{6}{|c|}{$\begin{array}{l}\text { On a scale from } 1-6,1 \text { being not at all and } 6 \text { being extremely, how likeable did you } \\
\text { find the musical excerpt? }\end{array}$} \\
\hline 1 & 2 & 3 & 4 & 5 & 6 \\
\hline 0 & 0 & 0 & 0 & 0 & 0 \\
\hline
\end{tabular}

Figure 1: Survey questions used for all stimuli presentations (audio-only and audio-visual).

\section{Stimuli}

Excerpts were taken from six string quartets, including two tonal excerpts (Haydn and Beethoven) and four atonal/post-tonal works (Berg, Webern, Ligeti, and Carter). The excerpts were selected based on the availability of video recordings each featuring a professional string quartet that did not differ greatly in terms of performance setting, and that were filmed in a way that presented a realistic audience perspective as closely as possible.

\section{Results}

There was no significant difference in overall ratings for familiarity, emotional engagement, mental engagement, or likability across the audio-only and audio-visual conditions (Figure 2). For the individual excerpts, the only significant difference for the ratings across the audio-only and audio-visual conditions was the familiarity rating for the Ligeti excerpt, which saw a significant $(p=.003)$ increase in familiarity in the audio- visual presentation. All other familiarity ratings across conditions remained consistent.

Table 1: Stimuli information.

\begin{tabular}{|l|l|l|l|}
\hline Composer & Piece & Period & Performer \\
\hline $\begin{array}{l}\text { Haydn, } \\
\text { Joseph }\end{array}$ & $\begin{array}{l}\text { Quartet in C } \\
\text { Major, } \\
\text { Op. 20/2, Mvt. I }\end{array}$ & $\begin{array}{l}\text { Classical/ } \\
1772\end{array}$ & $\begin{array}{l}\text { Quatuor } \\
\text { Ebène }\end{array}$ \\
\hline $\begin{array}{l}\text { Beethoven, } \\
\text { Ludwig } \\
\text { van }\end{array}$ & $\begin{array}{l}\text { Quartet in F Major, } \\
\text { No. 11 "Serioso," } \\
\text { Op. 95, Mvt. I }\end{array}$ & $\begin{array}{l}\text { Classical- } \\
\text { Romantic/ } \\
1810-1811\end{array}$ & $\begin{array}{l}\text { Zemlinsky } \\
\text { Quartet }\end{array}$ \\
\hline $\begin{array}{l}\text { Webern, } \\
\text { Anton }\end{array}$ & $\begin{array}{l}\text { Fünf Sätze (Five } \\
\text { Movements), } \\
\text { Op. 5, Mvt. I }\end{array}$ & $\begin{array}{l}\text { Atonal/ } \\
1909\end{array}$ & $\begin{array}{l}\text { Telegraph } \\
\text { Quartet }\end{array}$ \\
\hline $\begin{array}{l}\text { Berg, } \\
\text { Alban }\end{array}$ & $\begin{array}{l}\text { String Quartet, } \\
\text { Op. 3, Mvt. I }\end{array}$ & $\begin{array}{l}\text { Atonal/ } \\
1910\end{array}$ & $\begin{array}{l}\text { Telegraph } \\
\text { Quartet }\end{array}$ \\
\hline $\begin{array}{l}\text { Ligeti, } \\
\text { György }\end{array}$ & $\begin{array}{l}\text { String Quartet No. } \\
\text { 1 "Métamorphoses } \\
\text { nocturnes," Mvt. I }\end{array}$ & $\begin{array}{l}\text { Post-Tonal/ } \\
1953-1954\end{array}$ & $\begin{array}{l}\text { Altius } \\
\text { Quartet }\end{array}$ \\
\hline $\begin{array}{l}\text { Carter, } \\
\text { Elliot }\end{array}$ & $\begin{array}{l}\text { String Quartet } \\
\text { No. 5, Mvt. I }\end{array}$ & $\begin{array}{l}\text { Post-Tonal/ } \\
1995\end{array}$ & $\begin{array}{l}\text { Pacifica } \\
\text { Quartet }\end{array}$ \\
\hline
\end{tabular}

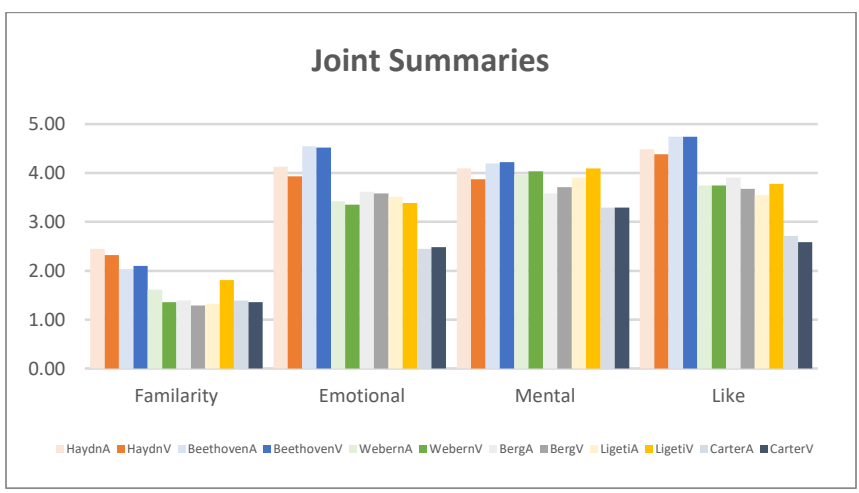

Figure 2: Chart with response averages for all excerpts across audio-only and audio-visual contexts. The " $A$ " at the end of composers' names indicates the audio-only context (lighter shading). The " $V$ " at the end of composers' names indicated the audio-visual context (darker shading).

Likability ratings were strongly correlated with emotional engagement ( $r=.830$ for audio-only; $r=.808$ for audio-visual) and mental engagement ( $r=752$ for audio-only; $r=.733$ for audio-visual). Familiarity was also significantly correlated, but was weaker than emotional and mental engagement $(r=.3)$.

One concern that arose from the general correlations was that the tonal excerpts were rated higher than the atonal/post-tonal excerpts, and that the higher ratings for tonal excerpts may be influencing the correlations. Additional post-hoc correlational analysis was conducted with excerpt groups to determine whether 
there was a difference in style across the tonal vs. atonal/post-tonal conditions. The tonal condition combined Haydn and Beethoven $(H B)$, atonal/post-tonal combined results for Webern, Berg, and Ligeti $(W B L)$, and the results for Carter were their own category $(C)$, since that excerpt's ratings across mental/emotional engagement and likability were significantly lower relative to the other excerpts. Table 2 provides the results of the ratings from these excerpt groups, with further commentary in the discussion.

Table 2: Results from excerpt group correlations.

\begin{tabular}{|l|c|c|c|c|c|c|}
\cline { 2 - 7 } \multicolumn{1}{c|}{} & \multicolumn{3}{c|}{ Audio-Only } & \multicolumn{3}{c|}{ Audio-Visual } \\
\cline { 2 - 7 } \multicolumn{1}{c|}{} & $H B$ & $W B L$ & $C$ & $H B$ & $W B L$ & $C$ \\
\hline Emotional & .745 & .805 & .844 & .741 & .757 & .832 \\
\hline Mental & .722 & .757 & .767 & .757 & .756 & .723 \\
\hline
\end{tabular}

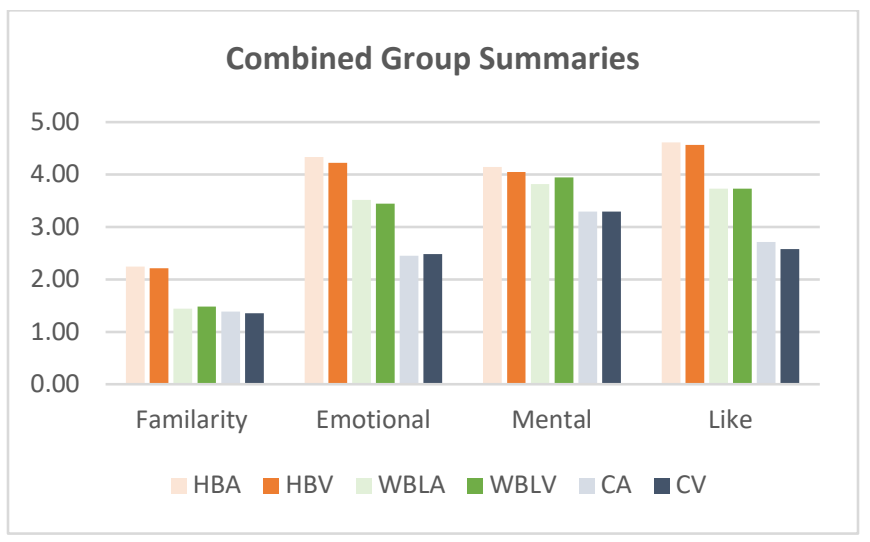

Figure 3: Chart with response averages for combined excerpt groups across audio-only and audio-visual contexts. HBA - Haydn and Beethoven ratings under the audio-only context; HBV - Haydn and Beethoven ratings under the audio-visual context. WBLA Webern, Berg, and Ligeti ratings under the audio-only context; WBLV - Webern, Berg, and Ligeti ratings under the audio-visual context. CA - Carter ratings under the audio-only context; $C V$-Carter ratings under the audio-visual context.

\section{Discussion}

While there were no significant effects of likability or emotional and mental engagement across the audio-only and audio-visual conditions, the relationship between the likability and emotional/mental engagement ratings themselves is more varied.
From the ratings for all excerpts across the audioonly and audio-visual conditions, the likability and emotional engagement ratings had a slightly higher correlation than likability and mental ratings, though both correlations were very strong. When divided into the tonal vs. atonal/post-tonal excerpt groups, the high correlation between likability and emotional engagement remained in the audio-only condition, with emotional engagement ratings again being stronger than mental engagement ratings, but in the audio-visual condition things were not as clear. For the Haydn and Beethoven $(H B)$ group the correlation between likability and mental engagement ratings was slightly higher than for likability and emotional engagement ratings; for the Webern, Berg, and Ligeti (WBL) group the correlations of likability and mental/emotional engagement were extremely close; the Carter $(C)$ sustained a higher correlation between likability and emotional engagement ratings than for likability and mental engagement ratings. This suggests that there may be a need for future research to examine the relationship between likability and emotional and mental engagement more closely to clarify that relationship.

Additional analysis was conducted as an exploratory look at response differences between participants with more musical training and those with less musical training. Preliminary results suggest no overall differences, with the notable exception for mental engagement across audio-only and audio-visual contexts; participants with more musical training selfreported significantly lower mental engagement ratings than less trained participants. Future designs of this experiment will develop more systematic parameters for studying the differences of emotional and mental engagement in highly vs. less trained participants.

Some limitations of the current study include a relatively small sampling of only six stimuli, with an exposure to each stimulus for only $30-40$ secs. In future experiments the number of stimuli would be increased to include more repertoire and potentially different exposure times. Another limitation is the different performance conditions, particularly in the audio-visual stimuli; ideally the recordings would be made by the same string quartet being recorded under the same performance conditions for all of the excerpts. Future studies may also investigate additional influences on the rating tasks, as well as differing representations of emotional and mental engagement. Such examples would include an expansion of the rating scale and the inclusion of more specific wording for the emotional engagement question to prevent participants from 
equating only positively valenced emotions with being emotionally engaged. Additional questions on listener bias to atonal/post-tonal works can be included to control for its effect on ratings of engagement and likability.

One motivation for this study was the pedagogical question of whether presenting audio-visual recordings of performances of atonal/post-tonal works in a classroom setting would increase student engagement and likability over audio-only presentations. While the answer seems like a definite no, the results have shed light on a potentially much more complicated listener relationship warranting further investigation.

\section{Conclusion}

Overall, there was no significant difference between ratings for likability, emotional engagement, or mental engagement for performances presented in audio-only and audio-visual conditions. Some complex interactions between likability ratings and the emotional/mental engagement ratings indicate the need for further exploration of how genre and musical training influence these ratings.

\section{References}

Behne, K. -E. (1990). “Blicken Sie auf die Pianisten?!” Zur bildbeeinflussten Beurteilung von Klaviermusik im Fernsehen [Do you watch the pianists? On visually influenced judgements of piano music]. Medienpsychologie, 2(2), 115-131.

Davidson, J. (1993). Visual perception of performance manner in the movements of solo musicians. Psychology of Music, 21(2), 103-113. https://doi.org/10.1177/030573569302100201

Griffiths, N., \& Reay, J. (2018). The relative importance of aural and visual information in the evaluation by musicians and non-musicians of classical music performance. Music Perception, 364-375. https://doi.org/10.1525/mp.2018.35.3.364

Huang, J., \& Krumhansl, C. L. (2011). What does seeing the performer add? It depends on musical style, amount of stage behavior, and audience expertise. Musicae Scientiae, 15(3), 343-364. https://doi.org/10.1177/1029864911414172

Vuoskoski, J. K., Thompson, M. R., Spence, C., \& Clarke, E. F. (2016). Interaction of sight and sound in the perception and experience of musical performance. Music Perception: An Interdisciplinary Journal, 33(4), 457-471. https://doi.org/10.1525/mp.2016.33.4.457 


\section{Regulating functional and hedonic emotions in the pursuit of musical practice goals \\ Gerard Breaden Madden ${ }^{1 \dagger}$ \\ Hans-Christian Jabusch ${ }^{1}$ \\ ${ }^{1}$ Institut für Musikermedizin (IMM), Hochschule für Musik Carl Maria von Weber Dresden, Germany \\ Corresponding author: gerard.madden@mailbox.hfmdd.de \\ Published 16 December 2021; https://doi.org/10.18061/FDMC.2021.0020 \\ Author video presentation and/or other conference material: https://doi.org/10.17605/OSF.IO/AJKPC}

\begin{abstract}
Individuals can regulate their emotions in order to feel better and avoid feeling worse. However, individuals can also regulate emotions if doing so is believed to be beneficial to the pursuit of a goal. When pursuing a long-term goal, an individual may choose to delay immediate hedonic emotional reward in order to maximize the functional influence of emotions. This study investigates emotion regulation in the context of musical practice. We examine whether musicians adopt specific emotional stances which support their goal orientation, and which are in line with their beliefs about the functional influence of emotions. Via an online questionnaire, musicians $(\mathrm{N}=421)$ reported their goals, meta-emotion beliefs, and emotion regulation behaviour. Musicians used affect-improvement strategies in their practice more often than affect-worsening strategies. Greater use of affect-worsening strategies was associated with stronger support for the utility of unpleasant emotions. Musicians who more strongly endorsed this belief more strongly pursed mastery goals than enjoyment goals. An examination of specific regulated emotions to support musical practice indicated that musicians generally sought to reduce unpleasant emotions, instead preferring pleasant, energizing emotions. However, a subgroup of masteryoriented musicians may seek a mixed emotional state, increasing anger and nervousness alongside a range of pleasant emotions. Musicians who pursue mastery may be motivated to experience emotions that combine functional and hedonic benefits. Functional emotions may be less relevant for musicians who practice for enjoyment. Research in this field may equip musicians with novel skills for better pursuit of their practice goals.
\end{abstract}

KEYWORDS: musical practice, emotion regulation, meta-emotion beliefs, practice goals

\section{Introduction}

Emotion regulation literature often assumes that individuals regulate their emotions for hedonic reasons (i.e., to feel better and avoid feeling worse). However, there is increasing support for an instrumental perspective, in which emotions are regulated if they are beneficial to the pursuit of personally-relevant goals. For example, Tamir et al. (2008) showed that individuals were motivated to experience anger when they anticipated a task for which anger was believed to be useful. In sport contexts, athletes often seek to experience whatever emotions will benefit their performance. Although most athletes seek pleasant and motivating emotions, some believe that performance is benefitted by increasing unpleasant emotions (Lane et al., 2011).

In everyday life, emotions that are pleasant to experience sometimes overlap with emotions that are useful. As a result, individuals may seek to experience emotions that maximise either hedonic rewards, or instrumental rewards, but also possibly both. The question of how individuals wish to feel in a given situation can depend on the goals they pursue. If individuals pursue a long-term goal, they may forego immediate hedonic rewards in order to maximise the instrumental rewards of emotions (Mischel et al., 1989). Regulating in this way may involve prioritizing unpleasant emotions. After all, up-regulated pleasant emotions can be both pleasant and useful, but up-regulated unpleasant emotions can only be useful.

The current study examines musicians' emotion regulation behaviour in musical practice. Although practice has many benefits, it is not always enjoyable, nor does it necessarily yield immediate progress (Ericsson et al., 1993). Musicians persevere through these challenges nonetheless. The extent to which musicians are focused on, for example, developing long-term expert skills, or on short-term enjoyment, depends on their goals. It is plausible to expect that musicians will adopt specific, regulated emotional stances in line with the goals they pursue and their beliefs about the functional influence of emotions.

The following hypotheses are investigated in this study: $H_{l}$ ) Musicians will use affect-improvement strategies in their musical practice more often than affect-worsening strategies. $H_{2}$ ) Greater use of affect- 
worsening strategies will be associated with stronger beliefs supporting the possible benefits of unpleasant emotions. $H_{3}$ ) In contrast to musicians that pursue short-term goals, musicians pursuing long-term goals may seek to experience unpleasant emotions in order to support their musical practice.

\section{Method}

\section{Participants, Procedure \& Materials}

Four hundred and twenty-one participants (female $=254$ ) were recruited from music institutions around the world, including professional orchestras, conservatoires, and music universities. Participants provided informed consent before completing an online questionnaire. Information was collected on the following topics: musical background, practice goals, emotion regulation strategies (two subscales: affectimprovement and affect-worsening), and meta-emotion beliefs (beliefs concerning the functional influence of emotions on musical practice). Participants also indicated how much they would like to increase or decrease different emotions in order to best support their musical practice.

The median age of musicians in this sample was 23 years, and they began playing music at a median age of 7 years. In a ranking of musical styles, participants indicated that they were predominantly engaged in classical music. Participants had a median of 16 years of experience playing music.

\section{Analysis Strategy}

The data were analyzed in several steps. First, Principle Component Analysis (PCA) was used to investigate factors underlying musicians' metaemotion beliefs and practice goals, respectively. Second, MANOVA was used to investigate differences in A) emotion regulation strategies used by musicians with different meta-emotion beliefs and B) differences in the meta-emotion beliefs held by musicians who pursue different goals. Third, musicians were classified into ad hoc subgroups on the basis of their metaemotion beliefs and practice goals. Descriptive statistics and MANOVA were used to examine the specific emotions that musicians in these subgroups sought to regulate in order to best support their musical practice.

\section{Results}

PCA of 1) meta-emotion beliefs and 2) practice goals PCA identified two factors underlying musicians' meta-emotion beliefs. The first factor, "Emotion-
Driven Practice" $(29.90 \%$ variance $)$ refers to a musicians' belief that effective musical practice requires the right emotional state, and that they actively seek to experience whatever emotions (positive and/or negative) help to best support their practice. The second belief factor, "Non-Hedonic Driven Practice" $(23.08 \%)$ refers to the belief that a musician does not necessarily have to feel good in order to practice effectively, and that unpleasant emotions may help to improve musical practice. The two belief factors include an overlap regarding the possible benefits of unpleasant emotions. This overlap was reflected in a weak, positive, statistically significant correlation (Spearman's $\rho=.16, \mathrm{p}<.01$ ).

PCA also identified two factors underlying musicians' musical practice goals. The first factor, "Mastery" (33.55\%) refers to practicing music in order to develop expert musical and instrumental skills. The second factor, "Enjoyment" (30.99\%) refers to practicing music for enjoyment and recreation. There was a weak, negative, non-significant correlation between the Mastery and Enjoyment factors (Spearman's $\rho=-.05, \mathrm{p}<.26$ ), suggesting that these goal pursuits are relatively discrete.

Using a median split, each factor was divided into a high and low category. These categories were used as between-subjects factors for subsequent MANOVA.

Emotion regulation strategies used by musicians holding different meta-emotion beliefs

MANOVA examining the effect of Emotion-Driven Practice (two levels - high/low endorsement) on emotion regulation strategies showed a significant main effect of belief (Wilks $\lambda_{1,420}=6.67 ; \mathrm{p}<.05$; $\left.\eta_{\mathrm{p}}^{2}=.03\right)$ with univariate effects for both affectimprovement and affect-worsening strategies. Musicians who strongly endorsed Emotion-Driven Practice showed greater use of affect-worsening strategies, and less use of affect-improvement strategies. MANOVA examining the effect of NonHedonic Driven Practice beliefs (two levels - high/low endorsement) on regulation strategies also revealed a significant main effect of belief (Wilks $\lambda_{1,420}=16.04$; $\left.\mathrm{p}<.01 ; \eta_{\mathrm{p}}{ }^{2}=.07\right)$ with a univariate effect only for affect-worsening strategies. Musicians who more strongly endorsed Non-Hedonic Driven Practice showed greater use of affect-worsening strategies.

There was no significant interaction between Emotion-Driven Practice and Non-Hedonic Driven Practice for either regulation strategy. 
Meta-emotion beliefs of musicians pursing different musical practice goals

MANOVA examining the effect of Mastery orientation (two levels - high/low pursuit) on meta-emotion beliefs showed a significant main effect of goal orientation (Wilks $\lambda_{1,420}=45.91 ; \mathrm{p}<.01 ; \eta_{\mathrm{p}}{ }^{2}=.18$ ) with univariate effects for Emotion-Driven Practice and Non-Hedonic Driven Practice. Stronger pursuit of Mastery was associated with stronger endorsement of both beliefs. MANOVA examining the effect of Enjoyment orientation (two levels - high/low pursuit) also showed a significant main effect of goal orientation (Wilks $\lambda_{1,420}=5.57 ; \mathrm{p}<.01 ; \eta_{\mathrm{p}}^{2}=.02$ ) with univariate effects for Emotion-Driven Practice and Non-Hedonic Driven Practice. Stronger pursuit of Enjoyment was associated with weaker endorsement of both beliefs.

There was a significant interaction between Mastery and Enjoyment goals, with a univariate effect evident only for Non-Hedonic Driven Practice $\left(F_{1,420}=12.00\right.$; $\left.\mathrm{p}=.001 ; \eta_{\mathrm{p}}{ }^{2}=.02\right)$. Musicians who strongly pursued Mastery but not Enjoyment goals showed the highest endorsement of Non-Hedonic Driven Practice beliefs.

\section{Specific regulated emotions to support practice}

Two constrasting subgroups were derived to address whether musicians selected to experience unpleasant emotions in order to support their musical practice.

Subgroup $\mathbf{M}^{*} \mathbf{N H}$ (Mastery*Non-Hedonic): This subgroup contains those musicians that strongly pursue Mastery only, and who also strongly endorsed NonHedonic Driven Practice.

Subgroup E*P (Enjoyment*Positive): This subgroup contains those musicians that strongly pursue Enjoyment only, and who do not endorse Non-Hedonic Driven Practice.

Table 1. Demographic and musical experience characteristics of subgroup musicians

\begin{tabular}{lll}
\hline & M*NH & E*P \\
\cline { 2 - 3 } & $\begin{array}{l}\text { Median } \\
\text { or n }\end{array}$ & $\begin{array}{l}\text { Median } \\
\text { or n }\end{array}$ \\
\cline { 2 - 3 } & $57 / 27$ & $11 / 61$ \\
Status (Professional / Student) & 28 & 22 \\
Age (years) & 21 & 15 \\
Years of playing (years) & 15.8 & 5.6 \\
$\begin{array}{l}\text { Cumulative lifetime practice } \\
\text { hours (1000hours)* }\end{array}$ & & \\
\hline${ }^{*} p<.01$ & &
\end{tabular}

The intensity of emotions preferred by both subgroups is shown in Figure 1. Two main points of similarity are evident: 1) The substantial increase of Concentration, Energy, Calmness, and Happinesss, 2) The substantial reduction of Gloom, Downheartedness, Anxiety, and Sluggishness. In general, the $\mathrm{E}^{*} \mathrm{P}$ group preferred a significantly greater intensity of pleasant emotions, whereas the $\mathrm{M}^{*} \mathrm{NH}$ preferred a significantly greater reduction of unpleasant emotions.

The two subgroups differed with respect to Anger and Nervousness. MANOVA indicated that the $\mathrm{M}^{*} \mathrm{NH}$ subgroup sought to increase these emotions to a significantly greater intensity compared to the $\mathrm{E} * \mathrm{P}$ subgroup, in addition to preferring significantly less reduction of Anger (Wilks $\lambda_{1,420}=14.42 ; \mathrm{p}<.01$; $\left.\eta_{\mathrm{p}}^{2}=.50\right)$.

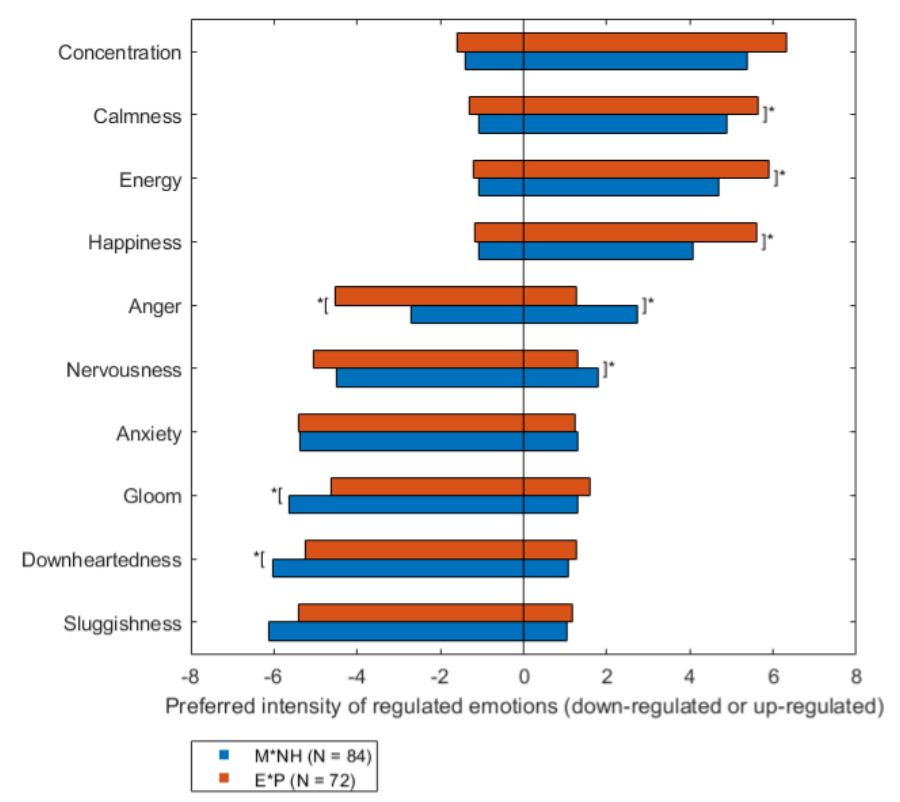

Figure 1: Subgroups' preferred intensity of emotions to support musical practice (mean ratings; ${ }^{*} p<.01$ )

\section{Discussion}

With respect to the aforementioned hypotheses, the following summary is provided:

Findings support $H_{l}$ : Musicians used affectimprovement strategies in their musical practice more often than affect-worsening strategies.

Findings support $H_{2}$ : Greater use of affectworsening strategies was associated with stronger endorsement of Non-Hedonic Driven Practice.

Findings support $H_{3}$ : Musicians in the $\mathrm{M} * \mathrm{NH}$ subgroup sought to experience Anger and Nervousness in order to support their musical practice. These emotions were sought in conjuction with several pleasant emotions. 
Emotion regulation strategies \& Meta-Emotion Beliefs Musicians in the current sample used affectimprovement strategies more often than affectworsening strategies. However, greater use of affectworsening strategies was associated with stronger endorsement of Non-Hedonic Driven Practice. A component of this belief involves support for the benefit of unpleasant emotions in musical practice. This is consistent with findings reported by Lane et al. (2011) who demonstrated that some athletes reported the belief that increasing anger and/or anxiety would benefit their performance.

\section{Meta-Emotion Beliefs and Goal Orientation}

Strong Enjoyment pursuit was associated with weaker endorsement of Non-Hedonic Driven Practice, whereas strong Mastery pursuit was associated with stronger endorsement of this belief. Research shows that Mastery-oriented musicians use more diverse learning strategies in their musical practice (Lehmann \& Papoušek, 2003). Associations regarding performance and the functional influence of emotions develop over the course of time and experience (Hanin, 2010). It is therefore possible that Mastery-oriented musicians may also develop more diverse emotion-beliefs during the course of their practice. Support for the functional influence of unpleasant emotions may arise as a consequence of greater exposure to the challenges of mastering musical and instrumental skills.

\section{Regulating specific emotions to support practice}

Emotions can impact our cognitive processes and provide the energy to pursue our goals (Mazur \& Laguna, 2019). If we assume that musicians have a genuine interest in their own musical practice goals, then it is reasonable to expect that they will seek out the emotional states that will help them achieve these goals. Assigning musicians into subgroups on the basis of their goals and meta-emotion beliefs allowed contrasting orientations toward musical practice to be examined. When asked to report what specific emotions they would seek to experience in order to support their musical practice, points of convergence and contrast were observed:

Avoidance of unpleasant emotions: Both the $\mathrm{M} * \mathrm{NH}$ and $\mathrm{E}^{* \mathrm{P}}$ subgroups sought to decrease Gloom, Sluggishness, and Downheartedness. Reducing these emotions may be advantageous as are they associated with an inability to regulate other emotional states which may be more useful (Kaleńska-Rodzaj, 2018). Furthermore, these emotions may prompt passive behavoural responses that are not conducive to effective musical practice. Musical practice may be better served by emotions that prompt a musician to be proactive.

Prioritizing positive affect: Both subgroups sought to substantially increase Happiness, Energy, and Calmness. While these emotions offer clear hedonic rewards, they may also offer functional benefits. Positive affect can lead to greater cognitive flexibility, and improves performance on tasks requiring creativity and innovative problem solving (Estrada et al., 1994). These benefits are not limited to performance contexts, but also to practice. Altenmüller \& Jabusch (2013) emphasise the importance of practicing music with joy, noting faster and more enduring adaptation in the central nervous system if dopamine is released in the limbic system.

Increasing Anger and Nervousness: Some musicians in the $\mathrm{M}^{*} \mathrm{NH}$ subgroup reported that they would seek to increase anger and nervousness to a moderate degree. This was not observed in the $\mathrm{E}^{*} \mathrm{P}$ group. Unpleasant emotions are generally viewed as counterproductive to good performance, although there is a mixed body of evidence on this topic (Lane et al., 2012). In certain tasks, unpleasant emotions are seen as more useful than pleasant emotions (Davis et al., 2010). Additionally, if anger and nervousness are experienced in conjunction with pleasant emotions (as preferred by the $\mathrm{M}^{*} \mathrm{NH}$ subgroup), this may in fact represent an advantage to having a mastery perspective in musical practice.

The mixed mindset of mastery: Compared to the E*P subgroup, $\mathrm{M}^{*} \mathrm{NH}$ musicians were older, and had greater lifetime involvement in music (see Table 1). With this in mind, it is possible that a mixed emotional state may be a regulatory decision that longer lifetime involvement with music-making has brought to light. A mixed emotional state may support the long-term development of musical skills, given that the majority of musicians in the $\mathrm{M} * \mathrm{NH}$ subgroup also identified as professional musicians. Mixed emotions are said to increase an individual's focus, and help to give a task greater meaning. In the case of Mastery-oriented musicians, mixed emotional experiences may in fact even be more enjoyable than just positive emotional experiences (Mukherjee et al., 2012).

\section{Limitations \& Directions for Development}

This study did not include any measure of musical practice outcome. As a result, this study cannot claim 
specific outcome advantages associated with any particular regulation behaviours.

Future research could address 1) developing a better understanding of the emotional mindsets that may support specific musical outcomes, and 2) the development of meta-emotion beliefs over a life-time perspective.

Given the challenging reality of musicianship, we argue that the emotional regulation behaviour of musicians should additionally be considered from the health and well-being perspective. Although unpleasant emotions may support the pursuit of Mastery, these emotions may also be accompanied by muscular tension and can possibly lead to playingrelated physical disorders and psychological strain. Identifying regulation strategies that can bring functional emotions to optimal levels, might help to minimize the risk of physical and psychological harm.

\section{Conclusion}

Musicians generally prefer to use affect-improvement strategies during their musical practice. However, some musicians strongly endorse the functional influence of unpleasant emotions. These musicians may be willing to experience unpleasant emotions as a tool to support the pursuit of expert musical and instrumental skills.

It is hoped that this study, and future research on this topic will provide musicians with novel skills for better pursuit of their practice goals, and maybe help to maximise health and well-being in musical practice.

\section{Endnote}

Theory and findings presented here are adapted in an abbreviated format from the full length journal article: Instrumental and Hedonic Motives for Emotion Regulation in Musical Practice. Frontiers in Psychology (Breaden Madden \& Jabusch, 2021, in revision).

\section{Acknowledgements and Funding Statement}

The authors wish to thank: Dorothea Schmitt, Maja Grützmann and Lucas Hoffman for their assistance throughout this project, Dr. Bryony Buck and Prof. Norman Shetler for helpful and insightful discussions during preparation of the study, and Dr Andy Lane and Prof. Maya Tamir for their advice.

This work was financially supported by the German Federal Ministry of Education and Research - BMBF (\#01PL17063). Additionally, this study was financed by the Saxon State government out of the State budget approved by the Saxon State Parliament.

\section{References}

Altenmüller, E. \& Jabusch, H-C. (2013). Musik lernen Musik machen. Was sind optimale Bedingungen aus neurowissenschaftlicher Sicht? I Rückblick auf das 12. Symposium der Deutsche Gesellschaft fur Musikphysiologie und Musikermedizin

Davis, P. A., Woodman, T., \& Callow, N. (2010). Better out than in: the influence of anger regulation on physical performance. Personality and Individual Differences, 49(5), 57-460. https://doi.org/10.1016/j.paid.2010.04.017

Ericsson, K. A., Krampe, R. Th., \& Tesch-Romer, C. (1993). The role of deliberate practice in the acquisition of expert performance. Psychological Review, 100: pp.363-406. https://doi.org/10.1037/0033-295X.100.3.363

Estrada, C., Young, M., \& Isen, A. M. (1994). Positive affect influences creative problem solving and reported source of practice satisfaction in physicians. Motivation and Emotion, 18, 285-299. https://doi.org/10.1007/BF02856470

Hanin, Y. L (2010). Coping with anxiety in sport. In: Nicholls A. R. (Editor) Coping in sport: theory, methods, and related constructs. Hauppauge, New York, USA; Nova Science

Kaleńska-Rodzaj, J. (2018). Waiting for the Concert. PrePerformance Emotions and the Performance Success of Teenage Music School Students. Polish Psychological Bulletin, 49(3), 322-331.

Lane, A. M., Beedie, C. J., Devonport, T. J., \& Stanley, D. (2011). Instrumental emotion regulation in sport: relationships between beliefs about emotion and emotion regulation strategies used by athletes. Scandinavian Journal of Medicine and Science in Sports, 21(6): 445-51. https://doi.org/10.1111/j.16000838.2011.01364.x

Lane, A. M., Beedie. C. J., Jones, M. V., Uphill, M., \& Devonport, T. J. (2012) The BASES Expert Statement on emotion regulation in sport. Journal of Sport Sciences, $\quad 30(11), \quad 1189-1195$. https://doi.org/10.1080/02640414.2012.693621

Lehmann, A. C. \& Papoušek, S. (2003). Self-reported performance goals predict actual practice behaviour among adult piano beginners. Proceedings of the $5^{\text {th }}$ Triennial ESCOM Conference.

Mazur, Z. \& Laguna, M. (2019). The role of affect in practicing a musical instrument: A systematic review of the literature. Psychology of Music. Vol. 47(6), 848863. https://doi.org/10.1177/0305735619861831

Mischel, W., Shoda, Y., \& Rodriguez, M. L. (1989). Delay of gratification in children. Science, 244(4907): 933938. https://doi.org/10.1126/science.2658056

Mukherjee, S., Kramer, T., \& Lau-Gesk, L. (2012). Finding meaning in mixed affective experiences. North American - Advances in Consumer Research, 40, 276-289.

Tamir, M., Mitchell, C., \& Gross, J. J. (2008). Hedonic and instrumental movies in anger regulation. Psychological Science. 19(4): 324-328. https://doi.org/10.1111/j.1467$\underline{9280.2008 .02088 . \mathrm{x}}$ 


\title{
Virtual Harmony: Music interaction with virtual reality to reduce stress
}

\author{
Alena Miskinis $^{1 \dagger}$ \\ Xiangxu Lin $^{2}$ \\ Shadi Kanaan ${ }^{3}$ \\ ${ }^{1}$ Hugh A. Glauser School of Music, Kent State University, Kent, Ohio, USA \\ ${ }^{2}$ Department of Computer Science, Kent State University, Kent, Ohio, USA \\ ${ }^{3}$ College of Nusing, Kent State University, Kent, Ohio, USA \\ ${ }^{\dagger}$ Corresponding author: amiskini@kent.edu \\ Published 16 December 2021; https://doi.org/10.18061/FDMC.2021.0021 \\ Author video presentation and/or other conference material: https://doi.org/10.17605/OSF.IO/BQX2P
}

\begin{abstract}
This paper presents a demonstration of a newly created device called Virtual Harmony, designed to address and reduce stress by applying Virtual Reality (VR) in a Music Therapy (MT) environment. The treatment combines VR and MT to stimulate audition through background music, vision through three-dimensional VR, and touch through virtual percussive instruments.

A pilot study was conducted among 19 high school and college students, in which participants used Virtual Harmony and provided pre- and post-exposure questionnaire responses about their experiences. $90 \%$ of participants reported that Virtual Harmony was effective and worth purchasing and $32 \%$ of participants reported a significant decrease in stress levels after having used the device. Only 5\% reported that their stress increased which may be related to past experiences with severe vertigo. Further controlled experimentation is needed, but these early results are consistent with Virtual Harmony being a promising, affordable, and accessible way for users to manage their stress.
\end{abstract}

KEYWORDS: music therapy, virtual reality, stress management, cognitive load

\section{Introduction}

Stress is a common experience in daily life for most people. $75 \%$ of adults reported feeling stressed in the previous month (APA 2017). Despite the ubiquity of stress, not all people know how to mitigate it. Unfortunately, in many instances, the professional treatments for reducing stress are expensive and inaccessible. One of the most affected populations of stress is college students, reaching as high as $89 \%$ feeling stressed 2 to 4 times per semester (MentalHelp 2016). Not only do Millenials report the highest levels of stress, they also seem to rely on sedentary stress management techniques - listening to music being the most common (APA 2015). In addition to the financial struggle of affording therapies, students also face stigmas against psychological services, a lack of transportation, and a limited amount of time.

This study explores a new application developed by the authors, Virtual Harmony, intended to supplement treatment options for stress. The application addresses obstacles to treatment by providing an accessible and affordable platform to reduce the effects of stress through the combination of Virtual Reality technology and Music Therapy practices. Moreover, like Music Therapy, the device can be used by musicians and nonmusicians alike. Figure 1 illustrates a user engaged with Virtual Harmony. ${ }^{1}$

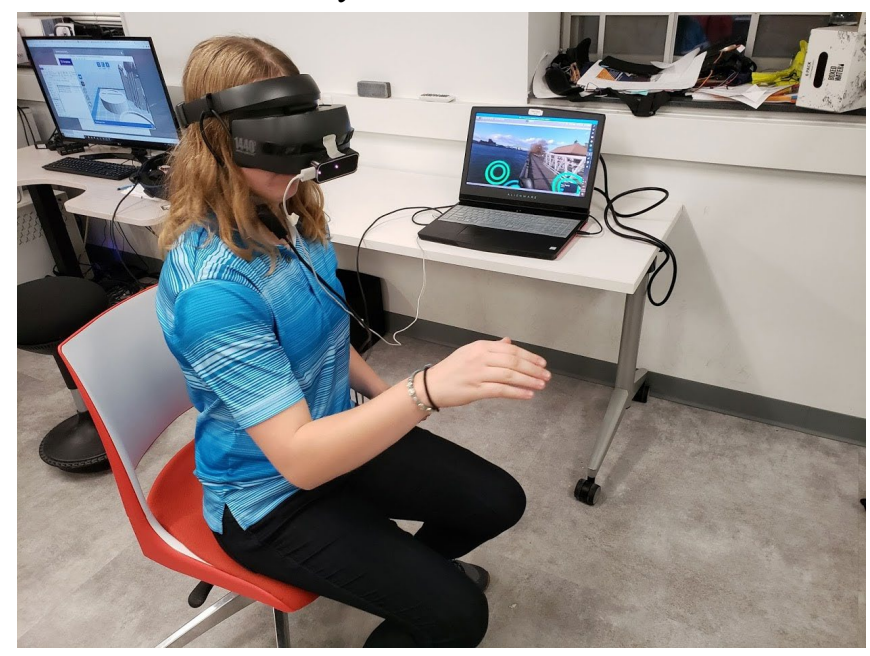

Figure 1: A user engaged with Virtual Harmony.

\section{Application Design}

\section{Related Devices}

Other developers have created similar devices to try to mitigate stress for their users. Mitrpanont et. al. (2017) created the iCare-Stress, a device that processes brainwaves to determine the user's stress levels, which then suggests music therapy practices based on the 
results. Pingle (2016) developed an Internet of Things (IOT) that recommends music through a web interface after collecting biodata. Mahalil et. al (2014) created a stress relieving therapy that combines VR and music therapy-based visualization using forest scenes coupled with audio elements such as a waterfall and animals. Similarly, Thoondee and Oikonomou (2017) also proposed a VR based system to reduce stress and provoke relaxation at work during breaks. Jiang et. al. (2013) designed the Intelligent Move Therapy System for Mental Disease Treatment to calm the stress and emotions of users through a movie, music, and a plot.

In contrast to these many devices, the proposed Virtual Harmony device provides both active and receptive music therapy in a peaceful VR threedimensional environment with soothing music. Virtual Harmony's most novel feature is its combination of several therapeutic techniques. Further, studies reveal that listening to music is the most common form of coping with stress, particularly among Millenials (APA 2015). While listening to music and watching videos is certainly a common destressing tool, Virtual Harmony provides yet another sensual interaction through touch.

\section{Music Therapy}

In recent years, music therapy has become the most commonly prescribed treatment for complementary and alternative medicine (CAM), as it affects both the central and peripheral nervous systems (Ferrer 2014).

One benefit of music in this kind of application is that it can manipulate time through beat, rhythm, and pulse. To encourage time-based manipulations, the Virtual Harmony prototype offers virtual percussive instrument selections, namely piano, xylophone, and a drum set, as shown in Figure 2. Furthermore, the auditory cortex, located deep within the cerebrum, is very close to the limbic system and hippocampus where the brain stores memories. Thus, the auditory cortex may be able to retrain neural and behavioral functions to maintain relief and comfort even outside of the therapeutic environment (Ferrer 2014).

The decision was made to include background music to simulate the experience of music therapy, in which the patient musically interacts with the music production of the therapist. Specifically, the Virtual Harmony prototype uses the second movement of Beethoven's 7th Symphony. There are several reasons why this musical selection is appropriate to simulate a music therapy session. First, the slow tempo of 72 beats per minute is similar to the average heart rate, so elevated heart rates due to stress may slow down to match the tempo, conducive for reducing stress. Second, as with several of Beethoven's slow movements, the melody cycles in about ten seconds - the amount of time it takes a signal to travel from the brain to the heart to regulate a healthy blood pressure (Young 2015). Lastly, less raucous and more tranquil music stimulates the brain, triggering chemicals that suppress the production of adrenaline and corticosteroids, hormones directly related to high levels of arousal and stress (Dove 2009).

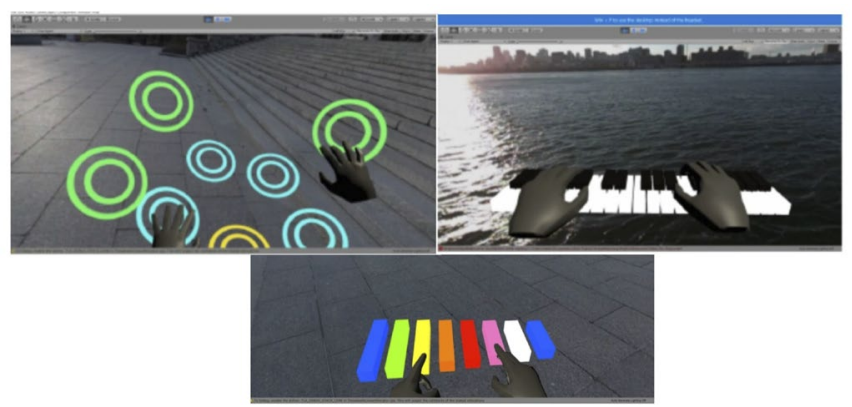

Figure 2: Examples of the interface of Virtual Harmony with the three instrument options.

Virtual Reality

Virtual Reality (VR) is a newly refined technology which immerses the user in a transformed, present threedimensional environment. Not only does VR provide an accessible and affordable vacation from collegiate chaos, recently it has also emerged as a therapeutic device, specifically as an antidepressant (Lindner et. al. 2019). Furthermore, in a study conducted comparing 3D videos and 2D videos, participants reported feeling more distracted when watching a 3D video (Nanda 2011).

\section{Cognitive Load Theory}

The most important feature of Virtual Harmony is the virtual percussive instruments (see Figure 2), because it provides yet another stimulation to distract the brain. Educational psychologist, John Sweller, (1988) developed the cognitive load theory which refined the understanding that sensory register memory can only intake a limited number of perceptions at once. Therefore, by distracting three of the five senses, Virtual Harmony creates a virtually real environment that medializes the participant before reaching the threshold of being overwhelmed.

\section{Cognitive Behavioral Therapy}

Virtual Harmony follows Cognitive Behavioral Therapy practices which focus on changing unhelpful cognitive 
distortions and behaviors, improving emotional regulation, and developing personal coping methods (Otte 2011). First of all, unlike a traditional music therapy setting, the environment is customizable. Secondly, the system operates in both receptive and active modes. In the receptive mode of operation, users experience the $360^{\circ}$ panoramic video and background music. Once accustomed to the new interface, users can participate in the active mode of operation with virtual percussive instruments. In this way, users can "communicate" with the background music, much like a typical music therapy setting.

\section{Technology Design}

Virtual Harmony uses two types of interfaces: the headmounted display (HMD) and the hand-tracker. The HMD, or the headset, is used to engage the user fully in the virtual experience. Once wearing the headset and in the virtual world, users cannot see anything in the real world. Furthermore, through the HMD, users can look around the environment which gives them control of the scene and feedback for motion. See Figure 3 for examples of the two environments used in the prototype.
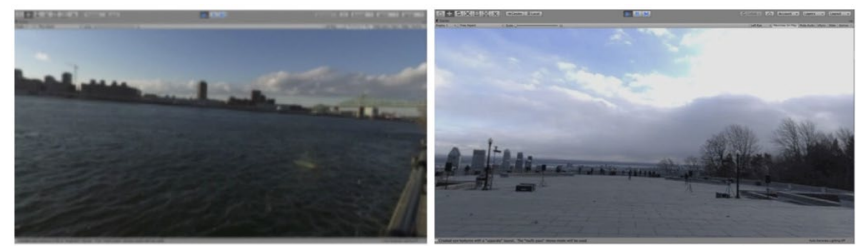

Figure 3: The two virtual environments used in the Virtual Harmony prototype.

After putting on the headset, users will see their virtual hands through the Leap Motion hand-tracker. This innovative controller-less system replaces the traditional joystick or keyboard to give the user a more realistic sensation (see Figure 4). After putting on the headset, users will see two virtual hands and the Leap Motion captures the movements of their real hands. Users are able to move their hands as well as each finger freely. When the left hand is facing palm up, a menu of background music, 3D videos, and instruments will open. Using the right hand, users can select a background piece, a video, and as many instruments as they prefer. Once in the virtual world, users can interact with background music as they wish with the instruments provided, or they can turn to observe what is happening visually in their surroundings. Even if the background music or video ends, they automatically repeat.
While not a component of the final prototype used in the study, we prerecorded a voice that guides users through navigating the virtual world as well as suggesting meditative relaxing techniques such as breathing deeply and closing the eyes.

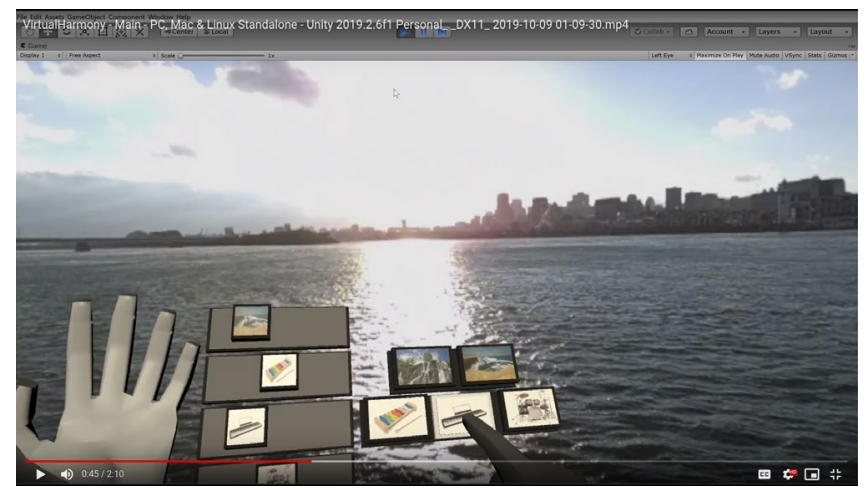

Figure 4: The menu shown on the left palm of a user in Virtual Harmony.

\section{Hardware}

The Virtual Harmony prototype was developed with a VR Ready gaming laptop, specifically Intel(R) Core(TM) i7-7820HK CPU @ 2.90GHz (8 CPUs), 32768MB RAM, NVIDIA GeForce GTX 1080 and Windows 10. In addition, it also uses a professional video game engine called Unity for the software development platform. The head mount display device is the consumer grade VR headset, HP Windows Mixed Reality Headset, with $1440 \times 1400$ resolution per eye and $90 \mathrm{HZ}$ refresh rate. Leap Motion, a world-leading hardware used for the hand tracking system, was attached to the front of the headset. The current marketing prices for these two devices are $\$ 179$ and $\$ 89$ respectively. However, while we used high performance systems, Virtual Harmony can still function under less powerful machines such as the Oculus Go, currently $\$ 149$.

\section{Method}

Due to the circumstances of the COVID-19 pandemic, thorough human participant research was not possible. Nevertheless, we conducted a pilot study of 19 volunteer participants, all high school and college students. Each participant was asked to complete a pre-survey regarding their stress level and general information such as age, education, level, and previous experiences with vertigo. After demonstrating how to use the device, we encouraged users to explore the virtual world presented. While we gave no time limit, participants spent an 
average of 11.2 minutes inside the simulation. Afterwards, participants filled out a post-survey regarding their experience and if their original level of stress was affected.

\section{Pre-Survey}

The pre-survey asked participants a range of background questions, including age, gender, and education level. It then asked several generalized questions about current stress levels, sources of stress, and strategies the participant used for managing stress. Specifically, we asked participants how they usually coped with stress from a list of options: music (listening or playing), reading, sports/exercise, eating, sleeping, socializing with friends, I don't feel as if I have any coping, and other. We also asked whether participants had visited a therapist, and if not, why not.

\section{Post-Survey}

Once the study was completed, participants were asked a number of follow-up questions about their experience with Virtual Harmony. On a scale of 1-10, they were asked to rate their experience with Virtual Harmony and their stress level after using it. They were then asked whether they would prefer using Virtual Harmony over seeing a therapist in person. Then they were asked whether they had any negative physical reactions to the application, including general discomfort, fatigue, headache, nausea, difficulty concentrating, or dizziness. Finally, they were asked how much they would be willing to pay for the system.

\section{Results}

The participants in the pilot study reported that music is indeed their most preferred coping method for dealing with stress (see Figure 5). Further, as shown in Figure 6, a majority of the participants reported that their overall stress levels significantly decreased after using Virtual Harmony $(p<.0001)$. Specifically, on a scale of $0-10$, mean participant stress decreased from a pre-test mean of 6.7 to a post-test mean of 3.5.

Notably, just one participant's stress level increased after using the device. However, this participant was also the only one to have reported suffering from severe vertigo, epilepsy, or a similar condition. Wearing the headset caused four additional participants to feel slight motion sickness. Even still, all five users were interested in participating in testing the next version of our system in the future.

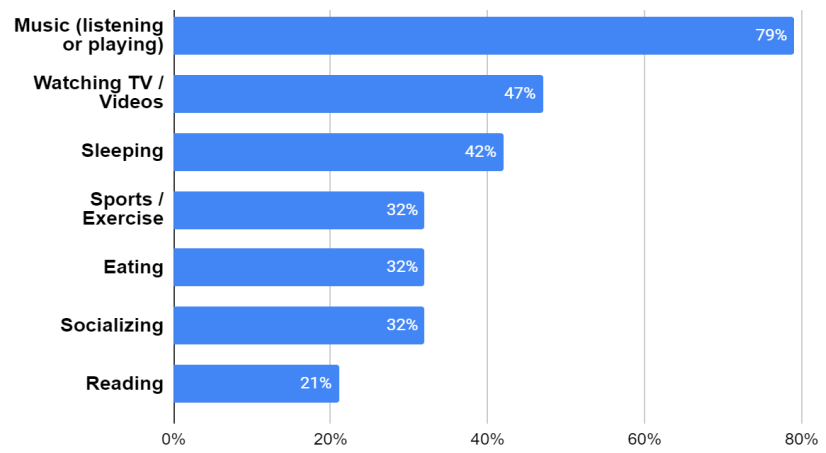

Figure 5: Preferred coping strategies of participants.

Pre- vs. Post-test Stress Rating

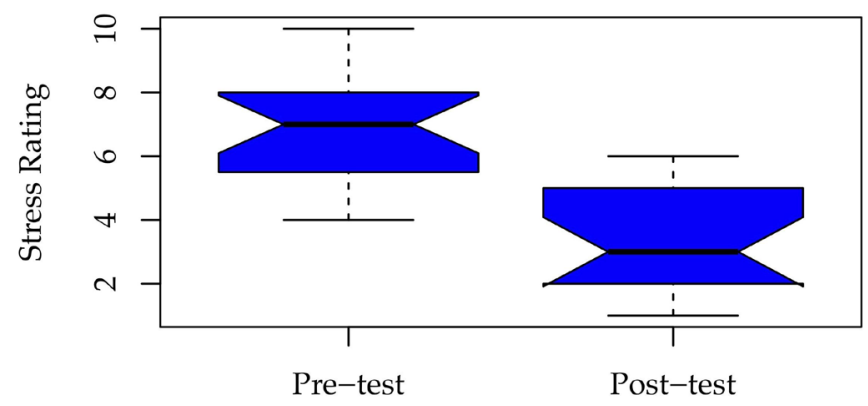

Figure 6: Self-reported post-test stress levels $(m=3.5)$ significantly lower than pre-test stress levels $(m=6.7)$, $\mathrm{p}<.0001$.

\section{Discussion}

The preliminary experimental results of Virtual Harmony are consistent with a positive effect of combining the two therapeutic techniques of Virtual Reality and Music Therapy to reduce participant stress levels. Some participants experienced motion sickness using Virtual Harmony, and of these participants, one experienced increased stress. While the occasions of motion sickness can be disheartening, as with any treatment, negative side effects can occur for some patients. Some participants may enjoy VR as a supplement to traditional therapies, but this technology may not be for all stress patients. Further, elements of the technology may have been cause for distress, especially the virtual piano, as the virtual keys are difficult to coordinate. This negative experience is likely exacerbated for those without prior musical experience. 


\section{Conclusion}

While Virtual Harmony is still in a prototypical stage, preliminary results promisingly affirm the therapeutic benefits demonstrated previously in Virtual Reality and Music Therapy practices. In future research, we hope to more thoroughly test the effectiveness of this treatment on stress through bio measures in comparison to traditional methods.

\section{Future Goals}

In addition to more formal experimentation, we have several visions for how we can expand user experience and improve the technology and technique. To begin with, the user interface (UI) design can be improved to better reflect human behavior. We will also consider increasing the size of the graphical user interface (GUI), so that users can understand and control the environment more simply. Secondly, we hope to develop a platform where users can personally interact with a professional music therapist through the virtual environment. Lastly, we want to design an application that allows users to select individualized music, $360^{\circ}$ videos, and virtual instruments. We would also offer packages of these three components as recommended by music therapists for purchase. Otherwise, users can simply upload their custom interests. Ultimately, we hope to one day implement Virtual Harmony on college campuses to reduce stress in a way that is affordable, accessible, and effective.

\section{Acknowledgements}

Many thanks to Kent State's Design Innovation for inspiring us to address mental health in the Mission Life Competition, to LaunchNet for guiding our entrepreneurial goals, and to our mentors Dr. J.R. Campbell, Dr. Edgar Kooijman, and Dr. Donna Lee.

\section{End Notes}

${ }^{1}$ https://www.youtube.com/watch? $\mathrm{v}=\mathrm{jirR}$ 6dUAbc\&t= $2 \mathrm{~s}$

\section{References}

American Psychology Association. (2017). By the numbers: Our stressed-out nation.

American Psychology Association. (2014). Paying with Our Health. Stress in America.

American Psychology Association. (2009). Stress
Management Techniques.

Dove, M. (2009). The Relationship of Rhythmic and Melodic Perception with Background Music Distraction in College Level Students. University of Missouri.

Ferrer, E.; Lew, P.; Jung, S. M.; Janeke, E.; Garcia, M.; Peng, C.; Poon, G.; Rathod, V.; Beckwith, S.; Tam, C. F. (2014). Playing Music to Relieve Stress in a College Classroom Environment. College Student Journal, 48(3), 481-494.

Jiang, J., Wang, Y., \& Yang, H. (2013). Towards designing an intelligent movie therapy system for mental disease treatment. 19th InternationalConference on Automation and Computing, 1-5.

Lindner, P.; Hamilton, W.; Miloff, A. \& Carlbring, P. (2019). How to Treat Depression With Low-Intensity Virtual Reality Interventions: Perspectives on Translating Cognitive Behavioral Techniques Into the Virtual Reality Modality and How to Make Anti-Depressive Use of Virtual Reality-Unique Experiences. Frontiers in Psychiatry, 10(792). https://doi.org/10.3389/fpsyt.2019.00792

Mahalil, I.; Rusli, M. E.; Yusof, A. M.; Yusof, M. Z. M. \& Zainudin, A. R. R. (2014). Virtual reality-based technique for stress therapy. 4th International Conference on Engineering Technology and Techno-preneuship (ICE2T), 295-300. https://doi.org/10.1109/ICE2T.2014.7006265

MentalHelp. (2016). Studies show students stress more during finals week. The Butler Collegian.

Mitrpanont, J.; Phandhu-Fung, J.; Klubdee, N.; Ratanalaor, S. \& Mitrpanont, T. iCare-stress: An integrated mental health software. 2nd International Conference on Information Technology (INCIT), 1-6.

Otte, C. (2011). Cognitive behavioral therapy in anxiety disorders: Current state of the evidence. Dialogues in clinical neuroscience, 13(4). https://doi.org/10.31887/DCNS.2011.13.4/cotte

Pingle, Y. (2016). IOT for music therapy. 3rd International Conference on Computing for Sustainable Global Development (INDIACom), 1453-55.

Sweller, John. (1988). Cognitive Load During Problem Solving: Effects on Learning. Cognitive Science, 12, 257-285. https://doi.org/10.1207/s15516709 $\operatorname{cog} 1202 \_4$

Thoondee, K. D. \& Oikonomou, A. (2017). Using virtual reality to reduce stress at work. Computing Conference, 492-9. https://doi.org/10.1109/SAI.2017.8252142

Young, E. (2015) "Healing Rhythms." New Scientist, 227(3038), 36-9. https://doi.org/10.1016/S0262-4079(15)31177-5 


\title{
Pleasurable music selects for enhanced music memory, hence music emotions: The evolutionary forces laid bare
}

\author{
Mark S. Riggle \\ Causal Aspects LLC, Charlottesville, VA, USA \\ markriggle@alumni.rice.edu \\ Published 16 December 2021; https://doi.org/10.18061/FDMC.2021.0022 \\ Author video presentation and/or other conference material: https://doi.org/10.17605/OSF.IO/RYP7N
}

\begin{abstract}
We have a phenomenal memory for music and seem highly motivated to remember pleasurable music. Since emotions greatly enhance memory, perhaps music evoked emotions are responsible for our substantial music memory. This makes music an evolutionary difficulty because of that consumed memory plus music's strong pleasures drive resource consuming behaviors that appear not useful to survival. Why should music evoked emotions, which enhance memory, exist and be so pleasurable? We introduce a new evolutionary framework for music where an important mechanism has been overlooked: Trait elaboration for sensory exploitation of a sensory preference. The theory shows that music selection developed cognitive neural capacities that are directly reusable for providing language. Music is not a fitness indicator, but it is attractive which makes evolution run.
\end{abstract}

KEYWORDS: evolution, emotions, memory, dance, language

\section{Introduction}

When looking at theories for the evolutionary origin of music, sexual selection for music has been taken to mean that music evolved as a fitness indicator of male mate quality. This has been a huge error and missed an important mechanism for the evolution of music; that is, sexual selection by female choice could have occurred for music (and likely did) without it having anything to do with being a fitness indicator. Calling it sensory exploitation of a sensory preference would be clearer (Ryan, 1998). How can that work and why the confusion?

\section{Trait Elaboration for Sensory Exploitation}

First, by sexual selection on female choice, no trait can really start off selected as a fitness indicator because there are several steps that must occur before that trait can become an indicator. While those steps are usually (and reasonably) ignored by evolutionary biologists (because they want to explain an existing sexually dimorphic trait - such as the peacock tail), the steps are critical in the analysis for music selection because music is not sexually dimorphic. The steps for sexual selection start with two initial parts: one, an initial pre-existing sensory preference (based on food, colors, warmth, or whatever environmental factors attract and reward individuals); and two, sensory exploitation where males evolve traits (by genetic mutations) to exploit that preference. So far, no sexual dimorphism, no indicator, sexes are equal. The details of the mechanism are important to see how music could evolve.

A sensory preference will attract all individuals, both males and females, to a particular environment. Now, males, who by new genetic mutations express a trait which stimulates that preference, will have greater reproductive success - this is sensory exploitation - and they will have more offspring carrying those genes. However, the genes responsible for that trait are not sexlinked and will express the phenotype in both the male and female offspring -- the genes do not 'know' they were selected by providing an advantage to a male. This selection will continue creating a stronger expressed (or more elaborated) trait(s) (and expressed in both males and females) until the expressed trait causes a reduced fitness to females. That is, while the males gain a reproductive advantage from the trait, females do not and will have a different cost/benefit tradeoff.

The critical point occurs when, for females, the trait's fitness costs exceed its benefits; at that point, natural selection will sex-link the trait to reduce its phenotypic expression in females (for example, a mutation occurs where testosterone presence regulates the trait's phenotypic expression - it turns the gene on, perhaps at puberty); So reducing the trait's expression in females will now increase the female's fitness. This sex-linking is when that trait becomes sexually dimorphic and sexual selection (sensory exploitation) can now continue to develop the trait at an exponential rate; this is because both sexes of offspring, now dimorphic, have increased fitness while carrying the same genotype.

For the evolutionary biologists, the next step of interest is why females maintain the strong preference for the displayed sexually dimorphic trait. We are finally at that last step to the trait being a fitness indicator: The 
female preference for the trait (the pleasure from) is maintained by natural selection only if the female gains in fitness (by more fit offspring) by keeping that preference. That is, the now sexually dimorphic male trait now indicates 'good-genes' - a fitness indicator.

The critical point is, if a trait provides fitness benefits to the female that exceeds the trait's costs, the trait will not become sex linked. That is, until the genes are sexlinked for expression, the trait, even though providing a reproductive advantage to males, will not be sexually dimorphic; thus, the trait cannot be a fitness indicator, and it cannot be even a sexual display.

\section{Applying This Principle to Music}

We can apply this principle to music, which is many traits and genes, and clearly creates strong sensory preferences (pleasures), and incurs high costs. Music capabilities can be developed by sensory exploitation by males, but still not be a fitness indicator, nor be sexually dimorphic, nor be a sexual display (thus even children will make and enjoy music, social groups will make music, and mothers will sing to infants because infants find it pleasing); these musical trait developments remain non-sex-linked and sexually monomorphic if and only if the music traits provide counterbalancing benefits to females.

\section{Counterbalancing Benefits from the Music Traits}

Those counterbalancing benefits fall into two categories: 1) the music traits develop the cognitive apparatus that can be reused for language (both males and females benefit greatly); 2) they provide infant protection from male conspecifics by female bonding [1].

For music, we have to show various elements for this framework:

1. the nature of the preexisting sensory preference that starts the selection process and what musiclike traits can exploit that initial preference

2. the additional cycles of new preferences and new exploitation traits that will occur for music

3. how those traits produce the cognitive apparatus usable for language which is the huge benefit offsetting the costs of the music traits - this narrows the concepts of what is language

4. why the various traits and pleasures of music are selected and developed including emotions induced by music (emotions were selected to enhance memory for music - and why that enhanced fitness)
Before going into these framework details, we need to discuss some of aspects of language and why the evolved music traits may provide the foundation for language (this is not by a music protolanguage).

\section{Language - What Is It and Why So Useful?}

The major issue for the origin of language (with a major simplification of the first extreme) is between the biolinguistic approach and the usage-based approach.

1. Biolinguistic approach - Is language an innate, syntax-specific, genetically endowed, 'language instinct', produced by evolution for communication (or even just some lesser combination of those elements)? Or the:

2. Usage-based approach -- Is language an emergent phenomenon based on latent cognitive capabilities where language is a learned skill in which linguistic structure is a product of culture (Christiansen \& Chater, 2016)?

The first approach has been described (perhaps a little unfairly) as "biologically implausible, unresponsive to cross-linguistic facts, theoretically inelegant, and unnecessary from the perspective of language acquisition". Of course, it is the latter view of language that fits with the music evolution framework here: where language is based on latent cognitive capabilities, a learned skill from the surrounding culture, and language acquisition occurs by building up a set of mental procedures for understanding and producing language (Christiansen \& Chater, 2016). An important question is why do those required cognitive capabilities seem to exist only in humans? We will show that the music framework may have created those cognitive capabilities that are needed for language. In this view, language is much more than just grammar and speech (there are more than a million published articles on aspects of language), rather, language is a powerful cognitive tool for problem solving, for reasoning about the world - and we know knowledge is power. Communication is not the driving force for language; however, the surrounding culture holds the knowledge to perform and use language to great effect. Therefore, communication is needed to learn language: to learn vocabulary, categories, and category features necessary for reasoning. This powerful problem-solving tool of language is the huge benefit that counterbalances the huge costs of music (and hence music stays monomorphic between the sexes). 


\section{Going from Music to Language and Cognition}

Music and language - a truly vast literature exists on how they overlap but not for the context of sensory exploitation with music selecting cognitive traits and where language is purely reusing those cognitive capabilities. Music in this framework could not be one genetic change but was many steps where each music capacity could grow in capability by additional genetic change. Similarly, because language is riding on those capacities, language would also grow in capability and grow in its useful power of reasoning and problem solving. Neither music nor language could be binary.

In sensory exploitation by males, any music trait that allows creating more a pleasurable music experience will attract females, which gives those males better reproductive success (making those genes spread in the population). Importantly, as long as that trait also supports language or other benefits (cognitive or infant protection), the trait will not become sex-linked and will express in all individuals: male, female, young, old, and will not become a sexual display. Furthermore, because that music trait is also expressed in females, it will change the sensory preference (or pleasures) from music; this feedback loop will strongly drive a growing complexity of many music traits (a classic runaway that maximizes the traits' development). The most influencing music traits would be the pleasure producing ones since they create that positive feedback loop for further sensory exploitation.

Of course, a large difference between language and music is the varied and intense pleasures of music, which seem absent or mostly absent from language. Music pleasure is dopamine related (Cardona et al., 2020) for example), and the more dopamine, the greater the reward. However, there is not just one monolithic music pleasure, there are multiple music pleasures that have originated over time. Some of the pleasures are: dancing to music or to rhythmic sounds; listening to music for the anticipation pleasure of music; learning new music; creating music; the emotions evoked by music; and music chills.

Each of these pleasures allow sensory exploitation and require cognitive developments to produce both the pleasure and the exploitation skill. Furthermore, those particular cognitive developments must then support a non-music benefit (cognitive, language related or infant protection) or else become sex-linked and sexually dimorphic. A brief and incomplete analysis of these pleasures follows.

\section{Dancing Pleasure}

Dancing is very pleasurable (Bernardi et al., 2017) and even for children. The pleasure of dancing to rhythmic sounds is very likely the first step in this music evolution, a pleasure that began independent from music (see its own section). Thus, before any music-like trait existed, because of sensory exploitation, dancing pleasure will give males who can create interesting rhythmic sounds a reproductive advantage. Creating those sounds requires some new cognitive abilities - a pattern generating ability and a memory for the created patterns and a memory for remembering the patterns produced by other individuals -- one can make more pleasing patterns by learning them from others. Additionally, a motivation to create rhythmic sounds is required, thus another trait under selection is a pleasure from making those sounds. [One benefit to females for creating those sounds is social bonding with other females to protect infant offspring.]

\section{Anticipation Pleasure of Music -- Needing Memory}

The pleasure of listening to music given by the music anticipation pleasure would have built on the prior pattern generation and the remembering of the patterns. This would become the 'rules' system for music along with the statistical analyses of auditory input and sensitivity to abstract patterns. This is the expectation system (Huron, 2006) which requires remembering much music, including the rules used during listening. The rules are acquired through mere listening to music, and those acquired rules are also used in creating music. Since not all music is pleasurable to listeners (culturally based), and because of the sensory exploitation principle, the learned rule system would be structured to remember pleasurable music. It learns what are pleasing music rules and uses them; as predicted, the greater the pleasure from music (more dopamine), the better its 'musical-memory' after a memory consolidation period (Cardona et al., 2020). This system will have been under selection through many generations producing greater capability in pattern generation, memory, and pleasure selectivity for creating more pleasurable music. This increase in pattern generation requires greater demands on the memory system for remembering music and its many (abstract) rules. For a review, see Ettlinger et al. (2011).

The benefit of the music rules system to language reuse is direct. Language learning is the building up of a set of procedures (rules) for understanding and producing language (Christiansen \& Chater, 2016); this includes memory intensive acquiring of vocabulary, 
categories, features and their relationships. As with the music elements, these language elements are also acquired from the surrounding culture of people. This language use is exactly like the learning of music rules and how memory for rules and pattern extraction is structured. Language development and use follows from the processing rules and regularities for the mapping between meanings and signals in the cognitive system provided by the music capabilities.

\section{Pleasure from Emotions Evoked by Music}

The pleasure from emotions evoked by music and from music chills become understandable in this framework; the memory requirements for learning the rules and patterns of music are huge, and emotions greatly enhance memory. Have a better and faster memory for music and the music rules system will then allow acquiring more rules (from listening to others); thus creating more pleasurable music. Hence, sensory exploitation will select for any traits that improve learning new music rules from other people; one of those traits is a better memory for music. Since emotions enhance memory greatly [happy and sad emotions use different memory systems (Kensinger \& Kark, 2018)], all emotional memory systems will eventually get selected for enhancing music memory -- memory for both what was heard veridically and the rules used in the anticipation. This means music is not for communicating emotions, but rather emotions are felt as a side effect of using them for memory enhancement (happy and sad music to the two memory systems). Furthermore, those music emotional responses will be selected to be pleasurable in order to motivate their important memory enhancing use.

Music chills are an extension of that memory enhancement by adding additional dopamine, epinephrine and cortisol, which strongly enhance memory (Kensinger \& Kark, 2018). Dopamine is critical in memory formation and is strongly present in the music evoked emotions (Cardona et al., 2020), as predicted here.

A problem is that the music evoked emotions appear specific to music and do not seem to occur with language in general. Hence, what is the benefit to language that is needed to prevent sex-linking of the traits for music emotion? The answer seems to be the exercising of the rule acquisition system with memory for what is heard along with the particular anticipation rules used. That is, this complex music rules system needs to acquire music rules developmentally in order to process language well; the pleasurable music emotions motivate practice on complex auditory music to hone the necessary learning skills; skills needed for language acquisition and use. This is supported by studies showing that music exposure may be needed to learn language well (Brandt et al., 2012). For example, informal music exposure at home impacts the development of complex language skills in 3 and 4year-old children (Politimou et al., 2019).

Interestingly then, young children must easily experience music evoked emotions and chills.

\section{Why should dancing be pleasurable?}

If the pleasure from dance is the initial sensory preference that can start the evolutionary cycle for music, where and why did the pleasure arise?

For humans, is moving in time to a beat an innate capability (perhaps a side-effect of vocal learning [2]) or is it a learned skill because some sensory sensation of dance is internally rewarded? We claim the latter. Dance is movement where the foot hits the ground or the head nods in time to music beats. Both of those movements will produce a vestibular jolt in time with an audible, rhythmic, musical beat. If some neural mutation caused that sensation to be internally rewarded - a Rhythmic Audible Vestibular Jolts (RAVJ) reward - dance would be rewarded and learned. Importantly, that mutation would have also produced human bipedalism; an advantage which would forever maintain that mutation (Riggle, 2018).

There is data supporting a RAVJ reward for motivating learning to dance (or operant conditioning). Infants (9-13 months old) will move to strongly rhythmic music (Zentner \& Eerola, 2010); however, their movements are jerky as they fling their limbs outwards and have a rapid stop at full extension (see the authors' video segments). That fling and stopping motion will produce a vestibular jolt. Importantly, and why this study is so critical, their movements were rarely in time to the music (period and phase), but when they were in time (on the beat), they smiled more $(\rho<0.01)$. Because smiling in infants mean they like what is occurring, the sensation must be pleasurable for them. This is consistent with a strong RAVJ reward motivating beginning to learn to move (to produce a vestibular jolt) in time to music.

Additional support comes from dancing children: dancing two-year-olds go in and out of phase; four-yearolds are much better while six-year-olds are near perfect. This is consistent with a rewarded learned skill; years of practice may be needed, but they really like to 
dance. Hence, the RAVJ reward is consistent as the initial reward for dancing pleasure which started the sensory exploitation cycles for creating the human music abilities and the side-effect of language.

\section{Conclusion}

We have shown that sensory exploitation of a sensory preference will drive evolution for rapid elaboration of traits that exploit the preference. This process is the first half of sexual selection, but the second half where sexlinking the trait occurs can be held off (and thus not be a fitness indicator trait). We have shown music can be developed under that evolutionary process as long as its developing cognitive machinery is also usable for language or other benefit to females.

Because pleasurable music is a sensory preference, the many developed cognitive abilities and pleasures for music are expected by a feedback loop of sensory exploitation. This theory explains music pleasures - why they exist and why there are so many. Additionally, this is the only theory that predicts music evoked emotions that enhance memory (and explains why happy and sad music emotions exist) and explains why music emotions are so prevalent and pleasurable (by dopamine). Furthermore, this theory explains why music, with emotions, interacts with language development, and why music exposure can improve language use in children.

We have shown music was not selected for communicating emotions (hard to show why that would be a benefit); in fact, music is not a communication system at all. However, because the emotional responses are selected to be so strong (for memory enhancement), human culture has figured out how to exploit that pleasurable emotional memory response (such as in film music). We also showed that language is consistent as an emergent capability based on the cognitive assets developed for music processing. This implies that language is also not for communication but supplies a benefit of a reasoning tool. Just as with music, language development needs to acquire the knowledge from the surrounding culture, hence the communication aspects of language.

Finally, we have shown that a simple neural reward mechanism for RAVJ may have started the dance pleasure which triggers the music evolution cycles.

\section{Endnotes}

1 Infanticide by males is common in chimpanzees but rare in bonobos. Bonobo females socially bond using sexual activity; hence, social bonding from dancing may have also prevented infanticide.

${ }^{2}$ Some issues on the vocal learning hypothesis: it does not explain the smiling babies when jolts are in time to the music -- the Zentner phenomena; why dance produces vestibular jolts; entrainment is a behavior and thus needs a reward motivation, left unexplained; why years are needed to become proficient; and the actual required relationship to language vocal use is unclear.

\section{References}

Bernardi, N. F., Bellemare-Pepin, A., \& Peretz, I. (2017). Enhancement of Pleasure during Spontaneous Dance. Frontiers in Human Neuroscience, 11. https://doi.org/10.3389/fnhum.2017.00572

Brandt, A. K., Slevc, R., \& Gebrian, M. (2012). Music and Early Language Acquisition. Frontiers in Psychology, 3. https://doi.org/10.3389/fpsyg.2012.00327

Cardona, G., Rodriguez-Fornells, A., Nye, H., Rifà-Ros, X., \& Ferreri, L. (2020). The impact of musical pleasure and musical hedonia on verbal episodic memory. Scientific Reports, 10(1), 16113. https://doi.org/10.1038/s41598$\underline{020-72772-3}$

Christiansen, M. H., \& Chater, N. (2016). Creating Language: Integrating Evolution, Acquisition, and Processing. MIT Press. https://doi.org/10.7551/mitpress/10406.001.0001

Ettlinger, M., Margulis, E. H., \& Wong, P. C. M. (2011). Implicit Memory in Music and Language. Frontiers in Psychology, 2. https://doi.org/10.3389/fpsyg.2011.00211

Huron, D. (2006). Sweet anticipation: Music and the psychology of expectation (pp. xii, 462). The MIT Press. https://doi.org/10.7551/mitpress/6575.001.0001

Kensinger, E. A., \& Kark, S. M. (2018). Emotion and Memory. In Stevens' Handbook of Experimental Psychology and Cognitive Neuroscience (pp. 1-26). American Cancer Society. https://doi.org/10.1002/9781119170174.epcn101

Politimou, N., Dalla Bella, S., Farrugia, N., \& Franco, F. (2019). Born to Speak and Sing: Musical Predictors of Language Development in Pre-schoolers. Frontiers in Psychology, 10. https://doi.org/10.3389/fpsyg.2019.00948

Riggle, M. (2018). A simple neural mutation that generates reward from rhythmic audible vestibular jolts fully explains all aspects of human bipedal gait development, including cerebral palsy gaits. Program No.495.12. 2018 Neuroscience Meeting Planner. San Diego, CA: Society for Neuroscience, 2018. Online., 898. 
Ryan, M. J. (1998). Sexual Selection, Receiver Biases, and the Evolution of Sex Differences. Science, 281(5385), 1999-2003. https://doi.org/10.1126/science.281.5385.1999

Zentner, M., \& Eerola, T. (2010). Rhythmic engagement with music in infancy. Proceedings of the National Academy of Sciences, 107(13), 5768-5773. https://doi.org/10.1073/pnas.1000121107 


\title{
Cortical representations of auditory perception using graph independent component analysis on EEG
}

\author{
Pranav Sankhe ${ }^{1 \dagger}$ \\ Ritik Madan ${ }^{1}$ \\ ${ }^{1}$ Indian Institute of Technology Bombay, Mumbai India \\ † Corresponding author: pranavsankhe40@gmail.com \\ Published 16 December 2021; https://doi.org/10.18061/FDMC.2021.0023 \\ Author video presentation and/or other conference material: https://doi.org/10.17605/OSF.IO/DTC3G
}

\begin{abstract}
Recent studies indicate that the neurons involved in a cognitive task aren't locally limited but span out to multiple human brain regions. We obtain network components and their locations for the task of listening to music. The recorded EEG data is modeled as a graph, and it is assumed that the overall activity is a contribution of several independent subnetworks. To identify these intrinsic cognitive subnetworks corresponding to music perception, we propose to decompose the whole brain graph-network into multiple subnetworks. We perform this decomposition to a group of brain networks by performing Graph-Independent Component Analysis. Graph-ICA is a variant of ICA that decomposes the measured graph into independent source graphs. Having obtained independent subnetworks, we calculate the electrode positions by computing the local maxima of these subnetwork matrices. We observe that the computed electrodes' location corresponds to the temporal lobes and the Broca's area, which are indeed involved in the task of auditory processing and perception. The computed electrodes also span the brain's frontal lobe, which is involved in attention and generating a stimulus-evoked response. The weight of the subnetwork that corresponds to the aforementioned brain regions increases with the increase in the music recording's tempo. The results suggest that wholebrain networks can be decomposed into independent subnetworks and analyze cognitive responses to music stimulus.
\end{abstract}

KEYWORDS: EEG, music perception, Graph Processing, ICA

\section{Introduction}

One of the most remarkable aspects of the human brain is its ability to recognize patterns and describe them. Among the amazing patterns we've tried to understand, music remains to be the most intensely thought and studied about. The process of studying ubiquitous properties of musical patterns is challenging because the interpretation of music has been inherently subjective through human history.
Our body carries out several varieties of tasks and almost all of those are carried directly or indirectly by the brain. An important question to ask is how neurons are allotted to a task and what is the relationship between neurons allotted to a particular task. This relationship can be modeled as a graph. From the neuroscientific studies of Bullmore (2009) and Bressler et al. (2010), we know that there exists a structure to function relationship for every cognition task. We consider this structural relationship from a network perspective. Studies show that there are a finite set of sub-networks which are dynamically allotted to cognitive tasks like listening to music.

There are many ways to study music perception. In our approach, we model brain activity as a graph. The hypothesis is that there are few underlying subnetworks that contribute to the overall functioning of the brain. To find out these underlying subnetworks, we perform Independent Component Analysis ICA on the EEG data recorded when the subjects were listening to music. Having obtained the subnetworks and thus the position of the electrodes, we intend to correspond them to the areas which according to literature, are indeed the regions who do the auditory processing.

\section{Method}

Dataset

We use Stanford's NMED-T dataset containing cortical (EEG) and behavioral data collected during natural music listening by Kaneshiro et al. (2017). EEG signals were recorded from 20 adult participants while listening to a set of 10 full-length songs with electronically produced beats at various tempos. The sampling rate of the EEG recording is $125 \mathrm{~Hz}$. The EEG data were preprocessed to remove artifacts.

Approach: Graph ICA (G-ICA)

To understand ICA, consider the cocktail party problem. Here, $\mathrm{n}$ speakers are speaking simultaneously at a party, 
and any microphone placed in the room records only an overlapping combination of the $n$ speakers' voices. Let us say we have $\mathrm{n}$ different microphones placed in the room, and because each microphone is a different distance from each of the speakers, it records a different combination of the speakers' voices. We ask whether using the recordings from microphones, can we separate out the original $\mathrm{n}$ speakers' speech signals? Independent Component Analysis essentially answers this question with a resounding yes.

Our approach is inspired by Park et al. (2014)'s approach to study networks using fMRI data for particular cognitive tasks. Graph-ICA is a variant of ICA that decomposes measured graphs into independent source graphs. We represent the graph by its adjacency matrix. We denote a graph (i.e. an adjacency matrix) with $\mathrm{L}$ nodes of the $\mathrm{i}$-th brain, a vector, $\mathrm{g}_{\mathrm{i}}$, with $\mathrm{K}=$ $\mathrm{L}(\mathrm{L}-1) / 2$ edges for elements. Assume $\mathrm{N}$ independent network components $\mathrm{sj}, \mathrm{j}=1, . ., \mathrm{N}$ exist in the brain. $\mathrm{M}$ graphs from $\mathrm{M}$ brains, i.e., gi, $\mathrm{i}=1, \ldots, \mathrm{M}$ were concatenated to a matrix $\mathbf{g}$.

$$
\mathbf{g}_{\mathbf{i}}=\sum^{\mathrm{N}} \mathrm{j}_{\mathbf{1}} \quad \mathbf{a}_{\mathbf{i j}} \mathbf{S i}
$$

where, $\mathbf{a}_{\mathbf{i j}}$ is the element of mixing matrix $\mathbf{A}$ indicating the contribution of (graph) source $\mathbf{S} \mathbf{j}$.

For this study, we assumed the number of ICs $(\mathrm{N})$ equaled the number of graphs (M), i.e., $\mathrm{N}=\mathrm{M}=20$, since we do not have a clear a priori knowledge of the number of ICs. The mixing matrix A can be estimated by an ICA algorithm, which maximizes mutual independence between estimated functional components.

For each participant, only upper diagonal elements of the adjacency matrix were used for graph-ICA since the adjacency matrix is symmetric in this study. The upper diagonal elements were vectorised. The subnetwork matrices obtained from ICA are also vectorised. To convert the vectorised subnetworks into matrix form, the vector is considered as a representative of the upper diagonal matrix. We employ ICA over these vectors. Having obtained the subnetworks, we identify the active EEG electrodes corresponding to the individual subnetworks and plot them on the surface of an inflated brain in order to study the cortical areas they correspond to.

\section{Results}

Once we get all the 20 subnetworks, we rank them based on their weights in the mixture. We observe that the subnetwork with the second-highest weight corresponds to the cortical regions, which are indeed responsible for audio processing in our brain. This observation does not change when we change the music stimuli (thus changing the tempo), which indicates the validity of the results.

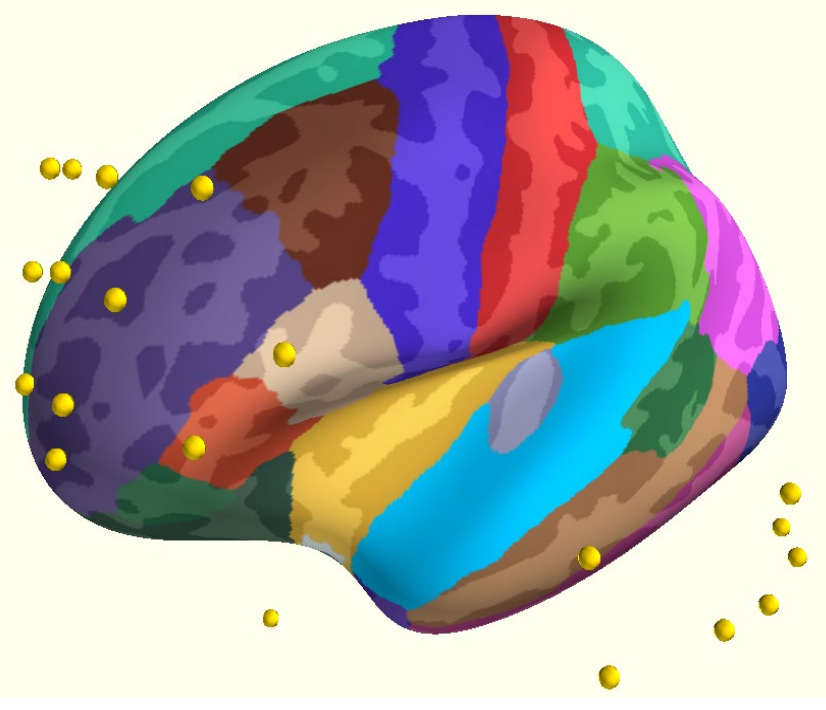

Figure 1: Subnetwork electrodes corresponding to auditory perception cortical areas

We observed that the subnetwork corresponding to auditory cortical areas covered in particular the Rostral Middle Frontal, Superior Frontal, Pars Opercularis, Pars Triangularis, Superior Temporal, and Transverse Temporal region of the brain. The Pars Opercularis, Pars Triangulairis regions are involved in semantic understanding of audio stimuli according to the study by Maess et al. (2001). The Superior Temporal and Transverse Temporal cortical areas belong to the auditory cortex, which is involved in the cognitive processing of incoming audio stimuli as found by Pickles at al (2012). The frontal cortical area carries out functions like coordinating sensory systems, emotion, and carrying out tasks involving past memory as reported by Goldberg et al. (2006).

After identifying the auditory subnetwork, we studied how its weight (obtained by G-ICA) varies with the tempo of the music stimuli. We observed that in almost all the cases, the weight of the auditory subnetwork increases proportionately with the tempo of the stimuli. 

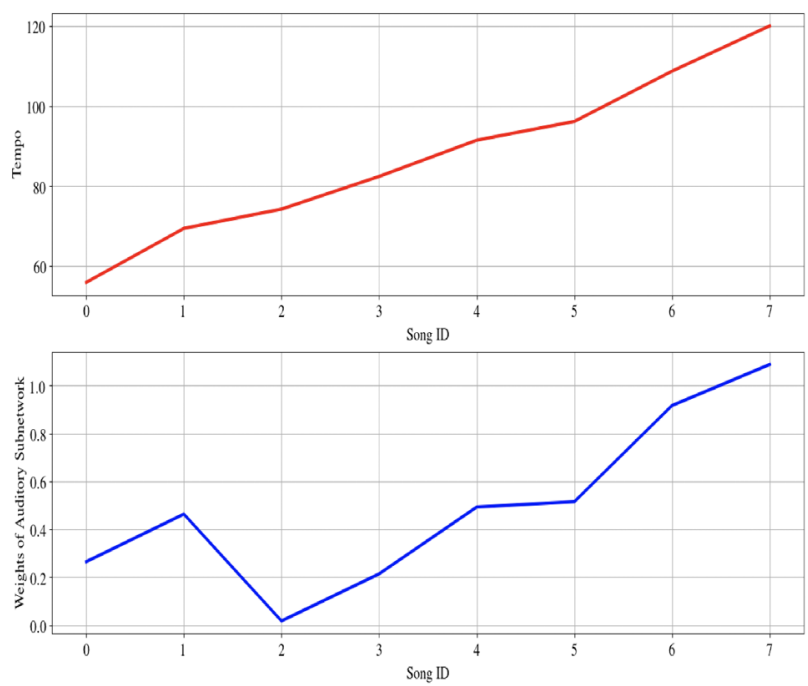

Figure 2: Subplot 1 shows how tempo varies with the audio stimuli. Subplot 2 depicts the variation of weight of auditory subnetwork with the audio stimuli.

\section{Conclusion}

We applied Graph Independent Component Analysis on EEG data to study brain activity networks for music perception. We identified the auditory subnetwork and studied its variation with the tempo of the music stimuli. The proportional relationship between the tempo and the weight of the subnetwork is encouraging for further investigation of different properties of music stimuli and understanding how they correspond with the subnetwork.

\section{References}

Bullmore E, Sporns O (2009) Complex brain networks: graph theoretical analysis of structural and functional systems. Nat Rev Neurosci 10: 186-198. https://doi.org/10.1038/nrn2575

Bressler SL, Menon V (2010) Large-scale brain networks in cognition: emerging methods and principles. Trends Cogn Sci 14: 277-290. https://doi.org/10.1016/j.tics.2010.04.004

Mesulam MM (1998) From sensation to cognition. Brain 121

1013-1052. https://doi.org/10.1093/brain/121.6.1013

Park, B., Kim, D. S., \& Park, H. J. (2014). Graph independent component analysis reveals repertoires of intrinsic network components in the human brain. PloS one, 9(1), e82873. https://doi.org/10.1371/journal.pone.0082873

Cf. Pickles, James O. (2012). An Introduction to the Physiology of Hearing (4th ed.). Bingley, UK: Emerald
Group Publishing Limited, pp. 215-217.

Goldberg I, Harel M, Malach R (2006). "When the brain loses itself: prefrontal inactivation during sensorimotor processing". $\quad$ Neuron. $50 \quad(2)$ : https://doi.org/10.1016/j.neuron.2006.03.015 


\title{
Sound and feeling: Musical training moderates the association between adult ADHD and emotion regulation
}

\author{
Michael Tate ${ }^{1 \dagger}$ \\ David M. Greenberg, $\mathrm{PhD}^{2,3}$ \\ Sarah O’Neill, $\mathrm{PhD}^{1,4}$ \\ ${ }^{1}$ The City College of New York, City University of New York, New York City, NY, USA \\ ${ }^{2}$ Bar-Ilan University, Ramat Gan, Tel Aviv, Israel \\ ${ }^{3}$ University of Cambridge, Cambridge, United Kingdom \\ ${ }^{4}$ The Graduate Center, City University of New York, New York City, NY, USA \\ ${ }^{\dagger}$ Corresponding author:mtate@gradcenter.cuny.edu \\ Published 16 December 2021; https://doi.org/10.18061/FDMC.2021.0024 \\ Author video presentation and/or other conference material: https://doi.org/10.17605/OSF.IO/75ZUS
}

\begin{abstract}
This study investigated whether adults with ADHD ("Cases") regulate emotions differently than their non-ADHD peers ("Controls"), and whether this effect is moderated by the number of years of musical training. We predicted that Cases would have poorer emotion regulation than Controls and the relation between ADHD and poor emotion regulation would be weakened with increased years of musical training. 1537 participants aged $18-65(\mathrm{M}=32, \mathrm{SD}=12$ years $)$ were recruited from the Music Universe project (www.musicaluniverse.org; Greenberg, \& Rentfrow, 2017). 57\% were female and $8 \%$ had a lifetime ADHD diagnosis. Emotion regulation was measured with the Mentalized Affectivity Scale (Greenberg et al., 2017), which assesses the ability to: identify, control and modulate, and express emotions inwardly or outwardly. More years of musical training was significantly associated with a better ability to identify emotions, irrespective of clinical status. Cases were significantly better at identifying emotions compared to Controls, but the groups did not differ in how they processed emotions. Adults with ADHD showed higher outward emotional expression, but only when number of years of music training was low/moderate. Musical training shows promise for improving how adults with ADHD express emotions, perhaps underpinned by stronger top-down mechanisms at work in emotional self- control.
\end{abstract}

KEYWORDS: ADHD, emotion regulation, musical training, self-regulation.

\section{Introduction}

Attention-deficit/ Hyperactivity Disorder (ADHD) is a common, impairing neurodevelopmental disorder characterized by symptoms of inattention, hyperactivity and impulsivity (American Psychiatric Association, 2013). ADHD affects approximately 7\% of children (Thomas et al., 2015) and 2.8\% of adults worldwide
(Fayyad et al., 2017). However, during recent years, the largest increases in ADHD prevalence have been observed in adults (not children) (Chung et al., 2019). Between 2007 and 2016, there was a 123\% increase in the prevalence of ADHD in adults (Chung et al., 2019).

In addition to the core symptoms of ADHD, 34 to $70 \%$ of adults with ADHD experience emotion regulation difficulties (Shaw et al., 2014). Emotion regulation has been defined "as an individual's ability to modify an emotional state so as to promote adaptive, goal-oriented behaviors" (Shaw et al., 2014, p. 279). The study of emotion regulation difficulties among adults with elevated ADHD behaviors is critical as emotion dysregulation is an independent predictor of their functional impairment, above and beyond inattention and hyperactivity/impulsivity (Barkley \& Fischer, 2010; O’Neill \& Rudenstine, 2019). For example, Barkley and Fischer (2010) found that among young adults with a childhood history of hyperactivity, emotional impulsiveness was an independent predictor of overall impairment, and specifically, their home life, intimate and other interpersonal relationships, financial management, driving, and engagement in community activities. Among adults seeking treatment at a community-based mental health clinic, O'Neill and Rudenstine (2019) found that emotion regulation deficits mediated the association between inattention and severity of anxiety and depression, as well as the relationship between inattention and problems with friends, family and romantic partners. Taken together, findings highlight the need for interventions to address not only the core symptoms of ADHD, but also the associated emotion regulation difficulties.

One intervention that may show some promise is music training. The music therapy and music cognition literature describe music's impact on various domains 
of cognitive functioning, including attention, language processing, working memory, as well as emotion regulation (see Moreno \& Bidelman, 2014 for a review). To date, however, no one has studied the impact of music training on emotion regulation among individuals with ADHD.

The aims of the current study were to examine if adults with a lifetime diagnosis of ADHD regulate emotions differently than their peers who have no mental health diagnosis, and if this effect is moderated by the amount years of musical training received. We hypothesized that adults with a lifetime diagnosis of ADHD ("Cases") would have poorer emotion regulation than adults with no history of mental health problems ("Controls"), and that this relation would be moderated by the number of years of music training. That is, the relation between ADHD and poor emotion regulation would be weakened as people had more years of music training.

\section{Method}

Participants

Participants are from the Musical Universe project, and self-reported measures voluntarily on www.musicaluniverse.org, from November 2015 and ending July 2016. Participants were recruited via international media outlets (e.g., CNN, IFLScience). The current sample comprises 2129 participants who ranged in age from $18-65$ years $(\mathrm{M}=32, \mathrm{SD}=12$ years) and who completed the Mentalized Affectivity Scale (Greenberg, Kolasi, Hegsted, Berkowitz, \& Jurist, 2017), the emotion regulation questionnaire utilized in the current study.

Just over half $(57 \%)$ of the sample identified as female and $43 \%$ identified as male. Nearly three quarters $(72.1 \%)$ of the participants identified as white; $1.7 \%$ identified as Black/African American; $4.4 \%$ as Latinx; $9.9 \%$ as Asian; $<1 \%$ as Native American/Pacific Islander; $11.4 \%$ identified as belonging to a racial group other than those listed. The sample was generally well educated with the majority of participants having an undergraduate degree $(35.4 \%)$ or graduate degree $(25.9 \%)$. Approximately one fifth of the sample had a High School diploma or equivalent (20.8\%); 16.5\% were currently attending high school, and $1.4 \%$ had not completed high school.

\section{Materials}

The Music Universe database comprises 17 sections that broadly measure a participant's musical preferences, personality, emotion regulation, and demographic information. ADHD diagnosis was assessed by a single question, which asked participants to indicate if they had ever been diagnosed with any of the mental health disorders listed, one of which was ADHD.

Musical training was assessed by a single self-report item. Participants stated how many years of musical training they had received in their lifetime.

Emotion Regulation was measured using the Mentalized Affectivity Scale (MAS; Greenberg et al., 2017). The 60-item self-report assessment measures three dimensions of emotion regulation, based on Jurist's (2005) theory of Mentalized Affectivity (MA): Identifying, Processing and Expressing emotions. Identifying emotions involves, at its most basic level, labeling emotions, but also includes exploring deeper emotional meaning such as, "Why am I feeling this way?" Processing Emotions measures one's ability to modulate or regulate the duration and intensity of an emotion. Expressing Emotions measures the ways in which one communicates emotions on a spectrum from outwardly to inwardly. Participants were asked to rate the extent they agreed or disagreed with each statement on a 7-point scale. High scores for both the Identifying and Processing domains represent a stronger ability to label emotions and modulate the intensity of those emotions respectively. For Expressing Emotions, lower scores reflect outward expression of emotions whereas higher scores reflect greater inward expression of emotions. The MAS was validated in a non-clinical sample of 2,000 individuals.

\section{Procedure}

Participants completed self-report measures voluntarily on the internet by going to www.musicaluniverse.org. The present study examined basic demographic information (e.g., age, gender, race/ethnicity), clinical diagnoses held by the individual, years of formal music training received, and emotion regulation capacity.

\section{Data Analysis}

Secondary analysis of the Musical Universe database was performed. Emotion regulation was parsed into three dimensions - Identifying, Processing and Expressing, each of which was normally distributed across the sample. Bivariate correlation analysis examined relations among the three MAS dimensions. An independent-samples t-test was conducted to compare each emotion regulation dimension between Cases and Controls. Years of formal music training was not normally distributed across the sample therefore a 
Mann-Whitney $U$ test examined whether there were differences in years of formal music education between Cases and Controls. Moderation analysis was conducted using Hayes' (2018) PROCESS to test whether ADHD Diagnosis (IV) was associated with differences in the three dimensions of Emotion Regulation (DV), and to see whether these relations were moderated by a continuous measure of the number of years of music training (M). Number of years of music training was mean centered and the HC3-estimator was used to account for non-normality of residuals. 10,000 bootstrap samples were taken to estimate the indirect effect.

\section{Results}

125 participants reported an ADHD diagnosis ("Cases"), and 1448 participants reports having no mental health diagnosis ("Controls"). A wide range of years of formal music training was observed from 0 years to 41 years of training. The median years of music training participants completed was $3(\mathrm{IQR}=0-7)$ but $35 \%$ of participants had 0 years of music training. A Mann-Whitney U test showed no significant differences in years of formal music education between Cases $(\mathrm{Mdn}=4.75)$ and Controls $(\mathrm{Mdn}=4.50), \mathrm{U}\left(\mathrm{N}_{\mathrm{ADHD}}=105\right.$, $\left.\mathrm{N}_{\text {Controls }}=1252\right)=69100, \mathrm{z}=.89, p=.37$.

Bivariate correlation analysis revealed significant, but weak relations among the three dimensions of emotion regulation. For Identifying and Processing emotions, $r=.24, p<.0001$; Identifying and Expressing emotions, $\quad r=-.24, \quad p<.001 ;$ and Processing and Expressing emotions, $r=-.22, p<.001$.

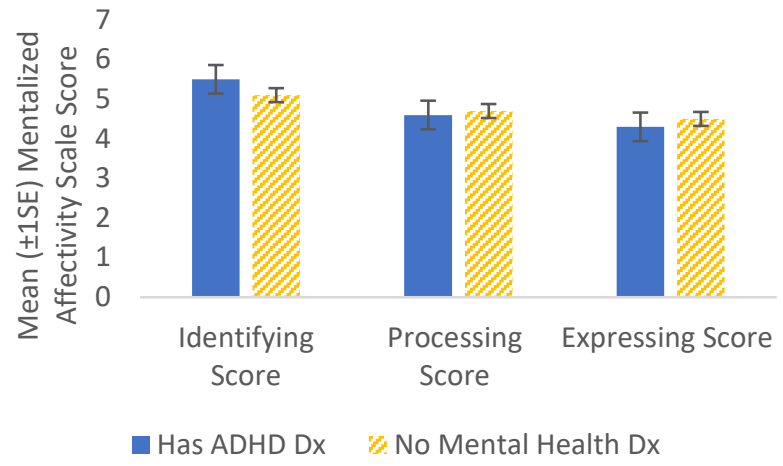

Figure 1: Mean $( \pm 1 S E)$ scores for the Mentalized Affectivity Scale domains - Identifying, Processing and Expressing - as a function of ADHD diagnosis.

Mean differences in the three domains of emotion regulation were calculated between Cases and Controls (see Figure 1). Compared to Controls, the Cases: scored higher on the Identifying emotions scale, $t(1571)=-4.66$, $p<.0001, \mathrm{~d}=.47$; showed no difference in Processing of emotions, $t(1571)=1.49, p=.14, \mathrm{~d}=.13$; and scored significantly lower on the Expressing emotions scale, $t(1571)=2.58, p=.01, \mathrm{~d}=.23$.

Moderation analyses were carried out to determine whether music training moderated the association between ADHD and emotion regulation (see Table 1).

Table 1: PROCESS Moderation Results

\begin{tabular}{|c|c|c|c|c|}
\hline $\begin{array}{l}\text { Model/ } \\
\text { Predictor }\end{array}$ & Coefficient & $t$ & $p$ & $\begin{array}{l}\text { 95\% CI } \\
\text { (Lower, } \\
\text { Upper) }\end{array}$ \\
\hline \multicolumn{5}{|l|}{ Identifying } \\
\hline Constant & 5.11 & 205.46 & $<.0001$ & $5.05,5.15$ \\
\hline $\begin{array}{l}\text { ADHD } \\
\text { diagnosis }\end{array}$ & .33 & 3.96 & .0001 & $.17, .49$ \\
\hline $\begin{array}{l}\text { Music } \\
\text { Training }\end{array}$ & .007 & 1.39 & .17 & $-.003, .02$ \\
\hline $\begin{array}{l}\text { ADHD x } \\
\text { Music }\end{array}$ & -.02 & -1.2 & .23 & $-.06, .01$ \\
\hline \multicolumn{5}{|l|}{ Processing } \\
\hline Constant & 4.17 & 214.87 & $<.0001$ & $4.67,4.77$ \\
\hline $\begin{array}{l}\text { ADHD } \\
\text { diagnosis }\end{array}$ & -0.14 & -1.52 & .13 & $-.31, .04$ \\
\hline $\begin{array}{l}\text { Music } \\
\text { Training }\end{array}$ & 0.006 & 1.49 & .14 & $-.002, .01$ \\
\hline $\begin{array}{l}\text { ADHD x } \\
\text { Music }\end{array}$ & -.02 & -.96 & .34 & $-.05, .02$ \\
\hline \multicolumn{5}{|l|}{ Expressing } \\
\hline Constant & 4.52 & 159.43 & $<.0001$ & $4.46,4.57$ \\
\hline $\begin{array}{l}\text { ADHD } \\
\text { diagnosis }\end{array}$ & -0.22 & -2.13 & .03 & $-.43,-.02$ \\
\hline $\begin{array}{l}\text { Music } \\
\text { Training }\end{array}$ & -0.006 & -1.14 & .26 & $-.02, .004$ \\
\hline $\begin{array}{l}\text { ADHD x } \\
\text { Music }\end{array}$ & 0.04 & 2.25 & .02 & $.005, .08$ \\
\hline
\end{tabular}

A significant model for Identifying emotions was seen, $F(3,1353)=6.61, p=.0002$. ADHD diagnosis was a unique predictor, such that Cases identified emotions significantly better than Controls. There was no main effect of Music Training on identifying emotions, and no significant ADHD x Music Training interaction.

A non-significant model for Processing emotions was obtained, $F(3,1353)=1.62, p=.18$ (see Table 1$)$. Neither ADHD diagnosis nor years of music training 
were associated with Processing and no significant ADHD diagnosis $x$ Music Training interaction emerged.

A significant model for Expressing emotions was obtained, $F(3,1353)=3.52, p=.015$ (see Table 1). A significant association between ADHD diagnosis and Expressing emotions was qualified by a significant ADHD diagnosis $x$ years of Music Training interaction (see Figure 2). Cases had significantly lower Expressing scores (i.e., more outward emotional expression) than Controls when years of formal music training was low $\left(16^{\text {th }}\right.$ percentile, $t(1353)=-3.18, p=.002,95 \%$ CI:-. 67 , .16.) or moderate $\left(50^{\text {th }}\right.$ percentile, $\mathrm{t}(1353)=-2.70$, $p=.007,95 \%$ CI:-.50, -.08.). When years of formal music training was high $\left(84^{\text {th }}\right.$ percentile, $t(1353)=.05$, $p=.96,95 \%$ CI: $-.29, .30)$, no differences in Expressing was seen between Cases and Controls.

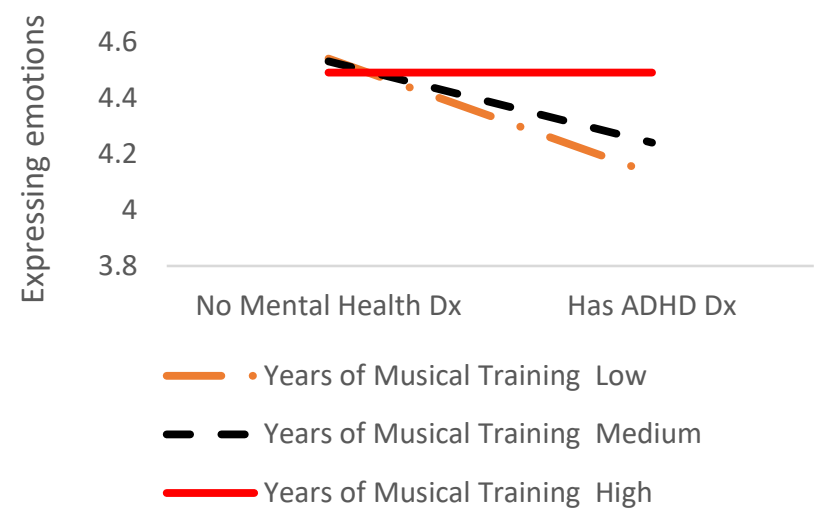

Figure 2: Interaction between ADHD diagnosis and years of music training on Expressing domain of Mentalized Affectivity Scale.

\section{Discussion}

The aim of the current study was to determine whether the association between ADHD and emotion regulation capacity is moderated by years of formal music training.

First, we found an association between ADHD diagnosis and Identifying emotions. Somewhat surprisingly, those with a lifetime history of ADHD ("Cases") were found to identify emotions significantly better than those without a mental health diagnosis ("Controls"). Music training was not associated with Identifying emotions, and nor did music training moderate the effect of ADHD on capacity to identify emotions. Greenberg and colleagues (2017) described one's ability to identify emotions as an ability to recognize emotions and make sense of emotions. It is possible that adults with a lifetime diagnosis of ADHD are attuned to their own emotion distress, but not to that of others. This is an area for future research.

The processing emotions domain measured an individual's ability to modulate and regulate emotions. This includes changing emotions in different ways, such as by duration or intensity (Greenberg et. al, 2017). Adults with a lifetime diagnosis of ADHD did not differ in how they process emotions when compared to individuals without a mental health diagnosis, which was surprising given the commonly reported emotion dysregulation among individuals with ADHD. For example, Shushakova, Ohrmann, and Pedersen (2017) found that compared to healthy controls, adults with ADHD showed more elevated emotional reactivity to negative stimuli, had decreased ability to evaluate their capacity to regulate emotions or to reappraise emotional situations, and that their use of emotion regulation strategies took greater cognitive effort.

In the present study, the lack of an effect of lifetime ADHD diagnosis on emotion Processing may be due to sample bias. It is possible that individuals who selfselected to take part in this study may have been relatively higher functioning than other adult ADHD samples.

Finally, research has shown that adults with a lifetime diagnosis of ADHD exhibited greater emotional impulsivity and emotion dysregulation, which is expressed primarily through irritability, aggression and temper outbursts (Mitchell, Robertson, Anastopolous, Nelson-Gray, \& Kollins, 2012; Shaw et al., 2014). Consistent with this literature, results of our analysis show that Cases showed greater outward emotion expression. This may reflect the inhibitory control deficits experienced by many with ADHD (Barkley, 1997).

Last, the relation between lifetime ADHD diagnosis and emotion expression was moderated by years of music training in a dose-dependent manner. When number of years of music training was high, no difference was observed between Cases and Controls, perhaps reflecting stronger top-down control of emotions after many years of training. Shaw and colleagues (2014) report that individuals with ADHD have deficits in their ability to adjust top-down regulation in response to different emotional stimuli. Research on music training has shown it can lead to improvement of top-down cognitive processes in general populations (e.g., Kim, Wigram \& Gold, 2008). Thus, it is possible that those adults with a lifetime 
diagnosis of ADHD who had many years of music training developed better self-regulation capacity.

\section{Conclusion}

To our knowledge this is one of the first studies to explore the effects of formal music training on emotion regulation among individuals with ADHD. Given the possible connection between deficits in cognitive processes, such as working memory and inhibitory control, and emotion dysregulation (Barkley, 1997; Shaw et al., 2014), future research should examine the extent to which inhibitory control mediates the effects of formal music training on emotion regulation among individuals with ADHD. Further, research should focus on whether music training affects symptom remittance thus providing evidence for changes in lifetime symptom trajectory. Also, future research should be conducted to determine if music training could serve as an efficacious intervention that improves both emotion dysregulation in individuals with ADHD as well as the core ADHD symptoms.

\section{References}

American Psychiatric Association. (2013). Diagnostic and statistical manual of mental disorders (5th ed.). https://doi.org/10.1176/appi.books.9780890425596

Barkley R. A. (1997). Behavioral inhibition, sustained attention, and executive functions: constructing a unifying theory of ADHD. Psychological bulletin, 121(1), 65-94. https://doi.org/10.1037/0033-2909.121.1.65

Barkley, R. A., \& Fischer, M. (2010). The unique contribution of emotional impulsiveness to impairment in major life activities in hyperactive children as adults. Journal of the American academy of child and adolescent psychiatry, 49(5), 503-513. https://doi.org/10.1016/j.jaac.2010.01.019

Chung, W., Jiang, S. F., Paksarian, D., Nikolaidis, A., Castellanos, F. X., Merikangas, K. R., \& Milham, M. P. (2019). Trends in the Prevalence and Incidence of Attention-Deficit/Hyperactivity Disorder Among Adults and Children of Different Racial and Ethnic Groups. JAMA network open, 2(11), e1914344. https://doi.org/10.1001/jamanetworkopen.2019.14344

Fayyad, J., Sampson, N. A., Hwang, I., Adamowski, T., Aguilar-Gaxiola, S., Al-Hamzawi, A., Andrade, L. H., Borges, G., de Girolamo, G., Florescu, S., Gureje, O., Haro, J. M., Hu, C., Karam, E. G., Lee, S., Navarro-Mateu, F., O'Neill, S., Pennell, B. E., Piazza, M., Posada-Villa, J., ... WHO World Mental Health Survey Collaborators (2017). The descriptive epidemiology of DSM-IV Adult ADHD in the World Health Organization World Mental Health Surveys. Attention deficit and hyperactivity disorders, 9(1), 47-65. https://doi.org/10.1007/s12402016-0208-3

Greenberg, D. M., Kolasi, J., Hegsted, C. P., Berkowitz, Y., \& Jurist, E. L. (2017). Mentalized affectivity: A new model and assessment of emotion regulation. PloS one, 12(10), e0185264. https://doi.org/10.1371/journal.pone.0185264

Jurist, E. L. (2005). Mentalized affectivity. Psychoanalytic psychology, 22(3), 426-444. https://doi.org/10.1037/07369735.22.3.426

Kim, J., Wigram, T., \& Gold, C. (2008). The effects of improvisational music therapy on joint attention behaviors in autistic children: a randomized controlled study. Journal of autism and developmental disorders, 38(9), 1758-1766. https://doi.org/10.1007/s10803-008-0566-6

Mitchell, J. T., Robertson, C. D., Anastopolous, A. D., Nelson-Gray, R. O., \& Kollins, S. H. (2012). Emotion dysregulation and emotional impulsivity among adults with attention- deficit/hyperactivity disorder: Results of a preliminary study. Journal of psychopathology and behavioral assessment, 34, 510-519. https://doi.org/10.1007/s10862-012-9297-2

Moreno, S., \& Bidelman, G. M. (2014). Examining neural plasticity and cognitive benefit through the unique lens of musical training. Hearing research, 308, 84-97. https://doi.org/10.1016/j.heares.2013.09.012

O’Neill, S., Rudenstine, S. (2019). Inattention, emotion dysregulation and impairment among urban, diverse adults seeking psychological treatment. Psychiatry research, 282, 112631. https://doi.org/10.1016/j.psychres.2019.112631

Shaw, P., Stringaris, A., Nigg, J., \& Leibenluft, E. (2014). Emotion dysregulation in attention deficit hyperactivity disorder. The American journal of psychiatry, 171(3), 276293. https://doi.org/10.1176/appi.ajp.2013.13070966

Shushakova, A., Ohrmann, P., \& Pedersen, A. (2018). Exploring deficient emotion regulation in adult ADHD: electrophysiological evidence. European archives of psychiatry and clinical neuroscience, 268(4), 359-371. https://doi.org/10.1007/s00406-017-0826-6

Thomas, R., Sanders, S., Doust, J., Beller, E., \& Glasziou, P. (2015). Prevalence of attention-deficit/hyperactivity disorder: a systematic review and meta-analysis. Pediatrics, 135(4), e994-e1001. 


\title{
Stimulus accessibility and music theory/therapy
}

\author{
Anna Yu Wang \\ Graduate School of Arts and Sciences, Harvard University, Cambridge, MA, United States \\ Corresponding author: anna wang@g.harvard.edu \\ Published 16 December 2021; https://doi.org/10.18061/FDMC.2021.0025 \\ Author video presentation and/or other conference material: https://doi.org/10.17605/OSF.IO/GXF6R
}

\begin{abstract}
This paper draws on music therapeutic, neuroscientific, and philosophical literature to posit three aspects of musical engagement that qualify music as an unusually accessible stimulus: 1) audition as a means of self-orientation 2) music's instigation of self-referential thought, and 3) the lower threshold required for processing musical meaning compared to linguistic meaning. This accessibility renders music a promising therapeutic stimulus for people living with a disorder of consciousness or other cognitive disorders, as clinical studies suggest. Moreover, this paper argues that culturally sensitive music theory and cognition can help maximize music's therapeutic potential by clarifying the variables that influence the accessibility of musical stimuli. Specifically, by complicating the research findings from participant cohorts dominated by members of Western, Educated, Industrialized, Rich, and Democratic (WEIRD) societies, music theory and cognition can illuminate how cultural context impacts the manner and extent to which listeners derive therapeutic benefit from musical structures. This suggests that there is fertile ground for future collaborative work between music therapists, cognitivists, and theorists.
\end{abstract}

KEYWORDS: music therapy, disorders of consciousness, accessibility, music theory, world music

\section{Introduction}

Disorders of consciousness (DOC) is an umbrella term that encompasses comatose, vegetative, and minimally conscious states, each of which severely reduces a person's ability to engage with their surroundings cognitively and physically. At the mild end of the DOC spectrum a person may not be able to comprehend spoken commands, while those more gravely affected require life-sustaining therapy and exhibit only the scarcest signs of awareness (Giacino et al., 2013). While pharmaceutical treatments for DOC remain limited, a growing body of literature points optimistically to the rehabilitative potential of music therapy (Grimm and Kreutz, 2018; Magee et al., 2016; Sun and Chen, 2015). This paper frames music's therapeutic efficacy as a matter of stimulus accessibility, which has been defined by Hansen (1959) as "the potential for interaction."

Along these lines, Perrin et al. (2015) have argued that "stimulus selection is critical for an accurate evaluation of the state of a patient with a disorder of consciousness as it determines the level of processing that a patient can have with his/her environment" (1). Identifying stimuli that are accessible enough to elicit cognitive processing and an agential response from DOC patients can be of seminal importance for their recovery. Patient responsiveness may improve the likelihood of an accurate diagnosis - with misdiagnosis potentially leading to substantial differences in the care team's allocation of rehabilitative and even lifesustaining resources (Edlow et al., 2017) - and it may help strengthen the patient's remaining neural connections (Langille and Brown, 2018).

This paper posits three reasons that qualify music as an unusually accessible stimulus for DOC patients: 1) the relationship between audition and self-orientation 2) music's instigation of self-referential thought, and 3) the lower threshold required for processing musical meaning compared to linguistic meaning. Clarifying the mechanism by which music is accessible to those with limited cognitive function will help clinicians more strategically harness music's therapeutic applications for people living with DOC and potentially other neurocognitive disorders such as dementia (El Haj et al. 2012).

This paper also draws on recent research in music theory and cognition to posit that not all music is equally accessible to every patient, and that accessibility depends on the degree of synergy that the chosen musical stimulus strikes with the patient's listening habits - those habits being socially and culturally formed. This adds a therapeutic imperative to the accumulating list of reasons for which the cultural scope of music theoretical and cognitive research should be expanded beyond the perceptual norms and preferred repertoires of WEIRD listeners-listeners from Western, Educated, Industrialized, Rich, and Democratic societies (Jacoby et al., 2020; Henrich et al., 2010). 


\section{Music as an Accessible Stimulus}

From the standpoints of diagnosis and recovery, it is crucial that DOC patients are invited to respond with their highest level of cognitive facility. For this, the appropriate selection of stimuli is of critical importance. The following broadly theorizes three ways that music represents an unusually accessible stimulus for individuals with limited consciousness on the grounds that being self-oriented within one's surroundings and having self-referential thought are requisites of conscious activity (Damasio, 1999; Morin, 2006; Kotchoubey, 2018).

\section{Audition and Self-Orientation}

Kotchoubey et al. (2015) collated ample neuroscientific evidence which suggest that audition implicates not only hearing but self-orientation. For example, they note that most of the input into the auditory cortex does not come directly from acoustic sub-cortical regions but from other cortices in the brain. They further point out that unlike the visual cortex, which pairs complex stimuli down to its basic components, the auditory system works on both complex and simple stimuli in tandem at nearly all levels of processing. For example, neurons on the auditory belt are not responsible for simple frequencies, but for harmonic spectra (Rauschecker, 1997). Moreover, upon observing mismatch negativity (MMN) elicited in the auditory cortex, Näätänen et al. (2001) speculated that the auditory cortex is also a site of "primitive" cognitive activity whereby sound patterns are tracked and anticipated. The auditory cortex's topdown heavy reception of information, its preference (in some regions) for complex tones rather than pure sine tones, and its ability to parse sonic patterns imply that the auditory cortex is more than a sensory processor. Rather, it performs a more holistic and associative task, appearing to be a site of context construction and of selfpositioning within an acoustic environment.

In this sense, audition can be likened to a form of proprioception, a haptic feedback which results in the awareness of oneself in relation to one's surrounding context. Music, then, as an auditory stimulus, taps into the proprioceptive system by which one gains awareness of the surrounding environment. Along these lines, Acitores (2011) drew on James Gibson's, Mark Johnson's, and Gerald Edelman's theories that consciousness requires proprioceptive awareness of the external environment to argue that conscious awareness resides within the body itself (as opposed to only in the "mind" in a mind/body dualist framework). She then connected this embodied mode of interacting with the world to the inextricability of body schema from musical engagement, as elaborated in Cox's (2011) mimetic hypothesis. Taken altogether, audition, the body, and music are related in their ability to foreground one's physical relationality with the environment, which in turn is an indispensable ingredient within consciousness and responsiveness.

\section{Music and Self-Referentiality}

Just as proprioception is a necessary condition for awareness, self-referential thought is necessary for the formation of conscious, agential responses. As such, neuroscientists working on DOC have devoted significant labor to the study of the default mode network (DMN) (e.g. Mäki-Marttunen et al., 2016; He et al., 2015; Bodien et al., 2019). The DMN is a network of neural structures hypothesized to be directly implicated in self-referential thought (Davey et al., 2016; Ino et al., 2011). Most recently, Bodien et al. (2019) studied the relationship between an intact DMN and a phenomenon known as covert consciousness. They concluded that while the DMN is a necessary condition for consciousness, it is not a sufficient condition: they found that there were some people with intact DMNs who did not exhibit conscious behavior. Nevertheless, there is much evidence that self-relevant stimuli are more cognitively accessible (Kempny et al., 2018; Perrin et al., 2015). For example, Howarth and Ellis (1961) found that there is a lower activation threshold (less energy required) for the perception of one's own name versus a random other first name. Given the limited neural resources of a DOC patient, self-referential stimuli appear to yield a higher likelihood of patient engagement because they require less mental exertion.

Several studies indicate that music is one type of self-referential stimulus. El Haj et al. (2012) showed that people with mild onset of Alzheimer's Disease were able to recollect more details about a past memory during an autobiographical interview after being played Vivaldi's "Spring." They subsequently found that the effect is amplified when music of the participant's own preference is played. Verger et al. (2014) also showed that DOC patients performed better on the Coma Recovery Scale-Revised (CRS-R) - the "gold standard" for determining consciousness in DOC patients - after listening to music, suggesting that music appreciably facilitates a patient's cognitive functioning. This phenomenon might be partially accounted for by the 
nature of musical semiosis - that is, musical meaning making - as described in the following section.

\section{The Semiosis of Music Versus Language}

Music, like language, is a semiotic system - a system in which meanings are extracted from signs. The process of translating signs into meanings is mediated by an interpreting mind and can result in traces of the interpreter's inner constructs - including their memories, predispositions, and cultural backgroundbeing encoded into the extracted meaning. Just as mirrors serve to reflect our outer appearances, semiotic systems can serve as reflective surfaces of our inner constructs.

However, the degree of "reflectivity" elicited by a semiotic system is not the same across all stimuli. Rather, it depends on the nature of the sign being parsed. Music, for instance, is composed primarily of Peircean "indices" (Atkin, 2013) - signs that do not come with a codified lexicon, and whose meanings are created on a case-by-case basis in relation to the interpreter's experiences (Zbikowski, 2011). Music is therefore a rather reflective stimulus. Meanwhile, language is not as reflective because it consists mainly of Peircean "symbols"-signs whose meanings are already predefined by convention (Table 1). In other words, while the semiosis of language requires engagement with an externalized database of meanings - found in a dictionary-musical semiosis draws heavily on an internalized database for its meanings, as furnished by one's own memories and experiences. Therefore, musical stimuli theoretically constitute the more direct and unfettered pathway to the mind.

The implications of this hypothesis, if it is empirically supported, are substantial. For one, there would be reason to redesign the CRS-R, which currently relies heavily on the patient's ability to comprehend verbal commands. We might instead move towards, or at least normatively incorporate, diagnostic tests that include musical stimuli such as the MATADOC (Magee et al., 2014), which appear to elicit higher degrees of arousal from DOC patients than CRS-R tasks (Bodine et al., 2020). Moreover, support for this hypothesis would also establish a concrete direction for the development of future DOC treatments: treatments would, as much as possible, capture and project aspects of the pre-injured mind back to the patient.
Table 1: Descriptions of a Peircean symbol and index.

\begin{tabular}{|l|l|}
\hline $\begin{array}{l}\text { Peircean Symbol } \\
\text { (Many linguistic signs) }\end{array}$ & $\begin{array}{l}\text { Peircean Index } \\
\text { (Many musical signs) }\end{array}$ \\
\hline Arbitrary, Conventional & Logical relationship \\
\hline $\begin{array}{l}\text { Defined through social } \\
\text { agreement }\end{array}$ & $\begin{array}{l}\text { Grounded in personal } \\
\text { experience }\end{array}$ \\
\hline
\end{tabular}

\section{Possibilities for Collaboration between Music Therapists, Cognitivists and Theorists}

Music therapy training generally requires practitioners to be fluent in Western musical idioms and Western harmonic instruments like piano and guitar (Wigram et al. 2002; Howland 2017). Practitioners are not typically required to develop a refined understanding of how music is structured and expressed in other cultures. This might be attributed, at least in part, to the academic standard set by music therapy's older sibling fields of music theory and cognition, which have also historically centered around the principles and perception of Western music.

However, if the efficacy of music therapy is rooted in music's accessibility as a stimulus, and accessibility is about the potential for interaction between the stimulus and the listener, then accessibility cannot be generalized across all listeners. Rather, to achieve optimal accessibility, musical stimuli and their strategic use must be tailored to the unique social and cultural bearings of each listener. While it is well within music therapy's modus operandi to find musical stimuli that are meaningful to individual patients, the current institutionalized knowledge about musics beyond the West is insufficient to truly allow for the strategic therapeutic use of such musics. For patients whose habits of listening differ from those represented in conventional, Western-focused music cognitive and theoretical research, the optimization of stimulus accessibility depends not only upon the choice of repertoire, but also on a nuanced understanding of how the patient is attending to the musical structures found in that repertoire.

Recently, a small but growing corpus of music theoretical and cognitive research has begun to challenge the assumed pan-applicability of Western listening habits by showing that the same musical structure can trigger very different listener responses depending on cultural context. For example, Margulis et al. (2019) showed that while one musical example prompted images of play for audiences in Dimen, China, the same example conjured frightening images for 
audiences in Arkansas, USA. Working along the parameters of pitch and rhythmic perception, McDermott et al. (2016) demonstrated that the degree of pleasure that isolated dissonant sounds invoke for a listener varies across cultures, while Jacoby and McDermott (2017) found that rhythmic priors may also be largely culturally determined. In the realm of music theory, scholars have attempted to trace divergent listening habits back to specific cultural and ideological forces (Yu Wang, in press; Stover, 2020; Goldberg, 2020; Tse and Wong, 2020).

This type of research is uncovering radical differences in the way musical sound is conceptualized and attended to, suggesting that a musical stimulus should not be expected to invoke similar experiences nor to present the same degree of accessibility to all listeners. In order to maximize the accessibility of musical stimuli, it is necessary that we answer the following questions: What are the factors that render a particular musical structure more or less accessible (i.e. along the parameters of self-orientation, selfreferentiality, and memory invocation) to a given listener? How can we strategically select culturally resonant musical stimuli to elicit a desired listener response?

The onus is largely on music theorists and cognitivists, as scholars with a critical ear towards the perceptual implications of musical structure, to be sensitive to the diversity of ways in which musical structures can be attended to. We need culturally sensitive contributions to music theory and cognition not only for the lasting rigor of these fields, but for the benefit of patients and their loved ones who are relying on the restorative effects of music therapy. This line of reasoning encourages music cognitivists and theorists to think of music therapists as both a potential audience for our research, and also perhaps as future collaborators.

\section{Conclusion}

Accessibility of stimuli is particularly critical for patients with DOC, for whom the primary medical challenge is to restore their ability to interact with their surroundings. Music cognitivists and theorists are poised to augment the efficacy of music therapy by contributing research on the factors by which a musical stimulus is made more or less accessible to a given listener, as impacted by cultural context. When these conditions are known, music therapists will be better equipped to design tailored therapy plans for patients with DOC and potentially a broader array of cognitive disorders. There are thus consequential grounds for cooperation between music theorists, cognitivists, and therapists.

\section{Acknowledgements}

I am grateful to Brian Harris, Alexander Rehding, and Leo Hanyi $\mathrm{Yu}$ for their constructive comments on this paper.

\section{References}

Acitores, A.P. (2012). In Clarke, D. \& Clarke E. (Eds.), Music and consciousness (pp. 215-230). Oxford University Press.

Atkin, A. 2013. Peirce's Theory of Signs. In E.N. Zalta (ed.), The Stanford encyclopedia of philosophy. Metaphysics Research Lab, Stanford University. https://plato.stanford.edu/archives/sum2013/entries/peirc e-semiotics/.

Bodien, Y.G., Threlkeld, Z.D., \& Edlow, B.L. (2019). Default mode network dynamics in covert consciousness. Cortex, 119 571-574.

https://doi.org/10.1016/j.cortex.2019.01.014

Bodine, C., Seu, A., Roth, E.A., Wegener, E.E., \& Magee, W.L. (2020). Examining the functionality of the MATADOC with the CRS-R: A pilot study. The Journal of Music Therapy, 57(4), 432-454. https://doi.org/10.1093/jmt/thaa010

Cox, A. (2011). "Embodying Music: Principles of the Mimetic Hypothesis.” Music Theory Online, 17(2): np. https://doi.org/10.30535/mto.17.2.1

Damasio, A. (1999). The feeling of what happens: Body and emotion in the making of consciousness. New York: Harcourt Brace.

Davey, C.G., Pujol, J., \& Harrison, B.J. (2016). Mapping the self in the brain's default mode network. NeuroImage, 132 , 390-397. https://doi.org/10.1016/i.neuroimage.2016.02.022

Edlow, B.L., Camille, C., Spencer, C.A., Chu, C.J., Bodien, Y.G., O'Connor, K. L., Hirschberg, R.E., Hochberg, L.R., Giacino, J.T., Rosenthal, E.S., \& Wu, O. (2017). Early detection of consciousness in patients with acute severe traumatic brain injury. Brain, (140)9, 2399-2414. https://doi.org/10.1093/brain/awx176

El Haj, M., Postal, V., \& Allain, P. (2012). Music enhances autobiographical memory in mild Alzheimer's Disease. Educational Gerontology, 38(1), 30-41. https://doi.org/10.1080/03601277.2010.515897

Giacino, J.T., Katz, D.I., \& Whyte, J. (2013). Neurorehabilitation in disorders of consciousness. Seminars in Neurology, 33(2), 142-156. https://doi.org/10.1055/s-0033-1348960

Grimm, T., \& Kreutz, G. (2018). Music interventions in disorders of consciousness (DOC) - A systematic review. Brain Injury, 32(6), 704-714. 


\section{https://doi.org/10.1080/02699052.2018.1451657}

Goldberg, D. (2020). What's the meter of Elenino Horo? Rhythm and timing in drumming for a Bulgarian folk dance. Analytical Approaches to World Music, 7(2), 69107.

Hansen, W.G. (1959). How accessibility shapes land use. Journal of the American Institute of Planners, 25(2), 7376. https://doi.org/10.1080/01944365908978307

He, J.H., Cui, Y., Song, M., Yang, Y., Dang, Y.Y., Jiang, T.Z, \& Xu, R.X. (2015). Decreased functional connectivity between the mediodorsal thalamus and default mode network in patients with disorders of consciousness. Acta Neurologica Scandinavica, 131(3), 145-151. https://doi.org/10.1111/ane.12299

Henrich, J., Heine, S. J., \& Norenzayan, A. (2010). The weirdest people in the world? Behavioral and Brain Sciences, 33, 61-135. https://doi.org/10.1017/S0140525X0999152X

Howarth, C.I., Ellis, K. (1961). The relative intelligibility threshold for one's own name compared with other names. Q. J. Exp. Psychol., 13 (4), 236-239. https://doi.org/10.1080/17470216108416500

Howland, K.M. (2016). Music therapy. In Encyclopedia Britannica. https://www.britannica.com/topic/musictherapy.

Ino, T., Nakai, R., Azuma, T., Kimura, T., \& Fukuyama, H.. (2011). Brain activation during autobiographical memory retrieval with special reference to default mode network. The Open Neuroimaging Journal, 5, 14-23. https://doi.org/10.2174/1874440001105010014

Jacoby, N., Margulis, E.H., Clayton, M., Hannon, E., Honing, H., Iverson, J., Klein, T.R., Mehr, S.A., Pearson, L., Peretz, I., Perlman, M., Polak, R., Ravignani, A., Savage, P.E., Steingo, G., Stevens, C.J., Trainor, L., Trehub, S., Veal, M. \& Wald-Fuhrmann, M. (2020). Cross-Cultural work in music cognition: Challenges, insights and recommendations. Music Perception, 37(3), 185-195. https://doi.org/10.1525/mp.2020.37.3.185

Jacoby, N., McDermott, J.H. (2017) Integer ratio priors on musical rhythm revealed cross-culturally by iterated reproduction. Current Biology, 27, 359-370. https://doi.org/10.1016/j.cub.2016.12.031

Kempny, A.M., James, L., Yelden, K., Duport, S., Farmer, S.F., Playford, E.D., \& Leff, A.P. (2018). Patients with a severe prolonged Disorder of Consciousness can show classical EEG responses to their own name compared with others' names. NeuroImage, 19, 311-319. https://doi.org/10.1016/j.nicl.2018.04.027

Kotchoubey, B. (2018). Human Consciousness: Where Is It From and What Is It for. Frontiers in Psychology, 9, 567. https://doi.org/10.3389/fpsyg.2018.00567

Kotchoubey, B., Pavlov, Y.G., \& Kleber, B. (2015). Music in Research and Rehabilitation of Disorders of
Consciousness: Psychological and Neurophysiological Foundations. Frontiers in Psychology, 6, 1763. https://doi.org/10.3389/fpsyg.2015.01763

Langille, J.J., \& Brown, R.E. (2018). The synaptic theory of memory: A historical survey and reconciliation of recent opposition. Frontiers in Systems Neuroscience, 12, 1-15. https://doi.org/10.3389/fnsys.2018.00052

Magee, W.L., \& O'Kelly, J. (2015). Music therapy with disorders of consciousness: Current evidence and emergent evidence-based practice. Annals of the New York Academy of Sciences, 13371(1), 256-62. https://doi.org/10.1111/nyas.12633

Magee, W.L., Siegert, R.J., Daveson, B.A., Lenton-Smith, G., \& Taylor, S.M. (2014). Music therapy assessment tool for awareness in disorders of consciousness (MATADOC): Standardisation of the principle subscale to assess awareness in patients with disorders of consciousness. Neuropsychological Rehabilitation, 24(1), 101-124. https://doi.org/10.1080/09602011.2013.844174

Mäki-Marttunen, V., Castro, M., Olmos, L., Leiguarda, R., \& Villarreal, M. (2016). Modulation of the default-mode network and the attentional network by self-referential processes in patients with disorder of consciousness. Neuropsychologia, $\quad 82, \quad 149-160$. https://doi.org/10.1016/j.neuropsychologia.2016.01.022

Margulis, E.H., Wong, P.C.M., Simchy-Gross, R. \& McAuley, J.D. (2019). What the Music Said: Narrative Listening Across Cultures. Palgrave Communications, 5, 146. https://doi.org/10.1057/s41599-019-0363-1

McDermott, J.H., Schultz, A.F., Unduragga, E.A. \& Godoy, R.A. (2016) Indifference to dissonance in native Amazonians reveals cultural variation in music perception. Nature, 535, 547-550. https://doi.org/10.1038/nature18635

Morin, A. (2006). Levels of consciousness and selfawareness: A comparison and integration of various neurocognitive views. Consciousness and Cognition, $15(2)$, 358-371. https://doi.org/10.1016/j.concog.2005.09.006

Näätänen, R., Tervaniemi, M., Sussman, E., Paavilainen, P. \& Winkler, I. (2001). 'Primitive intelligence' in the auditory cortex. Trends in Neurosciences, 24(5), 283288. https://doi.org/10.1016/S0166-2236(00)01790-2

Perrin, F., Castro, M., Tillmann, B. \& Luauté, J. "Promoting the use of personally relevant stimuli for investigating patients with disorders of consciousness. Frontiers in Psychology 6 (2015). https://doi.org/10.3389/fpsyg.2015.01102

Rauschecker, J.P., Tian, B., Pons, T. \& Mishkin, M. (1997). Serial and parallel processing in rhesus monkey auditory cortex. J. Comp. Neurol. 382, 89-103. https://doi.org/10.1002/(SICI)1096-9861(19970526)382 


\section{$: 1<89::$ AID-CNE6 $>3.0$. CO $; 2-\mathrm{G}$}

Sun, J. \& W. Chen. (2015). Music therapy for coma patients: Preliminary results. European Review for Medical and Pharmacological Sciences, 19(7), 1209-18.

Stover, C. (2020). Contextual theory, or theorizing between the discursive and the material. Analytical Approaches to World Music, 7(2), 13-40.

Tse, C.Y. and Wong, C.F. (2020). Metrical structure and freedom in qin music of the Chinese literati. Analytical Approaches to World Music, 8(2), 164-194.

Verger, J., Ruiz, S., Tillmann, B., Ben Romdhane, M., De Quelen, M., Castro, M., Tell, L., Luauté, J. \& Perrin, F. (2014). Effets bénéfiques de la musique préférée sur les capacités cognitives des patients en état de conscience minimale. Revue Neurologique, 170(11), 693-99. https://doi.org/10.1016/j.neurol.2014.06.005

Zbikowski, Lawrence M. (2011). Music, Language, and Kinds of Consciousness. In D. Clarke \& E. Clarke (Eds.), Music and consciousness (pp. 179-192). Oxford University Press. https://doi.org/10.1093/acprof:oso/ $\underline{9780199553792.003 .0058}$ 


\title{
Sonic intimacy in the music of Billie Eilish and recordings that induce ASMR
}

\author{
Lindsay Warrenburg, ${ }^{1 \dagger}$ Nathan Centa, ${ }^{1}$ Xintong Li, ${ }^{2}$ Hansae Park, ${ }^{2}$ Diana Sari, ${ }^{1}$ Feiyu Xie ${ }^{2}$ \\ ${ }^{1}$ School of Music, Ohio State University, Columbus, OH, USA \\ ${ }^{2}$ Department of Psychology, Ohio State University, Columbus, OH, USA \\ $\dagger$ Corresponding author: lindsay.a.warrenburg@gmail.com \\ Published 16 December 2021; https://doi.org/10.18061/FDMC.2021.0026 \\ Author video presentation and/or other conference material: https://doi.org/10.17605/OSF.IO/NWZ7Y
}

\begin{abstract}
This article explores similarities in the music of Billie Eilish and recordings that induce the Autonomous Sensory Meridian Response (ASMR). Two complementary approaches are presented. First, the methodology and preliminary results of an empirical study are reported, which investigates peoples' emotional responses to Eilish's music, mouth-related ASMR sounds (oral wetness cues, whispering, breathing sounds), and non-mouth ASMR sounds (tapping, scratching). Second, a new theory of sonic intimacy is presented that draws on similar electroacoustic techniques in the music of Billie Eilish and Bing Crosby and may account for their popularity during times of stress and isolation.
\end{abstract}

KEYWORDS: Billie Eilish, ASMR, emotion, sonic intimacy, musicovid

\section{Introduction}

Billie Eilish is a popular singer who rose to fame with her 2019 album WHEN WE ALL FALL ASLEEP, WHERE DO WE GO?. At the age of 17, she became the first Gen Z musician to hit No. 1 on the Billboard charts, where her single bad guy was in the Top 100 list for 49 weeks, with more than 25 of those weeks in the Top 10 . Eilish is the youngest person to win the 4 major Grammys in the same year, in 2019 winning Best New Artist, Record of the Year, Song of the Year, and Album of the Year, as well as Best Pop Vocal Album. Although iTunes classifies her music as "alternative," she describes her music as "genre-less" (Crozier, 2019).

The beginning of the music video for bad guy gives a taste of Billie's music: The video starts by focusing on saliva sounds as she takes out her Invisalign. Table 1 lists some of the techniques at which critics and audiences have marveled, including the use of close miking, breathing sounds, whispering, binaural effects, and the use of dentist drills and Easy-Bake Ovens (e.g., Connick, 2019).

Researchers, journalists, and audience members have noted that there are similar electroacoustic effects in Eilish's music and audiovisual recordings that induce ASMR-Autonomous Sensory Meridian Response (e.g., Geaghan-Breiner \& Gmoser, 2019). ASMR is a sensory response to certain auditory stimuli, which includes tingling and static-like sensations across the body, as well as feelings of relaxation, wellbeing, and psychological flow (Barratt, Spence, \& Davis, 2017). Videos and sounds that evoke ASMR are extremely popular among Millennials and Gen $Z$ listeners: As of February 2021, the YouTube channel "Gentle Whispering ASMR" has 2 million subscribers and 846 million views, and the Reddit page "ASMR. Sounds that feel good" has 229,000 members.

Sounds that elicit ASMR include whispering, speaking with less vocal energy, slow speaking rates, increased use of pauses, oral wetness cues, the greater use of silibants, sounds of breathing, alternating binaural stimuli, crisp sounds, and tapping or scratching sounds (Andersen, 2015; Kovacevich \& Huron, 2019). Broadly, ASMR-inducing clips can be broken into two categories - mouth-related sounds and non-mouth sounds. As might be expected, mouth-related sounds include features like audible breathing sounds or oral wetness cues (e.g., sounds of saliva), whereas nonmouth sounds include effects such as tapping or crinkling paper.

This article explores whether the popularity of ASMRtists and Billie Eilish can be attributed, in part, to shared sonic techniques that elicit feelings of intimacy and close physical proximity. First, the methodology and preliminary results of an empirical study will be described. Second, a new theory, called sonic intimacy, is proposed, which may account for the popularity of certain artists and genres during times of isolation and loneliness, including the current coronavirus pandemic.

Table 1: Some audio effects in Billie Eilish's music.

\begin{tabular}{|l|l|c|}
\hline \multicolumn{1}{|c|}{ Effect } & \multicolumn{1}{c|}{ Track } & Timing \\
\hline Close miking & goodbye & $00: 00-00: 30$ \\
\hline Whispering & goodbye & $01: 18-01: 48$ \\
\hline Binaural effects & bury a friend & $01: 04-01: 13$ \\
\hline Dentist drills & bury a friend & $01: 51-02: 00$ \\
\hline
\end{tabular}




\section{Methods}

\section{Stimulus Selection}

A team of researchers listened to Billie Eilish's album WHEN WE ALL FALL ASLEEP, WHERE DO WE GO? and to the three most popular albums on iTunes under the search term "ASMR": (1) FrivolousFox's ASMR Lollipop Trigger Words \& Up Close Mouth Sounds, (2) ASMR HeadHI, Asmr \& Asmr Sleep Sounds's Assorted Asmr: Slime, Tapping \& Whispers, and (3) GiBi ASMR's Extreme Secret Whispering. From the complete recordings, passages were selected that best corresponded to published features of ASMR (Andersen, 2015; Kovacevich \& Huron, 2019).

The resulting list of excerpts included 22 passages by Billie Eilish and 62 passages from an ASMR-designated album. Each member of a six-person research team rated a random subset of stimuli on certain criteria (described below). In order to better enable within-stimulus group comparisons, half of the researchers were randomly selected to rate Billie Eilish passages before ASMR passages; the other three researchers rated ASMR passages before Billie Eilish passages.

Each of the Billie Eilish passages was rated by two or three researchers on two criteria: (1) the percent of the clip that contained ASMR-related features $(0$ : very little ASMR, 100: exclusively ASMR) and (2) its overall quality as a stimulus, with regard to duration, loudness, and the inclusion of lyrics (0: very poor quality, 100: very high quality). An average "ASMR score" and "quality score" was calculated for each excerpt. The ASMR scores ranged from 36.6 to $86.6(\mathrm{M}=75.8, \mathrm{SD}$ $=13.1)$ and quality scores ranged from 33.3 to $88.3(\mathrm{M}$ $=76.8, \mathrm{SD}=10.8$ ). The 22 excerpts were then sorted by average quality score, followed by average ASMR score. The top ten excerpts from this ranked list were used as the final Billie Eilish stimuli. The length of these excerpts ranged from 5 to 56 seconds in duration. Because the experiment location was the local science museum, an a priori decision was made not to use any recording longer than 30 seconds. Therefore, for the three long passages, the first 30 seconds were used as the final stimulus. The final Billie Eilish stimuli had an average length of 19 seconds ( $\mathrm{SD}=1$ second).

Similarly, each of the ASMR-inducing passages was rated by two or three researchers on two criteria: (1) its similarity to Billie Eilish's music writ large (0: very little similarity, 100: very high similarity) and (2) its overall quality as a stimulus (0: very poor quality, 100: very high quality). An average "similarity score" and "quality score" was calculated for each excerpt. The similarity scores ranged from 7.5 to $91.7(\mathrm{M}=64.3, \mathrm{SD}$ $=20.2$ ) and the quality scores ranged from 52.5 to 91.7 $(\mathrm{M}=76.2, \mathrm{SD}=10.4)$. The passages were then sorted by average quality score, followed by average similarity score. Passages that included audible words or phrases were excluded. From this ranked list, the top ten "mouth-related" excerpts-ASMR sounds that were made by a person's mouth, such as whispering, tongue clicking, and oral wetness cues - and the top ten "nonmouth" excerpts - ASMR sounds that were not made by a person's mouth, such as tapping, scratching, crinkling, and bubbles - were used as stimuli in the experiment. Once again, the first 30 seconds of each excerpt longer than 30 seconds was used as the final stimulus. The "mouth-related" stimuli had an average length of 11 seconds (SD $=6$ seconds) and the "non-mouth" stimuli had an average length of 16 seconds ( $\mathrm{SD}=9$ seconds).

\section{Experimental Procedure}

The experiment was conducted at the Center of Science and Industry in Columbus, Ohio, USA. Researchers were stationed outside an exhibit and asked entering visitors if they would be interested in participating in a short music-related study. In order to participate, participants were required to be at least 18 years old and could not have a history of speech, language, or hearing problems. The Ohio State University Institutional Review Board \#2019E0809 approved all procedures.

Each participant heard a random selection of six stimuli (out of the 30 total stimuli). A randomizer was programmed to evenly present all 30 items, but there were no controls ensuring that everyone heard the same number of music and non-music stimuli. For each stimulus, participants were asked: While listening to this clip, how much did you experience the following things? (1) tingling, (2) relaxation or calm, (3) discomfort or anxiety, (4) enjoyment. Participants responded to each question on a $0-100$ continuous scale, anchored at 0 (none). They were also asked: Would you listen to this clip again? with the options of yes, maybe, and no.

In addition to these stimulus-related questions, participants were asked whether they had prior experiences of ASMR and synesthesia, as well as their overall level of musicianship (Ollen, 2006), age, preferred gender, race/ethnicity, first language, and zipcode. A series of personality-related questions included four questions from the Social Anxiety Questionnaire for Adults (regarding public teasing, talking to strangers, being asked a question by a superior, and looking in the eyes of a stranger while talking; Caballo et al., 2015), two questions from the 
Big-Five Trait Taxonomy (regarding their curiosity about many different things and their level of value for artistic and aesthetic experiences; John \& Srivastava, 1999), four questions from the Ten-Item Personality Inventory (regarding trait anxiety, openness to new experiences, emotional stability, and conventionality; Gosling, Rentfrow, \& Swann Jr., 2003), and their preference for Dance/Electronica music from the STOMP-R (Rentfrow, Goldberg, \& Levitin, 2011).

\section{Preliminary Results}

Of a planned cohort of 400 participants, only 17 competed the survey before the science museum closed due to COVID-19. Seven participants identified as female, four identified as male, and six did not answer. The average age was 30.5 years (range $=21$ to 40 ).

Because of the small sample size, no statistical tests were conducted. Figure 1 shows violin plots of the amount of anxiety, enjoyment, relaxation, and tingling sensations that participants felt when listening to Eilish's music, mouth-related ASMR sounds, and nonmouth ASMR sounds. Although more data is needed to substantiate these trends, the results could be consistent with the idea that people experience less anxiety and more relaxation and enjoyment when listening to Eilish's music than when listening to ASMR-related sounds.

\section{Discussion}

Certain acoustic characteristics, like whispering and breathing sounds, are characteristics of close physical proximity (Kovacevich \& Huron, 2019). Namely, when we hear sounds like someone breathing, our brain is wired to assume that there is a person physically close to us. Recordings created to elicit ASMR tend to draw on these sonic characteristics, which can result in feelings of intimacy or personal attention (Kovacevich $\&$ Huron, 2019). The current study aimed to test whether people experience similar affective states in response to the proximity-related cues in recordings by ASMRtists and in the music of Billie Eilish. The preliminary results of the current study suggest that even though there are shared sonic techniques in these two domains, people did not enjoy the experience of listening to ASMRrelated recordings as much as they enjoyed the music of Billie Eilish.

Once the COVID-19 lockdown is over, a larger cohort of participants will be used to test for the influence of demographic, health, and personality variables, including social anxiety and extraversion. Future research will also include participants who purposefully seek out ASMR-inducing recordings.
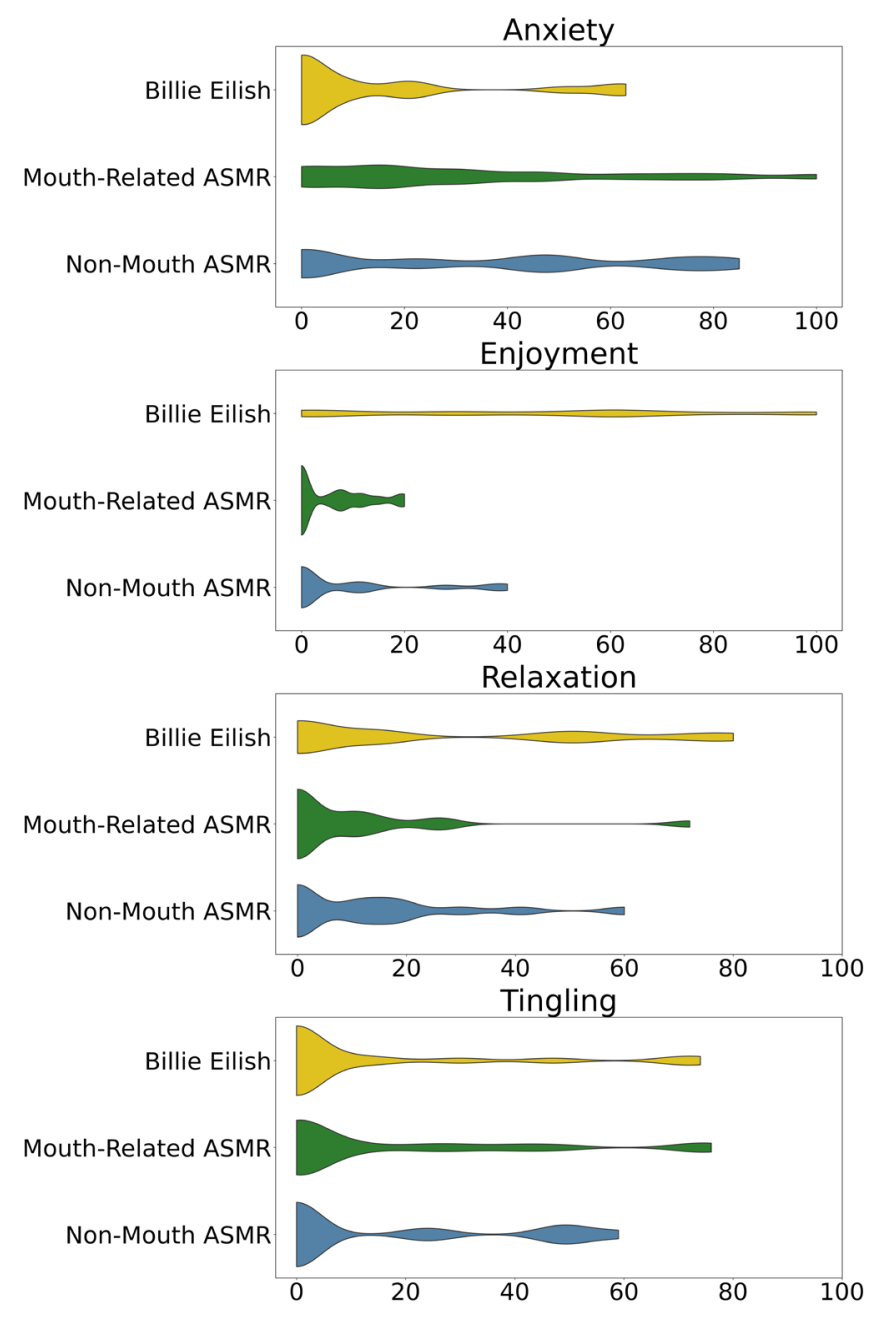

Figure 1: Violin plots of self-reported ratings of anxiety, enjoyment, relaxation, and tingling when listening to the music of Billie Eilish, mouth-related ASMR sounds, and non-mouth ASMR sounds (0: none, 100: high amount).

\section{Theoretical Implications: Sonic Intimacy}

Recordings by Billie Eilish and ASMRtists have soared in popularity among Gen $\mathrm{Z}$ and Millennial listeners. If this popularity can be explained, in part, through shared sonic techniques like close miking and whispering, the question arises if these techniques also contribute to the popularity of other artists. The following section explores the music of the American crooners in the first half of the twentieth century and proposes a theory that may account for the popularity of the crooners, Billie Eilish, and ASMRtists: sonic intimacy. 


\section{Crooning and Bing Crosby}

The recording techniques in the music of Billie Eilish share similarities with the music of the American crooners of the 1930s through the 1950s, such as Bing Crosby. Before the era of the crooners, much of the music that appealed to the American masses was cabaret-style music that involves loudly projecting your voice. Crooning, by contrast, involves quieter and more conversational styles of singing (McCracken, 1999). This crooning style became popular among the American public in the 1920s. The origins of the popular (white) crooning style are steeped in the appropriation of Black music and minstrel songs and by making this music popular among the dominant American culture (McCracken, 1999). Early white crooners, like Al Jolson and Eddie Cantor, even performed in blackface. The American public saw figures like Bing Crosby as "legitimiz[ing] crooning by connecting it to traditional notions of white masculinity: a good work ethic, patriarchy, religious belief, white supremacy, and contained emotion" (McCracken, 1999, p. 389).

Despite these racist origins, the American public embraced Crosby: At the height of his popularity, 25\% of Americans listened to his radio shows and he was voted as the most admired man in America (Barnes, 2001). Crosby was a source of comfort during the Great Depression and World War II, both in America and abroad. Crosby's beloved status lasted throughout his lifetime and he became the first recipient of the Grammy Lifetime Achievement Award in 1963.

McCracken (1999) notes that the development of microphone technologies, such as the ribbon dynamic microphone in 1924, may have helped contribute to the popularity of crooning, as these electronic recordings were able to convey wider frequency ranges and more complex sounds than the previous recordings. While performing, singers could use the improved microphone to convey a more personal tone. This intimate performance style, including close miking and conversational speaking, was Bing Crosby's signature.

\section{Sonic Intimacy}

Recall that certain features tend to induce ASMR: close miking, whispering, audible breathing, and oral wetness cues. These sonic effects may mimic neural cues of physical proximity and may even activate the parasympathetic nervous system (Andersen, 2015; Kovacevich \& Huron, 2019). We propose that these findings extend to the musical domain and, in particular, to the music of Billie Eilish and Bing Crosby. To introduce a new term, moments when music uses techniques like breathing sounds, close miking, whispering, and binaural effects can be described as exhibiting sonic intimacy. Using this definition, the music of Billie Eilish and Bing Crosby contains a multitude of sonically intimate sections (Table 2). When listening to sonically intimate moments, listeners may feel the same sense of relaxation and pleasure as being physically near a loved one.

In support of this idea, some snapshots of history indicate when musicians who use sonic intimacy become exceedingly popular. After the 1929 stock market crash, record sales, vaudeville houses, and nightclubs disappeared for most of the American public (McCracken, 1999). Crosby's use of inventions such as tape-based recording and the electronic microphone helped his popularity soar in the 1930s and 1940s (Hammar, 1989). Billie Eilish rose to fame among Gen $\mathrm{Z}$ listeners in 2019, which could suggest the need for new methods of intimacy after the rise of social media. Of course, the loneliness and isolation felt during the coronavirus crisis would also seem to be a good time to engage with sonically intimate music. Composer Scott Ordway recently described his series of compositions, called "Interiors," that was composed during the coronavirus crisis as works "created in isolation to be performed by musicians in isolation and heard by listeners in isolation. They are quiet, personal, fragile pieces whose medium is the microphone rather than the concert hall. You'll hear creaking piano benches, fingers brushing across clothing.... and the incredible quality of sounds produced at the edge of silence, tone colors which would never be audible in live performance. Music under a microscope" (Ordway, 2020).

\section{Conclusion}

During a time of social distancing and lockdown, such as the COVID-19 pandemic, listeners may turn to sounds that induce feelings of intimacy or closeness, even though the artist or performer may not be physically present. What we have defined as sonic intimacy includes electroacoustic features such as close miking, whispering, oral wetness cues, sounds of breathing, and binaural beats. These techniques are common among recordings that induce the sensory phenomenon of ASMR. We suggest that these moments of sonic intimacy may also contribute to the popularity of artists like Billie Eilish and Bing Crosby, who spoke to audiences nearly 100 years apart. 
Table 2: Sonic intimacy in Eilish and Crosby's music.

\begin{tabular}{|l|l|}
\hline \multicolumn{1}{|c|}{ Artist } & \multicolumn{1}{c|}{ Quotation } \\
\hline Eillish & $\begin{array}{l}\text { She's decisively young and CAREFREE in } \\
\text { that sort of neon bisexual lightning flashy } \\
\text { strobe streetwear instagram-story urgency } \\
\text { without being at all naive or Lolita-sexualized. } \\
\text { ALSO! I think gen z is extremely lonely!!!! } \\
\text { songs like 'When the Party's Over' get } \\
\text { RIGHT at the desperate, gnawing sense of } \\
\text { isolation social media has sewn into a } \\
\text { generation who are becoming increasingly } \\
\text { sequestered (Petrusich, 2019) }\end{array}$ \\
\hline $\begin{array}{l}\text { Billie } \\
\text { Eilish }\end{array}$ & $\begin{array}{l}\text { Everything is close-mic'd and whispered...the } \\
\text { goal is to always have Billie's vocals sound } \\
\text { super-present.' It's as if she's right there, } \\
\text { murmuring directly into your ear (Geaghan- } \\
\text { Breiner \& Gmoser, 2019) }\end{array}$ \\
\hline Billie & $\begin{array}{l}\text { Both oh-so-2019 products of the internet, the } \\
\text { respective rises of tingle-sensation du } \\
\text { jour ASMR and pop prodigy Billie Eilish have } \\
\text { run parallel to each other (Connick, 2019) }\end{array}$ \\
\hline Bing \\
Crosby & $\begin{array}{l}\text { [Crosby treated] a new invention, the } \\
\text { microphone, as if it were a friend's ear...[He] } \\
\text { shepherded America through the Depression } \\
\text { and World War II, then became a symbol of } \\
\text { postwar domestic stability (Gavin, 2018) }\end{array}$ \\
\hline Crooners \\
Crooners & $\begin{array}{l}\text { Radio allowed [the crooner] to create a sense } \\
\text { of intimacy with his listeners by evaporating } \\
\text { the physical boundaries between the performer } \\
\text { and the audience (McCracken, 1999) } \\
\text { Crooners are defined by their intimacy with } \\
\text { as microphone and...crooning is constructed } \\
\text { identification on the part of the individual } \\
\text { listener (McCracken, 1999) }\end{array}$ \\
\hline
\end{tabular}

\section{References}

Andersen, J. (2015). Now you've got the shiveries: Affect, intimacy, and the ASMR whisper

community. Television \& New Media, 16(8), 683-700.

https://doi.org/10.1177/1527476414556184

Barnes, K. (Host). (2001, December 29). Bing Crosby meets [Audio podcast episode]. In Archive on 4. BBC. https://www.bbc.co.uk/sounds/play/b09jvgph

Barratt, E. L., Spence, C., \& Davis, N. J. (2017). Sensory determinants of the autonomous sensory meridian response (ASMR): Understanding the triggers. PeerJ, 5, e3846. https://doi.org/10.7717/peerj.3846

Caballo, V. E., Arias, B., Salazar, I. C., Irurtia, M. J., Hofmann, S. G., \& CISO-A Research Team. (2015). Psychometric properties of an innovative self-report measure: The Social Anxiety Questionnaire for adults. Psychological Assessment, 27(3), 997-1012. https://doi.org/10.1037/a0038828

Crozier, S. (2019, October 24). Billie Eilish and the end of genre. Untitled Magazine. http://untitledmagazine.com/billie-eilish-and-the-end-of-genre

Connick, T. (2019, April 18). How Billie Eilish employed principles of ASMR in her spine-tingling horror-pop. NME. https://www.nme.com/blogs/nme-blogs/billieeilish-asmr-sounds-2478496

Gavin, J. (2019, November 28). How Bing Crosby changed the course of pop music. New York Times.

https://www.nytimes.com/2018/11/28/books/review/gar y-giddins-bing-crosby-biography.html

Geaghan-Breiner, M. \& Gmoser, J. (2019, November 25). How New Artist of the Year, Billie Eilish, harnesses the power of ASMR in her music. Insider.

https://www.insider.com/how-billie-eilish-harnessesthe-power-of-asmr-2019-10

Gosling, S. D., Rentfrow, P. J., \& Swann Jr, W. B. (2003). A very brief measure of the Big-Five personality domains. Journal of Research in Personality, 37(6), 504-528. https://doi.org/10.1016/S0092-6566(03)00046-1

Hammar, P. (1989). Jack Mullin: The man and his machines. Journal of the Audio Engineering Society, 37(6), 490-512.

John, O. P., \& Srivastava, S. (1999). The Big-Five Trait Taxonomy: History, measurement, and theoretical perspectives. In L. A. Pervin \& O. P. John (Eds.), Handbook of personality: Theory and research, Vol.2 (pp. 102-138). New York: Guilford Press.

Kovacevich, A., \& Huron, D. (2019). Two studies of Autonomous Sensory Meridian Response (ASMR): The relationship between ASMR and music-induced frisson. Empirical Musicology Review, 13(1-2), 39-63. https://doi.org/10.18061/emr.v13i1-2.6012

McCracken, A. (1999). "God's gift to us girls": Crooning, gender, and the re-creation of American popular song, 1928-1933. American Music, 17(4), 365-395. https://doi.org/10.2307/3052656

Ollen, J. E. (2006). A criterion-related validity test of selected indicators of musical sophistication using expert ratings (Doctoral dissertation). The Ohio State University, Columbus, $\mathrm{OH}$.

Ordway, S. (2020, May 13). This Friday at 12PM EDT I'm launching a new series of compositions called "Interiors" [Status update]. Facebook. https://www.facebook.com/profile.php?id=17701532

Petrusich, A. (2019, August 23). The Loneliest Generation embraces Billie Eilish. The New Yorker. https://www.newyorker.com/culture/culturalcomment/the-loneliest-generation-embraces-billie-eilish 
Rentfrow, P. J., Goldberg, L. R., \& Levitin, D. J. (2011). The structure of musical preferences: A five-factor model. Journal of Personality and Social Psychology, 100(6), 1139-1157. https://doi.org/10.1037/a0022406 


\title{
How musicality changes moral consideration: People judge musical entities as more wrong to harm
}

\author{
Tanushree Agrawal ${ }^{1 \dagger}$ \\ Joshua Rottman ${ }^{2}$ \\ Adena Schachner ${ }^{1}$ \\ ${ }^{1}$ Department of Psychology, University of California San Diego, La Jolla, CA, USA \\ ${ }^{2}$ Department of Psychology, Franklin \& Marshall College, Lancaster, PA, USA \\ ${ }^{\dagger}$ Corresponding author: t2agrawa@ucsd.edu \\ Author video presentation and/or other conference material: https://doi.org/10.17605/OSF.IO/B5ERK
}

A growing literature shows that music increases prosocial behavior (Clarke et al., 2015). Why does this occur? We propose a novel hypothesis: Evidence of others' musicality may promote prosociality by leading us to judge musical individuals as having enhanced moral standing. This effect may be largely indirect, by increasing perceptions of how intelligent and emotionally sensitive musical individuals are. If so, simply knowing about others' musicality should affect moral evaluations, such as wrongness-to-harm. Across four experiments (total $\mathrm{N}=550$ ), participants were introduced to a host of characters, and asked which of each pairing of characters felt more wrong to harm. We manipulated musicality across matched character pairs, with one character described as musical, one not described as musical, and one described as explicitly non-musical (all matched for length/style). In all experiments, we found supportive evidence (all $p \mathrm{~s}<$ 0.01). Information that an animal or person had the capacity and motivation to engage with music led participants to judge them as more wrong to harm than matched neutral or non-musical counterparts. Similarly, knowing that a person was not musical made people judge them as less wrong to harm than neutral or musical counterparts. As predicted, musicality was positively associated with perceptions of emotionality and intelligence; These broader factors partially mediated the relationship between musicality and wrongness to harm. Effects were not influenced by participants' own musicality. Thus, non-moral attributes like musicality are deeply interwoven with moral decision-making, carrying implications for social behavior and for interventions to promote prosociality.

KEYWORDS: moral standing, musicality, emotionality, agency, intelligence

\section{References}

Clarke, E., DeNora, T., \& Vuoskoski, J. (2015). Music, empathy and cultural understanding. Physics of Life Reviews, 15 , $61-88$. https://doi.org/10.1016/j.plrev.2015.09.001.

\section{Changing musical categorization between eras}

\author{
Benjamin Kelly ${ }^{1 \dagger}$ \\ Cameron Anderson ${ }^{1}$ \\ Mike Schutz ${ }^{1}$ \\ ${ }^{1}$ McMaster University, Hamilton, ON, CA \\ † Corresponding author: kellyb7@mcmaster.ca
}

Author video presentation and/or other conference material: https://doi.org/10.17605/OSF.IO/38DKY

Composers convey emotion using a variety of cues, including dynamics, timing, and mode. However, they vary their approaches to emotional communication, in part due to differences in the musical conventions of their time. The goal of this study is to build upon recent research examining systematic differences in the musical cues affecting emotional communication throughout different musical eras. Here we supplement musicological work with a perceptual complement exploring how listeners group historical music 
according to their emotional content. Participants listened to excerpts of the first 8 measures of 24 Prelude sets by either Bach (Baroque era) or Chopin (Romantic era). Each set includes pieces in all Western keys in both major and minor modes. After each excerpt participants selected a label for the piece, representing the five most common categories from Horn \& Huron (2015). Complementing previous findings, our results indicate differences in the categorization of each composer's work. For example, Chopin appears to have structured his pieces so that participants utilized fewer Joyful and Tender/Lyrical labels than Bach, and more Passionate labels. The labelling of Chopin's pieces are also more strongly aligned with the descriptions of each label provided by Horn \& Huron (2015) than Bach's. Together with their work, the current research highlights that analyzing "cues for emotion" overlooks that emotion is stylistically specific to compositional context. Our findings present a novel analysis of historic changes influencing perception and are consistent with ideas of great shifts in the use of musical cues to convey emotion between musical eras.

\section{References}

Horn, K., \& Huron, D. (2015). On the changing use of the major and minor modes 1750-1900. Music Theory Online, 21(1), 1-11. https://doi.org/10.30535/mto.21.1.4

\title{
Emotional outcomes of music listening on mobile phones
}

\author{
William M Randall ${ }^{1 \dagger}$ \\ ${ }^{1}$ University of Jyväskylä, Jyväskylä, Finland \\ † Corresponding author: will.m.randall@gmail.com
}

Author video presentation and/or other conference material: https://doi.org/10.17605/OSF.IO/AYHD2

Listening to music on mobile phones has grown rapidly to become a central component of everyday music listening. This personal style of listening allows for the immediate selection of music to fulfil emotional needs, calling for a greater understanding of how it influences emotional states. The current study aimed to determine the predictors of emotion change, and to update the model of personal music listening presented by Randall \& Rickard (2017). All data were collected through MuPsych, a mobile experience sampling app designed to collect ecologically-valid and real-time data during music listening. Participants were 735 young people (mean age $=22.4$ years) who responded to questions during listening experiences (total $=6,445$ ). These questions assessed change in emotional state (valence, arousal, and intensity) over a five-minute listening period, along with contextual variables, and reasons for listening. A multilevel structural equation model was constructed to determine the predictors of these changes in emotional states. Female listeners and those low in trait openness experienced more positive emotional outcomes from music listening. Younger listeners, along with those high in trait conscientiousness and extraversion experienced more enjoyment from listening. The features of the music selected were also predicted by personality traits, and by reasons for listening. These results give insight into how emotional outcomes are related to individual traits and contextual variables. Understanding these relationships will have implications for how music can be used to support emotional health, either through self-selection, or personalised scientific curation.

KEYWORDS: everyday music listening, emotions, experience sampling 


\section{Redefining music and emotion research through the adaptation of emotional granularity \\ Lindsay Warrenburg ${ }^{1 \dagger}$ \\ ${ }^{1}$ School of Music, Ohio State University, Columbus, OH, USA \\ † Corresponding author: lindsay.a.warrenburg@gmail.com \\ Author video presentation and/or other conference material: https://doi.org/10.17605/OSF.IO/79HC3}

A recent review of over 22,000 musical stimuli has shown that research through 2018 has only focused on 10 emotional terms and that these emotional terms are used inconsistently across researchers (Warrenburg, $2020 \mathrm{a}$; in press). In this paper, I argue that the variable use of emotional terminology could indicate that researchers have unintentionally conflated multiple emotional states. I present a novel methodology that can be used to identify emotional states and examine emotional behaviors in music research. The methodology draws on recent psychological work on emotional granularity, which describes the specificity with which a person labels their emotional states (Barrett, 2004; 2017). I describe how researchers and participants can be trained to utilize more specificity when describing emotions and can further learn to better differentiate emotions they feel and perceive in music listening. Using this methodology, it is possible to minimize the problem of semantic underdetermination discussed by Warrenburg (2020a). The implications of this research include the idea that meta-analyses and reviews can be interpreted in new ways using an emotionally granular framework. A series of five experiments consistent with this idea is reviewed, where participants were able to use emotionally granular terms to identify subgroups of music previously unrecognized in the music and emotion literature (Warrenburg, 2020b; 2020c). In considering the "future directions" of music and emotion research, it will be important to utilize methodology consistent with emotional granularity in order to discover (potentially) many more than 10 emotions that can be expressed and elicited by music.

KEYWORDS: emotion, emotional granularity

\section{References}

Barrett, L. F. (2004). Feelings or words? Understanding the content in self-report ratings of experienced emotion. Journal of Personality and Social Psychology, 87(2), 266-281. https://doi.org/10.1037/0022-3514.87.2.266
Barrett, L. F. (2017). How emotions are made: The secret life of the brain. Houghton Mifflin Harcourt.

Warrenburg, L. A. (2020a). Choosing the right tune: A review of music stimuli used in emotion research. Music Perception: An Interdisciplinary Journal, 37(3), 240-258. https://doi.org/10.1525/mp.2020.37.3.240

Warrenburg, L. A. (2020b). Redefining sad music: Music's structure suggests at least two sad states. Journal of New Music Research, 49(4), 373-386. https://doi.org/10.1080/09298215.2020.1784956

Warrenburg, L. A. (2020c). People experience different emotions from melancholic and grieving music. Music \& Science, 3, 1-23. https://doi.org/10.1177/2059204320977384

Warrenburg, L. A. (in press). The PUMS database: A corpus of previously-used musical stimuli in 306 studies of music and emotion. Empirical Musicology Review. 


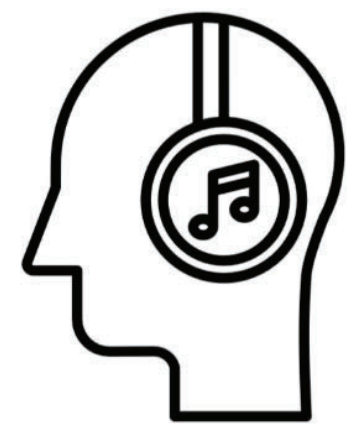

\section{Pedagogy}

Future Directions of Music Cognition February - May 2021

Ohio State University

Virtual 


\title{
Does Gestalt hearing exist?
}

\author{
Timothy Chenette ${ }^{1 \dagger}$ \\ Alexandra Phillips ${ }^{2}$ \\ Emily Wood ${ }^{3}$ \\ ${ }^{1,2,3}$ Utah State University, Logan, Utah, United States \\ $\dagger$ Corresponding author: timothy.chenette@usu.edu \\ Published 16 December 2021; https://doi.org/10.18061/FDMC.2021.0028 \\ Author video presentation and/or other conference material: https://doi.org/10.17605/OSF.IO/X62CZ
}

\begin{abstract}
Karpinski 2000 describes "Gestalt hearing," identifying chords instantly and holistically, as the ideal endpoint of training in harmonic dictation. Yet the subdominant chord (e.g.) is not a single object but a collection of objects that differ in timbre, texture, inversion, spacing, etc. Is it really possible to become so acquainted with this group that one perceives it as a Gestalt? If so, what experiences and abilities are necessary to develop Gestalt hearing? This article draws on relevant results from an observational, exploratory study of harmonic listening $(N=73)$ to suggest preliminary answers to these questions and paths for future research in this area. Our results suggest that Gestalt hearing, if it exists, may not be available in college-level instruction.
\end{abstract}

KEYWORDS: harmonic dictation, pedagogy, Gestalt, bass

\section{Introduction}

Karpinski (2000) describes "Gestalt hearing," identifying chords instantly and holistically, as the ideal endpoint of training in harmonic dictation. Rogers (2001) describes an incremental strategy to meet this goal, starting by asking students to identify cadences, then in later dictations gradually adding tonic and dominant expansions, followed by a series of predominants; this strategy is similar to the "chord-at-atime" approach taken in many current textbooks. Jarvis (2015) suggests a slightly different approach, using an analogy with language immersion to propose that students can develop "Gestalt" chord identification through extensive listening.

Yet it is not clear that Gestalt hearing - if it existswould operate in the ways Karpinski, Rogers, and Jarvis describe. After all, the subdominant chord (e.g.) is arguably not a single object but a collection of objects that differ in timbre, texture, inversion, spacing, function, and placement within a phrase. Principles from Gestalt psychology are also not clearly relevant here, as Karpinski describes Gestalt listening as emerging after an extended period of listening through other methods, while Gestalt psychologists asserted that perception of wholes is more immediate than perception of parts.

The study presented in this article was thus motivated by this question: does Gestalt hearing exist? That is, is Gestalt hearing actually a different mode of perception, learnable over time, that does not require other strategies such as listening for bass lines or chord qualities, or is it simply a way to describe using these other strategies efficiently and accurately in situationally-appropriate combinations? Subsidiary questions include: If this ability exists, what experiences and abilities are necessary in order to acquire it? Is this capacity available to everyone, or just to people with (e.g.) absolute pitch or extensive experience with a chord instrument?

Unfortunately, foundational experimental evidence about harmonic dictation is lacking. Butler (2000), reviewing the "Harmonic Dictation" portion of Karpinski (2000), lamented the lack of such data. But while scholars have suggested methods of instruction in the years since (Jarvis 2015; Sayrs 2019; Stevens 2016, 2020), there have been no studies about how individuals learn to hear harmony. Of course, these questions are difficult to address in a controlled, randomized study, both because of the difficulty of observing aural perception and because of the myriad of relevant intertwined variables that can be operational over large spans of an individual's life: level of absolute pitch, instruments played, years of experience, listening habits, working memory capacity, level of education, and more.

Facing the lack of existing experimental data on this subject, we conducted a survey in order to collect a wide variety of data that can be used to form hypotheses and support future controlled experiments. We approached the interpretation of this information as we might an experience with a student in the classroom: we made our best educated guesses, based on our knowledge and 
teaching experience. Nevertheless, more specific and controlled studies will be necessary in the future to confirm or disprove our preliminary observations and hypotheses.

\section{Method}

Our study, an anonymous online survey developed in Qualtrics, explored harmonic listening through a questionnaire about participants' abilities and musical experiences, three chord-identification exercises, and a self-reflection survey, resulting in both quantitative and qualitative data. We circulated the study through emails to aural skills classes at Utah State University and a post on the SMT Discuss online forum inviting other instructors to do the same at their own institutions.

\section{Participants}

73 participants completed the study; an additional 26 participants did not complete the entire study and are excluded from our results. The vast majority of participants reported being a current music major (58\%) or having completed a music major $(21 \%)$. The most common reported primary instrument was piano (34\%), followed by voice $(16 \%)$; the remaining participants were fairly evenly spread among a variety of wind, brass, and string instruments.

\section{Chord Identification Excerpts}

Participants were asked to identify the chords of several excerpts "in your own words," though example answers were given that suggested the desirability of Roman numerals. Three contrasting examples were chosen: a nearly-homophonic four-part excerpt composed explicitly for dictation and played on piano (Harmonic Dictation 42.6 from Karpinski 2017), a four-chord loop from a popular song (Beyoncé, "Halo"), and a short excerpt from a Classical piano sonata with an Alberti bass (the first two measures of Mozart, K. 332, mvmt. 2). The Karpinski and Mozart examples were embedded as .mp3s, and the Beyoncé as a Youtube video; each could be played and replayed at will.

\section{Participant Self-Reflections}

After the first chord identification exercise, and then again after the third, participants were asked to reflect on the process of identifying chords. First, they were asked to describe in their own words "how you typically identify a chord progression." Then they were asked to select the strategies they use from a provided list. These choices, distilled from a variety of textbooks that address harmonic dictation, included "Expectation of what is likely to come next," "Chord quality (major, minor, etc.)," "Bass line," "The feeling of a chord (the 'one-ness' or 'it feels dominant-y')," "Solfege syllables or scale degrees in the chords," "Cadences," "Imagining playing the chord on some instrument," and "Other."

\section{Scoring}

In order to categorize and compare participant results in the harmony identification section, we graded each participant's answer on each excerpt. To enable comparisons across examples, we set the maximum possible score for each at 100 percent; each chord in the excerpt, then, was worth $100 / C$ percentage points, where $C$ represents the number of chords in the excerpt. Incorrect inversions were given half credit, and a superfluous chord added to an otherwise correct progression also resulted in a deduction of half the points allotted to a correct chord for that excerpt. While most participants used Roman numerals to answer these questions, a few used other systems. Where these gave clear and specific answers (for example, one participant used notation such as " $1 / 3$ " to indicate a tonic chord in first inversion) we scored them similarly; where answers were vague or nonspecific, we left them out of our results. We also figured, for each participant, their average score across all three examples.

\section{Results}

Our survey participants had a wide range of average scores across the three chord-identification exercises (Figure 1). We analyzed the results through three lenses: first, demographic data; second, evidence from participant reflections on chord identification; and finally, corroboration from analysis of incorrect chord identifications.

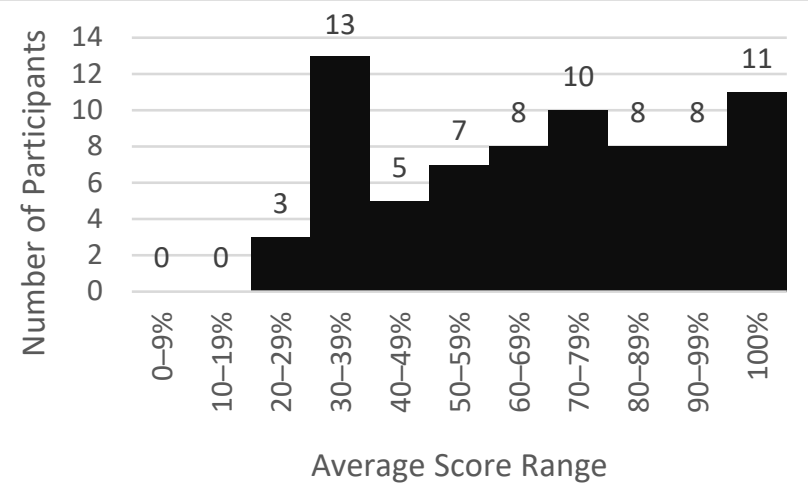

Figure 1: Histogram of participants' average scores (expressed as percent correct) across the three harmonic identification excerpts. 
Among demographic factors that may affect participants' chord-identifying abilities, we focused on three that seem to hold the potential for building conceptual models of chord Gestalts. First, presence of absolute pitch, including both Heightened Tonal Memory (HTM) and Absolute Pitch (AP; Ross et al. 2005; Figure 2). Second, playing a polyphonic primary instrument, though we tracked only piano because very few participants listed other polyphonic instruments as primary (Figure 3). Finally, years of formal lessons on the primary instrument (Figure 4) and years of formal lessons on piano regardless of primary instrument (Figure 5). Note that excluding the top-right-most two data points in Figure 4 would result in a less-sloped trend line, while in Figure 5 the outliers appear to be the two low-performing participants with 15 years of piano instruction.

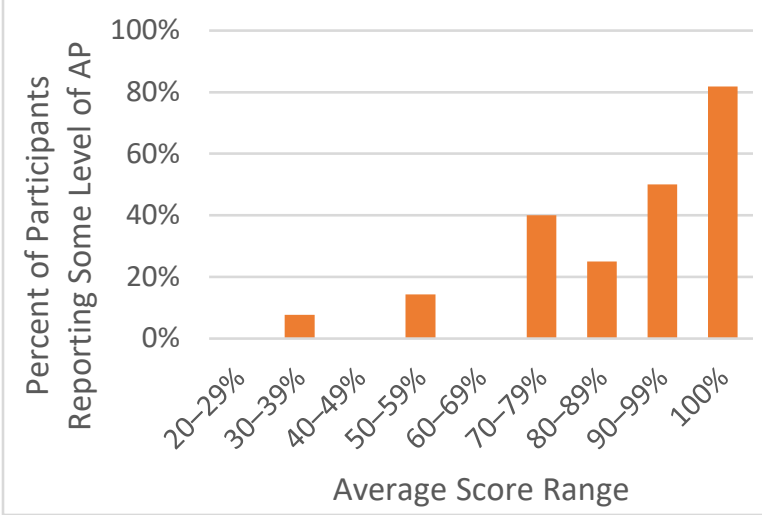

Figure 2: Percent of participants in each average-score category that reported some level of absolute pitch.

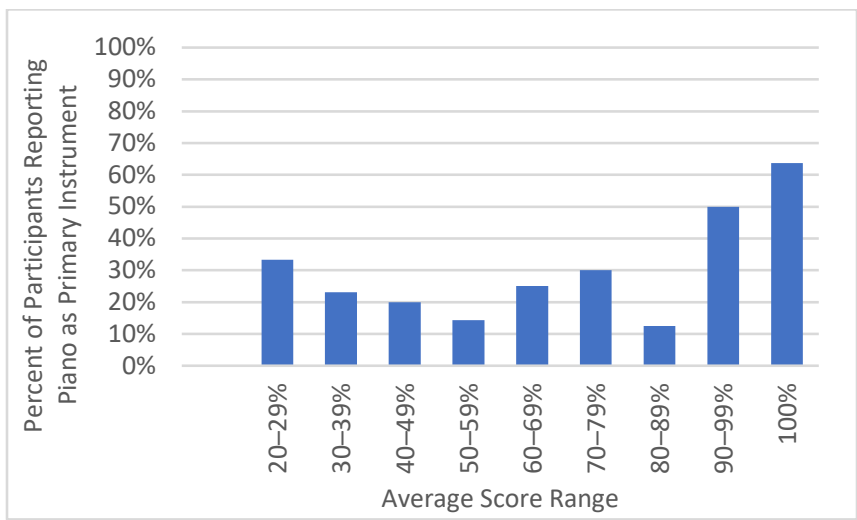

Figure 3: Percent of participants in each average-score category that reported piano as primary instrument.

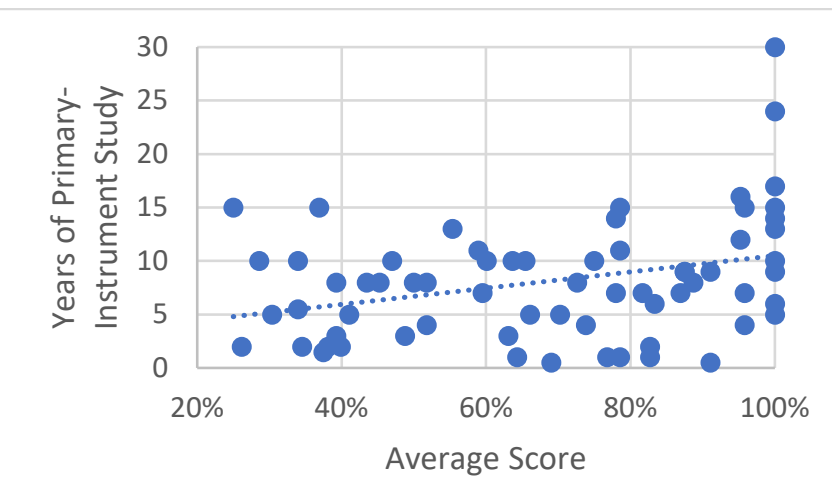

Figure 4: Scatterplot of average score on harmonic identification tasks against years of formal primaryinstrument study.

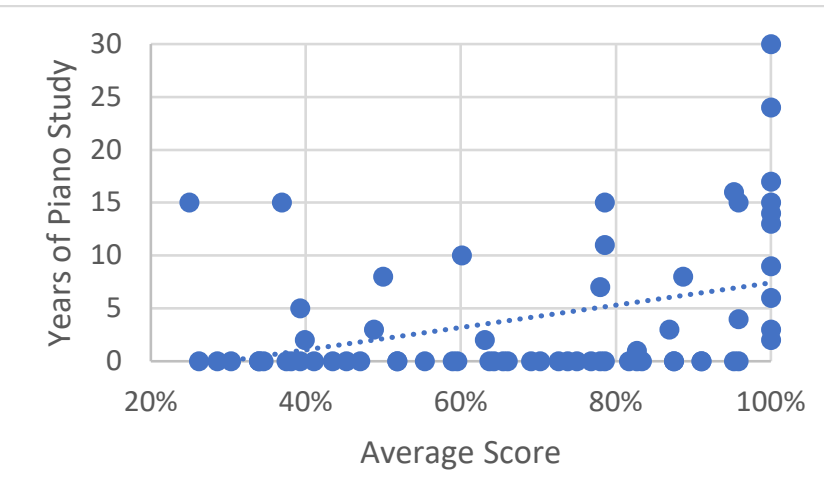

Figure 5: Scatterplot of average score on harmonic identification tasks against years of formal piano study.

When asked to select from a list those strategies that they use when identifying chords, $51 \%$ of participants selected "The feeling of the chord (the 'one-ness' or 'it feels dominant-y')," the choice intended to communicate Gestalt listening. Yet only "Imagining playing the chord on some instrument" was chosen by less participants, and several other strategies were far more popular (Figure 6). Even among the 11 participants who scored $100 \%$ across all three excerpts, who would presumably be those most likely to experience Gestalt hearing, only 55\% selected "The feeling of the chord" while $73 \%$ selected "Bass line." Participants' free-response reflections similarly tended to emphasize listening for the bass. 


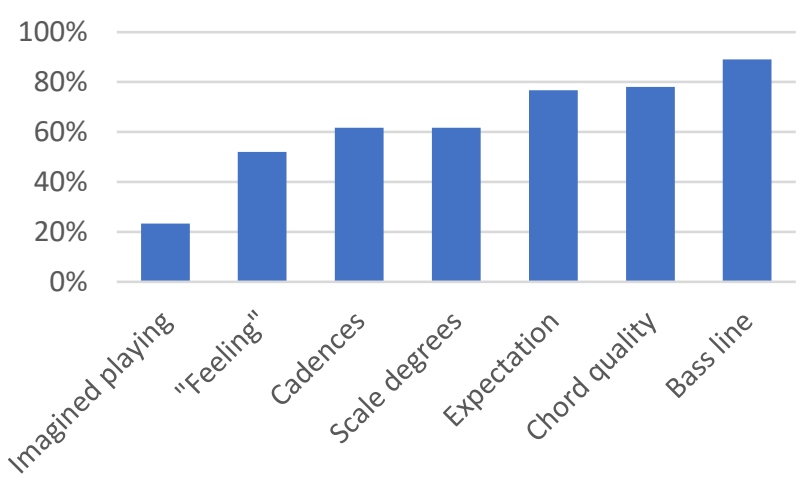

Figure 6: Percent of participants that indicated they use each chord-identification strategy.

Nevertheless, the free-response reflections of some participants who scored $100 \%$ did use wording that may suggest a truly different mode of (Gestalt) perception. Only two did not mention any other strategy: one, who has AP, wrote "I hear simultaneously in absolute pitches, Roman numeral function in relation to a local tonic, and figured bass symbols"; the other, who does not have AP, wrote, "I've internalized a kind of mental space for diatonic harmonies and outer-voice counterpoint, so I usually think about chord progressions traversing that space to identify them." Intriguingly, these were the two participants who reported the highest number of years of piano study (30 and 24, respectively). A few other participants among those who scored $100 \%$ combined similar statements with additional strategies; the one that most strongly suggested Gestalt hearing was "Sometimes, I can just tell instantly - other chords will require listening closely to individual notes within to figure them out." The remaining descriptions focused on component-based strategies such as "I follow the bass and try to identify its solfege, then I listen to the melody and its solfege."

Finally, common types of errors on the chordidentification exercises suggest that even highperforming chord identifiers rely heavily on an excerpt's bass line. For example, the most common error in the Karpinski dictation was labelling $\mathrm{ii}^{6}$ as IV, an error made even by half of the 8 participants whose average scores were between 90-99\%. The excerpt with the highest average score across all participants $(87.5 \%)$ was the Beyoncé song, in which all chords were in root position, while the lowest overall average score $(62.5 \%)$ was for the Mozart example, whose functional bass line was integrated into a potentially distracting Alberti bass. While incorrect answers in the examples with distinct bass lines (Karpinski and Beyoncé) typically preserved the correct bass contour, this was not true for the Mozart example. In fact, though our sample size is not large enough to establish this clearly, preliminary observations suggest that one of the primary factors that distinguished higher-performers (who scored an average of 80-99\%) from lower-performers (those with an average score of $<79 \%$ ) may have been the ability to successfully extract the bass line in the Mozart example.

\section{Discussion}

Drawing on these preliminary results, we propose a number of hypotheses and questions for future study.

First, with regard to our motivating question, we hypothesize that Gestalt hearing is not attainable as a primary mode of attending for most musicians at the college level. Nevertheless, there is tantalizing evidence that this kind of Gestalt hearing is available to some listeners, perhaps, as Karpinski (2000) suggests, as a result of other techniques practiced over "weeks, months, or even years" (p. 119). If so, then it is still an open question whether this is a skill available to all or whether it is only available to those with certain kinds of experiences or abilities. Notably, the two respondents whose wording strongly suggests Gestalt hearing reported 30 and 24 years (respectively) of formal piano instruction.

Second, we note that prevalence of AP/HTM, prevalence of piano as primary instrument, and years of formal piano instruction all appear to be higher among those who scored well at harmonic identification. We encourage further study to determine whether these are simply correlations or whether some combination significantly affects harmonic identification.

Third, we hypothesize that the most important strategy for chord identification is listening for bass lines. As noted above, this strategy was selected the most often by both our participant population as a whole and our highest-scoring group, and it was mentioned frequently in free responses. If this is true, then instruction in harmonic dictation would likely benefit from greater emphasis on bass-listening strategies. While current textbooks do often ask for bass- or outervoice dictation to accompany harmonic dictation, they also tend to organize their chapters/units around Roman numerals regardless of inversion/bass, and typically do not include advice on how to listen to bass lines. Ideas here might be derived from Sayrs 2019.

It is possible that our traditional method of judging harmonic hearing through Roman numerals with inversion symbols artificially inflated the apparent 
importance of listening for the bass; after all, to determine the correct inversion symbol, one must detect the bass. Nevertheless, participant free responses suggested that the bass was often used as a primary tool for detecting chords, not simply as a necessary step for determining inversions (e.g., "I listen for the bass note, and spell up the triad by note from that pitch to find the chord identity and inversion," and "I listened to the bass line, mostly, and thought about characteristic progressions in the common-practice style"). It is also likely that there is some circularity here: that the inversion of a chord (and thus its implied bass) is part of our traditional chord symbol because it is important to perception of chord identity. Still, it might be helpful for future research to examine whether musicians still listen for bass lines to this degree even when they are not asked to identify chord inversions.

One particularly ripe area for future study is the effects of formal instruction/experience with a chord instrument on harmonic listening. Again, our two participants who seemed to indicate Gestalt hearing had extensive piano experience, and piano experience was common among high-scoring participants. While this might suggest the importance of piano study to harmonic dictation skills, it is also possible that these participants benefitted from the presence of two excerpts in the study performed on piano, or that the prevalence of piano-based excerpts in current textbooks means that current teaching methods work best for pianists. In addition, it would be useful to study whether there are differences in how polyphonic-instrument players beyond pianists-guitarists, harpists, marimbists, etc.-perceive harmony; our sample size for each of these instruments was too small to suggest even preliminary conclusions.

\section{Conclusion}

This is the first relatively large-scale study of harmonic hearing, and as such, our focus has been on gathering a wide range of data that will be useful in planning future studies. Nevertheless, these data allow us to make some educated guesses about a few matters relevant to the teaching of this skill. In particular, our study suggests that "Gestalt hearing"-as appealing as it is - may be more complicated and less universally available than is sometimes assumed. If this is true, then the field of aural skills pedagogy might benefit from focusing instruction in harmonic listening more on strategies (particularly listening for bass lines, but also listening for chord qualities, characteristic inner-voice scale degrees, cadences, etc.) rather than on Roman numerals. We hope that future studies on harmonic listening follow, and we look forward to the results.

\section{References}

Butler, D. (2000). Review of Gary Karpinski, Aural Skills Acquisition: The Development of Listening, Reading, and Performing Skills in College-Level Musicians. Journal of Music Theory Pedagogy, 14, 105-114.

Jarvis, B. E. (2015). Hearing Harmony Holistically: Statistical Learning and Harmonic Dictation. Engaging Students: Essays in Music Pedagogy, 3. https://doi.org/10.18061/es.v3i0.7197

Karpinski, G. S. (2000). Aural Skills Acquisition. Oxford University Press.

Karpinski, G. S. (2017). Instructor's Dictation Manual for Manual for Ear Training and Sight Singing (2nd ed.). W.W. Norton.

Rogers, N. (2001). Review of Gary S. Karpinski, Aural Skills Acquisition: The Development of Listening, Reading, and Performing Skills in College-Level Musicians. Indiana Theory Review, 22(2), 83-93.

Sayrs, E. (2019). The Case For More Bass in the Aural Skills Curriculum. Pedagogy Into Practice: Teaching Music Theory in the Twenty-First Century, Santa Barbara.

Stevens, D. B. (2016). Symphonic Hearing: Mastering Harmonic Dictation Using the Do/Ti Test. Journal of Music Theory Pedagogy, 30, 111-176.

Stevens, D. B. (2020). In Search of Hidden Treasures. In The Routledge Companion to Music Theory Pedagogy (pp. 211-216). https://doi.org/10.4324/9780429505584-34
Routledge. 


\author{
What is "musical ability" and how do we measure it? \\ Brooke M. Okada ${ }^{1}$ \\ L. Robert Sleve ${ }^{1 \dagger}$ \\ ${ }^{1}$ Department of Psychology, University of Maryland, College Park, MD, USA \\ ${ }^{\dagger}$ Corresponding author: slevc@umd.edu \\ Published 16 December 2021; https://doi.org/10.18061/FDMC.2021.0029
}

\begin{abstract}
There is little consensus on what exactly constitutes musical ability and how to best measure it. Past research has used various tasks; most commonly assessing perceptual skills (e.g., same/different judgments in sequentially presented melodies), but also sometimes production tasks (e.g., singing a series of pitches or tapping along with a musical sequence). Outcome measures have ranged from single indices (e.g., "pitch ability") to composite scores from multiple tasks (e.g., pitch, rhythm, loudness, timbre, etc.). To date, it remains unclear how these different measures/scores relate to one another, limiting the ability to generalize across tasks and results. To address these issues, we assessed 165 participants' performance on 15 representative musical ability tasks to model the unity and diversity of musical abilities. Latent variable model comparisons suggest that musical ability is best represented by related but separable pitch, timing, perception, and production factors.
\end{abstract}

KEYWORDS: musical ability, individual differences, factor analysis

\section{Introduction}

Researchers have been measuring musical ability for at least the last hundred years (e.g., Seashore, 1915), and these measures have been used to investigate a variety of topics, including the innate vs. acquired nature of musical skill, relationships between musical and nonmusical abilities, and the components of musical ability and their dissociations in amusic patients (e.g., Ayotte, Peretz, \& Hyde, 2002; Mosing, Madison, Pedersen, Kuja-Halkola, \& Ullén, 2014; Okada \& Slevc, 2018). Given the breadth and duration of study, one might assume that we have a pretty good idea what musical ability is. However, despite the relatively large literature on individual differences in musical ability, there has been little consensus on what exactly constitutes musical ability and how to best measure it (see, e.g., Hallam \& Prince, 2003).

Certain debates about the nature of musical ability are perennial. For example, musical ability has often been described as an innate predisposition for music:
Seashore (1915) defined it as the "inborn psycho-physic and mental capacities distinguished from skills acquired in training" (p. 129) [1], and Law and Zentner (2012) similarly define it as the "potential for learning music before formal training and achievement" (p. 2). In contrast, others argue that musical ability is experiencebased. For example, Shinichi Suzuki claimed that "there is no such thing as an innate aptitude for music" (Hermann, 1981, p. 137).

Other debates are, perhaps surprisingly, less prominent. Most relevant to this paper: does a single factor underlie musical ability, as has been argued for general intelligence (e.g., Carroll, 1997; Spearman, 1904)? Or is what we call "musical ability" actually a disparate set of unrelated (or weakly related) skills?

Most research (including some of our own) has employed a circular definition of musical ability by assuming (at least implicitly) that musical ability corresponds to the task(s) used to measure it. For example, some studies rely on a single score of musical ability, effectively assuming a single underlying factor, while other studies calculate separate scores for melody and rhythm perception tasks, effectively assuming two separable latent perceptual factors. This conflation of task design with underlying constructs is common in individual differences research (take Boring's (1923) oft-cited claim that "intelligence is what is measured by intelligence tests") but is nevertheless problematic.

More broadly, assessing individual differences in musical ability requires the assumption that "musical ability" is a valid construct. However, there may very well be no natural kind (or at least no unitary construct) that corresponds to the term "musical ability." It is easy to assume that musical ability is a psychologically real concept because of the existence of the label "musical ability" (and measures thereof) but this, of course, is a fallacy (Brick et al., in press; Malt \& Majid, 2013).

Even setting these theoretical / philosophical issues aside, there has been little commonality in the tasks researchers have used to measure (and so define) musical ability. (For short taxonomies of commonly used tasks, see Law \& Zentner, 2012; Okada, 2018). On 
one hand, this is a problem because it makes it difficult to reconcile findings across studies (both practically and because it suggests studies are using different underlying conceptions of musical ability). On the other hand, one advantage of this variety of tasks in the literature is that they may offer a way, albeit indirect, to investigate the unity and diversity of the underlying construct(s) of musical ability.

The work described here is a step in this direction: to assess the unity and diversity of musical ability as defined by the measures typically used in the literature. To this end, we briefly describe an individual differences study investigating the relationships between performance on 15 different musical ability tasks; specifically assessing if performance on these tasks is better explained as an underlyingly unitary ability or as separable abilities for pitch and timing and/or perceptual and production tasks. (Note that methods and results are presented only briefly here; for further details, see Okada, 2018).

\section{Method}

We conducted a relatively large, pre-registered (https://osf.io/jwyhu) individual differences study using multiple tasks to measure pitch perception, pitch production, timing perception, and timing production. Because tasks are indirect measures of underlying cognitive constructs (the "task impurity problem" - i.e., even if performance on a task does reflect a specific construct of interest, it involves other processes as well), the analyses below rely on latent variable analysis to estimate the latent ability underlying performance on a set of theoretically related tasks (cf. Miyake et al., 2000; Okada \& Slevc, 2018). That is, because a single task measuring a construct of interest may not be indicative of someone's true score (e.g., could include measurement error), multiple measures of each construct of interest were administered. By using latent variable analysis, one can estimate what is common between the tasks measuring a given construct and better estimate the underlying component of interest removed from task-specific effects.

More specifically, a series of confirmatory factor analyses (CFA) were used to assess whether individual differences in these abilities fit better with a unitary model of musical ability (cf. general intelligence), a model with separable abilities for pitch- and timingrelated abilities, a model with separable perceptual and productive abilities, or some combination of separable pitch/timing and perception/production abilities.

\section{Participants}

165 participants were recruited from the University of Maryland's undergraduate research pool and received class credit for participation.

\section{Measures and Procedure}

Participants completed a battery of 15 musical tasks measuring both receptive and productive pitch ability and receptive and productive timing ability (see Table 1 for a summary; for additional information, see Okada (2018) and https://osf.io/mp3u7/).

Table 1: Musical ability tasks, split by perception- and production-based, and by pitch-and timing-based tasks.

\begin{tabular}{|c|c|c|}
\hline & Pitch & Timing \\
\hline \multirow{5}{*}{ } & Pitch Discrimination $^{\mathrm{a}}$ & Rhythm Discrimination \\
\hline & Melody Discrimination ${ }^{a}$ & Tempo Discrimination $^{a}$ \\
\hline & Tuning Discrimination ${ }^{\mathrm{a}}$ & Beat Perception $^{\mathrm{d}}$ \\
\hline & Chord Analysis ${ }^{b}$ & Timing Thresholds ${ }^{\mathrm{c}}$ \\
\hline & Pitch Thresholds ${ }^{c}$ & \\
\hline \multirow{6}{*}{ 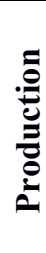 } & Familiar Song & Synchronization to a \\
\hline & Imitation $^{\mathrm{e}}$ & Metronome $^{\mathrm{d}}$ \\
\hline & Melody Imitation (note, & Synchronization \\
\hline & interval, melodies) $)^{\mathrm{e}}$ & Continuation $^{\mathrm{d}}$ \\
\hline & Tonal Memory ${ }^{\mathrm{f}}$ & Synchronization to \\
\hline & & Musical Passage \\
\hline
\end{tabular}

a. PROMS-S (Zentner \& Strauss, 2017); b. Wing (1962); c. Soranzo \& Grassi (2014); d. Beat Alignment Test (BAT, Iverson \& Patel, 2008); e. Pfordresher et al. (2010); f. Mowrer (1994); g. H-BAT (Fujii \& Schlaug, 2013)

\section{Results}

In general, task reliabilities were acceptable and comparable to past work (but see discussion below). Individual tasks were generally correlated significantly, but the magnitude of these correlations was not especially high (most correlations ranged from about .2 to .5). This suggests that these tasks are tapping related abilities but are not completely redundant measures.

A single-factor model of musical ability (i.e., one latent factor underlying performance on all 15 musical tasks) showed acceptable model fit, but fit was significantly improved with a two-factor model including separate, but correlated Pitch and Timing factors. Model fit was additionally improved by including both pitch/timing and perception/production factors, supporting related, but dissociable abilities in 
perception and production of both pitch and timing aspects of music (see Figure 1 for the best fitting model).

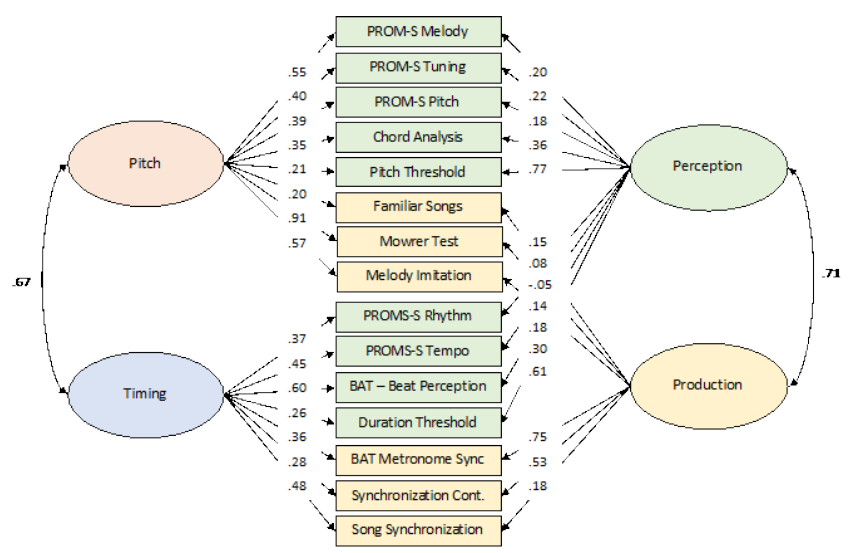

Figure 1: Best fitting CFA model. Observed task scores are represented with rectangles and estimated latent factors with ovals. Single headed arrows from latent factors to measured variables represent standardized factor loadings. Double headed arrows between latent factors represent correlations between the factors.

\section{Discussion}

The data presented (briefly) here suggest that individual differences in measures of musical ability are not best explained by a unitary underlying "musical ability" factor. Instead, individuals' performance fit best with a model assuming related but separable underlying latent factors for pitch and timing abilities and for perception and production abilities.

Most research measuring musical abilities has relied on perceptual measures, probably because they are relatively easy to administer and score. However, this may come at a cost: in these data, some of the more commonly used perceptual discrimination tasks had the lowest reliabilities (e.g., the Chronbach's alpha for the PROMS-S measures and the beat perception task from the BAT were below .6). In addition, the "best" individual measure (in terms of being most closely related to the overall latent structure from all tasks) was the Mowrer Test of Tonal Memory, a production task in which participants heard and sung back short melodies (Mowrer, 1996). Of course, production tasks like this are more challenging to administer than perception tasks (e.g., because some participants can be hesitant to sing) and also more challenging to evaluate/score, but we suggest the extra effort may be worthwhile.
Of course, this and all of our conclusions are limited by the tasks we chose to include, which measure only a subset of the types of abilities that are likely relevant to musical ability more broadly (see, e.g., Hallam \& Prince, 2003). An additional limitation is that our data come from a group of roughly 20-year-old Western college undergraduates (WEIRD people; Henrich, Heine, \& Norenzayan, 2010), and it is not at all obvious that the same pattern would emerge in other demographics.

A broader limitation of this type of work is that it assumes that performance on a task reflects an underlying ability (i.e., a stable trait) but of course task performance is also influenced by dynamic changes in the individuals' current state. For example, people regularly show temporary attention lapses during cognitive tasks (e.g., Smallwood et al., 2008) and task engagement can be influenced by a variety of factors such as motivation, mood, and task difficulty (e.g., Chiew \& Braver, 2014). Thus, a task score from a single point in time may not accurately reflect an individual's ability even on that specific task.

Despite all these issues, it seems likely that researchers will continue to measure musical ability. Of course, we do not suggest that researchers should conduct hours of cognitive testing to extract latent factors (as we did here). We do, however, hope that the diversity among measures that we highlight will encourage more careful consideration of what measures to include should one decide to measure musical ability. In addition, we hope this type of work motivates future research working to better understand the complex nature of musical ability.

\section{Conclusion}

Despite over a hundred years of research on individual differences in musical ability, we still do not have a very good idea what it is. Here, we suggest that performance on most current measures of musical ability do not reflect a single underlying factor, but instead reflect (at minimum) separable, but related, musical pitch, timing, perception, and production abilities. While this is certainly not a comprehensive model, we hope it is a useful step toward a better understanding of the structure and diversity of musical abilities.

\section{End Notes}

[1] It is worth noting that Seashore's interest in measuring musical ability dovetailed with his interests in eugenics (see, e.g., Koza, 2007), paralleling the 
somewhat ignoble history of intelligence testing in the USA (e.g., Kamin, 1974).

\section{References}

Ayotte, J., Peretz, I., \& Hyde, K. (2002). Congenital amusia: A group study of adults afflicted with a music-specific disorder. Brain, 125(2), 238-251. https://doi.org/10.1093/brain/awf028

Boring, E. G. (1923). Intelligence as the tests test it. New Republic, 35-37.

Brick, C., Hood, B., Ekroll, V., \& de-Wit, L. (in press). Illusory essences: A bias holding back theorizing in psychological science. Perspectives on Psychological Science. https://doi.org/10.31234/osf.io/eqma4

Carroll, J. B. (1997). Psychometrics, intelligence, and public perception. Intelligence, 24(1), 25-52. https://doi.org/10.1016/S0160-2896(97)90012-X

Chiew, K. S., \& Braver, T. S. (2014). Dissociable influences of reward motivation and positive emotion on cognitive control. Cognitive, Affective, \& Behavioral Neuroscience, 14(2), 509-529. https://doi.org/10.3758/s13415-0140280-0

Fujii, S., \& Schlaug, G. (2013). The Harvard Beat Assessment Test (H-BAT): A battery for assessing beat perception and production and their dissociation. Frontiers in Human Neuroscience, 7, 1-16. https://doi.org/10.3389/fnhum.2013.00771

Hallam, S., \& Prince, V. (2003). Conceptions of Musical Ability. Research Studies in Music Education, 20(1), 222. https://doi.org/10.1177/1321103X030200010101

Henrich, J., Heine, S. J., \& Norenzayan, A. (2010). The weirdest people in the world? Behavioral and Brain Sciences, 33(2-3), 61-83. https://doi.org/10.1017/S0140525X0999152X

Hermann, E. (1981). Shinichi Suzuki: The Man and His Philosophy. Miami, FL: Summy-Birchard Inc.

Iversen, J. R, \& Patel, A. D. (2008). The Beat Alignment Test (BAT): Surveying beat processing abilities in the general population. In K. Miyazaki et al. (Eds.), Proceedings of the 10th International Conference on Music Perception \& Cognition (ICMPC10). Adelaide, Australia: Causal Productions.

Kamin, L. J. (1974). The science and politics of IQ. Psychology Press.

Koza, J. E., (2007). In sounds and silences: Acknowledging political engagement. Philosophy of Music Education Review, 15(2), 168-176. https://doi.org/10.2979/PME.2007.15.2.168

Law, L. N., \& Zentner, M. (2012). Assessing musical abilities objectively: Construction and validation of the Profile of Music Perception Skills. PloS one, 7(12), e52508. https://doi.org/10.1371/journal.pone.0052508

Malt, B. C. and Majid, A. (2013). How thought is mapped into words. WIREs Cognitive Science, 4, 583-597. https://doi.org/10.1002/wcs.1251

Miyake, A., Friedman, N. P., Emerson, M. J., Witzki, A. H., Howerter, A., \& Wager, T. D. (2000). The unity and diversity of executive functions and their contributions to complex "frontal lobe" tasks: A latent variable analysis. Cognitive Psychology, 41(1), 49-100. https://doi.org/10.1006/cogp.1999.0734

Mosing, M. A., Madison, G., Pedersen, N. L., Kuja-Halkola, R., \& Ullén, F. (2014). Practice does not make perfect: no causal effect of music practice on music ability. Psychological Science, 25(9), 1795-1803. https://doi.org/10.1177/0956797614541990

Mowrer, A. (1996). Tonal Memory as an Audition Factor for Choral Ensembles (Doctoral dissertation). Temple University.

Okada, B. M. (2018). Towards a Comprehensive Model of Musical Ability (Doctoral dissertation). University of Maryland. https://doi.org/10.13016/cajm-89aw

Okada, B. M., \& Slevc, L. R. (2018). Individual differences in musical training and executive functions: A latent variable approach. Memory \& Cognition, 46(7), 10761092. https://doi.org/10.3758/s13421-018-0822-8

Pfordresher, P. Q., Brown, S., Meier, K. M., Belyk, M. Liotti, M. (2010). Imprecise singing is widespread. Journal of the Acoustical Society of America, 128(4), 2182-2190. https://doi.org/10.1121/1.3478782

Seashore, C. E. (1915). The measurement of musical talent. The Music Quarterly, 1(1), 129-148. https://doi.org/10.1093/mq/I.1.129

Smallwood, J., Beach, E., Schooler, J. W., \& Handy, T. C. (2008). Going AWOL in the brain: Mind wandering reduces cortical analysis of external events. Journal of Cognitive Neuroscience, 20(3), 458-469. https://doi.org/10.1162/jocn.2008.20037

Soranzo, A., \& Grassi, M. (2014). Psychoacoustics: A comprehensive Matlab toolbox for auditory testing. Frontiers in Psychology, 5:712. https://doi.org/10.3389/fpsyg.2014.00712

Spearman, C. (1904). General intelligence objectively determined and measured. American Journal of Psychology, 15, 201-293. https://doi.org/10.2307/1412107

Wing, H. D. (1962). A Revision of the "Wing Musical Aptitude Test". Journal of Research in Music Education, 10(1), 39-46. https://doi.org/10.2307/3343909

Zentner, M., \& Strauss, H. (2017). Assessing musical ability quickly and objectively: Development and validation of the Short-PROMS and the Mini-PROMS. Annals of the New York Academy of Sciences, 1400, 33-45. https://doi.org/10.1111/nyas.13410 


\section{Does cognitive load differ among sight-singers? An exploratory study using pupillometry and interviews \\ Justine Pomerleau-Turcotte ${ }^{1 \dagger}$ \\ Maria Teresa Moreno Sala ${ }^{1}$ \\ Francis Dubé \\ François Vachon ${ }^{2}$ \\ ${ }^{1}$ Faculty of Music, Université Laval, Quebec City, QC, Canada \\ ${ }^{2}$ School of Psychology, Université Laval, Quebec City, QC, Canada \\ $\dagger$ Corresponding author: justine.pomerleau-turcotte.1@ulaval.ca \\ Published 16 December 2021; https://doi.org/10.18061/FDMC.2021.0030 \\ Author video presentation and/or other conference material: https://doi.org/10.17605/OSF.IO/BZVQP}

\begin{abstract}
Sight-singing is challenging for many music students, yet they can experience various difficulties with this task. To explore how cognitive load (CL) might differ among students, we combined two approaches: 1) a quantitative approach using pupil size diameter - a psychophysiological indicator of $\mathrm{CL}$ - to see whether CL differed as a function of sight-singing achievement and experience; 2) a qualitative approach to learn student's challenges when sight-singing and verify how such challenges reflect on sight-singing scores. We asked 56 post-secondary music students to complete a musical background questionnaire and to complete a sight-singing exercise, while an eye-tracker gathered data about their pupil size. After that, we interviewed them about the difficulties they experienced. The results revealed that CL did not vary between sight-singing performance and musical experience levels. However, we found a tendency suggesting that students with the highest intonation scores and lowest intonation scores both experienced a lower CL. On the contrary, CL was higher for students with average intonation scores. Interviews also revealed that many students experienced information overload while sight-singing, and students who shared such perception obtained, on average, lower sight-singing scores. Future studies should include qualitative data collection to deepen our understanding of learners' experiences.
\end{abstract}

KEYWORDS: Aural skills, Sight-singing, Higher education, Eye-tracking, Cognitive load

\section{Introduction}

Sight-singing is an essential dimension of aural skills classes included in music programs in higher education. However, sight-singing can be challenging for many students: some have trouble reading music (Asmus, 2004), suffer from a lack of preparation (Anderman, 2011), or experience anxiety (Buonviri, 2014; Fournier et al., 2019). Furthermore, students begin their studies with various musical backgrounds (Buonviri, 2015; Teixeira dos Santos \& Puchalski dos Santos, 2020). Consequently, students might experience differently the tasks their instructors choose. Indeed, some students will sight-sing easily, while others will have to put a lot of effort into this task.

Cognitive load (CL) is the relationship between a task's demands and the mental resources available (Wickens \& Hollands, 1999). A higher CL can hinder improvisers' creativity (Norgaard et al., 2016), instrumentalists' expressivity (Çorlu, Muller, et al., 2015), and singers' timing (Çorlu, Maes, et al., 2015). To our knowledge, no study so far has investigated the relationship between sight-singing performance and CL. However, it remains unknown whether pupil size can also fluctuate as a function of sight-singing achievement or musical background.

Advanced musicians usually sight-read better. For example, Kopiez and Lee (2006) found that sightreading experience acquired before the age of 15 was a strong predictor of sight-reading performance. Also, Arthur et al. (2020) found that sight-reading expertsthose able to play a $6^{\text {th }}$ Grade sight-reading exercise from the Australian Examination Board-were more likely to have had formal training for more than 10 years and to have begun learning music before the age of seven. One possible explanation is that experienced musicians can access schemas from their long-term memory and, therefore, process the score more easily (Sheridan et al., 2020). The amount of previous musical experience is also related to better sight-singing performance (Fournier, 2020). Nevertheless, the question as to whether sight-singing requires less effort-i.e., imposes a lower CL-for experienced musicians remained unanswered. 
This study aimed to determine whether CL varied as a function of musical experience and sight-singing performance. We also wanted to know which challenges, notably related to mental effort and subjective perception of CL, post-secondary music students experienced while sight-singing and if they could relate to sight-singing performance.

One way to assess CL objectively is to measure pupil size. Variations in pupil diameter are deemed to reflect changes in CL (Beatty, 1982; Einhäuser, 2017). For music reading, pupil size varies depending on task difficulty. For example, pupil size tends to be larger in harder tonalities (Chitalkina et al., 2020) or when reading unusual chord progressions (Hadley et al., 2018).

\section{Method}

Participants

After obtaining ethical approval, we recruited 56 music students from three post-secondary institutions in the authors' urban area. All participants had normal or corrected-to-normal vision. As compensation for their participation in the study, they were offered free aural skills tutoring by the first author.

Of that number, 39 were students from CEGEP level - a 2-year post-secondary training in Quebec between high school and university - and 17 were university students. Participants were between 17 and 67 years old $(M=22.88, M d n=19.00, S D=11.03)$. They had accumulated between 3 and 26 years of musical experience $(M=10.61, M d n=9.00, S D=5.22)$. Regarding the main instrument played, 18 reported a harmonic instrument (e.g., piano), while 38 reported a non-harmonic instrument (e.g., trumpet) or voice.

\section{Material}

Using Google Forms, participants first completed a homemade survey aiming at gathering information about their musical background. It included open-ended questions about when they began learning music, the instruments they played, their main instrument, their post-secondary education, and their number of years of musical experience. The sight-singing exercise consisted of an 8-measure, medium difficulty melody, adapted and transposed from École préparatoire de musique de l'Université Laval (1999).

The survey and the melody were presented on a Dell Precision T5810 computer screen. While they sang, a FOVIO eye-tracker recorded their pupil diameter. The eye-tracker was on the desk and centered below the computer screen. Eye-tracking data were processed with the software EyeWorks (EyeTracking Inc., 2019). The sampling rate was $60 \mathrm{~Hz}$. A Yamaha NP11 electronic piano keyboard was located in front of the participant. The semi-structured interview included questions about the students' difficulties with sight-singing.

\section{Procedure}

The experimenter met students individually for a single session in a dimly lit soundproof room. They first completed the questionnaire, which lasted about 15 minutes. After that, the experimenter launched EyeWorks and assisted participants with the calibration of the eye tracker. Instructions for the sight-singing task appeared on the screen, followed by the score. Participants could play the starting pitch on the keyboard and could rehearse mentally for as long as they wanted. Their performance was audio recorded. This segment of the data collection lasted from 5 to 10 minutes. After they completed the task, the first author came back into the room to conduct the interview, which lasted approximately 10 minutes.

\section{Scoring and data preparation}

We used five dimensions of sight-singing performance. Three of them were objective: pitch, rhythm, and combined scores. Each note was worth two points: one for pitch, one for rhythm. The combined score was the sum of the two. Two measures were subjective and assessed on a four-point scale: rhythmic fluidity and intonation accuracy. The experimenter rated the recordings, and an aural skills teacher with a Ph.D. in Music Education scored ten sight-singing performances to validate our rating scales. Both scoring correlated strongly and significantly, for the combined score, $r(8)$ $=.987, p<.0001$, the rhythmic fluidity score, $r(8)=$ $0.962, p<.0001$, and for the intonation accuracy score, $r(8)=0.871, p=.001$. Therefore, we considered the experimenter's scoring as valid.

We averaged pupil size diameter between both eyes. Measurements were restricted to the exercise's four central measures because we wanted to obtain data for the sight-reading task's cognitive load while accounting for the time the pupil takes to adjust. This portion of the sight-singing task lasted about eight seconds.

We excluded three participants whose pupil diameter data was more than 1.5 times outside the interquartile range for analyses involving cognitive load, as well as 11 subjects for which we did not have pupillometric data for the time interval we studied. 


\section{Analyses}

We conducted statistical analyses with RStudio (R Core Team, 2019), with the packages $l s r$ (Navarro, 2015) and car (Fox \& Weisberg, 2019). We created groups to compare different levels of sight-singing (low-, average-, and high-performing groups. We made those three groups based on quantiles for rhythm score, pitch score, and combined score to obtain approximately equal groups. We assessed rhythmic fluidity and intonation accuracy with scales ranging from zero to three, and we used these scores to create groups to compare. We collapsed the two highest scores and created three groups for these variables too.

We used a similar process to compare groups based on musical experience. We used quantiles to create groups based on age when participants began learning music and number of years of experience. For the academic level, we compared students from CEGEP and university. For the main instrument, we compared students who played a harmonic instrument with students who played a non-harmonic instrument.

With regards to the interviews, we conducted a thematic content analysis (Krippendorff, 2013). We listed every theme related to challenges students faced when performing the sight-singing task and, more generally, sight-singing in their aural skills classes.

\section{Results}

After checking for variance homogeneity and normality of residuals, we used analyses of variance (ANOVAs) to compare pupil diameter between different performance levels for pitch (low: $n=14$; average: $n=$ 13; high: $n=15$ ), rhythm (low: $n=15$; average: $n=15$; high: $n=12$ ), combined score (low: $n=14$; average: $n$ =13; high: $n=15$ ), rhythmic fluidity (low: $n=9$; average: $n=14$; high: $n=19$ ), and intonation accuracy (low: $n=10$; average: $n=11$; high: $n=21$ ). No significant pupil diameter difference was found for any of these performance dimensions. However, as shown in Figure 1, differences almost reached significance for intonation accuracy, $F(2,39)=2.98, p=.062$.

Moreover, we conducted ANOVAs to compare pupil diameter between groups based on the age when participants began learning music (participants who began before eight years old: $n=20$; between eight and ten years old: $n=9$; after ten years old: $n=13$ ). We also compared pupil diameter based on the number of years of musical experience (participants with less than eight years of experience: $n=17$; between eight and eleven years of experience: $n=10$; and with more than eleven years of experience: $n=15$ ). These analyses revealed no significant difference between groups. After checking for variance homogeneity, we then conducted $t$-tests to compare pupil diameter according to study level (CEGEP, $n=28$ vs. university, $n=14$ ) and primary instrument type (harmonic, $n=15$ vs. non-harmonic, $n$ $=27$ ). No significant differences arose between groups.

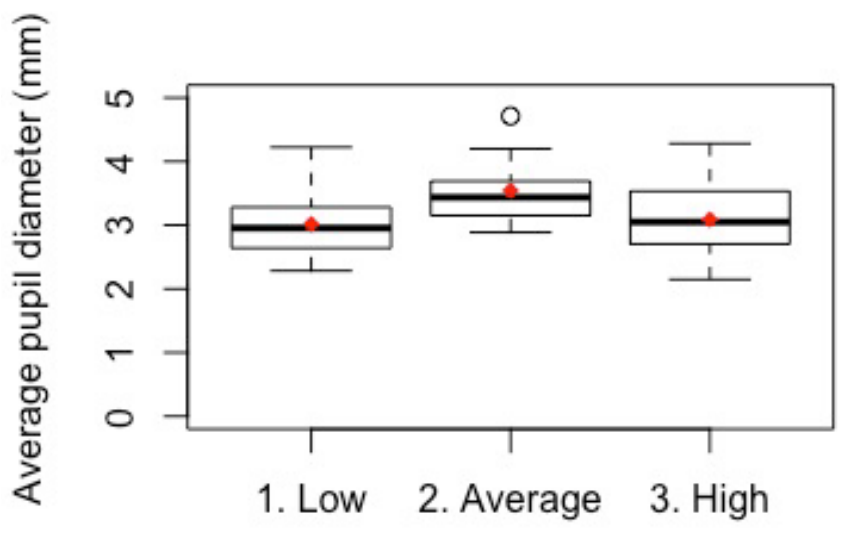

\section{Intonation accuracy}

Figure 1: Average pupil diameter (in $\mathrm{mm}$ ) as a function of intonation accuracy. The bold line in each box indicates the median, while red lozenges show the mean for each intonation accuracy level.

Interviews about students' difficulties with the sightsinging task revealed that at least 20 participants had difficulties managing multiple information types simultaneously, indicating that they felt high levels of subjective cognitive load, as Participant 3 reports: "...there was just too much information, I was overloaded, and then I couldn't keep up." More precisely, singing the right pitch while keeping up with the rhythm is also an issue, as described by Participant 16: "I was just messing up with the rhythm, even if it was simple. Then I focused on the pitches, and I knew I was messing up, so I just dropped the ball...so rhythm was nonsense." Making conducting gestures while singing to help maintain the pulse is also hindered by this overload, as Participant 54 suggests: "First time I sight-sing a melody, I can't conduct because it confuses me, it's too much to handle." Other challenges many participants shared included difficulty to sing intervals (29 participants), understanding rhythm figures (25 participants), and lacking experience with sight-singing (20 participants).

We compared results between the 20 participants who reported difficulties managing multiple 
information types simultaneously and those who did not. After checking for variance homogeneity, we used $t$-tests to compare rhythm, pitch, combined scores, rhythmic fluidity, and intonation accuracy. For rhythm, there was no significant difference between participants who experienced subjective overload $(M=17.50, S D=$ $3.32)$ with participants who did not $(M=19.53, S D=$ $4.54), t(54)=1.62, p=.110$, Cohen's $d=.47$. However, we found significant differences for pitch score between subjects who reported subjective overload $(M=10.94$, $S D=5.64)$ and those who did not $(M=16.03, S D=$ $7.49), t(54)=2.61, p=.012$, Cohen's $d=.75$. We also found significant differences for the combined score between overloaded subjects $(M=28.44, S D=8.13)$ and those who were not $(M=35.55, S D=10.10), t(54)=$ $2.67, p=.010$, Cohen's $d=.76$ ). For rhythmic fluidity, there was a significant difference between those who reported subjective overload $(M=0.89, S D=0.75)$ and those who did not $(M=1.79, S D=1.13), t(54)=3.03, p$ $=.005$, Cohen's $d=.87$ ). Finally, for intonation accuracy, there was no significant difference between subjects who perceived an information overload $(M=$ $0.94, S D=1.00)$ and those who $\operatorname{did} \operatorname{not}(M=1.34, S D=$ $0.60), t(54)=1.67, p=.101$, Cohen's $d=.48)$.

\section{Discussion}

In this study, we wanted to verify if cognitive load (CL) while sight-singing varied between students as a function of sight-singing performance and musical experience. We also wanted to explore which difficulties they experienced during the sight-singing task. In terms of sight-singing performance, we did not find significant pupil diameter differences between participants for rhythm, pitch, and combined scores. We also did not find significant differences based on rhythmic fluidity and intonation accuracy. In terms of musical experience, we also did not find significant differences in pupil diameter based on the number of years of experience, the age when participants began learning music, their level of study, or their primary instrument. These results suggest that cognitive load does not vary as a function of sight-singing performance and musical experience.

Although not significant, the differences we observed in pupil diameter as a function of intonation accuracy performance, i.e., that pupil size diameter was lower for the low- and the high-performing group and higher for the average performing group is something that we should explore more in-depth in the future. It suggests that there might be an ideal CL when sight- singing. A cognitive overload could be detrimental, as other studies demonstrated (Çorlu, Maes, et al., 2015; Çorlu, Muller, et al., 2015; Norgaard et al., 2016), but a load that is not sufficiently high could reflect lower engagement in the task. Consequently, this could explain how a lower load could be linked to poorer results. Pupil size diameter differences could be tested with a larger sample to see how it relates to intonation accuracy. Indeed, due to data loss during the short interval for which we measured pupil size diameter, we could only conduct our analyses with 42 participants. Furthermore, this problem could have been avoided with a more extended task, allowing more time to obtain pupil size data. Future studies could also use sightsinging tasks of various difficulties to manipulate cognitive load and measure its impact on performance.

Interestingly, participants' testimonies suggest that cognitive overload is an issue when sight-singing. It can even be an obstacle to using strategies many authors view as efficient, like using conducting gestures to help maintain the pulse (e.g., Karpinski, 2000). Indeed, some students might not have sufficient cognitive resources to simultaneously read music, sing, understand rhythms, and maintain the pulse with gestures that are potentially not fully automated. From a pedagogical standpoint, this suggests that learners should rehearse some difficulties separately (executing rhythms, keeping a pulse while conducting, singing chords) before they are integrated into more complex sight-singing exercises.

Because we found that many students reported problems managing multiple information sources simultaneously, suggesting a feeling of cognitive overload, we investigated if sight-singing scores were lower for students who talked about such difficulties. We found participants who reported a higher subjective CL had lower sight-singing scores for some dimensions only: pitch, combined score, and rhythmic fluidity. It could be because when the task is too hard, it is still possible for students to maintain correct rhythmic durations, but pitches might be erroneous and rhythmic fluidity might suffer. Future studies could measure the impact of a higher cognitive load of sight-singing performance, for example, in adding progressive difficulties to a task and observing which sight-singing performance dimensions are affected. It also seems that qualitative reports can deepen our understanding of how students experience mental effort, and studies about cognitive load in that context should include them. 


\section{Conclusion}

Our results suggest that musical background and sightsinging performance are not related to cognitive load when sight-singing. However, participants reported feelings of information overload when singing, which should be taken into account by instructors while designing learning activities, as it can be associated with lower performances.

\section{References}

Anderman, M. A. (2011). Musicianship instruction in California Community Colleges (Publication No. 3483464) [Doctoral dissertation, Boston University]. ProQuest Dissertations \& Theses Global.

Arthur, P., McPhee, E., \& Blom, D. (2020). Determining what expert piano sight-readers have in common. Music Education Research, 22(4), 447-456. https://doi.org/10.1080/14613808.2020.1767559

Asmus, E. P. (2004). Music teaching and music literacy. Journal of Music Teacher Education, 13(2), 6-8. https://doi.org/10.1177/10570837040130020102

Beatty, J. (1982). Task-evoked pupillary responses, processing load, and the structure of processing resources. Psychological Bulletin, 91(2), 276-292. https://doi.org/10.1037/0033-2909.91.2.276

Buonviri, N. O. (2014). An exploration of undergraduate music majors' melodic dictation strategies. Update: Applications of Research in Music Education, 33(1), 2130. https://doi.org/10.1177/8755123314521036

Buonviri, N. O. (2015). Three music education majors' journeys through Aural Skills 101. Journal of Music Teacher Education, 25(1), 95-106. https://doi.org/10.1177/1057083714552328

Chitalkina, N., Puurtinen, M., Gruber, H., \& Bednarik, R. (2020). Handling of incongruences in music notation during singing or playing. International Journal of Music Education. https://doi.org/10.1177/0255761420944036

Çorlu, M., Maes, P.-J., Muller, C., Kochman, K., \& Leman, M. (2015). The impact of cognitive load on operatic singers' timing performance. Frontiers in Psychology, 6, 429. https://doi.org/10.3389/fpsyg.2015.00429

Çorlu, M., Muller, C., Desmet, F., \& Leman, M. (2015). The consequences of additional cognitive load on performing musicians. Psychology of Music, 43(4), 495-510. https://doi.org/10.1177/0305735613519841

École préparatoire de musique de l’Université Laval. (1999). Solfèges mélodiques 10e degré. Université Laval.

Einhäuser, W. (2017). The pupil as marker of cognitive processes. In Q. Zhao (Ed.), Computational and Cognitive Neuroscience of Vision (pp. 141-169). Springer. https://doi.org/10.1007/978-981-10-0213-7_7

EyeTracking Inc. (2019). EyeWorks (3.0) [Computer software]. eyetracking.com

Fournier, G. (2020). L'apport des stratégies dans l'apprentissage du solfège chez les étudiants en musique de niveau collégial [Doctoral dissertation, Université Laval]. CorpusUL.

https://corpus.ulaval.ca/jspui/handle/20.500.11794/39089

Fournier, G., Moreno Sala, M. T., Dubé, F., \& O’Neill, S. (2019). Cognitive strategies in sight-singing: The development of an inventory for aural skills pedagogy. Psychology of Music, 47(2), 270-283. https://doi.org/10.1177/0305735617745149

Fox, J., \& Weisberg, S. (2019). An $\{R\}$ Companion to Applied Regression, Third Edition. Sage. [Computer software] https://socialsciences.mcmaster.ca/jfox/Books/Companion

Hadley, L. V., Sturt, P., Eerola, T., \& Pickering, M. J. (2018). Incremental comprehension of pitch relationships in written music: Evidence from eye movements. The Quarterly Journal of Experimental Psychology, 71(1), 211219. https://doi.org/10.1080/17470218.2017.1307861

Karpinski, G. S. (2000). Aural skills acquisition: The development of listening, reading, and performing skills in college-level musicians. Oxford University Press.

Kopiez, R., \& Lee, J. I. (2006). Towards a dynamic model of skills involved in sight reading music. Music Education Research, 8(1), 97-120. https://doi.org/10.1080/14613800600570785

Krippendorff, K. (2013). Content analysis: An introduction to its methodology (3rd ed.). SAGE.

Navarro, D. J. (2015). Learning statistics with R: A tutorial for psychology students and other beginners (0.5) [Computer software]

Norgaard, M., Emerson, S. N., Dawn, K., \& Fidlon, J. D. (2016). Creating under pressure. Music Perception: An Interdisciplinary Journal, 33(5), 561-570. https://doi.org/10.1525/mp.2016.33.5.561

R Core Team. (2019). R: A language and environment for statistical computing. [Computer software] https://www.Rproject.org/.

Sheridan, H., Maturi, K. S., \& Kleinsmith, A. L. (2020). Eye movements during music reading: Toward a unified understanding of visual expertise. In K. D. Federmeier \& E. R. Schotter (Eds.), Psychology of Learning and Motivation (Vol. 73, pp. 119-156). Academic Press. https://doi.org/10.1016/bs.plm.2020.07.002

Teixeira dos Santos, R. A. T., \& Puchalski dos Santos, R. P. (2020). Developing a tool for music theory placement: An emphasis on implicitly learned abilities. Journal of Research in Music Education. https://doi.org/10.1177/0022429420949963

Wickens, C. D., \& Hollands, J. G. (1999). Engineering psychology and human performance ( $3^{\text {rd }}$ ed.). PrenticeHall. 


\section{Reaching across the aisle: The essential need for collaboration among researchers, medical personnel, therapists, musicians, and educators \\ Martha Summa-Chadwick, DMA \\ Music Therapy Gateway in Communications, Signal Mountain, TN, USA \\ summa@marthasumma.com \\ Published 16 December 2021; https://doi.org/10.18061/FDMC.2021.0031 \\ Author video presentation and/or other conference material: https://doi.org/10.17605/OSF.IO/Q2G36}

\begin{abstract}
A remarkable body of excellent research verifying the benefits of music for the brain has already been published, but is generally unknown to the consumer [1]. If this knowledge could be channeled directly to consumers, the result would be a revolution of the health-care system, as millions of consumers learn of the confirmed benefits of science-based music protocols as described by Thaut (2014) to help persons with motor, speech, cognition, and pain management challenges. Requests for services that deliver research-based protocols would be greatly expanded, and upon delivery of these services, the societal paradigms of how music is perceived would expand. In addition, accompanying metadata would be produced to support further organizational growth of music into health care. To accomplish this transformation, new paths of dissemination and advocacy must be created to channel the research information to a general audience (Summa-Chadwick, 2020).
\end{abstract}

KEYWORDS: music therapy, silo effect, professional group collaboration, opinion paper

\section{Discussion}

Current research is acknowledged predominantly in the upper circles of neuroscience, and little information is available to medical CEOs or university chancellors to develop business plan models justifying expanding programs to include music therapy or other sciencebased music procedures. Therefore, only a few medical professionals are gradually becoming interested in implementing music in their own regional hospitals, therapy centers, and educational systems. In order to define pathways to reach the consumer directly with this knowledge, roadblocks and controversies stifling this information must be identified. Four known issues that prevent information flow are defined here.

\section{Known Issues}

Issue 1: Misinformation and old conceptions about music in the brain and music for therapy are still prevalent. When music therapy was established as a vocation midway through the last century, the profession was based on the social science known at the time. This origin led to some inaccurate results and the idea that music therapy was not a science-based profession. With the advent of imaging tools such as fMRI in the 1990s, scientists were able to see directly into the brains of persons interacting with music, and music therapy made the leap from social science to neuroscience. Unfortunately, some of the lesser reputation of music therapy persists from earlier random social science initiatives that were perceived as ineffective solutions.

Issue 2: Collection of cost-effectiveness data is inconsistent or nonexistent regarding the amount of money that hospitals and clinics can expect to pay out or recoup as a result of adding music therapy to their programs. The American Music Therapy Association, the parent organization of music therapy in the United States, maintains few studies on the subject, and those that are available are not current. Lack of such data and few advocacy efforts from this organization prevent a general knowledge flow to the consumer of the benefits of music therapy. In addition, political issues within the organization divide groups of music therapists who define their vocation as either a science or an art. Until this internal divide within the profession is resolved, it will be more difficult for the profession to thrive.

Issue 3: The university systems that drive massive research programs maintain a tenure-track system that rewards those who successfully publish their research results. This system creates a huge incentive to publish, but little to no provision is made to disseminate the outcomes upon publication to the doctors or therapists who could apply the new knowledge with their patients. Therefore, much of the research remains generally unknown to the consumer. 
Issue 4: Skilled researchers, medical personnel, and music educators are some of the groups producing research studies regarding music and the brain, but these professional factions tend to overlook collaboration outside their own working groups. This creates the "silo effect," defined by examples of doctors working only with doctors or researchers working only with other researchers. Perceived boundaries between professions must be overcome in order to work together and share the strengths of individual expertise that might not be found within another unit of experts.

\section{Proposed Solutions}

With the above challenges defined, successful pathways to carry knowledge of music and the brain directly to the consumer can now be established. The following three solutions are recommended to circumvent the issues previously specified.

Solution 1: Establish Centers of Excellence in Music Science or other similar institutions where representatives of medicine, therapy, education, and music can sit together and contribute their own expertise in presenting medical, therapeutic, and educational solutions based on music science. Such centers in key locations throughout the world would encourage collaboration between universities, communities, and medical centers to facilitate an atmosphere where the different professions can sit around the same table to discuss a problem, thus eliminating the "silo effect." The centers can house clinical work, research, performances, education, and community outreach to those in need. They will provide a professional venue that will encourage specialists from diverse vocations involved in music science to respectfully work together and contribute their own expertise in finding dynamic resolutions to therapeutic or research problems. This solution clearly addresses the need in issue \#4, and the respect garnered in the form of elevated public opinion regarding music science from such institutions will also apply to resolving issue \#1. In addition, the information output from such establishments could also be utilized to create data for business models in music therapy as a solution to issue \#2.

Solution 2: Create global models for performing arts organizations to educate their audiences about the therapeutic as well as aesthetic benefits of music. This goal can be artistically accomplished by programming literature that directs a unique twist to what an audience member learns about a composer or a style.
For example, Holm-Hadulla and Koutsoukou-Argyraki (2017) make the case that Robert Schumann is thought to have had bipolar disorder. Schumann composed with the help of his two internal personalities of Florestan (exuberant, funny, and passionate) and Eusebius (thoughtful, romantic, and reflective). By adding a pre-concert lecture or program notes discussing Schumann's story and the science of how his neural condition affected him, the audience can learn a great deal about music and the brain.

Solution 3: Harness the power of online information and social media by introducing "outside-the-box" ideas that can easily go viral in order to reach the consumer. As an example, the author is collaborating with the Erie (PA) Philharmonic in creating a unique video learning series that fits neatly into the intersection of music, science, technology, and health; these modules weave together the diverse elements of aesthetic and therapeutic musical attributes. This learning series is a free resource on their website (Neural Tango, 2021) and can be utilized as a model for all orchestras to reach their audience and communities. The webinars feature leading experts in various musical, medical, therapeutic, nonprofit, and administrative professional areas giving in-depth conversations to provide the audience a broader view of the positive aesthetic and health benefits of music. Following the discussion chapters of each unit, musicians from the Erie Philharmonic will perform a composition chosen for relevance to the discussions, in order to merge the knowledge with an experiential musical performance. Upon publication to their website, this information can be advertised on social media for an immediate potential to raise global consciousness regarding these very important topics.

\section{Conclusion}

An astounding amount of high-quality science-based research regarding music and the brain is already in place. The mission is to translate it from the upper realms of neuroscience and place it in the hands of the consumer, where awareness of such research outcomes could generate positive changes by promoting music in the medical community. Health care can truly be revolutionized by the addition of music to medical practice. Raising awareness directly with the consumer is key to making this happen, and collaboration between crucial vocational groups to work together and create powerful advocacy solutions is critical to a successful result. 


\section{Acknowledgements}

I am deeply grateful to Erie Philharmonic Executive Director Steve Weiser and the Erie Philharmonic musicians for their collaborative efforts in creating an online learning series regarding music and the brain.

\section{End Notes}

1. The definition of "consumer" for this proposal is anyone who would directly benefit from these research outcomes, including doctors, patients, therapists, and many others.

\section{References}

Holm-Hadulla RM, Koutsoukou-Argyraki A. Bipolar Disorder and/or Creative Bipolarity: Robert Schumann's Exemplary Psychopathology - Combining Symptomatological and Psychosocial Perspectives with Creativity Research. Psychopathology. 2017;50(6):379388. https://doi.org/10.1159/000479883

Neural Tango. (n.d.). Retrieved January 20, 2021, from https://eriephil.org/neuraltango

Society for Education and Music Psychology Research Forthcoming Associated. (n.d.). Retrieved January 20, 2021, from

https://www.sempre.org.uk/conferences/forthcomingassociated - conference paper in press

Summa-Chadwick, M. (2020, November). The Neural Tango: A Musical Transformation in Healthcare [Video file]. $\quad$ Retrieved from https://www.ted.com/talks/martha summa chadwick the neural tango a musical transformation in healthcare

Thaut, M. H., \& Hoemberg, V. (Eds.). (2014). Handbook of neurologic music therapy. Oxford University Press. 


\title{
Future directions for musical imagery development: Contributions from pedagogy and cognitive science
}

\author{
Sarah Gates ${ }^{\dagger}$ \\ ${ }^{1}$ Bienen School of Music, Northwestern University, Evanston, Illinois, USA \\ $\dagger$ Corresponding author: sarahgates2015@u.northwestern.edu
}

Author video presentation and/or other conference material: https://doi.org/10.17605/OSF.IO/CQ4ME

In this talk, I integrate scholarship from aural skills pedagogy and cognitive science to advance both the study and practice of musical imagery development. I propose that musical imagery as developed in North American aural skills pedagogical approaches is a form of expert memory, or long-term working memory (LTWM, Ericsson \& Kintsch, 1995; Ericsson, 2018). My analysis of the literature shows that pedagogical methods discussed by scholars in the North American tradition can be divided into four discrete categories each with their own distinct function in expertise acquisition. These include schema/chunk formation, semantic encoding, construction of retrieval cues and retrieval structures, and the gradual improvement of memory skill (or LTWM formation). I also integrate insights from auditory imagery psychometrics (Halpern, 2015) through a mapping of imagery processes (generation, manipulation) and their corresponding subjectively available properties (perceived vividness, control) onto the established LTWM framework. To conclude, I introduce my recent work operationalizing musical imagery LTWM as a form of multimodal dualcoding fluency (Paivio, 2007).

KEYWORDS: musical imagery, expertise, long-term working memory, dual-coding theory

\section{Electrophysiological Correlates of Style-Based Harmonic Expectation \\ Terrence Gatton ${ }^{1}$ \\ Bryn Hughes ${ }^{2}$ \\ Dominique T. Vuvan ${ }^{1 \dagger}$ \\ ${ }^{1}$ Skidmore College, Saratoga Springs, New York, USA \\ ${ }^{2}$ University of Lethbridge, Lethbridge, Alberta, Canada \\ † Corresponding author: dvuvan@gmail.com \\ Author video presentation and/or other conference material: https://osf.io/2qzxu/}

Previous research on the effect of musical style on harmonic expectancy indicates that listeners are differentially sensitive to certain chord progressions depending on the stylistic context in which they are presented (Vuvan \& Hughes, 2019). Despite this, there is little research on the neural basis of listener expectation as a function of musical style. The current study focused on the event related potential known as the early anterior negativity (EAN, Loui et al., 2009), which has been shown to be correlated with perception of musical syntax violations. Trials were blocked by style (Classical, Rock), and contained a four bar-long excerpt ending in either a V-I or a bVII-I cadence. We hypothesized that the bVII-I cadence would evoke an EAN in the classical context, where it is unexpected, but not the rock context, where it is expected. Preliminary data seems to confirm this hypothesis, providing evidence for the effect of musical style on the early nonconscious neural processing of harmonic syntax.

KEYWORDS: musical style, event-related potentials, early anterior negativity, harmony, expectancy, rock, classical, popular music 


\title{
How learned schema influence melodic phrase perception
}

\author{
Preeti $\mathrm{Rao}^{1 \dagger}$
}

Kaustuv Kanti Ganguli ${ }^{1}$

${ }^{1}$ Dept. of Electrical Engineering, I.I.T. Bombay, Mumbai, India

${ }^{\dagger}$ Corresponding author: prao@ee.iitb.ac.in

Author video presentation and/or other conference material: https://doi.org/10.17605/OSF.IO/SN67T

Music across genres comprises of specialized schemas at both large and small scales. In the case of Indian art music, musical phrases form categories as defined in the melodic framework of the raga. The phrases, defined by their melodic shapes or time-pitch contours, are salient cues to raga identity and the associated semantics such as affect. The semantic-cognitive association of the raga-characteristic motifs, linked to long-term memory for trained musicians, makes them akin to linguistic constructs. We present a corpus study of melodic phrase shape as a cue to raga identity to illustrate the invariance of certain aspects such as rhythmic timing and nonstandard intonation of specific notes in the presence of the overall context-dependent variability of the given phrase. A perceptual experiment paradigm is drawn from speech perception studies where the categorical perception of acoustic phenomena influence judgements of similarity. While tonal intervals and chords have been part of categorical perception studies in music, there is no similar work involving continuous melodic shape. Synthetic stimuli representing the melodic shape are used to elicit distinctly different behaviors between participants highly trained in the genre and those who are not similarly trained. This raises the potential of the presented work for applications in pedagogy.

KEYWORDS: categorical perception, raga phrase, melodic shape 


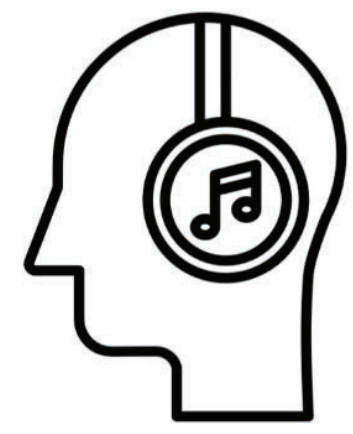

Perception

Future Directions of Music Cognition February - May 2021

Ohio State University

Virtual 


\title{
The effect of gesture on the perception of linearity in instrumental music
}

\author{
Samuel Gardner ${ }^{1 \dagger}$ \\ Vita Berezina-Blackburn ${ }^{1,2}$ \\ Daniel Shanahan ${ }^{1}$ \\ ${ }^{1}$ Ohio State University, Columbus, Ohio, USA \\ ${ }^{2}$ Advanced Computing Center for the Arts and Design, Ohio State University, Columbus, Ohio, USA \\ $\dagger$ Corresponding author: gardner.1162@,osu.edu \\ Published 16 December 2021; https://doi.org/10.18061/FDMC.2021.0033 \\ Author video presentation and/or other conference material: https://doi.org/10.17605/OSF.IO/U9WH5
}

\begin{abstract}
Given that music performances are made up of gestures, we might ask how the movements of an individual can alter how one perceives music. To address this question, this paper examines a hypothesis concerning the gestural priming of melodic events, and the role of this priming on the perceived continuation of the melody. When primed with a linear gesture, we hypothesize that participants will be more likely to select the continuation of a melodic idea, that is to say the melody keeps moving in the same direction. Conversely, when primed with circular gestures, participants will be more likely to select musical ideas that reverse and return to the starting pitch. Our results show that there was no significant effect of gesture, but there was a significant effect of musical scale when diatonic scales were used alongside the gesture. It appears that gestural priming is not a predictor of whether participants selected a musical gesture that continued or returned. These results suggest that familiarity with a musical context is perhaps more predictive of melodic expectation than gesture.
\end{abstract}

KEYWORDS: gesture, perception, motioncapture, melodic expectancy

\section{Introduction}

A musical performance comprises gestures, be they the performative gestures of playing an instrument, when a conductor leads an ensemble, when a dancer engages their entire body, or the responsive gestures one makes as they listen and perceive music. It would therefore seem that every aspect of making and engaging with music results in gestures. If gesture is important to how one makes and engages with music, can it inform one's understanding of music?

Previous research has discussed the role of crossmodal interactions between music and gesture. Schutz and Lipscomb (2007) found that, in regard to the marimba, "while longer gestures do not make longer notes, longer gestures make longer sounding notes through the integration of sensory information" (pg. 888). Previous research has also shown the importance of the visual modality when listening to music. Jane Davidson (1993) found that when a listener observes the musician performing when listening to music, that the visual perception more clearly specifies "manner" (defined as a way of doing a thing, in this case exaggerated, deadpan, and projected) than the other modalities. Similarly, Robert Gillespie (1997) finds that violin teachers, when only given the visual cue for vibrato, overcompensate on judging said vibrato, suggesting visual cues inadvertently influence their perception. The overall relationship between music and gesture is considered by Lawrence Zbikowski (2017) in his book Foundations of Musical Grammar, wherein he writes "[language and gesture are] built on an infrastructure of common conceptual ground and shared intentionality...music, puts greater emphasis on shared feelings and attitudes" (pp. 113-114). He claims that the shared "feelings and attitudes" have their basis in grounded bodily experience. It is this bodily experience that is considered in this paper.

Perhaps the most important claim of this paper is that gesture shapes thought. Most relevant to this particular study is the work of Barbra Tversky and her colleagues, particularly Tversky and Jamalian (2002) in which the authors found that gesture can alter how one perceives (non-musical) time as either linear or cyclical. In this study, participants were primed with gestures from the experimenter which were either depicting a line or a clock before being presented with a narrative. The participants were then asked to draw what they felt conveyed the narrative they were given, and in a second experiment they were asked what they felt came next. The results demonstrated that the gestural priming of a linear motion or a cyclical motion was highly predictive of the narrative selected. Similarly, Tversky's work with 
Seokmin Kang (2016) demonstrated that when gestures look like they thought that they represented, they can be even more powerful. If gestures are more effective when they resemble the linguistic goal, then this asks the question, are they also more effective when they resemble the musical goal? Susan Goldin-Meadow (2006) also writes about the importance of gestures in communication, arguing that when gesture is used on its own, it is able to function fully as communication, however, when used in tandem with language, gesture is then able to take imagistic forms, highlighting information not present in speech. This might be the case because, as David McNeill (1985) argues, gesture and speech are a part of the same "psychological structure" and that they share the same computational stage in thought (also see McNeill, 1992). Adam Kendon (1980) makes a similar argument that both gestures are "so intimately connected with the activity of speaking that we cannot say that one is dependent upon the other" (pg. 208).

Gesture is also shown to have an important role in explaining information that is difficult to conceptualize. In a study by Hostetter et al. (2007), they find that when participants are presented with a series of dots, or a series of dots and shapes, participants gesture more with the ambiguous series of dots in order to try to convey what they think the dots represent. Goldin-Meadow et al. (2009) found when learning a math lesson, nine and ten year olds who were forced to make the correct gesture with the problem learned more than those who were forced to make partially correct gestures, who in turn learned more than children who made no gestures.

Another aspect of the relationship between gesture and thought is the specific role of cognitive offloading. As Goldin-Meadow et al. (2001) are able to show, when participants are tasked with remembering a list of letters or words while forced to do another task, those participants who were able to gesture during their explanation of the task remembered more of the letters or words than those who were unable to gesture during the task. This suggests that gestures are able to allot more resources to memory than simply having to do the task. Further work continues to point to the power of gesture and cognitive load. For example, Ping and Goldin-Meadow (2010) show the role of gesture and cognitive load with objects that are not literally present, Wagner et al. (2004) shows that gesture is used significantly more in both visuospatial and verbal working memories, Gillespie et al. (2014) show that those with less working memory have higher rates of gesture, and Melinger and Kita (2007) highlight that gesture increases when the cognitive load needed for information processing is higher.

These ideas clearly place an importance on gesture and its role in communication, learning, and conceptualizing information, but gesture is also rooted in spatial thinking (for a detailed review see Alibali 2005; Hostteter and Alibali, 2008). One study by Ehrlich et al. (2006) shows that when children gestured about moving pieces when solving transformation puzzles, performed better than those who did not. Spatial orientation has recently been connected with musical motion and communication about music time. Cox (2016) for example argues that spatiality and music events/relationships are conceptually blended in the same mental domain (pg. 122-3). Godoy et al. (2016) take a similar approach and argue that it is shape similarity that is the common element in cross-modal research involving sound and space involving motion. Further, that, "for the implementation of shape cognition, we believe that body motion is necessary, and hence we locate the basis for amodal shape cognition in so-called motor theory. Motor theory is that which can encompass most (or most relevant) modalities by rendering whatever is perceived (features of sound, textures, motion, postures, scenes and so on) as actively traced shape images" (pg. 213).

\section{Method}

\section{Hypothesis}

We hypothesized that there would be a correspondence between the gestural priming condition (circular or linear gestures) and the perceived continuation of melodic tones. Put more formally:

H1a: When primed with linear gestures, participants will be more likely to select the continuation of melodic ideas (a continuously ascending scale, for example).

H1b: Conversely, when primed with circular gestures, participants will be more likely to select musical ideas that return (such as a change in direction, with a melody ascending and then descending).

\section{Participants}

113 participants (47 Male, 58 Female, 1 participant who identified as non-binary, and 7 participants who preferred not to answer; $\mathrm{M}=24.01$ years; $\mathrm{SD}=10.87$ ) completed an experiment online. Some were given course credit, whereas others volunteered. We asked participants a question "What title best describes you? 
(professional musician, semi-professional musician, serious amateur musician, amateur musician, musicloving nonmusician, or nonmusician)", taken from the Ollen Musical Sophistication Index (Ollen, 2006). Zhang and Schubert (2019) identified this as the most predictive single question of musical ability. 16 participants described themselves as amateur musicians, 30 as serious amateurs, 13 as professional musicians, 46 as semi-professional, and 8 as music-loving nonmusicians.

\section{Procedure and Design}

The participants were split into four groups, based on two factors, audio and gesture. Half of the participants were played the examples with audio from a piano, and the other half were given the examples as sine tones. Within each of those groups they were then presented with the same five audio examples accompanied by a motion-capture avatar motioning their hand with either a moving line across its body, or by circling around its body. A Picture of this avatar is seen in FIGURE 1. Participants were then asked "I play for you a piece of music. I'd like you to think about this music while watching this video. I will then play two more examples, and I would like for you to choose the one that is closest to your expectation." All groups were played the same five melodies and were asked to choose what they think came next. Two examples were the Western diatonic major scale, one was based on the whole-tone scale, one was based on the octatonic scale, and the final example was taken from the thirteen note Bohlen-Pierce scale.

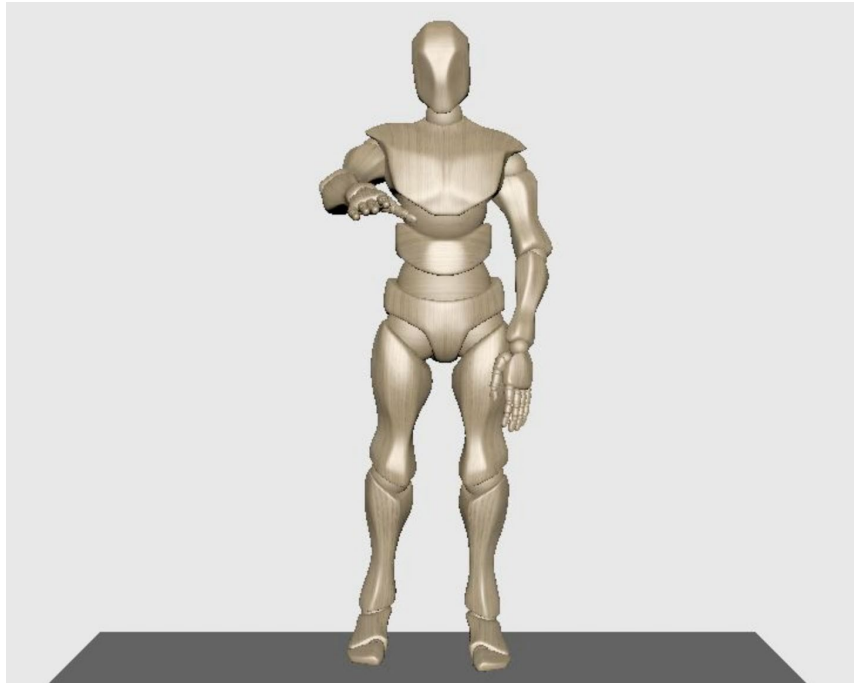

Figure 1: Motion-capture Avatar used in the experiment.

\section{Results}

Employing a multinomial logistic regression in which the participant response was predicted through both the gesture and the type of musical scale, we found no effect of gesture, but a significant effect of musical scale, specifically with the diatonic musical scale only $(\mathrm{p}=$ .006). It would appear that the gestural priming was not a predictor of whether participants selected a musical gesture that continued or returned.

Table 1: Results of a logistic regression predicting the correspondence of gesture and melodic idea.

\begin{tabular}{|l|l|l|l|l|}
\hline & $\begin{array}{l}\text { Estimat } \\
\text { e Std. }\end{array}$ & Error & $z$ Value & $\operatorname{Pr}(>|z|)$ \\
\hline Intercept & 0.4465 & 0.2347 & 1.902 & 0.05713 \\
\hline Linear & 0.2318 & 0.1826 & 1.269 & 0.20433 \\
\hline Diatonic & -0.7017 & 0.2573 & -2.728 & $\begin{array}{l}0.00638^{*} \\
*\end{array}$ \\
\hline Octatonic & -0.2811 & 0.2975 & -0.945 & 0.34476 \\
\hline $\begin{array}{l}\text { Whole } \\
\text { Tone }\end{array}$ & -.03396 & 0.2941 & -1.1155 & 0.24823 \\
\hline
\end{tabular}

\section{Discussion}

Judging from these results, it would seem that gesture does not play a significant role in the nature of melodic expectation, contrary to our hypotheses. There is a possibility that the prevalence of the major diatonic scale means that listeners are likely to infer a specific continuation, and that the priming plays no role in any scale whatsoever.

\section{Conclusion}

Future work will explore the interaction of musical events in such a way that listeners might be less accustomed to musical events, and will also explore the broader cross-cultural application of the effects of gesture on melodic expectation, as well as musical expectation more broadly.

\section{Acknowledgements}

This project could not have been completed without the support of Ohio State University's Center for Cognitive 
and Brain Sciences Summer Graduate Research Award. Support also comes from Ohio State University's Advanced Computing Center for the Arts and Design. Federico Camara-Halac assisted with the construction of the audio stimuli.

\section{References}

Alibali, M. W. (2005). Gesture in Spatial Cognition: Expressing, Communicating, and Thinking About Spatial Information. Spatial Cognition \& Computation, 5(4), 307-331. https://doi.org/10.1207/s15427633scc0504_2

Cox, Arnie. (2016). Music and Embodied Cognition. Indiana University Press. https://doi.org/10.2307/j.ctt200610s

Davidson, J. W. (1993). Visual Perception of Performance Manner in the Movements of Solo Musicians. Psychology of Music, 21(2), 103-113. https://doi.org/10.1177/030573569302100201

Ehrlich, S. B., Levine, S. C., \& Goldin-Meadow, S. (20061106). The importance of gesture in children's spatial reasoning. Developmental Psychology, 42(6), 1259. https://doi.org/10.1037/00121649.42.6.1259

Gillespie, M., James, A. N., Federmeier, K. D., \& Watson, D. G. (2014). Verbal working memory predicts co-speech gesture: Evidence from individual differences. Cognition, 132(2), 174-180. https://doi.org/10.1016/j.cognition.2014.03.012

Gillespie, R. (1997). Ratings of Violin and Viola Vibrato Performance in Audio-Only and Audiovisual Presentations. Journal of Research in Music Education, 45(2), 212-220. https://doi.org/10.2307/3345581

Godøy, R. I., Song, M., Nymoen, K., Haugen, M. R., \& Jensenius, A. R. (2016). Exploring Sound-Motion Similarity in Musical Experience. Journal of New Music Research, 45(3), 210-222. https://doi.org/10.1080/09298215.2016.1184689

Goldin-Meadow, S. (2006). Talking and Thinking With Our Hands. Current Directions in Psychological Science, 15(1), 34-39. https://doi.org/10.1111/j.0963-7214.2006.00402.x

Goldin-Meadow, S., Cook, S. W., \& Mitchell, Z. A. (2009). Gesturing Gives Children New Ideas About Math. Psychological Science, 20(3), 267-272. https://doi.org/10.1111/j.1467-9280.2009.02297.x

Goldin-Meadow, Susan, Nusbaum, Howard, Kelly, Spencer, \& Wagner, Susan. (2001). Explaining Math: Gesturing Lightens the Load. Psychological
Science, 12(6), 516-522. https://doi.org/10.1111/1467-9280.00395

Hostetter, A. B., \& Alibali, M. W. (2008). Visible embodiment: Gestures as simulated action. Psychonomic Bulletin \& Review, 15(3), 495-514. https://doi.org/10.3758/PBR.15.3.495

Hostetter, A. B., Alibali, M. W., \& Kita, S. (2007). I see it in my hands' eye: Representational gestures reflect conceptual demands. Language and Cognitive Processes, 22(3), 313-336. https://doi.org/10.1080/01690960600632812

Iverson, J. M., \& Thelen, E. (1999). Hand, mouth and brain. The dynamic emergence of speech and gesture. Journal of Consciousness Studies, 6(1112), 19-40.

Jamalian, A., \& Tversky, B. (n.d.). Gestures Alter Thinking About Time. 7.

Kang, S., \& Tversky, B. (2016). From hands to minds: Gestures promote understanding. Cognitive Research: Principles and Implications, 1(1), 4. https://doi.org/10.1186/s41235-016-0004-9

Kendon, A. (1980). Gesticulation and speech: Two aspects of the process of utterance. In The Relationship of Verbal and Nonverbal Communication (pp. 207-228). Walter de Gruyter. https://doi.org/10.1515/9783110813098.207

Kita, S. (2000). How Representational gestures help speaking. In Language and Gesture (pp. 162-185). Cambridge University Press. https://doi.org/10.1017/CBO9780511620850.011

McNeill, D. (n.d.). So You Think Gestures Are Nonverbal? 22.

McNeill, D. (1996). Hand and Mind: What Gestures Reveal about Thought (New edition). University of Chicago Press.

Melinger, A., \& Kita, S. (2007). Conceptualisation load triggers gesture production. Language and Cognitive Processes, 22(4), 473-500. https://doi.org/10.1080/01690960600696916

Ollen, J. E. (2006). A criterion-related validity test of selected indicators of musical sophistication using expert ratings

Ping, R., \& Goldin-Meadow, S. (2010). Gesturing Saves Cognitive Resources When Talking About Nonpresent Objects. Cognitive Science, 34(4), 602$619 . \quad$ https://doi.org/10.1111/j.15516709.2010.01102.x

Schutz, M., \& Lipscomb, S. (2007). Hearing Gestures, Seeing Music: Vision Influences Perceived Tone Duration. Perception, 36(6), 888-897. https://doi.org/10.1068/p5635 
Wagner, S. M., Nusbaum, H., \& Goldin-Meadow, S. (2004). Probing the mental representation of gesture: Is handwaving spatial? Journal of Memory and Language, 50(4), 395-407. https://doi.org/10.1016/j.jml.2004.01.002

Zbikowski, L. M. (2017). Foundations of Musical Grammar. Oxford University Press. https://doi.org/10.1093/oso/9780190653637.001.00 $\underline{01}$

Zhang, J. D., \& Schubert, E. (2019). A single item measure for identifying musician and nonmusician categories based on measures of musical sophistication. Music Perception: An Interdisciplinary Journal, 36(5), 457-467. https://doi.org/10.1525/mp.2019.36.5.457 


\title{
Rethinking gesture theory via embodiment and acousmatic music
}

\author{
Hubert $\mathrm{Ho}^{1 \dagger}$ \\ ${ }^{1}$ Northeastern University, Boston, MA, USA \\ $\dagger$ Corresponding author: h.ho@northeastern.edu
}

Published 16 December 2021; https://doi.org/10.18061/FDMC.2021.0034

Author video presentation and/or other conference material: https://doi.org/10.17605/OSF.IO/G3YRP

\begin{abstract}
The crucial role of embodiment in perceiving music is increasingly well documented by cognitive scientists and music theorists. Less thoroughly explored is the role that embodied cognition plays in our hearing of acousmatic music. Primarily produced on digital audio workstations, and diffused through loudspeakers, this genre of music has often been critiqued because the sources producing the sound do not neatly map onto specific sound-producing actions.

This paper proposes an analytical model utilizing gesture theory (Leman and Godøy, 2010) in framing an embodied listening approach to acousmatic music. Gesture, defined as a "pattern through which we structure our environment through actions," (Leman and Godøy, 2010), allows listeners to assign specific musical meaning to the disembodied sounds. The model focuses specifically on sound-tracing gestures that "follow the contour of sonic elements" (Godøy et al., 2006) of the acousmatic track. A multidimensional analytic approach focused on three parameters crucial to contour understanding (localization, causality, mobility) (Frengel, 2010) in acousmatic music is applied to Jonathan Harvey's Mortuos Plango, Vivos Voco. This work thus broadens the scope of an embodied approach to music cognition as it applies to music analysis.
\end{abstract}

KEYWORDS: embodied cognition, gesture, embodiment, electroacoustic music, acousmatic music

\section{Literature Review}

Researchers have noted how embodied cognition plays an increasingly important role in music perception. Perceiving music activates the action-perception network (Maes et al., 2014) in listeners. Expert pianists demonstrate greater levels of connectivity in brain regions linked to the mirror neuron system (Gallese et al., 1996) while observing other pianists (Haslinger et al., 2005). Other studies demonstrate listening subjects' ability to match body movement to specific acoustical traits (Eitan and Granot, 2006). Leveraging this evidence, music theorist Arnie Cox (2016) proposes that mimetic motor imagery (MMI) and mimetic motor action (MMA) underlie musical experience and has developed a number of frameworks for situating the action-perception network at the core of musical analytical activity.

The research on musical gesture from a variety of perspectives (music cognition, embodiment, music theory, computer music) is increasingly varied. Leman and Godøy (2010) define a musical gesture as a "pattern through which we structure our environment through action." Jensenius et al. (2010) outlines four categories of gesture; the categories "point out ... the different functions of gestures" rather than substantiate any "absolute" classification scheme: 1) Sound-producing (sub-divided into actions that excite the sound, and actions that modify sound), 2) Sound-facilitating, 3) Sound-accompanying and 4) Communicative. Gestures might function in more than one way; and gestures can overlap, blending from one to another.

Gestural conceptualization of music operates from the perspective of both the musical agent (composer, performer) and listener. Cox (2016) proposes that listeners enact a surreptitious simultaneous performance while listening to music, a phenomenon he calls "subvocal mimetic participation." This process creates "mimetic musical imagery" in listeners' mental representations as if the listener imagined what the process of performance might be. Thus, according to Cox, musical meaning is derived from the ability to link musical phenomena to action-based correlates.

Prior research in computer music has wrestled with the distinction between a gesture that a musician or composer enacts, and that of a programmer. For Cadoz (1998), "[i]n the case of musical writing, the subject intervenes to decode the signs and interpret them in function of established conventions ... the instrumental gesture is a direct causal component of the sound phenomenon."

Electroacoustic (EA) music presents an ontological problem from the perspective of the action-perception cycle, as the agents who produced the sounds in the first place (developer of synthesis programs, or artists who recorded sound samples) are not visible to the listener in 
the performance space. EA work is typically diffused through loudspeakers in indoor concert spaces in concert settings. Furthermore, spatialization and resonance are central to much EA and sound art aesthetics; composers and sound assistants consider the acoustics of performance spaces and loudspeaker placement when diffusing works of musique concrète.

\section{Method}

This initial study focuses on expanding and revising the gesture model of Leman and Godøy (2010) and Godøy et al. (2006) to account for situations highly salient to acousmatic (without live performers) music. Godøy et al. (2006) asks participants to draw out tracings of sonic gestures. This short paper will also center on issues of movement and tracing, drawing upon a multidimensional approach utilized to codify relationships between instruments and fixed media tracks (Frengel, 2010). It will also address issues of causality (sound sourcing) and propose embodied analogues for all three processes. Jonathan Harvey's Mortuos Plango, Vivos Voco will be referenced as an exemplification these processes.[1]

\section{Localization}

A CONTAINER [2] is a useful metaphor to describe how a listener might locate themselves in a musical work ("We hear the first theme in the exposition," "We are in the key of E minor."). Acousmatic musical audience members are physically situated in a space, putting themselves - as sentient, perceiving, and listening beings - inside of the space through which sound propagates. Listeners identify the sound sources and become attuned to their directionalities according to classical psychophysical principles determined by interaural time difference and interaural intensity difference. Harvey plays with localization techniques compositionally, noting that "[r]hythmic patterns of great subtlety were easy to devise, sometimes in interplay with programmed spatial movement" (Harvey, 1980) during a performance at IRCAM. Harvey also "individually distributed [partials of the bell] around the eight speakers, giving the listener the curious sensation of being inside the bell." That spatialization plays such a significant role within the confines of the performance space, does not negate the piece's ability to induce more metaphorical assertions of musical place in the piece. In fact, the co-existence of both modes of localization, the literal and the metaphoric, is grounds for further discussion.

\section{Mobility}

Auditory images can consist of sounds travelling within a concert or listening space, either as a result of a specific prerecorded mixing techniques or live diffusion techniques ("The bell sound moves from left to right."). But once again, as music itself is considered to both induce movement and represent motion ("The intervals are getting closer and closer"), the musical journey operates on both literal and metaphoric levels. This duality is demonstrated at 2:45, when the resynthesized spectra from vowels of a boy's voice move in contrary motion inwards as glissandi (Harvey, 24).

\section{Causality}

While loudspeakers serve as the units of sonic production in EA concert settings, audiences tend not to comment on their agency (or lack thereof) as musical units. They are more likely to focus on the indirect sources of the sound, either samples or synthesized materials. According to Frengel (2010), "Strong timbral associations are likely to occur whenever the non-live sounds are based on recordings." This enhances source identification of the sound, rendering the Schaefferian prescription for a "reduced listening" (that ignores such recognition) sometimes difficult. The opening of Harvey's work (0:00-0:24) presents both source sounds in highly recognizable fashion - a boy's voice that emerges from a group of bells sampled from the Winchester Cathedral, England - but eventually gives way to manipulations that render the sources unrecognizable (see example above from 2:45). Listeners, according to Cox, participate subvocal[ly] mimetic[ally], regardless of whether the sounds they hear remain untransformed or digitally processed.

At 6:34-6:54, Harvey reverts to the recognizable bell timbre and treats it as an isochronous repeating rhythmic percussive unit, supporting chorusing effects. Interestingly, this section resembles instrumental writing, as the timing of the onsets and the instrumental nature of the bell sound might be perceived as an ensemble.

\section{Conclusion}

This paper provides the basis of a revised model of embodied cognitive approaches to the analysis of acousmatic music, taking into account the compositional techniques, aesthetics, and listening practices of sonic localization, mobility, and causality. It focuses on how models of musical gesture can be refined to take into account more literal presentations of 
what is often conceived of as metaphoric. Further research will consider other dimensionalities of acousmatic music - reverberation, accretion/addition, looming-sense, timbre (beyond source recognition), sonic morphologies - and incorporate them into the model.

\section{End Notes}

[1] A detailed analysis of the piece is given in Dirks (2007)

[2] Here the convention of labelling metaphor types using small caps follows that set out by Lakoff and Johnson (1980).

\section{References}

Cadoz, C. (1988). Instrumental Composition and Musical Gesture. In Proceedings of the 1988 International Computer Music Conference. The Hague, The Netherlands, 60-73.

Cox, A. (2016). Music and Embodied Cognition. Oxford: Oxford UP. https://doi.org/10.2307/j.ctt200610s

Dirks, P. L. (2007). An Analysis of Jonathan Harvey's "Mortuos Plango, Vivos Voco." eContact! 9(2). https://econtact.ca/9_2/dirks.html

Eitan, Z., and Granot, R. Y. (2006). How music moves: musical parameters and listeners images of motion. Music Percept. 23, 221-248. https://doi.org/10.1525/mp.2006.23.3.221

Frengel, M. (2010). A Multidimensional Approach to Relationships between Live and Non-live Sound Sources in Mixed Works. Organised Sound 15(2), 96-106. https://doi.org/10.1017/S1355771810000087

Gallese, V., Fadiga, L., Fogassi, L., \& Rizzolatti, G. (1996). Action recognition in the premotor cortex. Brain 119, 593-609. https://doi.org/10.1093/brain/119.2.593

Godøy, R. Haga, E. \& Jensenius, A. (2006). Exploring music-related gestures by sound-tracing: a preliminary study. In K. Ng (Ed.), Proceedings of the COST287ConGAS $2^{\text {nd }}$ International Symposium on Gesture Interfaces for Multimedia Systems. Leeds, UK, 27-33.

Harvey, J. (1980). Mortuos Plango, Vivos Voco. On Mortuos Piango, Vivos Voco: The Essential Works Of Jonathan Harvey [MP3 file]. London, UK: Sargasso. (2013)

Harvey, J. (1981). "Mortuos Plango, Vivos Voco": A Realization at IRCAM. Computer Music Journal 5(4), 22-24. https://doi.org/10.2307/3679502

Haslinger, B., Erhard, P., Altenmüller, E., Schroeder, U., Boecker, H., \& Ceballos-Baumann, A. O. (2005). Transmodal sensorimotor networks during action observation in professional pianists. J. Cogn. Neurosci.

\section{7, 282-293. https://doi.org/10.1162/0898929053124893}

Jensenius, A. R., Wanderley, M. M., Godøy, R. I., \& Leman, M. (2010). Musical gestures: Concepts and methods in research. In R. I. Godøy \& M. Leman (Eds.), Musical gestures: Sound, movement, and meaning. New York: Routledge, 12-35.

Lakoff, G. and Johnson, M. (2003). Metaphors We Live By. Chicago: Chicago UP. (Original work published 1980). https://doi.org/10.7208/chicago/9780226470993.001.00 $\underline{01}$

Leman, M. and Godøy, R. (2010). Why study musical gestures? In R. Godøy and M. Leman (Eds.), Musical Gestures: Sound, Movement, and Meaning. New York: Routledge, 3-11.

Maes, P., Leman, M., Palmer, C., \& Wanderley, M. (2014). Action-based effects on music perception. Front. Psych. 4: 1008. https://doi.org/10.3389/fpsyg.2013.01008 


\title{
Identifying Beatles songs from their chord progressions: New evidence of the effect of specialized harmonic familiarity, melodic cues, and transposition on the identification of songs from chord progressions
}

\author{
Tuire Kuusi ${ }^{1}$ \\ Ivan Jimenez ${ }^{2 \dagger}$ \\ Matthew D. Schulkind ${ }^{3}$ \\ ${ }^{1}$ Sibelius Academy, University of the Arts Helsinki, Finland \\ ${ }^{2}$ Department of Psychology, Amherst College, Amherst, MA, USA \\ † Corresponding author: ivan.jimenez.rodriguez@uniarts.fi \\ Published 16 December 2021; https://doi.org/10.18061/FDMC.2021.0035 \\ Author video presentation and/or other conference material: https://doi.org/10.17605/OSF.IO/M4ZVJ
}

\begin{abstract}
Listener and musical factors influence the identification of songs from chord progressions. Having played and being able to write out the chords of the target song from longterm memory (hereafter, specialized harmonic familiarity) facilitate the identification of jazz standards from their chord progressions among Jazz musicians. Additionally, both musicians and non-musicians find it easier to identify popular songs and pieces of classical music from chord progressions when stimuli are played using piano tones as opposed to Shepard tones, an effect that may be at least partially driven by the melodic ambiguity that Shepard tones create. The present study investigated whether similar and additional effects can be observed under different experimental conditions. Adopting a gating paradigm, this new study tested the ability of 303 Beatles fans to identify four well-known Beatles songs from chord progressions played using piano tones. Results confirm previous findings regarding the effect of melodic cues, provide some evidence of the effect of transposition, and show that specialized harmonic familiarity has an effect on the identification of songs from chord progressions for repertoires other than jazz standards and among listeners who have very diverse musical backgrounds.
\end{abstract}

KEYWORDS: memory for harmony, popular music, Beatles, transposition, gating paradigm

\section{Introduction}

There is plenty of research showing that listeners, regardless of their musical training, can identify pieces of music from the melody. Identification is possible even if the musical material is manipulated in one way or another (for a review, see Halpern \& Bartlett, 2010). Some studies also indicate that timbre may facilitate the identification of pieces of music, especially if the excerpts are very short (Krumhansl, 2010;
Schellenberg, Iverson, \& McKinnon, 1999). There has been considerably less research on the contribution of harmony to the identification of music, which might be due to the fact that aural focus on harmony is not easy because of the extra-harmonic features affecting in perception (Cullimore, 1999; Farbood, 2012; Halpern, 1984; Mélen \& Deliège, 1995; Williams, 2005).

It has been shown that various aspects of musical training sharpen listeners' attention to harmony (Farbood, 2012; Norgaard, 2017; Sears, Caplin, \& McAdams, 2014; Williams, 2005). For example, identifying well-known pieces of classical music as well as pop and rock songs from the harmony appears to be easier for musicians than for non-musicians (Jimenez \& Kuusi, 2018; Jimenez, Kuusi, \& Doll, 2020), and having played the target piece and being able to retrieve the chord labels of the pieces from the long-term memory (referred to as "specialized harmonic familiarity") facilitate the identification of jazz standards from their chord progressions among jazz musicians (Jimenez \& Kuusi, 2020). Additionally, identifying popular songs and pieces of classical music from chord progressions has been shown to be easier when stimuli are played using piano tones as opposed to Shepard tones, an effect that may be at least partially driven by the melodic ambiguity that Shepard tones create (see, e.g., Jimenez \& Kuusi, 2018).

The present study aimed at expanding our understanding of listeners' ability to identify songs from chord progressions. Specifically, we tested whether specialized harmonic familiarity has an effect on the identification of songs from chord progressions for repertoires other than jazz standards and among listeners who have more diverse musical backgrounds than the professional jazz musicians and advanced jazz students previously tested. Therefore, we decided to use well-known Beatles songs and a large number of 
non-professional participants who were familiar enough with the target songs to name all of them from commercial recordings. We, further, tested the following musical factors: melodic cues, transposition, and the number of chords.

\section{Methods}

Altogether 303 Beatles fans (246 male, 54 female, and 3 other; mean age $=37.2, S D=14.4$ ) participated. Males were overrepresented, but we do not know of any research showing that gender has any effect on harmonic awareness. The task was to identify four well-known Beatles songs from chord progressions (up to 16 chords from the beginning of the song) played using piano block chords. The songs were chosen according to their popularity (number of Last.fm listeners), harmonic rhythm (isochronous) and chord duration (IOI between 1.95 and 2.925 seconds), harmonic uniqueness (not only I, IV and V), and chord texture (not identical to the texture of our chordal stimuli). The four Beatles that fulfilled our selection criteria were "Let It Be," "Lucy in the Sky with Diamonds," "While My Guitar Gently Weeps," and "A Day in the Life." We refer to these four songs in the rest of the text as LET, LUCY, GUIT, and DAY, respectively.

Four different versions of each chord progression were prepared: (1) melodic cues in top voice and original key, (2) melodic cues in top voice but transposed, (3) no melodic cues but original key, (4) no melodic cues and transposed. The top voice in the melodic-cues version was composed to represent the most prominent pitches of the original vocal melody. The transposed version was always a tritone down from the original key. The chord progressions were recorded with a Steinway grand piano sound using GarageBand software (Apple Inc.). The duration of the chords matched the harmonic tempo from the best well-known commercial recording of each song. Each participant heard all four songs and the conditions, but only one condition per song.

A gating paradigm was used: Chord progressions were presented multiple times, increasing the number of chords with each new presentation. We created a gating sequence with five conditions, using $2,3,4,8$, and 16 chords from the beginning of each song. The participants were encouraged to listen to the excerpts until they were sure that they had identified the song, and then provide the name of the song or words from the lyrics as a response. After finishing the gating experiment, the participants heard the songs as 15- second excerpts from commercial recordings and were asked to name them and to provide information about whether they had ever played the chords of the song in question. At the end of the experiment the participants were asked to write from memory (using chord symbols or other types of chord labels) the first six chords of the songs used in the experiment.

\section{Results}

Song identification from chords was possible even for non-professional listeners. As little as two chords was enough for song identification in some cases. LET was the most easily identified while DAY was the most difficult (Table 1).

Table 1: Number of participants who identified the songs from their chord progressions.

\begin{tabular}{lcc}
\hline $\begin{array}{l}\text { Target } \\
\text { piece }\end{array}$ & $\begin{array}{c}\text { Participants who } \\
\text { identified the song }\end{array}$ & $\begin{array}{l}\text { Participants who did not } \\
\text { identify the song }\end{array}$ \\
\hline LET & 259 & 44 \\
GUIT & 198 & 105 \\
LUCY & 109 & 194 \\
DAY & 91 & 212 \\
\hline
\end{tabular}

We were interested in the extent to which identification was influenced by the participant's specialized harmonic familiarity with the songs, that is, whether they had played the target piece and how well they had been able to write the chords of the song from memory. We identified the following three participant groups: PW (P standing for Play and $\mathrm{W}$ for Write), comprising participants who reported having played the target piece at least three times and were able to write the chords; PnW (Play, not Write), those who reported having played the target piece at least three times but were unable to write the chords; and $\mathrm{nPnW}$ (neither Play nor Write). We did not use the nPW group because there were very few cases in which the participant was able to write the chords without having played the piece.

In order to analyze the number of chords needed for identification, we used conditional testing, the conditions being 2, 3, 4, 8, and 16 chords. The conditional ID percentage (ID\%) was calculated separately for each target piece and participant group. The ID\% (shown in Figure 1) indicates the number of 
participants who identified the target piece at the condition of $\mathrm{x}$ chords as a percentage of all participants who had not yet identified the piece. As the figure indicates, having played a piece's chords and being able to write them appeared to help with the identification of the piece from its chord progression, at least to some extent.

LET Conditional ID\%

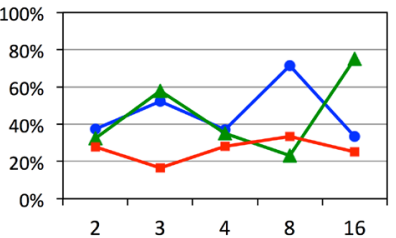

LUCY Conditional ID\%

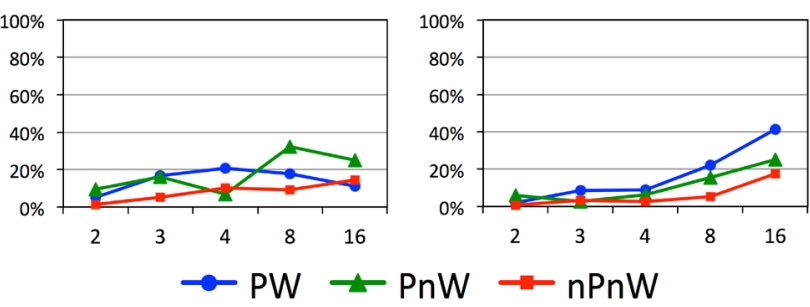

Figure 1: The conditional ID\%s for the three groups of participants, separately for each target piece. The horizontal axis shows the conditions.

An independent-samples Kruskal-Wallis test showed that there were statistically significant differences between the participant groups for LET ( $p$ $=.046)$, and marginally significant differences for GUIT ( $p=.056$ ) but not for the other two pieces. The pairwise comparisons showed that the differences were between participant groups $\mathrm{PW}$ and nPnW. The standard test statistics were as follows: PW$\mathrm{nPnW}($ LET $)=2.406, p=.048$ and $\mathrm{PW}-\mathrm{nPnW}($ GUIT $)$ $=2.404, p=.049$.

Figure 1 also shows that adding more chords did not systematically increase the ID\%. Instead, there are conditions at which identification seems to be easier than at some other points. DAY(16), for example, seems to be a point of identification in all the participant subgroups, and the identification could be attributable to the distinctive chord succession bVII(add\#11)-vi. In the case of GUIT, specialized harmonic familiarity had a general effect, but the identification points were seemingly similar for all groups of participants. As the figures show, there was a small peak at 3 chords and a higher one at 8 chords, both of which were most likely related to the occurrence of the major IV chord (D chord in A minor), which contains a raised $6^{\text {th }}$ scale-degree (F\# in A minor).

We also analyzed the effect of transposition and melodic cues on the participants' ability to identify each song. We found that the chord progressions from LET and GUIT played in the original key were identified more frequently than the transposed chord progressions. On the other hand, chord progressions from GUIT, LUCY and DAY without melodic cues were identified less frequently than progressions with melodic cues. In sum, the results show that the original key and melodic cues tended to facilitate identification, but the effect depended on the song (for details of all analyses, see Kuusi, Jimenez, \& Schulkind, 2021).

\section{Discussion and Conclusions}

We showed that it is possible for listeners other than professional musicians to identify pieces of music from very little harmonic information, even without correct melodic cues and heard in a key that is distant from the original. The number of chords needed for identification was not the same for all pieces or participants, varying from as little as two to as many as 16. The extent to which adding chords made the songs easier to identify also varied. Of the participant background variables, the specialized harmonic familiarity (the participants' ability to write the chord labels from memory and their having played the target piece) was the only one that had an effect on identification. Generally, the results are in line with our earlier findings (Jimenez \& Kuusi 2018, 2020), although there was variation between the individual songs.

Some of the identification patterns are consistent with the view that harmonic uniqueness facilitates song identification (Coker, Knapp, \& Vincent, 1997). However, harmonic uniqueness did not seem to be required or sufficient for the identification of these songs. For instance, $58 \%$ of the participants were able to identify LET from just two chords, I-V, one of the most common chord successions in tonal music. Moreover, relatively distinctive harmonic events such as the shift from emphasizing $\mathrm{C}$ major to emphasizing its relative minor in LET, the shift from the key of A minor to its parallel major in GUIT, and the modulation from A Mixolydian to Bb Lydian in LUCY did not increase identification rates compared to other 
less distinctive harmonic events in the progressions. Future research could focus on the time course of the identification of songs from the harmony, and specifically the potential role of harmonic uniqueness in that process.

In our study, transposition seemed to affect the identification of LET and GUIT, but not of the other two songs. We do not know whether our participants had been exposed to only one transposition (which could explain the result) of the songs - especially if they had played the songs themselves. Correct melodic cues tend to help identification, and this was the case in our study, except for LET. Although the versions with and without melodic cues were created following the same rules, these rules seem to have resulted in the uncued version of LET still having melodic cues, the melodically downgraded upper voice of the in-cued LET started with scale-degrees 3-2-1 and happened to be similar to the original vocal melody for the fifth (I), sixth (V), seventh (IV), and eighth chords (I) of the song.

One possible factor affecting the identification of LET could have something to do with the popularity of the song. Participants in an experiment concerning famous Beatles songs would probably expect to hear "Let It Be". If a participant was expecting to hear the words "Let it be" before hearing the first chord, and tried mentally to sing "Let it be" on top of the chords to see if the melody matched the stimuli, that top-down strategy would be affected by downgraded melodic cues to a lesser extent than a bottom-up strategy that allowed him or her to rely on the melodic features of the stimuli only in activating long-term-memory traces of the song (e.g., Schellenberg, Iverson, \& McKinnon, 1999). This strategy could have been strengthened by the timbral similarity between the original and the stimulus played on piano.

In sum, our study shows that there are various factors affecting song identification from the harmony. As correct melodic cues were found to help identification, the roles of transposition and specialized harmonic familiarity were not as clear, and our results imply that they may only have an impact on songs that are relatively easy to identify from their chord progressions. Our chordal stimuli were more similar to the original commercial recordings in LET and GUIT, the two most often identified songs in our experiment, than in LUCY and DAY in terms of timbre (piano tones) and texture (one unembellished block chord per chord change). Therefore, our findings indicate that specialized harmonic familiarity and transposition are more likely to affect the identification of songs from chords when the extra-harmonic similarity between the chordal stimuli and the original is relatively high. One possible explanation for this is that participants who are very familiar with a song and its harmony have a higher likelihood of using top-down identification strategies such as singing the melody on top of the chord or recollecting the chord labels of the song. Close transposition and extra-harmonic similarity between stimuli and the original could also facilitate the success of such top-down strategies. We must point out, however, that we only tested four songs representing a particular segment of popular music, and that further testing of these ideas would require a larger set of songs. Future research is needed to assess the effect of these and other aspects of musical and participant factors on the identification of songs from harmony.

\section{Acknowledgements}

We thank the editors of the DocMus Research Publications for kindly permitting us the possibility to publish this shortened version of the original article.

\section{References}

Coker, J, Knapp, B., \& Vincent, L. (1997). Hearin' the changes: Dealing with unknown tunes by ear. [Rottenburg:] Advance music.

Cullimore, J. R. (1999). Harmonic hierarchies as distinctive abstractions that listeners may derive from musical surface structure. MA Thesis, Queen's University at Kingston (Canada).

Farbood, M.M. (2012). A parametric, temporal model of musical tension. Music Perception: An Interdisciplinary Journal 29(4), 387-428. https://doi.org/10.1525/mp.2012.29.4.387

Halpern, A. R. (1984). Perception of structure in novel music. Memory \& Cognition 12(2), 163-70. https://doi.org/10.3758/BF03198430

Halpern, A. R., \& Bartlett, J. C. (2010). Memory for melodies. In M. R Jones. R.R. Fay, \& A. N. Popper (Eds.), Music Perception, 233-58. New York: Springer. https://doi.org/10.1007/978-1-4419-6114-3_8

Jimenez, I. \& Kuusi, T. (2018). Connecting chord Progressions with specific pieces of music. Psychology of Music 46(5), 716-33. https://doi.org/10.1177/0305735617721638

Jimenez, I. \& Kuusi, T. (2020). What helps jazz musicians name tunes from harmony? A study of the effect of general and specific Work with harmony on the ability to identify music from chord progressions. 
Psychology of Music 48 (2), 215-31. https://doi.org/10.1177/0305735618793005

Jimenez, I., Kuusi, T., \& Doll, C. (2020). Common chord progressions and feelings of remembering. Music \& Science $3, \quad 1-16$. https://doi.org/10.1177/2059204320916849

Krumhansl, C. L. (2010). Plink: "Thin slices" of music. Music Perception: An Interdisciplinary Journal 27(5), 337-54. https://doi.org/10.1525/mp.2010.27.5.337

Kuusi, T., Jimenez, I., Schulkind, M. (2021). Revisiting the effect of listener and musical factors on the identification of music from chord Progressions. In J. Ojala and L. Suurpää (Eds.) Musical Performance in Context: A Festschrift in Celebration of Doctoral Education at the Sibelius Academy. DocMus Research Publications 17. Helsinki: Sibelius Academy.

Mélen, M., \& Deliège, I. (1995). Extraction of cues or underlying harmonic structure: Which guides recognition of familiar melodies? European Journal of Cognitive Psychology 7(1), 81-106. https://doi.org/10.1080/09541449508520159

Norgaard, M. (2017). Descriptions of improvisational thinking by developing jazz improvisers. International Journal of Music Education 35(2), 259-71. https://doi.org/10.1177/0255761416659512

Schellenberg, E. G., Iverson, P., \& Mckinnon, M. C. (1999). Name that tune: Identifying popular recordings from brief excerpts. Psychonomic Bulletin \& Review 6(4), 641-46. https://doi.org/10.3758/BF03212973

Sears, D., Caplin, W. E., and McAdams, S. (2014). Perceiving the classical cadence. Music Perception: An Interdisciplinary Journal 31(5), 397-417. https://doi.org/10.1525/mp.2014.31.5.397

Williams, L. R. (2005). Effect of music training and musical complexity on focus of attention to melody or harmony. Journal of Research in Music Education 53(3), 210-21. https://doi.org/10.1177/002242940505300303 


\title{
Music surface and musical structure: The role of abstraction in musical processing
}

\author{
Mark A. Schmuckler ${ }^{1 \dagger}$ \\ ${ }^{1}$ University of Toronto Scarborough, Scarborough, ON, Canada \\ † Corresponding author: mark.schmuckler@utoronto.ca \\ Published 16 December 2021; https://doi.org/10.18061/FDMC.2021.0036 \\ Author video presentation and/or other conference material: https://doi.org/10.17605/OSF.IO/3EQ69
}

\begin{abstract}
Because of its temporal nature, music presents a unique challenge to the perceptual systems. To understand music one must infer underlying musical structure based on a musical surface that is constantly changing. Accordingly, a central component of musical behavior involves the abstraction of underlying musical structure from the musical surface. The following paper discusses the central importance of such abstraction, looking at examples of the role of abstraction based on a variety of underlying representational structures (tonal hierarchies, tonal-metric hierarchies, melodic patterns). These examples support the idea that musical understanding is fundamentally driven by the apprehension of structural patterns, and not by auditory surface information.
\end{abstract}

KEYWORDS: Abstraction, Tonality, Tonalmetric hierarchies, Melodic processing, Prototype formation

\section{Introduction}

Because of its ephemeral nature, music represents a challenge to the perceptual systems. Even with reference to other temporal arts (drama, dance, poetry) music is unique. Both drama and dance retain critical visual elements, whether they involve delineating event sequences such as in drama (time provides a framework for a series of actions) or a sequence of movements such as in dance. Even in poetry, in which visual information is minimized, the critical emphasis is on language. Its "structure does not rely solely on the sounds of the words, but rather on a poetic juxtaposition of meanings and connotations" (Stambaugh, 1964, p. 266). Thus, music is unique in its fundamental reliance on the temporal dimension for appreciation of its structure.

Nowhere is this unique nature of audition and music more obvious than in attempts to delineate what defines an auditory object (Brefczynski-Lewis \& Lewis, 2017; Kubovy \& Van Valkenburg, 2001; O'Callaghan, 2008). Because of the centrality of objects to our experience of the world, the concept of objecthood should translate across perceptual dimensions. Nevertheless, objecthood is in most ways visually-oriented (Kubovy \& Van Valkenburg, 2001; O'Callaghan, 2008).

One component of auditory object formation consists of explaining the mechanism(s) by which such objects might be formed. Most commonly researchers point to the process of auditory scene analysis (Alain \& Bernstein, 2015; Bregman, 1990, 2005; Carlyon, 2004) as a mechanism underlying auditory object formation. Although clearly a central process in organizing auditory experience, this framework is limited in equating auditory objects with auditory sources. Within a musical context, such a relation is at best incomplete, overlooking other musical structures that might be critical in understanding music.

One mechanism for creating musical objects involves the abstraction of underlying structural organizations from the musical surface. Interestingly, abstraction of underlying structure is a topic that has only been investigated sporadically over the years (Barsalou, 2005; Posner \& Keele, 1968), and within music processing (Deliège, 1996; Deutsch, 1969). According to Barsalou (2005), a critical feature of abstractions is their organization into structured representations. Such ideas lead to the intriguing realization that structured schematic representations could indeed form the basis of musical objects, with such objects consisting of abstracted, schematic musical structures drawn from the musical surface.

Critical to this idea is that abstract musical schematic representations do indeed exist, and that such representations are central to our experience of music. The goal of this paper is to address this issue, reviewing a set of investigations of musical experience in which the primary object apprehended by individuals are schematic representations abstracted out of the musical surface information.
Abstraction in Tonality
Tonality and Key-Finding. In Western music, tonality, or the organization of the chromatic set around a central reference pitch, exists as a central structural principle for musical experience. Classic work by Krumhansl and colleagues (Krumhansl \& Cuddy, 2010; Krumhansl \& Kessler, 1982; Krumhansl \& Shepard, 1979) demonstrated the psychological existence of this theoretic structure, producing the well-known "tonal hierarchy" findings shown in Figure 1. This figure graphs the perceived stability ratings of the chromatic 
set with reference to a major and minor tonal context. Subsequent work hypothesized that these ratings could be used as an idealized template for the duration of notes in a tonal context, with tonally stable notes occurring with a higher total duration than tonally unstable tones. The result of this work was the Krumhansl-Schmuckler (KS) key-finding algorithm (Krumhansl, 1990; Krumhansl \& Schmuckler, 1986; Schmuckler \& Tomovski, 2005), which has become one of the preeminent approaches for key-finding in music (Albrecht \& Shanahan, 2013; Quinn \& White, 2017; Temperley, 1999, 2001, 2008; Temperley \& Marvin, 2008).
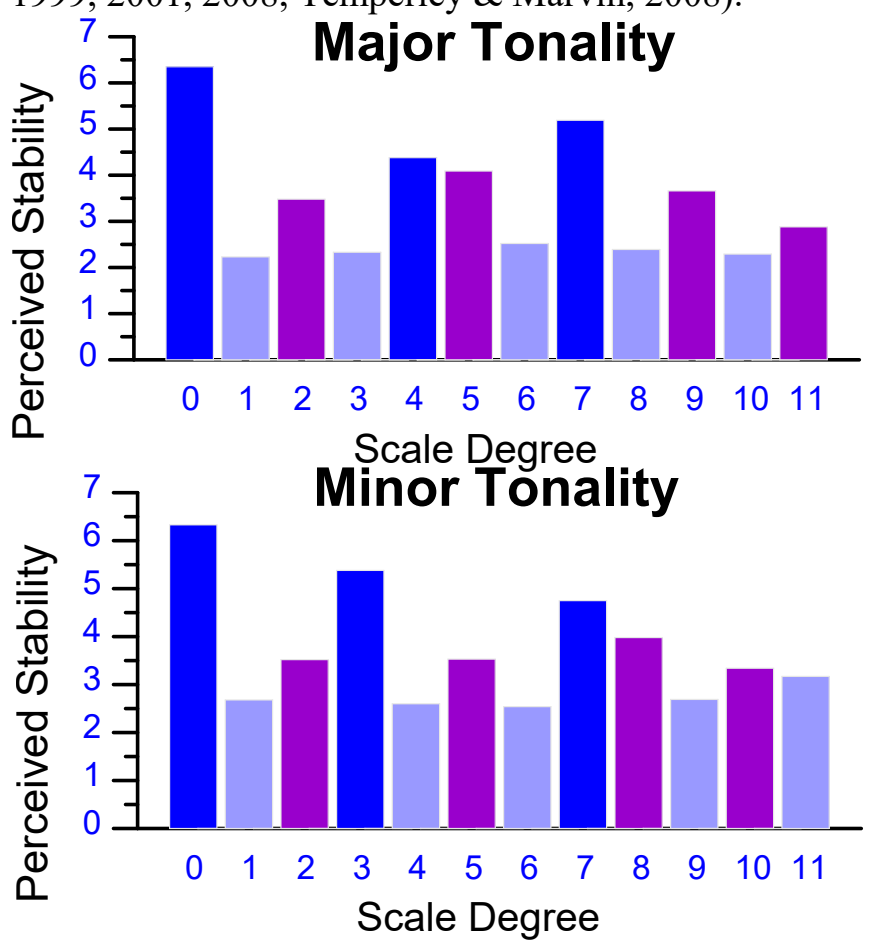

Figure 1: The major and minor tonal hierarchies, adapted from Krumhansl \& Kessler (1982). Tonic triad members appear in blue, diatonic scale degrees in purple, and non-diatonic scale degrees in lilac.

Why is the KS algorithm relevant to this discussion? Simply put, this algorithm operates by abstracting total note durations from the musical surface, collapsing across temporal ordering of tones. Although the KS algorithm has been criticized by multiple authors on a variety of points (Albrecht \& Shanahan, 2013; Quinn \& White, 2017; Temperley, 1999, 2001, 2002, 2004), this abstraction process ironically has rarely been the focus of such criticism (but see Butler, 1989 for an exception). Regardless, because the KS algorithm operates by creating a global distribution of durations, it is a prime example of the role of abstraction in music processing, one that is fundamental for perceiving tonality.
Tonal-Metric Processing. Recent years have extended this approach by exploring the co-occurrence of idealized tonal and metric information. Palmer and Krumhansl (1990) demonstrated that there also exists a hierarchy in metric perception, with some metric positions heard as psychologically strong beats within a meter, and other positions heard as weak beats.

Based on a corpus analysis, Prince and Schmuckler (2014) demonstrated the existence of an alignment between tonal and metric hierarchies, with tonally important tones occurring at metrically strong positions, and vice versa. Figure 2 shows these findings, graphing the frequency of co-occurrence of pitch and metric information as a function of time signature and musical mode. Overall, these results are compelling - tonal and metric structures aligned, with tonally strong notes occurring at metrically strong positions, and vice-versa.
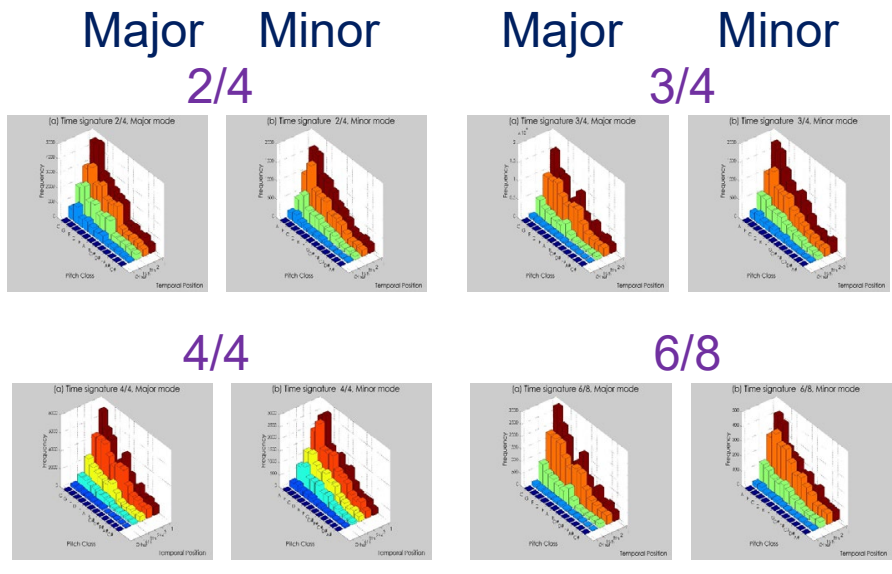

Figure 2: Co-occurrence of tonal and metric hierarchies, taken from Prince and Schmuckler (2014).

Recently, Prince et al. (2020) investigated whether this tonal-metric co-occurrence influenced listeners' percepts of musical passages. In this work, melodies were created in which the tonal-metric hierarchy was either aligned (correlated tonal-metric events) or misaligned (uncorrelated tonal-metric events); Figure 3 presents sample melodies from this study.

Aligned melody (tonal-metric $r=.61$ )

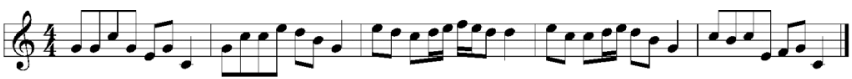

Misaligned melody (tonal-metric $r=-.65$ )

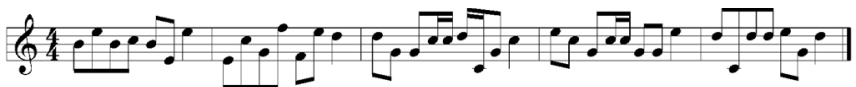

Figure 3: Sample aligned and misaligned melodies from Prince et al. (2020). 
Across a series of studies participants made melodic goodness and metric clarity ratings, and generally found that tonal-metric aligned melodies received higher ratings than misaligned melodies. As with the previous work, this research is important in demonstrating the critical influence of abstracted hierarchical information on general aspects of musical apprehension.

\section{Abstraction in Melodies}

Tonal Melodies. Abstraction also plays a significant role in melodic processing For example, Vuvan et al. (2014) examined whether the underlying tonal structure of melodies would drive false memory in listeners, causing them to "fill-in" tone information that did not occur in the melodies, but that was nevertheless consistent with the tonal structure of these melodies. Across multiple studies, listeners showed evidence of such false memories, incorrectly indicating the occurrence of tonally consistent information that was not present in the original melodies. Interestingly, this false memory effect decreased systematically with the psychological stability of the contexts, with major melodies leading to strong tonal frameworks and evidence of false memories, followed by minor melodies, and then finally atonal melodies, which produced no evidence of false memories. Even more fascinating was a subsequent reanalysis of previously unpublished pilot data from this project by Schmuckler et al. (2020). This project examined an earlier version of the atonal study that inadvertently instantiated a pair of tonal centers when one looked at the tonal implications of the entire set of atonal melodies, but that was not apparent on an individual melodic basis. Interestingly, this pilot data did produce false memories for musical information related to these tonal centers. Once this inadvertent tonal information was removed from the stimuli, however, the evidence for these false memories disappeared.

Relevant to the current framework, these studies demonstrate the importance of abstraction in at least two ways. First is the result that abstracted tonal structure of individual melodies led to listeners' false remembering. The confusion of abstract tonal information with actually occurring musical surface information represents the main thesis at hand. Second, and even more fascinatingly, is the demonstration that listeners responded to tonal implications that were only available by aggregating across a set of atonal melodies, but was not present in any individual melodies. Together, these results indicate that abstraction occurs across multiple time scales and stimulus sets, highlighting the potential of this process for influencing musical behavior.
Atonal melodic prototypes. Finally, a recently completed set of experiments examined the abstraction of melodic prototypes, based on hearing distortions of these prototypes. This research was predicated on the work of Posner and Keele (Posner et al., 1967; Posner $\&$ Keele, 1968, 1970), who explored abstraction of prototypic visual patterns by training observers to categorize distortions of different prototype patterns, and then testing generalization of this initial learning as a function of the degree of distortion present in the initial learning set. Posner and Keele found that observers who experienced more distorted learning sets showed better generalization of learning. These authors suggested that these more varied learning sets afforded better abstraction of the prototypic patterns, ultimately leading to more robust generalization.

In current work by Schmuckler et al. (in preparation), a melodic analogue was produced by creating four different random note "prototype" melodies, along with a set of distortions varying in their degree of distortion by manipulating by the size and frequency of the distorted pitch intervals in these melodies. Figure 4 shows a sample pair of prototypes, along with different levels of distortions for these melodies.

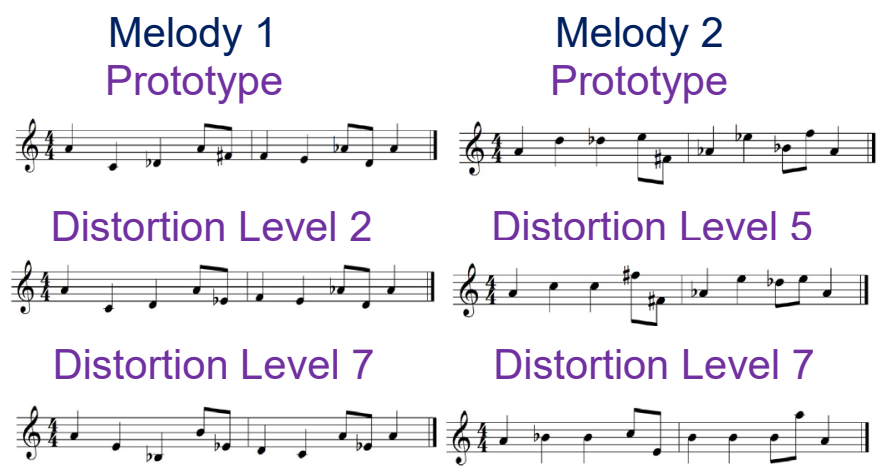

Figure 4: Sample melodic prototypes and distortion levels, for categorization and recognition memory.

Different listeners were trained to categorize varying degrees of distortions of these prototypes, and were then tested for generalization of learning using melodies with a higher distortion level than previously experienced. Figure 5 shows categorization accuracy in the initial learning and generalization phases, and reveals that the difference in performance between these phases decreased systematically with increasing distortion level of the initial learning set. Thus, the more difficult the initial learning was, the better able listeners were to generalize their learning to a subsequent set of even more distorted melodies. 


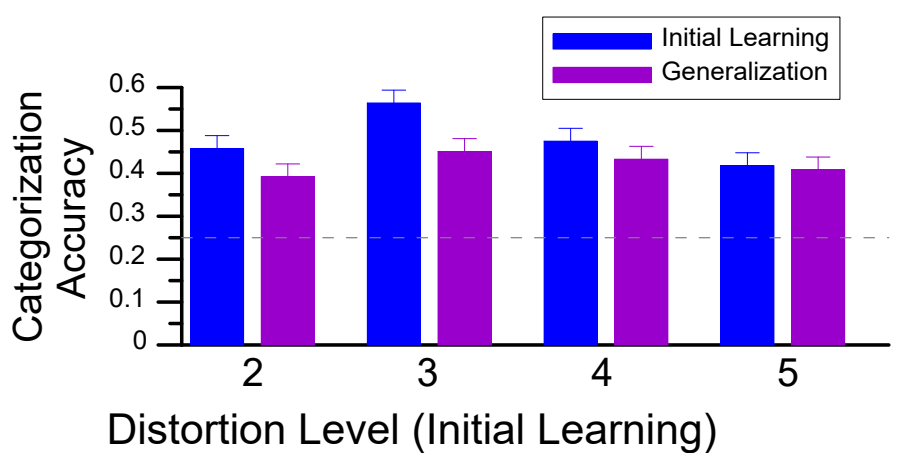

Figure 5: Categorization accuracy for initial learning and generalization trials. Initial training occurred at distortion levels of 2, 3, 4, or 5 for different listeners, with generalization employing a common distortion level of 7. Chance performance (.25) is shown.

It is important to note that although these findings suggest that listeners abstract melodic prototypes, this paradigm does not explicitly test such abstraction. This question was addressed in a subsequent recognitionmemory paradigm. Specifically, listeners received multiple blocks of study-test trials. In the study phases, listeners heard 9 melodies, consisting of 3 exemplars of a prototype at distortions levels of 3,5 , and 7 . Following the study phases, listeners were tested with 20 melodies, consisting of the original 9 study melodies, 9 new distortions ( 3 new exemplars at these same 3 distortion levels), and 2 repetitions of the original prototype, and were asked to say whether the test melody had been presented in the previous study phase. Figure 6 displays the average accuracy for the old distortions, the new distortions, and the prototypes. Although clearly a difficult task for listeners, the most critical finding was that recognition accuracy for the previously unheard prototype was significantly lower than both the old and new distortions, and was significantly less than chance performance. In other words, listeners consistently misidentified the prototype as being heard in the previous study phase. Such inaccuracies would arise through abstraction of the prototype based on the distortions, producing the mistaken belief that this melody had been previously presented. Interestingly, this finding converges with Vuvan et al. (2014), extending the demonstration of false memory from single tones to entire melodies. More generally, these studies provide another example of the importance of abstraction in musical processing, demonstrating that listeners' memory for musical surface information can be significantly distorted by abstracted representational structure of these materials.

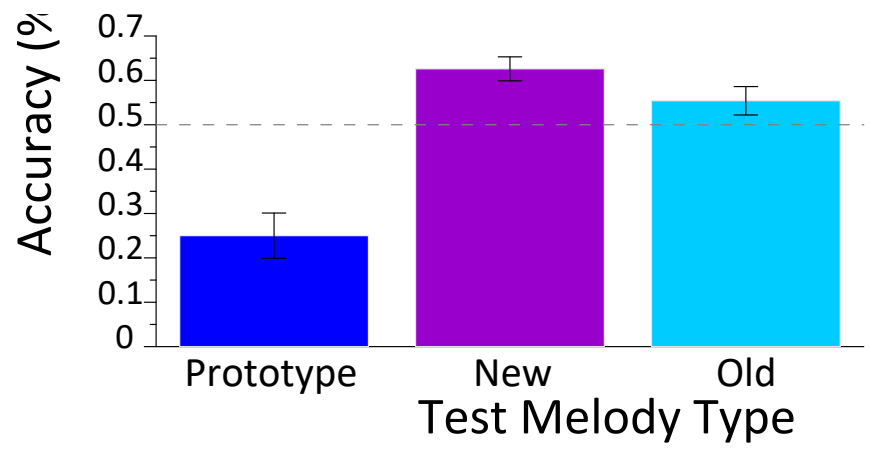

Figure 6: Recognition memory for test melodies across test melody type. Chance performance (.5) is shown.

\section{Discussion}

This paper has provided an array of evidence that abstracting schematic structures is a fundamental process driving musical apprehension. This abstraction has been observed across a range of domains, and included a range of abstracted structures such as tonality, the co-occurrence of tonal and metric hierarchies, contour processes, and so on. In this regard, it is interesting that the process of abstraction has been so neglected in the auditory and music literature, with the notable exception of the voluminous work on statistical learning (e.g., Saffran \& Kirkham, 2018).

The recognition of abstraction as a central process of musical processing leads to an array of questions for future work. For instance, what are the mechanisms involved in abstraction? Throughout this paper it has been assumed that simple exposure to musical patterns enables abstraction. This assumption, however, begs the question of the nature of such exposure. For instance, how much exposure is necessary to enable abstraction, and what types of experiences are necessary for such abstraction of patterns out of stimuli? And finally, what types of structure can be abstracted out of such stimuli? There are potentially an infinite set of organizational principles available for abstraction, yet individuals do not show equal facility in abstracting all such structures. As an example, consider 12-tone serial music, which presents a remarkably coherent theoretical structure available for abstraction by listeners. And yet, research with serial patterns has demonstrated that listeners are relatively insensitive to such structure (Krumhansl et al., 1987), suggesting that there is something in such structure that is simply not amenable to the abstraction process. Investigation of such issues, along with a host of related topics, has the potential to provide important additional insights into this fundamental process of musical abstraction. 


\section{Acknowledgements}

This research was supported by a grant from the Natural Sciences and Engineering Research Council of Canada to Mark A. Schmuckler.

\section{References}

Alain, C., \& Bernstein, L. J. (2015). Auditory scene analysis: Tales from cognitive neurosciences. Music Perception, 33, 70-82. https://doi.org/10.1525/mp.2015.33.1.70

Albrecht, J., \& Shanahan, D. (2013). The use of large corpora to train a new type of key-finding algorithm: An improved treatment of the minor mode. Music Perception, 31, 5967. https://doi.org/10.1525/mp.2013.31.1.59

Barsalou, L. W. (2005). Abstraction as a dynamic intepretation in perceptual symbol systems. In $\mathrm{L}$. Gershkoff-Stowe \& D. H. Rakison (Eds.), Building object categories (pp. 389-431). Carnegie Symposium Series. Erlbaum.

Brefczynski-Lewis, J. A., \& Lewis, J. W. (2017). Auditory object perception: A neurobiological model and prospective review. Neuropsychologia, 105, 223-242. https://doi.org/10.1016/j.neuropsychologia.2017.04.034

Bregman, A. S. (1990). Auditory scene analysis: The perceptual organization of sound. The MIT Press. https://doi.org/10.7551/mitpress/1486.001.0001

Bregman, A. S. (2005). Auditory scene analysis and the role of phenomenology in experimental psychology. Canadian Psychology, 46, 32-40. https://doi.org/10.1037/h0085822

Butler, D. (1989). Describing the perception of tonality in music: A critique of the tonal hierarchy theory and a proposal for a theory of intervallic rivalry. Music Perception, 6, 219-241. https://doi.org/10.2307/40285588

Carlyon, R. P. (2004). How the brain separates sounds. Trends in Cognitive Sciences, 8, 465-471. https://doi.org/10.1016/j.tics.2004.08.008

Deliège, I. (1996). Cue abstraction as a component of categorisation processes in music listening. Psychology of Music, 24, 131-156. https://doi.org/10.1177/0305735696242007

Deutsch, D. (1969). Music recognition. Psychological Review, 76, 300-307. https://doi.org/10.1037/h0027237

Krumhansl, C. L. (1990). Tonal hierarchies and rare intervals in music cognition. Music Perception, 7, 309-324. https://doi.org/10.2307/40285467

Krumhansl, C. L., \& Cuddy, L. L. (2010). A theory of tonal hierarchies in music. In M. R. Jones, R. R. Fay, \& A. N. Popper (Eds.), Music Perception (pp. 51-87). Springer. https://doi.org/10.1007/978-1-4419-6114-3 3

Krumhansl, C. L., \& Kessler, E. J. (1982). Tracing the dynamic changes in perceived tonal organization in a spatial representation of musical keys. Psychological Review, 89, 334-368. https://doi.org/10.1037/0033-295X.89.4.334

Krumhansl, C. L., Sandell, G. J., \& Sargeant, D. C. (1987).
The perception of tone hierarchies and mirror forms in twelve-tone serial music. Music Perception, 5, 31-78. https://doi.org/10.2307/40285385

Krumhansl, C. L., \& Schmuckler, M. A. (1986). Key-finding in music: An algorithm based on pattern matching to tonal hierarchies 19th annual Mathematical Psychology Meeting, Cambridge, MA.

Krumhansl, C. L., \& Shepard, R. N. (1979). Quantification of the hierarchy of tonal functions within a diatonic context. Journal of Experimental Psychology: Human Perception and Performance, 5, 579-594. https://doi.org/10.1037/0096-1523.5.4.579

Kubovy, M., \& Van Valkenburg, D. (2001). Auditory and visual objects. Cognition, 80, 97-126. https://doi.org/10.1016/S0010-0277(00)00155-4

O'Callaghan, C. (2008). Object perception: Vision and auditory. Philosophy Compass, 3/4, 809-829. https://doi.org/10.1111/j.1747-9991.2008.00145.x

Palmer, C., \& Krumhansl, C. L. (1990). Mental representations for musical meter. Journal of Experimental Psychology: Human Perception and Performance, $\quad 16, \quad 728-741$. https://doi.org/10.1037/0096-1523.16.4.728

Posner, M. I., Goldsmith, R., \& Welton, K. E. J. (1967). Perceived distance and the classification of distorted patterns. Journal of Experimental Psychology, 73, 28-38. https://doi.org/10.1037/h0024135

Posner, M. I., \& Keele, S. W. (1968). On the genesis of abstract ideas. Journal of Experimental Psychology, 77, 353-363. https://doi.org/10.1037/h0025953

Posner, M. I., \& Keele, S. W. (1970). Retention of abstract ideas. Journal of Experimental Psychology, 83, 304-308. https://doi.org/10.1037/h0028558

Prince, J. B., \& Schmuckler, M. A. (2014). The tonal-metric hierarchy: A corpus analysis. Music Perception, 31, 254270. https://doi.org/10.1525/mp.2014.31.3.254

Quinn, I., \& White, C. (2017). Corpus-derived key profiles are not transpositionally equivalent. Music Perception, 34, 531-540. https://doi.org/10.1525/mp.2017.34.5.531

Saffran, J. R., \& Kirkham, N. Z. (2018). Infant statistical learning. Annual Review of Psychology, 69, 181-203. https://doi.org/10.1146/annurev-psych-122216-011805

Schmuckler, M. A., Johanis, T. C., \& Lutian, J. (in preparation). Abstraction of melodic prototypes.

Schmuckler, M. A., \& Tomovski, R. (2005). Perceptual tests of an algorithm for musical key-finding. Journal of Experimental Psychology: Human Perception and Performance, 31, 1124-1149. https://doi.org/10.1037/0096-1523.31.5.1124

Schmuckler, M. A., Vuvan, D. T., \& Lewandowski, O. P. (2020). Aggregate context effects in music processing. Attention, Perception, \& Psychophysics, 82, 2215-2229. https://doi.org/10.3758/s13414-020-02003-4

Temperley, D. (1999). What's key for key? The KrumhanslSchmuckler key-finding algorithm reconsidered. Music Perception, 17, 65-100. https://doi.org/10.2307/40285812 
Temperley, D. (2001). The cognition of basic musical structures. The MIT Press.

Temperley, D. (2002). A Bayesian approach to key-finding. In C. Anagnostopoulou, M. Ferrand, \& A. Smaill (Eds.), Music and artificial intelligence (pp. 195-206). Springer. https://doi.org/10.1007/3-540-45722-4_18

Temperley, D. (2004). Bayesian models of musical structure and cognition. Musicae Scientiae, 8, 1061-1069. https://doi.org/10.1177/102986490400800204

Temperley, D. (2008). A probabilities model of melody perception. Cognitive Science, 32, 418-444. https://doi.org/10.1080/03640210701864089

Temperley, D., \& Marvin, E. W. (2008). Pitch class distribution and the identification of key. Music Perception, 25, 192-212. https://doi.org/10.1525/mp.2008.25.3.193

Vuvan, D. T., Podolak, O. M., \& Schmuckler, M. A. (2014). Memory for musical tones: The impact of tonality and the creation of false memories. Frontiers in Psychology: Auditory Cognitive Neuroscience, 5, 1-18. https://doi.org/10.3389/fpsyg.2014.00582 


\author{
Post-tonal harmonic tension and theoretical hybridity \\ Yvonne $\mathrm{Teo}^{1 \dagger}$ \\ ${ }^{1}$ Durham University, England \\ ${ }^{\dagger}$ Corresponding author: yvonne.teo@durham.ac.uk \\ Published 16 December 2021; https://doi.org/10.18061/FDMC.2021.0037
}

\begin{abstract}
Despite the large body of research that has examined tonal and atonal harmonies to our perception of tension, there is no work that describes or explores the perception of post-tonal chords, but more specifically, chords that contain both tonal and post-tonal features.

As there is no theoretical model that fully accounts for neoclassical practices, some have responded to this problem is by extending the individual methods' application Schenkerian, neo-Riemannian, and pitch class set theories or by simultaneously using more than one analytical approach, both of which invokes theoretical hybridity.

This paper applies the concept of calculating the total amount of voice-leading movement, to examine its relationship to our perception of tension and release. To do this, three neoclassical pieces by Hindemith, Ravel, and Stravinsky are selected to analyze the relationship between theoretical and perceived tension. The findings suggest that in addition to calculating the horizontal motion between harmonies, physical and acoustical factors play a critical role in relating theoretical to perceived tension. This approach is adaptable to other neoclassical works and in addition, this study could have implications in other musical fields such as performance practices and analyzing formal functions in posttonal repertoire.
\end{abstract}

KEYWORDS: neoclassical, tension, perception, music theory, music analysis

\section{Introduction}

Understanding how one perceives musical tension can vary significantly depending on the readers' lens, a music-theoretical or psychological perspective. On the one hand, Bigand, Parncutt and Lerdahl state that musical tension from a purely theoretical perspective can be generally explained by a few variables, such as the function of chords within a tonal context (Riemann, 1893; Schenker, 1979), acoustical or sensory consonance (Rameau, 1971) and its melodic organization (Bigand, Parncutt and Lerdahl, 1996). On the other hand, a psychological perspective of musical tension investigates other factors such as cognitive approaches that justify the importance and role of tonal contexts (how chords have specific functions in tonal contexts) (Bharucha, 1984; Bigand, 1990; Krumhansl, 1990; Lerdahl, 1988; Lerdahl, 2001), and theories in musical perception that reveal the chords' psychoacoustical features (Parncutt, 1989; Roberts \& Shaw; 1984). This psychological approach can also be extended to the study perceptual aspects of auditory perception and its impact in music perception such as dynamics and timbral elements such as pitch register, roughness, brightness and density (Burnsed \& Sochinski, 1988-2001; Granot \& Eitan, 2011; Hutchinson \& Knopoff, 1978; Ilie and Thompson, 2006; Krumhansl, 1996; Misenhelter, 2001; Pressnitzer, McAdams, Winsberg, \& Fineberg, 2000; Plomp \& Levelt, 1965).

One common trait between these perspectives is the emphasis of establishing a stable tonal center to derive sentiments of musical tension (Lerdahl and Jackendoff, 1983; Bigand, 1993; Bigand, Parncutt, \& Lerdahl, 1996). These studies and observations have undoubtedly stemmed from the analysis of tonal repertoire and although some work has been extended to atonal repertoire (Dibben, 1994; Lerdahl, 1989; Krumhansl, Sandel and Sargeant 1987; Dibben 1999), understanding musical tension in repertoire that embodies both tonal and atonal elements, such as neoclassical repertoire, is an area of research that remains to be explored.

Despite the large body of research that has delved the various different aspects of music that attribute to our perception of tension, there is no work that describes or explores the perception of post-tonal chords, but more specifically, how these chords will be perceived without reference to a tonal center. The appraised literature therefore points out two key points: one, a lack of research into understanding and measuring post-tonal tension, a series of chords that encompass atonal and tonal elements and two, the importance of using psychoacoustical factors such as roughness to support empirical analyses into chordal tension. This study therefore seeks to explore a different way to measure musical tension in post-tonal repertoire, by taking the approach of calculating the total amount of voice- 
leading movement between chords - and extending Lerdahl's model of calculating tonal tension (Lerdahl \& Krumhansl, 2007; Lerdahl, 1988; Lerdahl, 1996). The aim of the experiment is to establish if the values from the model approach have a direct relationship to how we perceive tension and release specifically in post-tonal repertoire.

\section{Method}

The design of this experiment derives from one component from a "hybrid" theoretical model, which encompasses elements from three different theories Schenkerian method, neo-Riemannian theory and pitchclass set theory (Baker, 1980 \& 1990; Hicken, 1974; Lewis, 1981, Morrison, 1991; Baker, 1983; Cinnamon, 1993; Pople, 1989; Travis 1959; Wilson, 1984). This model was created to address the harmonic issues posed by post-tonal repertoire, more specifically neoclassical works, as these works contains vestiges of tonality as well as post-tonal elements. Rather than applying the conventional Roman numeral labelling, calculating the total amount of minimal movement between each voice in the chords or more loosely, pitch collections (PCns), will provide a different and concise representation of the harmonies. This will be known as the Aggregated Voice-Leading (AVL) movement. (The AVL differs to voice-leading movement as AVL is the sum of all the voice-leading movement whilst voice-leading movement denotes the individual movements.) This aspect of the model suggests that there are potentially correlations between tension and release to the numerical fluctuation within the harmonic contents. In order to test this empirically, a number of extracts taken from the model's application - are tested for listeners to rate the amount of tension through the use of a tension rating scale, from 1 to 5 .

In addition, several commonly used descriptors of music associated with measuring tension were extracted to provide an alternative or broader account of the tension within the music. Tonal stability, roughness, and several variables denoting other characteristics of the pitch collections such as mean pitch, median range, and the number of notes in a PCn. Tonal stability and roughness were extracted from the last pitch collection of the clip using MIR Toolbox (Lartillot \& Toiviainen, 2007). The other calculations of the other properties of the pitch collections was carried out with MIDI toolbox (Eerola \& Toiviainen, 2004).

\section{Results}

The main results from the study are summarized as follows, with regard the relationship between MTR and AVL values. Firstly, there are instances where the model calculations (AVL) would depict a higher amount of tension whilst the perceived tension (MTR) is much lower. This simply demonstrates that VL calculations and assessing the PCns is not sufficient in determining real-time tension and release in music. Secondly, the arrangement of the PCns could also play a factor in participants' ratings. Additionally, the number of notes within the PCn may have had an effect on how listeners perceived tension. A movement from a PCn with more notes to less notes may result in a lower MTR but the AVL might project the opposite result. Thirdly, there may also be a correlation between listeners' preconceived notions of consonance and dissonance to release and tension. This could suggest the importance of the PCn's contents rather than its movement, as its unique quality may hold the key in influencing listeners' perception rating. For instance, there are cases of PCns that contain no voice-leading changes yet they are represented with a higher MTR.

\section{Discussion}

On the whole, this study has shown that listeners' perception of tension is dependent on a few factors, ones that go beyond the mere use of AVL calculations. External factors include register (e.g. shift from higher to lower registers may result with a small MTR), number of notes (e.g. shift from a larger to smaller number of pitches in a $\mathrm{PCn}$ ) and its pitch organization (e.g. root position triads tend to hold less tension whilst its inversions tend to be more tense).

Other variables that may better account for posttonal tension can include chord size, range of the PCns, mean pitch and applying Krumhansl and Kessler's (1982) key profiling system. A comparative analysis between these variables (as well as MTR, AVL and roughness) illustrating the means, standard deviations and correlations with confidence intervals demonstrates this. One can observe the following points: firstly, that the roughness has a high correlation with the size of chords, thereby suggesting that the varying number of notes has an impact on listeners' perception; secondly, that there is a strong correlation between roughness and chord size to mean tension ratings, which then reinforces the importance of physical factors in perception of post-tonal harmonies; thirdly, other physical variables such as the registral range and the 
tonal stability of the chords, also have a strong correlation to tension ratings as well.

Perhaps post-tonal features could be described from analyzing other physical factors such as roughness, chord size and range. Unfortunately, based on the analysis of these additional variables, there is a stronger correlation between these physical factors to perception than simply using the AVL and roughness.

One step that can be taken further with these additional variables is to perform a regression analysis. The regression analysis suggests that an adequate model can be created to account about $72 \%$ of the variance in the tension ratings. This analysis suggests that the two factors that are most crucial in accounting for the tension ratings are the chord size and tonal stability. The signs of the beta coefficients suggest that tension is negatively linked to tonal stability whereas higher chord size, roughness, mean pitch seem to contribute to higher tension ratings. Despite the overall success of the regression model with these variables for this material, it is important to realize that the model would require more data in order to form generalized statements, for different contexts. Although the AVL loses out in the process, what this ultimately demonstrates is that unlike tonal tension, other physical and psychoacoustical factors must be analyzed and accounted for to understand the treatment of post-tonal tension, to create a better measurement.

\section{Conclusion}

This study examined listener judgements of musical tension for selected segments from three selected neoclassical works: the first movement from Hindemith's Second Piano Sonata, Mouvement de Menuet from Ravel's Sonatine, and the first movement from Stravinsky's Concerto for Piano and Wind Instruments. The focal point of this study was to investigate an unexplored aspect of tension: the perception of harmonies in music that embodies both tonal and post-tonal features, how listeners would perceive tension without reference to a tonal center. The methodology employed is novel, drawing upon the AVL, an element from a hybrid theoretical model (comprising elements from Schenkerian, neoRiemannian and pitch-class set theories), by calculating the individual VL movement between each segmented vertical sonority, PCn. This therefore provided a renewed perspective on how tension is reflected in listeners' musical perceptions from this repertoire.

As this study focuses purely on one parameter harmony - in an attempt to measure how we perceive post-tonal tension, future studies can be explored and expanded to several directions. It is clear from established studies that other parameters such as rhythmic and metric influences need to be accounted for when analyzing our perception from tension. One possibility could be through Volk's (2008a, 2008b) computational model, "Inner Metric Analysis," and combining the model with the voice-leading calculational approach set out in this study. The method of this study can be adapted in future studies to utilize continuous measurements instead of discrete measurements. As discussed by Britten and Duke (1997), continuous measurements may be a more beneficial measurement tool to better understand participants' changing perceptions during the listening experience.

As only one instrumental timbre was used for this study, future studies can extend to the use of other instrumentation to explore how perception responses changes depending on the timbre, exploring how this can have an impact on perceived emotions, and how a spectral analysis can further enhance or reinforce the findings. The stimuli set can also be expanded to include other works by different composers, but of the same time period, to explore the relationship between these composers' harmonic idiom to listeners' perception. Another possibility is to extend the AVL method, comparing the perception of the PCns to its original stimuli, from the music, bringing in the examination of other parameters. As this study focused on harmony to measure tension, this can be extended to include other parameters to further support this notion. BaileyShea and Monahan (2018) for instance consider a new method for describing musical energetics (relating to musical tension as perceptions of musical movement or force) that is not dependent on tonality.

Correlating tension ratings to physical and psychoacoustical features is perhaps the next step one should take in theorizing post-tonal harmonic tension, as accounting for the AVL simply does not suffice in such repertoire. In creating an abstract model, there will be potential implications in music analysis as this could ultimately be a tool to assist in explaining formal functions such as closure in post-tonal music. Although additional follow-up experiments would undoubtedly reinforce the findings, the analysis and observations from this study demonstrate a new perspective on perceiving tension that neither belong to the tonal nor post-tonal realm. The study has shed light on the complexity of these harmonies and the various external factors that affect listeners' perception. Further 
examination into post-tonal tension perception will in the bigger picture assist in our understanding of the interrelationships between performance practices, refining theoretical and perceived interpretations and musical structures.

\section{Acknowledgements}

The author wishes to thank Tuomas Eerola for his invaluable assistance throughout the entire study and his insightful feedback.

\section{References}

Baker, J.M. (1980). Scriabin’s Implicit Tonality. Music Theory Spectrum, 2, 1-18. https://doi.org/10.2307/746177

Baker, J.M. (1983). Schenkerian Analysis and Post-Tonal Music. In D. Beach (Ed.), Aspects of Schenkerian Theory (pp. 153-186). New Haven: Yale University Press.

Baker, J.M. (1990). Voice-Leading in Post-Tonal Music: Suggestions for Extending Schenker's Theory. Music Analysis, 9(2), 177-200. https://doi.org/10.2307/854227

Bigand, E. (1993). The influence of implicit harmony, rhythm and musical training on the abstraction of "tension-relaxation schémas" in tonal musical phrases. Contemporary Music Review, 9, 123-137. https://doi.org/10.1080/07494469300640391

Bigand, E., Parncutt, R., \& Lerdahl, F. (1996). Perception of musical tension in short chord sequences: The influence of harmonic function, sensory dissonance, horizontal motion, and musical training. Perception and Psychophysics, 58(1), 125-141. https://doi.org/10.3758/BF03205482

Cinnamon, H. (1993). Tonal Elements and Unfolding Nontriadic Harmonies in the Second of Schoenberg's "Drei Klavierstücke. Theory and Practice, 18, 127-170.

Dibben, N. (1999). The perception of structural stability in atonal music: The influence of salience, stability, horizontal motion, pitch commonality, and dissonance. Music Perception, 16(3), 265-294. https://doi.org/10.2307/40285794

Hicken, K. (1974). Schoenberg's 'Atonality': Fused Bitonality? Tempo, 109, 27-36. https://doi.org/10.1017/S0040298200055522

Krumhansl, C., Sandel G., \& Sargeant, D. (1987). The perception of tone hierarchies and mirror forms in twelve-tone serial music. Psychological Review, 89, 3178. https://doi.org/10.2307/40285385
Lerdahl F., \& Jackendoff, R. (1983). A generative theory of tonal music. Cambridge: MIT Press.

Lerdahl, F. (1989). Atonal prolongational structure. Contemporary Music Review, 4, 64-87. https://doi.org/10.1080/07494468900640211

Lewis, C. (1981). Tonal Focus in Atonal Music: Berg's op. 5/3. Music Theory Spectrum, 3, 84-97. https://doi.org/10.2307/746136

Morrison, C.D. Prolongation in the Final Movement of Bartók's String Quartet No. 4. Music Theory Spectrum, 13(2),179-96. https://doi.org/10.1525/mts.1991.13.2.02a00030

Pople, A. (1989). Skryabin and Stravinsky 1908-1914: Studies in Theory and Analysis. New York: Garland Publishing

Salzer, F. (1962). Structural Hearing. New York: Dover. 


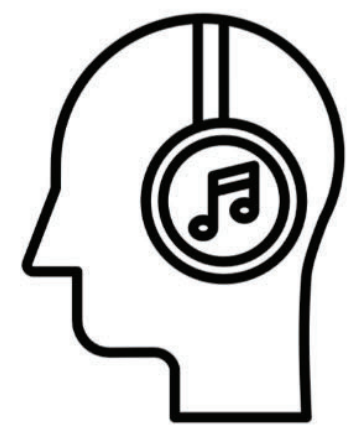

Rhythm \& Meter

Future Directions of Music Cognition February - May 2021

Ohio State University Virtual 


\title{
Pitch cues to hierarchical metric structure in children's poetry
}

\author{
Mara Breen ${ }^{1 \dagger}$ \\ Ahren Fitzroy ${ }^{1,2}$ \\ ${ }^{1}$ Mount Holyoke College, South Hadley, MA, USA \\ ${ }^{2}$ University of Massachusetts Amherst, Amherst, MA, USA \\ $\dagger$ Corresponding author: mbreen@mtholyoke.edu \\ Published 16 December 2021; https://doi.org/10.18061/FDMC.2021.0038 \\ Author video presentation and/or other conference material: https://doi.org/10.17605/OSF.IO/HZVJU
}

\begin{abstract}
We investigated whether speakers use pitch to signal hierarchical metric structure in productions of Dr. Seuss's The Cat in the Hat, by modeling fundamental frequency (F0) of monosyllabic words as a function of metric strength and a set of control parameters. We modeled maximum F0 of $\sim 25000$ words in a corpus of book productions from 17 speakers, comparing a 3-level musical metric model and a 5-level linguistic metric model. Results demonstrate that speakers consistently realized two levels of musical metric strength, as words corresponding with downbeats were produced with higher maximum F0 than all other beats. In addition, speakers simultaneously realized three levels of linguistic metric strength, as maximum F0 decreased linearly across the three highest linguistic metric levels. These results are consistent with previous work in both prose speech production and Western music composition, demonstrating that poetic speech uses pitch variation in ways that are consistent with both music and speech, and they complement prior demonstrations that duration and intensity variation signal musical and linguistic metric structure in the same corpus.
\end{abstract}

KEYWORDS: speech, pitch, meter, rhyme, intonation

\section{Introduction}

Children's poetry shares many structural features with music (Lerdahl, 2001), but there has been little empirical investigation of how these features are realized in production. This project assesses how speakers use pitch variation to signal both musical and linguistic metric structure in children's poetry by analyzing productions of The Cat in the Hat (Dr. Seuss, 1957), a quintessential example of children's poetry with regular hierarchical metric structure.

Prior work demonstrates that music performers signal hierarchical metric structure with duration (Palmer, 1996; Todd, 1985) and intensity variation (Drake \& Palmer, 1993). Our previous work demonstrates that, in productions of The Cat in the Hat, speakers systematically signal hierarchical musical metric structure with intensity variation (Fitzroy \& Breen, 2020) and hierarchical linguistic metric structure with duration variation (Breen, 2018). The goal of the current study is to investigate how a third acoustic variable - pitch - is manipulated by speakers to signal hierarchical musical and linguistic metric structure in productions of The Cat in the Hat.

There is considerable evidence from psycholinguistics that speakers signal metric structure with pitch variation. Pitch accents, which are aligned with metrically strong syllables, are generally signaled by a local increase in pitch (Breen et al., 2010). Music cognition makes similar claims about how metric structure is realized in Western music; the Generative Theory of Tonal Music predicts that metrically strong positions are more likely to correspond with pitch excursions (Lerdahl \& Jackendoff, 1983). Moreover, analyses of Western music reveal that pitch accents frequently occur at metrically strong positions (Huron \& Royal, 1996). In addition, musical phrase boundaries, which typically coincide with metric unit boundaries (Temperley, 2003), are often signaled with falling pitch (Huron, 2006), and, in Western music, "late phrase compression" in which the pitch interval size tends to decline toward the end of a phrase (Shanahan \& Huron, 2011).

The current paper investigates how speakers use pitch variation to signal both musical and linguistic hierarchical metric structure in productions of childdirected poetic speech. To do this, we modeled the pitch of each word using two hierarchical models of metric structure (Figure 1): a 3-level musical metric model based on a $6 / 8$ measure structure, and a 5-level linguistic metric model based on cross-linguistic metrical poetry (e.g., Fabb \& Halle, 2008). Based on prior work in music and speech production, we predict that speakers will signal metrically strong syllables with higher pitch than metrically weak syllables, and that speakers will signal ends of metric units with decreases in pitch.

\section{Method}

\section{Participants}

In the current study, we analyzed productions from the The Cat in the Hat corpus (Breen, 2018) from 17 female native speakers of American English. 


\section{Stimuli}

Participants read aloud from a hardcover copy of The Cat in the Hat (Dr. Seuss, 1957) - a 61-page, illustrated children's book written in rhyming anapestic tetrameter, which is widely read by English-speaking caregivers to 0-3 year old children (Hudson Kam \& Matthewson, 2017). The book consists of 1625 words (1576 monosyllabic words, 236 unique lexemes) organized primarily into 70 stanzas; each stanza contains two lines of four anapests each (as in (1)). The first line in each stanza ends with a rhyme prime and the second line ends with a phonologically predictable rhyme target.

(1)

$$
\begin{aligned}
& \text { "Put me down!" said the fish. } \\
& \text { "This is no fun at all! } \\
& \text { Put me down!" said the fish. } \\
& \text { "I do NOT wish to fall!" }
\end{aligned}
$$

\section{Acoustic Measures}

Word and silence boundaries (Figure 2) were identified by automatic force-alignment of the audio productions with the text in Praat (Boersma \& Weenink, 2018) using the Prosodylab-Aligner (Gorman et al., 2011), then manually adjusted as needed. F0 values were identified using Praat's auto-correlation algorithm. The maximum F0 value of each word was defined as the parabolically interpolated maximum pitch (Figure 2). Multisyllabic words were excluded from analysis, because the unstressed syllables have reduced pitch for reasons unrelated to metric structure (Fry, 1958). Limiting investigation to one-syllable words resulted in exclusion of 16 of 236 unique lexemes (49/1625 words). Disfluent and incorrect word productions were also excluded, resulting in 473 out of 26,792 possible monosyllabic word productions $(1.77 \%)$. The remaining maximum F0 values of monosyllabic words were centered and scaled to standard deviation units (i.e., converted to z-scores) separately within each participant.

\section{Text Annotation}

All words were first annotated for a set of control factors: a) number of phonemes $(\mathrm{M}=2.98, \mathrm{SD}=0.8)$, b) word class (542 open-class, 1034 closed-class), c) $\log$ lexical frequency $(\mathrm{M}=5.82, \mathrm{SD}=2.15)$, d) syntactic dependency structure, e) text emphasis (26 words in SMALL CAPS), and f) intra-stanza repetition. Next, metric structure was annotated in two ways (Figure 1): with a 3-level musical metric structure based on $6 / 8$ musical meter, where performers signal greatest prominence on beat 1 , intermediate prominence on beat 4 , and lowest prominence on beats 2, 3, 5, and 6 (Drake
\& Palmer, 1993); and a linguistic metric structure where metric feet are iteratively grouped in pairs, creating a 5level hierarchical structure (Fabb \& Halle, 2008).

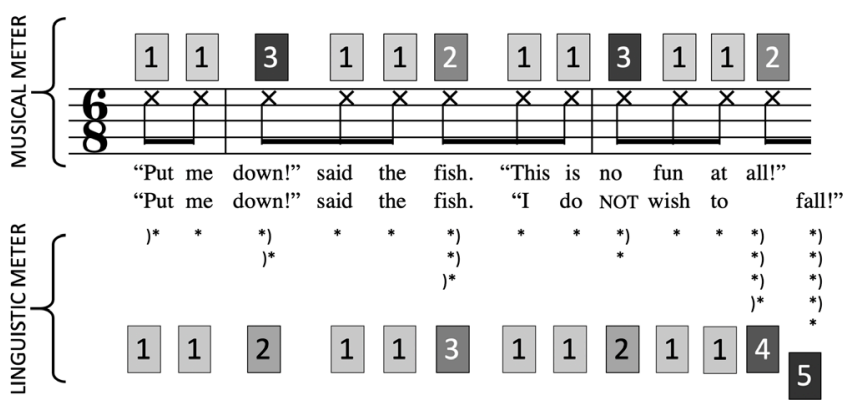

Figure 1: One stanza of The Cat in the Hat annotated with the musical metric model (top) and the linguistic metric model (bottom).

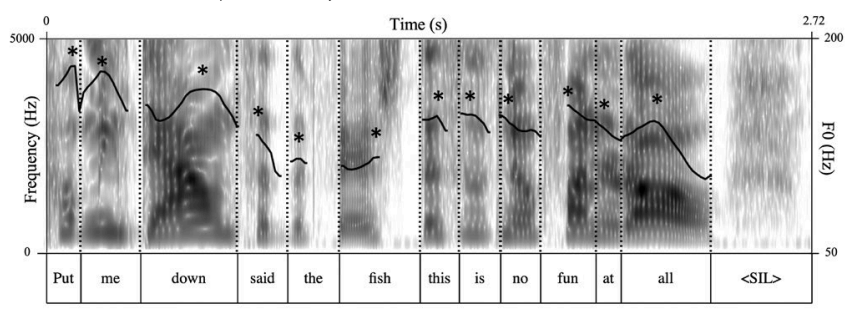

Figure 2: Word F0 measurement. An excerpt from one production is plotted as a time-frequency spectrogram. Identified word and silence boundaries are indicated by dashed vertical lines. The F0 contour generated for this excerpt is plotted in black over the spectrogram, with the parabolically-interpolated maximum FO for each word indicated with an asterisk.

Analysis

Using linear mixed-effects regression, we fit a model of maximum F0 using the control factors. The data were fit on a word-by-word basis. The fully-saturated model included all control fixed effects, a random effect of speaker, and random slopes over speaker for each fixed effect. This model did not converge, so we iteratively removed random slopes accounting for the least variance and refit the model until it converged. Fixed effects were then individually removed and the simpler model was compared to the more complex model using a likelihood ratio test (Baayen et al., 2008). Factors accounting for significantly more variance in the more complex model remained in the final control model.

We then fit an experimental model by adding fixed effects corresponding to the predictions of musical meter and linguistic meter. We added musical metric strength as both a fixed effect and random slope over 
participant, coded using simple contrast coding with metric strength level 2 (intermediate) as the reference level. We added linguistic metric strength as both a fixed effect and random slope over participant, coded using backward difference coding where each higher level is contrasted with the level below.

\section{Results}

Normalized maximum F0 is shown for words at each level of the musical metric hierarchy in Figure 3, and at each level of the linguistic metric hierarchy in Figure 4. The final model parameters appear in Table 1. Results demonstrate the speakers consistently signal two levels of musical metric strength with pitch: words aligned with metric strength level 3 (beat 1 in a 6/8 measure structure) are produced with higher pitch than words aligned with the other levels. Although words aligned with metric strength level 2 were produced with numerically higher F0 than level 1, this contrast did not reach significance in the model when including control parameters. Results also demonstrate that speakers use pitch to signal the end of large linguistic metric constituents, as evidenced by significant pitch decreases from metric strength level 3 to level 4, and from level 4 to level 5. Control parameters indicate that speakers use higher pitch to cue open class words (as opposed to closed class words), lower frequency words, words written in SMALL CAPS, and the first mention of words (as opposed to repeated mentions).

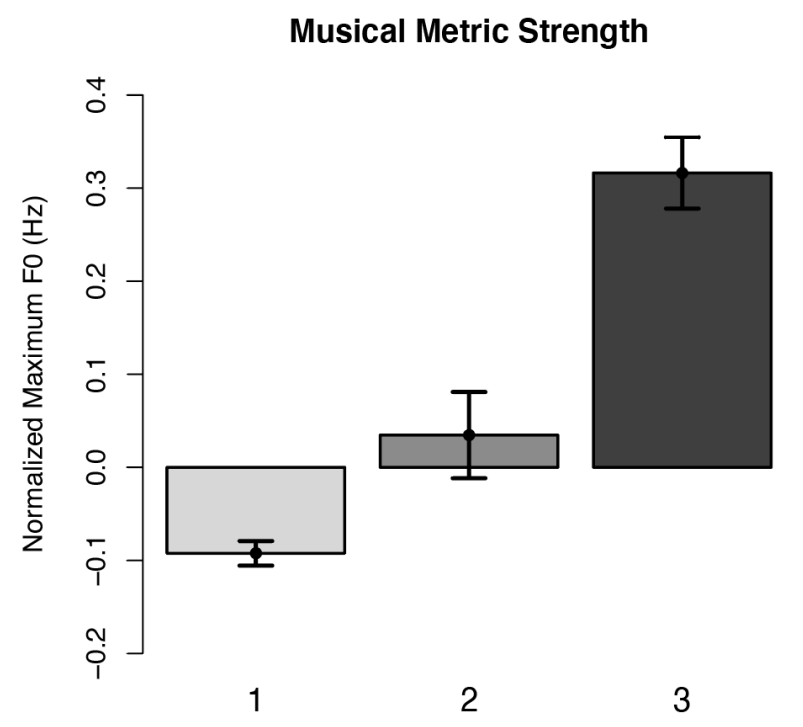

Figure 3. Values of normalized maximum F0 calculated across three levels of musical metric strength. Error bars indicate standard error.

\section{Linguistic Metric Strength}

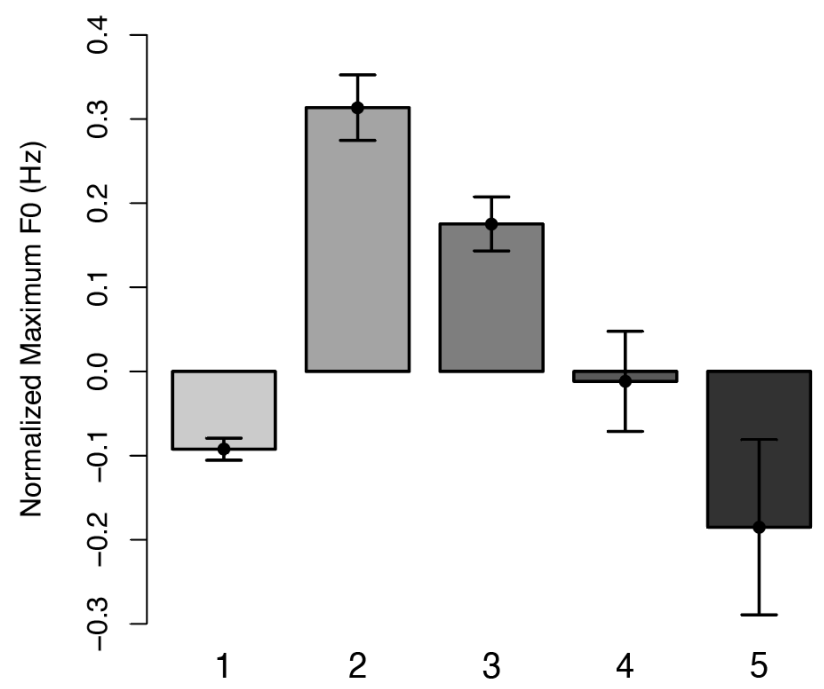

Figure 4. Values of normalized maximum FO calculated across five levels of linguistic metric strength. Error bars indicate standard error.

Table 1: Fixed effects in the model of normalized maximum FO by linguistic metric structure and control predictors

\section{Normalized Maximum Fo}

\begin{tabular}{lcccc} 
Fixed Effects & Estimates & $S E$ & $t$ & $p$ \\
\hline (Intercept) & 0.71 & 0.06 & 11.51 & $<\mathbf{0 . 0 0 1}$ \\
Music Meter 3v2 & 0.44 & 0.17 & 2.60 & $\mathbf{0 . 0 0 9}$ \\
Music Meter 1v2 & 0.28 & 0.24 & 1.16 & 0.245 \\
Ling Meter 5v4 & -0.15 & 0.04 & -3.79 & $<\mathbf{0 . 0 0 1}$ \\
Ling Meter 4v3 & -0.18 & 0.04 & -4.92 & $<\mathbf{0 . 0 0 1}$ \\
Ling Meter 3v2 & 0.25 & 0.17 & 1.50 & 0.134 \\
Ling Meter 2v1 & 0.12 & 0.17 & 0.72 & 0.472 \\
Word Class & -0.18 & 0.02 & -8.66 & $<\mathbf{0 . 0 0 1}$ \\
Lexical Frequency & -0.08 & 0.00 & -18.26 & $<\mathbf{0 . 0 0 1}$ \\
Font Emphasis & 0.41 & 0.05 & 8.36 & $<\mathbf{0 . 0 0 1}$ \\
$\begin{array}{l}\text { Intra-stanza } \\
\text { repetition }\end{array}$ & -0.10 & 0.02 & -6.76 & $<\mathbf{0 . 0 0 1}$ \\
\hline
\end{tabular}




\section{Discussion}

The current study was designed to investigate the signaling of metric structure through pitch variation in child-directed productions of highly metric poetry. Results demonstrate that speakers use pitch variation to signal metric structure in poetic production in multiple ways, consistent with work in both music performance and prose speech production. Specifically, readers cue metrically strong syllables (i.e., beat 1 in a $6 / 8$ musical meter) with higher pitch than metrically weak syllables (all other beats). In addition, speakers cue three hierarchical levels of metric units by decreasing pitch.

Although it has long been observed that poetry shares features with both music and language (Lerdahl, 2001), there have been few empirical investigations of this claim. The current study provides such empirical support by demonstrating that models of both musical and linguistic metric structure simultaneously account for pitch variation in child-directed productions of poetry. Interestingly, this hybrid realization of musical and linguistic metric structure in pitch differs from the patterns we have previously demonstrated for word intensity and duration in this corpus, which respectively predominantly signal either musical or linguistic metric structure alone.

For musical metric structure, pitch signaled a twolevel metric hierarchy with sole accent on the downbeat (strength level 3). This contrasts with our previous investigations of both intensity, which signaled a threelevel hierarchy with primary accent on the downbeat and secondary accent on strength level 2 (Fitzroy \& Breen, 2020), and word duration, which signaled a three-level hierarchy with primary accent on strength level 2 and secondary accent on the downbeat (Fitzroy $\&$ Breen, 2018). The realization of downbeat accent in pitch suggests that, like intensity, musical metric structure is signaled via pitch variation. However, the realization of only two levels of musical metric strength in pitch indicates that this aspect of metric structure is realized with lower fidelity in pitch than in intensity.

For linguistic metric structure, pitch marked differences between the three highest strength levels. This clearly contrasts with our previous investigation of duration, which unambiguously signaled five levels of linguistic metric strength (Breen, 2018), and differs somewhat from our previous findings for intensity, which marked differences between strength levels 2 and 3 , and between strength levels 4 and 5 (Fitzroy \& Breen, 2018). The similarity of linguistic metric structure realization in pitch and intensity is consistent with prior findings that physical aspects of these prosodic channels lead them to correlate somewhat in speech production (e.g., Gramming et al., 1988). However, the clearer distinction between linguistic metric levels in pitch than in intensity suggests that linguistic metric structure is realized more clearly in pitch. Taken together, our present pitch results and prior investigations of metric realization in word intensity and duration in this same corpus demonstrate that metric structure is realized in poetic production in a manner that reflects both musical and linguistic features of poetry, but that the balance of these features differs across prosodic channels.

The realization of metric structure through pitch and other acoustic cues provides important temporal information to child listeners of poetic texts like The Cat in the Hat. Specifically, they can use the metric structure to generate temporal expectations about upcoming information. For example, event-related potential studies demonstrate that listeners use metric structure, cued by in part by pitch variation, to direct their attention to metrically strong events in both music (Fitzroy \& Sanders, 2015) and speech (Breen et al., 2014). Child listeners who hear metrically-regular poetry like The Cat in the Hat with clear prosodic cues to metric structure can direct their attention to metrically strong moments, facilitating phonological learning.

\section{Conclusion}

The current study demonstrates that speakers of childdirected poetic text use pitch variation to consistently signal two levels of musical metric structure and three levels of linguistic metric structure. These results are consistent with previous work in both prose speech production and Western music composition. Moreover, these results complement prior demonstrations that duration and intensity variation signal musical and linguistic metric structure in the same corpus, while providing further evidence that metric structure is realized differently across these prosodic channels.

\section{Acknowledgements}

The authors wish to thank Kathryn Guarino, who collected the recordings and provided initial analysis of the data, Sarah Weidman, who provided assistance with analysis and inspired discussion of the corpus, and Michele Cubillo, who implemented the syntactic analysis. This work was supported by the James S. McDonnell Foundation [Understanding Human Cognition Scholar Award to MB]. 


\section{References}

Baayen, R. H., Davidson, D. J., \& Bates, D. M. (2008). Mixed-effects modeling with crossed random effects for subjects and items. Journal of Memory and Language, 59(4), 390-412. https://doi.org/10.1016/j.jml.2007.12.005

Boersma, P., \& Weenink, D. (2018). Praat: Doing phonetics by computer [Computer software]. Version 6.0. 43.

Breen, M. (2018). Effects of metric hierarchy and rhyme predictability on word duration in The Cat in the Hat. Cognition, 174, 71-81. https://doi.org/10.1016/j.cognition.2018.01.014

Breen, M., Dilley, L. C., McAuley, J. D., \& Sanders, L. D. (2014). Auditory evoked potentials reveal early perceptual effects of distal prosody on speech segmentation. Language, Cognition and Neuroscience, 29(9), 1132-1146.

https://doi.org/10.1080/23273798.2014.894642

Breen, M., Fedorenko, E., Wagner, M., \& Gibson, E. (2010). Acoustic correlates of information structure. Language and Cognitive Processes, 25(7-9), 10441098. https://doi.org/10.1080/01690965.2010.504378

Dr. Seuss. (1957). The cat in the hat (Vol. 1). Random House Books for Young Readers.

Drake, C., \& Palmer, C. (1993). Accent Structures in Music Performance. Music Perception: An Interdisciplinary Journal, 10(3), 343-378. https://doi.org/10.2307/40285574

Fabb, N., \& Halle, M. (2008). Meter in poetry: A new theory. Cambridge University Press. https://doi.org/10.1017/CBO9780511755040

Fitzroy, A. B., \& Breen, M. (2018). Readers use duration and intensity variation to signal hierarchical metric structure in child-directed poetic speech. 15th International Conference on Music Perception and Cognition, Montreal, Quebec, Canada.

Fitzroy, A. B., \& Breen, M. (2020). Metric Structure and Rhyme Predictability Modulate Speech Intensity During Child-Directed and Read-Alone Productions of Children's Literature. Language and Speech, 63(2), 292-305. https://doi.org/10.1177/0023830919843158

Fitzroy, A. B., \& Sanders, L. D. (2015). Musical meter modulates the allocation of attention across time. Journal of Cognitive Neuroscience, 27(12), 2339-2351. https://doi.org/10.1162/jocn a 00862

Fry, D. B. (1958). Experiments in the perception of stress. Language and Speech, 1(2), 126-152. https://doi.org/10.1177/002383095800100207

Gorman, K., Howell, J., \& Wagner, M. (2011). Prosodylab-aligner: A tool for forced alignment of laboratory speech. Canadian Acoustics, 39(3), 192-193.
Gramming, P., Sundberg, J., Ternström, S., Leanderson, R., \& Perkins, W. H. (1988). Relationship between changes in voice pitch and loudness. Journal of Voice, 2(2), 118-126. https://doi.org/10.1016/S0892-1997(88)80067-5

Hudson Kam, C. L., \& Matthewson, L. (2017). Introducing the infant bookreading database (IBDb). Journal of Child Language, 44(6), 1289-1308. https://doi.org/10.1017/S0305000916000490

Huron, D. (2006). Sweet anticipation. Cambridge, MA: MIT Press. https://doi.org/10.7551/mitpress/6575.001.0001

Huron, D., \& Royal, M. (1996). What is melodic accent? Converging evidence from musical practice. Music Perception, 13(4), 489-516. https://doi.org/10.2307/40285700

Lerdahl, F. (2001). The sounds of poetry viewed as music. Annals of the New York Academy of Sciences, 930(1), 337-354. https://doi.org/10.1111/j.1749-6632.2001.tb05743.x

Lerdahl, F., \& Jackendoff, R. (1983). A Generative Theory of Tonal Music. MIT Press.

Palmer, C. (1996). Anatomy of a performance: Sources of musical expression. Music Perception, 13(3), 433-453. https://doi.org/10.2307/40286178

Shanahan, D., \& Huron, D. (2011). Interval Size and Phrase Position: A Comparison between German and Chinese Folksongs. https://doi.org/10.18061/1811/52948

Temperley, D. (2003). End-accented phrases: An analytical exploration. Journal of Music Theory, 47(1), 125-154. https://doi.org/10.1215/00222909-47-1-125

Todd, N. (1985). A model of expressive timing in tonal music. Music Perception, 3(1), 33-57. https://doi.org/10.2307/40285321 


\author{
Information specification during singing: A theoretical approach to music performance \\ Lana J. Delasanta ${ }^{1 \dagger}$ \\ ${ }^{1}$ Department of Psychological Sciences, University of Connecticut, Storrs, CT, USA \\ $\dagger$ Corresponding author: lana.delasanta@uconn.edu \\ Published 16 December 2021; https://doi.org/10.18061/FDMC.2021.0039 \\ Author video presentation and/or other conference material: https://doi.org/10.17605/OSF.IO/3VPZQ
}

\begin{abstract}
Self-organized systems emphasize Gibson's (1966) proposal that organisms and the environment are one coupled system. As a result, energy flow throughout the system allows its subsystems to utilize it in a meaningful way. Specifically, emergent collective organization provides information about and specific to the world around us. During music performance, this is especially important for both performers and listeners alike. This theoretical proposal discusses how we can consider examining ecological physics and the theory of the global array through music performance. If we consider a group of singers as a self-organized system, it opens the door to understanding the dynamics and information flow within and around it. The goal of this paper is to explore an unorthodox approach to examining the perception of music performance.
\end{abstract}

KEYWORDS: collective organization, ecological psychology, emergent dynamics, energy flow, information, music performance, perception and action

\section{Ecological Framework}

The field of ecological psychology, brought to the forefront by J. Gibson, emphasizes the epistemology and intentionality of organized systems through perception-action coupling. Gibson (1966) noted that the appropriate scale of investigation for living organisms in their environment is structured differently at each level - from microscopic amoeba to entirely coordinated animal herds. Importantly, organisms in their environment are not isolated from it, and for a system to maintain its organization, energy must always be expended or dissipated (Gibson, 1966; Walton et al., 2014). The self-organizing system demonstrates the necessity of energy flow throughout the system which allows its subsystems to utilize it in a meaningful way.

Anytime an open non-equilibrium system does work, energy dissipates through the system and provides constraints in a reciprocal manner - this affords selforganization (Walton et al., 2014). When many of these systems (or subsystems) maintain each other's mutual constraints, it is considered an autocatakinetic system where circular causality and emergent collective organization occurs (Swenson, 1997).

One such example of this phenomenon could be theorized as a group of singers. If we consider each singer individually as an open non-equilibrium subsystem that transforms energy throughout an intentional performance, emergent collective organization occurs as each person constrains the other in unique ways that result in a dynamic yet synchronized performance. This paper is meant to bring attention to the possible theoretical consideration of a music performance as part and parcel of an emergent collective organization. First, let's discuss the properties of emergent collective organization that provide the means for information flow.

\section{Open Systems and Ambient Energy Arrays}

Energy expenditure is the fundamental foundation of nonlinear system dynamics. Ecological physics, similarly, defines the nature of the organismenvironment interaction and how it is specified to an organism. Gibson (1966) introduces ambient arrays as a source of information - structured energy by the animal-environment interaction governed by physical laws which give rise to unique structures and patterns of ambient energy (Stoffregen \& Bardy, 2001). This structuring of energy specifies information to an organism because each particular pattern defines a unique relationship between the organism and their environment. Specification is described as a lawful, 1to-1 relationship between patterns in ambient energy arrays and aspects of the organism-environment interaction that give rise to them (Shaw et al., 1982; Stoffregen \& Bardy, 2001).

When considering the perceptual system, the orthodox perspective is that different stimuli in the environment activate specific sensory receptors which then send signals to the central nervous system where meaning is then interpreted (i.e., Dewey, 1896). Gibson 
(1986), on the other hand, argued that perception is based on ambient energy where the variations in sensory information are related directly to physical reality through natural law. Further, Stoffregen and Bardy (2001) make the argument that behavior is perceived and controlled relative to real and lawful aspects of the animal-environment system rather than sensory representations or internal models. By introducing ecological physics, we can move away from the focus on how the world around us becomes structured inside the brain or nervous system. Instead, the activity of an organism can be understood in relation to many independent referents that result in various patterns of ambient energy arrays which provide information that is inherently structured (Stoffregen et al., 2017).

Gibson (1966) originally described each sensory system to be stimulated by specific forms of energy (i.e., the auditory system stimulated by an acoustic ambient array). However, it has been theorized that direct perception must be based on sensitivity to emergent higher order patterns of multiple ambient energy arrays because perceivers seek information about behavior relative to multiple referents at once (Stoffregen et al., 2017). Thus, because perception is not comprised of multiple, distinct sensory systems that respond to a only one distinct form of energy, we can instead conclude that there is only one irreducible perceptual system where information is specified by a single, global energy array.

\section{Theory of the Global Array}

To better understand the theory of a global array as information that specifies the system-environment relationship of a system, let's assess the act of walking as an example. If multiple ambient arrays were sources of specific sensory information (i.e., the eyes respond to the ambient light array or the ears respond to the acoustic array), what happens if what we perceive is not consistent across all ambient arrays? When a person walks, footfalls compress receptors on the feet (mechanical) but there is a very different pattern of stimulation on the vestibular system (proprioceptive) and through optical changes in the layout of the environment (visual) without stimulation on the mechanical or vestibular systems. Here, it would not be feasible for there to exist a 1-to-1 specification of "walking" in a single ambient array since each one is specifying unique information. The act of perceiving must provide rich information about aspects of the animal-environment system relevant to a specific action (Stoffregen et al., 2017).

Stoffregen and Bardy (2001) maintain that emergent, higher order patterns across multiple forms of ambient energy are all that meet the criteria for lawful 1-to-1 specification. The global array offers a solution to this discrepancy. Here, information exists as high order invariant relations between other ambient energy arrays (Stoffregen et al., 2017). Accordingly, while single ambient arrays may provide specification of a particular form of energy in relation to the actor, the global energy array provides unique specification of all possible ambient arrays in relation to each other and to the actor. The global array is an inherently compound invariant that emerges from patterns of single-energy arrays which are, by nature, irreducible and directly perceivable (Stoffregen et al., 2017).

This theory is consistent with Gibson's (1966) claim as follows: "If the invariants in this structure can be registered by the perceptual system, the constants of neural input will correspond to the constants of stimulus energy. [...] The brain is relieved of the necessity of constructing such information by any process" (pp. 267). Thus, we rid ourselves of the necessity for integration or computation within the perceptual system through the coupling of the system to the global energy array embedded in lawful physical relations.

\section{Proposed Experimental Application}

While the existence of a global array has much theoretical support from an ecological perspective, research conducted to test this theory is severely lacking. Many questions remain as to what features of the global array allow us to specify relational information in the world around us, as previous research on the topic emphasizes single forms of energy stimulating specific, isolated sensory systems. The current proposed experimental application offers an applied approach to understanding how the perceptual system might access specific information in structured energy emergent in the global array.

\section{Emergent Collective Organization in Choir}

\section{Musical Semiotics}

Considering a group of singers as a self-sustaining system which has emergent collective organization has often been discussed in terms of musical semiotics (Walton et al., 2014). Musical semiotics, as defined by Tarasti et al. (1996), is music production as a system of signs where others seek to understand how sound events 
come to signify musical meaning through their relationship with instruments and other performers. As Rappaport (1974) expertly notes, communication not only includes what is said, but also includes certain sorts of 'doing' as well. This coincides with Pierce's (1960) classification of indexicality - where an action is caused by part of what it indicates (Rappaport, 1974). For example, a singer performs a piece which, in turn, embodies the meaning of that piece. Sawyer (1995) notes that a musician's actions within a performance contribute to the evolving emergent - the musical output of that interaction, which reciprocally constrains the performance. More specifically, there are kinesthetic constraints that affect the ways that the performance is generated and able to be interpreted by both the audience and other performers (Walton et al., 2014).

\section{Perception-Action Coupling During Performance}

Music performance, like other behavior, involves both perception and action couplings continuously informed by specific and lawful information. While auditory information seems to be primarily what we perceive, it is affordances that are the true objects of perception according to Gibson (1986). Affordances are possibilities (or lack thereof) for action specified by lawful and relational information structured in patterned energy. In a musical performance, for example, the information indicating the dynamics of the performance specify the affordances for continuing to perform (i.e., speeding up or getting softer). It is clear why this relationship is circularly causal and impredicative.

In the framework of Stoffregen et al.'s (2017) theory of the global array, we can conceive that the information specified during music performance is much richer than the single-energy array containing acoustics. Windsor and de Bezenac (2012) explain that the significance of music, or any sound, does not lie in an abstract, auditory phenomenon but instead lies in the manner in which it directly specifies interactions among people in the environment.

Group music performance, in particular, requires excellent coordination and regulation of action among members to achieve a common goal, a property of selforganizing systems (Kugler \& Turvey, 1988). Behaviors of each individual solicit particular patterns of behavior in others. Interacting musicians are not motivated and constrained only by the sound produced by other musicians, but also by the specific actions and gestures that provide richer information about the performance. Windsor and de Bezenac (2012) go further to note that, "Music presents a limitless array of highly structured information about the bodies and environments of those that produce it" (pp. 111). The musical performance itself may encompass its own global array that captures all dynamics of the system.

As discussed by Schögler and Trevarthen (2007), there has been research that demonstrates how an expressive musical performance can provide perceptual information in various modalities through physical movement (Juslin \& Sloboda, 2001; Todd, 1994). When singing or performing with others we inform others about our action in how we move, which can act as an invitation for others to join in (Schögler \& Trevarthen, 2007). This information that specifies the possible intent of other performers must be of various modalities, not simply auditory or visual. Thus, an expressive musical performance is a complex array of energy as emphasized by the theory of the global array.

Evidence has shown that movement is more strongly associated with the auditory system than the visual system (Patel et al., 2005). In addition, other research has emphasized the temporal patterns of musical performance giving rise to beat perception. For example, Toiviainen et al. (2010) conducted a kinetic analysis of peaks in mechanical energy during music listening which showed that participants embodied the musical stimulus on multiple metrical levels. This provides further evidence that there are various ways to parse information specific to a music performance.

Research is warranted to explore the information that emerges among a group of performers. The various possibilities of information coupling may offer enhanced information about the piece being performed (i.e., acoustical timbre, stylistic details, variations in breath control). Experiments that involve music listening offer insight into how it is heard, but when manipulating sound production and feedback in realtime we can uncover if changes in coupling strategies impact information flow.

One previous study by D'Amario and colleagues (2019) manipulated visual contact among singers and used the audio recordings as stimuli for listeners. Results demonstrated that participants listening to the recordings were able to perceive differences in synchrony in correspondence with the visual contact conditions (D'Amario, Daffern, \& Bailes, 2019). This finding demonstrates the possible suggestion that manipulating information available to the singers in each condition changes how that performance was perceived by listeners.

The research application discussed here offers an empirical lens to investigate how changing the 
structured information of the performers' global array may change how they perform, how synchronized they perform, and how the performance is perceived by listeners.

\section{Proposed Study}

The current proposal aims to investigate how mechanical coupling affects listeners' perception of a performance using a similar paradigm as D'Amario and colleagues (2019). However, in addition to visual coupling, a subsequent question of interest is whether varying mechanical coupling among singers will produce similar results.

\section{Experiment 1}

Four singers will be recruited from the University of Connecticut to partake in the study. The participants will be members of an already existing performance group that have a simple chorale or carol in their repertoire that they can perform comfortably acapella. They will be positioned so each singer can see and reach each other during baseline (a small square).

The performance conditions will be as follows:

1. Visual contact (eyes open)

a. No mechanical coupling

b. Holding hands

2. No visual contact (eyes-closed)

a. No mechanical coupling

b. Holding hands

Audio recordings will be recorded for each condition and serve as stimuli for Experiment 2. Additionally, movement data will be recorded and analyzed to examine the impact of the visual and/or mechanical manipulations on movement synchrony of the singers during the performance. Singers will also be asked to rate their performance after each condition as to how well they did to gauge perceptions in real-time. All data will be used to compare relationships with participants' perceptual ratings of each performance condition during Experiment 2.

We predict that movement during the visually and mechanically coupled condition will result in the most synchrony between singers (and best performance ratings), while the least synchronized condition will be the performance with no mechanical coupling with eyes closed (and least performance ratings).

\section{Experiment 2}

Using the recordings from Experiment 1, we will recruit students from the University of Connecticut participant pool to come into the lab and listen to all of the condition recordings. The order of the audio files will be randomized to avoid any order bias. We will ask participants to rate how well each performance was performed, how synchronized they thought each performance was, and other exploratory questions (i.e., "How many singers did you hear?").

In conjunction with the data collected from the performance in Experiment 1, we will analyze the effect of condition on listeners' ratings and compare the results to the movement data collected from the singers. This will provide evidence, if any, of coherence in ratings and the mechanical or visual coupling manipulations.

We predict that perceptual ratings of each performance should correspond with the motion synchrony of each condition. Further, we predict that mechanical coupling may provide richer information for performers and listeners alike, which may influence listeners to have more aligned ratings with the performance data for the mechanically coupled conditions.

\section{Discussion}

The results of this research will contribute to our understanding of how performance manipulated by mechanical or visual coupling may specify information about the performance to listeners. Further, it is of interest to theorize how information contained in the global energy array may or may not be limited when compressed into a different domain - auditory listening. Future research may consist of Experiments 1 and 2 concurrently and capture the performer-audience dynamic.

The proposed study will provide much needed empirical data to explore the global array while adding to the vast amount of existing literature on synchrony during music performance. If participants' experience listening to each performance recording reflects our coupling manipulation, it would provide quantitative evidence that mechanical information can be picked up in the auditory domain. Thus, individual single-energy arrays would be affecting each other in an interactive way - evidence for an emergent global array, rather than individualized sensory pathways.

\section{Conclusion}

An empirical approach to investigating the dynamics of group singing embedded in a noteworthy theoretical framework provides a novel way to quantify the complexities of the shared collective experience. Ecological physics and the theory of the global array has 
not received much attention from experimental work. However, the benefits of examining real-world experiences like group singing from an ecological physics perspective allows for us to explore the rigor of proposed theories and move research on music cognition forward in an innovative way.

\section{Acknowledgements}

Thank you to the Center for the Ecological Study of Perception and Action for providing me with the perspective that inspired the ideas explored herein.

\section{References}

D’Amario, S., Daffern, H., \& Bailes, F. (2019). Perception of synchronization in singing ensembles. Plos one, 14(6), e0218162. https://doi.org/10.1371/journal.pone.0218162

Dewey, J. (1896). The reflex arc concept in psychology. Psychological review, 3(4), 357. https://doi.org/10.1037/h0070405

Gibson, J. J. (1966). The Senses Considered as Perceptual Systems. United States: Houghton Mifflin.

Gibson, J. J. (1986). The Ecological Approach to Visual Perception. United Kingdom: Lawrence Erlbaum Associates.

Juslin, P. N., \& Sloboda, J. A. (2001). Music and emotion: Theory and research. Oxford University Press.

Kugler, P. N., \& Turvey, M. T. (1988). Self-organization, flow fields, and information. Human Movement Science, 7(2-4), 97-129. https://doi.org/10.1016/01679457(88)90009-7

Patel, A. D., Iversen, J. R., Chen, Y., \& Repp, B. H. (2005). The influence of metricality and modality on synchronization with a beat. Experimental brain research, 163(2), 226-238. https://doi.org/10.1007/s00221-004-2159-8

Rappaport, R. A. (1974). Obvious aspects of ritual. Cambridge Anthropology, 3-69.

Sawyer, R. K. (1995). Creativity as mediated action: A comparison of improvisational performance and product creativity. Mind, culture, and activity, 2(3), 172-191. https://doi.org/10.1080/10749039509524698

Schogler, B., \& Trevarthen, C. (2007). To sing and dance together: From infants to jazz. Advances in consciousness research, 68, 281. https://doi.org/10.1075/aicr.68.22sch

Shaw, R. E., Turvey, M. T. \& Mace, W.M. (1982). Ecological psychology: The consequence of a commitment to realism. In W. Weimer \& D. Palermo (Eds.) Cognition and the symbolic processes. Vol. 2, 159226.

Stoffregen, T. A., \& Bardy, B. G. (2001). On specification and the senses. Behavioral and Brain Sciences, 24(2),
195-213. https://doi.org/10.1017/S0140525X01003946

Stoffregen, T. A., Mantel, B., \& Bardy, B. G. (2017). The senses considered as one perceptual system. Ecological Psychology, 29(3), 165-197. https://doi.org/10.1080/10407413.2017.1331116

Swenson, R. (1997). Autocatakinetics, evolution, and the law of maximum entropy production: A principled foundation towards the study of human ecology. Advances in Human Ecology, 6, 1-48.

Tarasti, E., Forsell, P., \& Littlefield, R. (Eds.). (1996). Musical semiotics in growth (Vol. 4). Indiana University Press.

Todd, N. P. M. (1994). The auditory "primal sketch": A multiscale model of rhythmic grouping. Journal of new music Research, 23(1), 25-70. https://doi.org/10.1080/09298219408570647

Toiviainen, P., Luck, G., \& Thompson, M. R. (2010). Embodied meter: hierarchical eigenmodes in musicinduced movement. Music Perception, 28(1), 59-70. https://doi.org/10.1525/mp.2010.28.1.59

Walton, A., Richardson, M. J., \& Chemero, A. (2014). Selforganization and semiosis in jazz improvisation. International Journal of Signs and Semiotic Systems (IJSSS), 3(2), 12-25. https://doi.org/10.4018/IJSSS.2014070102

Windsor, W. L., \& De Bézenac, C. (2012). Music and affordances. Musicae scientiae, 16(1), 102-120. https://doi.org/10.1177/1029864911435734 


\title{
Form and intertextuality in movie music videos
}

\author{
Brent Ferguson, Ph.D. ${ }^{1 \dagger}$ \\ ${ }^{1}$ Washburn University, Topeka, Kansas, United States \\ $\dagger$ Corresponding author: brent.ferguson@washsburn.edu \\ Published 16 December 2021; https://doi.org/10.18061/FDMC.2021.0040 \\ Author video presentation and/or other conference material: https://doi.org/10.17605/OSF.IO/8DHBZ
}

\begin{abstract}
This paper addresses a phenomenon I call the Movie Music Video (MMV henceforth). A MMV is a music video for a soundtrack song displaying intertextual relationships with its corresponding film, such as intercutting clips of the film in the mise en scene, using costumes from the film, or telling a film's narrative through lyrics. My primary goal in this paper is to calculate the frequency of certain intertextual relationships, or types between the music video and the film. For the secondary goal, I demonstrate a methodology for the analysis of this novel medium, focusing particularly on form and intertextuality. To do this, I create an analytical style borrowing from multi-domain form graphs to display song form aligned with visual information and types of intertextuality.

Most MMVs contain four independent texts in its mise en scene: its video diegesis, the corresponding film, its song and performing artist. Just as Lafrance and Burns (2017) emphasizes that the analysis of any music video should include visual, lyrical, and musical domains, my examples can be broken down into three categories based on whether the MMV references the corresponding film through visual, lyrical, or aural domains. My graphs highlight film references in the music video. Such references tighten intertextual bonds between multimedia, serving as tethers between texts. Using form graphs of MMVs, my paper demonstrates the rich interplay between texts executed through music form.
\end{abstract}

KEYWORDS: corpus study, music video, film, form, intertextuality

\section{Introduction}

Movie music videos (MMVs henceforth) feature scenes of the artist performing their song intercut with clips from the film. In MMVs, elements of the song or video reference the film through lyrical content, musical quotes, and visual stimuli. Presentations of different intertexts in MMVs are often associated with sections of the song form. The types of visual intertextuality this article covers include the use of intercuts, superimpositions (typically of intercuts), as well as film settings, costumes, actions, and celebrities from the film. Lyrical intertextuality includes the employment of a film's title as the song's title, alluding to the film's themes or actions in the lyrics, and the lyrics telling the film's narrative. Types of aural intertextuality includes the quotation of theme songs from a another medium (for films based off other multimedia), the film composer as a producer of the song recycling content from the film's musical score, the use of dialogue and sound effects from the film, and covering or sampling other music.

The goal of this research is to document the three types of intertexts in a corpus-driven study of 174 MMVs. I adapt a type of multi-domain form graph (see Burns, 2017; Osborn, 2020) to exhibit how song form organizes these intertexts within the MMV. This research builds on, and contributes to, musicological intertextuality by adapting pre-existing models to create a new method of analysis designed specifically to highlight textual relationships in MMVs. As a multimedia chimera, MMVs often provide intertextual links between the song, multiple visual worlds, celebrities, and sometimes brands. These various texts interact in counterpoint exclusive to the medium. With the recent interest in music video scholarship and intertextuality of popular music-evidenced by the publication of The Bloomsbury Handbook of Popular Music Video Analysis (2019) and The Pop Palimpsest (2018), respectively - this work adds to these growing canons including research from film and media studies, as well as recent literary scholars.

\section{Method}

Selecting videos for this corpus was built from three methods: the author's personal memory, the memory of their acquaintances, and searching the internet. YouTube yielded all but two of the additional examples. For every MMV found, details were sought related to it including: its title, artist, film title, song's release date, video's release date, film's release date, the MMV's director, any featured artists, and additional information through interviews, analyses, and other metatexts. I stopped collecting MMVs for the corpus on November 11, 2019. Within this corpus, which includes MMVs produced between 1980 and 2019, the span of 2000- 
2013 is represented with the fewest videos per year. This drought could relate to the decline of music television followed by music video's recent resurgence on the internet.

After the corpus was established, a spreadsheet was created with all the information and blank columns with the following headings for visual film intertextuality, lyrical intertextuality with narrative, and aural intertextuality, along with their subtypes. Each of the 174 MMVs were viewed at least twice. The first viewing consisted of concentrating on all aspects of the video without taking notes. My second viewing involved putting a " 1 " in the respective box of the categories present in the MMV. Additional viewings were warranted depending on the quantity of intertextuality in the MMV or the depth of analysis provided.

\section{Results}

Table 1 displays the total amounts of intertextual types between visual, lyrical, and aural. Only two MMVs did not exhibit visual intertextuality. Almost half of the MMVs possessed aural intertextuality with the film or other musical sources. This does not account for the MMV song's usage in the corresponding film's soundtrack. Under a third of the MMVs in the study utilized lyrical intertextuality.

Table 1: MMV Intertextuality Types and Results

$\begin{array}{ll}\text { Intertextuality Type } & \text { Total (Percentage) } \\ \text { Visual } & 172(98.85 \%) \\ \text { Lyrical } & 53(30.46 \%) \\ \text { Aural } & 83(47.7 \%)\end{array}$

Table 2: MMV Intertextuality Subtypes and Results

\begin{tabular}{|c|l|l|l|}
\hline Intertext Type & \multicolumn{1}{|c|}{ Subtype } & Total & Percent \\
\hline Visual & Intercuts & 141 & $80.6 \%$ \\
& Superimpositions & 56 & $32 \%$ \\
& Setting & 94 & $53.7 \%$ \\
& Costume & 43 & $24.6 \%$ \\
& Action & 46 & $26.3 \%$ \\
& Brands & 37 & $21.1 \%$ \\
& Celebrities & 50 & $28.6 \%$ \\
\hline Lyrical & Title Songs & 24 & $13.7 \%$ \\
& Allusions & 10 & $5.7 \%$ \\
& Narratives & 20 & $11.4 \%$ \\
\hline \multirow{2}{*}{ Aural } & Theme Quote & 7 & $4 \%$ \\
& Score Quote & 24 & $13.7 \%$ \\
& Dialogue/SFX Quote & 39 & $22.3 \%$ \\
& Other Music Quote & 26 & $14.9 \%$ \\
\hline
\end{tabular}

Specific subtypes of intertextuality and their results are shown in Table 2. Intercuts of the cotextual film are the most common intertextual binds in MMVs with just over $80 \%$. Utilizing film settings in MMVs occur in more than half of MMVs. While only occurring in roughly $13 \%$ of MMVs, employing the film's title as the song's title (and usually a line in the chorus) is the prevailing lyrical intertextuality, and just under that are songs that tell the film's narrative through its lyrics. Most aural intertextualities are samples of the film's dialogue and sound effects to accompany intercuts during the MMV; these do not occur on the soundtrack album version of the songs.

\section{Discussion}

Visual intertextuality serves as the vital narrative link between MMVs and film, because film is most prominently visual multimedia. One of Claudia Gorbman's main arguments in the groundbreaking Unheard Melodies (1987) is that music is secondary to the visual domain in the classical Hollywood film. We can see intertextual tethers between the song and film because of the MMV. Our eyes make an immediate connection between intercuts (or superimpositions) and the MMV diegesis. Citing costumes, settings, and characters in the video diegesis elicits a synergetic narrative world within the MMV. The mise en scene showing the artist performing actions from the film casts the artist as an actor in the film's narrative world without actually being cast in the film. Thus, MMVs become part of the film's lore - the world of the narrative not shown through the film.

Lyrical intertextuality combines the verbal and aural realms; a lyricist writes the lyrics and a singer performs them. Linking the film to the song through lyrical allusions and narratives provide similar intertextual tethers to the film. Text painting, or allusion, in MMVs through aligning certain visuals with lyrics further spins the intertextual web between multimedia. Presenting lyrical narratives and complex visual intertextualities secures the MMV as a convergent advertisement for the film and song, respectively.

Unlike lyrical or visual intertextuality, aural intertextuality does not always depend on references to the film. A MMV can utilize other texts related to the film, such as films based on other media, in the case of a producer sampling or covering older music. Importing dialogue and sound effects from the film push the MMV more towards "movie trailer" territory, but it also increases advertising quality of the MMV. Referencing 
outside musical works pushes the MMV's marketing value to include the previous media as well.

While I did not document the amount of times song form organized intertextualities, this article includes two form graphs that display how this works in MMVs. Table 3 displays a form graph for the MMV for Will Smith's Wild Wild West from the film of the same title. The symbol $>$ notates a change of setting, and this happens during each Chorus in Wild Wild West. Each verse and chorus pairing organizes both visual setting and lyrical narratives. The three settings are displayed in Figure 1. The first pairing displays a burning town with the exposition of the story and a description of its characters. During the second pairing, the visual setting shifts to the insides of a mechanical spider along with the lyrics describing the main conflict in the film and its antagonist. The final pairing (Verse 3 and Chorus 4), with the non-musical episode beforehand utilizes a mansion setting with lyrical allusions to the filmic action.

Table 4 shows a similar alignment of settings and song form in Puff Daddy's Come With Me, and it also displays the sections sampled from the Led Zeppelin song Kashmir (sections shown in Figure 1). Like Wild Wild West, Come With Me features rapid changes of scenery until halfway through the song. At this point, the camera centers on Puff Daddy in Times Square with an orchestra behind him. Tables 3 and 4 display a sample of multi-domain analyses.

\section{Conclusion}

Visual intertextuality prevails as the most common referential device in MMVs due to its format as visual medium. Intercuts being the preferred visual intertextuality is attributed to the affection for this format in its early days. MMVs are a type of advertisement for a film (see Gray, 2010; Hayward \& Fitzgerald, 2016; Summers, 2020), and intercutting parts of the film in an MMV mise en scene makes the advertising intent clear-it takes on the persona of a trailer while being simultaneously a music video. Employing the same titles between film and MMV refers to a practice in Hollywood that predates MTV: the commission of songs for film and television. Unique to MMV, apart from its corresponding soundtrack song, is its ability to capture film sound and dialogue occurring with intercuts. This pushes the MMV further into the territory of a trailer advertisement. Other MMVs play on the consumer's nostalgia by sampling popular songs in recent memory. In all, MMVs are effective musical

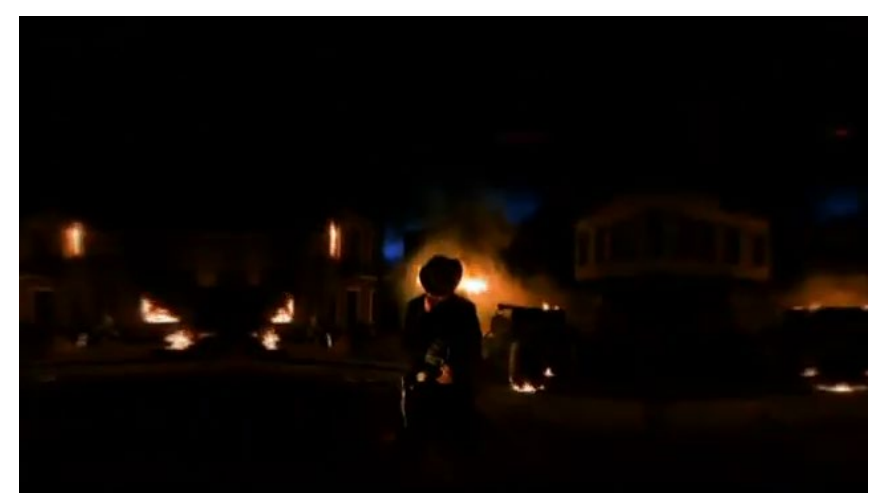

Burning Town

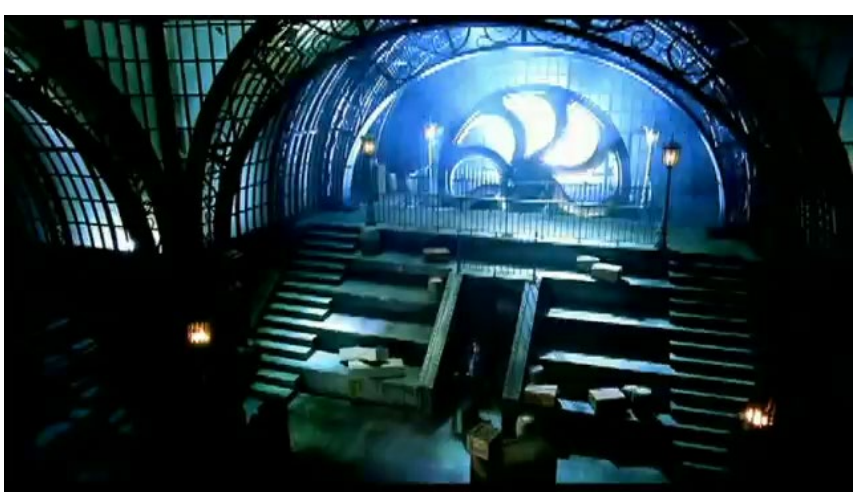

Mechanical Spider (Inside)

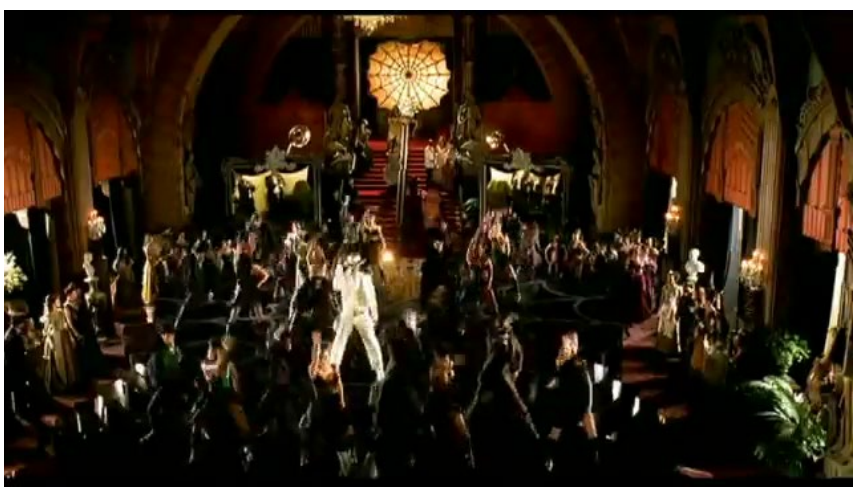

Mansion

Figure 1 : Settings in Wild Wild West MMV

advertisements that not only market the film with trailer like references, it markets the artist and their song. Due to this, MMVs might be more effective advertisements than movie trailers. This is apparent with a rise in the use of MMVs in the last five years alongside the use of samples or covers of popular music in blockbuster trailers. I predict that MMVs will continue to be a preferred advertising method for films and artists, and studios will continue to find more ways to expose audiences to these marketing entities. 
Table 3: Wild Wild West MMV Form Graph

\begin{tabular}{|c|c|c|}
\hline $\begin{array}{l}\text { Song } \\
\text { Section }\end{array}$ & Mise en scene & Intertextuality \\
\hline Introduction & $\begin{array}{l}\text { Desert }>\text { Burning } \\
\text { Town }\end{array}$ & $\mathrm{N} / \mathrm{A}$ \\
\hline Verse 1 & Burning Town & $\begin{array}{l}\text { Lyrical Narrative - } \\
\text { Character description } \\
\text { of Jim West } \\
\text { Intercuts }\end{array}$ \\
\hline Chorus 1 & $\begin{array}{l}\text { Burning Town > } \\
\text { Mechanical } \\
\text { Spider (inside) }\end{array}$ & Title Lyric \\
\hline Verse 2 & $\begin{array}{l}\text { Mechanical } \\
\text { Spider (inside) }\end{array}$ & $\begin{array}{l}\text { Lyrical Narrative - } \\
\text { Character description } \\
\text { of Arliss Loveless } \\
\text { and conflict } \\
\text { Superimpositions }\end{array}$ \\
\hline Chorus 2 & $\begin{array}{l}\text { Mechanical } \\
\text { Spider (inside) > } \\
\text { Mansion }\end{array}$ & $\begin{array}{l}\text { Title Lyric } \\
\text { Superimpositions }\end{array}$ \\
\hline $\begin{array}{l}\text { Dramatic } \\
\text { Break }\end{array}$ & Mansion & $\begin{array}{l}\text { Other celebrities } \\
\text { Sampled Artist } \\
\text { Character - Arliss } \\
\text { Loveless } \\
\text { Aural - Film score }\end{array}$ \\
\hline Chorus 3 & & $\begin{array}{l}\text { Title Lyric } \\
\text { Sampled Artist }\end{array}$ \\
\hline Interlude & & N/A \\
\hline Verse 3 & & Lyrical Allusions \\
\hline $\begin{array}{l}\text { Chorus } 4 \\
\text { Outro } \\
\end{array}$ & & Title Lyric \\
\hline
\end{tabular}
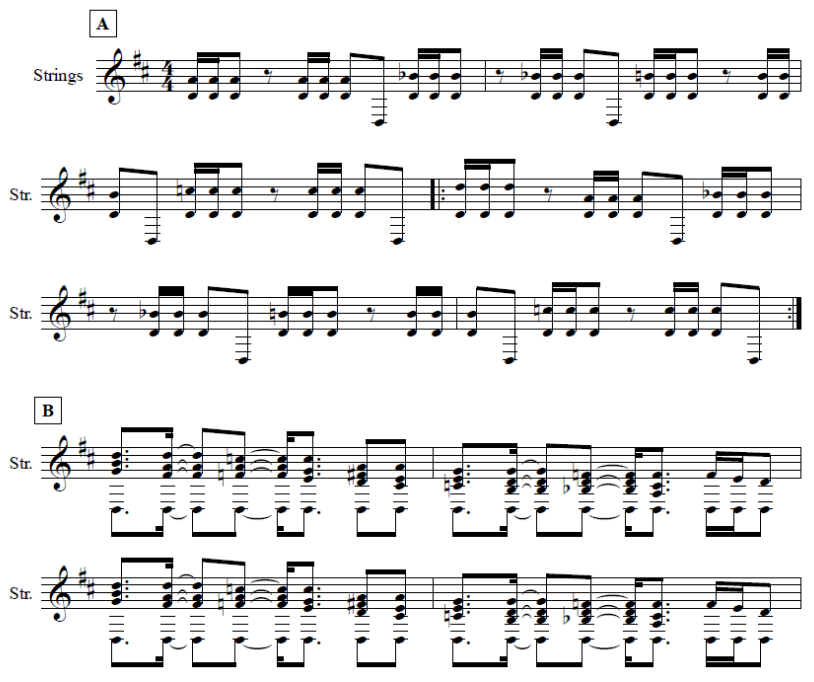

C

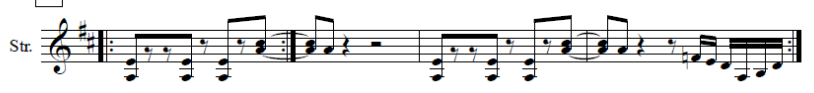

Figure 2 : Sections of Kashmir in Come With Me

Table 4: Come With Me MMV Form Graph

\begin{tabular}{|c|c|c|}
\hline Song Section & Sampled Section & $\begin{array}{l}\text { Video Diegesis } \\
\text { Setting }\end{array}$ \\
\hline $\begin{array}{l}\text { Dramatic } \\
\text { Introduction }\end{array}$ & No Music & \multirow[t]{3}{*}{ Apartment } \\
\hline $\begin{array}{l}\text { Musical } \\
\text { Introduction }\end{array}$ & \multirow[t]{2}{*}{$\mathrm{A}$} & \\
\hline Verse 1 & & \\
\hline Chorus 1 & $\mathrm{~B}$ & Apartment $>$ Elevator \\
\hline Verse 2 & $\mathrm{~A}$ & Elevator \\
\hline Chorus 2 & $\mathrm{~B}$ & Elevator $>$ Sky \\
\hline Bridge & Original & Sky \\
\hline Interlude 1 & \multirow[t]{2}{*}{ A } & \multirow{8}{*}{$\begin{array}{l}\text { Stage (in Times } \\
\text { Square) }\end{array}$} \\
\hline Verse 3 & & \\
\hline Chorus 3 & $\mathrm{~B}$ & \\
\hline Interlude 2 & $\mathrm{C}$ & \\
\hline Verse 4 & $\mathrm{~A}$ & \\
\hline Chorus 4 & B & \\
\hline Verse 5 & \multirow[t]{2}{*}{$\mathrm{A}$} & \\
\hline Outro & & \\
\hline
\end{tabular}

\section{Acknowledgements}

I want to thank all of the people who helped shape and edit my research, and this includes T.J. Laws-Nicola, Matthew Ferrandino, and Brad Osborn. 


\section{References}

Burns, L. (2017). Multimodal analysis of popular music video: genre, discourse, and narrative in Steven Wilson's "Drive Home." In C.X. Rodriguez (Ed.) Coming of Age: Teaching and Learning Popular Music in Academia. Michigan Publishing Services.

Burns, L. \& Hawkins, S. (Eds.). (2019). The Bloomsbury Handbook of Popular Music Video Analysis. Bloomsbury Academic. https://doi.org/10.5040/9781501342363

Burns, L. \& Lacasse, S. (2018). The Pop Palimpsest: The Intertextuality in Recorded Popular Music. University of Michigan Press. https://doi.org/10.3998/mpub.9755813

Fitzgerald, J. \& Hayward, P. (2016). Paranormal product: the music and promotion of Ghostbusters. In M. Evans \& P. Hayward (Eds.) Sounding Funny: Sound and Comedy Cinema. Equinox Publishing.

Genette, G. (1987). Palimpsestes: La littérature au second degré. Seuil.

Gorbman, C. (1987). Unheard Melodies. Indiana University Press.

Gray, J. (2010). Show Sold Separately: Promos, Spoilers, and Other Media Paratexts. University of New York Press.

Hatten, R. (1985). The place of intertextuality in music studies. American Journal of Semiotics, 3(4), 69-82.

Klein, M. (2005). Intertextuality in Western Art Music. Indiana University Press.

Kristeva, J. (1969). The Kristeva Reader, ed. Toril Moi. Columbia University Press.

Lafrance, M. \& Burns, L. (2017). Finding love in hopeless places: complex relationality and impossible heterosexuality in popular music videos by P!nk and Rihanna. Music Theory Online 23(2). https://doi.org/10.30535/mto.23.2.5

Osborn, B. (2020). Risers, drops and a fourteen-foot cube: a transmedia analysis of Emil Nava, Calvin Harris and Rihanna's This Is What You Came For. In C. Vernallis, H. Rogers, and L. Perrott (Eds.) Transmedia Directors: Artistry, Industry and New Audiovisual Aesthetics. Bloomsbury Academic. https://doi.org/10.5040/9781501339295.0020

Summers, T. (2018). From "Sabotage" to "Sledgehammer": trailers, songs, and the musical marketing of Star Trek Beyond (2016)." Music and the Moving Image 11(1), 4065. https://doi.org/10.5406/musimoviimag.11.1.0040

Vernallis, C. (2004). Experiencing Music Video: Aesthetics and Cultural Context. Columbia University Press. 


\title{
Computational models of temporal expectations
}

\author{
Lauren K. Fink ${ }^{1,2}$ \\ ${ }^{1}$ Department of Music, Max Planck Institute for Empirical Aesthetics, Frankfurt am Main, Germany \\ ${ }^{2}$ Max Planck - NYU Center for Language, Music, and Emotion (CLaME), New York, USA \\ $\dagger$ Corresponding author: lauren.fink@ae.mpg.de \\ Published 16 December 2021; https://doi.org/10.18061/FDMC.2021.0041
}

\begin{abstract}
With Western, tonal music, the expectedness of any given note or chord can be estimated using various methodologies, from perceptual distance to information content. However, in the realm of rhythm and meter, the same sort of predictive capability is lacking. To date, most computational models have focused on predicting meter (a global cognitive framework for listening), rather than fluctuations in metric attention or expectations at each moment in time. This theoretical contribution reviews existing models, noting current capabilities and outlining necessities for future work.
\end{abstract}

KEYWORDS: rhythm, meter, percussion, salience, prediction error, novelty, surprise

\section{Introduction}

One major goal of music cognition research is to understand how certain properties of an acoustic signal give rise to specific physiological, psychological, and behavioral responses. Such an endeavor is not straightforward, as the body and nervous system filter the incoming acoustic signal, suppressing certain aspects, amplifying others, and creating emergent percepts dependent on different temporal windows of integration. While many mysteries remain regarding the transformation from acoustic signal to psychological percept or subjective experience, numerous strides have been made, particularly in the realm of predicting listeners' tonal or harmonic expectations (e.g., Janata et al., 2002; Pearce \& Wiggins, 2012; Cancino-Chacón, Grachten, \& Agres, 2017).

While most Western music does organize itself around a tonal center - allowing for specific harmonybased expectations - plenty of music does not. Because one ultimate goal of the field should be a model of expectancy that can operate on never-before-heard music, especially from styles outside of Western, dominant culture (Jacoby et al., 2020; Baker et al., 2020), I focus here on the case of percussive music. Independent of musical culture or style, percussive music may or may not be pitch-based. Hence, I ask which computational models can provide continuous, meaningful predictions of listeners' physiological responses or psychological expectations while listening to a drum set solo, a tabla performance, a dùndún ensemble, a marching drumline, or pieces like John Cage's Credo in US?

At present, there exist a variety of impressive models to extract the beat or meter from a given piece (e.g., Klapuri, Eronen, \& Astola, 2006; Volk, 2008; Smith \& Honig, 2008; Tomic \& Janata, 2008; Temperley, 2010; Grosche \& Muller, 2010; Large, Herrero, \& Valesco, 2015; van der Weij, Pearce, \& Honing, 2017; Boenn, 2018; Lartillot \& Grandjean, 2019), with annual competitions held (e.g., MIREX Audio Beat Tracking Competition; Holzapfel et al., 2012), and algorithms constantly improving. While beat and meter provide an important foundation for when listeners might perceive certain moments to be more salient than others, they are not necessarily clearly present in every style of music. Also, beat and meter can fluctuate over time, such that one may need to conceive of salience within different metric frameworks of listening at different points in time (an approach exemplified via theoretical analysis in London's Hearing in Time, Ch. 7, 2012).

Can shifting metric frameworks of listening be modelled online in a manner that allows for updating predictions, especially as a function of repeatedly violated expectations? How might we account for the likelihood of certain perceived meters over others, given the number of present pulse layers (see Gotham, 2015, for discussion)? Can our models incorporate asymmetries in perception with regard to increases vs. decreases in certain features (e.g., regularity, loudness, etc.)? What about the role of listener familiarity and enculturation in predisposing a listener to certain tempometrical types (London, 2012; London, Polak, Jacoby, 2016; Holzapfel, 2015)? Or the role of repeated exposures in shaping the temporal context (e.g., local vs. global) over which listeners are attending (Margulis, 2014)? Finally, how might we model temporal 
expectations when no clear beat, meter, or typical tonal relationships exist?

This short article is not meant to be an exhaustive review of state-of-the-art models, but rather an entry point for interested researchers and students. Below, I provide an overview of relevant criteria when considering models of musical expectation more broadly. I then briefly outline a few models possibly able to meet the previously defined task of generating meaningful predictions in the case of percussive music. In so doing, I simultaneously highlight strengths, limitations, and directions for future modeling efforts.

\section{Model Considerations}

\section{Biological Plausibility}

One important note at the outset is that not all models of musical expectations aim to be biologically plausible. While biologically plausible models of auditory processing, from the cochlea to the cortex, do underpin some existing models of various aspects of music cognition (e.g., Collins et al., 2014; Large, Herrera, Velasco, 2015), biological plausibility is not necessarily a requirement (i.e., one could build a model that accurately predicts human perception or behavior by using signal transformations very different from those thought to be employed by the nervous system). In general, the validation of computations on a neural level is likely relegated to, or informed by, research in animal models (e.g., Elie \& Theunissen, 2019) and/or computational neuroscience (e.g., Tomková, 2015; Masquelier, 2018). Whether biological plausibility matters depends on one's specific research question and context.

\section{Mathematical Framework}

Related to, but not synonymous with the notion of biological plausibility, is the mathematical framework in which a model of musical expectations operates. The current leading models come from a variety of mathematical stances, from probabilistic frameworks to non-linear oscillatory ones. These approaches reflect more than just a mathematical means to an end; they often signify the fundamental concept and perhaps even level of explanation at play in the model.

Oscillatory models take a bottom-up approach, closely aligned with Dynamic Attending Theory (Large \& Kolen, 1994; Large \& Jones, 1999). Specifically, the idea is that periodicities (oscillations) in music can entrain oscillations of the nervous system (bottom-up). In some models, the predicted perceived meter (as determined by the most active oscillators) can then direct attention to the incoming stimulus (see e.g., Hurley, Fink, Janata, 2018). Such models have a sort of short-term memory built in, in the form of the decay time of the oscillators, but do not (at present) integrate over longer time scales in the way that Bayesian or Markov models can. Nonetheless, enculturation can be built into oscillatory models in the form of pre-set couplings between oscillators of different frequencies, which reflect the metric norms of the listeners' culture, as in the model of Large, Herrera, and Valesco (2015).

Alternatively, prior experience can be modelled in the form of a Bayesian prior. As such, Bayesian models can easily account for accumulated sensory evidence over time (at short and long time scales; see, e.g., Skerritt-Davis \& Elhilali, 2018), as well as listener enculturation (see e.g., van der Weij, Pearce, \& Honing, 2017). Bayesian approaches fit well within predictive coding theory (Friston, 2002; Vuust \& Witek, 2014), which is compatible with Dynamic Attending Theory, and reflect the idea that past experience is used to generate predictions about incoming stimuli. The mismatch between what was expected and what occurs is used to update a probability distribution and thereby modify future predictions. Some Bayesian models also include parameters to reflect human memory, as well as observation noise (e.g., Skerritt-Davis \& Elhilali, 2018).

\section{Input Signal}

Many leading models of melodic (e.g., Pearce, 2005), harmonic (e.g., Harrison, Bianco, Chait, Pearce, 2020), and temporal expectations (e.g., Forth et al., 2016) or meter (e.g., Large, Herrera, \& Velasco, 2015) operate on symbolic (e.g., **kern, MIDI, etc.) data. Databases of music meticulously converted into such formats exist (e.g., http://kernscores.stanford.edu/), which allow users of models requiring them to test their predictions on these specific corpora. However, these symbolic representations require transcription or automated conversation, which costs additional time and is subject to inaccuracies. Further, by reducing music to constituent features, some critical information that drives human perception may be lost (i.e., features are treated discretely, rather than continuously; aspects of timbre and expressivity cannot be represented). Most important for the present account, unless one is studying tonal music played on mallet instruments, percussive music is not so simply translated into discrete alphabets, as it might involve different ways of striking or preparing the same instrument to generate subtly different timbres, the incorporation of human voice, 
pitch bends, water gongs, and so on. Thus, a model that can operate on raw audio files, or continuously extracted acoustic features, would be ideal.

One additional issue with regard to input signal is not the data file format, but rather the amount of training data required to produce an estimate of expectation over time. Some models require training on a corpus of music to statistically learn the syntax of particular musical styles, while others can operate only a single piece in a purely bottom-up manner. Still others can operate on single pieces (taking into account only what has been heard of the piece so far) or use a previously trained model for defining initial probabilities. Again, whether these aspects are important, depends on one's question (e.g., modeling a naïve vs. well-experienced listener), and music of interest (e.g., monophonic MIDI representations from a style with copious examples vs. percussive music from a style which may not be represented by available corpora).

\section{Salience, Information Content, Surprisal, Novelty}

While each of the words in the above heading have slightly different meanings, especially when considered with respect to the mathematical equations they represent, their underlying basic concept is the same: a continuous (symbol-wise, or sample-wise) prediction of the allocation of a listeners' attention or expectations.

In some cases, however, such words may index slightly different constructs. For instance, in the model utilized in Hurley et al. (2018), high values of salience indicate a prediction of increased attention to that moment in time. On the other hand, in the model of Skerritt-Davis \& Elhilali (2018) high values of surprisal indicate moments when expectations have been violated. Information content (e.g., as implemented in van der Weij, 2017) indexes a construct synonymous to surprisal (i.e., an unexpected event likely to elicit a prediction error). All of these measures, in essence, should be correlated, in that moments of expectation violation re-orient attention. However, moments of high salience could be expected (as a culmination of the preceding context) and do not necessarily evoke an event-related potential in physiological data, as would be expected by moments of high surprisal.

Taking a slightly different, but related, semantic stance, the novelty metric (Lartillot, Cereghetti, Eliard \& Grandjean, 2013) from the music information retrieval (MIR) toolbox (Lartillot \& Toiviainen, 2007), is not so much grounded in ideas about listeners' attention but rather in notions of segmentation (i.e., how listeners might parse an acoustic signal into different sections). Novelty thus represents how likely one is to perceive a transition at any particular moment. This construct is related to attention / expectation, but will likely show a somewhat different relationship to physiological and behavioral activity than the previously defined measures.

\section{Models of Temporal Expectations}

I now turn to the task set forward at the beginning of this article: a model that can provide a continuous prediction of listener expectation over time for a recording of percussive music. Without finding some way to transcribe such a recording into a symbolic format, we are left with few options. I will briefly summarize three relevant models that can, in theory, accomplish the task, but, in practice, may require additional validation.

From a bottom-up, oscillatory framework, the extension of the Beyond the Beat model (Tomic \& Janata, 2008) validated in Hurley et al. (2018) and Fink et al. (2018) functions in the current context. Raw audio can be input to generate a prediction of salience over time. The prediction is calculated through a series of steps involving a cochlear model, amplitude envelope extraction and onset detection in multiple frequency bands, feeding onsets through a bank of oscillators (tuned to frequencies up to $10 \mathrm{~Hz}$ and exhibiting decay), and averaging the activity of only the most active oscillators at each point in time. This model has been shown to predict fluctuations in listeners' attention, measured via perceptual thresholds (Hurley et al., 2018) and pupil size (Fink et al., 2018), but still requires further validation against a broader range of stimuli and against other models (discussed below).

From the realm of music information retrieval, mirnovelty (MIR toolbox) may be useful in the current context, as it reflects changes in the high-level structure of music, by taking into account contrasts in temporal scale on an instant-by-instant basis. It can be calculated in a kernel-based or multi-granular (Lartillot et al., 2013) way, from the similarity matrix of the acoustic signal or particular acoustic features (i.e., by taking into account one, or many different, temporal windows of integration). Given that this measure indexes largerscale moments of structural change (i.e., the probability and importance of transitions between sections), one might expect behavioral or physiological measures related to "chunking" to most closely follow this prediction, though extensive testing against human behavior (Hartmann, Lartillot, \& Toiviainen, 2017) and/or physiology has yet to be conducted. 
From a Bayesian probabilistic framework, the Dynamic Regularity Extraction (D-REX) model of Skerritt-Davis \& Elhilali (2018) predicts changes in the underlying statistical structure of a stimulus. Its predictions can be based only on what has been processed for the current stimulus or informed by a previously trained model. D-REX builds probabilities about current events based on 1) the current context and 2 ) the probability that the context (underlying statistics) has changed. The model incorporates both a memory and noise parameter to more accurately simulate human perception. Input to the model is any continuous acoustic feature of choice; output is prediction error, or surprisal, over time. D-REX predictions have been validated against electroencephalographic data using stochastic, random, and regular monophonic stimuli. To date, only predictions based on pitch have been employed (see Skerritt-Davis \& Elhilali, 2018; 2019), though predictions based on other acoustic features, or even combined over multiple features is possible.

\section{Discussion}

At present, few models exist that can make continuous predictions of listener expectations from continuous audio stimuli. A systematic comparison of all relevant models, using the same stimuli, participants, and physiological experiments is required. It would also be ideal to also include symbolic models (e.g. Harrison et al., 2020) in such a comparison. However, this task is not simple, as equivalence needs to be achieved regarding discretized symbols vs. continuous acoustic features and model outputs. It may be the case that such comparisons are not so meaningful and that a more fruitful approach involves characterizing the particular research questions and musical styles for which each model is most well-suited. Future work along such lines will move the field forward.

Additionally, future models might incorporate deep learning approaches. At present, such models are used to generate novel music from training on tokens (e.g., MuseNet; Payne, 2019) or, impressively, on raw audio (e.g., WaveNet, Oord et al., 2016; Jukebox, Dhariwal et al., 2020). These models could be adapted to generate predictions about what will happen next in a given piece of music, rather than to generate novel music.

\section{Conclusion}

Inspiring computational advances have been made in the past decades to predict listeners' expectations while engaging with music. Future advances may involve:
- Generating predictions across multiple time scales, acoustic features, and perceptual classes (e.g., streams, textures).

- Pooling temporal expectations across multiple modalities. Musical engagement often involves a strong visual component, which can also shape expectations. Perhaps models of acoustic and visual saliency could be combined to study expectations in audiovisual contexts.

- Filtering predictions based on physiological limitations and processing asymmetries.

- Tuning predictions based on listener familiarity (both in terms of exposure to the particular stimulus in question, as well as the cultural context of the stimulus and listener).

\section{Acknowledgements}

I thank Lea Fink, Angela Nazarian, and Lindsay Warrenburg for helpful comments on an earlier version of this manuscript.

\section{References}

Baker, D. J., Belfi, A., Creel, S., Grahn, J., Hannon, E., Loui, P., ... \& Vuvan, D. T. (2020). Embracing Anti-Racist Practices in the Music Perception and Cognition Community. https://doi.org/10.1525/mp.2020.38.2.103

Boenn, G. (2018). Computational Models of Rhythm and Meter (pp. 1-187). Springer. https://doi.org/10.1007/9783-319-76285-2

Cancino-Chacón, C., Grachten, M., \& Agres, K. (2017). From Bach to the Beatles: The simulation of human tonal expectation using ecologically-trained predictive models. arXiv preprint arXiv:1707.06231.

Collins, T., Tillmann, B., Barrett, F. S., Delbé, C., \& Janata, P. (2014). A combined model of sensory and cognitive representations underlying tonal expectations in music: From audio signals to behavior. Psychological review, 121(1), 33. https://doi.org/10.1037/a0034695

Dhariwal, P., Jun, H., Payne, C., Kim, J. W., Radford, A., \& Sutskever, I. (2020). Jukebox: A generative model for music. arXiv preprint arXiv:2005.00341.

Elie, J. E., \& Theunissen, F. E. (2019). Invariant neural responses for sensory categories revealed by the timevarying information for communication calls. PLoS computational biology, 15(9), e1006698. https://doi.org/10.1371/journal.pcbi.1006698

Forth, J., Agres, K., Purver, M., \& Wiggins, G. A. (2016). Entraining IDyOT: Timing in the Information Dynamics of Thinking. Front Psychol, 7, 1575. https://doi.org/10.3389/fpsyg.2016.01575

Grosche, P., \& Muller, M. (2010). Extracting predominant 
local pulse information from music recordings. IEEE Transactions on Audio, Speech, and Language Processing, 19(6), 1688-1701. https://doi.org/10.1109/TASL.2010.2096216

Holzapfel, A. (2015). Relation between surface rhythm and rhythmic modes in Turkish Makam music. J. New Music Res. 44, 25-38. https://doi.org/10.1080/09298215.2014.939661

Holzapfel, A., Davies, M. E., Zapata, J. R., Oliveira, J. L., \& Gouyon, F. (2012). Selective sampling for beat tracking evaluation. IEEE Transactions on Audio, Speech, and Language Processing, 20(9), 2539-2548. https://doi.org/10.1109/TASL.2012.2205244

Harrison, P. M., Bianco, R., Chait, M., \& Pearce, M. T. (2020). PPM-Decay: A Computational Model of Auditory Prediction with Memory Decay. PLoS computational biology. https://doi.org/10.1371/journal.pcbi.1008304

Hartmann, M., Lartillot, O., \& Toiviainen, P. (2017). Musical feature and novelty curve characterizations as predictors of segmentation accuracy. In Proceedings of the Sound and Music Computing Conferences, Aaltoyliopisto.

Hurley, B., Fink, L., \& Janata, P. (2018). Mapping the dynamic allocation of attention in musical patterns. Journal of Experimental Psychology: Human Perception \& Performance, 44(11), 1694-1711. https://doi.org/10.1037/xhp0000563

Jacoby, N., Margulis, E. H., Clayton, M., Hannon, E., Honing, H., Iversen, J., ... \& Wald-Fuhrmann, M. (2020). Cross-cultural work in music cognition: Challenges, insights, and recommendations. Music Perception, 37(3), 185-195. https://doi.org/10.1525/mp.2020.37.3.185

Janata, P., Birk, J. L., Van Horn, J. D., Leman, M., Tillmann, B., \& Bharucha, J. J. (2002). The cortical topography of tonal structures underlying Western music. Science, 298(5601), https://doi.org/10.1126/science.1076262

$2167-2170$.

Klapuri, A., Eronen, A., \& Astola, J. T. (2006). Analysis of the meter of acoustic musical signals. IEEE Trans. Audio, Speech, Lang. Process. 14, 342-355. https://doi.org/10.1109/TSA.2005.854090

Large, E. W., Herrera, J. A., \& Velasco, M. J. (2015). Neural networks for beat perception in musical rhythm. Frontiers in systems neuroscience, 9, 159. https://doi.org/10.3389/fnsys.2015.00159

Large, E. W., \& Jones, M. R. (1999). The dynamics of attending: how people track time-varying events. Psychol. Rev. 106, 119-159. https://doi.org/10.1037/0033-295X.106.1.119

Large, E. W., and Kolen, J. F. (1994). Resonance and the perception of musical meter. Conn. Sci. 6, 177-208. https://doi.org/10.1080/09540099408915723
Lartillot, O., \& Grandjean, D. (2019). Tempo and metrical analysis by tracking multiple metrical levels using autocorrelation. Applied Sciences, 9(23), 5121. https://doi.org/10.3390/app9235121

Lartillot, O., \& Toiviainen, P. (2007). "A Matlab Toolbox for musical feature extraction from audio." International Conference on Digital Audio Effects, Bordeaux.

London, J., Polak, R., and Jacoby, N. (2016). Rhythm histograms and musical meter: a corpus study of Malian percussion music. Psychon. Bull. Rev. 24, 474-480. https://doi.org/10.3758/s13423-016-1093-7

London, J. (2012). Hearing in time: Psychological aspects of musical meter. Oxford University Press. https://doi.org/10.1093/acprof:oso/9780199744374.001. $\underline{0001}$

Margulis, E. H. (2014). On repeat: How music plays the mind. Oxford University Press. https://doi.org/10.1093/acprof:oso/9780199990825.001. 0001

Masquelier T. (2018). STDP allows close-to-optimal spatiotemporal spike pattern detection by single coincidence detector neurons. Neuroscience, 389, 133140. https://doi.org/10.1016/j.neuroscience.2017.06.032

Oord, A. V. D., Dieleman, S., Zen, H., Simonyan, K., Vinyals, O., Graves, A., ... \& Kavukcuoglu, K. (2016). Wavenet: A generative model for raw audio. arXiv: 1609.03499 .

Payne, Christine. "MuseNet." OpenAI, 25 Apr. 2019, openai.com/blog/musenet

Pearce, M. T. (2005). The construction and evaluation of statistical models of melodic structure in music perception and composition (Doctoral dissertation, City University London).

Pearce, M. T., \& Wiggins, G. A. (2012). Auditory expectation: the information dynamics of music perception and cognition. Topics in cognitive science, $4(4), \quad 625-652 . \quad$ https://doi.org/10.1111/j.17568765.2012.01214.x

Skerritt-Davis, B., \& Elhilali, M. (2018). Detecting change in stochastic sound sequences. PLoS computational biology, 14(5), e1006162. https://doi.org/10.1371/journal.pcbi.1006162

Skerritt-Davis, B., \& Elhilali, M. (2019). A model for statistical regularity extraction from dynamic sounds. Acta Acustica united with Acustica, 105(1), 1-4. https://doi.org/10.3813/AAA.919279

Smith, L., \& Honig, H. (2008). Time-frequency representation of musical rhythm by continuous wavelets. Journal of Mathematics and Music, 2(2), 81-97. https://doi.org/10.1080/17459730802305336

Temperley, D. (2010). Modeling common-practice rhythm. Music Perception, 27(5), 355-376. https://doi.org/10.1525/mp.2010.27.5.355 
Tomic, S. T., \& Janata, P. (2008). Beyond the beat: Modeling metric structure in music and performance. The Journal of the Acoustical Society of America, 124(6), 4024-4041. https://doi.org/10.1121/1.3006382

Tomková, M., Tomek, J., Novák, O., Zelenka, O., Syka, J., $\&$ Brom, C. (2015). Formation and disruption of tonotopy in a large-scale model of the auditory cortex. Journal of computational neuroscience, 39(2), 131-153. https://doi.org/10.1007/s10827-015-0568-2

van der Weij, B., Pearce, M. T., \& Honing, H. (2017). A probabilistic model of meter perception: Simulating enculturation. Frontiers in psychology, 8, 824. https://doi.org/10.3389/fpsyg.2017.00824

Volk, A. (2008). The study of syncopation using inner metric analysis: Linking theoretical and experimental analysis of metre in music. Journal of New Music Research, 37(4), 259-273. https://doi.org/10.1080/09298210802680758

Vuust, P., \& Witek, M. A. (2014). Rhythmic complexity and predictive coding: a novel approach to modeling rhythm and meter perception in music. Frontiers in psychology, 5, 1111. https://doi.org/10.3389/fpsyg.2014.01111 


\title{
Social music: Investigating the link between personal liking and perceived groove
}

\author{
Douglas Kowalewski ${ }^{1 \dagger}$ \\ Taylor Kratzer ${ }^{1}$ \\ Ronald Friedman ${ }^{1}$ \\ ${ }^{1}$ University at Albany, SUNY, Albany, NY, USA \\ $\dagger$ Corresponding author: dkowalewski@albany.edu \\ Published 16 December 2021; https://doi.org/10.18061/FDMC.2021.0042 \\ Author video presentation and/or other conference material: https://doi.org/10.17605/OSF.IO/Z8E35
}

\begin{abstract}
Integrating methods from experimental social psychology and music perception, we tested the hypothesis that when listeners personally like a musician, they will be more inclined to experience his or her music as both provoking movement and as subjectively pleasurable, the two core features of perceived groove. In Experiment 1, participants were exposed to a set of moderately-syncopated, high-groove drum-breaks which they were led to believe were either produced by a relatively likable or unlikable musician. In line with predictions, participants led to find the musician more versus less likable rated the same drum-breaks as more evocative of both the urge to move and of feelings of pleasure. When participants in a follow-up study (Experiment 2) were administered the exact same manipulation of likability, but exposed to highly syncopated, low-groove drum-breaks, these effects were eradicated, suggesting that the results of Experiment 1 were not merely due to demand characteristics or response biases. Together, these findings support the notion that listeners are more responsive to "participating in the music" when they are relatively motivated to affiliate with the musician(s). Methodological limitations and directions for future research on the social psychology of "the groove" are discussed.
\end{abstract}

KEYWORDS: syncopation, groove, affiliation, liking, movement

\section{Introduction}

In a seminal article, Janata et al. (2012) defined musical groove as a pleasurable urge to move the body in response to music. In recent years, a number of studies have been conducted to identify the aspects of musical structure that promote the experience of groove. For instance, Witek and her colleagues (Witek, 2017; Witek et al., 2014) have focused on the role of syncopationthe appearance of musical onsets at metrically unexpected moments - showing that the pleasurable desire to move is most associated with moderately syncopated music. According to Witek (2017), a common source of groove-inducing syncopation is created by the "...superimposition of two rhythms with different metric tendencies, such as $4 / 4$ against 3/4" (p. 141). She theorizes that such polyrhythmic structures create "gaps" in which metric accents are not accompanied by objective auditory onsets, motivating listeners to move their bodies in order to physically enact the missing beats. Notably, Witek (2017) proposes that gaps in the musical surface do not compel movement, but rather, invite listeners' participation in the music, implying that listeners must choose whether to accept this invitation or not (see also, Levitin et al., 2018; Senn et al., 2018). [1]

Given Witek's (2017) analysis, the question arises: What determines whether listeners will answer this implicit call to participate in the music? One potential clue may be found in the theorizing of Janata et al. (2012), who posit that since "...music implies the actions of a group (the musicians)... high-groove music [may serve] as an invitation to join the group by virtue of inducing an urge to move along with [their] actions" (p. 71; emphasis added). Restated, the groove may be at least partially driven by a desire to affiliate with the musician(s). This suggests that the experience of groove should be heightened when affiliation motives are stronger. One well-established predictor of the desire to affiliate is personal liking-individuals are most inclined to connect with others whom they like and to avoid interacting with those whom they dislike (e.g., Likowski et al., 2008).

Interestingly, a general link between affiliation motives and body movement has been established in social psychological research on behavioral mimicry. Specifically, Likowski et al. (2008) have found that when individuals like others, they are automatically more inclined to imitate their facial expressions of emotion. Likewise, Lakin and Chartrand (2003) have shown that when individuals are induced to adopt the goal of affiliating with others, they are subsequently more likely to mimic a stranger's body language. 
According to Duffy and Chartrand (2015), mimicry of this sort has important social functions, helping individuals to understand the behavior that they are mimicking and allowing them to nonverbally convey this understanding to those whom they emulate. As a result, mimicry often fosters liking, rapport, and prosociality among interacting individuals, and is therefore associated with a range of social rewards.

Integrating these observations, we hypothesize that when listeners find musicians more likable, they will be more motivated to affiliate with them and thereby more responsive to participating in the music that they create or perform. In turn, this should facilitate the urge to move, at least when the music invites "gap filling" via moderate syncopation (Witek, 2017). Inasmuch as moving to the music may be construed as a form of mimicry - of moving along with the virtual actions of the musician (Janata et al., 2012) - experiencing a heightened urge to move may also engender positive affect inasmuch as mimicry is often socially rewarding (Duffy \& Chartrand, 2015). In sum, we predicted that when they like the musician, individuals will be more likely to experience their music as stimulating movement and as subjectively pleasurable, the two core features of musical groove (Janata et al., 2012). We tested this proposition in a large-scale lab experiment, in which we manipulated the likability of the musician and measured the amount of groove participants perceived in the music that he had ostensibly produced.

\section{Experiment 1: Method}

\section{Participants}

Participants were 201 students from the University at Albany (114 female; Age: $M=19.14, S D=2.82$ ) who completed the study for partial course credit in an introductory psychology course. Fifty-one (25.4\%) reported having at least one year of formal training in music theory and $122(60.7 \%)$ reported at least one year of formal training on a musical instrument.

\section{Materials}

Musical stimuli consisted of 6 moderately syncopated, two-measure-long drum-breaks adapted from Witek et al. (2014; see Supporting Information for musical notation and syncopation measures; see also Kowalewski et al., 2020 for full listing of individual stimuli). Each drum-break was looped four times and played at $120 \mathrm{BPM}$ for a total duration of $16.5 \mathrm{~s}$. The stimuli were transcribed into Sibelius First music notation software (v. 2018.7) and exported as MIDI files, which were played through Sibelius' virtual instrument sample banks (high-hat, snare, and bass drum) and recorded as WAV files.

\section{Procedure}

Upon arrival at the lab, participants were seated at visually isolated computer workstations and instructed to put on a pair of headphones (Koss UR-20). They were then provided with the cover story:

The music you will be hearing today has been made by students at UAlbany who produced music for an electronic music course they took last semester. The students were also asked about their experiences at UAlbany and their impressions of the music course. You will later be asked about these details and how they compare to your own experiences. The names of the students have been changed to protect their identity. You will listen to several rhythms made by each student. Please listen to each rhythm and answer the questions that follow.

Participants were then sequentially presented with the names (Matthew, Jacob, and Martin) and classes (sophomore or junior) of each musician, as well as their purported comments regarding the music course. The comments made by the first two musicians were simply included to bolster the plausibility of the cover story: However, the comments of the third musician (Martin) were designed to manipulate his likability by suggesting that he either loved or hated the university that participants were attending and toward which they tend to have highly favorable attitudes. Specifically, for participants randomly assigned to the High Likability group, Martin commented, "I love UAlbany! I have always had a passion for music and I feel at home in this class!"; whereas, for those assigned to the Low Likability group, Martin instead commented, "Honestly, I hate UAlbany. I have always had a passion for music, but I don't feel at home in this class." Decades of social psychological research has established that attitudinal (dis)similarity robustly predicts (dis)liking (e.g., Byrne, 1971).

As the information regarding a particular musician remained on screen, participants listened to two of the abovementioned drum-breaks, which were ostensibly created by this musician. Following the procedure of Witek et al. (2014), to assess perceived groove, after each drum-break, participants were asked, "To what extent did this rhythm make you want to move?" and "How much pleasure did you experience listening to this rhythm?" on a 7-point Likert scale anchored at 1 (not at all) and 7 (very much). After evaluating Martin's rhythms, as a manipulation check, participants were 
asked to rate their attitudes toward all of the musicians in reverse order of appearance. Finally, participants completed measures of age, gender, and music training (years of formal training in music theory and on a musical instrument), as well as a measure of attitudes toward the University at Albany (see Kowalewski et al., 2020 for exact wording of all items).

\section{Experiment 1: Results}

According to independent samples $t$-tests, compared to those in the Low Likability $(\mathrm{n}=101)$ group, participants in the High Likability group $(\mathrm{n}=100)$ rated themselves as more apt to like the critical musician $\left(M_{\text {High }}=4.79\right.$, $S D=1.22 ; M_{\text {Low }}=3.88, S D=1.44 ; t[194.05]=4.83, p$ $\left.<.0001, \eta_{\mathrm{p}}{ }^{2}=.11\right)$, and to like interacting with him $\left(M_{\text {High }}=4.58, S D=1.11 ; M_{\text {Low }}=3.65, S D=1.45\right.$; $\left.t[187.18]=5.08, p<.0001, \eta_{\mathrm{p}}{ }^{2}=.12\right)$, confirming that the manipulation of likability was successful. In addition, participants' self-reported liking for $(M=2.68$, $S D=1.51)$ and feelings about $(M=2.41, S D=1.29)$ the University at Albany were significantly below the (reverse-coded) scale midpoint, $t$ 's $>12.42, p$ 's $<.0001$, suggesting highly favorable attitudes toward the institution. This supports the assumption that (dis)liking for the critical musician was shaped by perceived differences in attitudinal (dis)similarity.

As in prior research (e.g., Witek et al., 2014), ratings of the two components of groove, urge to move and listening pleasure, were highly correlated overall, $r(199)$ $=.86, p<.0001$. However, following Witek et al. (2014), we analyzed these measures separately. Consistent with predictions, analyses of variance (ANOVAs) revealed that participants in the High versus Low Likability group rated the drum rhythms ostensibly produced by the critical musician as eliciting a significantly greater urge to move $\left(M_{\mathrm{High}}=4.49, S D=\right.$ $1.36 ; M_{\text {Low }}=4.09, S D=1.42 ; F[1,199]=4.09, p<.05$, $\left.\eta_{\mathrm{p}}^{2}=.02\right)$, as well as more listening pleasure $\left(M_{\mathrm{High}}=\right.$ $4.44, S D=1.42 ; M_{\text {Low }}=3.96, S D=1.52 ; F[1,199]=$ $5.19, p<.03, \eta_{\mathrm{p}}{ }^{2}=.03$ ). Groove ratings for rhythms ostensibly produced by control musicians (i.e., those for whom liking was not manipulated) did not differ between groups, all $p$ 's $>.17$.

Although these initial results were consistent with our hypothesis, they did leave open at least two alternative explanations. First, the findings may have stemmed from a "halo" effect (Thorndike, 1920), in which participants were biased to rate the music of the likable musician more favorably, irrespective of its structure and, most critically, whether or not it invited movement via moderate syncopation (Witek, 2017).
Second, it is possible that despite our attempts to obscure the true purpose of the study using a deceptive cover story and a between-participants likability manipulation, participants were nonetheless able to infer the hypothesis, leading them to artificially modify their groove ratings as a response to perceived experimenter demand. To address these concerns, we conducted a follow-up experiment in which high syncopation stimuli (i.e., low groove; Witek et al., 2014) were used. In addition, to measure levels of suspicion, we appended a funnel debriefing to the end of the experimental procedure (e.g., Blackhart \& Clark, 2019).

\section{Experiment 2: Method}

\section{Participants}

Participants were 181 students from the University at Albany (134 female; Age: $M=18.76, S D=1.22$ ) who completed the study for partial course credit in an introductory psychology course. Fifty-six (30.9\%) reported having at least one year of formal training in music theory and $110(60.8 \%)$ reported at least one year of formal training on a musical instrument. Thirteen participants were excluded from the analysis based on the results of the suspicion check (see below), leaving 168 participants (125 female). None of the participants had participated in Experiment 1.

\section{Materials and Procedure}

Materials and procedure for Experiment 2 were identical to those for Experiment 1 with two exceptions: First, the moderately syncopated drum-breaks used for the critical musician were replaced with 2 highly syncopated, lowgroove drum-breaks composed by Witek (see Kowalewski et al., 2020). Again, the order of presentation of these drumbreaks was counterbalanced between participants. Second, immediately prior to debriefing, participants were administered a "funneled" suspicion check in which general questions about the study gradually gave way to a more direct inquiry regarding suspicions, thereby giving participants a set of graded opportunities to "confess" any doubts regarding the cover story as well to reveal any beliefs regarding the true nature of the hypothesis (see Bargh \& Chartrand, 2000; Blackhart \& Clark, 2019).

\section{Experiment 2: Results}

Examination of responses to the post-experimental suspicion check revealed that 13 participants $(7.2 \%)$ correctly inferred that the study was aimed at testing 
whether attitudes toward the musician influenced ratings of his music. Although this lends credence to the possibility that experimenter demand is capable of influencing responses within this experimental paradigm, it indicates that participants generally appear to have believed the cover story and failed to guess the hypothesis. As alluded to above, the responses of these 13 participants were excluded from the analyses. The results reported below did not differ based on these exclusions. As in Experiment 1, independent samples $t$ tests revealed that the likability manipulation was successful (see Kowalewski et al., 2020 for detailed statistics on this manipulation check).

Groove ratings were analyzed exactly as in Experiment 1. In contrast to the results of the first experiment, ANOVAs revealed no significant differences between participants in the High and Low Likability groups either for ratings of the urge to move $\left(M_{\text {High }}=1.94, S D=1.19 ; M_{\text {Low }}=1.73, S D=0.98 ; F[1\right.$, $\left.166]=1.70, p=.20, \eta_{\mathrm{p}}{ }^{2}=.01\right)$ or listening pleasure $\left(M_{\text {High }}=1.89, S D=1.12 ; M_{\text {Low }}=1.66, S D=0.97 ; F[1\right.$, $\left.166]=1.88, p=.17, \eta_{\mathrm{p}}{ }^{2}=.01\right)$. This failure to replicate the results of Experiment 1 using highly syncopated stimuli suggests that the impact of Likability on groove ratings is sensitive to musical structure and is not merely an artifact of halo effects or demand characteristics.

\section{Discussion}

In this study, we tested the hypothesis that when listeners have relatively positive attitudes toward a musician, they will be more inclined to experience his or her music as provoking movement and as subjectively pleasurable, the two core features of perceived groove (Janata et al., 2012). To this end, in Experiment 1, we exposed participants to a set of moderately-syncopated drumbreaks and experimentally manipulated the likability of the musician who had ostensibly created them. In line with predictions, participants led to find the musician more versus less likable rated the same drumbreaks as more evocative of both the urge to move and of feelings of pleasure.

Results of a follow-up experiment using highsyncopation, low-groove stimuli (Experiment 2) revealed no effect of induced motivation to affiliate with the musician on either the urge to move or feelings of pleasure, suggesting that participants did not indiscriminately rate the music of more likable musicians more favorably. An added suspicion check did show that at least some participants were capable of explicitly guessing the connection between the experimental manipulation and the dependent measures. However, the null results of Experiment 2 suggest that the demand characteristics associated with the likability manipulation cannot account for the significant impact of likability on perceived groove found in Experiment 1. As such, the present findings remain consistent with the notion that moderately syncopated music extends an invitation to move (Witek, 2017) and that this invitation is more likely to be accepted when the music is associated with someone with whom listeners would prefer to affiliate.

Although the present study may be the first to empirically support a link between personal liking and groove, it is also subject to a number of methodological limitations that will need to be addressed in future research. First and foremost, it did not gauge actual movement in response to the musical stimuli. In at least a handful of studies, self-reported groove ratings have been supplemented with objective measurements of body movement based on video coding (e.g., Janata et al., 2012) or psychophysiological indices of motor system activation (e.g., motor-evoked potentials; Stupacher et al., 2013). Conceptual replication of the present findings using a measure of overt movement would provide more conclusive evidence for our hypothesis by objectively confirming that the selfreported urge to move is not merely a response bias.

Although the present study only focused on affiliation motives based on attitudinal (dis)similarity, a number of other social psychological factors might also bear on the desire to affiliate with musicians, thereby impacting the urge to move to their music. For instance, listeners may be more inclined to form a personal connection with, and thereby to participate in the music produced by a musician with whom they share social group membership based on ethnicity, gender, race, or class. Likewise, the (un)favorability of the stereotypes that individuals possess regarding members of different groups may affect whether they will emotionally and/or physically engage with music produced by a member of the group, particularly when "individuating" information regarding the musician is limited.

The specific contents of stereotypes might contribute to perceptions of groove more directly, irrespective of their influence on affiliation motives. For example, research has shown that African Americans are commonly stereotyped as superior in rhythmic ability (Plous \& Williams, 1995). This raises the possibility that listeners may be, however implicitly, biased to perceive music produced by black musicians as higher in groove simply due to their expectation that such 
music is likely to be more "rhythmic" or danceable ("positive" stereotypes; see Czopp et al., 2015).

\section{Conclusion}

The present study suggests that the experience of groove does not merely involve an automatic compulsion to move elicited by particular musical structures. Rather, it at least partially reflects the desire to affiliate with the musician(s) by synchronizing one's movement with the sounds that they produce - the stronger the motive to affiliate, the greater the urge to move to the music and the more pleasure it evokes. To be clear, this does not imply that the structure of the music itself is irrelevant to groove - as discussed earlier, some forms of music may be more likely to set the stage for movement, for instance, by creating "gaps" within a syncopated metrical framework that can be filled by moving the body in synchrony with the beat (Witek, 2017). Consistent with this assumption, we found no effect of musician likability when the critical musical stimuli were too high in syncopation to elicit an urge to move. This suggests that the impact of musical structure on groove may be moderated by the implicit or explicit "sense of social interaction" (Janata et al., 2012, p. 71) inherently associated with musical engagement. We hope that our findings will spur additional research aimed at elucidating the potential social-psychological underpinnings of "the groove".

\section{Acknowledgements}

We thank Faith Gaillard, Tinotenda Muchenje, Hannah Pell, Julian Pezzulo, Ryley Scott, Estelle Song, and Sara Wosnjuk for their help running these experiments.

\section{End Notes}

[1] This proceedings article is based on Kowalewski et al. (2020), which contains a more comprehensive description of the research program outlined here.

\section{References}

Bargh, J. A., \& Chartrand, T. L. (2000). The mind in the middle: A practical guide to priming and automaticity research. In H. T. Reis \& C. M. Judd (Eds.), Handbook of research methods in social and personality psychology (pp. 253-285). Cambridge University Press.

Blackhart, G. C., \& Clark, T. D. (2019). Suspicion probes and debriefing in the social and behavioral sciences. In J. Edlund \& A. Nichols (Eds.), Advanced research methods for the social and behavioral sciences (pp. 129-144). Cambridge University

Press. https://doi.org/10.1017/9781108349383.011

Byrne, D. (1971). The ubiquitous relationship: Attitude similarity and attraction: A cross-cultural study. Human Relations, 24, 201-207. https://doi.org/10.1177/001872677102400302

Czopp, A. M., Kay, A. C., \& Cheryan, S. (2015). Positive stereotypes are pervasive and powerful. Perspectives on Psychological Science, 10, 451-463. https://doi.org/10.1177/1745691615588091

Duffy, K. A., \& Chartrand, T. L. (2015). The extravert advantage: How and when extraverts build rapport with other people. Psychological Science, 26, 1-8. https://doi.org/10.1177/0956797615600890

Janata, P., Tomic, S. T., \& Haberman, J. M. (2012). Sensorimotor coupling in music and the psychology of the groove. Journal of Experimental Psychology: General, 141, 54-75. https://doi.org/10.1037/a0024208

Kowalewski, D. A., Kratzer, T. M., \& Friedman, R. S. (2020). Social music: Investigating the link between personal liking and perceived groove. Music Perception, 37, 339-346. https://doi.org/10.1525/mp.2020.37.4.339

Lakin, J. L., \& Chartrand, T. L. (2003). Using nonconscious behavioral mimicry to create affiliation and rapport. Psychological Science, 14, 334-339. https://doi.org/10.1111/1467-9280.14481

Levitin, D. J., Grahn, J. A., \& London, J. (2018). The psychology of music: Rhythm and movement. Annual Review of Psychology, 69, 51-75. https://doi.org/10.1146/annurev-psych-122216-011740

Likowski, K. U., Mühlberger, A., Seibt, B., Pauli, P., \& Weyers, P. (2008). Modulation of facial mimicry by attitudes. Journal of Experimental Social Psychology, 44, 1065-1072. https://doi.org/10.1016/j.jesp.2007.10.007

Plous, S. \& Williams, T. (1995). Racial stereotypes from the days of American slavery: A continuing legacy. Journal of Applied Social Psychology, 25, 795-817. https://doi.org/10.1111/j.1559-1816.1995.tb01776.x

Senn, O., Kilchenmann, L., Bechtold, T., \& Hoesl, F. (2018). Groove in drum patterns as a function of both rhythmic properties and listeners' attitudes. PLoS ONE, 13, 1-33. https://doi.org/10.1371/journal.pone.0199604

Stupacher, J., Hove, M. J., Novembre, G., Schütz-Bosbach, S., \& Keller, P. E. (2013). Musical groove modulates motor cortex excitability: A TMS investigation. Brain and Cognition, 82, 127-136. https://doi.org/10.1016/j.bandc.2013.03.003

Thorndike, E. L. (1920). A constant error in psychological ratings. Journal of Applied Psychology, 4, 25-29. https://doi.org/10.1037/h0071663

Witek, M. A. G. (2017). Filling in: Syncopation, pleasure and distributed embodiment in groove. Music Analysis, 36, 138-160. https://doi.org/10.1111/musa.12082 
Witek M. A. G., Clarke, E. F.,Wallentin, M., Kringelbach, M. L., \& Vuust, P. (2014). Syncopation, body-movement and pleasure in groove music. PLOS ONE, 9, 1-12. https://doi.org/10.1371/journal.pone.0094446 


\title{
Effect of metrical primes on perceived complexity of 2:3 and 3:4 polyrhythms
}

\author{
Zachary Lookenbill ${ }^{1 \dagger}$ \\ Leigh VanHandel ${ }^{2}$ \\ ${ }^{1}$ Michigan State University, East Lansing, MI, USA \\ ${ }^{2}$ University of British Columbia, Vancouver, BC, Canada \\ ${ }^{\dagger}$ Corresponding author: lookenb1@msu.edu \\ Published 16 December 2021; https://doi.org/10.18061/FDMC.2021.0043 \\ Author video presentation and/or other conference material: https://doi.org/10.17605/OSF.IO/GXM8C
}

\begin{abstract}
Previous empirical studies concerning the perception of polyrhythms have isolated the rhythms from a metrical context to observe the meter associated with each rhythm. These studies show the important effect pitch and tempo have on identifying meter (Handel \& Oshinsky, 1981; Handel \& Lawson, 1983). For example, listeners tend to tap the 3-pulse layer in a 2:3 polyrhythm except at fast tempos where the 2pulse layer is preferred. (Handel \& Oshinsky, 1981). This study extends prior research on polyrhythms by focusing on the metrical context in which they are presented, emulating realistic musical environments. The current methodology has participants rating the complexity of the pattern and how well the polyrhythm fits with the metrical prime. My hypothesis is that a metrical prime will overwhelm any previous preference for meter, and patterns placed in simple meters will be perceived as less complex than in compound meters. Results indicate listeners do have a preference of meter in both $2: 3$ and 3:4 polyrhythms. Listeners also rated the complexity of the stimuli lower in simple meters for $2: 3$, but this relationship is not clear in 3:4. Discussion considers the implication of the results on music pedagogy and performance of polyrhythms.
\end{abstract}

KEYWORDS: polyrhythm, meter, perception, complexity, performance strategies

\section{Introduction}

The existing music cognition literature on polyrhythms has illuminated the complexity and variability involved in a person's ability to interpret or replicate multiple rhythmic streams. Previous experiments vary in their methodology; some require participants to replicate polyrhythmic patterns to observe performance and attentional strategies, while others explore beat perception and meter entrainment by having subjects tap a steady beat to various polyrhythms. I will review a variety of literature exploring both polyrhythm performance and perception to identity some general trends that have formed the present understanding of this research area, but I will also identify some gaps in the literature that this experiment hopes to answer.
Handel and colleagues (Handel \& Oshinsky, 1981; Handel \& Lawson, 1983) conducted multiple studies observing meter perception in various polyrhythms. Since a stream of two rhythms may suggest two meters, these studies examined which meter a listener might hear for specific polyrhythms. They asked participants to tap a steady beat along with the rhythmic stimuli at a variety of tempos. Many participants tapped along with a single pulse stream, but some tapped a replication of the stimuli, and others tapped hypermetrically. Often, their metrical preference was influenced by the construction of the polyrhythm, the tempo, the pitch of each stream, and the patterns of accents added to the rhythm, demonstrating the number of variables affecting our perception of meter in polyrhythms. Other researchers have used similar methodologies and agree that there are many factors influencing beat perception in polyrhythms (Beauvillain, 1983; Moelants \& Noorden, 2005).

A later study by Jones et. al. (1995) suggests two attentional strategies when listening to two rhythmic streams: integrative or streaming. They found integrating both streams into one coherent pattern was only useful with a small pitch interval between the rhythmic streams, while larger pitch intervals resulted in streaming, and the listener would track one of the two streams. Fidali, Poudrier, and Repp (2013) expanded this study to include more complex polyrhythms to see if integrative attending can handle the cognitive load created by the complex stimuli. They found integrating a polyrhythmic pattern typically results in a more acute attention, although this strategy was not able to handle the memory demands created by complex polyrhythms.

In contrast to these perceptual and attentional studies, other researchers have focused on replication and performance accuracy of polyrhythms. Pressing, Summers, and Magill (1996) demonstrate one strategy available to musicians performing polyrhythms, figureground separation. "For a polyrhythm consisting of two separate rhythmic streams, the elements of one stream act as a ground, whereas the elements of the other stream 
act as figural elements perceived in relation to the ground" (pg. 1127). They had participants perform a 4:3 polyrhythm using different combinations of the figureground model and found that subjects were most accurate in their performance of the ground figure.

Similarly, a study by Peters and Schwartz (1989) had participants tap a 2:3 polyrhythm with both hands while counting aloud to one of the two streams. They found that polyrhythmic performance was most accurate when attending to and counting the 3-stream. They also considered the method by which participants determined the placement of each onset. Their results suggested that the timing of each onset of the figure hand was dependent upon the placement of the previous onset from the ground hand. Peters and Schwartz conclude that "subjects do not perform the two sequences independently; the initiation of movement in one hand is more clearly depended on the preceding movement in the other hand than on the preceding movements by the same hand" (pg. 215). Krampe et. al. (2000) elaborates on this approach and suggests two models: integrated timing and parallel timing. Integrated timing allows performers to find the placement of one note on one hand in relation to the previous note, regardless of which stream it is in. In the parallel timing model, each hand tracks the intervals of its own streams, both at the same time.

This brief survey demonstrates several key principles, including the variability and complexity of mental and motor processes involved with polyrhythms. I have observed however, that with the exception of a few studies (Keller \& Burnham, 2005; Poudrier, 2017) most experiments used polyrhythmic stimuli in isolation, rather than in a musical context. To address this gap in the experimental literature, this study focuses on polyrhythmic patterns presented in metrical contexts to determine how ecological validity affects polyrhythmic perception. Ultimately, I hope to inform musicians in their musical practice. The conclusions from this study may not only serve as a glimpse into the process of hearing and performing polyrhythms, but also encourage different strategies of approaching polyrhythms when learning and performing them.

\section{Method}

Participants

Forty subjects were recruited through multiple institutions and streams of social media $(22 \mathrm{~F}, 17 \mathrm{M}, 1$ unreported). The participants' average age was 21.6 years $(S D=3.57)$. Six participants did not provide their age. Most participants had some musical training; the average number of years of private music lessons was 9 $(S D=6.13)$ and the average number of semesters of music theory coursework was 3.6 semesters $(S D=4.65)$.

\section{Stimuli}

Rhythmic stimuli were created using a TR-808 virtual drum machine from onemotion.com (n.d.). The stimuli were comprised of a single rhythmic stream consisting of the composite rhythm of $2: 3$ or $3: 4$ polyrhythms. These composite rhythms were created using the snare drum sound.

The composite rhythm, which is a result of two unequal isochronous streams, can be perceived in one of two metrical contexts. Metrical primes were given in two of the three conditions which suggested one of the two possible meters in order to observe which metrical framework was preferred by the subject. The metrical prime used the hi-tom sound which was distinct in pitch and timbre from the snare drum. In the A condition, no metrical prime was used and the polyrhythms were repeated eight times. In the $\mathrm{B}$ and $\mathrm{C}$ conditions different metrical primes preceded the polyrhythms for four measures of the suggested meter and continued throughout the eight repetitions of the polyrhythm. The $\mathrm{B}$ condition received a prime of the faster of the two streams. The $\mathrm{C}$ condition received a prime of the slower of the two streams. The B prime for 2:3 implies a simple triple meter, $3 / 4$, while the $\mathrm{C}$ prime implies compound duple meter, 6/8. The $\mathrm{B}$ prime for $3: 4$ implies a compound quadruple meter, $12 / 8$, and the $\mathrm{C}$ prime implies a simple triple meter, $3 / 4$. Musical notation of the stimuli are shown in Figure 1. Stimuli were presented at three different rates and are described in BPM of the slower of the two streams. The 2:3 polyrhythm was presented with the 2-stream $=50,80$, and 120 BPM. The 3:4 polyrhythm was presented with the 3 -stream $=60,90$, and $136 \mathrm{BPM}$.

\section{Procedure}

Participants completed the experiment on their own devices through a Qualtrics survey and were encouraged to use headphones and locate themselves in a quiet place. They completed a short demographic survey that included questions about musical expertise. Subjects then proceeded through two blocks of stimuli and questions. The first block had 2:3 and 3:4 polyrhythms without a metrical prime, or condition $\mathrm{A}$, at all three tempos. The order of stimuli was randomized. Participants were asked to listen to the stimuli and rate how complex the rhythm is. They were told to think how complicated it would be to replicate the rhythm and 
were encouraged to tap a steady beat with the rhythm. The six-point complexity scale was labeled with descriptors of very simple - simple - somewhat simple - somewhat complex - complex - very complex.

In the second block, 2:3 and 3:4 polyrhythms were played with either of the metrical primes, $\mathrm{B}$ or $\mathrm{C}$, at three tempos, again presented in a random order. Participants were asked to tap along with the metrical prime, rate how well the rhythm fits with the provided steady beat, and rate the complexity of the rhythm. The fit of the rhythm with the prime was on a six-point numerical scale with 1 described as "does not fit" and 6 described as "fits extremely well."

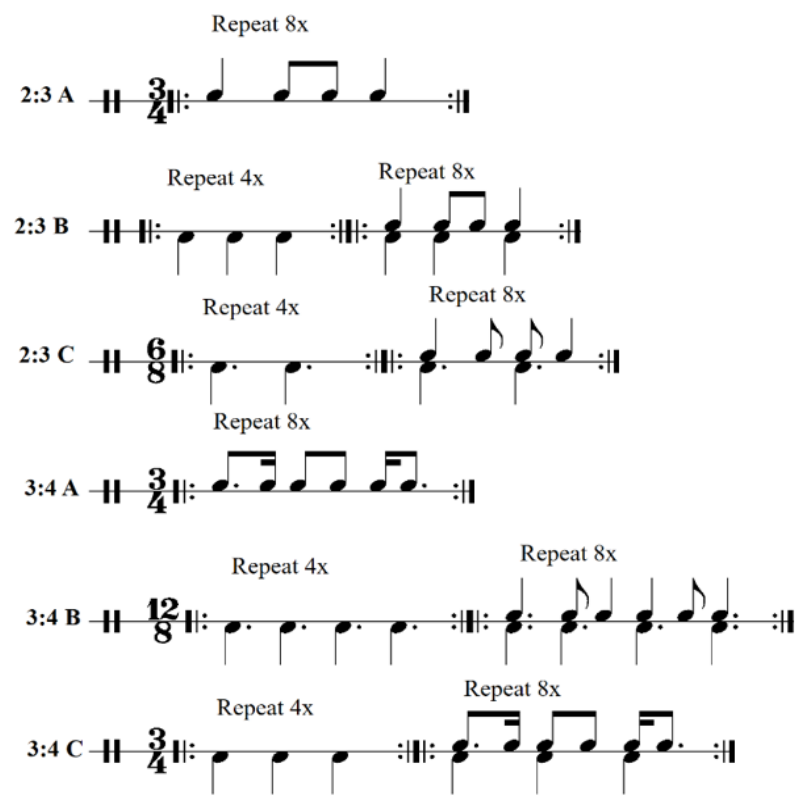

Figure 1: Notated stimuli. Composite polyrhythm is above the line, metrical prime is below.

\section{Results}

Table 1 shows the average fit and complexity ratings in all conditions. See Figures 2-5 for visual representations of the data.

\section{Metrical Preference}

For the 2:3 polyrhythm, participants rated the stimuli as fitting with the 3-layer prime significantly better than the 2-layer prime in the slow and medium tempo conditions, with $p<.05$ for both tempo conditions. However, for the 2:3 polyrhythm at the fast tempo, participants did not show any preference for metrical prime, $p=1$. Additionally, there was a significant effect of tempo on fit ratings with the 3-layer prime, which at the fast tempo is significantly lower than at the medium and slow tempos, $(p<.05$ for both $)$.

For the 3:4 polyrhythm participants rated the rhythm as fitting better with the 3-layer than the 2-layer in all tempo conditions, with $p<.05$ for all tempo conditions. There was no significant effect of tempo on the fit ratings for 3:4 polyrhythms, $p>.05$.

Table 1: Average fit and complexity ratings for $2: 3$ and 3:4 stimuli in all context and tempo conditions.

\begin{tabular}{|c|c|c|c|c|c|c|}
\hline & & & \multicolumn{2}{|c|}{ Complexity } & \multicolumn{2}{|c|}{ Fit } \\
\hline & & & $M$ & $S D$ & $M$ & $S D$ \\
\hline \multirow{9}{*}{$2: 3$} & \multirow{3}{*}{ Slow } & No Prime & 1.38 & 0.70 & --- & -- \\
\hline & & 2-Layer & 2.48 & 1.26 & 3.95 & 1.74 \\
\hline & & 3-Layer & 1.53 & 0.72 & 5.58 & 0.71 \\
\hline & \multirow{3}{*}{ Medium } & No Prime & 1.98 & 0.95 & -- & -- \\
\hline & & 2-Layer & 2.85 & 1.14 & 4.15 & 1.59 \\
\hline & & 3-Layer & 1.98 & 0.77 & 5.60 & 0.74 \\
\hline & \multirow{3}{*}{ Fast } & No Prime & 2.48 & 0.99 & --- & --- \\
\hline & & 2-Layer & 3.23 & 1.07 & 4.05 & 1.52 \\
\hline & & 3-Layer & 2.48 & 1.06 & 4.10 & 1.28 \\
\hline \multirow{9}{*}{$3: 4$} & \multirow{3}{*}{ Slow } & No Prime & 2.33 & 1.10 & --- & --- \\
\hline & & 3-Layer & 2.73 & 1.06 & 5.10 & 1.06 \\
\hline & & 4-Layer & 2.95 & 0.72 & 3.88 & 1.79 \\
\hline & \multirow{3}{*}{ Medium } & No Prime & 2.73 & 1.06 & -- & -- \\
\hline & & 3-Layer & 2.83 & 1.06 & 5.25 & 0.87 \\
\hline & & 4-Layer & 3.38 & 0.77 & 4.03 & 1.59 \\
\hline & \multirow{3}{*}{ Fast } & No Prime & 2.18 & 1.08 & -- & -- \\
\hline & & 3-Layer & 3.35 & 1.29 & 5.08 & 1.00 \\
\hline & & 4-Layer & 3.80 & 1.06 & 3.45 & 1.69 \\
\hline
\end{tabular}

\section{Complexity}

Complexity ratings were converted to a 6-point numerical scale for analysis. For the $2: 3$ polyrhythm participants rated the stimuli with the 2-layer prime as significantly more complex than with no prime and with the 3-layer prime at the slow and medium tempo conditions, with $p$ $<.05$ for all comparisons. However, for the 2:3 polyrhythm at the fast tempo, the stimuli with the 2-layer prime was rated as significantly more complex than with no prime, $p<.05$, and approaching but not significantly more complex than with the 3-layer, $(p=.062)$.

For the 3:4 polyrhythm, the complexity ratings in only one condition are significantly different from the others. The complexity rating of the stimuli with the 4layer prime at the medium tempo is significantly higher than with the 3-layer, $(p<.05)$. 


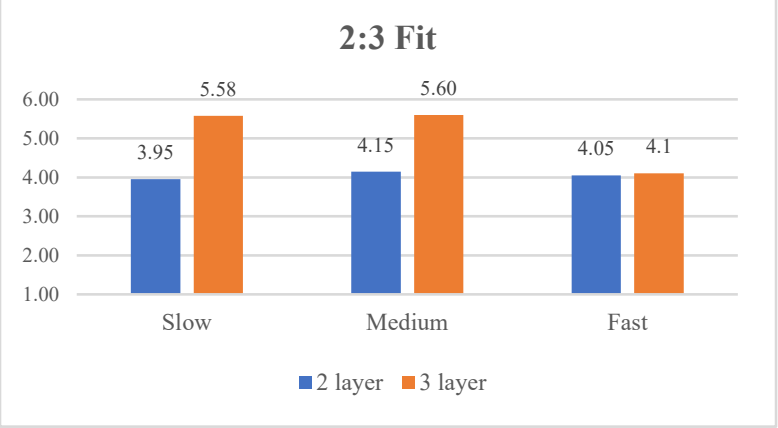

Figure 2: Average metrical fit ratings for 2:3.

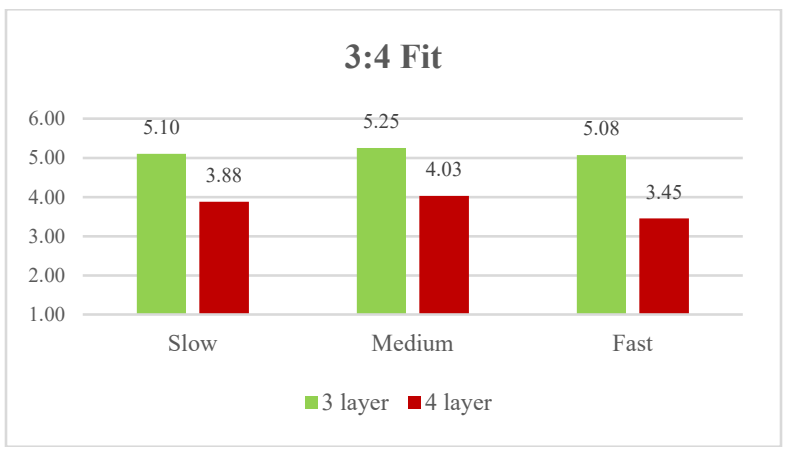

Figure 3: Average metrical fit ratings for 3:4.

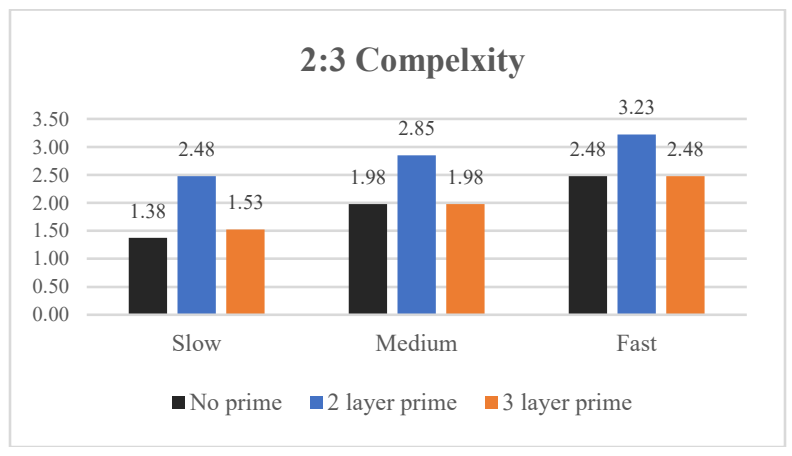

Figure 4: Average complexity ratings for 2:3.

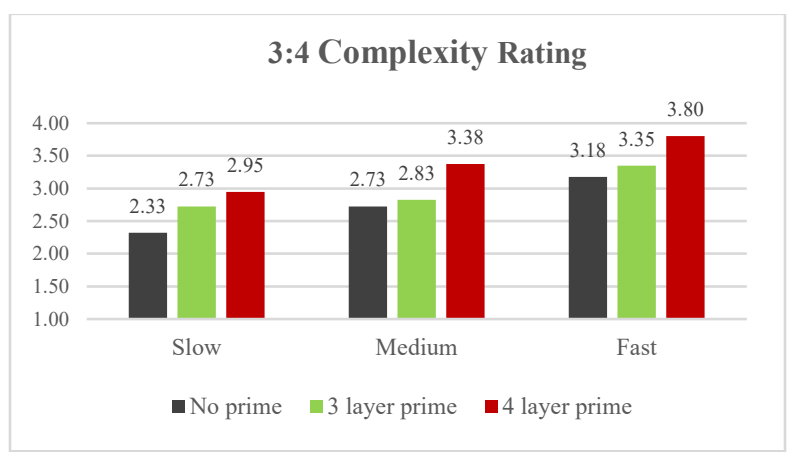

Figure 5: Average complexity ratings for 3:4.

\section{Discussion}

Preferred Meter

I predicted that any inherent preference for meter in $2: 3$ and 3:4 polyrhythms would be ignored when the rhythms are played in the context of a clear meter. Having provided two metrical primes that elicit the two possible meters of each polyrhythm, I expected no preference to be given to the fit of the polyrhythms in either of the contexts. However, results show a preference for the 3-layer (3/4 meter) with 2:3 polyrhythms at slow and medium tempos, and a preference for the 3-layer (3/4 meter) with 3:4 polyrhythms at all tempos. These results do bear some similarity to the tapping patterns of the subjects in Handel and Oshinsky's (1981) study. They found most participants tapped the 3-layer in the 2:3 polyrhythm except at faster tempos, where some began tapping the 2-layer. This is mirrored by the metrical fit ratings in the present study with the $2: 3$ polyrhythm rated as fitting the best with the 3-layer except at the fast tempo.

Results from the $3: 4$ polyrhythm are less similar to Handel and Oshinsky (1981). Their participants preferred tapping the 3-layer at slow tempos, but tapped the 4-layer at the equivalent of this study's medium tempo. No clear preference was shown at fast tempos. However, the results of the fit ratings show participants preferred the 3:4 polyrhythm in the context of the 3layer at all three tempos. While there there are some similarities between these perceptual ratings and previous tapping studies, metrical fit ratings may not be able to replace or replicate tapping behaviour.

\section{Complexity}

The two metrical primes used in this study are suggestive of either a simple or a compound meter. Since compound meters require more subdivisions than duple meter, I predicted polyrhythms placed in the context of a compound meter would be rated as more complex than the simple meter. This was true for the $2: 3$ polyrhythm with the 2-layer prime (with the implication of a compound meter of 6/8) since it was rated as significantly more complex than the 3-layer prime (3/4 simple meter) and no prime. However, this relationship is not observed in the 3:4 polyrhythm, except at the medium tempo where the 4-layer prime suggesting a compound meter of $12 / 8$ was rated as significantly more complex than the 3-layer prime (3/4 simple meter).

The insignificant differentiation of complexity ratings in the 3:4 polyrhythm could be a result of the higher note per second density compared to the $2: 3$ 
polyrhythm. A greater number of onsets in the pattern means the listener must process more information. Note density has been shown to correlate with complexity ratings by (Eerola et. al., 2006). This correlation can also be observed in the different tempo conditions. As the tempo increases, so does the note density and complexity ratings. For the $2: 3$ polyrhythm, there was a moderate positive correlation between tempo and complexity rating $(r=.338, p<.05)$ and for the $3: 4$ polyrhythm there was a weak positive correlation between tempo and complexity rating $(r=.262, p<.05)$.

\section{Conclusion}

This study further demonstrates the complex metrical structures of polyrhythms, but suggests there might be conditions in which listeners can more easily interpret these rhythms. This can serve to better inform musicians and music educators when studying and performing polyrhythms. For example, since the data here suggests 2:3 polyrhythms are perceived to fit best with the 3layer, musicians might try practicing this polyrhythm in a 3/4 metrical context where the 3-layer is the main pulse and the 2-layer is subdivided from the ground layer, similar to the ground-figure model in Peters and Schwartz (1989) and Pressing, Summers and Magill (1996).

Future directions of this study might be to investigate the figure-ground model further and include subdivisions in the metrical prime to reinforce the intervals between onsets of the polyrhythm. This could be used to develop a model of polyrhythmic subdivision that would advance our understanding of the perception of these challenging rhythmic patterns and help musicians better understand how to perform polyrhythmic patterns accurately.

\section{References}

Beauvillain, C. (1983). Auditory perception of dissonant polyrhythms. Perception \& Psychophysics, 34(6), 585592 https://doi.org/10.3758/BF03205915

Eerola, T., Himberg, T., Toiviainen, P., \& Louhivuori, J. (2006). Perceived complexity of western and African folk melodies by western and African listeners. Psychology of Music, 34(3), 337-371. https://doi.org/10.1177/0305735606064842

Fidali, B C., Poudrier, È., \& Repp, B. H. (2013). Detecting perturbations in polyrhythms: effects of complexity and attentional strategies. Psychological Research 77, 183 195. https://doi.org/10.1007/s00426-011-0406-8

Handel, S., \& Lawson, G. R. (1983). The contextual nature of rhythmic interpretation. Perception \& Psychophysics, 34 (2), 103-120. https://doi.org/10.3758/BF03211335

Handel, S., \& Oshinsky, J. S. (1981). The meter of syncopated auditory polyrhythms. Perception and Psychophysics, 30(1), 1-9. https://doi.org/10.3758/BF03206130

Jones, M. R., Jagacinski, R. J., Yee, W., Floyd, R. L., \& Klapp, S. T. (1995). Tests of attentional flexibility in listening to polyrhythmic patterns. Journal of Experimental Psychology: Human Perception and Performance, 21(2), 293-307.

https://doi.org/10.1037/0096-1523.21.2.293

Keller, P., \& Burnham, D. (2005). Musical Meter in Attention to Multipart Rhythms. Music Perception 22(4), 629-666. https://doi.org/10.1525/mp.2005.22.4.629

Krampe, R. T., Kliegl, R., Mayr, U., Engbert, R., \& Vorberg, D. (2000). The fast and the slow of skilled bimanual rhythm production: parallel versus integrated timing. Journal of experimental psychology: Human perception and performance 26(1) 206-233. https://doi.org/10.1037/0096-1523.26.1.206

Moelants, D., \& Noorden, L. V. (2005). The Influence of Pitch Interval on the Perception of Polyrhythms. Music Perception: An Interdisciplinary Journal 22(3), 425-440. https://doi.org/10.1525/mp.2005.22.3.425

OneMotion. (n.d.). Drum Machine. https://www.onemotion.com/drum-machine/

Peters, M., \& Schwartz, S. (1989). Coordination of the Two Hands and Effects of Attentional Manipulation in the Production of a Bimanual 2:3 Polyrhythm. Australian Journal of Psychology 41(2), 215-224. https://doi.org/10.1080/00049538908260084

Poudrier, È. (2017). Tapping to Carter: Mensural Determinacy in Complex Rhythmic Sequences. Empirical Musicology Review 12(3-4), 277-315. https://doi.org/10.18061/emr.v12i3-4.5814

Pressing, J., Summers, J., \& Magill, J. (1996). Cognitive Multiplicity in Polyrhythmic Pattern Performance. Journal of Experimental Psychology: Human Perception and Performance 22(5), 1127-1148. https://doi.org/10.1037/0096-1523.22.5.1127 


\title{
Find your groove: a pilot study on the influences of beat salience and social connectedness on groove perception
}

\author{
Hannah Percival ${ }^{1 \dagger}$ \\ ${ }^{1}$ School of Music, Texas Tech University, Lubbock, TX, USA \\ ${ }^{\dagger}$ Corresponding author: percival.piano@gmail.com \\ Published 16 December 2021; https://doi.org/10.18061/FDMC.2021.0044 \\ Author video presentation and/or other conference material: https://doi.org/10.17605/OSF.IO/J9832
}

\begin{abstract}
Is beat salience an accurate way to measure groove? Does personal identification with the music affect groove ratings? Weigl (2016) lists 24 17-second clips from Rock/Pop/Electronic Dance Music genres in categories of high, medium, and low beat salience. To determine if beat salience influences groove perception, participants in my pilot study completed a Likert scale for each of Weigl's clips, ranging from " $1=$ no need to move" to " $7=$ dance party!"; the results suggest that this phrasing is an effective operationalized proxy for groove and beat salience. Participants also filled out social surveys to see if a propensity towards social connectedness would influence groove perception (GP). There was a weak correlation between GP and feeling connected to the musical environment. This connected feeling was correlated with prior emotional engagement with music. Self-construal as an independent or interdependent person did not have any correlation with GP; this result suggests that the intersection of musical and social elements in groove should focus on the musical environment, rather than self-construal elements.
\end{abstract}

KEYWORDS: beat salience, groove perception, musical-social entrainment, self-construal.

\section{Introduction}

Groove tends to elicit a desire to move through a combination of both musical elements and social elements (Senn et al., 2018). It has been defined as "crystallizations of collaborative expectancies in time" (Feld, 1988, p.74) and "the bodily experience of shared timing" (Doffman, 2013, p.62). As a type of "musicspecific social entrainment" (Kim et al., 2019, p. 11), groove perception may rely on both musical perception and social elements. Many performance environments where groove can be observed involve many contributing factors that can complicate experimental control. In this pilot study, participants evaluated one element of musical groove, beat salience, alone in a sound attenuation booth; afterwards, participants also filled out surveys that investigate engagement tendencies in musical and social situations. This provided separate assessment of both musical perception and social tendencies as agents in groove perception.

\section{Method}

Twenty participants listened to randomized playlists of Weigl's (2016) dataset of 17 second excerpts from Rock/Pop/Electronic Dance Music (EDM) genres. These 24 stimuli fall equally into three categories of high, medium, and low beat salience. During the nonperiodic stimuli after each musical stimulus, participants rated how much the music made them want to move using the following Likert scale: "On a scale of $1-7$, how much did this music make you want to move?" where $1=$ "no movement needed" and $7=$ "dance party!" After completing the playlist, participants completed the following post-test surveys: SelfConstrual Scale (SCS) that evaluates the participant's independent and interdependent tendencies in social situations (Singelis, 1994); Inclusion of Other in the Self (IOS) that shows seven pairs of increasingly overlapping circles (Aron et al., 1992), where other in this case means "experience with the musical environment in the booth"; and Goldsmith's Musical Sophistication Index (Gold-MSI) that assesses different elements of musical sophistication (Müllensiefen et al., 2013).

\section{Results}

Participants rated the music stimuli with higher beat salience as prompting more of a need to move, or groove. The mean of each stimulus's groove rating was calculated. The ratings were categorized into three grand means, with a maximum of 7.00, that represented each level of beat salience according to Weigl's dataset, see Figure 1. The high beat salience condition [music stimuli 1-8] had a grand mean of $3.948(\mathrm{SD}=0.807)$, the medium beat salience condition [music stimuli 9-16] had a grand mean of $3.077(\mathrm{SD}=0.681)$, and the low beat salience condition had a grand mean of 2.084 (SD $=0.727$ ). This slight decrease in grand means as the beat salience decreases suggests that asking participants to rate the need to move may be an accurate proxy for assessing participant's perception of beat salience. 


\section{Mean of beat salience categories}

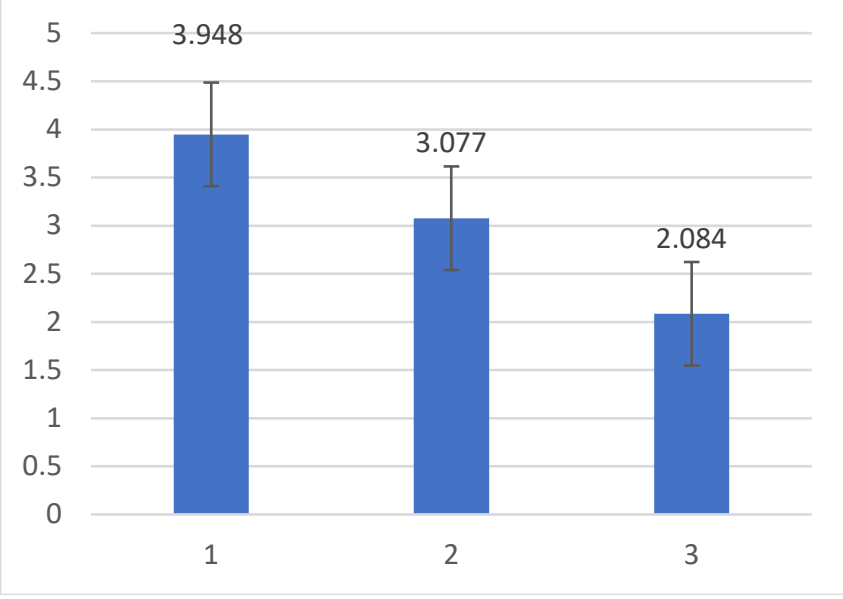

Figure 1: Means of groove ratings for each of Weigl's (2016) beat salience categories

Each participant's groove responses were averaged to create their Groove Perception (GP) score. The GP score indicates the participant's propensity to want to move with the music. For example, if a participant had a high GP score, it indicates that the musical stimuli frequently prompted them to move. The GP scores were then correlated with the participant's other scores from the post-test surveys using the Pearson correlation coefficient, $r$.

GP had a weak positive correlation with IOS ratings, $[r(18)=.46, \mathrm{p}=.039]$, as shown in Figure 2. This indicates that individuals who tended to rate music high for groove also tended to feel more included in the overall musical experience.

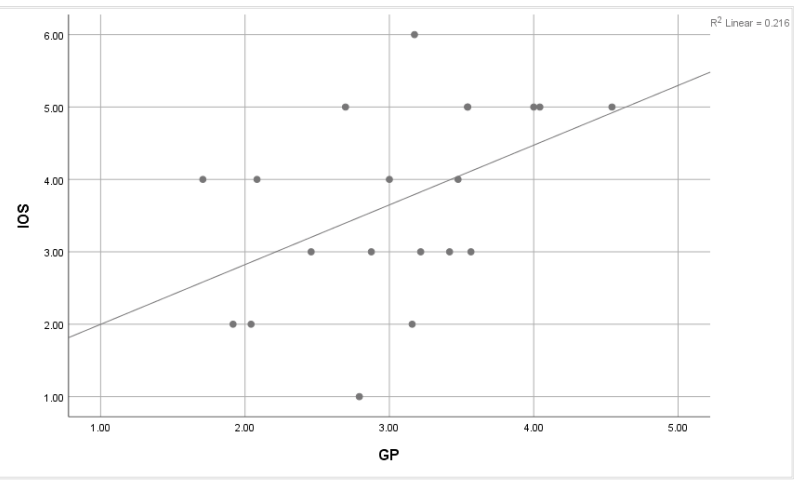

Figure 2: Groove Perception (GP) has a weak positive correlation with Inclusion of Other in Self (IOS) [r (18) $=.46, p=.039]$
IOS scores also had a weak positive correlation with the Emotional Engagement subscale of the Gold-MSI, $[r(18)=.46, \mathrm{p}=.042]$, as shown in Figure 3. This suggests that individuals who felt most connected with the musical environment in the experiment also recalled feeling emotionally connected to music overall in previous experiences. There were no other significant correlations between IOS ratings and Gold-MSI scores.

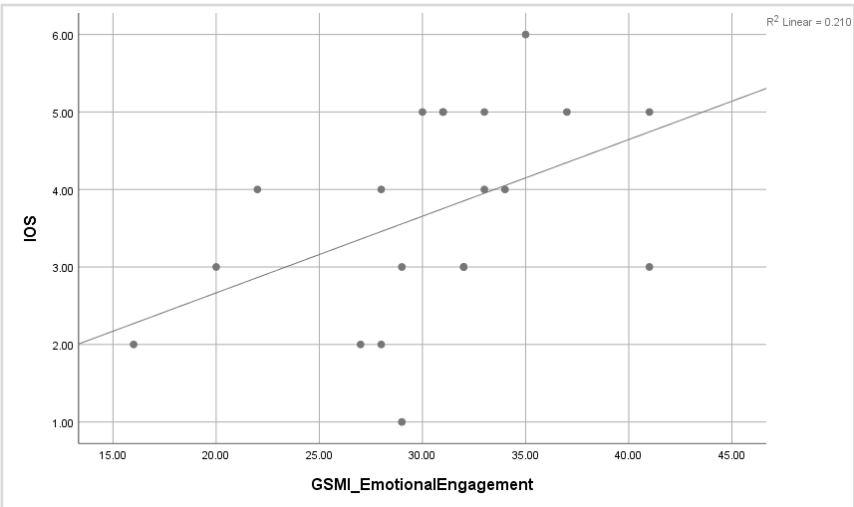

Figure 3: Inclusion of Other in Self (IOS) has a weak positive correlation with Gold-MSI's Emotional Engagement subscale $[r(18)=.46, p=.042]$.

There were no statistically significant correlations between GP and either independent $[r(18)=.28, p=$ .230] or interdependent $[r(18)=-29, p=.219] \mathrm{SCS}$ scores. While it is fascinating that an independent selfconstrual seems to be positively correlated with groove perception while an interdependent self-construal seems to be negatively correlated with groove perception, neither are statistically significant. This suggests that self-construals of independent or interdependent approaches to social situations may not impact groove perception.

\section{Discussion}

The participant responses validated the phrasing of "need to move" as a proxy for beat salience in groove. While beat salience is not the only important musical element in groove, these results show that this description of a need to move produces similar results as assessing beat salience. It is possible that this embodied definition of beat salience may be more accessible to participants without musical training.

These results suggest that feeling connected to the musical environment may be correlated with groove perception. This correlation may indicate why 
participants in intense musical environments have difficulty distinguishing the music from the environment: "[the experience at raves] melds into one cosmic soup and everything is one and you can't separate the music or the moves or which came first" (Hutson, 2000, p. 42).

This connection to the musical environment may be connected by previous emotional engagement with music. Lee and Robins' (1995) article on social belonginess shows that emotional engagement is an important element for inclusion. Perhaps prior emotional engagement with music predicts the propensity for feeling included in a current musical environment. As there is not a significant correlation between GP ratings and Gold-MSI Emotional Engagement, it does not follow that emotional engagement will necessarily lead to more groove perception.

The lack of significant correlation of self-construal suggests that larger-scale social interactions may not be a fruitful path for groove perception. Rather, future research on groove perception should focus on prior emotional engagement with music along with current feelings of being connected to the environment.

\section{Conclusion}

Participant ratings of the music's ability to prompt movement, an element of groove, were similar to previous assessments of beat salience. This suggests that "the need to move" is an appropriate operationalized proxy for beat salience in groove. Participant's individual groove perception was linked with feeling included in the musical environment; this inclusion in the musical environment was linked with prior emotional engagement with music. Neither the independent nor the interdependent self-construal had any correlation with groove perception. The results of this pilot study suggest that future work on the intersection of musical and social elements at work in groove perception should focus on relationships to the musical environment rather than self-construal.

\section{References}

Aron, A., Aron, E.N., \& Smollan, D. (1992). Inclusion of other in the self scale and the structure of interpersonal closeness. Journal of Personality and Social Psychology, 63,(4), 596-612. $\quad$ https://doi.org/10.1037/00223514.63.4.596

Doffman, M.R. (2013). Groove: Temporality, awareness and the feeling of entrainment in jazz performance. In $\mathrm{M}$.
Clayton, B. Dueck, \& L. Leante (Eds.), Experience and meaning in music performance (pp. 62-85). Oxford University Press. https://doi.org/10.1093/acprof: oso/9780199811328.003.0004

Feld, S. (1988). Aesthetics as iconicity of style, or 'lift-upover sounding': Getting into the kaluli groove. Yearbook for Traditional Music, 20, 74-113. https://doi.org/10.2307/768167

Hutson, S. R. (2000). The rave: Spiritual healing in modern western subcultures. Anthropological Quarterly, 73 (1), 35-49.

Kim, J.H., Reifgerst, A., \& Rizzonelli, M. (2019). Musical social entrainment. Music \& Science, 2, 205920431984899. https://doi.org/10.1177/2059204319848991

Lee, R.M. \& Robbins, S.B. (1995). Measuring belongingness: The social connectedness and the social assurance scales. Journal of Counseling Psychology , 42 (2), 232-41. https://doi.org/10.1037/0022-0167.42.2.232

Müllensiefen, D., Gingras, B., Musil, J., \& Stewart, L. (2014). The musicality of non-musicians: An index for assessing musical sophistication in the general population. PLOS ONE, 9(2), e89642. https://doi.org/10.1371/journal.pone.0089642

Senn, O., Kilchenmann, L., Bechtold, T., \& Hoesl, F. (2018). Groove in drum patterns as a function of both rhythmic properties and listeners' attitudes. PLoS One, 13(6), e0199604. https://doi.org/10.1371/journal.pone.0199604

Singelis, T. M. (1994). The measurement of independent and interdependent self-construals. Personality and Social Psychology Bulletin, https://doi.org/10.1177/0146167294205014

Weigl, D. M. (2016). Rhythmic information as a relevance criterion for music information retrieval. $\mathrm{PhD}$ Dissertation, McGill University. 


\title{
Rhythm contour drives musical memory
}

\author{
Mark A. Schmuckler ${ }^{1 \dagger}$ \\ Rebecca Moranis ${ }^{2}$ \\ ${ }^{1}$ University of Toronto Scarborough, Toronto, ON, Canada \\ ${ }^{2}$ University of Toronto, Toronto, ON, Canada \\ † Corresponding author: mark.schmuckler@utoronto.ca \\ Published 16 December 2021; https://doi.org/10.18061/FDMC.2021.0045 \\ Author video presentation and/or other conference material: https://doi.org/10.17605/OSF.IO/M3RG5
}

\begin{abstract}
Two experiments examined listeners' use of contour information to drive memory for rhythmic patterns; these experiments were distinguished by the use of metric rhythms (Experiment 1) and ametric rhythms (Experiment 2). Both experiments employed a typical short-term memory task in which listeners heard a standard rhythm followed by a comparison rhythm. Comparison rhythms could be one of three types: an exact repetition of the standard rhythm, a same contour rhythm in which the relative durations of successive notes were comparable to the standard, and a different contour rhythm in which the relative durations of successive notes were modified relative to the standard. Analyses of $d$ primes for same/different detection revealed that, for both studies, listeners performed better when the comparisons had different rhythm contours, relative to comparisons with the same rhythm contours. These findings converge with results investigating melodic contour, and suggest that listeners both form and use contours of novel rhythmic patterns.
\end{abstract}

KEYWORDS: Rhythm, Contour, Short-term Memory, D primes

\section{Introduction}

Multiple authors have proposed that contour, along with tonality, are fundamental components of musical processing (Dowling, 1978; Schmuckler, 2016). These factors, to varying degrees, drive online processing of musical information, including perceptual organization (Bregman, 1990; Krumhansl \& Schmuckler, 1986). complexity (Eerola et al., 2006; Schmuckler, 1999), similarity (Prince, 2014; Schmuckler, 2010), memory (Dowling \& Fujitani, 1971; Halpern \& Bartlett, 2010), and performance (Drake \& Palmer, 2000; Lewandowska \& Schmuckler, 2019). Given this wealth of evidence, it is clear that both of these components play central roles in all aspects of musical behavior.

One characteristic of tonality and contour is that the underlying structure of these components involves the organization of pitch information. Thus, work on tonality has been driven by investigation of the tonal hierarchy (Krumhansl, 1990, 2000; Krumhansl \&
Cuddy, 2010). Work on contour has similarly focused on characterizing models of pitch structure (Adams, 1976; Friedmann, 1985; Marvin \& Laprade, 1987; Quinn, 1999; Schmuckler, 1999). Other work has extended the formal characterizations of tonality (i.e., its hierarchical structure) to other musical dimensions, such as meter and rhythm (Martin, 1972; Povel \& Essens, 1985; Povel, 1981, 1984). In contrast, although the concept of contour is discussed relative to other perceptible dimensions, most notably vision (e.g., Koenderink et al., 1997), it has only rarely been discussed with reference to other auditory and/or musical dimensions (but see Schmuckler \& Gilden, 1993, for one notable exception).

Given this paucity of previous work, what might be an appropriate context for investigating the formation and use of contour in the temporal domain? Methodologically, it would make sense to employ paradigms that have been used successfully to investigate perceived contour in other musical domains. Within this framework, it is most instructive to look at work investigating melodic contour. Specifically, work by Dowling and colleagues (e.g., Bartlett \& Dowling, 1980; Dowling, 1978, 1994; Dowling \& Bartlett, 1981; Dowling \& Fujitani, 1971; Dowling et al., 1995; Halpern \& Bartlett, 2010; Halpern et al., 1998) provides some of the most comprehensive and well-known research on melodic contour. This work has demonstrated that listeners make use of similar contour codes for both short- and long-term memory of musical melodies, although contour information is more critical for short-term melodic representations, and is enhanced when the melodies adhere to a coherent tonal framework (Dowling et al., 1995).

In Dowling's work, melodic contour is coded as a series of +'s and -'s, representing relative pitch differences between successive notes; this general form of contour coding (as well as its equivalent of 1's and -1's) has been employed by multiple authors (Friedmann, 1985; Marvin \& Laprade, 1987; Quinn, 
1999). Unfortunately, little work exists on how to characterize duration and/or rhythmic contours, with the majority of work analyzing rhythm more focused on patterns of stress and intonation, as opposed to durations, likely due to the emphasis on rhythm and prosody in speech and language (e.g., Aiello, 1994; Cooper \& Meyer, 1960; Lerdahl \& Jackendoff, 1983; Patel et al., 2006; Thaut, 2008). Cooper and Meyer's (1960) classic text on the rhythmic structure of music, for instance, explicitly relates musical rhythmic structure to accented and unaccented groupings, using terminology drawn from work in prosody.

Marvin (1991) proposed a characterization of rhythmic contours more consistent with the framework employed in Dowling's research. Specifically, Marvin suggests encoding rhythm contours "as analogous to melodic contours: they represent relative durations in much the same way that melodic contours represent relative pitch height, without a precise calibration of the intervals spanned" (Marvin, 1991, p. 64).

Based on this framework, one means for investigating whether contour plays a role in processing the temporal/rhythmic dimension in music thus makes use of the classic melodic contour paradigm pioneered by Dowling and colleagues (e.g., Dowling, 1984; Dowling et al., 2008). Although this research has employed a variety of paradigm variants in its explorations, one of the most basic procedures involves simply presenting an initial standard melody, followed (after a short interval) by a subsequent test melody, and asking participants if these two melodies were the same or different (e.g., Dowling et al., 2008). As shown in Figure 1, the relation between the standard and comparison melody can take multiple forms, with the comparison being an exact pitch transposition of the standard melody (Figure 1b), a same contour, but different pitch interval version of the standard (Figure 1c), and a different contour, and hence different pitch interval, version of the standard (Figure 1d). Findings from multiple studies have revealed listeners' accuracy in determining the relation between standard and comparison is driven by contour similarity, with listeners correctly discriminating melodies with different contours, but failing to discriminate melodies with the same contour. Interestingly, these findings hold for both tonal (Dowling, 1978) and atonal (Dowling \& Fujitani, 1971) melodies. The goal of the current study was to adapt this general framework for the perception of rhythmic contours, both within a metrical context (Experiment 1) and without a metrical framework (Experiment 2).
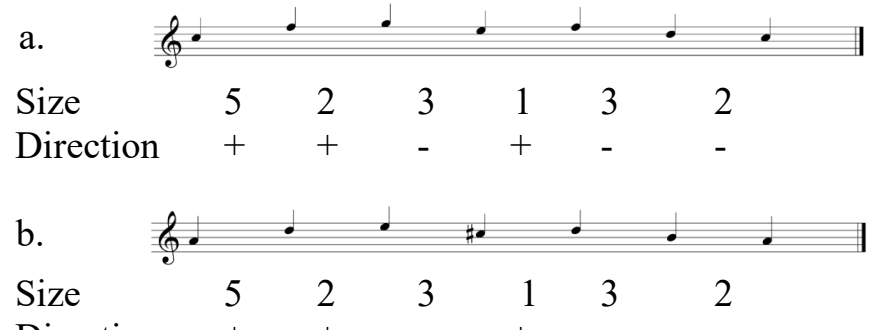
Direction $++\quad+\quad-\quad+$

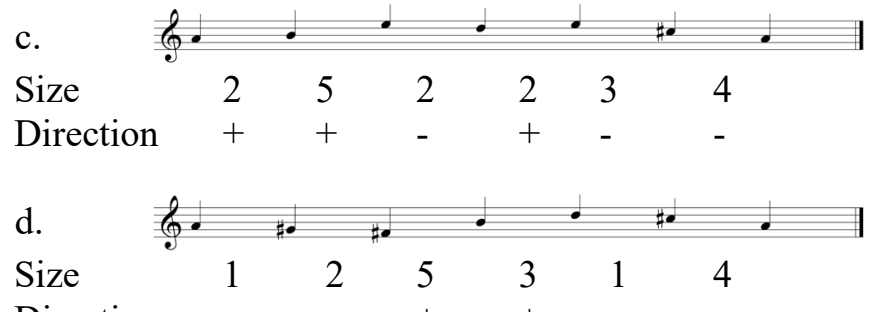
Direction - $\quad-\quad+\quad+\quad-\quad$ -

Figure 1: Sample melodic contours, adapted from Dowling (1994). Figure la shows the standard contour, separating out pitch interval and direction information. Figure $1 b$ shows a comparison contour that is pitch transposed having the same pitch interval and direction information. Figure 1c shows a comparison contour having different pitch interval information, but the same pitch direction information. Figure $1 d$ shows a comparison contour with different pitch interval and direction information. For all melodies, "size" refers to the number of semitones between notes, and "direction" indicates whether successive tones are higher (+) versus lower (-) in pitch.

\section{Method}

\section{Participants}

Participants were drawn from the Introductory Psychology subject pool at the University of Toronto Scarborough; participants were not selected based on their prior musical training. Overall, data from 20 participants in each of Experiments 1 and 2 were employed in this study. One additional participant in Experiment 1 completed the study, but this participant's data was removed for a failure to appropriately use the rating scale.

\section{Experimental Apparatus and Stimuli}

Stimuli for these studies consisted of either 8 note (Experiment 1) or 7 note (Experiment 2) rhythms, played using a piano sound, on a single tone $\left(\mathrm{F}_{4}\right)$. For the metric stimuli of Experiment 1, the meter was 
established by sounding 8 beats of a woodblock prior to the start of the rhythm, which was spread out over 8 subsequent beats. For the ametric stimuli of Experiment 2, no a priori framework was established, and the rhythms themselves occurred across variable timeframes, depending on the specific duration values of their component tones. For the ametric stimuli, the lengths of the standard, same, and different contours were roughly equivalent.

For both metric and ametric stimuli, a set of standard rhythmic contours were created. These standard contours then generated three comparison contours; these different comparisons appear in Figure 2a (metric rhythms) and Figure $2 \mathrm{~b}$ (ametric rhythms). The first comparison rhythm represents an exact repetition of the standard rhythm, played at a faster overall tempo (150 beats/min) than the standard (120 beats/min). The second comparison rhythm represents a same contour variant of the standard, again played at a faster overall tempo (150 beats/min). As seen in Figure 2 , the relative pattern of durations for this rhythm is equivalent to the pattern of durations in the standard. The third comparison rhythm represents a different contour variant of the standard, played at a faster overall tempo (150 beats/min), with the relative durations displaying a different pattern than the standard. Twenty different sets of standard and comparison rhythm contours were created for each of the metric and ametric experiments.

All listeners heard 80 trials of randomly ordered standard-comparison rhythm contour pairs. These pairs consisted of two repetitions of the exact repetition comparison, and one repetition each of the same contour and different contour comparisons, for each of the 20 rhythms. The two repetitions of the exact repetition were included to balance the number of objective "same" and "different" responses. Listeners were instructed to judge whether standard and comparison rhythms were exactly the same (ignoring the overall tempo). They made these judgments using a $1-6$ scale, with responses $1-3$ indicating the rhythms were different at varying levels of confidence ( 1 = very confident; $3=$ mildly confident $)$, and responses $4-6$ indicating the rhythms were the same at varying levels of confidence $(4=$ mildly confident; 6 = very confident).

\section{Results}

For each listener, the 6-point rating scale was used to calculate percent values (i.e., responding $1-3$ for same contour and different contour comparisons, and $4-6$ for the exact repetition contours). These percent values were then used to calculate $d$ prime scores separately for the same contour and different contour rhythms, using the percent correct for these two stimuli as their respective hit rate, and 1-percent correct for the exact repetition as the false alarm rate.

\section{a. Metric Rhythms (Experiment 1)}
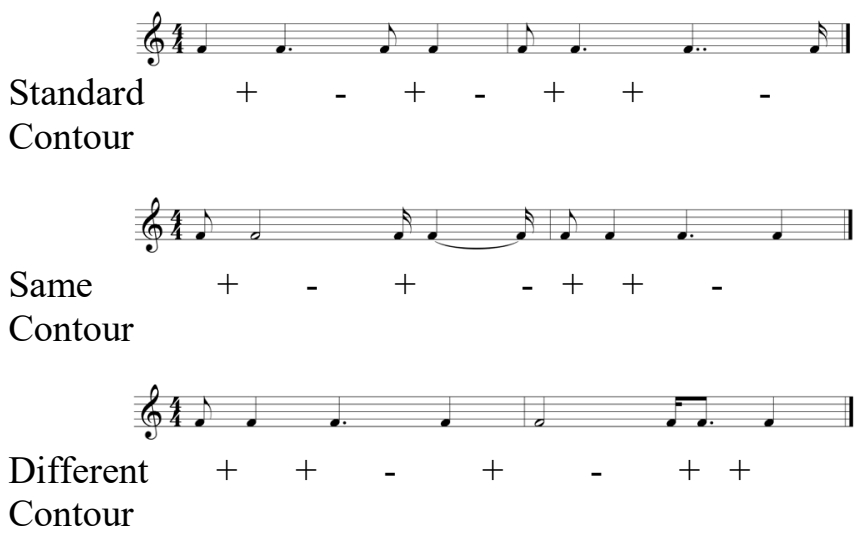

b. Ametric Rhythms (Experiment 2)

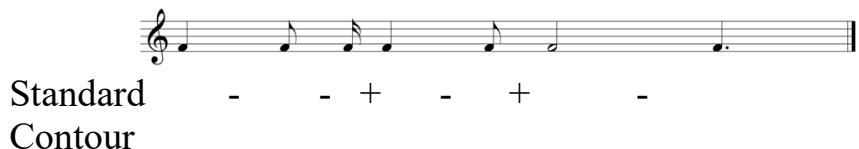

Same

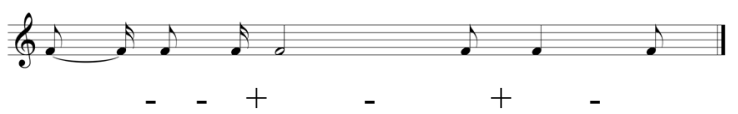

Contour

Different

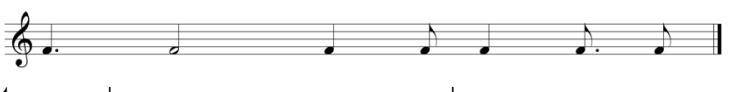

Contour

Figure 2: Standard, same, and different contours for the metric rhythms of Experiment 1 (a) and ametric rhythms of Experiment 2 (b). Increases in relative durations between notes are indicated with a "+", and decreases in relative durations are indicated with a "-".

D prime scores for metric (Experiment 1) and ametric (Experiment 2) rhythms were analyzed in oneway ANOVAs, with the within subjects factor of Contour Type (same contour, different contour); d prime measures incorporate the hit rate $(\%$ correct for exact repetitions) into the overall measure. For the metric stimuli, this analysis produced a main effect of Contour Type, $F(1,19)=5.74, M S E=0.266, p<.05, n p^{2}=.232$. 
Mean values for this analysis appear in Figure 3a. For the ametric stimuli, this analysis also revealed a main effect of Contour Type, $F(1,19)=18.04, M S E=0.072$, $p<.001, n p^{2}=.487$. Figure 3 b shows the mean values for this analysis. a. Metric Rhythms

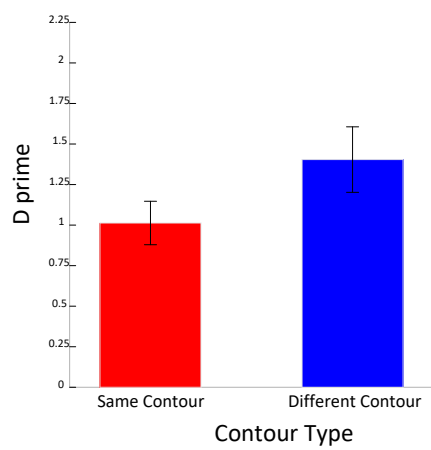

b. Ametric Rhythms

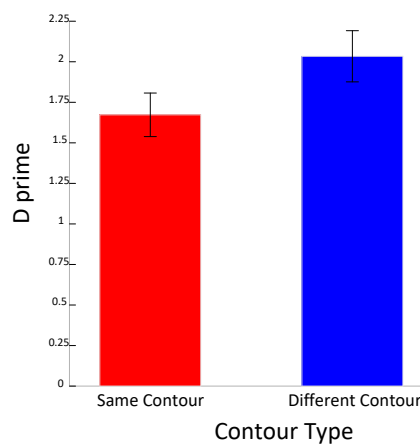

Figure 3: D primes (and SEs) for discrimination of same, and different contours for the metric rhythms of Experiment 1 (a) and ametric rhythms Experiment 2 (b).

\section{Discussion}

Two experiments investigated listeners' use of contour information in short-term memory for rhythmic patterns. These studies found that contour similarity led to memory confusions, such that listeners were less able to discriminate a new rhythmic pattern from an old pattern when the two rhythms shared the same pattern of relative durations. Thus, these findings suggest that listeners are able to form duration contours, and that when hearing a rhythm, listeners are encoding the relative patterns of durations, as opposed to the actual durations of the rhythms. This process, in many ways, is comparable to that observed in the processing of melodic contour, in which listeners encode the relative patterns of pitch changes, as opposed to the exact pattern of pitch intervals (Dowling, 1994).

Somewhat more surprisingly, these studies found little difference in rhythm encoding as a function of whether or not the underlying rhythm was set in a metric framework. Comparison of Experiments 1 and 2 showed that both metric and ametric rhythms produced equivalent influences on memory of same versus different contours. Although intuitively surprising, this finding converges with results from the melodic contour literature in which contour information plays a role in melodic memory for both tonal (Dowling, 1978) and atonal (Dowling \& Fujitani, 1971) melodies. Future work might profitably explore these results by investigating the impact of contour durations on pitch and rhythm processing simultaneously, as well as looking at factors such as short- versus long-term memory, which influences the relative impact of tonal and interval information.

\section{Acknowledgements}

This research was supported by a grant from the Natural Sciences and Engineering Research Council to Mark A. Schmuckler.

\section{References}

Adams, C. R. (1976). Melodic contour typology. Ethonomusicology, 20, 179-215. https://doi.org/10.2307/851015

Aiello, R. (1994). Music and language: Parallels and contrasts. In R. Aiello (Ed.), Musical perceptions (pp. 4063). Oxford University Press.

Bartlett, J. C., \& Dowling, W. J. (1980). Recognition of transposed melodies: A key-distance effect in developmental perspective. Journal of Experimental Psychology: Human Perception and Performance, 6, 501-515. https://doi.org/10.1037/0096-1523.6.3.501

Bregman, A. S. (1990). Auditory scene analysis: The perceptual organization of sound. The MIT Press. https://doi.org/10.7551/mitpress/1486.001.0001

Cooper, G., \& Meyer, L. B. (1960). The rhythmic structure of music. Chicago University Press.

Dowling, W. J. (1978). Scale and contour: Two components of a theory of memory for melodies. Psychological Review, 85, 341-354. https://doi.org/10.1037/0033295X.85.4.341

Dowling, W. J. (1984). Musical experience and tonal scales in the recognition of octave-scrambled melodies. Psychomusicology, 4, 13-32. https://doi.org/10.1037/h0094206

Dowling, W. J. (1994). Melodic contour in hearing and remembering melodies. In R. Aiello \& J. Sloboda (Eds.), Musical Perceptions (pp. 173-190). Oxford University Press.

Dowling, W. J., \& Bartlett, J. C. (1981). The importance of interval information in long-term memory for melodies. Psychomusicology, 1, 30-49. https://doi.org/10.1037/h0094275

Dowling, W. J., Bartlett, J. C., Halpern, A. R., \& Andrews, M. W. (2008). Melody recognition at fast and slow tempos: Effects of age, experience, and familiarity. Perception and Psychophysics, 70, 496-502. https://doi.org/10.3758/PP.70.3.496 
Dowling, W. J., \& Fujitani, D. S., (1971). Contour, interval and pitch recognition in memory for melodies. Journal of the Acoustical Society of America, 49, 524-531. https://doi.org/10.1121/1.1912382

Dowling, W. J., Kwak, S., \& Andrews, M. W. (1995). The time course of recognition of novel melodies. Perception and Psychophysics, 57, 150-158. https://doi.org/10.3758/BF03206500

Drake, C., \& Palmer, C. (2000). Skill acquisition in music performance: Relations between planning and temporal control. Cognition, 74, 1-32. https://doi.org/10.1016/S0010-0277(99)00061-X

Eerola, T., Himberg, T., Toiviainen, P., \& Louhivuori, J. (2006). Perceived complexity of western and African folk melodies by western and African listeners. Psychology of Music, 34, 337-371. https://doi.org/10.1177/0305735606064842

Friedmann, M. L. (1985). A methodology for the discussion of contour: Its application to Schoenberg's music. Journal of Music Theory, 29, 223-248. https://doi.org/10.2307/843614

Halpern, A. R., \& Bartlett, J. C. (2010). Memory for melodies. In M. R. Jones, R. R. Fay, \& A. N. Popper (Eds.), Music Perception (pp. 233-258). SpringerVerlag. https://doi.org/10.1007/978-1-4419-6114-3_8

Halpern, A. R., Bartlett, J. C., \& Dowling, W. J. (1998). Perception of mode, rhythm and contour in unfamiliar melodies: Effects of age and experience. Music Perception, 15, 335-355. https://doi.org/10.2307/40300862

Koenderink, J. J., van Doorn, A. J., Kappers, A. M. L., \& Todd, J. T. (1997). The visual contour in depth. Perception and Psychophysics, 59, 828-838. https://doi.org/10.3758/BF03205501

Krumhansl, C. L. (1990). Cognitive foundations of musical pitch. Oxford University Press.

Krumhansl, C. L. (2000). Rhythm and pitch in music cognition. Psychological Bulletin, 126, 159-179. https://doi.org/10.1037/0033-2909.126.1.159

Krumhansl, C. L., \& Cuddy, L. L. (2010). A theory of tonal hierarchies in music. In M. R. Jones, R. R. Fay, \& A. N. Popper (Eds.), Music Perception (pp. 51-87). Springer. https://doi.org/10.1007/978-1-4419-6114-3_3

Krumhansl, C. L., \& Schmuckler, M. A. (1986). The Petroushka chord: A perceptual investigation. Music Perception, 4, 153-184. https://doi.org/10.2307/40285359

Lerdahl, F., \& Jackendoff, R. (1983). A generative theory of tonal music. MIT Press.

Lewandowska, O. P., \& Schmuckler, M. A. (2019). Tonal and textural influences on musical sight-reading. Psychological Research, 84, 1920-1945. https://doi.org/10.1007/s00426-019-01187-1
Martin, J. (1972). Rhythmic (hierarchical) vs. serial structure in speech and other behavior. Psychological Review, 79, 487-509. https://doi.org/10.1037/h0033467

Marvin, E. W. (1991). The perception of rhythm in nontonal music: Rhythmic contours in the music of Edgard Varèse. Music Theory Spectrum, 13, 61-78. https://doi.org/10.2307/745974

Marvin, E. W., \& Laprade, P. A. (1987). Relating musical contours: Extensions of a theory for contour. Journal of Music Theory, 31, 225-267. https://doi.org/10.2307/843709

Patel, A. D., Iversen, J. R., \& Rosenberg, J. C. (2006). Comparing the rhythm and melody of speech and music: The case of British English and French. The Journal of the Acoustical Society of America, 119, 3034-3047. https://doi.org/10.1121/1.2179657

Povel, D.-J., \& Essens, P. J. (1985). Perception of temporal patterns. Music Perception, 2, 411-440. https://doi.org/10.2307/40285311

Povel, D. J. (1981). Internal representation of simple temporal patterns. Journal of Experimental Psychology: Human Perception and Performance, 7, 3-18. https://doi.org/10.1037/0096-1523.7.1.3

Povel, D. J. (1984). A theoretical framework for rhythm perception. Psychological Research, 45, 315-337. https://doi.org/10.1007/BF00309709

Prince, J. B. (2014). Contributions of pitch contour, tonality, rhythm, and meter to melodic similarity. Journal of Experimental Psychology: Human Perception and Performance, 40, 2319-2337. https://doi.org/10.1037/a0038010

Quinn, I. (1999). The combinatorial model of pitch contour. Music Perception, 16, 439-456. https://doi.org/10.2307/40285803

Schmuckler, M. A. (1999). Testing models of melodic contour similarity. Music Perception, 16, 295-326. https://doi.org/10.2307/40285795

Schmuckler, M. A. (2010). Melodic contour similarity using folk melodies. Music Perception, 28, 169-193. https://doi.org/10.1525/mp.2010.28.2.169

Schmuckler, M. A. (2016). Tonality and contour in melodic processing. In S. Hallam, I. Cross, \& M. Thaut (Eds.), The Oxford handbook of music psychology (2nd ed., pp. 143165). Oxford University Press. https://doi.org/10.1093/oxfordhb/9780198722946.013.1 4

Schmuckler, M. A., \& Gilden, D. L. (1993). Auditory perception of fractal contours. Journal of Experimental Psychology: Human Perception and Performance, 19, 641-660. https://doi.org/10.1037/0096-1523.19.3.641

Thaut, M. H. (2008). Rhythm, music, and the brain: scientific foundations and clinical applications. Routledge. 


\title{
The origins of dance in infancy: Characterizing the development of dance during the first two years of life
}

\author{
Minju Kim ${ }^{\dagger}$ \\ Adena Schachner ${ }^{\dagger}$ \\ ${ }^{1}$ Department of Psychology, University of California, San Diego, La Jolla, CA, United States \\ † Corresponding author: minjukim@ucsd.edu \\ Author video presentation and/or other conference material: https://doi.org/10.17605/OSF.IO/2DCMG
}

Dance is a universal human behavior, and a crucial component of human musicality. What drives and limits the earliest ages dance can appear? We aimed to characterize infants' earliest dance behavior, asking: When do infants begin to spontaneously dance to music, and how variable is this age of onset? What predicts whether an infant can dance: Is this simply a function of gross motor development? Does the nature of infants' dance change over the first two years, or remain stable? Parents of infants age $0-24$ months $(\mathrm{N}=278)$ completed an online survey to characterize their infants' dance behavior. We asked parents to consider movements that were produced by the child, occurred more when music is playing, and "looked like dance, to them". We found that dance begins early in life, and at a wide range of ages across individuals: from 1 to 20 months $(M=$
$9.5 \mathrm{~m})$. Once children can dance, they dance frequently, as a near-daily part of their behavioral repertoire. Motor development was not a major determinant of ability to dance: It did not account for more variance than age alone. Infants' dance changed dramatically with age: For example, infants increasingly used iconic gestures $\left(\chi^{2}(1)=45.4, p<0.001\right)$, and danced more frequently $(F(1)=16.4, p<0.001)$. These data provide an initial characterization of the developmental origins of dance, informing broad questions about the origins of human musicality and highlighting the prominent role of dance behavior in infancy.

KEYWORDS: music cognition, development, infancy, movement, dance

\section{Subjective beat perception develops through adolescence and is related to phonology}

\author{
Karli Nave ${ }^{1 \dagger}$ \\ Joel Snyder ${ }^{1}$ \\ Erin Hannon ${ }^{1}$ \\ ${ }^{1}$ University of Nevada Las Vegas, Las Vegas, Nevada, USA \\ $\dagger$ Corresponding author: karlinave@gmail.com \\ Author video presentation and/or other conference material: https://doi.org/10.17605/OSF.IO/DNHV5
}

Rhythm and beat perception are critical for synchronized musical movement (e.g., walking, dancing) and may also influence some aspects of speech perception. It is assumed that listeners experience the beat as a quasi-isochronous pattern of prominent time points, inferred from regularly occurring events in the musical surface, and that they can sustain this percept even in the absence of events that reinforce it. However, few studies have attempted to disentangle the surface information from the internal beat percept, and no studies to our knowledge have done so with young listeners. In this experiment, we presented listeners aged
4-23 with a rich musical excerpt that induced one of two beat patterns, followed by a prolonged ambiguous phase during which they heard a rhythm that could match either of the two beat patterns. In a final probe phase, listeners indicated whether a drummer did or did not match the beat of the music. Younger children (age 4-7 years) did not perform above chance. Children aged 817 and adults accurately matched the probe with the induced beat, and children aged 12-17 performed with no statistically significant difference from adults. Since previous studies have suggested that rhythm ability is related to phonological awareness, we also administered 
the Comprehensive Test of Phonological Processing (CTOPP). A hierarchical regression revealed that performance in our task was a significant predictor of phonology, accounting for $4.4 \%$ additional variance, above and beyond age (19\% variance) and music/dance training $(2.6 \%$ variance). Overall, these results suggest older (but not younger) children are capable of selfsustained and long-lasting beat perception, which is related to phonological language ability. However, self- sustained beat skills do not become adult-like until adolescence, suggesting that beat perception may have a more gradual developmental trajectory than has been previously assumed.

KEYWORDS: beat, meter, rhythm

\title{
Effects of Musicianship on Hypermetrical Interpretations of Rhythms
}

\author{
Nathalie Nordan ${ }^{1 \dagger}$ \\ Dr. Leigh VanHandel ${ }^{2}$ \\ Dr. Devin McAuley ${ }^{1}$ \\ ${ }^{1}$ Michigan State University, East Lansing, Michigan, United States \\ ${ }^{2}$ The University of British Columbia, Vancouver, British Columbia, Canada \\ ${ }^{\dagger}$ Corresponding author: nordanna@msu.edu \\ Author video presentation and/or other conference material: https://oi.org/10.17605/OSF.IO/AXQHC
}

Previous research reaches different conclusions on whether non-musicians or musicians are more likely to perceive the beat of a rhythm at a slower tempo. To investigate this, participants completed two tasks for thirty monotonic rhythms in either a Fast tempo (150 bpm, $400 \mathrm{~ms}$ inter-beat-interval) or Slow tempo condition (75 bpm, $800 \mathrm{~ms}$ inter-beat-interval). Their first task was to determine their preferred tempo of the rhythms; their second task was to tap along with what they felt was the beat of each rhythm. Participants did this both with and without an isochronous metrical context. Finally, participants' musical background was assessed using the Goldsmith's Musical Sophistication
Index (GMSI). The ratio of the determined best tempo to the tapped tempo was calculated for each participant and rhythm. Of interest were ratios of 0.5 and 0.25 , where participants tapped at half the tempo and a quarter the tempo of the beat unit, a phenomenon called hypermetrical interpretation. Overall, participants produced more hypermetrical interpretations in the Fast tempo condition and for rhythms presented with metrical context than without metrical context. Moreover, participants with higher GMSI scores produced more hypermetrical interpretations than participants with lower GMSI scores, but only in the Fast tempo condition. 


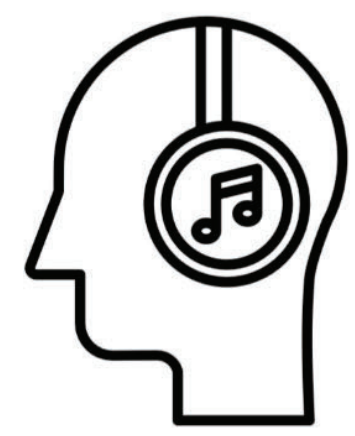

\section{Timbre}

Future Directions of Music Cognition February - May 2021

Ohio State University

Virtual 


\title{
An investigation of loudness effect on pitch and timbre discrimination
}

\author{
Wesley A. Bulla ${ }^{\dagger}$ and Song Hui Chon \\ Curb College, Belmont University, Nashville, TN, USA \\ †Corresponding author: wesley.bulla@belmont.edu \\ Published 16 December 2021; https://doi.org/10.18061/FDMC.2021.0047 \\ Author video presentation and/or other conference material: https://doi.org/10.17605/OSF.IO/MNHXG
}

\begin{abstract}
This study was a first-step investigation of the efficacy of signal loudness when engaging in listening tasks. Based on physiological principles, pitch and timbre discrimination were hypothesized to be more accurate when stimuli were at low excitation levels. Discrimination tests were carried out at near audible threshold and at a robust somewhat loud listening level. Comparative metrics and non-parametric analyses indicated a consistent negative influence of signal strength on measured performance. While promising, more work is needed to determine the practicality and application of the findings.
\end{abstract}

KEYWORDS: listening, cochlear tuning, discrimination, loudness, perception, pitch, timbre.

\section{Introduction}

The ability to discriminate fine details in pitch and timbre are among the most important skills required of musicians, music producers, and audio engineers. While organizations such as the International Telecommunications Union (ITU) and the Audio Engineering Society (AES) have standardized calibration levels for equipment testing, currently, only ad hoc and often conflicting recommendations exist for proper listening loudness levels. Suggestions and documented common practices range from "conversation" level (Swedien, 2009) to as loud as 85 dBA SPL where the so-called "equal-loudness" effects (Fletcher \& Munson, 1933; ISO, 2003; ITU, 2015) are believed to be beneficial in the discrimination process (Naqvi, 2013; Allen, 2014; Devine, 2017; McDonald, 2017).

Psychophysical evidence supporting claims of any case for the application of softer, intermediate, or louder listening levels when engaging in listening-oriented tasks is nonexistent. Additionally, the physiological mechanisms of cochlear tuning, loudness adaptation, and temporary threshold shift seem to be at odds with a tacit assumption that louder signals provide for easier, or more accurate, discrimination.

\section{The Frequency Threshold Curve}

Auditory coding begins when the inner hair cells in the Organ of Corti respond to fluid movement within the cochlea. Auditory nerve fibers are those afferent radial neural filaments exiting the vicinity of the inner sensory cells of the cochlear organ (Rosenzweig, Leiman, \& Breedlove, 1996). The Frequency Threshold Curve (FTC) of a cochlear nerve fiber is defined by its actionpotential response (Hodgkin \& Huxley, 1952) as charted against frequency sensitivity and signal strength (Matthews, 1999).

Investigations have shown that mammalian auditory nerve fibers exhibit a "V" shaped FTC where a fiber's most sensitive frequency, designated its "characteristic frequency" (CF), resides near the threshold of detection (Pickles, 1984; Evans, 1993). Referring to Figure 1, note the response area of the FTC expands with signal strength where an auditory fiber normally associated with a given $\mathrm{CF}$ might generate an action potential in response to a stimulus frequency not normally associated with its designated CF.

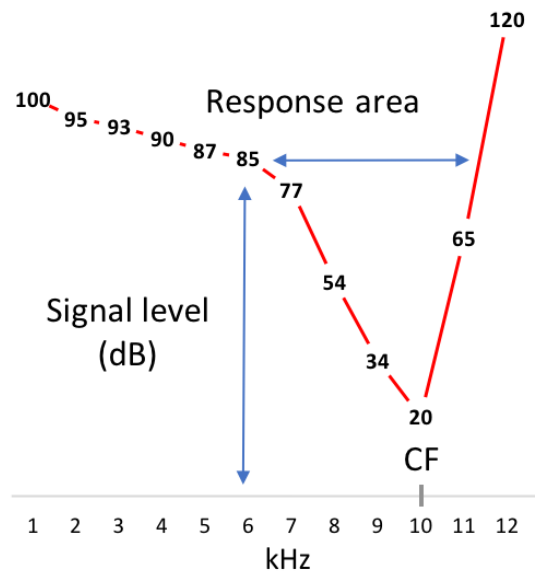

Figure 1. Stylized depiction of the FTC of a cochlear nerve fiber (adapted from Evans, 1993).

Of interest here is whether or not the variability in the FTC might interact with pitch, and by extension, timbre perception. If so, the perceptual outcome could manifest an increase in perceptual variability resulting in a decrease in discrimination accuracy commensurate with a rise in signal level.

\section{Method}

A four-part experiment was executed via headphone presentation in a semi-anechoic lab with a noise floor of 
21 dBA (IEC, 2013). The complete experiment comprised pitch- and timbre-discrimination tests with soft and loud conditions. Headphone output was calibrated for two levels where "soft" was associated with listening near hearing threshold and "loud" was referenced to a robust signal of $83 \mathrm{dBA}$ SPL. The loud listening level was chosen firstly because it was below the recognized action-level for mandated workplace hearing protections (OSHA, 2008); it matches well with popular press claims for so-called "best" listening levels when performing tasks such as blending musical instruments or making decisions regarding pitch and timbre; and finally, because it lies at a signal strength that is coincident with the observed broadening of the FTC.

The loud stimulus level was measured via ANSI/IEC 651 type-1 sound-level meter set to A-weighted, slow response (NMI, N.D.). Using the loud setting as a reference, the soft stimulus level was estimated via decrease of signal strength to just noticeable level within the noise floor of the room and headphone signal. The resulting signal was measured at $-68 \mathrm{~dB}$ VU (15 dBA SPL). The just noticeable level was initially obtained by investigators' audition and was confirmed via unanimous verbal acknowledgement from the subjects.

\section{Stimuli}

Stimuli were modeled on an aptitude test designed for fourth-grade assessment of basic music-oriented listening proficiency (Seashore, Lewis, \& Saetveit, 1960). Pitch test stimuli were seven $600 \mathrm{~ms}$ sinusoidal tones including 500, 508, 505, 504, 503, 502, and 501 Hz. The pitch stimuli set comprised sixty pairwise presentations where the $500 \mathrm{~Hz}$ stimulus was compared to each of the other six stimuli.

Stimuli for the timbre test were created by combining a $180 \mathrm{~Hz}$ sinusoidal tone with its first five harmonically related sinusoids (see Table 1). A resultant $1200 \mathrm{~ms}$ complex tone with phase-coincident harmonics at equal intensity was designated the "reference." Timbral quality of the reference tone was varied by reciprocal adjustment of the intensity of the 3rd and 4th harmonic sinusoids.

Table 1. Harmonic tones in the timbre stimuli.

\begin{tabular}{ccccccc}
\multicolumn{8}{c}{ Sinusoidal (Hz) } \\
Variation & $\mathbf{1 8 0}$ & $\mathbf{3 6 0}$ & $\mathbf{5 4 0}$ & $\mathbf{7 2 0}$ & $\mathbf{9 0 0}$ & $\mathbf{1 0 8 0}$ \\
\hline Reference & \multicolumn{7}{c}{ Equal intensity } & of all tones. \\
v1 & $\mathrm{nc}$ & $\mathrm{nc}$ & -10.0 & +9.0 & $\mathrm{nc}$ & $\mathrm{nc}$ \\
$\mathrm{v} 2$ & $\mathrm{nc}$ & $\mathrm{nc}$ & -8.5 & +4.0 & $\mathrm{nc}$ & $\mathrm{nc}$ \\
$\mathrm{v} 3$ & $\mathrm{nc}$ & $\mathrm{nc}$ & -7.0 & +2.5 & $\mathrm{nc}$ & $\mathrm{nc}$ \\
$\mathrm{v} 4$ & $\mathrm{nc}$ & $\mathrm{nc}$ & -5.5 & +1.2 & $\mathrm{nc}$ & $\mathrm{nc}$ \\
$\mathrm{v} 5$ & $\mathrm{nc}$ & $\mathrm{nc}$ & -4.0 & +0.7 & $\mathrm{nc}$ & $\mathrm{nc}$ \\
\hline "nc" $=$ no change of intensity \\
\hline \multicolumn{10}{c}{}
\end{tabular}

Five variations, each with greater differences in the reciprocal alternations were created. The timbre stimuli set comprised fifty pairwise presentations where the five variation stimuli (v1, v2, v3, v4, and v5) were each compared to the reference.

\section{Subjects}

Subjects were eleven graduate audio engineering students with ages clustered in the mid-twenties. All participants reported normal hearing and had completed a graduate course in ear-training for audio and music production. In a generalized sense, all subjects would be considered more experienced than "novice" but not yet "expert" listeners.

All subjects participated in all parts of the listening test. Subjects were randomly assigned to receive either the soft condition first with the loud condition following or vice versa. All four sessions were completed in one sitting. Instructions were given and sample stimuli were presented. However, there was no pre-test training. Each listening session lasted between six to seven minutes. Five-minute breaks were taken between sessions. The total testing session, inclusive of instructions, listening, breaks, and debriefing, lasted approximately $50 \mathrm{~min}$.

\section{Presentation}

In each session, every possible pairwise evaluation was presented ten times in a double-blind randomized sequence. All pairs were presented with $350 \mathrm{~ms}$ between stimuli and four seconds between trials. In the pitch test, the first and second stimulus orders, (i.e., higher or lower) were balanced with five of each (per stimulus) randomized across trials. Subjects were asked to "indicate if the second tone in a pair was higher or lower than the first." In the timbre test, the reference tone was presented first followed by one of the variations or a repeat of the reference. Subjects were then asked to indicate if the second tone in a pair "was the same or different from the first." Subject responses were recorded manually via Scantron answer sheet (Scantron, N.D.) and collated electronically.

\section{Results}

This experiment employed simple discrimination tests where subjects' per-trial responses were considered independent measures scored as either correct or incorrect. Repeated responses generated percentcorrect measures for categorical and grouped analyses. Due to the binomial origin of data, scalar response metrics were transformed for non-parametric statistical 
analyses (Peng, Lee, \& Ingersoll, 2002). Correct response measures (\%) are displayed for discussion.

Wilcoxon Matched-Pairs test indicated no significant difference between mean scores for soft VS loud condition order; therefore, data were combined without regard for condition order. Shapiro-Wilk tests rejected normality for all four data sets (pitch soft $W=$ $0.73, p<.001$; pitch loud $W=0.86, p<.001$; timbre soft $W=0.81, p<.001$; timbre loud $W=0.93, p=.002$ ) and all data sets demonstrated a strong negative skew. Loud response data exhibited lower kurtosis and were more broadly distributed than soft condition data for both tests (figures 2 and 3, rightward panels).
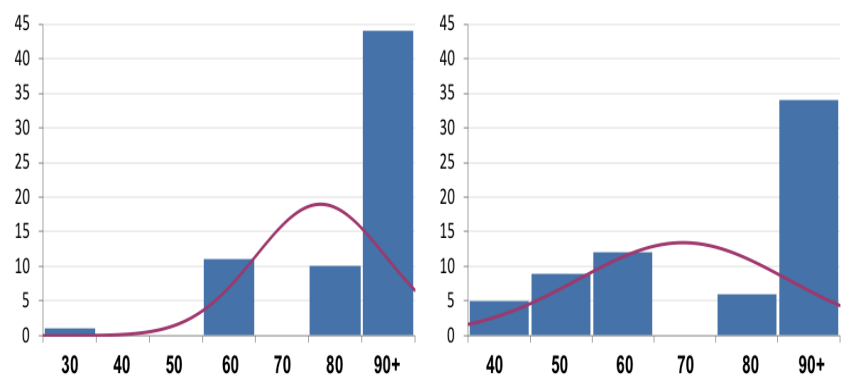

Figure 2. Percent correct score distributions for the pitch soft (left) and loud (right) data sets $(n=66)$.
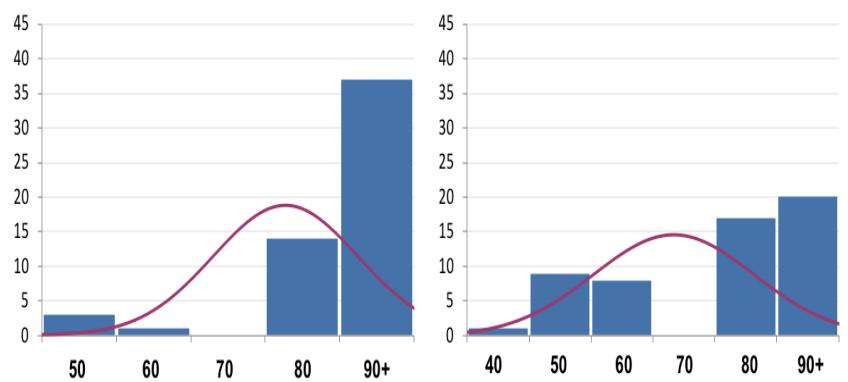

Figure 3. Percent correct score distributions for the timbre soft (left) and loud (right) data sets $(n=55)$.

Scores for both tests are plotted in figures 4 (pitch) and 5 (timbre). Here, each datum represents the mean of ten trials for each subject and each pair-wise stimulus combination. Comparative tests indicated significant differences $(Z=4.58, p<.001, n=66)$ between means of the soft $(M=.90, S D=.08)$ and loud $(M=.80, S D=$ .13) pitch trials and between the soft $(M=.89, S D=.05)$ and loud $(M=.73, S D=.14)$ timbre trials $(Z=4.26, p<$ $.001, n=55)$ indicating loud condition group scores were significantly lower than soft condition group scores for both types of stimuli (see tables 2 and 3 ).

A non-parametric analysis of variance (ANOVA) indicated a significant effect of pitch difference in both the soft $\left(X^{2}(5, \mathrm{~N}=11)=34.5, p<.001, w=.62\right)$ and loud $\left(X^{2}(5, \mathrm{~N}=11)=31.6, p<.001, w=.57\right)$ conditions suggesting pitch discrimination changed as differences $(\mathrm{Hz})$ decreased. ANOVA found no influence of timbre variation (v1-v5) in the soft condition. Here, scores resided predominantly above $80 \%$ suggesting all variations were equally identifiable. In contrast, a significant effect was indicated for variations of timbre in the loud condition $\left(X^{2}(4, N=11)=15.5, p=.003, w\right.$ $=.35$ ) where scores show increasing range and a greater number of incorrect judgements as differences between stimuli were decreased.

Table 2. Descriptive statistics of pitch discrimination performance.

\begin{tabular}{cll|ll} 
& \multicolumn{2}{c}{ Soft } & \multicolumn{2}{c}{ Loud } \\
Subject & $\boldsymbol{M}$ & $\boldsymbol{S D}$ & $\boldsymbol{M}$ & $\boldsymbol{S D}$ \\
\hline S-01 & 0.95 & 0.08 & 0.87 & 0.20 \\
S-02 & 0.95 & 0.08 & 0.87 & 0.15 \\
S-03 & 0.93 & 0.12 & 0.92 & 0.13 \\
S-04 & 0.92 & 0.12 & 0.90 & 0.20 \\
S-05 & 0.92 & 0.13 & 0.87 & 0.22 \\
S-06 & 0.78 & 0.12 & 0.52 & 0.13 \\
S-07 & 0.92 & 0.13 & 0.90 & 0.15 \\
S-08 & 0.72 & 0.23 & 0.73 & 0.16 \\
S-09 & 0.92 & 0.10 & 0.85 & 0.16 \\
S-10 & 0.93 & 0.12 & 0.82 & 0.10 \\
S-11 & 0.93 & 0.12 & 0.62 & 0.17 \\
\hline
\end{tabular}

Table 3. Descriptive statistics of timbre discrimination performance.

\begin{tabular}{ccc|ll} 
& \multicolumn{2}{c}{ Soft } & \multicolumn{2}{c}{ Loud } \\
Subject & $\boldsymbol{M}$ & $\boldsymbol{S} \boldsymbol{D}$ & $\boldsymbol{M}$ & $\boldsymbol{S D}$ \\
\hline S-01 & 0.90 & 0.10 & 0.74 & 0.15 \\
S-02 & 1.00 & 0.14 & 0.90 & 0.16 \\
S-03 & 0.90 & 0.07 & 0.80 & 0.13 \\
S-04 & 0.88 & 0.08 & 0.80 & 0.07 \\
S-05 & 0.88 & 0.08 & 0.70 & 0.12 \\
S-06 & 0.90 & 0.10 & 0.60 & 0.15 \\
S-07 & 0.90 & 0.14 & 0.80 & 0.08 \\
S-08 & 0.94 & 0.09 & 0.80 & 0.04 \\
S-09 & 0.82 & 0.15 & 0.90 & 0.15 \\
S-10 & 0.86 & 0.21 & 0.50 & 0.22 \\
S-11 & 0.84 & 0.15 & 0.50 & 0.18 \\
\hline
\end{tabular}

Grouped mean scores and standard deviations by stimulus are plotted in figures 6 (pitch) and 7 (timbre). Data here show increasing incorrect judgement trends consistent with those observed in the subject-bystimulus scores shown in figures 4 and 5 .

\section{Discussion}

Pitch test soft condition scores were above $90 \%$ for all but two subjects. In contrast, eight of eleven subjects 
scored $80 \%$ or lower in the loud condition. Subjects' timbre scores exhibited similar trends. Notably, the magnitude of difference between the conditions was $60 \%$ larger for the timbre test as compared to the pitch test indicating potentially greater influence of signal strength interaction with timbre perception.

As expected, scores for both conditions show increasing incorrect judgments as differences between stimuli were reduced. Comparatively, loud condition incorrect judgments are noticeably more broadly spread over a greater range across more stimulus pairs (figures 4 and 5). Additional indicators lie in the consistent across-stimuli higher scores and lower variability for the soft condition as contrasted against the loud condition for both sets of stimuli (figures 6 and 7).

Score distributions, response metrics, and statistical analyses all suggest a consistent negative change in performance for the loud condition. Nonetheless, this study is far from complete. Firstly, treatment conditions employed represent two rather unrealistic boundaries that are fundamentally unworkable for common practice. The requirement for extremely low background noise makes the soft listening level difficult

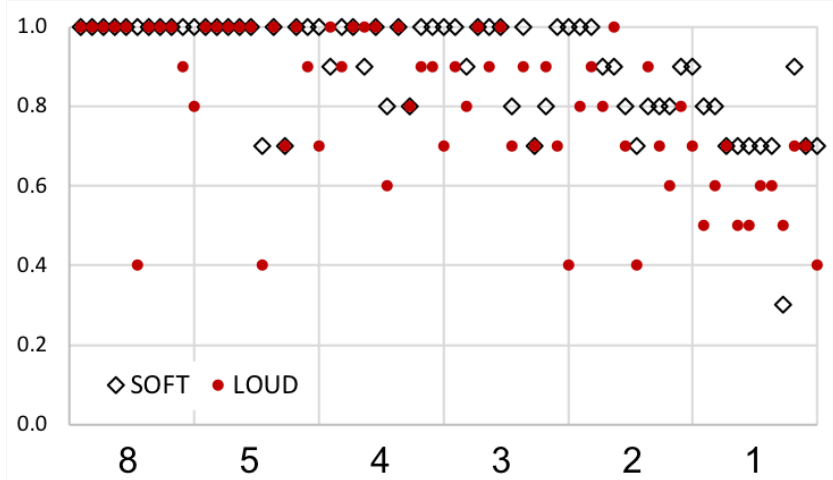

Figure 4. Subject scores by difference $(\mathrm{Hz})$ for the soft and loud pitch tests.

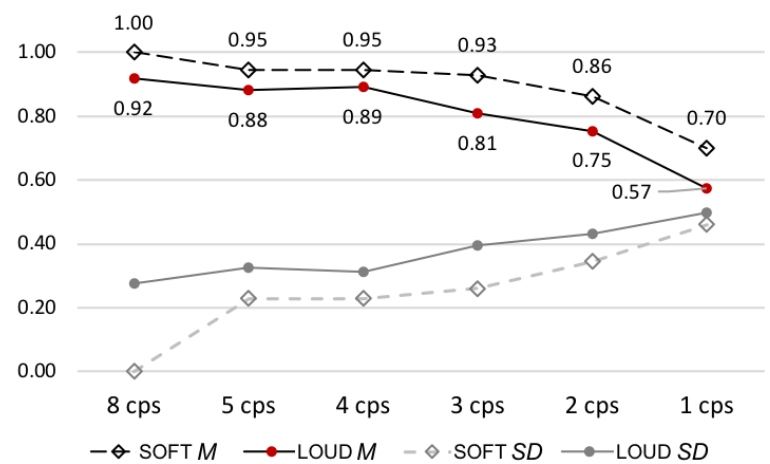

Figure 6. Group means $(M)$ and standard deviations (SD) by stimulus for pitch trials. to achieve and listening at or above the loud level is illadvised as doing so will likely induce auditory fatigue and temporary threshold shift during prolonged exposures (Hirsh, Bilgera, \& Burns, 2016; Regers, 2016). Secondly, from a psychophysical perspective, while neural fibers and their corresponding CFs may be associated with tonotopic theories of pitch perception (Moore, 2003), the auditory system employs a variety of mechanisms (e.g., inhibition, dampening, amplification, and neural timing) in a system vastly more complex than a simple band-pass filter might suggest (Matthews, 1999; Howard \& Angus, 2009). As a practical matter, one would expect cochlear loudness recalibration and adaptation (Scharf, 1997) to greatly influence the outcomes of any listening-focused task. An assumption of simple linearity of effect, i.e., "softer is better" would be, at least for now, vastly naïve.

This first experiment was a starting point in a broader investigation with many more intrinsic variables to consider and explore. While promising, clearly, more work is need in order to fully understand the influence of loudness when making critical auditory-based decisions.

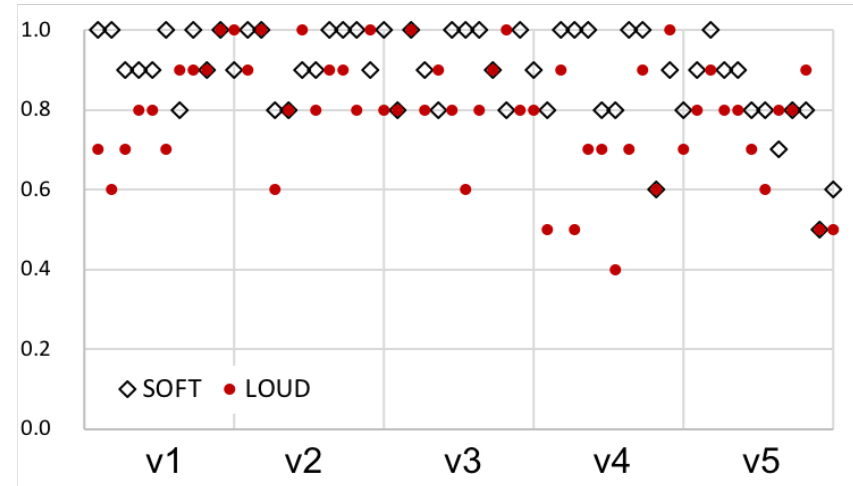

Figure 5. Subject scores by tonal variation for the soft and loud timbre tests.

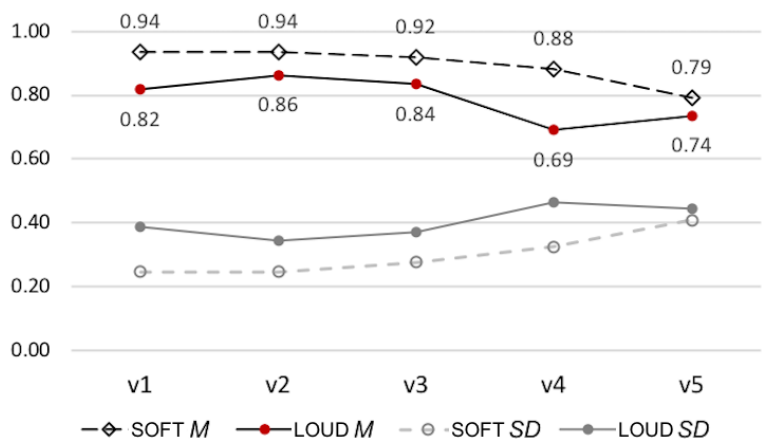

Figure 7. Group means (M) and standard deviations (SD) by stimulus for timbre trials. 


\section{Conclusion}

While outcomes here indicate a consistent negative influence of signal strength on the accuracy of both pitch and timbre perception, more investigation is needed to determine the practicality and application of the findings. Specifically, next steps should examine changes of signal strength across the auditory bandwidth to determine whether or not observations here may reliably apply across a range of related stimuli and listening levels. Additionally, future work should be varied enough to determine if there is an optimum loudness range for engaging in a broad variety of listening tasks such as identifying differences of pitch, discriminating fine details in timbre, or the blending and balancing of musical instruments.

\section{References}

Allen, R. (2014, November 9). The Best Listening Level for Mixing. (Rick Allen Creative Services, Inc. rickallencreative.com) Retrieved December 20, 2019, from https://www.rickallencreative.com/production-buzz/bestlistening-level-mixing

Devine, S. (2017, August 15). Best Studio Monitoring Levels For Mixing. (YouTube (at 5:23-5:41)) Retrieved December 20, 2019, from https://www.youtube.com/watch?v=wJfcxPEjdN8

Evans, E. (1993). Basic physiology of the hearing mechanism. AES 12th Conference: The Perception of Reproduced Sound. Paper \#12-002 (Permalink: http://www.aes.org/elib/browse.cfm? elib $=6253)$.

Fletcher, H., \& Munson, W. A. (1933). Loudness, its definition, measurement and calculation. J. Acoust. Soc. Am., 5(2), 82-108. https://doi.org/10.1121/1.1915637

Hirsh, I. J., Bilgera, R. C., \& Burns, N. W. (2016). AuditoryThreshold Recovery after Exposures to Pure Tones. ASA 49th Meeting (M6) (p. 1013). Acoustical Society of America.

Hodgkin, A. L., \& Huxley, A. F. (1952). A quantitative description of membrane current and its application to conduction and excitation in nerve. J. Physiol., 117(4), 500544. https://doi.org/10.1113/jphysiol.1952.sp004764

Howard, D., \& Angus, J. (2009). Chapter 2, Introduction to Hearing. In Acoustics and Psychoacoustics (4th ed.) (pp. 73-119). New York, NY: Focal Press. https://doi.org/10.1016/B978-0-240-52175-6.00002-8

IEC. (2013). IEC 61672-1 (2nd ed.) Electroacoustics - Sound level meters, ICS 17.140.50. International Electrotechnical Commission.

ISO. (2003). ISO 226:2003 Acoustics - Normal equalloudness-level contours (2nd ed.). Geneva, Switzerland: International Organization for Standardization.

ITU. (2015, Feburary 4). Methods for the subjective assessment of small impairments in audio systems. " Rec. ITU-R BS.1116-3 (02/2015) BS Series. Retrieved October
31, 2019, from International Telecommunications Union [https://www.itu.int/rec/R-REC-BS.1116-3-201502-I/en]

Matthews, M. (1999). The Auditory Brain. In P. Cook (Ed.), Music, Cognition, and Computerized Sound (pp. pp. 1-10). MIT Press.

Matthews, M. (1999). The Ear and How it Works. In P. Cook (Ed.), Music, Cognition, and Computerized Sound (pp. 1110). MIT Press.

McDonald, M. (2017, October 5). How Loud Should You Mix? (sweetwater.com) Retrieved December 20, 2019, from https://www.sweetwater.com/insync/how-loud-should-you$\underline{\operatorname{mix}}$

Moore, B. C. (2003). An introduction to the psychology of hearing. Academic Press (ISBN 0-12-505628-1).

Naqvi, R. (2013, September 18). PreSonus-How to calibrate your studio monitors. (presonus.com PreSonus Audio Products) Retrieved December 20, 2019, from https://www.youtube.com/watch? $\mathrm{v}=\mathrm{idGvZnSnPhs}$ (at 0:20$0: 43)$

NMI. (N.D.). Sound Level Meter Standards. Retrieved from Noise Meters, Inc., Berkley, MI: https://www.noisemeters.com/help/faq/standards/

OSHA. (2008). Occupational noise exposure. (URL: https://www.osha.gov/enforcement/directives/04-00-004): Occupational Safety and Health Admin., Federal Register, 29 CFR-2010, 1910-95.

Peng, C. Y., Lee, K. L., \& Ingersoll, G. M. (2002). An Introduction to Logistic Regression Analysis and Reporting. J. Edu. Resrch., 96(1), 3-14. https://doi.org/10.1080/00220670209598786

Pickles, J. O. (1984). Frequency threshold curves and simultaneous masking functions in single fibres of the guinea pig auditory nerve. Hearing Research, 14, 245-256. https://doi.org/10.1016/0378-5955(84)90053-4

Regers, S. N. (2016). Effects on Threshold Sensitivity of Exposing the Normal Ear to Pure Tones and White Noise over Sensation Levels of $10 \mathrm{db}$ to $100 \mathrm{db}$. ASA 49th Meeting (M7) (p. 1013). Acoustical Society of America. https://doi.org/10.1121/1.1918033

Rosenzweig, M. R., Leiman, A. L., \& Breedlove, S. M. (1996). Biological Psychology. Sunderland, MA: Sinauer Associates.

Scantron. (N.D.). Insight 20/30 form no. 223127, GPAS$100 Q \times 5$. store.scantron.com.

Scharf, B. (1997). Sequential Effects in Loudness. Boston, MA (URL: https://psychologie.lw.unileipzig.de/fechner/generalinfo/PDFs/BScharf.pdf): Department of Psychology and Institute for Hearing, Speech, and Language, Northeastern University.

Seashore, C., Lewis, D., \& Saetveit, J. G. (1960). Seashore Measures of Musical Talents (revised ed.). New York, NY: The Psychological Corp.

Swedien, B. (2009). Make Mine Music. NY: Hal Leonard. 


\section{Evaluating Tenney's critical band using a computational model of the human cochlea} Ashkan Fakhrtabatabaie ${ }^{1,2 \dagger}$

Skyler G. Jennings ${ }^{2}$

${ }^{1}$ College of Fine Arts, University of Utah, Salt Lake City, Utah, USA

${ }^{2}$ College of Health, University of Utah, Salt Lake City, Utah, USA

† Corresponding author: ashkan.fakhrtabatabaie@utah.edu

Published 16 December 2021; https://doi.org/10.18061/FDMC.2021.0048

Author video presentation and/or other conference material: https://doi.org/10.17605/OSF.IO/TMDFY

\begin{abstract}
To understand and formalize the perceptual outcomes produced by certain orchestration techniques, researchers studying the psychology of timbre rely largely on combining the listeners perception reports with audio analysis of musical excerpts. Despite their ability to predict many aspects of auditory perception, mathematical auditory models have been scarcely explored in understanding the neurophysiological basis of music perception. If implemented, these models can quickly simulate physiological responses thought to mediate the perception of various timbral effects. Using a computational auditory model (Zilany et al., 2014), we present a physiological analysis of an excerpt from Critical Band (Tenney, 1988/2000), which uses loudness fluctuations as a structural element to create implicit timbral phenomena such as beat frequency and roughness. In this study, the results of the score analysis, audio analysis, and auditory model predictions were compared. The score analysis (Fakhrtabatabaie, 2020) suggested an implicit timbral component that the audio analysis of a computer realization of the score was not able to demonstrate. However, the auditory model prediction supported the timbral effect observed in the score analysis. The results of this study suggest that the auditory model can successfully provide a neurophysiological correlate of an aspect of perception of a complex musical stimulus. The use of an auditory model in future research may help with interpreting the score and predicting what perceptual experiments are likely to reveal.
\end{abstract}

KEYWORDS: critical band, beat frequency, roughness, auditory filter, temporal envelope, inner hair cells, model simulation

\section{Introduction}

Critical Band is a music composition for any 16 or more sustaining instruments (Tenney 1988/2000). The title of Critical Band refers to a psychoacoustic concept with the same name, introduced by physicist Harvey Fletcher (1940). Fletcher used auditory masking techniques and established the concept of the critical band (CB) and auditory filters (AF) to account for the limitation of the auditory system in resolving simultaneous tones with a narrow frequency separation (e.g., 1-5\%). Our ears are sensitive to a wide range of frequencies and the basilar membrane (BM), located within the cochlea in the inner ear, plays a crucial role in separating input frequencies. Due to longitudinal variation of mass and stiffness along the BM, spatially separate locations on the BM vibrate independently from each other, and each location responds to a specific characteristic frequency (CF). Thus, the BM functions as a bank of bandpass AFs enabling the resolution of frequency components within complex input signals. The $\mathrm{CB}$ quantifies this frequency-tuning property of the inner ear: the $\mathrm{CB}$ is the width of frequencies that optimally pass through an $\mathrm{AF}$ without being attenuated. However, if two frequencies within an input signal are too close (e.g., 5\%), they cannot excite two fully independent $\mathrm{CF}$ locations on the BM. In other words, the excitation patterns elicited by these frequencies overlap (i.e., they pass through the same AF). One perceptual result of this overlap is that the excitation of the two frequencies will beat at a frequency equal to the frequency difference of the two components and give rise to the sensation of fluctuations in loudness or a roughness in timbre (Viemeister, 1979).

In the score analysis of Critical Band, Fakhrtabatabaie (2020) used intervallic analysis combined with phenomenology approaches and provided a detailed prediction of the anticipated timbral effects upon an accurate performance of the piece. According to Tenney's (1988/2000) program notes, the sensation of beat or roughness generated by simultaneous tones that are within the $\mathrm{CB}$ are two of the main timbral effects that Tenney desires to achieve in Critical Band. The timbral effects generated by this process are the perceptual results of destructive and constructive interferences of the amplitude envelopes of simultaneous tones within the $\mathrm{CB}$ also known as temporal envelope. The difference between the frequencies of the tones defines the frequency of the 
beat they generate. If the difference between the frequencies of two tones within the $\mathrm{CB}$ increases, the resulting beat become faster. When the frequency of the beat reaches above $\sim 20 \mathrm{~Hz}$ the beat is perceived as roughness (Plomp \& Steenken, 1968; Terhardt, 1974, 1978). This roughness has been described as the basis for the perception of sensory dissonance (Terhardt, 1976, 1984; Tramo et al., 2001). The roughness disappears and the sensory dissonance resolves as soon as the frequency difference exceeds the CB. In this study we implemented an auditory model to determine if physiological responses from the inner ear were consistent with the anticipated timbral effects in Critical Band. The perception of beats and roughness in response to tones within the $\mathrm{CB}$ may be associated with spectral distortion produced by inner hair cells (IHC) transduction, which is the transformation of the mechanical energy of the BM to electrical potentials that drive the response of the auditory nerve (Lins \& Picton, 1995).

Zilany et al. (2014) introduced a computational model of the human auditory system based on inner ear physiology measured from laboratory animals (e.g., Carney et al., 1993) and psychophysical findings in humans (Moore and Glasberg, 1993; Shera et al., 2002). The model includes stages that simulate middle ear, BM, IHC, synaptic, and auditory nerve function. Model simulations may be realized in MATLAB by inputting any acoustic waveform and obtaining the predicted response for an array of CFs for one or more model outputs including IHC, synapse, and auditory nerve stages. The simulated physiological responses from the model have been rigorously validated against a wide range of empirical data from animal physiology experiments (Zhang et al., 2001; Bruce et al., 2003; Jackson and Carney, 2005; Zilany et al., 2009), and experiments in simple psychophysics (e.g.., Jennings et al., 2011) and speech perception (e.g., Wirtzfeld et al., 2017).

We hypothesized that the timbral effects predicted in the score analysis will be revealed in the time-varying response of simulated IHCs from the auditory model.

\section{Method}

Using the program notes written by the composer and the interval analysis using frequency figures we (1) found a segment of Critical Band in which both beats and timbral roughness emerge, and (2) hypothesized a numerical measurement of beats and timbral roughness (discussed later and presented in Table I) to test by the physiological analysis.

Because the timbral effects explored in this study are the distortion products created by the nonlinearity of the inner ear we hypothesized that they would not appear in a spectral analysis of the acoustic signal. However, a spectral analysis of IHC responses to the acoustic signal should carry these timbral effects. To test this, a Fast Fourier Transformation (FFT) was employed to analyze the frequency components of both acoustic and physiological signals of Segment 5. A spectral analysis was used - as opposed to other analyses - as such analysis is physiologically realistic, given the known tonotopic representation of sound throughout the auditory system (Pickles, 2013). We performed the analysis on a $4000 \mathrm{~ms}$ slice of a digitally realized version of Segment 5 that we simulated using sine waves. This sine wave realization was done to eliminate any performance-related inconsistency in order to render the perceptual result in a condition that the tones were performed as accurately as possible. This slice was used as the acoustic input signal for acoustic and auditory model analysis. The output of an ensemble of 26 IHCs over an array of CFs spanning the frequency range between $125 \mathrm{~Hz}$ to $3 \mathrm{kHz}$ responding to this acoustic signal was calculated and used for the physiological analysis.

\section{Results}

Score Analysis

Segment 5 of Critical Band consists of a five-note chord built out of stacks of $13.8 \mathrm{~Hz}$ intervals. The chord tones are $\mathrm{G} \#(412.5 \mathrm{~Hz}), \mathrm{G} \#(426.2 \mathrm{~Hz}), \mathrm{A}(412.5 \mathrm{~Hz}), \mathrm{B}$ b $(453.8 \mathrm{~Hz})$, and $\mathrm{B} b(467.5 \mathrm{~Hz})$. The beat frequency column in Table 1 shows all possible intervals existing between the tones in Segment 5. Because of the symmetrical construction of this chord the $13.8 \mathrm{~Hz}$ interval and its first three harmonics were found four, three, two, and one time respectively. The occurrences column in Table 1 shows the number of times each interval was found in the intervallic analysis. We speculated that a larger number of occurrences should result in a larger amplitude of the physiological response of that beat frequency. 
Table 1: All possible intervals (left column) existing between the tones and number of occurrences (right column) in Segment 5 of Critical Band. From Fakhrtabatabaie (2020).

\begin{tabular}{|c|c|}
\hline \multicolumn{2}{|c|}{ Segment 5 } \\
\hline Beat frequency $(\mathbf{H z})$ & Occurrences \\
\hline 13.8 & 4 \\
27.5 & 3 \\
41.4 & 2 \\
55 & 1 \\
\hline
\end{tabular}

\section{Physiological Analysis}

Figure 1 shows the simulated waveform of $4000 \mathrm{~ms}$ of the audio signal of Segment 5. As you can see, the temporal envelope created by beats of the available tones in this segment is revealed by slower periodic dips and peaks. Figure 2 shows the frequency spectrum of Segment 5 using FFT. As expected, the temporal envelope associated with the implied beat frequencies discussed in the score analysis $(13.8,27.5,41.4$, and 55 $\mathrm{Hz}$ ) are not present in the frequency spectrum of the acoustic signal. FFT of the acoustic signal can only demonstrate the frequency components that exist in the acoustic signal, but is not capable of showing how the acoustic spectrum is modified by non-linear processes inherent in the peripheral auditory system and how these processes are expected to affect perception.

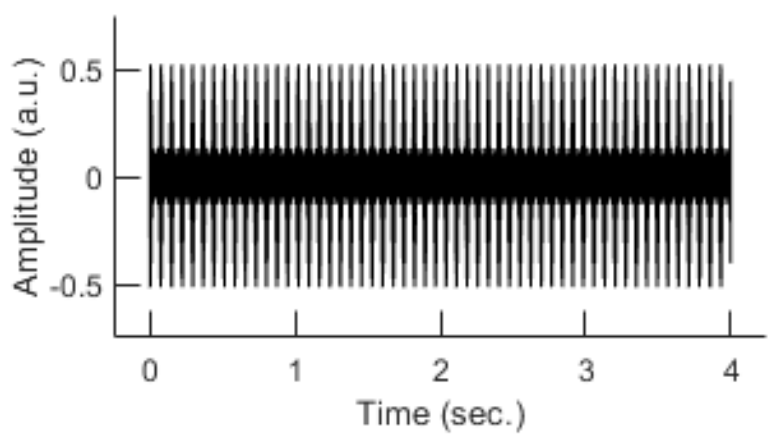

Figure 1: Simulated waveform of $4000 \mathrm{~ms}$ of the audio signal of Segment 5.

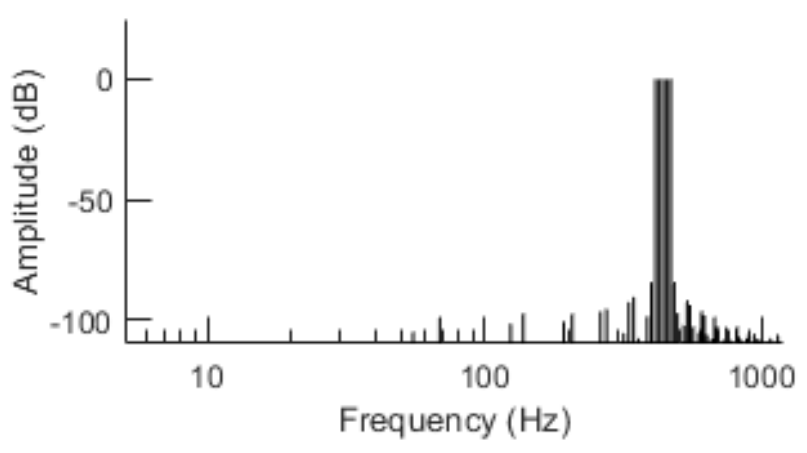

Figure 2: Frequency spectrum of the simulated waveform of Segment 5 calculated using FFT.

Figure 3 shows the output of IHCs of 13 different CFs ranging from 200 to $1000 \mathrm{~Hz}$ in response to Segment 5 of Critical Band. The output of the IHCs depends on the CF of the patch of BM to which the IHC resides within the inner ear. The IHCs located in close proximity to regions of the BM tuned to the input frequencies generate the strongest output. Thus, Figure 3 shows how only the IHCs located around the area of the $\mathrm{BM}$ responding to the characteristic frequencies ranging from $412.5 \mathrm{~Hz}$ to $467.5 \mathrm{~Hz}$ respond strongly to the inputs from Segment 5. Figure 4 shows the sum of the output signal of all individual IHCs simulated. The output of this population of IHC is very similar to the inputted acoustic signal, with one difference: the negative values of the amplitude fluctuation are clipped (i.e., rectified). This change in the physiological signal results in an output that contains frequency components not present in the acoustic input signal. Figure 5 shows the results of the spectral analysis of the physiological signal corresponding to Segment 5 using FFT, which was identical to the approach we employed in the acoustic signal analysis. The physiological signal contains the predicted beat frequencies from the score analysis. The results are also consistent with our second hypothesis that the number of iterations of each beat frequency is proportional to the relative amplitude of the physiological response. The results are consistent with the notion that nonlinear neural distortion produced by the transduction process of the IHCs contributes to the distinct beat percept experienced when listening to Critical Band. 


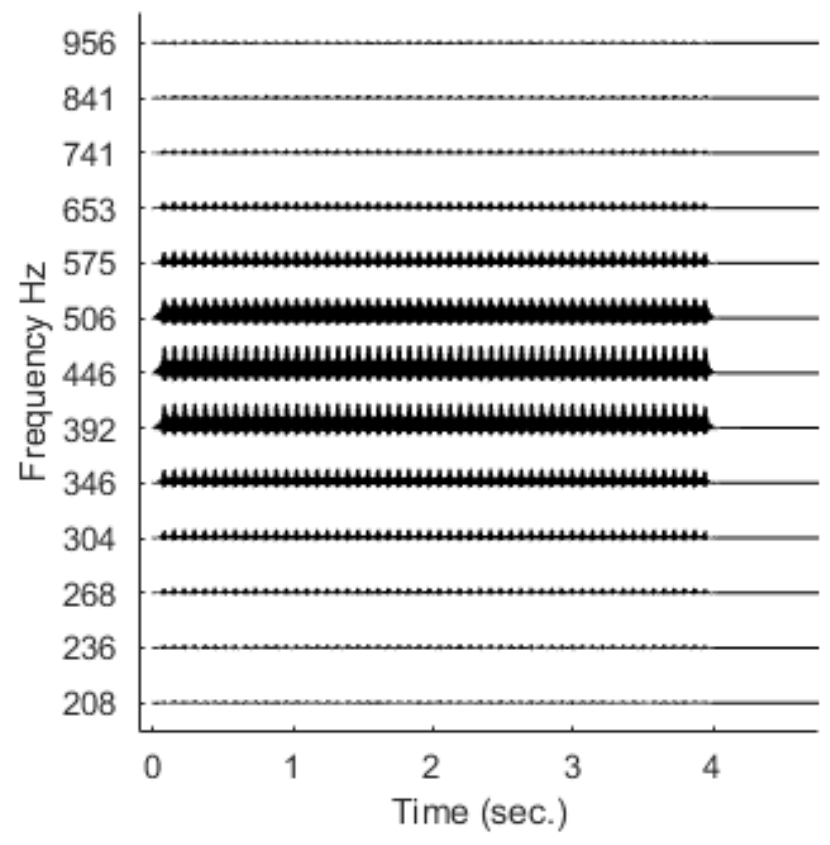

Figure 3: The output of individual IHCs of 13 different CFs ranging from 200 to $1000 \mathrm{~Hz}$ responding to Segment 5.

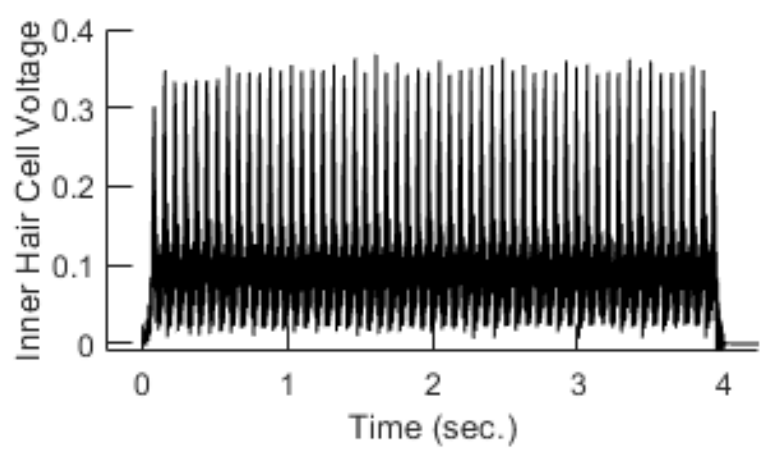

Figure 4: The sum signal of IHCs responding to Segment 5.

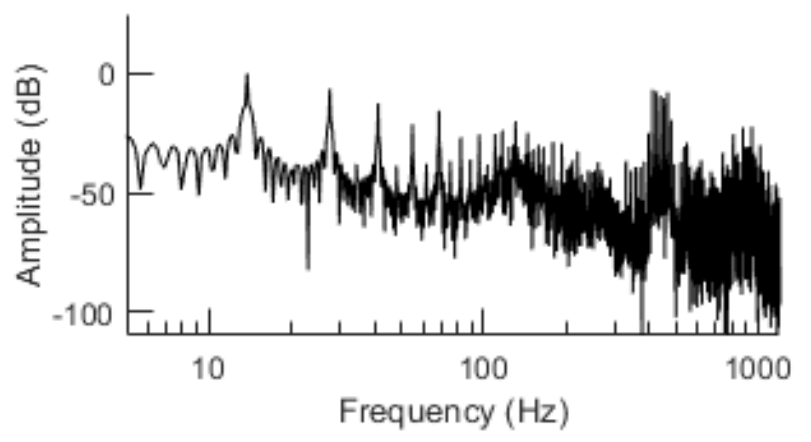

Figure 5: Frequency spectrum of the sum signal of IHCs calculated using FFT.

\section{Discussion}

The score analysis of Critical Band suggested implied envelope frequency components - resulting in beating and roughness in timbre - that a spectral analysis of the computer realization of the tones in the score was not able to detect. However, the auditory model prediction supported the implied frequency components observed in the score analysis. These results suggest that, if the computational auditory model employed in this study is accurate, the implied beats and timbral roughness suggested in the score analysis are generated in the inner ear and will be carried over to further stages of auditory processing.

\section{Conclusion}

A perceptual analysis can help theorists to understand and formalize the sonic outcomes that any acoustic stimulus will generate in the process of sound perception. In listeners' perception reports, audio segments are played for the listeners directly. Through a perceptual task or a survey that targets the expected perceptual outcomes of the audio segments, the listener's perception of these outcomes would be analyzed. The listener perception report method has some limitations, such as the lengthy process of data acquisition, the availability of a very controlled testing environment, as well as controlling for the many possible ways that the design of the survey or the perceptual task could create biases that may result in a false perceptual outcome. Our results show that a new model simulation combined with a thorough physiological understanding of a musical phenomenon could be used to predict some aspects of what perceptual studies are likely to reveal.

\section{References}

Bruce, V., Green, P. R., \& Georgeson, M. (2003). Visual perception : Physiology, psychology and ecology. (4th ed.) Psychology Press.

Fakhrtabatabaie, A (2020). Critical Band: A Continuum of Rhythm and Pitch Perception (Order No. 28153338) [Doctoral disertation, University of Utah]. ProQuest Dissertations and Theses Global.

Fletcher, H. (1940). Auditory Patterns. Reviews of Modern Physics, 12(1), 47-65. https://doi.org/10.1103/RevModPhys.12.47

Franklin, J. (2006). Settling Scores: A Life in the Margins of American Music. Sunstone Press.

Jackson, B. S., \& Carney, L. H. (2005). The spontaneousrate histogram of the auditory nerve can be explained by only two or three spontaneous rates and long-range 
dependence. Journal of the Association for Research in Otolaryngology: JARO, 6(2), 148-159. https://doi.org/10.1007/s10162-005-5045-6

Jennings, S. G., Heinz, M. G., \& Strickland, E. A. (2011). Evaluating adaptation and olivocochlear efferent feedback as potential explanations of psychophysical overshoot. Journal of the Association for Research in Otolaryngology: JARO, 12(3), 345-360. https://doi.org/10.1007/s10162-011-0256-5

Lins, O. G., \& Picton, T. W. (1995). Auditory steady-state responses to multiple simultaneous stimuli. Electroencephalography and Clinical Neurophysiology/Evoked Potentials Section, 96(5), 420432. https://doi.org/10.1016/0168-5597(95)00048-W

Moore, B. C. J. (2012). An Introduction to the Psychology of Hearing. Netherlands: Emerald.

Plomp, R., \& Steeneken, H. J. M. (1968). Interference between Two Simple Tones. The Journal of the Acoustical Society of America, 43(4), 883-884. https://doi.org/10.1121/1.1910916

Tenney, J. (2000). Critical Band: 1988/2000. Lebanon, NH: Frog Peak Music, 2000s.

Terhardt, E. (1974). On the Perception of Periodic Sound fluctuations (Roughness). Acta Acustica United with Acustica, 30(4), 201-213.

Terhardt, E. (1978). Psychoacoustic evaluation of musical sounds. Perception \& Psychophysics, 23(6), 483-492. https://doi.org/10.3758/BF03199523

Terhardt, E. (1984). The Concept of Musical Consonance: A Link between Music and Psychoacoustics. Music Perception: An Interdisciplinary Journal, 1(3), 276-295. https://doi.org/10.2307/40285261

Tramo, M. J., Cariani, P. A., Delgutte, B., \& Braida, L. D. (2001). Neurobiological foundations for the theory of harmony in western tonal music. Annals of the New York Academy of Sciences, 930, 92-116. https://doi.org/10.1111/j.1749-6632.2001.tb05727.x

Wirtzfeld, M. R., Pourmand, N., Parsa, V., \& Bruce, I. C. (2017). Predicting the quality of enhanced wideband speech with a cochlear model. The Journal of the Acoustical Society of America, 142(3), EL319. https://doi.org/10.1121/1.5003785

Zilany, M. S., Bruce, I. C., Nelson, P. C., \& Carney, L. H. (2009). A phenomenological model of the synapse between the inner hair cell and auditory nerve: long-term adaptation with power-law dynamics. The Journal of the Acoustical Society of America, 126(5), 2390-2412. https://doi.org/10.1121/1.3238250

Zilany, M. S. A., Bruce, I. C., \& Carney, L. H. (2014). Updated parameters and expanded simulation options for a model of the auditory periphery. The Journal of the Acoustical Society of America, 135(1), 283-286. https://doi.org/10.1121/1.4837815 


\title{
Timbre's function in the perception of affective intentions: Contextual information and effects of learning
}

\author{
Lena Heng ${ }^{1 \dagger}$ \\ Stephen McAdams ${ }^{2}$ \\ ${ }^{1,2}$ Schulich School of Music, McGill University, Montreal, Quebec, Canada \\ † Corresponding author: lena.heng@mail.mcgill.ca \\ Published 16 December 2021; https://doi.org/10.18061/FDMC.2021.0049 \\ Author video presentation and/or other conference material: https://doi.org/10.17605/OSF.IO/M45XA
}

\begin{abstract}
Timbre has been identified by music perception scholars as a component in the communication of affect in music. While its function as a carrier of perceptually useful information about sound source mechanics has been established, less is understood about whether and how it functions as a carrier of information for communicating affect in music. To investigate these issues, listeners trained in Chinese and Western musical traditions were presented with phrases, measures, and individual notes of recorded excerpts interpreted with a variety of affective intentions by performers on instruments from the two cultures. These excerpts were analyzed to determine acoustic features that are correlated with timbre characteristics. Analysis revealed consistent use of temporal, spectral, and spectrotemporal attributes in judging affective intent in music, suggesting purposeful use of these properties within the sounds by listeners. Comparison between listeners' perceptions across notes and longer segments also revealed greater accuracy in perception with increased musical context. How timbre is used for musical communication appears to be implicated differently across musical traditions. The important role timbre plays also appears to vary for different positions within a musical phrase, suggesting that patterns of change over time are crucial in emotional communication.
\end{abstract}

KEYWORDS: timbre, musical affect, acoustic features, cross-cultural differences

\section{Introduction}

Timbre has been shown to carry perceptually useful information about sound source mechanics, but while it has been identified by music perception scholars as a component in the communication of affect in music, there is still a lot to uncover about how it functions as a carrier of information for such communication. A recent definition by McAdams (2019) considers timbre as a "complex auditory attribute, or as a set of attributes, of a perceptually fused sound event in addition to those of pitch, loudness, perceived duration, and spatial position...[and] is also a perceptual property, not a physical one" (p.23). Even though timbre is a psychophysical attribute, and it is the perception of physical properties that defines timbre, it is undeniable that one cannot underestimate the importance of the physical acoustic properties that give rise to timbre perception. As surface acoustic properties carry important information for perception, a systematic approach to sound analysis that is "oriented towards human perception" (Peeters et al., 2011, p. 2902) and their relation to the communication of affective intentions in music will greatly aid the understanding of timbre's function in musical communication.

As Juslin and Timmers observed, "both the mean level of a cue and its variability throughout the performance may be important for the communicative process" (2010, p. 462). Therefore, it is likely that the amount of information timbre carries across different parts of a phrase varies according to musical context. Cultural noise might also be present because of "disparities which may exist between the habit responses required by the musical style and those which a given individual actually possesses" (Meyer, 1994, p. 16). As a result, how timbre is used for musical communication may also be different across musical traditions.

\section{Previous research}

Studies have revealed some differences between listeners from different cultures-Chinese and Western - in the multidimensional space obtained from rating dissimilarities of instrument sounds (e.g., Zhang and Xie, 2017). Although these differences might have been due to different sets of instruments used (Chinese vs. Western instruments), the different dimensions obtained from the multidimensional scaling could also imply a focus on different aspects of a sound by different groups of listeners (McAdams et al., 1995). 
With regards to attributing affective intentions to musical sounds, Scherer and Oshinsky (1977) systematically manipulated certain acoustic parameters to study listeners' ratings on both discrete emotions and dimensional affective intentions. Other researchers since then have also found consistent mappings of acoustic cues to affective responses (e.g., Schimmack \& Grob, 2000; Eerola et al., 2012; Bowman \& Yamauchi, 2016; McAdams et al., 2017). There are also many overlaps in the acoustic dimensions that seem to be involved in carrying these affective intentions, suggesting that affective content is not just related to a single acoustic dimension, but also communicated through the complex combinations and interactions of several acoustic parameters. Thompson and Balkwill (2010) also proposed in their cue redundancy model that listeners who are familiar with a musical style should be able to easily decode meanings in music of that style because "they can draw from both culture-specific and psychophysical cues" (p. 766).

\section{Research questions}

This study aims to look into how different aspects of a sound may be implicated in different affective intentions and how musical context provides varying amounts of information in musical communication. In addition, it also attempts to look at whether differences in musical experience and training influence both the ways acoustic cues are used by listeners and the accuracy of their responses.

\section{Method}

To investigate these issues, three groups of listeners with different musical backgrounds (Chinese musicians (CHM) and Western musicians (WM) and nonmusicians (NM), $n=30$ per group) from Singapore were recruited for listening experiments. The criterion for musicians during participant recruitment was to have more than five years of formal musical training in either the Chinese $(M=12.00, S D=2.98)$ or Western $(M=$ $12.13, S D=7.40)$ music tradition, and the criterion for nonmusicians was less than a year of formal training in any type of music $(M=0.2, S D=0.41)$. There was no significant difference between the number of years of musical training between the CHM and WM listeners, $\mathrm{F}(1,58)=1.41, \mathrm{p}=.24$. None of the WM listeners had any prior training in Chinese music while some CHM listeners had received formal instruction in Western music. However, all CHM listeners self-identified as being more proficient in Chinese music than Western music. All participants had casual exposure to both Chinese and Western art music, both being ubiquitous musical forms found in Singapore.

One professional musician for each instrument (dizi, flute, erhu, violin, pipa, and guitar) was recruited for the recording. The two-dimensional model of valence and arousal (Russell, 1980) was explained to the performers, and they were asked to interpret the excerpt of music in performance with five different affective intents: low valence and arousal, low valence and high arousal, high valence and arousal, high valence and low arousal, and neutral.

All of the listeners took part in two experimental sessions conducted at least a week apart. As the stimuli used for both experiments were obtained from the same recordings, this delay between the first and second experiments was to reduce any memory effects. Experiment A involved participants listening to individual notes extracted from the recorded excerpts, which were interpreted with a variety of affective intents by performers on Western and Chinese instruments, and then making judgments about each stimulus' perceived affective intent within a two-dimensional affective space of valence and arousal (Russell, 1980). Experiment B involved participants listening to measures and phrases of these same recorded excerpts and again making global judgments of the affective intent. Half of the participants were randomly assigned to experiment A first while the other half were assigned to experiment $\mathrm{B}$ first.

Using the Timbre Toolbox implemented in the MATLAB environment, individual notes were analyzed for their temporal, spectral, and spectrotemporal descriptors. Based on hierarchical clustering analyses done by Peeters and colleagues (2011), 13 acoustic descriptors that represent each cluster were selected. These acoustic descriptors included median and interquartile range of spectral centroid, spectral flatness, and RMS envelope, as well as the median for noisiness, harmonic spectral deviation, spectrotemporal variation, temporal centroid, frequency and amplitude modulations, and log attack time.

\section{Results}

To look into how much agreement there was between the listeners' perception of the affective intentions with what was intended by the performers, the average ratings of perceived affective intentions for each group of listeners were plotted with respect to their valence and arousal responses as shown in Figure 1. Due to 
space limitations, graphic representations of Figure 1 can be found at the following address: http://132.206.14.109/supplementaryMaterials/HengF DoMC2021/Fig1.pdf. Although each instrument has a slightly different pattern, arousal appears generally accurate with the data points more or less staying on the correct side of the space. Valence however is somewhat more ambiguous; positive-valence/low-arousal is often confused as negative-valence/low-arousal. With increasing musical context, valence appears to become more differentiated and becomes more accurate for the high-arousal conditions. The points also start to spread out more over the valence-arousal space.

The Kruskal-Wallis test on ranks was used to look at the main effects of listener groups with accuracy. Posthoc comparisons between the listener groups were done using the Mann-Whitney test with the Holm method used to adjust the alpha-level for multiple pairwise comparisons. Figure 2 shows the differences in accuracy for each affective intention, over notes, measures, and phrases. CHM listeners appear to be the most accurate. WM listeners generally perform more accurately than NM listeners with the exception of negative-valence, low arousal stimuli. All listeners also appear to fare badly for positive-valence, low-arousal stimuli. As can be seen in Figure 1, they tend to confuse this affective intention for negative-valence, lowarousal. Figure 2 can be accessed through this link: http://132.206.14.109/supplementaryMaterials/HengF DoMC2021/Fig2.pdf.

Next, the acoustic features listeners use to decode perceived affective intents are explored. This set of analyses focuses on whether listeners fluent in a particular musical tradition converge on a similar set of acoustic features they use in their decoding process, rather than on the accuracy. Instead of looking at the number of "correct" responses from the listeners, all the responses of the listeners in each group were coded into one of the four quadrants in the affective space, regardless of whether they were correct in their judgment of the performer's affective intent. The values of each acoustic descriptor for the notes in a particular quadrant are averaged. From this, four different sets of values for each acoustic descriptor are obtained over the 30-note excerpt. Similar procedures are used for listeners' responses from individual notes, measures, and phrases. Given that the sample size for each group of perceived affective intent can be very different and that consequently the assumptions for parametric tests might be violated, the Kruskal-Wallis test was used to test if the acoustic descriptors that are perceived as expressing different affective intents were significantly different between the groups of listeners, and post-hoc pairwise comparisons were performed using the MannWhitney test with Holm corrections.

Figures 3 to 5 shows the percentage of notes for each acoustic feature that are significantly different over the different affective intentions for each group of listeners. Figure 3 (CHM listeners): http://132.206.14.109/ supplementaryMaterials/HengFDoMC2021/Fig3.pdf.

Figure 4 (WM listeners): http://132.206.14.109/ supplementaryMaterials/HengFDoMC2021/Fig4.pdf.

Figure 5 (NM listeners): http://132.206.14.109/ supplementaryMaterials/HengFDoMC2021/Fig5.pdf.

As can be seen from these figures, differentiation between the different affective intentions increased with more musical context. However, it also appears that even when the notes are presented individually in a random order, listeners are quite consistent in their understanding of perceived affective intentions. This effect is even more pronounced for the CHM listeners where the values for several acoustic descriptors such as the spectral centroid median for dizi stimuli were all significantly different between the different affective intentions even at the note level. CHM listeners were generally more consistent in the acoustic features they used to determine the perceived affective intentions. There was also greater differentiation between the different affective intentions in the CHM listeners, followed by the WM listeners, whereas NM listeners were the least consistent and had the least differentiation. This trend was seen regardless of the musical tradition of the performer: CHM listeners performed with the greatest consistency in excerpts played by both Chinese and Western instruments.

\section{Discussion}

Increasing musical context provides listeners with more information for decoding affective intentions expressed by the performers and there was greater accuracy and clearer divergence between the different affective intentions. Listeners trained in Chinese music appear to be the most consistent, and this could be a result of differences in musical training. There may be differences in the emphasis on the use of timbre in the musical training of the Chinese, as compared to the Western musical tradition, and because of that, listeners trained in the Chinese music tradition may be more sensitive to minute changes in the way timbre is being manipulated in expressing an affective intent. Musical understanding is dependent on the performer, the 
stylistic characteristics of the composer, the musical tradition, and also on the experience and expertise of the listener and the listening process, just to name a few of these complex factors that might play a part in communicating musical intentions.

\section{Conclusion}

The function of timbre in communicating musical information is a highly complex process with many interactions involving not only different musical parameters, but also interactions between the multitude of acoustic features that make up the quality of a sound. Listeners with different musical backgrounds also appear to utilize the acoustic features to different extents which suggests that conventions regarding timbre function in musical communication are learned.

No continuous response was elicited from listeners with respect to changes in affective intents over the course of the excerpt in this experiment. Although the comparisons across responses for notes, measures, and phrases provide an indication of musical context providing increasing cues for understanding, future studies could attempt to look at continuous responses to better understand the function of timbre over the course of the music.

\section{Acknowledgements}

We thank CIRMMT for funding an Inter-Centre Research Exchange. We also thank Dr. Dorien Herremans (Singapore University of Technology and Design) for hosting LH for the Inter-Centre Research Exchange and for providing support in data collection. This research was supported by grants to SMc from the Canadian Social Sciences and Humanities Research Council (895-2018-1023) and the Fonds de recherche du Québec-Société et culture (017-SE-205667), as well as a Canada Research Chair (950-223484).

\section{References}

Bowman, C., \& Yamauchi, T. (2016). Perceiving categorical emotion in sound: The role of timbre. Psychomusicology: Music, Mind, and Brain, 26(1), 1525. https://doi.org/10.1037/pmu0000105

Eerola, T., Ferrer, R., \& Alluri, V. (2012). Timbre and affect dimensions: Evidence from affect and similarity ratings and acoustic correlates of isolated instrument sounds. Music Perception: An Interdisciplinary Journal, 30(1), 49-70. https://doi.org/10.1525/mp.2012.30.1.49

McAdams, S. (2019). The perceptual representation of timbre. In K. Siedenburg, C. Saitis, S. McAdams, A.
Popper, \& R. Fay (Eds.), Timbre: Acoustics, perception, and cognition (pp. 23-57). Springer. https://doi.org/10.1007/978-3-030-14832-4_2

McAdams, S., Douglas, C., \& Vempala, N. N. (2017). Perception and modeling of affective qualities of musical instrument sounds across pitch registers. Frontiers in Psychology, 8, 1-19. https://doi.org/10.3389/fpsyg.2017.00153

McAdams, S., Winsberg, S., Donnadieu, S., De Soete, G., \& Krimphoff, J. (1995). Perceptual scaling of synthesized musical timbres: Common dimensions, specificities, and latent subject classes. Psychological Research, 58(3), 177-192. https://doi.org/10.1007/BF00419633

Peeters, G., Giordano, B. L., Susini, P., Misdariis, N., \& McAdams, S. (2011). The timbre toolbox: Extracting audio descriptors from musical signals. The Journal of the Acoustical Society of America, 130(5), 2902-2916. https://doi.org/10.1121/1.3642604

Russell, J. A. (1980). A circumplex model of affect. Journal of Personality and Social Psychology, 39(6), 1161-1178. https://doi.org/10.1037/h0077714

Scherer, K. R., \& Oshinsky, J. S. (1977). Cue utilization in emotion attribution from auditory stimuli. Motivation and Emotion, 1(4), 331-346. https://doi.org/10.1007/BF00992539

Schimmack, U., \& Grob, A. (2000). Dimensional models of core affect: A quantitative comparison by means of structural equation modeling. European Journal of Personality, 14(4), 325-345. https://doi.org/10.1002/1099-0984(200007/08)14:4<325 :AID-PER380>3.0.CO;2-I

Thompson, W. F., \& Balkwill, L. (2010). Crosscultural similarities and differences. In P. N. Juslin \& J. A. Sloboda (Eds.), Handbook of Music and Emotion: Theory, Research, Applications (pp. 755-788). Oxford University Press. https://doi.org/10.1093/acprof:oso/9780199230143.003. $\underline{0027}$

Zhang, J., \& Xie, L. (2017). Analysis of timbre perceptual discrimination for Chinese traditional musical instruments. 2017 10th International Congress on Image and Signal Processing, BioMedical Engineering and Informatics (CISP-BMEI), 1-4. 


\title{
Chord spacing and quality: Lessons from timbre research
}

\author{
Noah Kahrs ${ }^{1 \dagger}$ \\ Matt Chiu ${ }^{1}$ \\ ${ }^{1}$ Eastman School of Music, University of Rochester, Rochester, NY, USA \\ ${ }^{\dagger}$ Corresponding author: nkahrs@u.rochester.edu \\ Published 16 December 2021; https://doi.org/10.18061/FDMC.2021.0050 \\ Author video presentation and/or other conference material: https://doi.org/10.17605/OSF.IO/G9DYF
}

\begin{abstract}
Although chords are often represented by pitch-class (chroma) content in computational research, chord spacing is often a more salient feature. This paper addresses this disparity between models and cognition by extending the discrete Fourier transform (DFT) theory of chord quality from pitch-classes to pitches. In doing so, we note a structural similarity between music theory's chord quality and audio engineering's timbral cepstrum: both are DFTs, performed in the pitch or frequency domains, respectively. We thus treat chord spacing as a hybrid of pitch-class and timbre.

To investigate the potential benefits of the DFT on pitch space (P-DFT), we perform two computational experiments. The first explores the P-DFT model theoretically by correlating chord distances calculated with a pitch-class model against those calculated with spacing. The second compares P-DFT estimations of chord distances against listener responses (Kuusi, 2005). Our results show that spacing is a salient feature of chords, and that it can be productively described by timbre-influenced methods.
\end{abstract}

KEYWORDS: chord spacing, chord quality, timbre, Discrete Fourier Transform, cepstrum

\section{Introduction}

Whether considering chords (Burgoyne et al., 2011), keys (Albrecht \& Shanahan, 2013; Temperley \& Marvin, 2008), or musical style (Yust, 2019), computational and corpus models of music tend to rely on pitch-classes (PCs, also known as pitch chroma), not pitches or frequencies. Although the assumption of octave equivalence may seem innocuous, given the crucial role of pitch chroma to perception (Krumhansl, 1979), listeners often identify chord similarity more by spacing than by PC content (Kuusi, 2005; Samplaski, 2001). To consider the role of spacing in chord quality, this paper takes advantage of a conceptual similarity between uses of the Discrete Fourier Transform (DFT) on pitch-classes in music theory and on spectra in computational timbre research.

The DFT is an equation that breaks a signal into periodic components. A DFT on a signal returns a set of values $f_{0}-f_{n}$ : $f_{n}$ (component $n$ ) represents a division of the signal's underlying space into $n$ equal parts. If a signal approximates an even division, then the magnitude for that component will be high. Figure 1 shows how DFTs are used in different disciplines. The DFT can be applied to a waveform to yield the pitch spectrum, which is commonly used in timbre research to yield cognitively salient timbre descriptors (Peeters et al., 2011). Shown on the same level, pitch and pitch-class content as used in music theory and pitch-based music cognition are conceptually equivalent in that they both use pitch data.

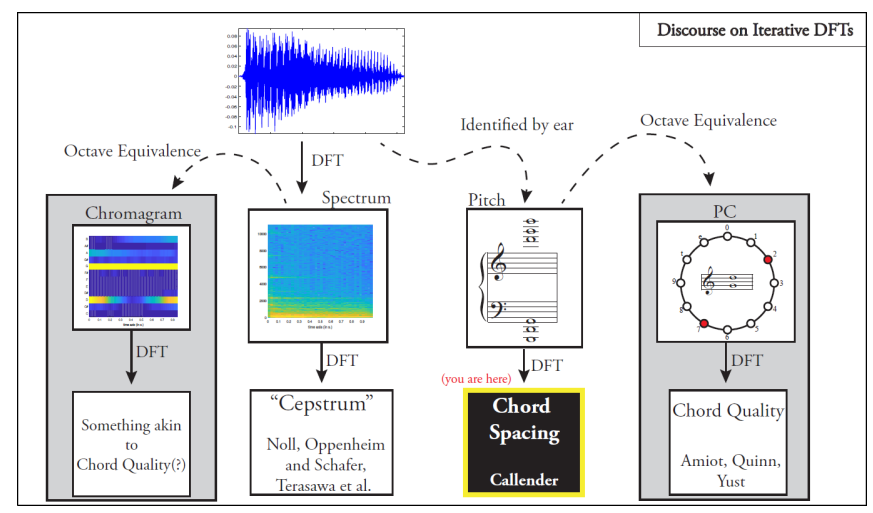

Figure 1: Summary of Previous DFT Research

Our paper introduces a hitherto unnoticed similarity between the cepstrum - a DFT on the spectrum's DFT data-and music theory's "chord quality," a DFT on pitch-class data (henceforth PC-DFT). The PC-DFT is used by mathematical music theorists to describe chord quality, which emerges from periodicities in pitch-class space (Amiot, 2016; Chiu, in press; Quinn, 2007; Yust, 2015). The cepstrum, the DFT of the spectrum, is also derived from periodicities in pitch data, albeit on all frequencies from the waveform rather than only on fundamentals. The cepstrum is used most often for timbre identification, (Aucoutrier \& Pachet, 2004; Herrera-Boyer et al., 2006), and despite its reputation for cognitive opacity, it does correlate with listener judgments of timbre similarity (Aucouturier \& Bigand, 
2012; Casey et al., 2012; Siedenburg et al., 2016; Terasawa et al., 2005).

Our P-DFT fuses these approaches by considering notated pitches, like the PC-DFT, but avoiding octave equivalence. Such a model was previously proposed by Callender (2007) in a continuous space; we expand on his work with both a more detailed examination of the P-DFT's relation to chord spacing, and a comparison to listener models of chord similarity. We thus study chord spacing using a combination of features from research in both pitch and timbre cognition, continuing in a tradition of investigating pitch-timbre relationships (Allen \& Oxenham, 2014; Hasegawa, 2019; Krumhansl \& Iverson, 1992; Saariaho, 1987).

To that end, we performed two exploratory computational studies to investigate how the P-DFT's spacing-oriented assessment of chord similarity compares to other measures, in both music-analytical and experimental contexts.

\section{General Method: Calculating and Interpreting P-DFT Components}

Both experiments used the same procedure to calculate chords' P-DFTs. First, chords were encoded as normalized characteristic functions in MIDI pitch space. For example, a closed-position C-major chord beginning on middle $\mathrm{C}$ would consist of notes $\mathrm{C} 4$, $\mathrm{E} 4$, and G4, or MIDI values 60,64 , and 67 . The corresponding characteristic function would be a 128place vector with the value .33 at positions 60,64 , and 67 , and 0 at all other positions. Then, the vectors were zero-padded (128 more zeros were appended) to avoid wrap-around effects.

A DFT was calculated on this vector of 256 values, yielding a new vector of 256 component magnitudes. Due to the Nyquist effect, only the first 128 of these components were non-redundant; the rest were discarded. Distances between chords were calculated as Euclidean distances (Albrecht \& Shanahan, 2013; Callender, 2007) between P-DFT vectors normalized in magnitude.

The $n$th component corresponds to chord spacing of $256 / n$ semitones. For example, an octave is 12 semitones, and $256 / 12=21+1 / 3$, so the corresponding components for the octave-21 and 22-would be high. Because this relationship is inversely proportional, low components correspond to wide spacing, whereas high components indicate specific smaller intervals. This parallels a similar situation in the cepstrum, in which only low components are used for timbre identification, whereas higher ones are more useful for pitch identification (Aucouturier \& Pachet, 2004; Muller \& Ewert, 2010).

Figure 2 shows the P-DFT component magnitudes for the iconic opening chord of Claude Debussy's "Sunken Cathedral" Prelude (henceforth the "Cathedral Chord"). We have circled peaks corresponding to the four-octave space between the two hands, as well as the open spacing, captured by peaks at the fourth, fifth, and tritone average within each hand.

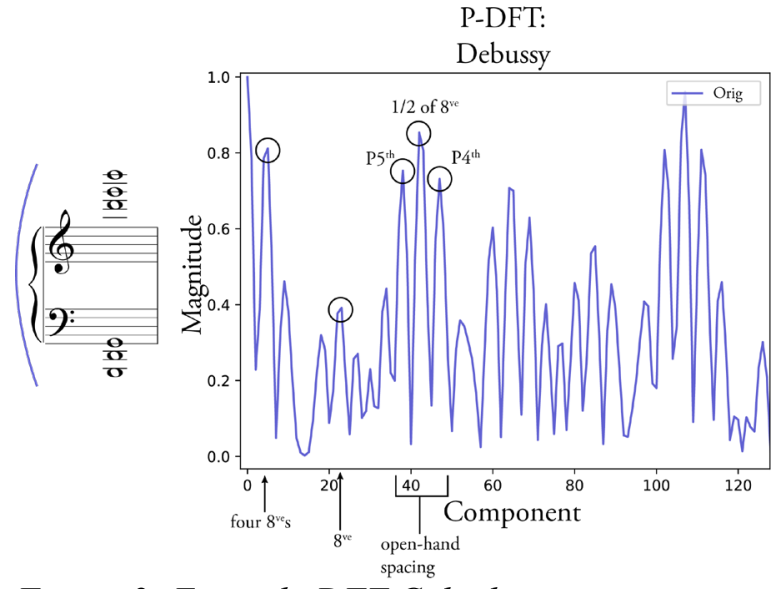

Figure 2: Example DFT Calculation

\section{Experiment 1: Similar Chords to Debussy's "Sunken Cathedral" under P- and PC-DFT}

Method

To determine what sorts of musical features are weighed by the P-DFT vs by the PC-DFT, we compared the opening chord of Debussy's "Sunken Cathedral" prelude to 50,000 random chords.

Chords were randomly generated by first selecting the number of pitches to be included (2 to 6), then independently selecting that many notes from the range of a standard grand piano (MIDI values 21 to 108) and removing duplicates. Distances were calculated from each random chord to Debussy's original chord as described above.

We hypothesized that chords similar to (that is, low distance from) Debussy's original chord by PC-DFT would be of a similar PC set-class (that is, related by transposition or inversion accounting for octave equivalence; see Morris, 1987), and that chords similar by P-DFT would have similar spacing on the piano keyboard. 
Results

Figure 3 plots dissimilarity of P- and PC-DFTs of all random chords, as measured against the Cathedral Chord. Although P- and PC-DFTs are correlated $(\mathrm{r}=.057, p<.001)$, there are clearly outliers. By investigating two outliers (selected by their maximal distance from the line extending from minimum to maximum P- and PC-DFT distances), we can see which features most directly affect assessed similarity.

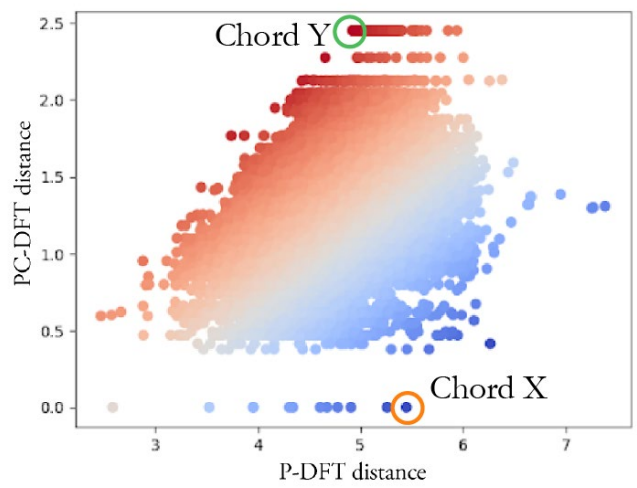

Figure 3: PC-vs P-DFT distances of random chords versus Cathedral Chord

Figure 4 compares the P-DFTs of the Cathedral Chord (Orig) and Chord X. Chord X is identical in PC content, and thus has PC-DFT distance 0. However, as an open eleventh, it has a completely different spacing, and thus its P-DFT has completely different components.

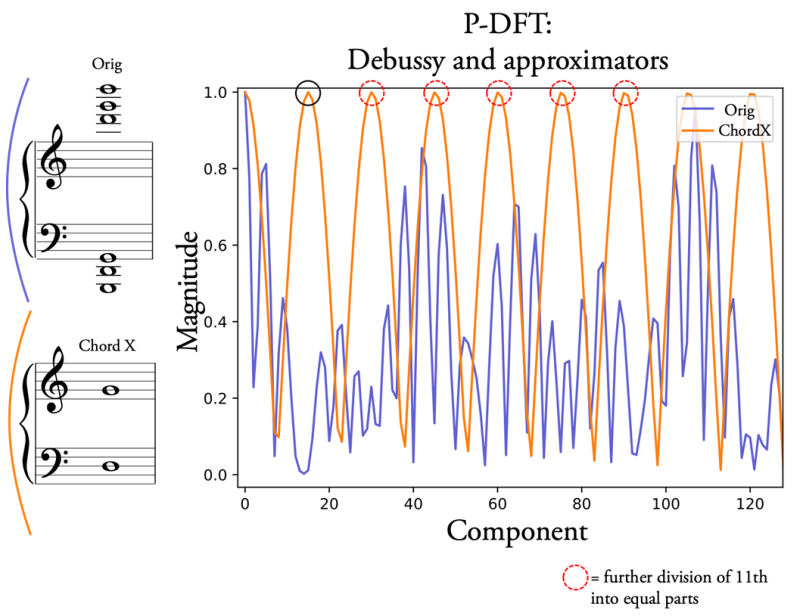

Figure 4: Cathedral Chord vs PC-similar chord

Figure 5 compares the Cathedral Chord to Chord Y, which is dissimilar in PC-DFT space, but somewhat similar in P-DFT space. It belongs to set-class (013579), which is almost a whole-tone scale and is very different from open fifths or fourths. However, its spacing is quite similar to that of the Cathedral Chord, as shown by shared peaks at components corresponding to wide spacing, octaves, and division of octaves into approximate halves (fifths/sixths).

\section{Discussion}

As we predicted, the P-DFT measures some aspect of chord spacing consistent with a subjective impression of Debussy's prelude; the prelude's opening, noted for its

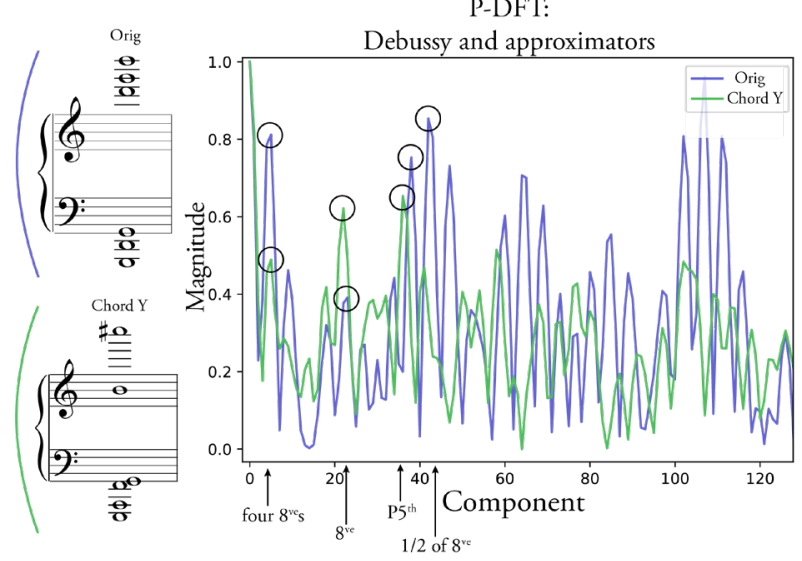

Figure 5: Cathedral Chord vs P-similar chord

wide spacing and "deep bass chords," (Roberts, 2001, p. 261) is reminiscent of a bell and the harmonic series. Such features are absent from the simple open eleventh of Chord X, but present in Chord Y despite its very different $\mathrm{PC}$ content.

These findings show that the P-DFT captures a musically relevant aspect of a chord's quality. Because the Cathedral Chord and Chord Y shared several similar peaks among low components, the P-DFT specifically captures wide-interval spacing. As low components measure timbre in the cepstrum, these components show that the P-DFT's chord spacing is sonically similar to timbre.

\section{Experiment 2: Correlations of P-DFT Similarity with Listener Judgments}

\section{Method}

In order to verify the model's cognitive salience, we compared P-DFT components with perceptual similarity ratings for chord distances from Kuusi (2005).

Kuusi's experiment explored correlations between subject responses and set-class similarity measures in a post-tonal setting. Her stimuli consisted of 16 chords: 4 different set classes each with 4 different spacings. Subjects were asked to rate the similarity of all possible 
pairs of chords on a Likert scale of 1-7. Subject ratings were correlated with set-class similarity measures; the measure yielding the highest correlation with subject responses was CSATSIM (Buchler, 1997) $(\mathrm{r}=.43$ ). Kuusi's subjects included both trained and less-trained musicians; we considered only the data from trained musicians. In a post-hoc analysis, Kuusi found that the subject-rated similarity between two chords increased with the difference in chord span (distance between the outer two notes of the chord).

Seeing the impact of chord spacing, we hypothesized that the Euclidean Distance between chords' P-DFT components would correlate more with subject data than set-class measures do. Such a finding would support our argument that the P-DFT, in measuring chord spacing, captures a cognitively meaningful aspect of chords erased by octave equivalence.

Results

The Pearson correlation between P-DFT distances and subject distances were significantly correlated $(\mathrm{r}=.38$, $p<.001$ ), but less so than CSATSIM. However, a stepwise regression yielded a subset of 49 components $(1-5,7-18,21,25,30,45,61-66,70,84-88,93,96$, $100,105-110,114,122-126,128)$ that was more correlated with subject responses $(\mathrm{r}=.74, p<.001)$.

\section{Discussion}

As the P-DFT components describe different elements of chord spacing, we expected distances between PDFTs to correlate with Kuusi's subject ratings. However, distances between raw P-DFT calculations are still outperformed by some set-class measures, perhaps because subjects use asymmetrical weightings for chord distances: P-DFT distances compare all 128 components equally rather than weighting particular features, whereas listeners might prioritize certain intervals over others. To identify such intervals in the experiment, we isolated and correlated individual component similarities with listener ratings of chord similarity.

Figure 6 shows that the 49 components selected by stepwise regression tend to cluster where components are particularly correlated or uncorrelated with listener ratings. Out of the 49 components, $32 \%$ corresponded to spacing wider than an octave (between $f_{0}-f_{20}$ ) something that could not be captured by PC measures. Later components clustered around smaller intervals that listeners may have found crucial in chord identification, and which were likely accounted for in PC measures such as CSATSIM. Because this specific combination of wide spacing and specific intervals better predicted correlations with listener data than PC similarity measures did $(r=.74)$, this result implies that our P-DFT model captures subjects' dual reliance on general spacing and on specific interval content.

Kuusi's experiment intended to produce a non-tonal environment, but, depending on the musical context, listeners may rely on different paradigms for evaluating chord proximity. Future experiments should explore this disparity, and the post-hoc exploration from Experiment 2 suggests that the P-DFT might be an effective tool for doing so. Furthermore, as listeners might prioritize certain intervals over others, future computational work might see if adjusted component weights better predicts listener responses, or if pitch-class measures provide additional non-redundant information.

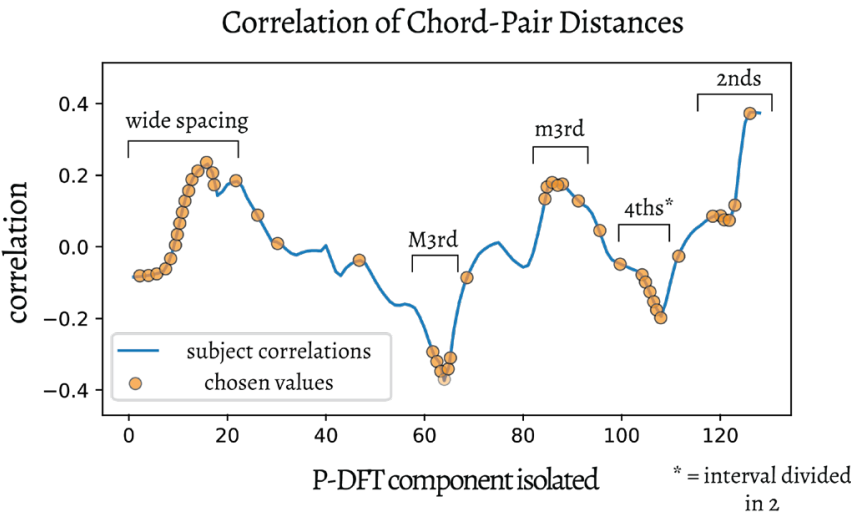

Figure 6. P-DFT Individual component correlations

\section{Conclusion}

Both of our experiments show that the P-DFT captures aspects of chord spacing that are salient, whether in the historical and music-theoretical reception of a piece or when comparing chords in isolation. As our theoretical framework demonstrates a formal similarity between chord quality and timbre similarity, our results fit with the growing literature on interactions between pitch, harmony, and timbre. Furthermore, by associating certain P-DFT components with specific chord spacings as well as listener similarity, we suggest that cepstrumlike methods may in fact be of potential use in cognitive studies.

\section{Acknowledgements}

We would like to thank Tuire Kuusi for providing us with data from her 2005 study, and David Temperley for providing comments on a draft of this article. 


\section{References}

Albrecht, J., \& Shanahan, D. (2013). The use of large corpora to train a new type of key-finding algorithm: An improved treatment of the minor mode. Music Perception, 31(1), 59-67. https://doi.org/10.1525/mp.2013.31.1.59

Allen, E. J., \& Oxenham, A. J. (2014). Symmetric interactions and interference between pitch and timbre. The Journal of the Acoustical Society of America, 135(3), 1371-1379. https://doi.org/10.1121/1.4863269

Amiot, E. (2016). Music through Fourier space. Springer. https://doi.org/10.1007/978-3-319-45581-5

Aucouturier, J., \& Pachet, F. (2004). Improving Timbre Similarity: How high's the sky? Journal of negative results in speech and audio sciences, 1(1).

Aucouturier, J.-J., \& Bigand, E. (2013). Seven problems that keep MIR from attracting the interest of cognition and neuroscience. Journal of Intelligent Information Systems, 41(3), 483-497. https://doi.org/10.1007/s10844-0130251-X

Buchler, M. H. (1997). Relative saturation of subsets and interval cycles as a means for determining set-class similarity [Doctoral dissertation, University of Rochester]. ProQuest Dissertations Publishing.

Burgoyne, J. A., Wild, J., \& Fujinaga, I. (2011). An Expert Ground Truth Set for Audio Chord Recognition and Music Analysis. ISMIR, 11, 633-638.

Callender, C. (2007). Continuous harmonic spaces. Journal of Music Theory, 51(2), 277-332. https://doi.org/10.1215/00222909-2009-004

Casey, M., Thompson, J., Kang, O., Raizada, R., \& Wheatley, T. (2012). Population codes representing musical timbre for high-level fMRI categorization of music genres. In Georg Langs, Irina Rish, Moritz GrosseWentrup, \& Brian Murphy (Eds.), Machine Learning and Interpretation in Neuroimaging (pp. 34-41). Springer. https://doi.org/10.1007/978-3-642-34713-9 5

Chiu, M. (in press). Macroharmonic Progressions through the Discrete Fourier Transform: An Analysis from Maurice Duruflé's Requiem. Music Theory Online, 27(3).

Hasegawa, R. (2019). Timbre as Harmony-Harmony as Timbre. In E. I. Dolan \& A. Rehding (Eds.), The Oxford Handbook of Timbre. Oxford University Press. https://doi.org/10.1093/oxfordhb/9780190637224.013.11

Herrera-Boyer, P., Klapuri, A., \& Davy, M. (2006). Automatic classification of pitched musical instrument sounds. In Signal processing methods for music transcription (pp. 163-200). Springer. https://doi.org/10.1007/0-387-32845-9 6

Krumhansl, C. L. (1979). The psychological representation of musical pitch in a tonal context. Cognitive psychology, 11(3), 346-374. https://doi.org/10.1016/00100285(79)90016-1
Krumhansl, C. L., \& Iverson, P. (1992). Perceptual interactions between musical pitch and timbre. Journal of Experimental Psychology: Human Perception and Performance, 18(3), 739. https://doi.org/10.1037/00961523.18.3.739

Kuusi, T. (2005). Chord span and other chordal characteristics affecting connections between perceived closeness and set-class similarity. Journal of New Music Research, 34(3), 259-271. https://doi.org/10.1080/09298210500236325

Morris, R. (1987). Composition with pitch-classes. Yale University Press. https://doi.org/10.2307/j.ctt1xp3ss4

Muller, M., \& Ewert, S. (2010). Towards Timbre-Invariant Audio Features for Harmony-Based Music. IEEE Transactions on Audio, Speech, and Language Processing, 18(3), 649-662. https://doi.org/10.1109/TASL.2010.2041394

Peeters, G., Giordano, B. L., Susini, P., Misdariis, N., \& McAdams, S. (2011). The Timbre Toolbox: Extracting audio descriptors from musical signals. The Journal of the Acoustical Society of America, 130(5), 2902-2916. https://doi.org/10.1121/1.3642604

Quinn, I. (2007). General equal-tempered harmony: Parts 2 and 3. Perspectives of New Music, 4-63. https://doi.org/10.1353/pnm.2007.0016

Roberts, P. (2001). Images: the piano music of Claude Debussy. Hal Leonard Corporation.

Saariaho, K. (1987). Timbre and harmony: Interpolations of timbral structures. Contemporary Music Review, 2(1), 93133. https://doi.org/10.1080/07494468708567055

Samplaski, A. G. (2000). A comparison of perceived chord similarity and predictions of selected twentieth-century chord-classification schemes, using multidimensional scaling and cluster analysis. [Doctoral dissertation, Indiana University]. ProQuest Dissertations Publishing.

Siedenburg, K., Fujinaga, I., \& McAdams, S. (2016). A Comparison of Approaches to Timbre Descriptors in Music Information Retrieval and Music Psychology. Journal of New Music Research, 45(1), 27-41. https://doi.org/10.1080/09298215.2015.1132737

Temperley, D., \& Marvin, E. W. (2008). Pitch-class distribution and the identification of key. Music Perception, 25(3), 193-212. https://doi.org/10.1525/mp.2008.25.3.193

Terasawa, H., Slaney, M., \& Berger, J. (2005). The thirteen colors of timbre. In IEEE Workshop on Applications of Signal Processing to Audio and Acoustics, 2005. (pp. 323-326).

IEEE. https://doi.org/10.1109/ASPAA.2005.1540234

Yust, J. (2015). Schubert's harmonic language and Fourier phase space. Journal of Music Theory, 59(1), 121-181. https://doi.org/10.1215/00222909-2863409 
Yust, J. (2019). Stylistic information in pitch-class distributions. Journal of New Music Research, 48(3), 217-231. https://doi.org/10.1080/09298215.2019.1606833 


\title{
A new auditory theory and its implications for the study of timbre
}

\author{
Braden N. Maxwell ${ }^{1}$ \\ Johanna B. Fritzinger ${ }^{2}$ \\ Laurel H. Carney ${ }^{2,3 \uparrow}$ \\ ${ }^{1}$ Eastman School of Music, University of Rochester, Rochester, NY, USA \\ ${ }^{2}$ School of Medicine and Dentistry, University of Rochester, Rochester, NY, USA \\ ${ }^{3}$ Hajim School of Engineering and Applied Sciences, University of Rochester, Rochester, NY, USA \\ $\dagger$ Corresponding author: Laurel_Carney@,rochester.edu \\ Published 16 December 2021; https://doi.org/10.18061/FDMC.2021.0051 \\ Author video presentation and/or other conference material: https://doi.org/10.17605/OSF.IO/XA2TZ
}

\begin{abstract}
A relatively new auditory theory describes how representations of the spectrum are transformed and sharpened in the early (below the cortex, or sub-cortical) auditory system (Carney, 2018). The current article introduces this theory and considers implications for timbre using computer model simulations. Models suggest that between two locations in the early auditory system, the auditory nerve and the midbrain, the neural representation of spectral peaks (the representation in overall activity along the tonotopic axis) becomes more precise. This peak-sharpening process depends on timing patterns of nerve activity called neural fluctuations. Neural fluctuations are comparable to temporal amplitude modulation but are, in some cases, created and modified within the auditory system rather than simply reflecting the stimulus itself. After the peaksharpening process, a center of mass of the most prominent spectral peaks - as encoded in the midbrain - serves as a neural representation of brightness. This work suggests that brightness may be fundamentally related to the concepts of locally prominent spectral peaks (spectral irregularity) and temporal modulation.
\end{abstract}

KEYWORDS: timbre, centroid, modulation, computational modeling, spectral irregularity

\section{Introduction}

The spectral centroid and similar acoustic descriptors have been established as important to the perceptual representation of musical instrument sounds, and specifically the percept of brightness, in numerous previous studies (McAdams, 2019). Here we discuss how a neural representation of spectral centroid may arise in the auditory system, according to a new theory of sub-cortical auditory representations (Carney, 2018). The primary goal of our present work is not to offer an alternative calculation for the centroid (see for example various centroid definitions discussed in Marozeau et al., 2003), but rather to investigate how the representation of the spectral centroid changes throughout the early auditory system. Understanding encoding of timbre at these early stages is an important open problem, especially for the purpose of restoring the perception of timbre for individuals with hearing loss.

The theory of sound representations proposed by Carney (2018) suggests a loose analogy between the auditory midbrain and the optic nerve in the visual system. In the visual system, contrast across the visual field is more important than brightness at any given point; similarly, this auditory theory suggests that contrast in the temporal features of neural signals (properties related to their timing) across the tonotopic axis (the axis describing neural tuning, from low to high frequencies) is more important than energy at a given frequency in a sound spectrum.

A particular category of temporal features of neural signals, referred to as 'neural fluctuations,' is foundational to this theory. Neural fluctuations are relatively low-frequency $(\sim 10-200 \mathrm{~Hz})$ time-varying changes in the statistics of auditory-nerve activity (Carney, 2018). This range of frequencies extends higher than the range of temporal modulation frequencies typically considered most essential for speech (Elhilali, 2019), and is generally much lower than the tonotopic 'tuning' of the neurons that carry them. These time-varying changes are often superimposed on higher-frequency changes in a neuron's activity (for example, phase locking), shaping the temporal envelope of the neural signal. Fluctuations can occur for a variety of reasons. Although they can result directly from amplitude modulation in a stimulus, they can also result from the filtering process. Beating between the components of a complex tone, for example, can produce fluctuations at the beating frequency. (Relatively high neural beating frequencies are possible at the high sound levels used for music listening (Epstein et al., 2010) because cochlear filters are wider at higher than at lower sound levels, see Figure 1 in Rose et al., 1971). Nonlinearities associated with the conversion of mechanical sound signals to neural signals strongly affect these neural fluctuations, making them substantially different from pre-neural 
representations of the sound (Carney, 2018). An important property of fluctuations, owed in part to these nonlinearities, is that they are relatively consistent if the sound is rescaled to a different sound level within the music-listening range.

Most midbrain neurons are sensitive to neural fluctuations (meaning their amount of activity increases or decreases as fluctuations change). In fact, the range of frequencies we have specified for neural fluctuations is derived from midbrain properties (see Figure 9 in Joris et al., 2004). The midbrain converts differences in fluctuations across the auditory-nerve tonotopic axis (passed through several intermediate neural stages) into different amounts of activity across its own tonotopic population of neurons. Here we focus on one type of midbrain neuron, called 'band-suppressed,' that has decreased response activity when fluctuation amplitudes increase, and vice versa.

What does this theory mean for the sub-cortical encoding of spectral centroid? The first step toward answering this question is to understand how neural fluctuations can facilitate sharper representations of spectral peaks. Figure 1 walks through this peaksharpening process as shown in model responses (models described in more detail in Method), beginning with a stimulus from Allen and Oxenham (2014) that has a single spectral peak (Figure 1A). Figure 1B shows the long-term average of auditory-nerve activity across a tonotopic population (the closest thing to a Fourieranalysis representation in the early auditory system) in response to the Allen and Oxenham stimulus. Each point represents the activity of a nerve fiber tuned to a unique tonotopic frequency. Note that this representation of the energy at different frequencies is broad and "blurred," and previous work has suggested that this representation is inadequate for center-of-mass estimates consistent with the percept of brightness (Maxwell et al., 2020).

Figure $1 \mathrm{C}$ shows activity of three auditory-nerve fibers from $1 \mathrm{~B}$, but zoomed to reveal detailed timing patterns (instead of the average response over time, as in 1B). For nerve fibers at points on the tonotopic axis below or above the spectral peak - in this case, $1000 \mathrm{~Hz}$ (left) and $1400 \mathrm{~Hz}$ (right), the activity is 'bumpy:' there are neural fluctuations due to beating between harmonics (4 cycles shown). However, at the peak $(1200 \mathrm{~Hz})$ the fluctuations are substantially reduced because the highest-magnitude harmonic dominates: there is no beating, and the envelope of the neural signal is nearly 'flat.' Importantly, this 'flatness' only occurs for nerve fibers very close to the spectral peak on the tonotopic axis.
A: Spectrum of Allen and Oxenham Stimulus

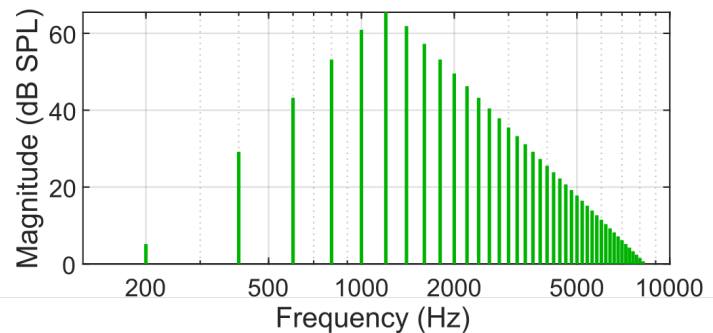

B: Auditory-Nerve Model

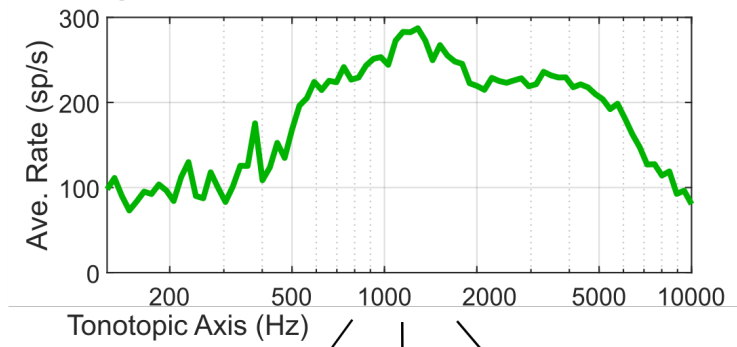

C: Neural Fluctuations

Below spectral peak: At spectral peak: Above spectral peak: Fluctuating 'Flat' Fluctuating

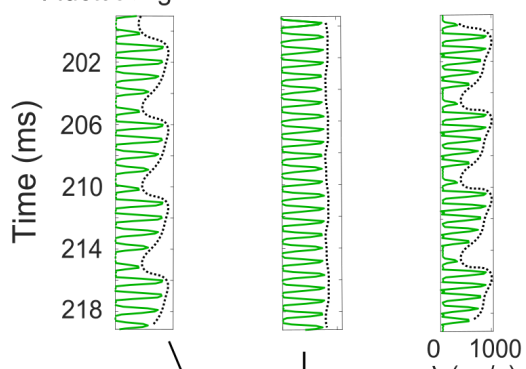

D: Midbrain Model

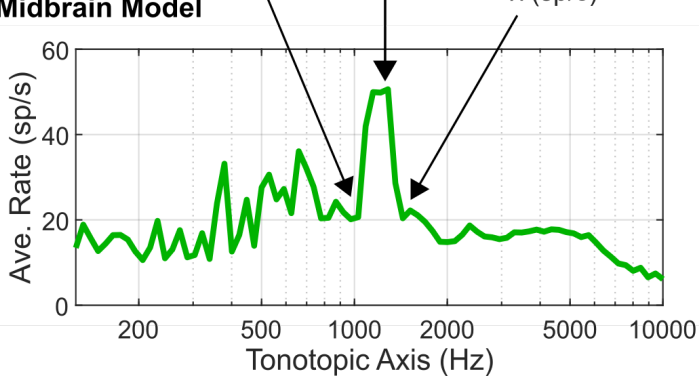

Figure 1: (A) Spectrum of a stimulus from Allen and Oxenham (2014): $F 0=200 \mathrm{~Hz}$, spectral peak at $1200 \mathrm{~Hz}$, $70 \mathrm{~dB}$ SPL overall level. (B) The overall amount of activity (averaged over $500 \mathrm{~ms}$ ) along the auditorynerve tonotopic axis in response to (A). (C) Another perspective on the activity of the same nerve fibers represented in (B), but focused on short-term timing patterns (neural fluctuations). Fluctuations are decreased at the spectral peak. For more detail on $\lambda$, see [1]. (D) Midbrain activity is sharply increased where fluctuations are decreased, and vice versa, precisely encoding the spectral peak. 
After transformation by intermediate stages of the auditory system, the midbrain receives fluctuating or flat signals (Figure 1C). Midbrain band-suppressed neurons exhibit an overall decrease in activity when fluctuations increase, so they respond strongly at the spectral peak, but not above and below it (Figure 1D).

These simulations emphasize the precision of midbrain encoding of spectral features and the potential for a precise representation of brightness to emerge at the midbrain stage. In the case of a stimulus with a single spectral peak, shifting the peak (and the peak in midbrain activity) shifts the centroid. However, some spectra have multiple peaks. In results shown here, we propose that the center of mass of the peaks of the midbrain representation, regardless of how many peaks there are, serves as a sub-cortical representation of the brightness of musical sounds (a midbrain-based 'centroid'). Our results include midbrain-based centroid estimates for several instrument samples (a basic test of feasibility of this theory) and one case study, a multipeak violin spectrum.

\section{Method}

Stimuli

For Figure 1, the stimulus was a 500-ms duration complex tone with a fundamental frequency of $200 \mathrm{~Hz}$, all harmonics up to $10 \mathrm{kHz}$, a band-pass spectral envelope shape centered at $1200 \mathrm{~Hz}$ rolling off at 24 $\mathrm{dB} /$ octave, and 20-ms onset and offset ramps (Allen and Oxenham, 2014). For the simulation results shown below, stimuli were from the pre-2012 University of Iowa Musical Instrument Samples (Fritts, 1997). All instrument samples, perfomed at mezzo-forte, had a fundamental frequency of $311 \mathrm{~Hz}$ ( E b 4 ). Guitar and violin samples were performed on the $\mathrm{B}$ and $\mathrm{D}$ strings, respectively. Stereo recordings were converted to mono before simulations, and all stimuli were scaled to an overall level of $70 \mathrm{~dB}$ SPL at the input of the auditorynerve model (this scaling is necessary because the auditory-nerve model properties change realistically with level). For simulations in which pink noise was added, the noise lasted throughout the simulation and had an approximate spectrum level of either 7 or $13 \mathrm{~dB}$ re $20 \mu \mathrm{Pa}$ at $100 \mathrm{~Hz}$. Both levels of noise were used for Figure 2; the lower level was used in Table 1. Instrument samples were scaled to $70 \mathrm{~dB}$ SPL before adding noise.

\section{Models}

Computational models simulated the responses of the auditory nerve (Zilany et al., 2014) and midbrain band- suppressed neurons (Carney, et al. 2015; Maxwell et al., 2020). Eighty tonotopic frequency channels (logarithmically spaced between $125 \mathrm{~Hz}$ and $10 \mathrm{kHz}$ ) were included in each simulation. The output of each model auditory-nerve fiber was passed to brainstem and midbrain stages that inherited their input's tonotopic frequency; there was no interaction between tonotopic channels. The auditory-nerve model simulated highspontaneous-rate fibers, the most common type of fiber (Liberman, 1978). High-spontaneous-rate fibers, with their low thresholds and saturated average response rates at high sound levels, present a challenge for traditional rate-based codes of the spectrum (Carney, 2018). Midbrain best modulation frequency (BMF, or the fluctuation frequency to which the midbrain is most sensitive) was set to $100 \mathrm{~Hz}$ for model midbrain neurons above $400 \mathrm{~Hz}$ on the tonotopic axis. Below $400 \mathrm{~Hz}$, BMF was set to the tonotopic frequency divided by 4 . These settings reflect a plausible range of modulation tuning frequencies for midbrain neurons (Langner et al., 2002). User-friendly software and instructions for conducting these simulations and producing similar figures is available at https://osf.io/2gpz6/. This software may be used with any input stimulus.

\section{Neural Estimate of Centroid}

To estimate the centroid as encoded in the model midbrain response (Table 1), we used a process described in detail in a previous proceedings article (Maxwell et al., 2020). Briefly, we calculated an estimate of the center of mass of the average amount of activity along the midbrain tonotopic axis (the axis shown in Figures 1D and 2C). The activity for each tonotopic location was averaged over $500 \mathrm{~ms}$ beginning just before the stimulus onset.

\section{Results and Discussion}

Figure 2A shows the spectrum of a violin recording with multiple local peaks (blue arrows) - two low harmonics that are higher than the others and are widely separated on a log scale (making them each a 'local peak' of sorts), and two peaks above $2000 \mathrm{~Hz}$ that are prominent relative to surrounding harmonics. This simulation included three versions (see Method), one without any added background noise (light gray), one with low-level pink noise (dark gray), and one with slightly higherlevel pink noise (green, shown in 2A). As in Figure 1, the average responses of the model auditory-nerve fibers along the tonotopic axis provide a broad, non-specific 
representation of the spectral peaks (Figure 2B). Figure $2 \mathrm{C}$ shows model midbrain activity.

In the simulation without pink noise (light gray), several harmonics are clearly represented in the midbrain, but the local spectral peaks do not stand out clearly from the other harmonics in every case. Because the violin fundamental frequency is higher than the fundamental frequency in Figure 1 (311 instead of 200 $\mathrm{Hz}$ ) there is more empty space in the spectrum - the wider spacing of harmonics prevents beating (fluctuation) frequencies that are constructive for the peak-sharpening process. However, in many realistic listening situations this space is filled by the harmonics of other instruments or low-level ambient noise. When a low-level pink noise is introduced ( $2 \mathrm{C}$; dark gray line) and then a higher-level pink noise (2C; green line) the spectral peak code becomes sharper, emphasizing the four peaks identified by blue arrows in the spectrum. Counterintuitively, a small amount of noise actually enhances this representation by increasing the amount of neural fluctuations in the auditory-nerve responses at tonotopic locations below and above the spectral peaks, while leaving fluctuations in nerve fibers near the spectral peaks unchanged (flat).

If we calculate a center of mass of this model midbrain representation (the green line in Figure 2C), the result is a 'centroid' in which locally prominent spectral peaks play a more influential role than individual harmonics. Moreover, the absolute magnitude of harmonics near the peaks matters less than their local prominence; note that the peak in the midbrain graph near $4000 \mathrm{~Hz}$ on the tonotopic axis is just as high as the peak near $600 \mathrm{~Hz}$ (Figure 2C), even though the harmonic near $600 \mathrm{~Hz}$ has a higher magnitude (Figure 2A). In this way, our proposed encoding of brightness is connected to the notion of spectral jaggedness or irregularity (Caclin et al., 2005).

This theoretical account of a sub-cortical neural representation of brightness suggests interesting relationships between brightness, spectral irregularity, and temporal modulations underlying the fluctuations that drive the peak-sharpening process. An important question is whether this representation can actually account for human perceptual results. We have shown previously that this simulated neural representation can potentially explain some brightness results in a psychophysical study involving single spectral peaks (Maxwell et al., 2020; Allen and Oxenham, 2014), but can the midbrain representation account for the frequently observed brightness-based perceptual organization of musical instrument timbres?

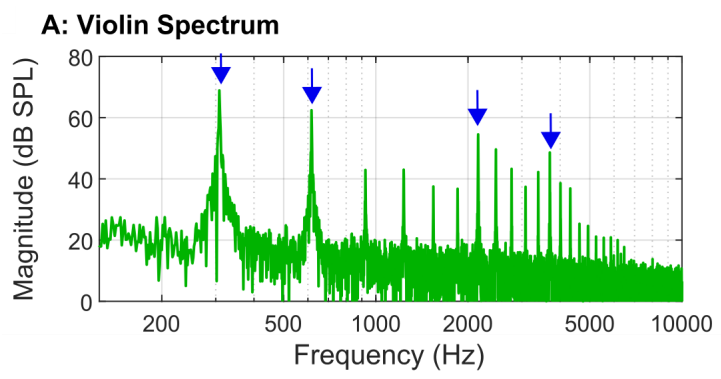

B: Auditory-Nerve Model
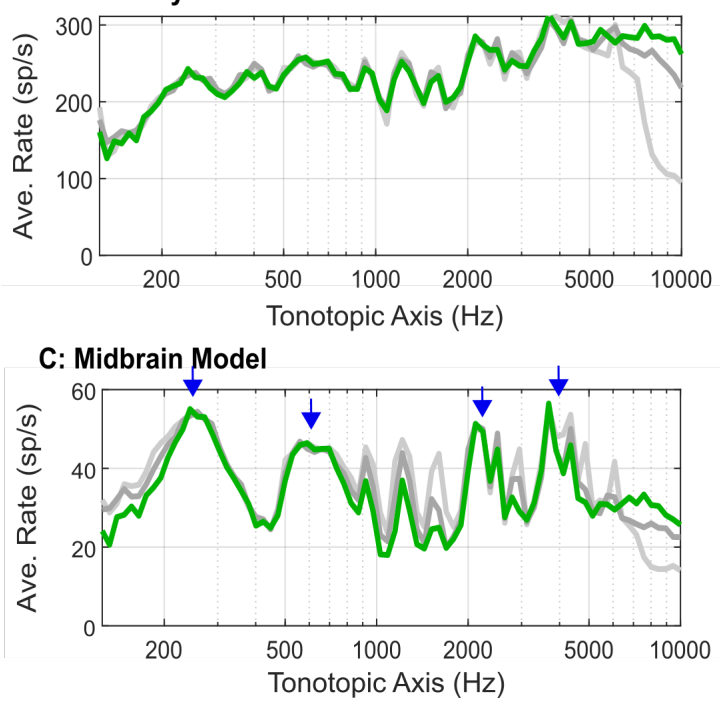

Figure 2: (A) Spectrum of violin stimulus (70 dB SPL) during steady state, with the higher-level pink noise added (see Method). (B) Auditory-nerve activity in response to (A), averaged over $500 \mathrm{~ms}$, with no noise (light gray), low-level noise (dark gray), and the higherlevel noise (green). (C) Elevated midbrain activity encodes prominent spectral peaks (blue arrows). Representation of spectral peaks is sharper with added noise (green, dark gray) than without noise (light gray).

The results in Table 1 offer a preliminary test of this question. 'Centroids' were estimated for simulations of midbrain responses to several different instrument samples. As in Figure 2 (dark gray lines), a low-level pink noise was added to these simulations to reflect realistic listening environments. Generally, these results are consistent with previous studies of perceptual timbre spaces: trumpet, oboe, and violin have higher values; vibraphone, clarinet, and horn have lower values; and piano and guitar are in between (compare to brightness axis in Figure 2.4 of McAdams 2019). Note that in this preliminary test, the stimuli were not directly matched to samples used in a previous perceptual study, and brightness can vary substantially for a given instrument depending on details of the sample itself (e.g. 
Schoonderwaldt, 2009). Furthermore, modeling these general trends is not a very specific test of this theory of brightness encoding; as such, these results constitute a baseline test of feasibility rather than strong evidence for this specific explanation of neural timbre encoding.

Table 1: Midbrain-model centroid estimates for selected instrument samples from the University of Iowa MIS, ordered from lowest centroid estimate to highest. *[2]

\begin{tabular}{|c|c|}
\hline $\begin{array}{c}\text { Instrument } \\
\text { Sample }\end{array}$ & $\begin{array}{c}\text { Midbrain-Model Centroid Estimate } \\
\text { (Hz) }\end{array}$ \\
\hline Vibraphone & 330 \\
\hline Horn & 521 \\
\hline Clarinet & 522 \\
\hline Bassoon & 581 \\
\hline Trombone & 701 \\
\hline Piano & 721 \\
\hline Oboe & 913 \\
\hline Trumpet & 1186 \\
\hline Violin & 1202 \\
\hline$*$ Guitar & $* 530$ (for $500 \mathrm{~ms}) * 1092$ (for 200 ms) \\
\hline
\end{tabular}

\section{Conclusion}

The neural fluctuation theory of Carney (2018) offers a novel explanation of how timbral brightness may be encoded through the sharpening of spectral-peak representations in the sub-cortical auditory system. This theoretical account suggests potentially fundamental neural relationships between brightness, temporal modulation, and spectral irregularity. Given the substantial role that time-varying changes in neural signals play in this theory, future work may examine implications for spectral flux and attack time as well.

\section{Acknowledgements}

Supported by NIH-DC010813 \& NIH-DC001641.

\section{End Notes}

[1] $\lambda$ can be understood as the instantaneous likelihood of an auditory-nerve spike (a single action potential), expressed here in units of spikes per second ( $\mathrm{sp} / \mathrm{s}$ ). If the value of $\lambda$ was $100 \mathrm{sp} / \mathrm{s}$ for a full second, 100 spikes would occur, on average, during that second.

[2] Estimated guitar centroid varied substantially depending on how much decay was included. Both timespans began at the same point before note onset.

\section{References}

Allen, E. J., \& Oxenham, A. J. (2014). Symmetric interactions and interference between pitch and timbre. JASA, 135(3), 1371-1379.

https://doi.org/10.1121/1.4863269
Caclin, A., McAdams, S., Smith, B. K., \& Winsberg, S. (2005). Acoustic correlates of timbre space dimensions: A confirmatory study using synthetic tones. JASA, 118(1), 471-482. https://doi.org/10.1121/1.1929229

Carney, L. H. (2018). Supra-threshold hearing and fluctuation profiles: Implications for sensorineural and hidden hearing loss. Journal of the Association for Research in Otolaryngology, 19(4), 331-352. https://doi.org/10.1007/s10162-018-0669-5

Carney, L. H., Li, T., and McDonough, J. M. (2015). Speech coding in the brain: Representation of vowel formants by midbrain neurons tuned to sound fluctuations. Eneuro. 2(4). https://doi.org/10.1523/ENEURO.0004-15.2015

Elhilali, M. (2019). Modulation representations for speech and music. In K. Siedenburg, C. Saitis, S. McAdams, A. Popper, R. Fay. (Eds.), Timbre: Acoustics, perception, and cognition (pp. 335-359). Springer. https://doi.org/10.1007/978-3-030-14832-4_12

Epstein, M., Marozeau, J., \& Cleveland, S. (2010). Listening habits of iPod users. Journal of Speech, Language, and Hearing Research, 53, 1472-1477. https://doi.org/10.1044/1092-4388(2010/09-0059)

Fritts, L. (1997). University of Iowa musical instrument samples database. Retrieved January 14, 2021 from theremin.music.uiowa.edu/MIS.html

Joris, P. X., Schreiner, C. E., and Rees, A. (2004). Neural processing of amplitude-modulated sounds. Physiological Reviews, 84, 541-577. https://doi.org/10.1152/physrev.00029.2003

Langner, G., Albert, M., \& Briede, T. (2002). Temporal and spatial coding of periodicity information in the inferior colliculus of awake chinchilla (Chinchilla laniger). Hearing Research, 168(1-2), 110-130. https://doi.org/10.1016/S0378-5955(02)00367-2

Liberman, M. C. (1978). Auditory-nerve response from cats raised in a low-noise chamber. JASA, 63(2), 442-455. https://doi.org/10.1121/1.381736

Marozeau, J., de Cheveigné, A., McAdams, S., \& Winsberg, S. (2003). The dependency of timbre on fundamental frequency. JASA, 114(5), 2946-2957. https://doi.org/10.1121/1.1618239

Maxwell, B. N., Fritzinger, J. B., Carney, L. H. (2020). Neural mechanisms for timbre: spectral-centroid discrimination based on a model of midbrain neurons. timbre2020.mus.auth.gr/assets/papers/22.Maxwell.pdf

McAdams, S. (2019). The perceptual representation of timbre. In K. Siedenburg, C. Saitis, S. McAdams, A. Popper, R. Fay. (Eds.), Timbre: Acoustics, perception, and cognition (pp. 23-57). Springer. https://doi.org/10.1007/978-3-030-14832-4 2

Rose, J. E., Hind, J. E., Anderson, D. J., \& Brugge, J. F. (1971). Some effects of stimulus intensity on response of auditory nerve fibers in the squirrel monkey. Journal of 
Neurophysiology, 34(4), 685-699. https://doi.org/10.1152/jn.1971.34.4.685

Schoonderwaldt, E. (2009). The violinist's sound palette: spectral centroid, pitch flattening and anomalous low frequencies. Acta Acustica United with Acustica, 95(5), 901-914. https://doi.org/10.3813/AAA.918221

Zilany, M. S., Bruce, I. C., \& Carney, L. H. (2014). Updated parameters and expanded simulation options for a model of the auditory periphery. JASA, 135(1), 283-286. https://doi.org/10.1121/1.4837815 


\title{
Kuvunga: Timbre, interlocking, and composite melodies in Zambian Luvale ngoma
}

\author{
Jason Reid Winikoff ${ }^{1 \dagger}$ \\ ${ }^{1}$ School of Music, The University of British Columbia, Vancouver, British Columbia, Canada \\ ${ }^{\dagger}$ Corresponding author: jason.winikoff@alumnni.ubc.ca \\ Published 16 December 2021; https://doi.org/10.18061/FDMC.2021.0052 \\ Author video presentation and/or other conference material: https://doi.org/10.17605/OSF.IO/F9J6S
}

\begin{abstract}
This paper is an attempt to explicate the concept of "kuvunga," an important term to Zambian Luvale drummers. Drums kuvunga when they produce a composite melody. While these composites have long been understood as vital to African percussion, the processes that create them - both performed and perceived - have been relatively ignored. To help illustrate these processes, I introduce new analytic terminology that organizes rhythms along timbral lines. When these types of rhythms interact in specific ways, the drums kuvunga. In this study I will explain how Luvale musicians achieve this interlocking resultant by manipulating timbre through technical control. I combine research on auditory stream segregation, African musicology, and ethnographic methods to show that making drums kuvunga involves performed and perceived attention to timbre. Ultimately, my analysis draws attention to the vitality of timbre in African percussion, explicates the emergence of an important perceptual construct, and provides a new set of analytic tools.
\end{abstract}

KEYWORDS: timbre, Luvale, perception, percussion, Zambia

\section{Introduction}

In scholarly discourse and general belief, drumming has often been reduced to a solely rhythmic artform. This is perhaps most manifest in sub-Saharan African music where, as Agawu (1995) convincingly argues, scholars' preoccupation with rhythm has led to an excessive othering of African musicians - rhythmic complexity separates Africans from everyone else. With this "invention of African rhythm" comes an oversight of other sonic parameters in drumming such as pitch and timbre. Although scholars like Nzewi (1974) have begun to draw attention to the melodic dimensions of African drumming, timbre has remained comparatively absent from this discussion. This is unfortunate as composite drum melodies are vital to these music systems, and the performed and perceived processes that create them heavily involve timbre. What follows in this study is an analysis of drum timbre and its impact on these composites in Zambian Luvale percussion.

At the heart of this study is an examination of the Luvale verb "kuvunga." Luvale musicians often invoke the word kuvunga when discussing supporting drums. On the surface, this verb refers to making drums sound good together. A deeper probing of the word, however, reveals information about emic timbral aesthetics. Ultimately, I present an understanding of this term as composite melodic-rhythms constructed through performed and perceived attention to timbre.

Kubik (1994) labeled a Kigandan xylophone version of this phenomenon the IP Effect. Fiagbedzi (1980) demonstrated how compositional practices yield the effect in Anlo-Ewe dance-drumming. In this study I argue that a Luvale version of the IP Effect, encoded in the concept of kuvunga, emerges not solely because of how the music was composed, but also in conjunction with performance of the composition and, importantly, perceptual processes of reorganization.

The Luvale people predominately live in present-day northwestern Zambia, eastern Angola, and southern Democratic Republic of the Congo. They are closely related to the Chokwe, Mbunda, and Luchazi peoples and together make up a relatively homogenous regional cultural grouping. Although relatives of the Luvale have been the focus of much musicological literature, research on Luvale music is relatively scarce. The little that does exist primarily focuses on the voice with only one study dedicated to Luvale drums (Tsukada, 1997).

Percussion ensembles play important roles in Luvale traditional festivals, initiation ceremonies, healing rituals, and entertainment. The Luvale percussion ensemble consists of a lead drum (ngoma pwita, ngoma ya shina, or jingoma jya kachacha), the mikakaji (two sticks struck against the body of a drum), and two to three supporting drums: ngoma yahakachi, ngoma yakusongo, and occasionally ngoma yakasumbi. [1] Supporting drums essentially produce three different sounds: an open tone and two different closed tones (a muffled stroke and a slap stroke). Proper hand technique is vital to producing these distinct timbres, and proper timbre is essential for making drums kuvunga.

\section{Method}

This study is based on ethnographic methods involving participant-observation, interviews, and experience as 
both a student and performer of this music. I conducted on-site research in Zambia in 2013-2014, 2015, 2017, and 2019. I also investigate the concept of kuvunga through deep readings of dictionary definitions, music analysis including the introduction of new analytical terminology, and research in the fields of African (ethno)musicology and cognitive psychology.

When investigating timbre, Fales (2002) stresses the importance of distinguishing between the acoustic and perceptual domains. I follow her lead by splitting my timbral inquiry into two levels: that which deals with the production of acoustic stimuli and that of perception.

The terminology in my theory classifies drum parts along timbral and melodic lines. These categorical terms provide information on technique, the instrument itself, and how the part is likely to interact with other musical ideas. This study utilizes three of these categories: durarhythm, timbre-rhythm, and melo-rhythm.

A dura-rhythm (short for durational-rhythm) is a part whose events are only differentiated (if at all) through rhythmic means; there are no timbral or melodic dissimilarities. This usually implies that each moment of attack involves the same technique and part of the drum. In the Luvale repertoire, dura-rhythms are almost exclusively used by supporting drums. Figure 1 (A-B) provides two examples of common Luvale durarhythms. Note that each example only includes one timbre (note-head) and one pitch (vertical position).

A timbre-rhythm is a musical idea in which a drummer rhythmically moves between notes that vary only in timbre (and, perhaps, duration); it is a timbral unfolding of notes, not a melodic one. These different timbres are produced through differences in technique and/or the instrument itself (e.g., hitting a different part of the drum or using a different kind of stick). Timbrerhythms usually have a hierarchy of tones, what Knight (1974) calls "a carefully balanced system of strong and weak accents...[a] system of inherent accents" (p. 29). In this way, the other timbre(s) support or embellish the dominant one. Frequently the open tones provide the instrument's main musical contribution to the ensemble's gestalt. Other timbres thus help provide musical interest or, as Locke (1998) notes, help the performer. Timbre-rhythms are quite common in both Luvale supporting and lead drumming. In examples CD of Figure 1, note the variety of note-heads despite the sometimes-absent durational variety. [2]

A melo-rhythm (short for melodic-rhythm) is a musical idea in which a drummer rhythmically moves between various pitches of the same timbre. [3] As melo-rhythms only include one timbre, they usually involve only one type of hand technique. Since a single ngoma drum is essentially only capable of producing one definite pitch (that of the open stroke), melorhythms usually require several drums. In the Luvale repertoire they are thus uncommon for supporting instruments, instead only featured in a specialized genre of lead drumming/dance known as Kachacha. In Figure 1 (E-F) note that while rhythm and pitch may change, each event has the same note-head and, thus, timbre.

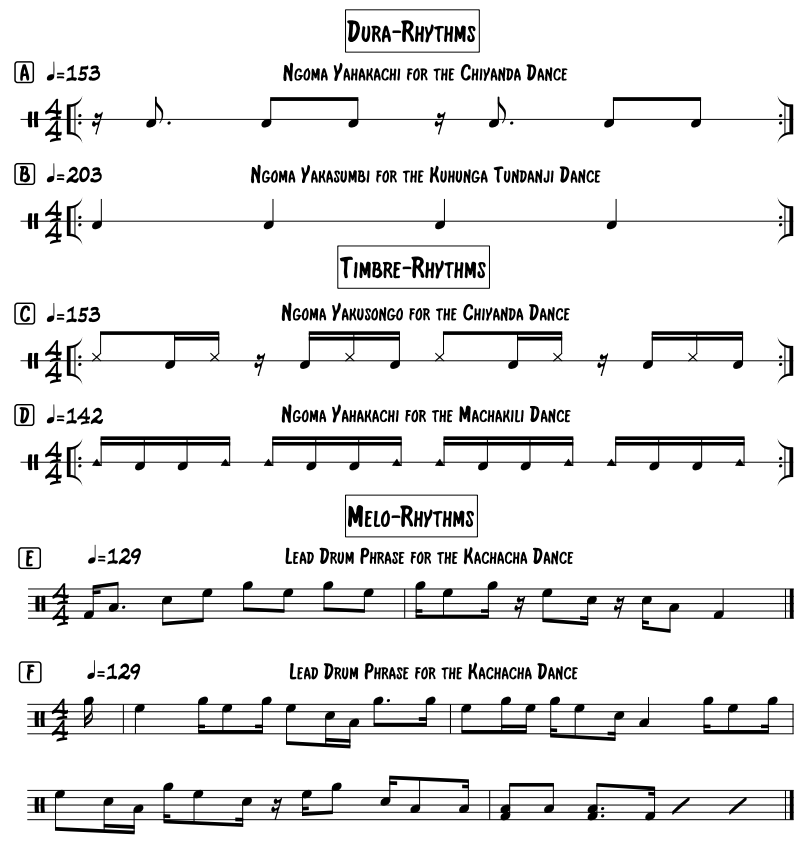

Figure 1: Examples of my terminology in the Luvale percussion ensemble repertoire. (E-F) show lead drum phrases Kapalu Lizambo often plays. Normal noteheads represent open strokes. " $X$ " note-heads represent slaps. Triangular note-heads represent muffled tones.

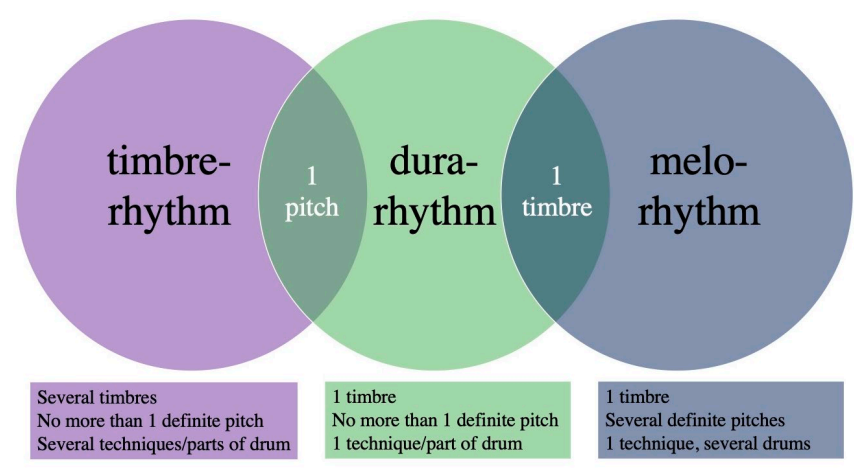

Figure 2: A visual aid for understanding my analytic terminology. The three categories of parts are organized along timbral and melodic lines. 


\section{Results}

In one of the only Luvale dictionaries, Horton (1975) defines kuvunga: "Carry the air of a song. Play the first or treble drum...To do something turn and turn about, alternate with one another or others" (p. 434). Here kuvunga involves playing a single drum in a way that carries the music whilst alternating with another.

In an interview, Kapalu Lizambo (arguably the most respected performer in this cultural region) partially contradicted Horton, instead stating that kuvunga involves the relationship between several drums/drummers. To Lizambo, this relationship is so vital that no one else (e.g., lead drummer, singer, dancer) can enter until the drums kuvunga. He explained, "You hear the kuvunga for three, two $n g o m a . .$. if the kuvunga is going in the same way or good like that." I interjected, "So if the drums are marrying." He eagerly agreed, happy to hear me using a metaphor he (and others) taught me years before. "Yeah. Now, ok, that's kuvunga, it's now straight. Then you can now come in. Because you can't come in if the kuvunga is not straight." (personal communication, August 1, 2019). Lizambo also mentioned that the different timbres (open and closed) of several drums connect as one in the process of kuvunga. "Kuvunga...that's different sounds. Yes. So the kuvunga is coming to the different $n g o m a . .$. so that one now you are connected for that sound" (personal communication, August 1, 2019). But how do several drums provide different timbres in a way that unifies the foundational layer?

In another interview, William Vunda, the leader of the Chota Culture Group, spoke on the importance of alternating timbres in kuvunga. He explained: "Opening and closing, you are the one who is giving your friend time also to breathe with their drums. So once you just open it - continuously open it - then these guys, the other drummers won't close...they won't kuvunga well. Yes. It's you who is supposed to open and close, open and close. So once you open, then them they close. You you close, then them they open. So it's like changing" (personal communication, July 17, 2019). Perhaps the alternation that Horton refers to is one of timbre.

If we place the words of Horton, Lizambo, and Vunda together, we can make several observations about the act of kuvunga. (1) It is the essence of a piece in that it both carries the music and is the foundation for other layers (e.g., lead drumming, song, dance). (2) Several drums are involved. (3) It involves not just the presence of several timbres, but the structured alternation of them. (4) The alternation of different tone colors leads to a unified whole. This process of unification, though, is not just created in the productive domain through technical control but also in the perceptual domain through specific cognitive processes.

Although the principles of kuvunga that I outline here apply to many pieces of Luvale music, I will focus on one example. Mokolo is an important and popular item of Luvale dance music. Example A of Figure 3 represents the supporting drum parts for that dance while Example B illustrates listeners' aural image of those same drums. In the remaining sections I will show how performance of Example A leads to perception of Example B. I argue that this entire chain of events, from drum stroke to perception, is the process of kuvunga.

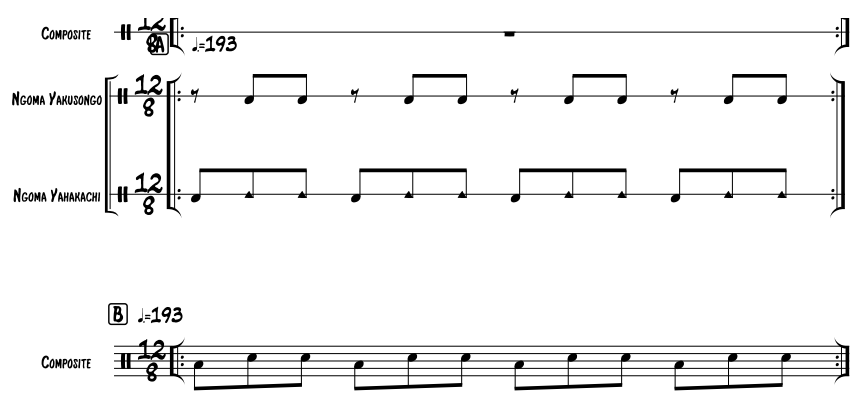

Figure 3: (A) The supporting drums for Mokolo and (B) their perceived composite.

\section{Discussion}

In the Mokolo example, the supporting drums do not rhythmically interlock as they frequently overlap; each ngoma yakusongo event occurs at the same time as a ngoma yahakachi note. Despite this, these drums still kuvunga well. Structured timbral alternation allows the listener to only perceive open strokes. This happens because open tones mask simultaneously occurring closed tones. Fales and McAdams (1994) define "masking" as the phenomenon when one timbre is perceived more stronger than other simultaneously occurring timbres. Bregman (1990) notes that lower tones (like an open stroke) are more likely to mask higher ones (e.g., slap or muffled stroke). He adds that masking is strengthened when the sources are physically close. This is the case with supporting drums as they are always adjacent in the ensemble's setup. An upward spread of masking, aided by the ensemble's physical orientation, allows for simultaneously sounding drums to contribute to the music in a cooperative manner.

An interlock, then, can work even if there is rhythmic overlap. The key lies in vertical timbral differentiation. At a moment of simultaneous onset, one drummer 
"breathes" through an open tone while the other gives space through a closed (slapped or muffled) timbre. This works not only because the closed stroke has a different timbre from the open one, but also because it is a secondary timbre - one that cedes its power to the primary, open strokes. This is a defining feature of a timbre-rhythm. The ngoma yahakachi of Mokolo plays a timbre-rhythm and its muffled tones are secondary to open strokes. This allows perception of every simultaneously occurring open tone of ngoma yakusongo's dura-rhythm. The principles of masking allow these dura- and timbre-rhythms to cooperate and are partially responsible for the perception of the composite melo-rhythm represented in Figure 3 (B).

Lizambo's point on how supporting drums must be unified in order for any other layer to enter is supported by cognitive psychology research. Bregman (1990) found that background notes (in our case, those provided by supporting drums) must be precise in order for the foreground to have its effect.

Although melo-rhythms are relatively rare in the productive domain of Luvale traditional music, they are ubiquitous in the perceptual domain and vital to the concept of kuvunga. The other two types of drum parts (dura- and timbre-rhythms) interact with each other to create a composite melo-rhythm. These composites result from perceptual grouping along timbral lines. But what must happen in the productive domain for the listener to reorganize the material as such in the perceptual domain? Scholars agree that notes from different sources are likely to segregate from their own musical units and form a new stream if the target notes: have a similar timbre (Bregman, 1990; Bregman \& Pinker, 1978; Kubik, 1994, 2010; Goodchild \& McAdams, 2018), are temporally close (Bregman 1990), are close in frequency (Bregman 1990; Kubik 2010; Goodchild \& McAdams, 2018), are part of a fast sequence (Bregman, 1990), emerge from sources that are physically near each other (Bregman, 1990; Goodchild \& McAdams, 2018), or belong to repeating sequences (Bregman, 1990; Kubik, 2010). Luvale supporting drums meet these criteria as different drums are capable of producing sounds with similar quality, are temporally close due to tight interlocking, are tuned closely and relative to each other, are played quickly, are set up next to each other, and repeat their parts.

Musical listening (as opposed to non-musical listening) is especially conducive to this type of auditory stream segregation along timbral lines. Fales (2002) notes that, when listening to music, we ignore source characteristics and, subconsciously, naturally hear the whole. Locke (1998) argues that acoustically prominent "bounce [or open] strokes constitute the player's "speech," his contribution to the ensemble's musical conversation...the contribution to the call-andresponse" (pp. 41-42, 47). Since tonal events segregate from noise-like events (Bregman \& Pinker, 1978), the pitched open strokes of each Luvale supporting drum will segregate from the noisy slaps, instead joining the other drums' open strokes of similar timbre. In order for these strokes to contribute as they do, they must segregate from and mask other types of strokes. When all of these findings are placed in conversation with one another, we can draw some valuable conclusions in a chain that lead us to a melo-rhythmic composite.

(1) When listening to music, we naturally perceive some version of the sum, not the individual parts. (2) Since the process of musical perception is distanced from that of source recognition, listeners will subconsciously seek out auditory stream segregation based on acoustic similarities; the sources of these sonic events are secondary at best. (3) If these sonic events are fast, repeated, close in timbre, pitch, time, and physical proximity then we are likely to group them together. (4) The sonic events we are likely to integrate into one unit are the open strokes (which segregate from and mask the other timbres). This means our composite is a series of pitches that have the same timbre, likely produced by similar techniques. (5) The composite is a melo-rhythm, born when several drummers contribute at least one pitch each with a similar timbre.

The composite melo-rhythm that is so vital to African drumming is a result of musicians' execution of timbre and listeners' timbrally-sensitive perceptual processes. In the Mokolo example, timbre- and durarhythms contribute to the perceptual melo-rhythm. The entirety of dura-rhythms contributes while only the primary, open tones of timbre-rhythms are included. The listener's mind extracts the open tones - creating a drummed version of Kubik's inherent pattern or Nzewi's complementary melo-rhythmic theme. This perceived melo-rhythm is the unified whole, born out of the alternation of several drums' timbres, that is the essence of the piece. Providing the appropriate acoustic stimuli to yield a composite melo-rhythm foundation in the perceptual domain is the process of kuvunga.

\section{Conclusion}

In this study I attempted to present an understanding of the Luvale musical term kuvunga. I looked to the productive domain to see how technique influences and reinforces Luvale drummers' definitions of the word. 
This revealed that kuvunga cannot just refer to interlocking rhythms because of these moments of simultaneous attack, because of these notions of open and closed timbres. If we ignore timbre, the parts do not interlock; there would be frequent overlap and no space for the drums to speak and breathe. Drummers must also alternate timbres. Dura- and timbre-rhythms are differentiated and defined through timbral means. By carefully relating these types of parts to one another in the productive domain, Luvale drummers help suggest a composite melo-rhythm for listeners in the perceptual domain. This composite, I argue, is the essence of kuvunga, and supporting drums that kuvunga are the foundation of Luvale music. Kuvunga references a specific type of auditory stream segregation, a drummed inherent timbre-pattern that is articulated through timbral control. An analysis of the processes, both performed and perceived, that yield composite melorhythms reveals the vitality of timbre to drumming.

This study also offers just a glimpse of what we can learn through ethnography and by placing our work within the frame of non-Western epistemologies. Cognitive psychology would benefit from investigating timbre beyond both the lab and Western culture. I suggest that we incorporate ethnographic methods and data, if nothing else, as a collaborative counterpart to quantitative-data driven studies. I call on scholars to expand the cultural and geographic scopes of their work and to apply their findings to non-Western case-studies.

\section{Acknowledgements}

This study would not have been possible without the aid of Kapalu Lizambo, William Vunda, the members of Lenga Navo and Chota culture groups, the Likumbi lya Mize Cultural Association, and the late Senior Chief Ndungu VIII. I would also like to thank Michael Tenzer for his supervision and help with early drafts. A version of this paper was presented at the 2020 SEM meeting.

\section{End Notes}

1. These drum names are not fully standardized and vary among cultural groups and areas of Luvale settlement. Ensembles may also contain additional instruments such as a njenje, ngezo, and/or sangu/jisangu.

2. Notes with different timbres may also have a slight perceived difference in pitch (this perceived difference may be a result of timbre). Because of this, I not only represent timbral difference through note-heads, but also through vertical position.

3. My "melo-rhythm" is inspired by Nzewi's (1974) concept of "melo-rhythmic essence." Although similar, our terms should not be used interchangeably. His term is applied to (what I term) "timbre-rhythms" that also have perceived melodic form. Nzewi's term serves his purposes as it is not part of a larger theory. My theory, however, is concerned with the different effects that result from timbral and melodic variation. As such, my term is more specific and restricted in its use.

\section{References}

Agawu, K. (1995). The Invention of "African Rhythm". Journal of the American Musicological Society, 48(3), 380-395. https://doi.org/10.2307/3519832

Bregman, A. S. (1990). Auditory Scene Analysis: The Perceptual Organization of Sound. MIT Press. https://doi.org/10.7551/mitpress/1486.001.0001

Bregman, A. S., \& Pinker, S. (1978). Auditory Streaming and the Building of Timbre. Canadian Journal of Psychology, 32(1), 19-31. https://doi.org/10.1037/h0081664

Fales, C. (2002). The Paradox of Timbre. Ethnomusicology, 46(1), 56-95. https://doi.org/10.2307/852808

Fales, C., \& McAdams, S. (1994). The Fusion and Layering of Noise and Tone: Implications for Timbre in African Instruments. Leonardo Music Journal, 4, 69-77. https://doi.org/10.2307/1513183

Fiagbedzi, N. (1980). A Preliminary Inquiry into Inherent [sic] Rhythms in Anlo Dance Drumming. Journal of the Performing Arts, 1(1), 82-92.

Goodchild, M., \& McAdams, S. (2018). Perceptual Processes in Orchestration. In E. Dolan \& A. Rehding (Eds.), The Oxford Handbook of Timbre. Oxford University Press. https://doi.org/10.1093/oxfordhb/ 9780190637224.013.10

Horton, A. E. (1975). A Dictionary of Luvale. Rahn Bros. Print \& Lithographing Co.

Knight, R. (1974). Mandinka Drumming. African Arts, 7(4), 24-35. https://doi.org/10.2307/3334885

Kubik, G. (1994). Theory of African Music (Vol. 1). The University of Chicago Press.

Kubik, G. (2010). Theory of African Music (Vol. 2). The University of Chicago Press. https://doi.org/10.7208/ chicago/9780226456928.001.0001

Locke, D. (1998). Drum Gahu: An Introduction to African Rhythm. White Cliffs Media.

Nzewi, M. (1974). Melo-Rhythmic Essence and Hot Rhythm in Nigerian Folk Music. The Black Perspective in Music, 2(1), 23-28. https://doi.org/10.2307/1214145

Tsukada, K. (1997). Drumming, Onomatopoeia and Sound Symbolism Among the Luvale in Zambia. In J. Kawada (Ed.), Cultures Sonores d'Afrique (pp. 349-393). Institute for the Study of Languages \& Cultures of Asia \& Africa, Tokyo University of Foreign Studies. 


\title{
The role of timbre, envelope and movement on audio-visual integration of musicians movements
}

\author{
Liam Foley ${ }^{1 \dagger}$ \\ Laura Schachtler ${ }^{1}$ \\ Michael Schutz ${ }^{1,2}$ \\ ${ }^{1}$ Department of Psychology, Neuroscience, and Behaviour, McMaster University, Hamilton, ON L8S 4L8, Canada \\ 2School of the Arts, McMaster University, Hamilton, ON L8S 4L8, Canada \\ $\dagger$ Corresponding author: foleyt@mcmaster.ca \\ Author video presentation and/or other conference material: https://doi.org/10.17605/OSF.IO/ZWSXH
}

Specific acoustic properties meaningfully shape the perceptual system's binding of sight and sound (Vatakis \& Spence, 2007, 2008). One understudied acoustic property in cross-modal integration is amplitude envelope, the way in which a sounds loudness changes over time. Here we examine the effect of amplitude envelope (flat versus percussive) on binding. Participants completed a temporal order judgement (TOJ) task, indicating which sensory modality was presented first. We hypothesized better binding for percussive envelope natural sounds compared to flat pure tones. Our preliminary results show a larger binding window for natural percussive tones. Further research into the role of timbre, temporal variation, and movement in cross-modal integration will help further our understanding on binding of natural stimuli.
KEYWORDS: cross-modal integration, unity assumption, amplitude envelope, timbre

\section{References}

Vatakis, A., \& Spence, C. (2007). Crossmodal binding: Evaluating the "unity assumption" using audiovisual speech stimuli. Perception \& Psychophysics, 69(5), 744-756. https://doi.org/10.3758/BF03193776

Vatakis, A., \& Spence, C. (2008). Evaluating the influence of the "unity assumption" on the temporal perception of realistic audiovisual stimuli. Acta Psychologica, 127(1), 12-23. https://doi.org/10.1016/j.actpsy.2006.12.002

\section{The effect of timbral and non-timbral cues on the rapid recognition of songs from single opening chords}

\author{
Ivan Jimenez ${ }^{1 \dagger}$ \\ Tuire Kuusi ${ }^{1}$ \\ Christoph Reuter ${ }^{2}$ \\ Isabella Czedik-Eysenberg ${ }^{2}$ \\ ${ }^{1}$ Sibelius Academy, University of the Arts Helsinki, Finland \\ ${ }^{2}$ Musicology Department, University of Vienna, Austria \\ † Corresponding author: ivan.jimenez.rodriguez@uniarts.fi
}

Author video presentation and/or other conference material: https://oi.org/10.17605/OSF.IO/DMEY2

It is currently believed that timbre plays a primary role in the identification of songs from very brief excerpts of music (McAdams \& Siedenburg, 2019; Wallmark, Iacoboni, Deblieck, \& Kendall, 2018). However, the specific contribution of timbre and other parameters to that type of identification remains unclear. By investigating the identification of songs from single piano and piano-driven block chords, the present study focuses on the role of timbre, vertical pitch structures, and duration in the rapid recognition of songs. Ninetythree participants were asked to identify 20 well-known songs from their opening piano or piano-driven block 
chord. We evaluated the contribution of 10 chord characteristics to song identification. Chord characteristics related to timbral brightness, pitch register, and duration were chosen due to their high perceptual salience according to a chord similarity estimation test that the participants completed after the main task. The other variables were selected based on theoretical predictions regarding auditory long-term memory. While participants' musical background did not affect their ability to identify the songs, the chords' timbral brightness and, to a lesser extent, their attack time, chord-type commonness, duration, and the songs' year of release contributed to the songs' recognition rate. This study shows that participants with and without musical training are able to identify songs from a single piano chord and suggests that both timbral and non-timbral cues contribute to this remarkable ability.

\title{
Partial-oriented listening and the timbre-pitch perceptual continuum
}

\author{
Jordan Lenchitz ${ }^{1 \dagger}$ \\ ${ }^{1}$ College of Music, Florida State University, Tallahassee, Florida, United States \\ $\uparrow$ Corresponding author: jlenchitz@,fsu.edu \\ Author video presentation and/or other conference material: https://doi.org/10.17605/OSF.IO/KAVJY
}

Partial-oriented listening is a mode of listening that entails attending to timbral upper partials as potential pitches, hearing out spectral prominences distinctly from timbral aggregates. Questions such as "What is the highest pitch you hear?" and "How many pitches do you hear?" invite partial-oriented listening, which can be considered a "top-down"- that is, attend to high frequencies first and then to low frequencies-top-down listening strategy. I posit the existence of a timbre-pitch continuum of percepts of upper partials arising from the confluence of top-down listening strategies (such as partial-oriented listening) and bottom-up acoustic features (such as spectral fission, my theorization of the other side of the coin to McAdams's "spectral fusion" that describes situations in which many listeners are likely to perceive a timbral upper partial as a discrete pitch). I argue that in order to best account for both flexibility of listening behaviors for any given listener as well as individual differences between listeners, it is most productive to center variance and variety along this continuum in terms of modes of listening and listening behaviors rather than "types of listeners." In other words, any individual listener is better represented by a band of percepts along the continuum than by any one individual percept on it. I present this theoretical framework to facilitate study of the role of individual differences in timbre and pitch perception and to embrace the diversity of potential perceptual experiences that can arise from different modes of listening to the same sound.

KEYWORDS: pitch perception, partialoriented listening, timbre-pitch continuum, spectral fission, auditory attention, individual difference, timbre 


\title{
Towards a theory of instrument-specific absolute pitch: Effects of timbre and motor imagery
}

\author{
Lindsey Reymore ${ }^{1 \dagger}$ \\ Niels Chr. Hansen ${ }^{2}$ \\ ${ }^{1}$ Schulich School of Music, McGill University, Montréal, Québec, Canada \\ ${ }^{2}$ Aarhus Institute for Advanced Studies \& Center for Music in the Brain, Aarhus University, Aarhus, Denmark \\ $\dagger$ Corresponding author: lindsey.reymore@mail.mcgill.ca \\ Author video presentation and/or other conference material: https://doi.org/10.17605/OSF.IO/QB7C6
}

While absolute pitch (AP) - the ability to name musical pitches without reference-is rare in expert musicians (Levitin \& Rogers, 2005; Ward, 1999), anecdotal evidence suggests that some musicians may better identify pitches played on their primary instrument than pitches played on other instruments. We call this phenomenon "instrument-specific absolute pitch" (ISAP) and offer the first theory of underlying mechanisms (Reymore \& Hansen, 2020). This theory is situated in neuroscientific research on the multimodal nature of expertise (e.g., Krishnan et al., 2018; Proverbio \& Orlandi, 2016). We propose that informative timbral cues arise from performer- or instrument-specific idiosyncrasies or from timbrefacilitated tonotopic representations and that sounds of one's primary instrument may activate kinaesthetic memory and motor imagery, aiding pitch identification (Hansen \& Reymore, 2021). Hypotheses derived from this theory are tested in two professional oboists. Only one of the two oboists showed an advantage for identifying oboe tones over piano tones. For this oboist, pitch-naming accuracy decreased and variance around the correct pitch value increased as an effect of transposition and motor interference, but not of instrument or performer. These results suggest that some musicians possess instrument-specific absolute pitch while others do not and that candidate mechanisms behind this ability capitalize on timbral cues and motor imagery. In a Registered Report (Hansen \& Reymore, 2021), we plan to extend these findings to a larger population of oboists. A deeper understanding of instrument-specific absolute pitch has theoretical implications for research on musical expertise, absolute pitch, timbre and pitch cognition, and musical embodiment, as well as practical implications for musical practice and pedagogy. Finally, the theory offers several directions for future research, employing behavioral, neuroimaging, and brain stimulation methods.
KEYWORDS: absolute pitch, timbre, motor planning, pitch perception, oboe

\section{References}

Hansen, N.C., \& Reymore, L. (2021). Articulatory motor planning and timbral idiosyncrasies as underlying mechanisms of instrument-specific absolute pitch in expert musicians. PLOS ONE, $16(2)$ e0247136. https://doi.org/10.1371/journal.pone.0247136

Krishnan, S., Lima, C. F., Evans, S., Chen, S., Guldner, S., Yeff, H., et al. (2018). Beatboxers and guitarists engage sensorimotor regions selectively when listening to the instruments they can play. Cerebral Cortex, 28, 4063-4079. https://doi.org/10.1093/cercor/bhy208

Levitin, D. J., and Rogers, S. E. (2005). Absolute pitch: perception, coding, and controversies. Trends in Cognitive Science, 9, 26-33. https://doi.org/10.1016/j.tics.2004.11.007

Proverbio, A. M., and Orlandi, A. (2016). Instrumentspecific effects of musical expertise on audiovisual processing (clarinet vs. violin). Music Perception, 33 , 446-456. https://doi.org/10.1525/mp.2016.33.4.446

Reymore, L., \& Hansen, N.C. (2020). A Theory of Instrument-Specific Absolute Pitch. Frontiers in Psychology, 11, 2801. https://doi.org/10.3389/fpsyg.2020.560877

Ward, W. D. (1999). “Absolute pitch," in D. Deutch (ed.), The Psychology of Music (pp. 265-298). New York, NY: Academic Press https://doi.org/10.1016/ B978-012213564-4/50009-3 


\title{
Coda: Charting Future Directions of Music Cognition in turbulent times
}

\author{
Joshua Albrecht ${ }^{\dagger}$ \\ ${ }^{1}$ Hugh A. Glauser School of Music, Kent State University, Kent, OH, USA \\ Published 16 December 2021; https://doi.org/10.18061/FDMC.2021.0054 \\ †Corresponding author: jalbrec6@kent.edu
}

\begin{abstract}
The Future Directions of Music Cognition conference and speaker series incorporated hundreds of scholars presenting their research and dialoguing about what future directions the field of music cognition may take. This proceedings volume fairly represents much of the activity at the conference proper. However, it is difficult for a conference about the future of an academic field to live up to its name during a time of such rapid societal change. This article attempts to partially address this gap by thinking through the implications current events may have on future directions of music cognition. It is a speculative investigation of three primary issues: social justice and anti-racism, the changing role of science in society, and the challenges of fostering an environment of healthy dialogue in inhospitable times.
\end{abstract}

\section{Music cognition's possible future directions}

Now that Future Directions of Music Cognition has finally come to a close at the end of a tumultuous two years, it is reasonable to ask whether this conference and speaker series has led us any closer to understanding what the future of music cognition might hold. Of course, a lot more has happened in the last two years than just this conference; the last eighteen months have been marked more by unpredictability than stability. Undoubtedly, this turbulent time has carried many powerful lessons that are worthy of careful thought and reflection, not least of which is to be cautious about being too certain about the future.

Many recent publications in 2020 and 2021 are in the unfortunate position of being immediately out of date at the moment of their publication. Unfortunately, Future Directions is not immune from this problem, having been conceived before COVID and many other significant changes that have left sizeable impacts on society. At the same time, many things about the conference were able to pivot in response to current events, especially the speaker series, as the introduction outlines (pp. 1-3). However, these proceedings mostly represent the conference submissions, which were all received before the end of 2019, and so several current research trends are not reflected in these pages. To give just one example, a wealth of recent research explores ways in which the pandemic, social distancing, and online behaviors have interacted with our musical behaviors and cognition.

Future Directions is by any account an ambitious project, and even with content contributed by 150 authors, these proceedings provide just a slice of the significant current and future trends in music cognition. Consequently, in planning the conference we were careful to omit the definite article "the" from the title of this conference. Even at that time, we knew there would be many other future directions of music cognition not represented in these pages. This is certainly more true in 2021 than it was in 2019. Even still, I believe we may detect some clues about directions the field might be moving in by looking at patterns of what was presented at the conference and the articles in this proceedings.

In this essay, I aim to explore some of the possible future directions of music cognition by attempting to retrospectively connect the work represented in this volume to some of the current societal forces that seem to be shaping not only the future of society at large, but also specifically of music cognition. Rather than simply reviewing the contents of these proceedings, I propose the slightly more ambitious goal in this essay of thinking through the intersections between current events and music cognition as a way of bringing the mission of the conference full circle. I believe that the research presented at the Future Directions conference interfaces in important ways with some of these current events. Having said that, it is important to underline that the following are my own thoughts that may not represent those of other conference participants. Of course, the COVID-19 pandemic is only one of many major societal shifts in the last eighteen months that have impacted the future of music cognition. The last couple of years represent an inflection point for the arts, sciences, and society at large.

I do not have the capacity here to do justice to all the factors likely to impact music cognition in the 
future. For example, concerns about climate change have already influenced the way that conferences are organized, and even after the pandemic has subsided efforts will undoubtedly continue to reduce the carbon footprint of music cognition societies and conferences. The financial impacts of the pandemic on universities has greatly reduced the amount of professional development and travel funds open to faculty and has resulted in hiring freezes. The dearth of new jobs has hit recent graduates the hardest. Coupled with the lack of networking opportunities through the near elimination of face-to-face conferences, these changes threaten the careers of a generation of researchers. In the United States, the realities of upcoming demographic changes mean the probable reduction of the number of students attending universities, which will also negatively impact research funding and productivity.

These and many other significant issues will all impact future directions of music cognition, but this essay will focus on three main issues: social justice and anti-racism, the changing role of science in society, and the challenges of fostering an environment of healthy dialogue at a time in which intolerance to others' perspectives and moral outrage are growing, while listening to others and compassion are declining.

\section{Calls for social justice and anti-racism}

In the wake of the murder of George Floyd in May 2020, a worldwide movement sprang up to grapple with the realities of a still all-too-pervasive racism in the United States and across the world. Unprecedented levels of demonstrations, activism, and protests calling for society to be more just for all people marked the summer of 2020 and beyond. Protests emerged in over 2,000 cities and towns and all 50 states in the following year related to civil rights, criminal justice, policing, and racism (Crowd Counting Consortium, 2021). The voices of individuals who have not been heard for too long suddenly had a bigger platform.

Many within music cognition have also begun actively working toward greater social justice. Recognizing the reality that there is no such thing as pure scientific objectivity and that echoes of racism continue to influence even the field of music cognition, the SMPC executive board together published an article in Music Perception urging the research community to embrace anti-racist practices (Baker et $a l ., 2020)$. Following up on this article as part of the Future Directions speaker series, several members of the SMPC board led a participatory town hall discussion about anti-racism (Gather Town, 2021). This session generated discourse and ideas among conference participants to stimulate change in the Society, focusing on problems with representation among music cognition researchers and publications, the 'leaky pipeline' of training underrepresented students, past explicit racism in methods used, and a lack of diversity and inclusion among participants that has led to results that do not readily generalize to all people.

There are already signs that researchers are taking these calls to action seriously by challenging prior research practices and broadening the scope of current and future research. During the speaker series, Justin London (London, 2021) argued that corpus studies are weakened by over-reliance on the same canonical works by Bach, Haydn, Mozart, and Beethoven, leading to results that are overfit to just these composers and do not represent broader compositional activity. Daniel Shanahan (Shanahan, 2021) provocatively suggested that the heavily Western European-centric Essen folksong database be retired for corpus research or used only as a sandbox dataset, due to its overuse and monocultural representation. Both speakers suggested a broader sample of music that should be encoded, and they also discussed important efforts already underway to diversify corpora, as well as the importance of centering the voices of scholars from underrepresented groups and being cognizant of the biases inherent in such empirical approaches to music research.

In these proceedings, two articles in particular explicitly study the music cognition of Black musicians. Jason Winikoff (pp. 262-266) explores the mental processes that Zambian Luvale ngoma drummers rely on to create interlocking composite melodies from multiple different types of drums. Hannah Merseal, et al. (pp. 91-92) conducted a corpus study on jazz solos from the Weimar Jazz Database and found that jazz musicians tend to retrieve "easier" melodic sequences in jazz solos before moving on to more complex ideas. It is likely that future music cognition research will continue to push into more diverse research topics and repertoire, incorporate more research on/by/with individuals from underrepresented groups, and further grapple with the role of anti-racism in music cognition. 


\section{"Following the science"}

Living in a worldwide pandemic has brought science into the popular imagination in a much more immediate way than it has been in recent memory. The breathtakingly rapid development of several COVID19 vaccines in record time has highlighted the important role that scientific research plays in the health and wellbeing of the world's population. Hearing directly from scientific leaders almost daily through interviews and press briefings has suddenly become a common experience for the general public, as many search out the best scientific evidence regarding policies like wearing masks, being vaccinated, and optimal distancing to avoid spreading the virus. At the same time, public debate has erupted over some of these policies, leading to a much stronger public interest in the nature of scientific claims. Consequently, opinions about scientific research among the public have rapidly multiplied and these opinions are more fiercely held now than they have been in recent memory. In the United States and elsewhere, the phrase "follow the science" has entered the common vernacular. Even political slogans have embraced the phrase as a way of combating what some see as "science deniers."

One consequence of the sudden celebrity or notoriety of science (depending on one's perspective) is that the public is now at once much more aware of some of the issues surrounding scientific findings and methodology. Another consequence is that scientific evidence and reporting has become politicized, pushing scientists into the forefront of public awareness, sometimes in ways that can be unwelcome for researchers. Aware of these forces, the Organization for Economic Co-operation and Development's "Science, Technology and Innovation Outlook" has recently published advice for scientists to communicate with the public in ways that are both effective and foster trust in science (OECD, 2021).

Music cognition research represents a tiny fraction of the global scientific community. However, the current public interest in science presents both opportunities and risks for the future of music cognition research. One side effect of greater public interest in science generally is a corresponding greater public exposure to music cognition research. Public exposure both raises researchers' responsibilities to conduct sound, reproducible research, and also enhances the possible public benefits of music cognition research. Those who seek to see their research directly benefit real people have wonderful new opportunities to have their work platformed in ways that echo Psyche Loui's (pp. 25-31) article on use-inspired music cognition. In it, she calls for a deeper integration of pure music cognition research with practical applications of music-based interventions that can make the world a better place.

Music cognition seems to be peculiarly well positioned to benefit people during a global pandemic, in which there are unprecedented levels of social isolation and emotional trauma. For example, Zachary Wallmark's speaker series talk (Wallmark, 2021) explores the way that music can enhance empathy and provide a sort of virtual human presence in times of social isolation. This kind of music research, geared toward addressing immediate societal needs, is likely to become more prevalent in upcoming years and is well suited to help people and influence public policy.

However, it is also important to recognize that "follow the science" as a slogan is somewhat misleading, erroneously suggesting that science speaks with one unified voice. On the contrary, science is a method, not a monolithic authority that makes pronouncements about what is definitively true. Consensus among researchers is certainly sometimes possible, but healthy scientific communities foster debate. The certainty suggested by the phrase "follow the science" and the kinds of popular news reporting of scientific results is a mischaracterization of the scientific enterprise, and if followed by limitations on the scope of debate it will only hinder scientists' ability to effectively generate research. Music cognition is an inherently interdisciplinary field and is a particularly striking illustration of the benefits of healthy dialogue and the convergence of multiple research methods across both the humanities and the sciences. This volume is a prime example of the intersection of multiple methods, containing research on similar topics supported by empirical studies, theoretical investigations, and policy proposals.

To take one example, there are still a variety of viable perspectives about the nature and origins of musical emotion, reflected in Future Directions contributions. One dominant paradigm conceives of musical emotion along dimensional lines, embraced by several researchers in this volume, such as Thery Al Alam and Nicola Dibben (pp. 97-101). By contrast, Lindsay Warrenburg (p. 139) argues that even researchers using discrete emotion terms have fallen prey to semantic underdetermination, conflating multiple emotions under the same term, and should 
increase the emotional granularity of their studies through more refined emotion terms. Mark Riggle (pp. 119-124) offers a theoretical evolutionary explanation of the role that musical pleasure plays in sexual selection, arguing that music evoked emotions serve as tools for memory enhancement. In her speaker series talk, on the other hand, Jonna Vuoskoski (Vuoskoski, 2021) focuses on the role that musical emotion plays in building social empathy, specifically exploring the more abstract feeling of 'being moved' as a way of understanding others' emotional states and activating deeper social bonds and prosocial behavior. Tanushree Agrawal, et al. (p. 145) experimentally explore this idea in a novel way, finding that participants are more reluctant to inflict physical harm on subjects that are perceived as more musical, underscoring music's prosocial role. Finding space for these types of vibrant discussions to take place is crucial to continuing to grow our understanding of the nature of musical emotion and the future of music cognition as a whole.

\section{Fostering healthy dialogue}

However, finding space for healthy, vibrant dialogue has been becoming more challenging lately. As the chair of the program committee, one of my responsibilities has been to foster dialogue and create space on the program for many diverse voices. Having such a complex group of viewpoints and subfields among the conference submissions offered its own set of programming challenges. However, recent years have witnessed the rise of other challenges to the kinds of free, open, and honest discussions out of which the best research and teaching flourishes.

In his monumental review of the history of ideas in the 20th century, Peter Watson argues that what is so refreshing about science is that, "[i]t has no real agenda. What I mean is that by its very nature science cannot be forced in any particular direction. The necessarily open nature of science...ensures that there can only ever be a democracy of intellect in this...To succeed, to progress, the world must be open, endlessly modifiable, unprejudiced" (Watson, 2001: 4). This idealistic understanding of science is attractive, but is unfortunately also overly simplistic and naïvescientists have complicated mixtures of motivations for the research they conduct, and there is a long history of documented methodological pitfalls, innocent mistakes, research prejudices, and publishing malpractices that undermines the objectivity of research and pushes it in biased directions.
Nevertheless, there is also an important underlying principle in Watson's comment that is embedded in the very nature of scientific inquiry itself - the freedom and honesty to pursue lines of inquiry wherever they lead. Those who have engaged in research long enough know that results are not always consistent with $a$ priori hypotheses. Research sometimes fails, and even well-loved theories can be overturned. Many of the scientific community's greatest successes and advancements of knowledge have flowed directly from the freedom to engage in controversial lines of research honestly, publish unpopular results, and pursue research that doesn't result in immediate commercial profit.

Unfortunately, in recent years there has been a surge of strong cultural undercurrents that work to shut down dialogue and silence voices. One of these undercurrents, made more concerning because it comes directly from the governments that are designed to protect freedom of speech themselves, is a spate of recent restrictions on teaching various elements about racial justice. As of this writing, eight states in the US have passed laws restricting the teaching or training of specific racial justice concepts or ideas. While most of these laws focus on public K-12 and charter schools, the laws in Idaho, Oklahoma, and Iowa pertain to public universities, as well (Legislature of the State of Idaho, 2021; House Bill 1775, 2021; The Iowa Legislature, 2021). Twenty additional states are considering similar legislation, with six of these bills (Alabama, Kentucky, Louisiana, Missouri, Pennsylvania, and Wisconsin) limiting speech in institutions of higher learning. Thankfully, none of these bills prohibits research on these topics, and so music cognition researchers who wish to engage with and publish these ideas in their research may continue to do so. But, many of the bills would prohibit this research from trickling down into coursework. As mentioned above and as reflected in the content of the Future Directions conference, many members of the SMPC community and others have begun examining racial justice issues, and these new state regulations could have a dampening effect on what is a burgeoning area of research. Beyond just this topic, though, any state restrictions on what can be taught sets a concerning precedent.

There are also broader cultural and institutional pressures limiting how academics communicate about research, especially with the general public. Rider and Peters (2018: 5) describe what they call a "post-truth" society in which traditional cultural reference points 
that once built shared meaning break down, such as the role universities once had as "truth tellers." In a posttruth society, individuals' conceptualizations of facts and evidence become politically and socially reconstructed, opening the possibility for the alt-right, neoliberalism, and illiberalism to take on a greater role as society's gatekeepers definers of truth. Oleksiyenko and Jackson (2021: 1058) have highlighted the way in which these movements have taken advantage of posttruth contexts to "pervert notions of freedom of speech, making universities battlefields of politicized emotions and expressions," using academic freedom as cover for new types of institutional proselytizing. An increasingly common response from legacy "truth teller" institutions to the fracturing of culturally-shared meaning is to restrict freedom of speech - according to Gibbs (2019), academic freedom has never been challenged as much as it is today. These overreactions against problematic speech in academia have led to broader "decline and corruptions of democratic free speech and academic freedom, or the forces to defend them" (Oleksiyenko and Jackson, 2021: 1058).

Under the circumstances, what is a scholar wishing to participate in Loui's (pp. 25-31) vision of communicating practical research to the public to do? Complicating issues even further are rapidly shifting norms of what is deemed appropriate public scientific discourse, especially in the realm of social media. The rigidity with which posts and users are banned from communicating about what in reality are still open scientific questions implies a scientific certainty that just does not exist in most cases. While much of the focus in the past year has rightly been on scientific misinformation surrounding COVID, other social and cultural issues have become flashpoints for public campaigns to officially sanction or even fire professors, and a university faculty's social media post that may be culturally acceptable when published could later become the impetus for public outcry. This problem is consistent with Gibbs's concept of an "erosion of trust [in universities] in a context where free speech interacts with social media and all are subject to the force of the transient present" (Gibbs, 2019: 502).

An atmosphere in which public opinion can shift so quickly and have such deep impacts on researchers can be particularly chilling for healthy academic discourse. To take one example, Väliverronen and Saikkonen (2021) documented the effect that the rise of the rightwing populist Finns Party has had on self-censorship among Finnish researchers. 881 surveys were collected in 2015, 2017, and 2019, during the period when the Finns Party first attained political power and then lost it again but remained publicly popular. Over this time, they documented strong concerns about "anti-science sentiment" among researchers, coming not only from the government, but also from "online hate, aggressive feedback, and politically motivated disparagement of scientists and experts" (Väliverronen and Saikkonen, 2021: 8). The high levels of self-censorship that often result from a culture of normalized aggressive attacks, whether online or not and coming from any political position, deeply undermines healthy scientific discourse and disincentivizes researchers from communicating with the public for fear of the negative consequences to their career and reputation.

Touching on these important but difficult issues in his article, David Huron (p. 13) applauds the intentions of the growing number of researchers who consider themselves activists to change the world for the better. However, he warns about the inherent danger this strategy results in of rushing to moral judgments of others as bad people rather than fairly and empirically testing the effectiveness of those ideas. Huron suggests that public musicologists who are trained as social activists but lack sound methodological training are more likely to fail in their efforts to transform society for the good.

Research flourishes in environments in which scholars are free to pursue whatever lines of research seem most pressing and to follow the evidence wherever it leads. Although it can sometimes be uncomfortable for voices with minority viewpoints to be heard, the alternative is to suppress the ideas of those with unpopular perspectives. As mentioned above, this is one of the very mistakes academia committed in the bad old days in which racism explicitly suppressed the speech of those not in power. When an individual's ability to speak in an academic discipline is dependent on how much power they have, academia trades in its lofty mission of pursuing knowledge wherever it leads for the much more impoverished mission of simply reinforcing popular, well-loved theories or the narratives of those already in power. A culture in which fear of reprisal prevents researchers from honestly engaging in controversial research or publishing unpopular results decimates healthy dialogue and undermines the scholar's ability to serve society. As the field of music cognition moves forward into the future, how it navigates the polarizing tensions pent up in these cultural and societal forces will not only influence the degree to which healthy 
dialogue will be fostered or hampered but also the field's ability to transform society for the good and benefit real people.

\section{Conclusion}

The Future Directions of Music Cognition conference and speaker series brought together hundreds of scholars from all over the world to hear about new research, learn about the most currently influential methods and approaches from several subfields, and to foster healthy conversations about the state of field in light of some of the issues that have recently become important. This sort of endeavor-scholars coming together to think and dream about the future of the field - is exceptionally important, especially in difficult times. The decentralized, international, interdisciplinary, collaborative, and methodologically and ideologically diverse nature of efforts like this conference and speaker series underscore the richness and diversity of music cognition, and the promise of the future of the field. Charting future directions of music cognition in turbulent times is fraught with many difficulties, but one of the lessons of this conference is that the effort is better met together in community.

\section{Acknowledgements}

I am deeply grateful to the conference participants, speaker series presenters, session moderators, and submission reviewers whose contributions comprise this whole endeavor. Lindsay Warrenburg and Lindsey Reymore deserve the most credit for the conference, serving as hosts and who were the original impetus for the conference. Many thanks to Daniel Shanahan for supervising the entire endeavor and providing his expertise in making this possible. I also want to thank the Ohio State University library and Knowledge Bank staff for all the work involved in publishing these proceedings. Deep gratitude to the team at the Open Science Framework for hosting conference presentations and supplemental materials for the indefinite future. Finally, we thank our generous sponsors, the Ohio State Energy Partners and Ohio State's Center for Cognitive and Brain Sciences for funding the conference and speaker series.

\section{References}

Baker, D.J, Belfi, A., Creel, S., Grahn, J., Hannon, E., Loui, P, Margulis, E.H., Schachner, A., Schutz, M., Shanahan, D., \& Vuvan, D. (2020). Embracing antiracist practices in the music perception and cognition community. Music Perception: An Interdisciplinary Journal 38(2): 103-105. https://doi.org/10.1525/mp.2020.38.2.103

Crowd Counting Consortium. (2021, September 9). CCC Data Dashboard; Civil Rights, Criminal Justice, Policing, and Racism protests in the US from May 26, 2020 through May 26, 2021. https://sites.google.com/view/crowdcountingconsortium/ home.

Gather Town. (2021). Town Hall on Anti-Racism in Music Cognition.

https://gather.town/app/BYatfy3d9rD3cwA0/FD_antirac ismTH

Gibbs, P. (2019). Why academics should have a duty of truth telling in an epoch of post-truth? Higher Education, 78, 501-510. https://doi.org/10.1007/s10734$\underline{018-0354-\mathrm{y}}$

House Bill 1775. (2001). An Act. http://webserver1.lsb.state.ok.us/cf pdf/202122\%20ENR/hB/HB1775\%20ENR.PDF

London, J. (2021). Music Theory as Junk Science. Open Science https://doi.org/10.17605/OSF.IO/Y5W2Z

Legislature of the State of Idaho. (2001). House Bill 377. https://legislature.idaho.gov/wpcontent/uploads/sessioninfo/2021/legislation/H0377.pdf

OECD. (2021). OECD Science, Technology and Innovation Outlook 2021: Times of Crisis and Opportunity. Paris: OECD Publishing. https://doi.org/10.1787/75f79015-en

Oleksiyenko, A.V. \& Jackson, L. (2021). Freedom of speech, freedom to teach, freedom to learn: The crisis of higher education in the post-truth era. Educational Philosophy and Theory, 53(11), 1057-1062. https://doi.org/10.1080/00131857.2020.1773800

Rider, S. \& Peters M.A. (2018). Post-truth, fake news: Viral modernity and higher education. In M.A. Peters, S. Rider, M. Hyvönen, \& Tina Besley (Eds.), Post-Truth, Fake News: Viral Modernity \& Higher Education (pp. 3-12). Singapore: Springer. https://doi.org/10.1007/978981-10-8013-5 1

Shanahan, D. (2021). What the history of computational musicology can tell us about the future of corpus studies. Open Science Framework. https://doi.org/10.17605/OSF.IO/CB26R

The Iowa Legislature. (2021, June 8). House File 802. https://www.legis.iowa.gov/legislation/BillBook?ga=89 $\& \mathrm{ba}=\mathrm{HF} 802$ 
Väliverronen, E. \& Saikkonen, S. (2021). Freedom of expression challenged: Scientists' perspectives on hidden forms of suppression and self-censorship. Science, Technology, \& Human Values, 46(6): 11721200. https://doi.org/10.1177/0162243920978303

Vuoskoski, J. (2021). From compassion to being moved: Social emotions evoked by music. Open Science Framework. https://osf.io/sb4hv/

Wallmark, Z. (2021). Future Directions presentation_Zachary Wallmark. Open Science Framework. https://doi.org/10.17605/OSF.IO/9MGHN

Watson, P. (2001). The Modern Mind: An intellectual history of the $20^{\text {th }}$ century. New York, NY: HarperCollins Publishers. 
Thank you to our sponsors!

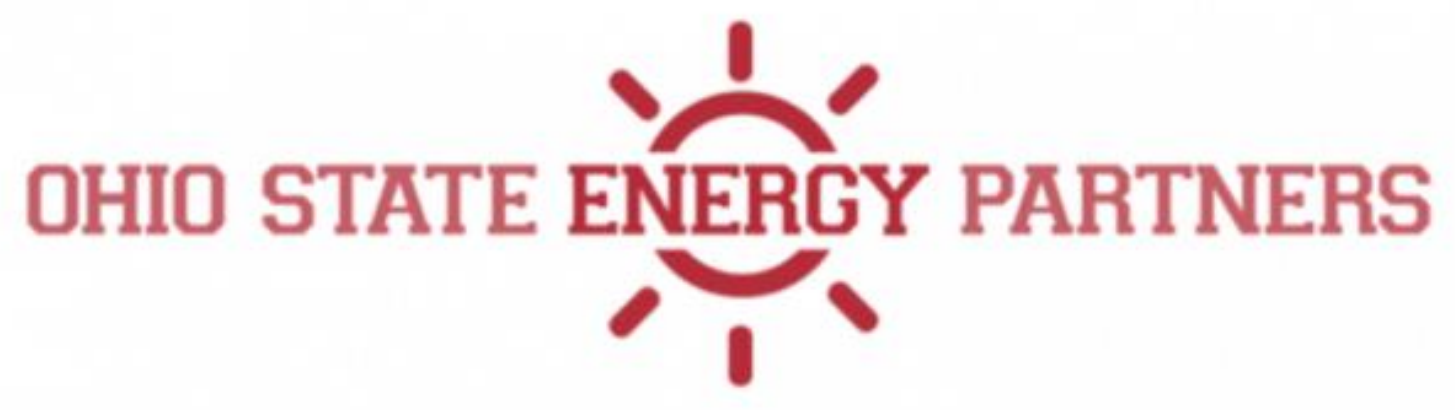

CENTER FOR COGNITIVE AND BRAIN SCIENCES 


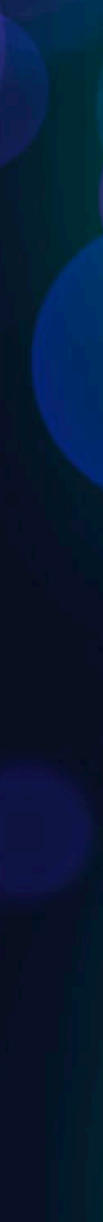

